Supporting Information for:

\title{
Asymmetric Catalysis with Organic Azides and Diazo Compounds Initiated by Photoinduced Electron Transfer
}

Xiaoqiang Huang, Richard D. Webster, Klaus Harms, and Eric Meggers*

*meggers@chemie.uni-marburg.de

1. General Information .....................................................................................................................52

2. Synthesis of Substrates..................................................................................................................33

3. General Procedure .......................................................................................................................57

4. Screening of Reaction Conditions ............................................................................................58

5. Mechanistic Studies.......................................................................................................................S11

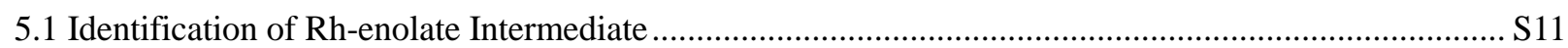

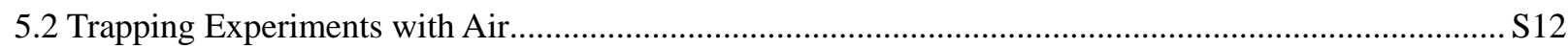

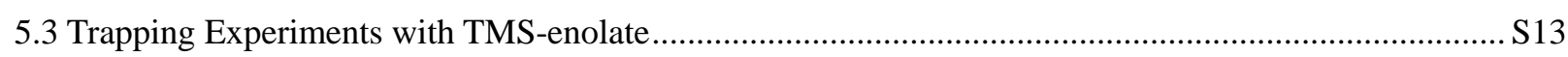

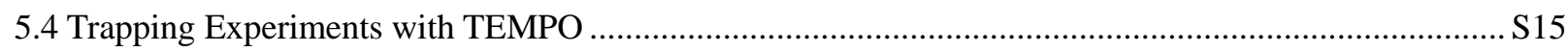

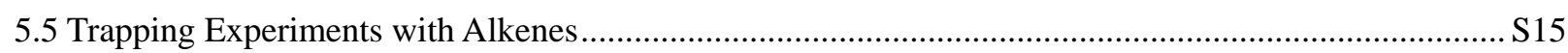

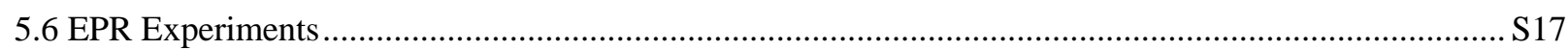

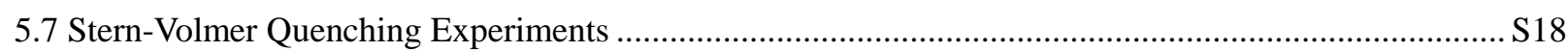

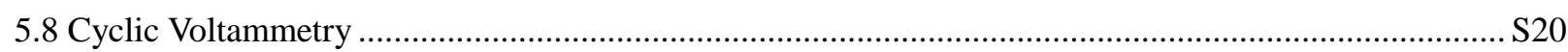

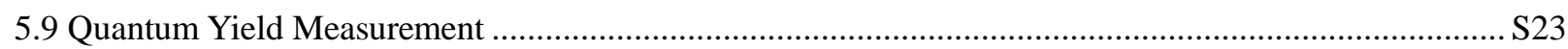

6. Experimental and Characterization Data of Products ......................................................S25

7. Removal of Directing Group ..........................................................................................................556

8. Enantioselectivities as Determined by Chiral HPLC ............................................................557

9. Single-Crystal X-Ray Diffraction Studies ............................................................................593

10. NMR Spectra of New Compounds ...................................................................................598

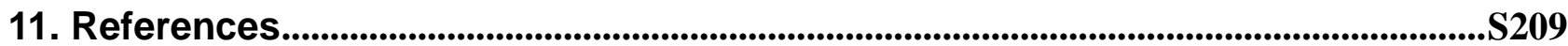




\section{General Information}

All reactions were carried out under an atmosphere of nitrogen with magnetic stirring. Catalytic reactions were performed in a Schlenk tube $(10 \mathrm{~mL})$. A $21 \mathrm{~W}$ compact fluorescent lamp (CFL) served as light source. The catalysts $\Delta-\mathbf{I r S},{ }^{1} \Delta-\mathbf{R h O},{ }^{2}$ and $\Delta / \Lambda-\mathbf{R h S}^{3}$ were synthesized according to our published procedures. Solvents were distilled under nitrogen from calcium hydride $\left(\mathrm{CH}_{3} \mathrm{CN}, \mathrm{CH}_{2} \mathrm{Cl}_{2}\right)$, sodium/benzophenone (THF). HPLC grade of acetone was used without further purification. Dry DMSO was bought from Sigma-Aldrich. Reagents that were purchased from commercial suppliers were used without further purification. Flash column chromatography was performed with silica gel $60 \mathrm{M}$ from Macherey-Nagel (irregular shaped, 230-400 mesh, $\mathrm{pH} 6.8$, pore volume: $0.81 \mathrm{~mL} \times \mathrm{g}^{-1}$, mean pore size: $66 \AA$, specific surface: $492 \mathrm{~m}^{2} \times \mathrm{g}^{-1}$, particle size distribution: $0.5 \%<25 \mu \mathrm{m}$ and $1.7 \%>71 \mu \mathrm{m}$, water content: $1.6 \%) .{ }^{1} \mathrm{H} \mathrm{NMR},{ }^{19} \mathrm{~F}$ NMR and proton decoupled ${ }^{13} \mathrm{C}$ NMR spectra were recorded on Bruker Avance 300 (300 MHz), or Bruker AM (500 MHz) spectrometers at ambient temperature. NMR standards were used as follows: ${ }^{1} \mathrm{H}$ NMR spectroscopy: $\delta=7.26 \mathrm{ppm}\left(\mathrm{CDCl}_{3}\right)$. ${ }^{19} \mathrm{~F} \mathrm{NMR}$ spectroscopy: $\delta=0 \mathrm{ppm}\left(\mathrm{CFCl}_{3}\right) .{ }^{13} \mathrm{C}$ NMR spectroscopy: $\delta=77.0 \mathrm{ppm}\left(\mathrm{CDCl}_{3}\right)$. IR spectra were recorded on a Bruker Alpha FT-IR spectrophotometer. High-resolution mass spectra were recorded on a Bruker En Apex Ultra 7.0 TFT-MS instrument using ESI/EI technique. Chiral HPLC chromatography was performed with an Agilent 1200, Agilent 1260 HPLC system or Shimadzu Lc2030c HPLC system. Optical rotations were measured on a Krüss P8000-T polarimeter with $[\alpha]_{D}^{22}$ values reported in degrees with concentrations reported in $\mathrm{g} / 100 \mathrm{~mL}$. The EPR spectrometer is from Bruker (model esp300), with a modified Varian rectangular X-band cavity and the modulation frequency was set to $100 \mathrm{kHz}$, the modulation amplitude was $0.1 \mathrm{mT}$. Luminescence quenching experiments were recorded on a Spectra Max M5 microplate reader in a $10.0 \mathrm{~mm}$ quartz cuvette. 


\section{Synthesis of Substrates}

2-Acyl imidazoles 1 were synthesized according to our recently published procedures. ${ }^{4}$ The experimental data of $\mathbf{1 a - b}, \mathbf{1 e - q}$ are in accord with our previous reports. ${ }^{4}$ The data of $\mathbf{1 c - d}$ are shown below.<smiles>COc1ccc(-n2ccnc2C(=O)Cc2ccccc2)cc1</smiles>

\section{1-(1-(4-Methoxyphenyl)-1H-imidazol-2-yl)-2-phenylethan-1-one (1c)}

A white solid.

${ }^{1} \mathrm{H}$ NMR (300 MHz, $\left.\mathrm{CDCl}_{3}\right) \delta$ 7.35-7.18 (m, 6H), 7.18-7.10 (m, 3H), 6.94-6.87 (m, 2H), $4.44(\mathrm{~s}, 2 \mathrm{H})$, $3.82(\mathrm{~s}, 3 \mathrm{H})$.

${ }^{13} \mathrm{C} \mathrm{NMR}\left(75 \mathrm{MHz}, \mathrm{CDCl}_{3}\right) \delta 188.5,159.7,142.9,134.5,131.1,129.9,129.5,128.4,127.7,126.9$, $126.7,114.1,55.5,45.6$.

IR (film): $v\left(\mathrm{~cm}^{-1}\right)$ 3105, 3030, 2914, 1685, 1592, 1494, 1452, 1390, 1340, 1307, 1208, 1147, 1079, 1023, 991, 958, 912, 887, 840, 789, 761, 721, 696, 590, 542, 512, 480, 454.

HRMS (ESI, $m / z$ ) calcd for $\mathrm{C}_{18} \mathrm{H}_{17} \mathrm{~N}_{2} \mathrm{O}_{2}[\mathrm{M}+\mathrm{H}]^{+}:$293.1285, found: 293.1286.<smiles>O=C(Cc1ccccc1)c1nccn1-c1ccc(F)cc1</smiles>

1-(1-(4-Fluorophenyl)-1H-imidazol-2-yl)-2-phenylethan-1-one (1d)

A white solid.

${ }^{1} \mathrm{H}$ NMR $\left(300 \mathrm{MHz}, \mathrm{CDCl}_{3}\right) \delta$ 7.37-7.17 (m, 9H), 7.16-7.08 (m, 2H), 4.48 (s, 2H).

${ }^{13} \mathrm{C}$ NMR $\left(75 \mathrm{MHz}, \mathrm{CDCl}_{3}\right) \delta 188.6,162.5(\mathrm{~d}, J=247.5 \mathrm{~Hz}), 142.9,134.3,134.2,129.91,129.86$, 128.5, $127.7(\mathrm{~d}, J=8.8 \mathrm{~Hz}), 127.4,126.8,115.9(\mathrm{~d}, J=23.0 \mathrm{~Hz}), 45.6$.

IR (film): $v\left(\mathrm{~cm}^{-1}\right)$ 3159, 3077, 1683, 1508, 1449, 1399, 1219, 1147, 1027, 962, 911, 842, 793, 727, $701,623,537$. 
HRMS (EI, $m / z$ ) calcd for $\mathrm{C}_{17} \mathrm{H}_{13} \mathrm{FN}_{2} \mathrm{O}[\mathrm{M}]^{+}:$280.1012, found: 280.1004 .

Azides $\mathbf{2} \mathbf{a}-\mathbf{b}^{5}, \mathbf{2} \mathbf{d}^{5}, \mathbf{2} \mathbf{e}^{6}$ were synthesized according to reported procedures. The experimental data are in accord with the literatures. The synthesis of $\mathbf{2 c}, \mathbf{2 f}, \mathbf{2 g}$, and $\mathbf{2 h}$ are shown below.<smiles>CCOC(=O)c1c(F)c(F)c(N)c(F)c1F</smiles>

\section{Ethyl 4-azido-2,3,5,6-tetrafluorobenzoate (2c)}

2c was synthesized by SNAr from corresponding ethyl 2,3,4,5,6-pentafluorobenzoate according to literature $^{7}$ as a yellow liquid.

${ }^{1} \mathrm{H}$ NMR $\left(500 \mathrm{MHz}, \mathrm{CDCl}_{3}\right) \delta 4.43(\mathrm{q}, J=7.0 \mathrm{~Hz}, 2 \mathrm{H}), 1.39(\mathrm{t}, J=7.3 \mathrm{~Hz}, 3 \mathrm{H})$.

${ }^{13} \mathrm{C}$ NMR (125 MHz, $\left.\mathrm{CDCl}_{3}\right) \delta$ 159.3, 146.4-146.1 (m), 144.3-144.0 (m), 141.6-141.3 (m), 139.6$139.3(\mathrm{~m}), 123.2-123.0(\mathrm{~m}), 108.3-107.9(\mathrm{~m}), 62.7,14.0$.

${ }^{19} \mathrm{~F}$ NMR (282 MHz, $\left.\mathrm{CDCl}_{3}\right) \delta-139.41--139.57$ (m, 2F), -151.45 - -151.60 (m, 2F).

IR (film): $v\left(\mathrm{~cm}^{-1}\right)$ 2990, 2170, 2126, 1733, 1645, 1484, 1419, 1368, 1325, 1250, 1201, 993, 915, 864, 748.

HRMS (ESI, $m / z)$ calcd for $\mathrm{C}_{9} \mathrm{H}_{5} \mathrm{~F}_{4} \mathrm{~N}_{3} \mathrm{O}_{2} \mathrm{Na}[\mathrm{M}+\mathrm{Na}]^{+}:$286.0210, found: 286.0210 .<smiles>N#Cc1cc(N)ccc1C(F)(F)F</smiles>

\section{5-Azido-2-(trifluoromethyl)benzonitrile (2f)}

2f was synthesized by diazotization-azidation from corresponding aniline according to literature ${ }^{5}$ as a grey solid.

${ }^{1} \mathrm{H}$ NMR $\left(300 \mathrm{MHz}, \mathrm{CDCl}_{3}\right) \delta 7.82(\mathrm{~d}, J=8.4 \mathrm{~Hz}, 1 \mathrm{H}), 7.38(\mathrm{~d}, J=2.1 \mathrm{~Hz}, 1 \mathrm{H}), 7.30\left(\mathrm{dd}, J_{1}=8.1\right.$ $\left.\mathrm{Hz}, J_{2}=2.1 \mathrm{~Hz}, 1 \mathrm{H}\right)$.

${ }^{13} \mathrm{C}$ NMR $\left(75 \mathrm{MHz}, \mathrm{CDCl}_{3}\right) \delta 145.6,136.3,135.3(\mathrm{q}, J=33.4 \mathrm{~Hz}), 122.3,121.8(\mathrm{q}, J=272.6 \mathrm{~Hz})$, $117.7(\mathrm{q}, J=5.2 \mathrm{~Hz}), 115.0,105.7(\mathrm{q}, J=1.7 \mathrm{~Hz})$.

IR (film): $v\left(\mathrm{~cm}^{-1}\right)$ 2387, 2226, 2113, 1606, 1493, 1432, 1314, 1262, 1178, 1124, 1044, 905, 839, 733, $675,634,548$.

HRMS (ESI, $m / z$ ) calcd for $\mathrm{C}_{8} \mathrm{H}_{3} \mathrm{~F}_{3} \mathrm{~N}_{4} \mathrm{Na}[\mathrm{M}+\mathrm{Na}]^{+}:$235.0202, found: 235.0202 . 
<smiles>N#Cc1ccc(N)cc1C#N</smiles>

\section{4-Azidophthalonitrile (2g)}

$\mathbf{2 g}$ was synthesized by diazotization-azidation from corresponding aniline according to literature ${ }^{5}$ as a grey solid.

${ }^{1} \mathrm{H}$ NMR $\left(300 \mathrm{MHz}, \mathrm{CDCl}_{3}\right) \delta 7.79(\mathrm{~d}, J=8.4 \mathrm{~Hz}, 1 \mathrm{H}), 7.40(\mathrm{~d}, J=1.8 \mathrm{~Hz}, 1 \mathrm{H}), 7.35\left(\mathrm{dd}, J_{l}=8.1\right.$ $\left.\mathrm{Hz}, J_{2}=2.1 \mathrm{~Hz}, 1 \mathrm{H}\right)$.

${ }^{13} \mathrm{C} \mathrm{NMR}\left(75 \mathrm{MHz}, \mathrm{CDCl}_{3}\right) \delta 146.0,135.1,123.8,123.3,117.7,115.0,114.5,111.3$.

IR (film): $v\left(\mathrm{~cm}^{-1}\right)$ 2233, 2122, 1764, 1589, 1558, 1483, 1413, 1306, 1263, 1212, 1173, 1131, 890, $843,753,605,521$.

HRMS (ESI, $m / z$ ) calcd for $\mathrm{C}_{8} \mathrm{H}_{3} \mathrm{~N}_{5} \mathrm{Na}[\mathrm{M}+\mathrm{Na}]^{+}:$192.0281, found: 192.0281 .<smiles>Nc1c(F)c(F)c(C(N)(F)F)c(F)c1F</smiles>

\section{1-(Azidodifluoromethyl)-2,3,4,5,6-pentafluorobenzene (2h)}

2h was synthesized by SN from corresponding perfluoro benzylic iodide according to literature ${ }^{8}$ as a yellow liquid.

${ }^{19} \mathrm{~F}$ NMR $\left(282 \mathrm{MHz}, \mathrm{CDCl}_{3}\right) \delta-64.19--64.92(\mathrm{~m}, 2 \mathrm{~F}),-139.56--140.22(\mathrm{~m}, 2 \mathrm{~F}),-149.90--$ $150.93(\mathrm{~m}, 2 \mathrm{~F})$.

IR (film): $v\left(\mathrm{~cm}^{-1}\right)$ 2126, 1651, 1490, 1426, 1334, 1250, 1164, 1054, 999, 975, 848, 782.

Diazo compounds 3 were synthesized according to reported literature. ${ }^{9}$ The experimental data of $\mathbf{3 c}, \mathbf{3 e - h}$ are in accord with the literatures. ${ }^{9}$ The data of $\mathbf{3 b}$ and $\mathbf{3 d}$ are shown below.<smiles>N#CC(=O)OCCCN1C(=O)c2ccccc2C1=O</smiles>

3-(1,3-Dioxoisoindolin-2-yl)propyl 2-diazoacetate (3b)

A yellow oil. 
${ }^{1} \mathrm{H}$ NMR $\left(300 \mathrm{MHz}, \mathrm{CDCl}_{3}\right) \delta$ 7.88-7.81 (m, 2H), 7.75-7.68 (m, 2H), $4.69(\mathrm{~s}, 1 \mathrm{H}), 4.21(\mathrm{t}, J=6.2 \mathrm{~Hz}$, 2H), $3.80(\mathrm{t}, J=6.9 \mathrm{~Hz}, 2 \mathrm{H}), 2.11-2.00(\mathrm{~m}, 2 \mathrm{H})$.

${ }^{13} \mathrm{C}$ NMR $\left(125 \mathrm{MHz}, \mathrm{CDCl}_{3}\right) \delta 168.2,134.0,132.0,123.3,62.2,46.2,34.9,27.7$. (Missing one ${ }^{13} \mathrm{C}$ signal)

IR (film): $v\left(\mathrm{~cm}^{-1}\right)$ 3104, 2955, 2110, 1771, 1703, 1616, 1464, 1441, 1395, 1362, 1240, 1184, 1048, $986,899,794,718,526,501$.

HRMS (ESI, $m / z$ ) calcd for $\mathrm{C}_{13} \mathrm{H}_{11} \mathrm{~N}_{3} \mathrm{O}_{4} \mathrm{Na}[\mathrm{M}+\mathrm{Na}]^{+}$: 296.0642, found: 296.0642 .

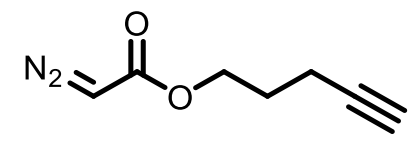

\section{Pent-4-yn-1-yl 2-diazoacetate (3d)}

A yellow liquid.

${ }^{1} \mathrm{H} \mathrm{NMR}\left(300 \mathrm{MHz}, \mathrm{CDCl}_{3}\right) \delta 4.73(\mathrm{~s}, 1 \mathrm{H}), 4.25(\mathrm{t}, J=6.2 \mathrm{~Hz}, 2 \mathrm{H}), 2.27\left(\mathrm{td}, J_{1}=7.2 \mathrm{~Hz}, J_{2}=2.7\right.$ $\mathrm{Hz}, 2 \mathrm{H}), 1.96(\mathrm{t}, J=2.7 \mathrm{~Hz}, 1 \mathrm{H}), 1.91-1.82(\mathrm{~m}, 2 \mathrm{H})$.

${ }^{13} \mathrm{C}$ NMR $\left(75 \mathrm{MHz}, \mathrm{CDCl}_{3}\right) \delta 166.6,82.8,69.0,63.3,46.0,27.7,15.1$.

IR (film): $v\left(\mathrm{~cm}^{-1}\right)$ 3296, 3114, 2961, 2107, 1682, 1443, 1396, 1359, 1296, 1239, 1179, 1086, 1035, $992,738,635,556,479$.

HRMS (ESI, $m / z$ ) calcd for $\mathrm{C}_{7} \mathrm{H}_{8} \mathrm{~N}_{2} \mathrm{O}_{2} \mathrm{Na}[\mathrm{M}+\mathrm{Na}]^{+}$: 175.0478 , found: 175.0478 . 


\section{General Procedure}

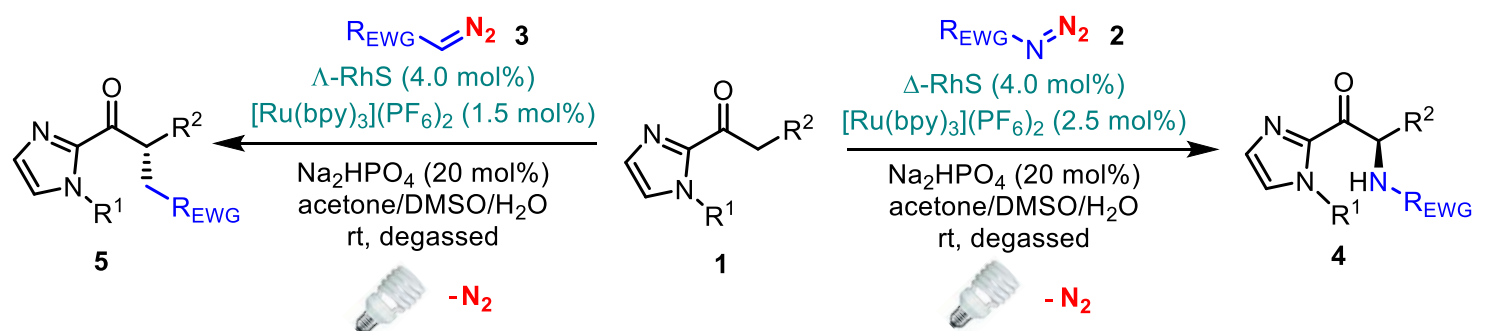

A dried $10 \mathrm{~mL}$ Schlenk tube was charged with 2-acyl imidazole $\mathbf{1}(0.10 \mathrm{mmol}), \Delta / \Lambda$ - RhS $(3.5 \mathrm{mg}, 4$ mol\%), $\left[\mathrm{Ru}(\mathrm{bpy})_{3}\right]\left(\mathrm{PF}_{6}\right)_{2}(2.2 \mathrm{mg}, 2.5 \mathrm{~mol} \%$ or $1.3 \mathrm{mg}, 1.5 \mathrm{~mol} \%)$ and $\mathrm{Na}_{2} \mathrm{HPO}_{4}(2.8 \mathrm{mg}, 20 \mathrm{~mol} \%)$. The tube was purged with nitrogen. Then acetone/DMSO $(9: 1,0.5 \mathrm{~mL}, 0.2 \mathrm{M})$ was added via syringe, followed by $\mathrm{H}_{2} \mathrm{O}$ (36.0 mg, 20 equiv). Azide 2 or diazo compound $\mathbf{3}$ (3.0 equiv) was added under nitrogen atmosphere with stirring. The reaction mixture was degassed via freeze-pump-thaw for three cycles. After the mixture was thoroughly degassed, the vial was sealed and positioned approximately $5 \mathrm{~cm}$ from a $21 \mathrm{~W}$ compact fluorescent lamp. The reaction was stirred at room temperature for the indicated time (monitored by TLC) under nitrogen atmosphere. Afterwards, the mixture was diluted with $\mathrm{CH}_{2} \mathrm{Cl}_{2}$. The combined organic layers were concentrated under reduced pressure. The residue was purified by flash chromatography on silica gel ( $n$-hexane/EtOAc) to afford the products $\mathbf{4}$ or $\mathbf{5}$. Racemic samples were obtained by carrying out the reactions with $r a c-\mathbf{R h S}$. The enantiomeric excess was determined by chiral HPLC analysis. 


\section{Screening of Reaction Conditions}

Table S1. Effect of Solvent Ratio ${ }^{a}$<smiles>O=C(Cc1ccccc1)c1nccn1-c1ccccc1</smiles>

1a<smiles>Nc1c(F)c(F)c(F)c(F)c1F</smiles>

$2 a$

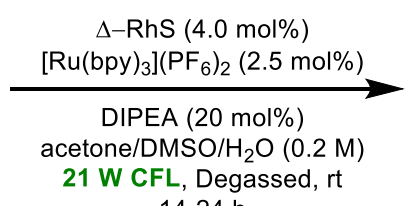

$14-24 \mathrm{~h}$

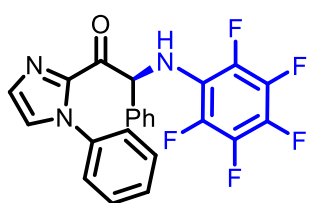

4aa

\begin{tabular}{lccc}
\hline entry & acetone/DMSO & $\mathrm{H}_{2} \mathrm{O}$ (equiv) & result \\
\hline 1 & $4: 1$ & 0 & $61 \%$ yield; $97.1 \%$ ee \\
2 & $4: 1$ & 5 & $64 \%$ yield; $97.7 \%$ ee \\
3 & $4: 1$ & 20 & $70 \%$ yield; $97.7 \%$ ee \\
4 & $4: 1$ & 50 & $68 \%$ yield; $97.9 \%$ ee \\
5 & $1: 0$ & 20 & $65 \%$ yield; $96.9 \%$ ee \\
6 & $9: 1$ & 20 & $77 \%$ yield; $98.1 \%$ ee \\
7 & $1: 1$ & 20 & $71 \%$ yield; $96.0 \%$ ee \\
\hline
\end{tabular}

${ }^{a}$ Reaction conditions: $\mathbf{1 a}(0.10 \mathrm{mmol}), \mathbf{2 a}(0.30 \mathrm{mmol}), \Delta-\mathbf{R h S}(4.0 \mathrm{~mol} \%),\left[\mathrm{Ru}(\mathrm{bpy})_{3}\right]\left(\mathrm{PF}_{6}\right)_{2}(2.5 \mathrm{~mol} \%)$, DIPEA (20 mol\%) and $\mathrm{H}_{2} \mathrm{O}$ in acetone/DMSO $(0.2 \mathrm{M})$ were stirred at room temperature for $14-24 \mathrm{~h}$ with visible light.

Table S2. Effect of Photoredox Catalysts ${ }^{a}$<smiles>O=C(Cc1ccccc1)c1nccn1-c1ccccc1</smiles>

1a<smiles>Nc1c(F)c(F)c(F)c(F)c1F</smiles>

2a

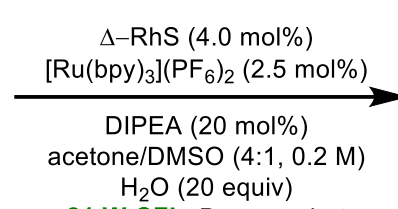

$\mathrm{H}_{2} \mathrm{O}$ (20 equiv)

21 W CFL, Degassed, rt 14-24 h

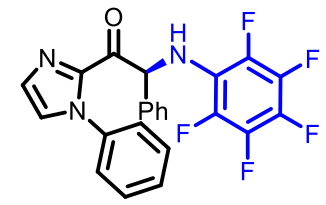

4aa

\begin{tabular}{lll}
\hline entry & photoredox catalyst & result \\
\hline 1 & {$\left[\mathrm{Ru}(\mathrm{bpy})_{3}\right]\left(\mathrm{PF}_{6}\right)_{2}$} & $70 \%$ yield; $97.7 \%$ ee \\
2 & {$\left[\operatorname{lr}(\mathrm{ppy})_{2}(\mathrm{dtbbpy})\right]\left(\mathrm{PF}_{6}\right)$} & $62 \%$ yield; $97.8 \%$ ee \\
3 & fac-lr(ppy $)_{3}$ & $37 \%$ yield; $89 \%$ ee \\
$4^{b}$ & $\Delta-\mathrm{IrS}$ & $0 \%$ yield \\
\hline
\end{tabular}

${ }^{a}$ Reaction conditions: $\mathbf{1 a}(0.10 \mathrm{mmol}), \mathbf{2 a}(0.30 \mathrm{mmol}), \Delta-\mathbf{R h S}(4.0 \mathrm{~mol} \%)$, photoredox catalyst $(2.5 \mathrm{~mol} \%)$, DIPEA (20 mol\%) and $\mathrm{H}_{2} \mathrm{O}$ in acetone/DMSO $(0.2 \mathrm{M})$ were stirred at room temperature for 14-24 h with visible light. ${ }^{b}$ Without RhS. 
Table S3. Effect of the Amount of Azide ${ }^{a}$

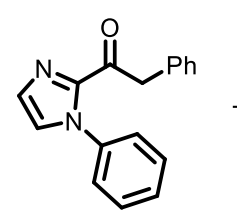

1a

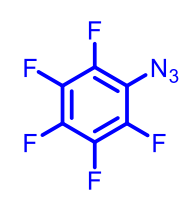

$2 a$

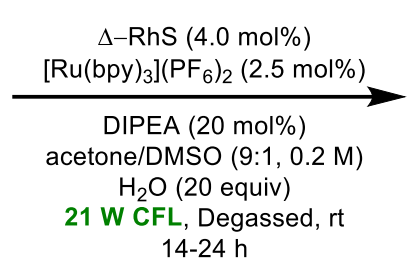

14-24 h

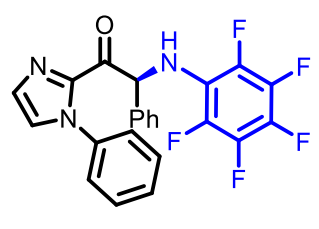

4 aa

\begin{tabular}{lll}
\hline entry & 2a (equiv) & result \\
\hline 1 & 1.2 & $51 \%$ yield; $97.8 \%$ ee \\
2 & 2.0 & $65 \%$ yield; $97.9 \%$ ee \\
3 & 3.0 & $\mathbf{7 7 \%}$ yield; $98.1 \%$ ee
\end{tabular}

${ }^{a}$ Reaction conditions: 1a $(0.10 \mathrm{mmol}), \mathbf{2 a}, \Delta-\mathbf{R h S}(4.0 \mathrm{~mol} \%),\left[\mathrm{Ru}(\mathrm{bpy})_{3}\right]\left(\mathrm{PF}_{6}\right)_{2}(2.5 \mathrm{~mol} \%)$, DIPEA $(20 \mathrm{~mol} \%)$ and $\mathrm{H}_{2} \mathrm{O}$ (20 equiv) in acetone/DMSO (9:1, 0.2 M) were stirred at room temperature for 14-24 h with visible light.

Table S4. Effect of Different Bases ${ }^{a}$<smiles>O=C(Cc1ccccc1)c1nccn1-c1ccccc1</smiles>

1a<smiles>Nc1c(F)c(F)c(F)c(F)c1F</smiles>

$2 a$

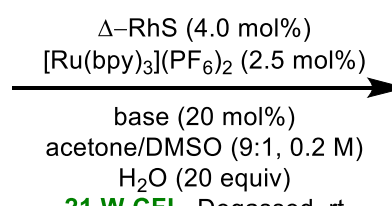

$\mathrm{H}_{2} \mathrm{O}$ (20 equiv)

21 W CFL, Degassed, rt $14-24 \mathrm{~h}$

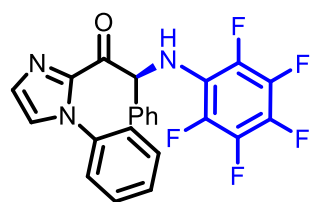

4 aa

\begin{tabular}{lll}
\hline entry & base $(20$ mol\%) & result \\
\hline 1 & DIPEA & $77 \%$ yield; $98.1 \%$ ee \\
2 & 2,6 -lutidine & $73 \%$ yield; $98.0 \%$ ee \\
3 & $\mathrm{Na}_{2} \mathrm{HPO}_{4}$ & $\mathbf{8 2} \%$ yield; $\mathbf{9 8 . 4} \%$ ee \\
$4^{b}$ & $\mathrm{NaOAC}$ & $<10 \%$ yield \\
$5^{c}$ & $\mathrm{Na}_{2} \mathrm{CO}_{3}$ & $<5 \%$ \\
$6^{c}$ & $\mathrm{~K}_{3} \mathrm{PO}_{4}$ & $0 \%$ \\
\hline
\end{tabular}

${ }^{a}$ Reaction conditions: 1a $(0.10 \mathrm{mmol}), \mathbf{2 a}(0.30 \mathrm{mmol}), \Delta-\mathbf{R h S}(4.0 \mathrm{~mol} \%),\left[\mathrm{Ru}(\mathrm{bpy})_{3}\right]\left(\mathrm{PF}_{6}\right)_{2}(2.5 \mathrm{~mol} \%)$, base $(20$ mol\%) and $\mathrm{H}_{2} \mathrm{O}$ (20 equiv) in acetone/DMSO (9:1, 0.2 M) were stirred at room temperature for 14-24 $\mathrm{h}$ with visible light. ${ }^{b}$ Low conversion. ${ }^{c}$ Full conversion with the decomposition of $\mathbf{4 a a}$. 
Table S5. Control Experiments of Alkylation ${ }^{a}$

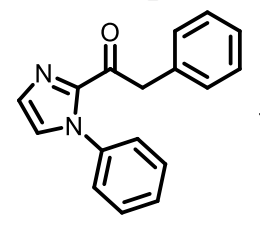

$1 \mathrm{a}$

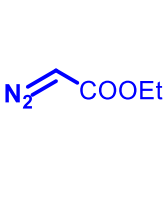

$3 a$

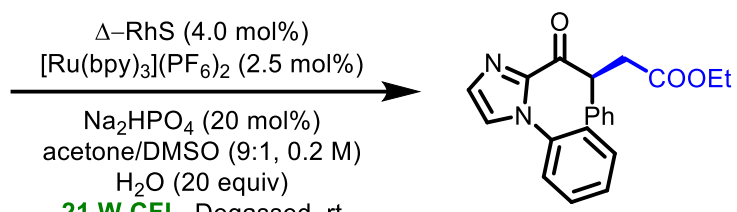

21 W CFL, Degassed, r

\begin{tabular}{cll}
\hline entry & variation & results \\
\hline 1 & none & $6 \mathrm{~h}, 93 \%$ yield, $92 \%$ ee \\
2 & without $\left[\mathrm{Ru}(\mathrm{bpy})_{3}\right]\left(\mathrm{PF}_{6}\right)_{2}$ & No reaction \\
3 & without 21 W CFL & No reaction
\end{tabular}

${ }^{a}$ Reaction conditions: $\mathbf{1 a}(0.10 \mathrm{mmol}), \mathbf{3 a}(0.30 \mathrm{mmol}), \Delta-\mathbf{R h S}(4.0 \mathrm{~mol} \%),\left[\mathrm{Ru}(\mathrm{bpy})_{3}\right]\left(\mathrm{PF}_{6}\right)_{2}(2.5 \mathrm{~mol} \%), \mathrm{Na}_{2} \mathrm{HPO}_{4}$ (20 mol\%) and $\mathrm{H}_{2} \mathrm{O}$ (20 equiv) in acetone/DMSO (9:1, 0.2 M) were stirred at room temperature for $15 \mathrm{~h}$ with visible light. 


\section{Mechanistic Studies}

\subsection{Identification of Rh-enolate Intermediate}

\subsubsection{Synthesis}
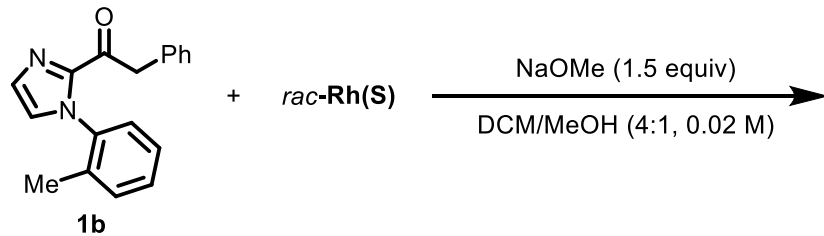

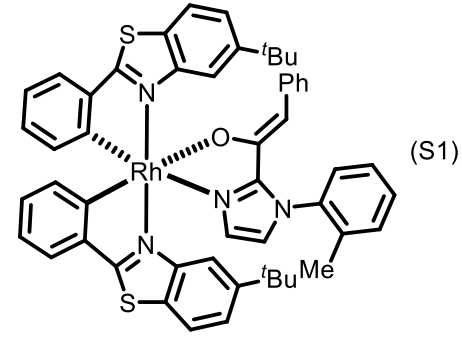

Rh-enolate

As shown in eq $\mathrm{S} 1$ : $\mathrm{MeOH}(0.4 \mathrm{~mL})$ and $\mathrm{DCM}(1.6 \mathrm{~mL})$ were added to a mixture of $\mathrm{rac}$ - $\mathbf{R h S}(86 \mathrm{mg}$, $0.1 \mathrm{mmol})$, imidazole $1 \mathbf{b}(33.1 \mathrm{mg}, 0.12 \mathrm{mmol})$ and $\mathrm{NaOMe}(8.1 \mathrm{mg}, 0.15 \mathrm{mmol})$ under $\mathrm{N}_{2}$ atmosphere The mixture was stirred at room temperature overnight. Then solvents were removed in vacuo and the mixture was filtered through a short silica column with $\mathrm{EA} / n$-hexane. The yellow elution fraction was collected, evaporated and the obtained yellow solid was recrystallized by $\mathrm{DCM} / n$-hexane giving pure Rh-enolate, which was characterized by X-ray diffraction (see part 9).

\subsubsection{Catalytic behavior}

Table S6. Catalytic Behavior of Rh-enolate

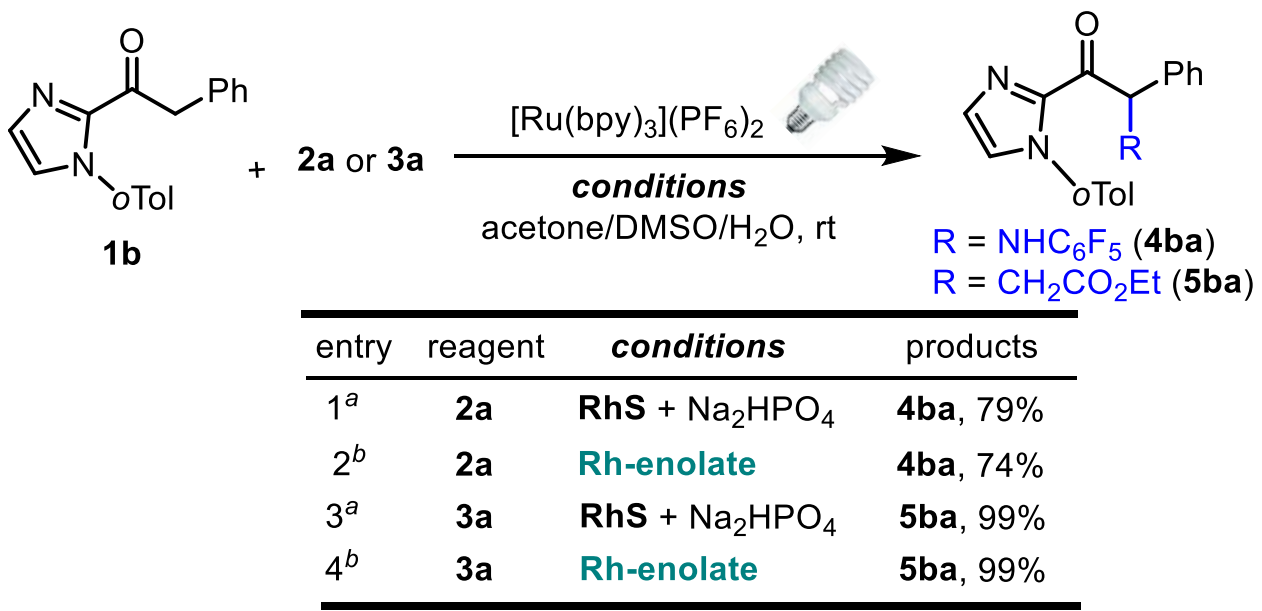

${ }^{a}$ Reaction conditions: $\mathbf{1 b}(0.10 \mathrm{mmol}), \mathbf{2 a} / \mathbf{3 a}(0.30 \mathrm{mmol}), \mathbf{R h S}(4.0 \mathrm{~mol} \%),\left[\mathrm{Ru}(\mathrm{bpy})_{3}\right]\left(\mathrm{PF}_{6}\right)_{2}(2.5 \mathrm{~mol} \%$ for entry $1,1.5 \mathrm{~mol} \%$ for entry 3), $\mathrm{Na}_{2} \mathrm{HPO}_{4}\left(20 \mathrm{~mol} \%\right.$ ) and $\mathrm{H}_{2} \mathrm{O}$ (20 equiv) in acetone/DMSO (9:1, $\left.0.2 \mathrm{M}\right)$ were stirred at room temperature with visible light. ${ }^{b}$ Reaction conditions: $\mathbf{1 b}(0.096 \mathrm{mmol}), \mathbf{2 a} / \mathbf{3 a}(0.30 \mathrm{mmol}), \mathbf{R h}-$ enolate $(0.004 \mathrm{mmol}),\left[\mathrm{Ru}(\mathrm{bpy})_{3}\right]\left(\mathrm{PF}_{6}\right)_{2}(2.5 \mathrm{~mol} \%$ for entry $2,1.5 \mathrm{~mol} \%$ for entry 4$), \mathrm{Na}_{2} \mathbf{H P O}_{4}(\mathbf{0} \mathbf{~ m o l} \%)$ and $\mathrm{H}_{2} \mathrm{O}$ (20 equiv) in acetone/DMSO (9:1, $0.2 \mathrm{M}$ ) were stirred at room temperature with visible light. 
As shown in Table S6, comparable yields to $\mathbf{R h S}$ /base were observed with rhodium-enolate as the catalyst in the absence of any base, providing alternative base-free conditions for these amination/alkylation. These results support that the rhodium-enolate serves as a key intermediate in these transformations.

\subsubsection{Superiority of Rh-base Lewis acid}

As shown in Table S7, chiral-at-metal Rh complex shows unique reactivity in this transformation. Other Lewis acid, such as $\mathrm{Sc}(\mathrm{OTf})_{3}, \mathrm{FeCl}_{3}$, and $\mathrm{Cu}(\mathrm{OAc})_{2}$ could not catalyze the reaction.

Table S7. Comparison of Lewis Acid ${ }^{a}$

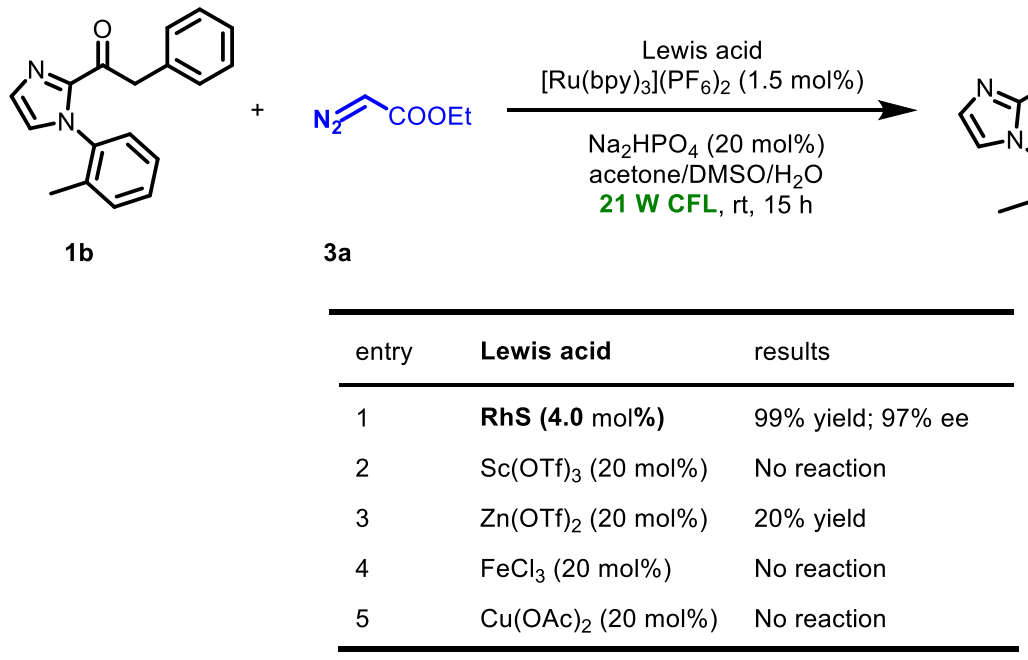

${ }^{a}$ Reaction conditions: $\mathbf{1 b}(0.10 \mathrm{mmol}), \mathbf{3 a}(0.30 \mathrm{mmol})$, Lewis acid, [Ru(bpy) $\left.{ }_{3}\right]\left(\mathrm{PF}_{6}\right)_{2}(1.5 \mathrm{~mol} \%)$, $\mathrm{Na}_{2} \mathrm{HPO}_{4}(20 \mathrm{~mol} \%)$ and $\mathrm{H}_{2} \mathrm{O}$ (20 equiv) in acetone/DMSO (9:1, $\left.0.2 \mathrm{M}\right)$ were stirred at room temperature for $15 \mathrm{~h}$ with visible light.

\subsection{Trapping Experiments with Air}

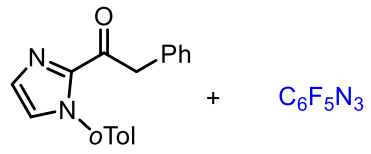

$1 b$

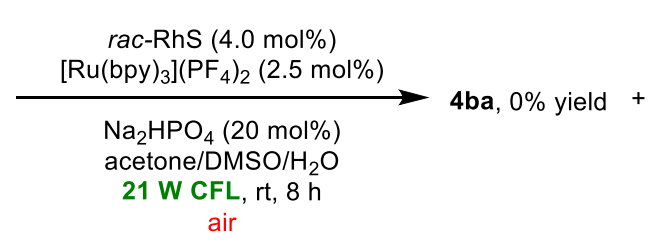

rac-RhS $(4.0 \mathrm{~mol} \%)$<smiles>O=C(C=N[18O-])O[Pb]</smiles>

3a
$\underset{\mathrm{Na}_{2} \mathrm{HPO}_{4}(20 \mathrm{~mol} \%)}{\left.\mathrm{Ru}(\mathrm{bpy})_{3}\right]\left(\mathrm{PF}_{4}\right)_{2}(1.5 \mathrm{~mol} \%)}$ 5ba, $0 \%$ yield $+\mathbf{6}$, quant. yield

$21 \mathrm{~W} \mathrm{CFL}, \mathrm{rt}, 15 \mathrm{~h}$
6, quant. yield<smiles>O=C(C(=O)c1nccn1[O-])c1ccccc1</smiles> 
As shown in eqs S2-S3, when the reaction was conducted under air atmosphere, no target molecules 4ba or $5 \mathbf{b a}$ was formed, respectively. Quantitative yield of diketone 6 was obtained, which implies that $\alpha$-carbonyl carbon radical might be involved.

\section{1-Phenyl-2-(1-(o-tolyl)-1H-imidazol-2-yl)ethane-1,2-dione (6)}

${ }^{1} \mathrm{H}$ NMR (300 MHz, $\left.\mathrm{CDCl}_{3}\right) \delta$ 7.97-7.90 (m, 2H), 7.67-7.58 (m, 1H), 7.53-7.20 (m, 8H), 2.14 (s, 3H).

${ }^{13} \mathrm{C}$ NMR $\left(125 \mathrm{MHz}, \mathrm{CDCl}_{3}\right) \delta 192.5,183.6,141.4,136.5,134.6,134.5,132.7,132.3,131.0,129.8$, $129.7,128.9,127.4,126.9,126.5,17.3$.

IR (film): $v\left(\mathrm{~cm}^{-1}\right)$ 3125, 3059, 2921, 1671, 1587, 1498, 1452, 1394, 1311, 1262, 1149, 1071, 860, 794, 761, 720, 663, 551, 527, 453.

HRMS (ESI, $m / z$ ) calcd for $\mathrm{C}_{18} \mathrm{H}_{15} \mathrm{~N}_{2} \mathrm{O}_{2}[\mathrm{M}+\mathrm{H}]^{+}: 291.1128$, found: 291.1128.

\subsection{Trapping Experiments with TMS-enolate}

\subsubsection{Trapping with amination reaction}

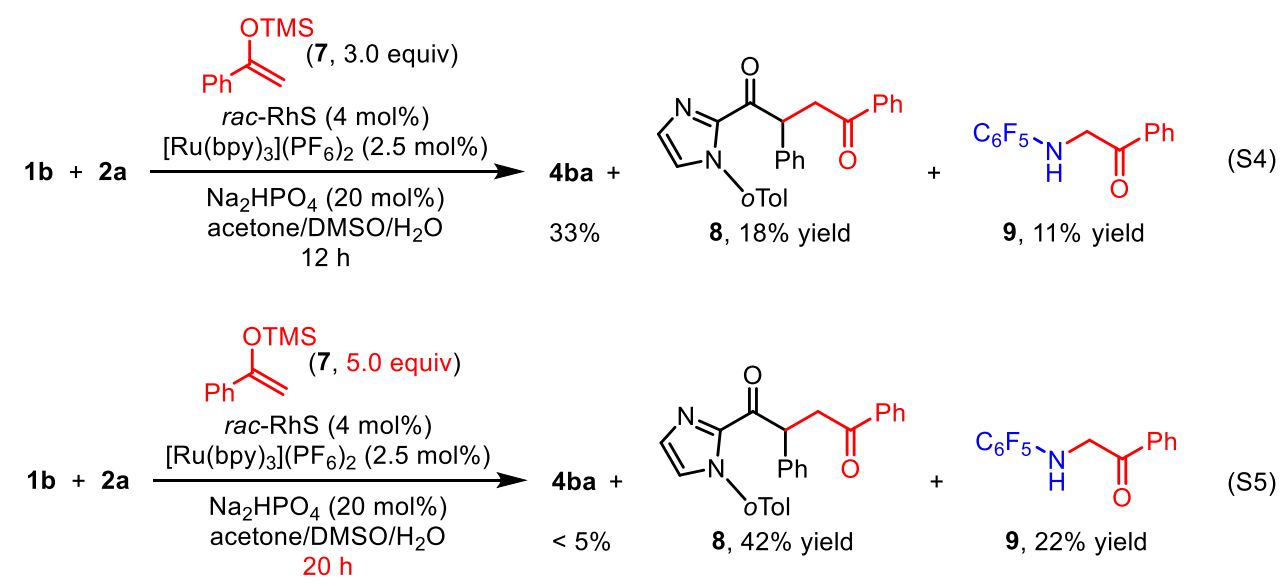

When the TMS-enolate $\mathbf{7}$ (3.0 equiv) was added to the reaction of $\mathbf{1 b}$ with $\mathbf{2 a}$, the $\mathrm{C}-\mathrm{C}$ and $\mathrm{C}-\mathrm{N}$ bond formation products 8 (18\% yield) and 9 (11\% yield) were isolated, respectively (eq S4). When 5.0 equiv of 7 was employed, 8 and 9 were isolated with improved yields (42\%, 22\% respectively) (eq S5). These results indicate the intermediate formation of an $\alpha$-carbonyl carbon radical and aminyl radical.

\section{2,4-Diphenyl-1-(1-(o-tolyl)-1H-imidazol-2-yl)butane-1,4-dione (8)}

${ }^{1} \mathrm{H}$ NMR $\left(300 \mathrm{MHz}, \mathrm{CDCl}_{3}\right) \delta$ 7.96-7.90 (m, 2H), 7.57-7.17 (m, 13H), 7.06-7.03 (m, 1H), $6.95(\mathrm{~d}, J$ $=7.8 \mathrm{~Hz}, 1 \mathrm{H}$, the other rotamer $), 6.84-5.75(\mathrm{~m}, 1 \mathrm{H}), 4.13-4.00(\mathrm{~m}, 1 \mathrm{H}), 3.42-3.30(\mathrm{~m}, 1 \mathrm{H}), 2.08(\mathrm{~s}$, 
$3 \mathrm{H}), 1.62$ (s, 3H, the other rotamer).

${ }^{13} \mathrm{C}$ NMR $\left(75 \mathrm{MHz}, \mathrm{CDCl}_{3}\right) \delta 197.5,189.6,189.5,143.2,143.1,138.3,138.2,138.1,138.0,136.53$, $136.49,135.2$, 134.3, 133.0, 130.6, 130.4, 130.2, 128.9, 128.8, 128.7, 128.4, 128.0, 127.2, 127.1, $126.7,126.5,126.3,126.21,126.19,126.1,48.10,48.06,43.2,42.7,17.1,16.5$. (Mixture of two rotation isomers).

IR (film): $v\left(\mathrm{~cm}^{-1}\right)$ 3060, 3031, 2913, 1679, 1591, 1495, 1451, 1403, 1360, 1306, 1247, 1203, 1150, $1015,989,944,907,759,726,692,591,552,523,456$.

HRMS (EI, $m / z$ ) calcd for $\mathrm{C}_{26} \mathrm{H}_{23} \mathrm{~N}_{2} \mathrm{O}_{2}[\mathrm{M}+\mathrm{H}]^{+}:$395.1754, found: 395.1756 .

\section{2-((Perfluorophenyl)amino)-1-phenylethan-1-one (9)}

${ }^{1} \mathrm{H}$ NMR $\left(300 \mathrm{MHz}, \mathrm{CDCl}_{3}\right) \delta$ 8.00-7.94 (m, 2H), 7.69-7.61 (m, 1H), 7.56-7.47 (m, 2H), 4.96 (br s, $1 \mathrm{H}), 4.83$ (br s, $2 \mathrm{H})$.

${ }^{13} \mathrm{C} \mathrm{NMR}\left(75 \mathrm{MHz}, \mathrm{CDCl}_{3}\right) \delta 194.5,134.3,134.2,129.0,127.8,52.0-51.7(\mathrm{~m})$.

${ }^{19} \mathrm{~F}$ NMR $\left(282 \mathrm{MHz}, \mathrm{CDCl}_{3}\right) \delta-160.78--160.95(\mathrm{~m}, 2 \mathrm{~F}),-165.05--165.26(\mathrm{~m}, 2 \mathrm{~F}),-172.40\left(\mathrm{tt}, J_{l}\right.$ $\left.=21.15 \mathrm{~Hz}, J_{2}=6.49 \mathrm{~Hz}, 1 \mathrm{~F}\right)$.

IR (film): $v\left(\mathrm{~cm}^{-1}\right)$ 3401, 2956, 2923, 2852, 1686, 1518, 1439, 1344, 1266, 1229, 1188, 1118, 995, $950,755,684,606,583,558,473$.

HRMS (EI, $m / z$ ) calcd for $\mathrm{C}_{14} \mathrm{H}_{8} \mathrm{~F}_{5} \mathrm{NO}[\mathrm{M}]^{+}:$301.0526, found: 301.0517 .

\subsubsection{Trapping with diazo compound}

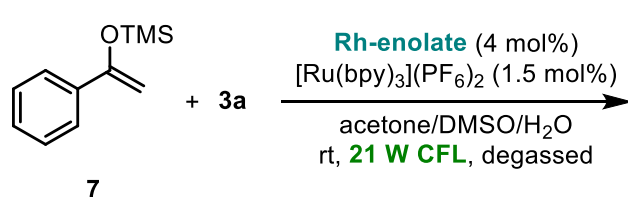

7

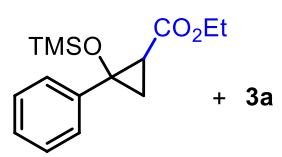

13

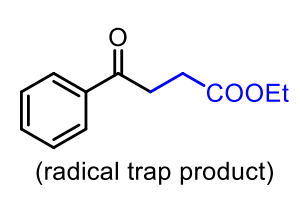

$12,68 \%$ yield<smiles>CCOC(=O)CCC(=O)c1ccccc1</smiles>

$12,<2 \%$ yield

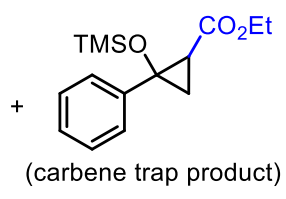

$13,0 \%$ yield

As shown in eq S6, when the TMS-enolate 7 was stirred with 3a under current conditions, 68\% of the radical addition product 12 was detected, instead of the cyclopropanation product 13. Furthermore, 13 cannot be converted into 12 under these reaction conditions (eq S7). These results exclude the 
possibility of carbene intermediates. 13 was synthesized according to literature report. ${ }^{10}$ All spectroscopic data of $\mathbf{1 2}^{11}$ and $\mathbf{1 3}^{10}$ are in agreement with previous reports.

\subsection{Trapping Experiments with TEMPO}

\subsubsection{Trapping with amination reaction}

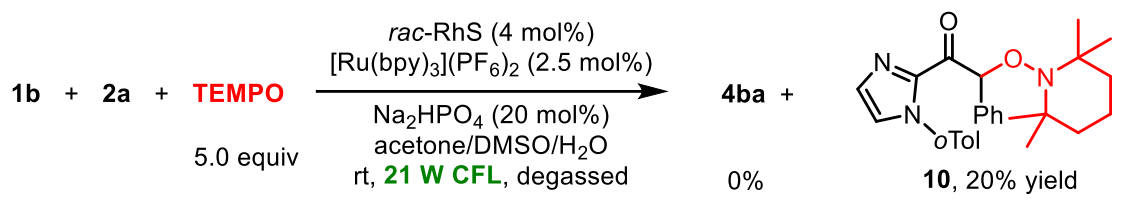

As shown in eq S8, when the TEMPO (5.0 equiv) was added to the reaction mixture of $\mathbf{1 b}$ with $\mathbf{2 a}$ under current conditions, the TEMPO adduct $\mathbf{1 0}$ was isolated in $20 \%$ yield and the formation of $\mathbf{4 b a}$ was totally inhibited, indicating that $\alpha$-carbonyl carbon radicals might be involved in amination reaction. All spectroscopic data of $\mathbf{1 0}$ are in agreement with our previous report. ${ }^{4 \mathrm{c}}$

\subsubsection{Trapping with alkylation reaction}

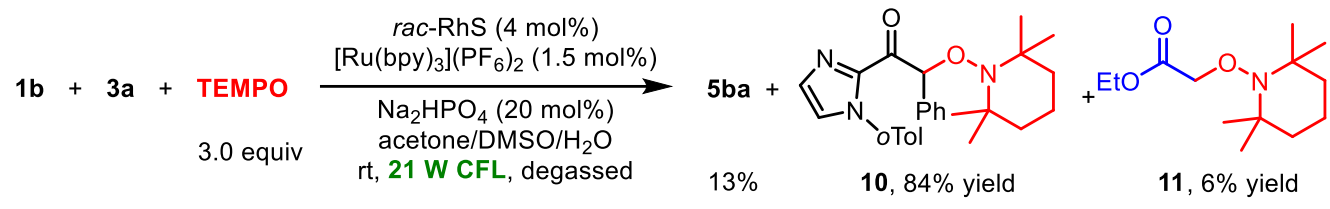

When the TEMPO (3.0 equiv) was added to the reaction mixture of $\mathbf{1 b}$ with $\mathbf{3 a}$ under current conditions, the TEMPO adducts 10 (84\% yield) and $\mathbf{1 1}$ (6\% yield) were isolated, respectively, being indicative for two types of intermediate $\alpha$-carbonyl carbon radicals (eq S9). All spectroscopic data of $\mathbf{1 1}$ are in agreement with literature report. ${ }^{12}$

\subsection{Trapping Experiments with Alkenes}

\subsubsection{Trapping with amination reaction}

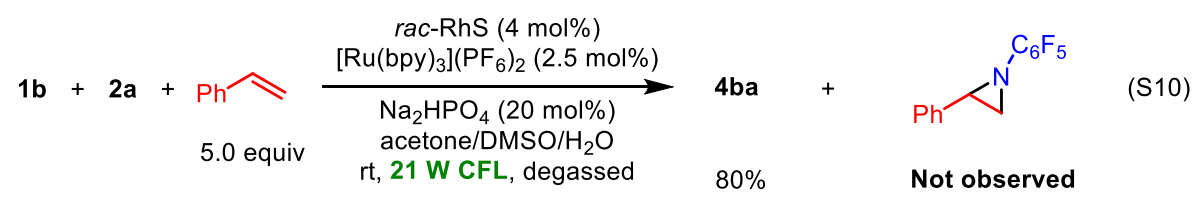

When the styrene (5.0 equiv) was added to the reaction mixture of $\mathbf{1 b}$ with $\mathbf{2 a}$ under current conditions, the amination product $\mathbf{4 b a}$ was detected in $80 \%$ yield, while aziridination product was not observed 
(S10). These results imply nitrene intermediate might not be involved in this transformation, which is thus distinct from Yoon's recent report. ${ }^{13}$

\subsubsection{Trapping with alkylation reaction}

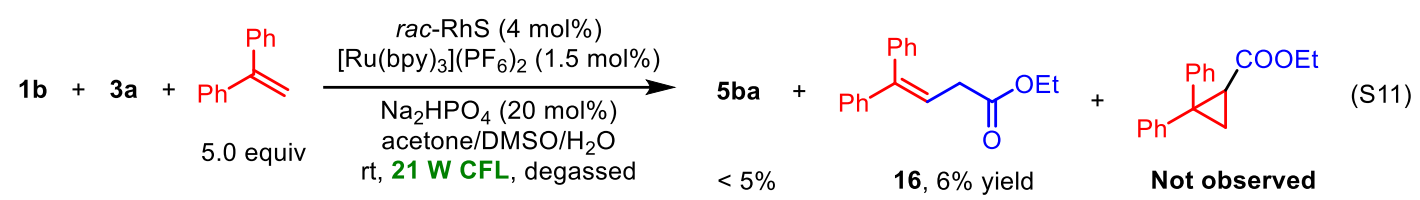

As shown in eq S11, when the 1,1-diphenylethene (5.0 equiv) was added to the reaction mixture of 1b with 3a under current conditions, the alkylation was totally inhibited. And $\mathbf{1 6}^{\mathbf{1 4}}$ was obtained in $6 \%$ yield via radical addition and subsequent oxidation processes, while cyclopropanation product was not observed. These results indicate that a radical process rather than a carbene insertion pathway is involved.
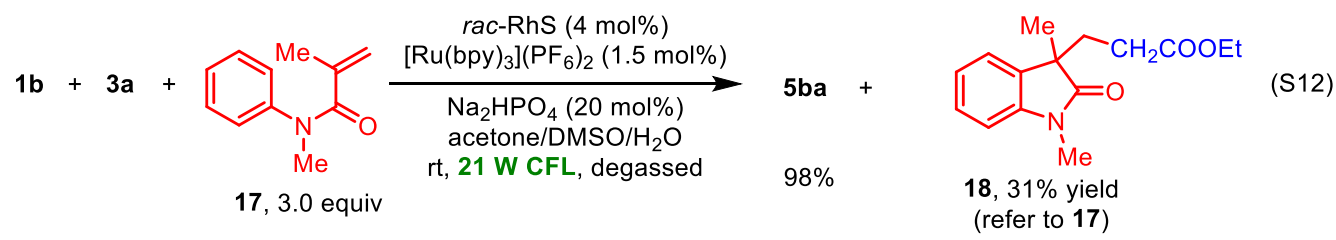

When the alkene 17 (3.0 equiv) was added to the reaction mixture of $\mathbf{1 b}$ with $\mathbf{3 a}$ under current conditions, the alkylation product $\mathbf{5} \mathbf{b a}$ was detected in $98 \%$ yield, together with the formation of radical cycloaddition product $\mathbf{1 8}$ (eq S12). These results support that an $\alpha$-ester carbon radical derived from diazo compound 3a was formed during the alkylation reaction. All spectroscopic data of $\mathbf{1 8}$ are in agreement with literature report. ${ }^{15}$

Substrate 3a with the relatively highly negative reduction potential $\left(E_{\mathrm{p}}{ }^{\text {red }}=-1.97 \mathrm{~V}, \mathrm{vs} \mathrm{Fc} / \mathrm{Fc}^{+}\right.$, see Figure S5) cannot quench the excited state of $\left[\mathrm{Ru}(\mathrm{bpy})_{3}\right]^{2+}\left(\mathrm{E}_{1 / 2}{ }^{\mathrm{III} / * \mathrm{II}}=-0.81 \mathrm{~V}\right.$, vs SCE in MeCN) via an oxidative quenching cycle. Therefore, the reaction of $\mathbf{1 7}$ and $\mathbf{3 a}$ under conditions listed in entry 1, Table S8, cannot deliver any product. When a catalytic amount of DIPEA or Rh-enolate was added as a reductive quencher, 18 can be observed in 20\% NMR yield (Table S8, entries 2-3). However, fac- $\operatorname{Ir}(\mathrm{ppy})_{3}$, which is a very strong SET reductant in excited state $\left[\operatorname{Ir}(\mathrm{IV}) / \operatorname{Ir}(\mathrm{III})^{*}=-1.73 \mathrm{~V}\right]$, failed to catalyze this reaction (entry 4). All these results support the conclusion that the Rh-enolate intermediate acts as a reductive quencher. 
Table S8. Investigation of Rh-enolate as a Reductive Quencher ${ }^{a}$
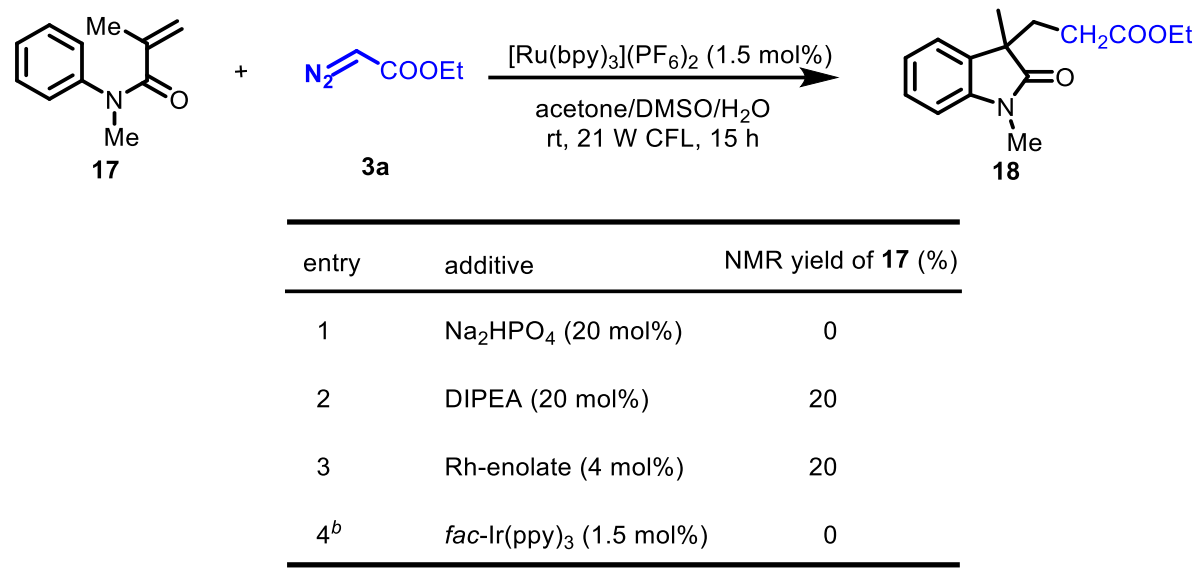

18

\begin{tabular}{llc}
\hline entry & additive & NMR yield of 17 (\%) \\
\hline 1 & $\mathrm{Na}_{2} \mathrm{HPO}_{4}(20 \mathrm{~mol} \%)$ & 0 \\
2 & DIPEA $(20 \mathrm{~mol} \%)$ & 20 \\
3 & Rh-enolate $(4 \mathrm{~mol} \%)$ & 20 \\
$4^{b}$ & fac-lr(ppy $)_{3}(1.5 \mathrm{~mol} \%)$ & 0 \\
\hline
\end{tabular}

${ }^{a}$ Reaction conditions: $17(0.10 \mathrm{mmol}), \mathbf{3 a}(0.30 \mathrm{mmol}),\left[\mathrm{Ru}(\mathrm{bpy})_{3}\right]\left(\mathrm{PF}_{6}\right)_{2}(1.5 \mathrm{~mol} \%)$, additives and $\mathrm{H}_{2} \mathrm{O}$ (20 equiv) in acetone/DMSO (9:1, $0.2 \mathrm{M}$ ) were stirred at room temperature for $15 \mathrm{~h}$ with visible light; ${ }^{b}$ Without $\left[\mathrm{Ru}(\mathrm{bpy})_{3}\right]\left(\mathrm{PF}_{6}\right)_{2}$, THF as solvent.

\subsection{EPR Experiments}

EPR spectra were recorded at room temperature using DMPO as free radical spin trapping agent. As shown in Figure S1, according to general procedure, the reaction of $\mathbf{1 b}$ and $\mathbf{3 a}$ was stirred under standard conditions for $60 \mathrm{~min}$. Then a portion of the reaction mixture was added to DMPO solution and measured by EPR. Signals with 6 lines $\left(\mathrm{g}=2.006 ; \alpha_{\mathrm{N}}=15.9 \mathrm{G}, \alpha_{\mathrm{H}}^{\beta}=22.5 \mathrm{G}\right)$ were observed and identified as EPR signals of adducts 14, which are in good agreement with literature (Figure S1). ${ }^{16}$ All these results support the formation of an ethyl acetate $\alpha$-carbon radical through single electron reduction of the diazo compound $\mathbf{3 a}$.

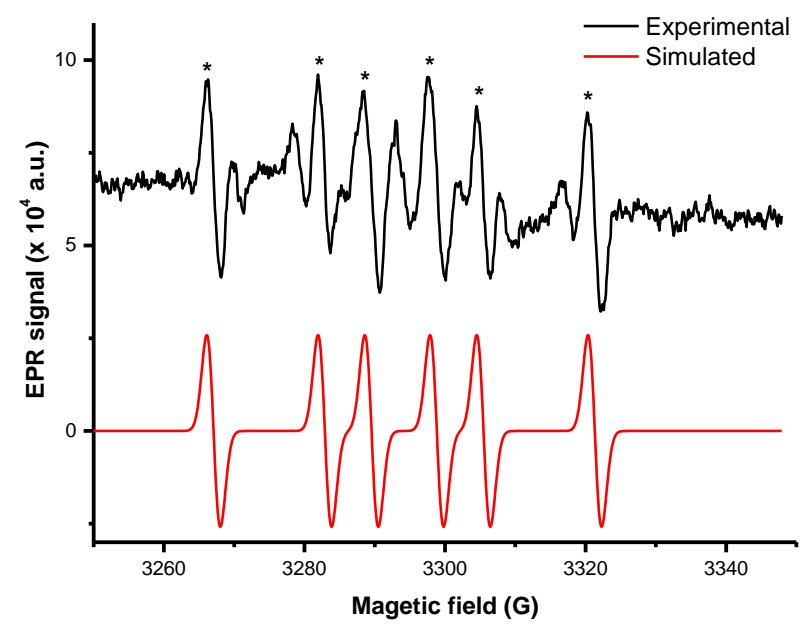

Figure S1. EPR experiments (X band, r.t.). After $60 \mathrm{~min}$ of irradiation, a portion of the reaction mixture was added to DMPO solution and then measured by EPR. 


\subsection{Stern-Volmer Quenching Experiments}

\subsubsection{UV-Vis absorption spectra and luminescence emission spectra}

As shown in Figure S2, Rh-enolate solution absorb light with wavelength $<475 \mathrm{~nm}$. In order to stimulate the reaction conditions, the luminescence quenching experiments were performed with the photoredox sensitizer $\left[\mathrm{Ru}(\mathrm{bpy})_{3}\right]^{2+}$ alone and with the mixture of $\left[\mathrm{Ru}(\mathrm{bpy})_{3}\right]^{2+}$ and $\mathbf{R h}$-enolate in a ratio of $1.5: 4.0$, respectively. Also in the luminescence quenching experiments with the $\left[\mathrm{Ru}(\mathrm{bpy})_{3}\right]^{2+}$ alone, different exciting light wavelengths were chosen.

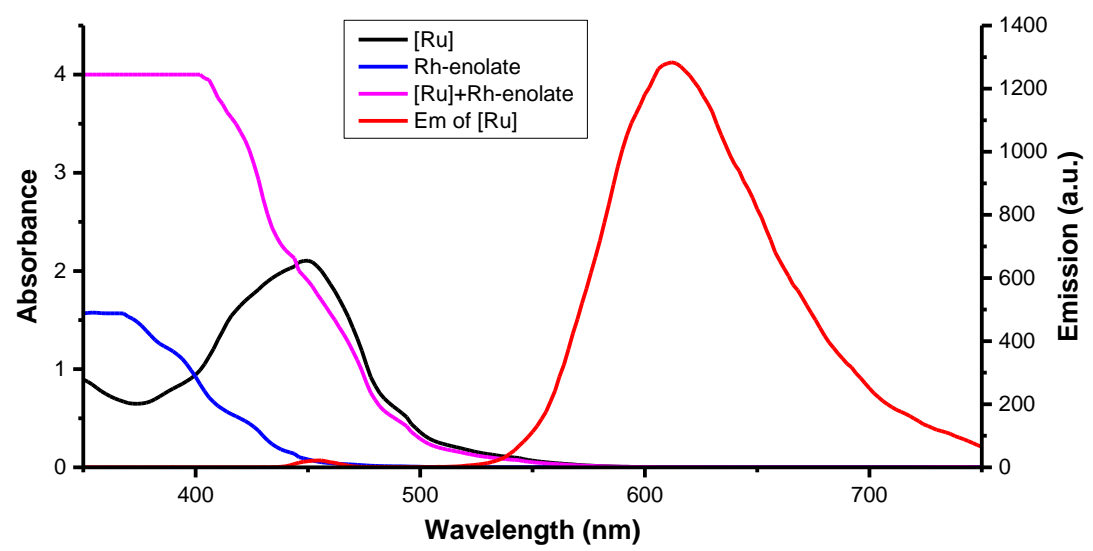

Figure S2. UV-Vis absorption spectra and luminescence emission spectra. $[\mathrm{Ru}]=\left[\mathrm{Ru}(\mathrm{bpy})_{3}\right]^{2+}$; Concentration in solution of acetone/DMSO/ $\mathrm{H}_{2} \mathrm{O}(9: 1: 0.72):[\mathrm{Ru}]=0.05 \mathrm{mM}$, Rh-enolate $=0.05$ $\mathrm{mM},[\mathrm{Ru}]+\mathbf{R h}$-enolate $=0.1 \mathrm{mM} / 0.27 \mathrm{mM}, \mathrm{Em}$ of $[\mathrm{Ru}]=0.10 \mathrm{mM}$.

\subsubsection{Quenching experiments with the mixture of $[\operatorname{Ru}(b p y) 3]^{2+}$ and Rh-enolate}

The solutions of $\left[\mathrm{Ru}(\mathrm{bpy})_{3}\right]^{2+}$ and $\mathbf{R h}$-enolate $\left(0.1 \mathrm{mM}\right.$ and $0.27 \mathrm{mM}$ in acetone/DMSO/ $\mathrm{H}_{2} \mathrm{O}$, respectively) were excited at $\lambda=470 \mathrm{~nm}$ and the emission was measured at $610 \mathrm{~nm}$ (emission maximum). For each quenching experiment, after degassed with a nitrogen stream for 5 minutes, the emission intensity of the solution $(1 \mathrm{~mL})$ of $\left[\mathrm{Ru}(\mathrm{bpy})_{3}\right]^{2+}$ and $\mathbf{R h}$-enolate with different concentration of quencher $(0,0.5,1.0,2.0,4.0 \mathrm{mM})$ in a screw-top $10.0 \mathrm{~mm}$ quartz cuvette was collected.

Results: imidazole $\mathbf{1 b}$, azide $\mathbf{2 a}$, diazo compound 3a, and TMS-enolate $\mathbf{7}$ could not quench the luminescence.

\subsubsection{Quenching experiments with $\left[\operatorname{Ru}(b p y)_{3}\right]^{2+}$ alone}

The solutions of $\left[\mathrm{Ru}(\mathrm{bpy})_{3}\right]^{2+}$ in acetone/DMSO/ $\mathrm{H}_{2} \mathrm{O}$ were excited at $\lambda=470 \mathrm{~nm}$ and the emission 
was measured at $610 \mathrm{~nm}$ (emission maximum). For each quenching experiment, after degassed with a nitrogen stream for 5 minutes, the emission intensity of the solution $(1 \mathrm{~mL})$ of $\left[\mathrm{Ru}(\mathrm{bpy})_{3}\right]^{2+}$ with different concentration of quencher $(0,0.5,1.0,2.0,4.0 \mathrm{mM})$ in a screw-top $10.0 \mathrm{~mm}$ quartz cuvette was collected.

Results: imidazole 1b, azide $\mathbf{2 a}$, diazo compound $\mathbf{3 a}$, and TMS-enolate $\mathbf{7}$ and rac-RhS could not quench the luminescence. Only Rh-enolate was capable of quenching the excited state of $\left[\mathrm{Ru}(\mathrm{bpy})_{3}\right]^{2+}$. In order to exclude the effect of competitive absorption by $\mathbf{R h}$-enolate, the solutions of $\left[\mathrm{Ru}(\mathrm{bpy})_{3}\right]^{2+}$ with different concentration of $\mathbf{R h}$-enolate $(0,0.5,1.0,2.0,4.0 \mathrm{mM})$ were excited at $\lambda$ $=470 \mathrm{~nm}, 510 \mathrm{~nm}$, and $530 \mathrm{~nm}$, respectively. At the same time, the absorbance of the mixture was measured as well. As shown in Figure S3, the absorption of the mixture kept constant with the increment of $\mathbf{R h}$-enolate indicating there is no competitive absorption by $\mathbf{R h}$-enolate in $530 \mathrm{~nm}$. And only the intermediate $\mathbf{R h}$-enolate, but not the catalyst $\mathbf{R h S}$ or the TMS-enolate $\mathbf{7}$, can quench the excited state of the $\left[\mathrm{Ru}(\mathrm{bpy})_{3}\right]^{2+}$. Furthermore, the quenching effect of $\mathbf{R h}$-enolate was similar in different exciting light wavelengths (Figure S4).

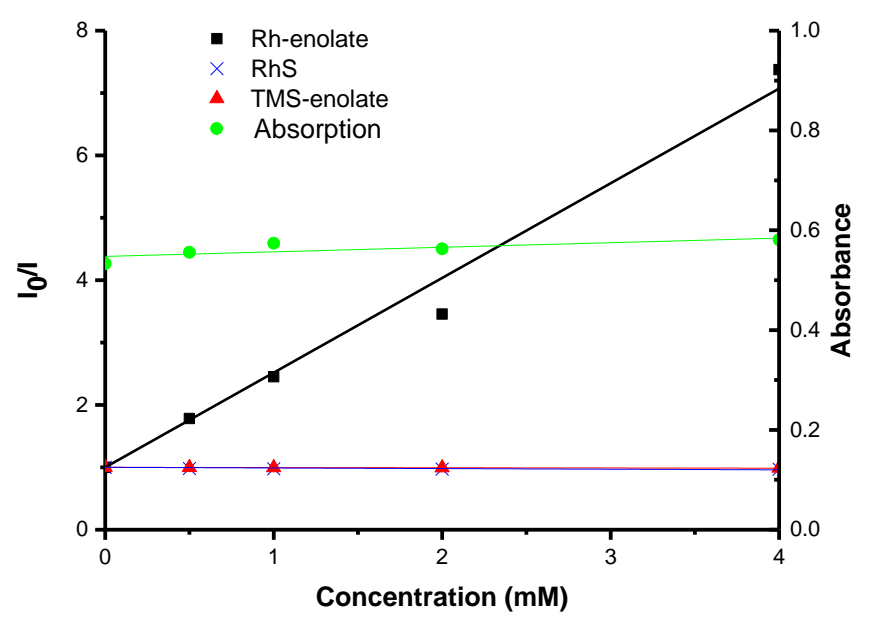

Figure S3. Stern-Volmer plots. $\mathrm{I}_{0}$ and I are respective luminescence intensities in the absence and presence of the indicated concentrations of the corresponding quencher. $[\mathrm{Ru}]=0.5 \mathrm{mM}, \mathrm{Ex}=530$ $\mathrm{nm}$, Absorption refer to the absorbance of the solutions of $\left[\mathrm{Ru}(\mathrm{bpy})_{3}\right]^{2+}$ with different concentration of Rh-enolate. 


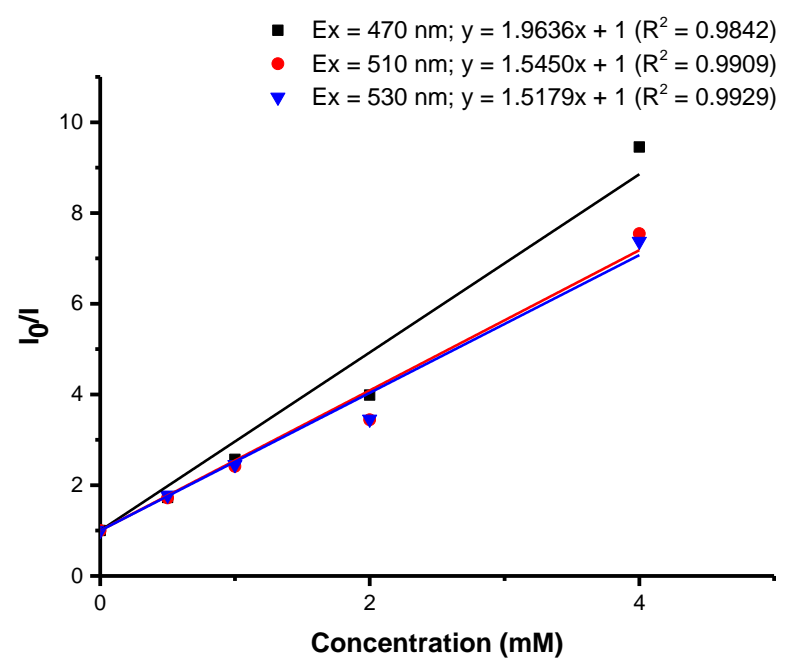

Figure S4. Quenching effect of Rh-enolate with different exciting light wavelengths.

\subsection{Cyclic Voltammetry}

Experiment details: Voltammetric experiments were conducted with a computer controlled Eco Chemie Autolab PGSTAT302N potentiostat in a Metrohm electrochemical cell containing $1 \mathrm{~mm}$ diameter planar platinum and glassy carbon (GC) disk electrodes (eDAQ), a platinum wire auxiliary electrode (Metrohm) and a silver wire miniature reference electrode (eDAQ) that was connected to the test solution via a salt bridge containing $0.5 \mathrm{M} n \mathrm{Bu}_{4} \mathrm{NPF}_{6}$ in $\mathrm{CH}_{3} \mathrm{CN}$. Accurate potentials were referenced to the ferrocene/ferrocenium $\left(\mathrm{Fc} / \mathrm{Fc}^{+}\right)$redox couple, which was used as an internal standard. All solutions used for the voltammetric experiments were deoxygenated by purging with high purity argon gas and measurements were performed in a Faraday cage at room temperature $\left(22 \pm 2{ }^{\circ} \mathrm{C}\right)$.

HPLC purity acetonitrile $\left(\mathrm{CH}_{3} \mathrm{CN}\right)$ was purchased from Macron. The supporting electrolyte, tetrabutylammonium hexafluorophosphate $\left(n \mathrm{Bu}_{4} \mathrm{NPF}_{6}\right)$, was prepared by reacting equal molar amounts of $n \mathrm{Bu} 4 \mathrm{NOH}\left(40 \%\right.$, Alfa Aesar) and $\mathrm{HPF}_{6}(65 \%$, Fluka), washing the precipitate with ultrapure water and recrystallizing three times with hot ethanol followed by drying under vacuum at $140{ }^{\circ} \mathrm{C}$ for 6 hours.

Results and discussion: As shown in Figure S5, both $\mathbf{R h S}$ and Rh-enolate were able to undergo an oxidation process in a chemically irreversible way at positive potentials between $\sim 1.0-1.4 \mathrm{~V}$ vs $\mathrm{Fc} / \mathrm{Fc}^{+}$. Importantly, Rh-enolate has an additional chemically irreversible oxidation peak at approximately $+0.08 \mathrm{~V} v s . \mathrm{Fc} / \mathrm{Fc}^{+}$, which means it could be easily oxidized at a relative low potential 
$\left(\sim 0.08 \mathrm{~V} \mathrm{vs} \mathrm{Fc} / \mathrm{Fc}^{+}\right)$to form a new compound that is itself further oxidized at more positive potentials. Different from $\mathbf{R h S}$, which could be reduced in a chemically irreversible process at $\sim-1.8--2.1 \mathrm{~V}$ vs $\mathrm{Fc} / \mathrm{Fc}^{+}$, no reduction process is seen for $\mathbf{R h}$-enolate unless the potential is first scanned in the positive direction past $0.08 \mathrm{~V}$ vs $\mathrm{Fc} / \mathrm{Fc}^{+}$. Therefore, it is likely that $\mathbf{R h}$-enolate undergoes SET oxidation at the enolate $\left(E_{\mathrm{p}}{ }^{\mathrm{ox}}=0.078 \mathrm{~V}, \mathrm{vs} \mathrm{Fc} / \mathrm{Fc}^{+}\right)$, generating $\mathrm{Rh}$-coordinated radical intermediate $\mathbf{B}_{\text {ox. }}$. And the subsequent gain of a hydrogen atom forms a Rh-coordinated substrate intermediate $\mathbf{A}$, which then can become reduced (eq S13).
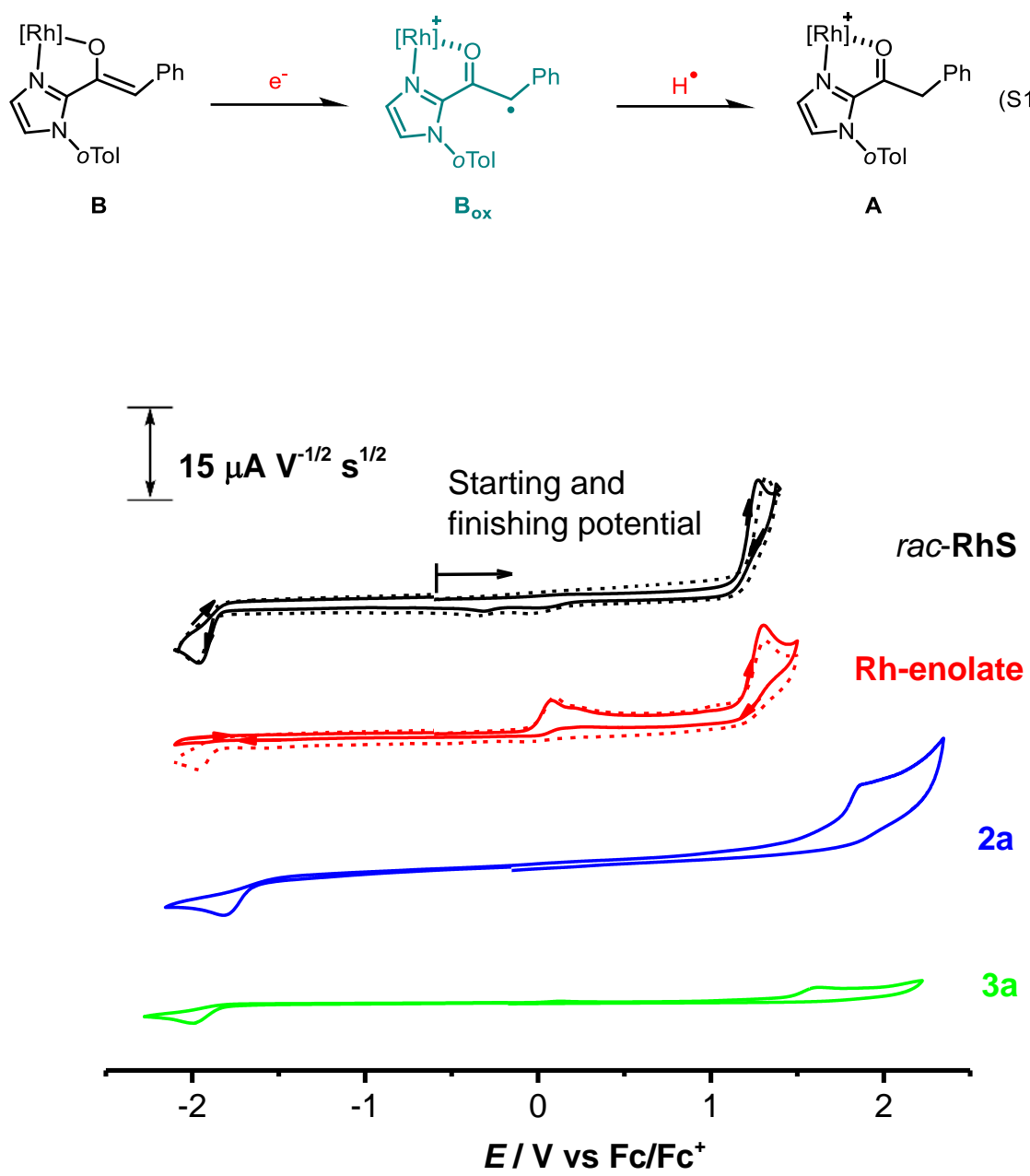

Figure S5. Cyclic voltammograms of $\mathbf{R h S}$, Rh-enolate, 2a and 3a. Recorded in $\mathrm{CH}_{3} \mathrm{CN}$ containing $0.1 \mathrm{M} n-\mathrm{Bu}_{4} \mathrm{NPF}_{6}$ at $22 \pm 2{ }^{\circ} \mathrm{C}$ at a $1 \mathrm{~mm}$ diameter $\mathrm{GC}$ electrode for $1 \mathrm{mM}$ solutions of the analytes. (-) Scan rate $=0.1 \mathrm{~V} \mathrm{~s}^{-1}$, and (…) scan rate $=1 \mathrm{~V} \mathrm{~s}^{-1}$. The current data were normalised by dividing by the square root of the scan rate.

The compounds $\mathbf{2 a}$ and $\mathbf{3 a}$ can both be reduced in a chemically irreversible way at relatively negative potentials that are similar to the other compound. As shown in Figure S5, 2a has a chemically 
irreversible reduction peak at $-1.82 \mathrm{~V}$ vs $\mathrm{Fc} / \mathrm{Fc}^{+}$, while 3a at $-1.97 \mathrm{~V}$ vs $\mathrm{Fc} / \mathrm{Fc}^{+}$. These results, in consistence with quenching experiments, demonstrate that the excited state of $\left[\mathrm{Ru}(\mathrm{bpy})_{3}\right]^{2+}\left(E_{1 / 2}^{\mathrm{III} / * \mathrm{II}}\right.$ $=-0.81 \mathrm{~V}$, vs $\mathrm{SCE}$ in $\mathrm{MeCN})^{17 \mathrm{a}}$ cannot reduce azide 2a or diazo compound 3a. On the contrary, the SET process from the strongly reducing $\left[\mathrm{Ru}(\mathrm{bpy})_{3}\right]^{+}\left(E_{1 / 2}^{\mathrm{II} / \mathrm{I}}=-1.33 \mathrm{~V}\right.$, vs SCE in MeCN; $<-1.7 \mathrm{~V}$, vs $\left.\mathrm{Fc} / \mathrm{Fc}^{+}\right)^{17}$, which could be formed via reductive quenching of $[\mathrm{Ru}]^{*}$ by $\mathbf{R h}$-enolate as demonstrated above, to the organic azide or diazo reagent is more feasible.

In summary, Rh-enolate $\left(E_{\mathrm{p}}{ }^{\mathrm{ox}}=0.078 \mathrm{~V}\right.$, vs $\mathrm{Fc} / \mathrm{Fc}^{+}$in $\left.\mathrm{MeCN}\right)$ has a significantly lower oxidative potential than $\mathbf{R h S}\left(E_{\mathrm{p}}^{\text {red }}=1.25 \mathrm{~V}\right.$, vs $\mathrm{Fc} / \mathrm{Fc}^{+}$of $\left.\mathrm{MeCN}\right)$. All these results, in combination with other mechanistic studies, strongly suggest that SET oxidation of $\mathbf{R h}$-enolate by excited $\left[\mathrm{Ru}^{\mathrm{II}}\right]^{*}\left(E_{1 / 2}{ }^{* \mathrm{II} / \mathrm{I}}=\right.$ $+0.77 \mathrm{~V}$, vs SCE in MeCN) is the key step of the photoredox cycle. A SET reduction of 2a or 3a by hereby generated $\left[\mathrm{Ru}(\mathrm{bpy})_{3}\right]^{+}$is also feasible. 


\subsection{Quantum Yield Measurement}

\subsubsection{General information}

All the light sensitive operations were processed in the darkroom under red light. A $150 \mathrm{~W}$ xenon lamp (50\% of light intensity, $420 \pm 5 \mathrm{~nm}$ bandpass filter) was used as the light source. The measured method was designed according to a published procedure with slight modifications. ${ }^{18}$ Photon flux of the spectrophotometer was determined as $7.32 \times 10^{-10}$ einstein/s by standard ferrioxalate actinometry.

\subsubsection{Determination of response factor for GC analysis}

Biphenyl was chosen as the internal standard, the amount of which remained constant for every GC measurement (FID detector, column: HP-5). The amount of product $\mathbf{5 b a}$ is related to the ratios of integrated areas (see figure below).

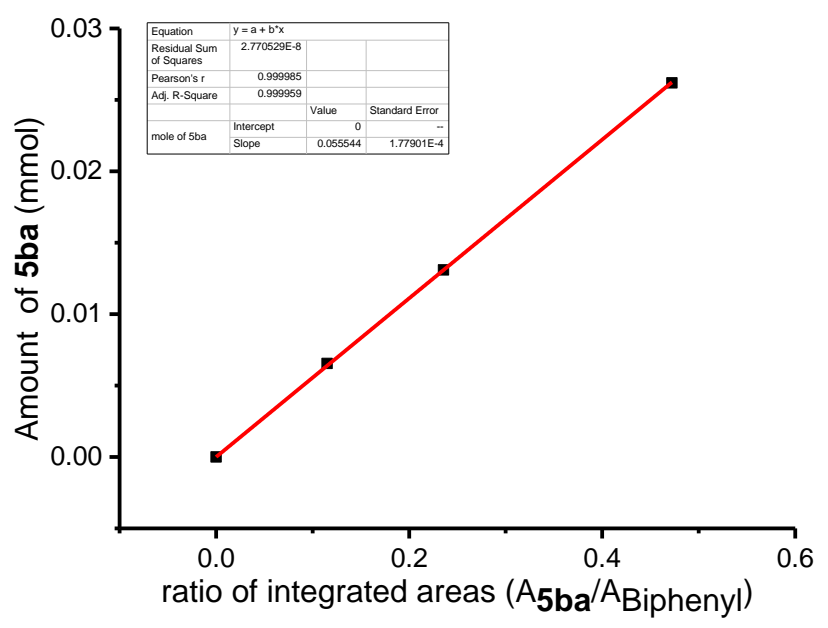

The relation formula was yielded as:

$y=5.554 \times 10^{-2} x$

Where $\mathrm{y}$ is the amount of product $\mathbf{5} \mathbf{b a}(\mathbf{m m o l}), \mathrm{x}$ is the ratio of integrated areas.

\subsubsection{Measurement of quantum yield}

Model reaction:

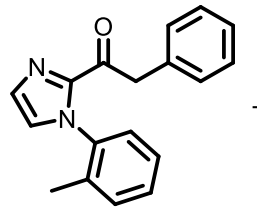

$1 \mathrm{~b}$

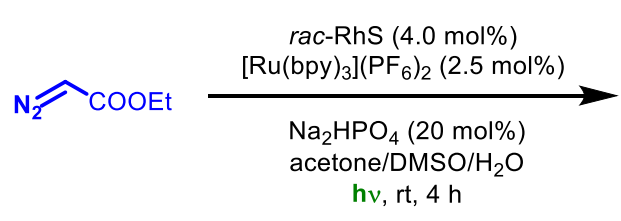

3a

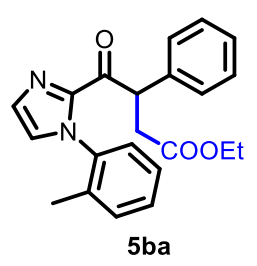

$5 \mathrm{ba}$ 
The Newport instrument for quantum yield determination was set up at a fixed position in a dark room (red light). A screw-top cuvette $(10.0 \mathrm{~mm})$ was charged with $\mathbf{1 b}(55.2 \mathrm{mg}, 0.2 \mathrm{mmol}), \mathbf{3 a}(68.4$ mg, 3.0 equiv), rac-RhS (6.9 mg, 4 mol\%), [Ru(bpy) $)_{3}\left(\mathrm{PF}_{6}\right)_{2}(4.4 \mathrm{mg}, 2.5 \mathrm{~mol} \%), \mathrm{Na}_{2} \mathrm{HPO}_{4}(5.6 \mathrm{mg}$, $20 \mathrm{~mol} \%)$ and $\mathrm{H}_{2} \mathrm{O}(72.0 \mathrm{mg}, 20$ equiv) in acetone/DMSO $(9: 1,1.0 \mathrm{~mL}, 0.2 \mathrm{M})$ and a small magnetic stir bar. The cuvette was degassed with an nitrogen stream for $10 \mathrm{~min}$. After the mixture was thoroughly degassed, the vial was sealed and fixed at the same position as the measurement of photon flux. The reaction mixture was stirred and irradiated for $4 \mathrm{~h}$. After irradiation, the reaction mixture was diluted with ethyl acetate. Then, Biphenyl was added and analyzed by GC.

\subsubsection{Results and discussion}

The amount of $\mathbf{5 b a}$ formed was measured by GC analysis as $1.046 \times 10^{-6} \mathrm{~mol}$.

Therefore, the quantum yield was calculated according to the following equation:

$\Phi=\frac{\text { moles of product }}{\text { moles of absorbed photons }}=\frac{\text { moles of product }}{\text { flux } * \mathrm{t} * \mathrm{f}}=\left(1.046 \times 10^{-6} \mathrm{~mol}\right) /\left(7.32 \times 10^{-10} \times 14400 \times 1\right)$ $=0.099$

Absorbance of at $420 \mathrm{~nm}$ is $>3$ demonstrating that the fraction of light absorbed is $>0.999(\mathrm{f}=1$ $\left.-10^{-\mathrm{A}}\right)$.

The photoredox sensitizer absorbs only a fraction of the overall light at $420 \mathrm{~nm}$ due to the competitive light absorption from present rhodium-enolate intermediate (inner filter effect, see Figure $\mathrm{S} 2)$. Thus, the quantum yield with respect to the photoredox sensitizer $\left[\mathrm{Ru}(\mathrm{bpy})_{3}\right]\left(\mathrm{PF}_{6}\right)_{2}$ at $420 \mathrm{~nm}$ is > 0.099. This value presents overall quantum yield and does not take into account that there is no chain process in this system. 


\section{Experimental and Characterization Data of Products}<smiles>O=C(Nc1c(F)c(F)c(F)c(F)c1F)C(P)c1nccn1-c1ccccc1</smiles>

(S)-2-((Perfluorophenyl)amino)-2-phenyl-1-(1-phenyl-1H-imidazol-2-yl)ethan-1-one (4aa)

According to the general procedure, the reaction of 2-phenyl-1-(1-phenyl-1H-imidazol-2-yl) ethan1-one 1a (26.2 mg, 0.10 mmol), 1-azido-2,3,4,5,6-pentafluorobenzene 2a (62.7 mg, 3.0 equiv), $\Delta$ RhS (3.5 mg, $4 \mathrm{~mol} \%$ ), [Ru(bpy) 3 ( $\left.\mathrm{PF}_{6}\right)_{2}(2.2 \mathrm{mg}, 2.5 \mathrm{~mol} \%), \mathrm{Na}_{2} \mathrm{HPO}_{4}(2.8 \mathrm{mg}, 20 \mathrm{~mol} \%)$ and $\mathrm{H}_{2} \mathrm{O}$ (36.0 mg, 20 equiv) in acetone/DMSO $(9: 1,0.5 \mathrm{~mL}, 0.2 \mathrm{M})$ under nitrogen atmosphere with visible light for 8 hours, afforded $36.3 \mathrm{mg}(82 \%)$ of 4 aa as a yellow oil. Enantiomeric excess was established by HPLC analysis using a Chiralpak IC column, ee = 98\% (HPLC: IC, $254 \mathrm{~nm}, n$-hexane/isopropanol $=95: 5$, flow rate $1 \mathrm{~mL} / \mathrm{min}, 25^{\circ} \mathrm{C}, \mathrm{t}_{\mathrm{r}}($ major $)=5.7 \mathrm{~min}, \mathrm{t}_{\mathrm{r}}($ minor $\left.)=4.9 \mathrm{~min}\right) .[\alpha]_{\mathrm{D}}^{22}=+150.0^{\circ}(c$ 1.0, $\left.\mathrm{CH}_{2} \mathrm{Cl}_{2}\right)$.

${ }^{1} \mathrm{H}$ NMR (500 MHz, $\left.\mathrm{CDCl}_{3}\right) \delta$ 7.51-7.54 (m, 5H), $7.33(\mathrm{~d}, J=1.0 \mathrm{~Hz}, 1 \mathrm{H}), 7.32-7.27(\mathrm{~m}, 2 \mathrm{H}), 7.26-$ $7.21(\mathrm{~m}, 1 \mathrm{H}), 7.18(\mathrm{~d}, J=0.5 \mathrm{~Hz}, 1 \mathrm{H}), 7.14-7.11(\mathrm{~m}, 2 \mathrm{H}), 6.74(\mathrm{~d}, J=9.0 \mathrm{~Hz}, 1 \mathrm{H}), 5.36(\mathrm{~d}, J=9.5$ $\mathrm{Hz}, 1 \mathrm{H})$.

${ }^{13} \mathrm{C} \mathrm{NMR}\left(125 \mathrm{MHz}, \mathrm{CDCl}_{3}\right) \delta 188.1,140.8,137.7,136.8,130.5,129.11,129.07,128.8,128.4,128.1$, $127.7,125.5,63.4-63.2(\mathrm{~m})$.

${ }^{19} \mathrm{~F} \mathrm{NMR}\left(282 \mathrm{MHz}, \mathrm{CDCl}_{3}\right) \delta-157.35--157.51(\mathrm{~m}, 2 \mathrm{~F}),-164.25--164.46(\mathrm{~m}, 2 \mathrm{~F}),-170.41\left(\mathrm{tt}, J_{1}\right.$ $\left.=22.28 \mathrm{~Hz}, J_{2}=5.92 \mathrm{~Hz}, 1 \mathrm{~F}\right)$.

IR (film): $v\left(\mathrm{~cm}^{-1}\right)$ 3367, 1688, 1596, 1517, 1449, 1398, 1346, 1305, 1263, 1188, 1154, 1107, 1072 , $1021,976,911,839,761,732,694,625,585,548,526,498,461$.

HRMS (ESI, $m / z$ ) calcd for $\mathrm{C}_{23} \mathrm{H}_{14} \mathrm{~F}_{5} \mathrm{~N}_{3} \mathrm{ONa}[\mathrm{M}+\mathrm{Na}]^{+}$: 466.0949, found: 466.0946.

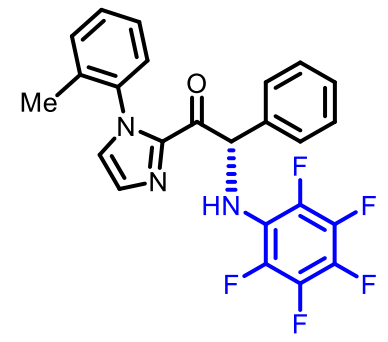




\section{(S)-2-((Perfluorophenyl)amino)-2-phenyl-1-(1-(o-tolyl)-1H-imidazol-2-yl)ethan-1-one (4ba)}

According to the general procedure, the reaction of 2-phenyl-1-(1-(o-tolyl)-1H-imidazol-2-yl)ethan1-one 1 b (27.6 mg, $0.10 \mathrm{mmol}), 1$-azido-2,3,4,5,6-pentafluorobenzene 2a (62.7 mg, 3.0 equiv), $\Delta$ RhS (3.5 mg, 4 mol\%), [Ru(bpy $\left.)_{3}\right]\left(\mathrm{PF}_{6}\right)_{2}(2.2 \mathrm{mg}, 2.5 \mathrm{~mol} \%), \mathrm{Na}_{2} \mathrm{HPO}_{4}(2.8 \mathrm{mg}, 20 \mathrm{~mol} \%)$ and $\mathrm{H}_{2} \mathrm{O}$ (36.0 mg, 20 equiv) in acetone/DMSO $(9: 1,0.5 \mathrm{~mL}, 0.2 \mathrm{M})$ under nitrogen atmosphere with visible light for 13 hours, afforded $36.1 \mathrm{mg}(79 \%)$ of $\mathbf{4 b a}$ as a white solid . Enantiomeric excess was established by HPLC analysis using a Chiralpak OD-H column, ee = 99.1\% (HPLC: OD-H, $254 \mathrm{~nm}$, $n$-hexane/isopropanol $=95: 5$, flow rate $1 \mathrm{~mL} / \mathrm{min}, 25^{\circ} \mathrm{C}, \mathrm{t}_{\mathrm{r}}($ major $)=7.5 \mathrm{~min}, \mathrm{t}_{\mathrm{r}}($ minor $\left.)=6.8 \mathrm{~min}\right)$. $[\alpha]_{\mathrm{D}}^{22}=+156.4^{\circ}\left(c 1.0, \mathrm{CH}_{2} \mathrm{Cl}_{2}\right)$.

${ }^{1} \mathrm{H}$ NMR $\left(500 \mathrm{MHz}, \mathrm{CDCl}_{3}\right) \delta$ 7.47-7.31 (m, 5H), 7.30-7.18 (m, 5H), $7.09(\mathrm{~d}, J=1.0 \mathrm{~Hz}, 1 \mathrm{H}), 6.78$ $(\mathrm{d}, J=8.0 \mathrm{~Hz}, 1 \mathrm{H}$, other rotamer), 6.76-6.68 $(\mathrm{m}, 1 \mathrm{H}), 5.41-5.28(\mathrm{~m}, 1 \mathrm{H}), 2.04(\mathrm{~s}, 3 \mathrm{H}$, other rotamer), $1.41(\mathrm{~s}, 3 \mathrm{H})$.

${ }^{13} \mathrm{C}$ NMR $\left(125 \mathrm{MHz}, \mathrm{CDCl}_{3}\right) \delta 186.0,185.9,141.3,141.2,137.2,137.1,136.7,134.7,134.2,130.81$, $130.78,130.7,129.5,129.4,128.8,128.7,128.4,128.3,128.1,128.0,127.18,127.16126 .7,126.6$, 126.4, 126.0, $63.3(\mathrm{t}, J=3.4 \mathrm{~Hz}), 63.0$ (t, $J=3.7 \mathrm{~Hz}), 17.2$, 16.1. (Mixture of two rotation isomers). ${ }^{19} \mathrm{~F}$ NMR $\left(282 \mathrm{MHz}, \mathrm{CDCl}_{3}\right) \delta-157.30--157.68(\mathrm{~m}, 2 \mathrm{~F}),-164.31--164.51(\mathrm{~m}, 2 \mathrm{~F}),-170.31--$ $170.69(\mathrm{~m}, 1 \mathrm{~F})$.

IR (film): $v\left(\mathrm{~cm}^{-1}\right)$ 3370, 2923, 1300, 1686, 1515, 1459, 1400, 1023, 980, 959, 768, 700, 673, 647, $618,558$.

HRMS (ESI, $m / z$ ) calcd for $\mathrm{C}_{24} \mathrm{H}_{16} \mathrm{~F}_{5} \mathrm{~N}_{3} \mathrm{ONa}[\mathrm{M}+\mathrm{Na}]^{+}$: 480.1106 , found: 480.1102 .

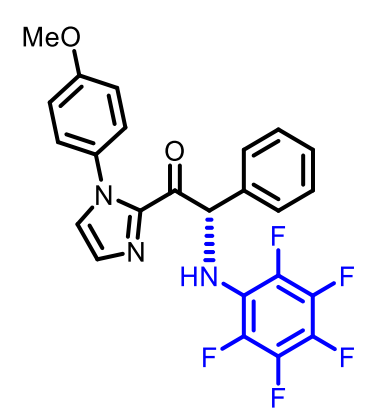

(S)-1-(1-(4-Methoxyphenyl)-1H-imidazol-2-yl)-2-((perfluorophenyl)amino)-2-phenylethan-1one (4ca)

According to the general procedure, the reaction of 1-(1-(4-methoxyphenyl)-1H-imidazol-2-yl)-2phenylethan-1-one 1c (29.2 mg, $0.10 \mathrm{mmol}), 1$-azido-2,3,4,5,6-pentafluorobenzene 2a (62.7 mg, 3.0 
equiv), $\Delta$-RhS (3.5 mg, 4 mol\%), [Ru(bpy) 3 ] $\left(\mathrm{PF}_{6}\right)_{2}(2.2 \mathrm{mg}, 2.5 \mathrm{~mol} \%), \mathrm{Na}_{2} \mathrm{HPO}_{4}(2.8 \mathrm{mg}, 20 \mathrm{~mol} \%)$ and $\mathrm{H}_{2} \mathrm{O}$ (36.0 mg, 20 equiv) in acetone/DMSO (9:1, $\left.0.5 \mathrm{~mL}, 0.2 \mathrm{M}\right)$ under nitrogen atmosphere with visible light for 6 hours, afforded $37.7 \mathrm{mg}(80 \%)$ of $\mathbf{4 c a}$ as a yellow oil. Enantiomeric excess was established by HPLC analysis using a Chiralpak IC column, ee $=99 \%$ (HPLC: IC, 254 nm, $n$ hexane/isopropanol $=90: 10$, flow rate $1 \mathrm{~mL} / \mathrm{min}, 25^{\circ} \mathrm{C}, \mathrm{t}_{\mathrm{r}}($ major $)=6.3 \mathrm{~min}, \mathrm{t}_{\mathrm{r}}($ minor $\left.)=5.3 \mathrm{~min}\right)$. $[\alpha]_{\mathrm{D}}^{22}=+173.0^{\circ}\left(c 1.0, \mathrm{CH}_{2} \mathrm{Cl}_{2}\right)$.

${ }^{1} \mathrm{H}$ NMR $\left(500 \mathrm{MHz}, \mathrm{CDCl}_{3}\right) \delta$ 7.48-7.44 (m, 2H), 7.32-7.27 (m, 3H), 7.25-7.20 (m, 1H), $7.14(\mathrm{~d}, J=$ $1.0 \mathrm{~Hz}, 1 \mathrm{H}), 7.07-7.02(\mathrm{~m}, 2 \mathrm{H}), 6.97-6.91(\mathrm{~m}, 2 \mathrm{H}), 6.73(\mathrm{~d}, J=9.0 \mathrm{~Hz}, 1 \mathrm{H}), 5.36(\mathrm{~d}, J=9.0 \mathrm{~Hz}, 1 \mathrm{H})$, $3.86(\mathrm{~s}, 3 \mathrm{H})$.

${ }^{13} \mathrm{C} \mathrm{NMR}\left(125 \mathrm{MHz}, \mathrm{CDCl}_{3}\right) \delta 186.0,159.8,140.8,136.8,130.4,128.8,128.3,128.10,128.06$ 126.6, 114.2, $63.2(\mathrm{t}, J=3.5 \mathrm{~Hz}), 55.5$. (Missing one ${ }^{13} \mathrm{C}$ signal)

${ }^{19} \mathrm{~F} \mathrm{NMR}\left(282 \mathrm{MHz}, \mathrm{CDCl}_{3}\right) \delta-157.74--157.90(\mathrm{~m}, 2 \mathrm{~F}),-164.67--164.89(\mathrm{~m}, 2 \mathrm{~F}),-170.85\left(\mathrm{tt}, J_{1}\right.$ $\left.=22.00 \mathrm{~Hz}, J_{2}=5.78 \mathrm{~Hz}, 1 \mathrm{~F}\right)$.

IR (film): $v\left(\mathrm{~cm}^{-1}\right)$ 3367, 2964, 2791, 1687, 1608, 1515, 1453, 1397, 1346, 1298, 1249, 1177, 1109, $1071,1020,976,911,836,779,732,698,675,625,585,499$.

HRMS (ESI, $m / z$ ) calcd for $\mathrm{C}_{24} \mathrm{H}_{16} \mathrm{~F}_{5} \mathrm{~N}_{3} \mathrm{O}_{2} \mathrm{Na}[\mathrm{M}+\mathrm{Na}]^{+}$: 496.1055 , found: 496.1054 .

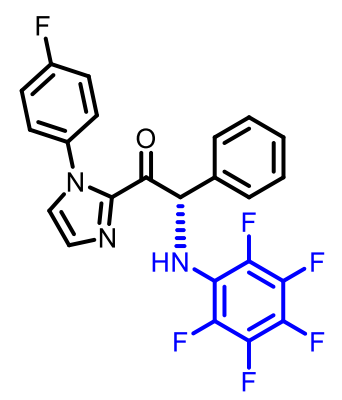

(S)-1-(1-(4-Fluorophenyl)-1H-imidazol-2-yl)-2-((perfluorophenyl)amino)-2-phenylethan-1-one (4da)

According to the general procedure, the reaction of 1-(1-(4-fluorophenyl)-1H-imidazol-2-yl)-2phenylethan-1-one 1d (28.0 mg, $0.10 \mathrm{mmol}), 1$-azido-2,3,4,5,6-pentafluorobenzene $2 \mathbf{a}$ (62.7 mg, 3.0 equiv), $\Delta$-RhS (3.5 mg, 4 mol\%), [Ru(bpy) 3 ] $\left(\mathrm{PF}_{6}\right)_{2}(2.2 \mathrm{mg}, 2.5 \mathrm{~mol} \%), \mathrm{Na}_{2} \mathrm{HPO}_{4}(2.8 \mathrm{mg}, 20 \mathrm{~mol} \%$ ) and $\mathrm{H}_{2} \mathrm{O}$ (36.0 mg, 20 equiv) in acetone/DMSO (9:1, $\left.0.5 \mathrm{~mL}, 0.2 \mathrm{M}\right)$ under nitrogen atmosphere with visible light for 14 hours, afforded $34.9 \mathrm{mg}$ (76\%) of 4da as a yellow oil. Enantiomeric excess was established by HPLC analysis using a Chiralpak AD-H column, ee = 98\% (HPLC: AD-H, 254 nm, $n$ - 
hexane/isopropanol $=90: 10$, flow rate $1 \mathrm{~mL} / \mathrm{min}, 25^{\circ} \mathrm{C}, \mathrm{t}_{\mathrm{r}}($ major $)=7.0 \mathrm{~min}, \mathrm{t}_{\mathrm{r}}($ minor $\left.)=5.4 \mathrm{~min}\right)$. $[\alpha]_{\mathrm{D}}^{22}=+150.0^{\circ}\left(c 1.0, \mathrm{CH}_{2} \mathrm{Cl}_{2}\right)$.

${ }^{1} \mathrm{H}$ NMR (500 MHz, $\left.\mathrm{CDCl}_{3}\right) \delta$ 7.48-7.43 (m, 2H), 7.32 (d, J=1.0 Hz, 1H), 7.31-7.27 (m, 2H), 7.26$7.21(\mathrm{~m}, 1 \mathrm{H}), 7.16-7.09(\mathrm{~m}, 5 \mathrm{H}), 6.72(\mathrm{~d}, J=9.5 \mathrm{~Hz}, 1 \mathrm{H}), 5.32(\mathrm{~d}, J=9.5 \mathrm{~Hz}, 1 \mathrm{H})$.

${ }^{13} \mathrm{C}$ NMR (125 MHz, $\left.\mathrm{CDCl}_{3}\right) \delta 186.2,162.6(\mathrm{~d}, J=249.3), 140.9,136.7,133.6(\mathrm{~d}, J=3.3 \mathrm{~Hz}), 130.7$, $128.9,128.4,128.1,127.5,127.4(\mathrm{~d}, J=8.4 \mathrm{~Hz}), 116.1(\mathrm{~d}, J=22.9 \mathrm{~Hz}), 63.3(\mathrm{t}, J=3.3 \mathrm{~Hz})$.

${ }^{19} \mathrm{~F}$ NMR (282 MHz, $\left.\mathrm{CDCl}_{3}\right) \delta-111.60(\mathrm{~s}, 1 \mathrm{~F}),-157.36--157.51(\mathrm{~m}, 2 \mathrm{~F}),-164.17$ - -164.38 (m, $2 \mathrm{~F}),-170.23\left(\mathrm{tt}, J_{l}=21.86 \mathrm{~Hz}, J_{2}=6.06 \mathrm{~Hz}, 1 \mathrm{~F}\right)$.

IR (film): $v\left(\mathrm{~cm}^{-1}\right) 3368,1687,1514,1452,1399,1345,1310,1227,1154,1099,1070,1023,978$, $914,842,784,735,699,625,585,545,464,423$.

HRMS (ESI, $m / z$ ) calcd for $\mathrm{C}_{23} \mathrm{H}_{14} \mathrm{~F}_{6} \mathrm{~N}_{3} \mathrm{O}[\mathrm{M}+\mathrm{H}]^{+}$: 462.1036, found: 462.1031 .

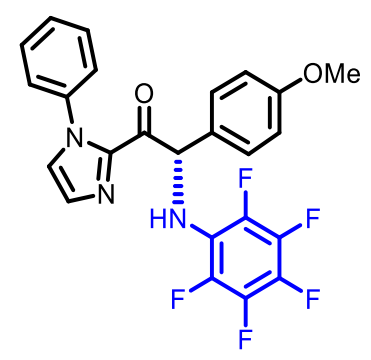

(S)-2-(4-Methoxyphenyl)-2-((perfluorophenyl)amino)-1-(1-phenyl-1H-imidazol-2-yl)ethan-1one (4ea)

According to the general procedure, the reaction of 2-(4-methoxyphenyl)-1-(1-phenyl-1H-imidazol2-yl)ethan-1-one 4e (29.2 mg, $0.10 \mathrm{mmol})$, 1-azido-2,3,4,5,6-pentafluorobenzene 2a (62.7 mg, 3.0 equiv), $\Delta$-RhS (3.5 mg, 4 mol\%), [Ru(bpy) 3 ](PF6)2 (2.2 mg, $2.5 \mathrm{~mol} \%)$, DIPEA (2.6 mg, $20 \mathrm{~mol} \%)$ and $\mathrm{H}_{2} \mathrm{O}$ (36.0 mg, 20 equiv) in acetone/DMSO (9:1, $\left.1.0 \mathrm{~mL}, 0.1 \mathrm{M}\right)$ under nitrogen atmosphere with visible light for 17 hours, afforded $33.6 \mathrm{mg}(71 \%)$ of $\mathbf{4 e a}$ as a yellow oil. Enantiomeric excess was established by HPLC analysis using a Chiralpak OD-H column, ee = 99.1\% (HPLC: OD-H, $254 \mathrm{~nm}$, $n$-hexane/isopropanol $=95: 5$, flow rate $1 \mathrm{~mL} / \mathrm{min}, 25^{\circ} \mathrm{C}, \mathrm{t}_{\mathrm{r}}($ major $)=10.1 \mathrm{~min}, \mathrm{t}_{\mathrm{r}}($ minor $\left.)=8.6 \mathrm{~min}\right)$. $[\alpha]_{\mathrm{D}}^{22}=+202.6^{\circ}\left(c 1.0, \mathrm{CH}_{2} \mathrm{Cl}_{2}\right)$.

${ }^{1} \mathrm{H}$ NMR (500 MHz, $\left.\mathrm{CDCl}_{3}\right) \delta$ 7.51-7.42 (m, 3H), 7.39-7.34 (m, 2H), 7.32-7.30 (m, 1H), 7.18-7.10 (m, 1H), 7.15-7.10 (m, 2H), 6.84-6.78 (m, 2H), 6.67 (br s, 1H), 5.30 (br s, 1H), 3.75 (s, 3H).

${ }^{13} \mathrm{C} \mathrm{NMR}\left(125 \mathrm{MHz}, \mathrm{CDCl}_{3}\right) \delta 186.0,159.5,140.8,137.7,130.5,129.4,129.11,129.06,128.6,127.6$, 
$125.6,114.3,62.6(\mathrm{t}, J=3.3 \mathrm{~Hz}), 55.1$.

${ }^{19} \mathrm{~F} \mathrm{NMR}\left(282 \mathrm{MHz}, \mathrm{CDCl}_{3}\right) \delta-157.30--157.47(\mathrm{~m}, 2 \mathrm{~F}),-164.32--164.55(\mathrm{~m}, 2 \mathrm{~F}),-170.46\left(\mathrm{tt}, J_{1}\right.$ $\left.=22.00 \mathrm{~Hz}, J_{2}=5.78 \mathrm{~Hz}, 1 \mathrm{~F}\right)$.

IR (film): $v\left(\mathrm{~cm}^{-1}\right)$ 3353, 2931, 2963, 1670, 1603, 1512, 1468, 1446, 1399, 1306, 1258, 1175, 1098, 1023, 979, 956, 913, 818, 786, 761, 731, 691, 573.

HRMS (EI, $m / z$ ) calcd for $\mathrm{C}_{24} \mathrm{H}_{16} \mathrm{~F}_{5} \mathrm{~N}_{3} \mathrm{O}_{2}[\mathrm{M}]^{+}$: 473.1163, found: 473.1173 .

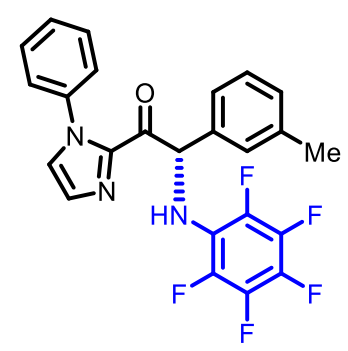

(S)-2-((Perfluorophenyl)amino)-1-(1-phenyl-1H-imidazol-2-yl)-2-(m-tolyl)ethan-1-one (4fa)

According to the general procedure, the reaction of 1-(1-phenyl-1H-imidazol-2-yl)-2-( $m$-tolyl) ethan1-one 1 f (27.6 mg, $0.10 \mathrm{mmol}), 1$-azido-2,3,4,5,6-pentafluorobenzene 2a (62.7 mg, 3.0 equiv), $\Delta$ RhS (3.5 mg, $4 \mathrm{~mol} \%)$, [Ru(bpy) 3 ] $\left(\mathrm{PF}_{6}\right)_{2}(2.2 \mathrm{mg}, 2.5 \mathrm{~mol} \%), \mathrm{Na}_{2} \mathrm{HPO}_{4}(2.8 \mathrm{mg}, 20 \mathrm{~mol} \%)$ and $\mathrm{H}_{2} \mathrm{O}$ (36.0 mg, 20 equiv) in acetone/DMSO (9:1, $0.5 \mathrm{~mL}, 0.2 \mathrm{M})$ under nitrogen atmosphere with visible light for 7 hours, afforded $35.1 \mathrm{mg}(77 \%)$ of $\mathbf{4 f a}$ as a brown solid. Enantiomeric excess was established by HPLC analysis using a Chiralpak AD-H column, ee = 98\% (HPLC: AD-H, $254 \mathrm{~nm}, n$ hexane/isopropanol $=90: 10$, flow rate $1 \mathrm{~mL} / \mathrm{min}, 25^{\circ} \mathrm{C}, \mathrm{t}_{\mathrm{r}}($ major $\left.)=6.4 \mathrm{~min}, \mathrm{t}_{\mathrm{r}}(\operatorname{minor})=4.7 \mathrm{~min}\right)$. $[\alpha]_{\mathrm{D}}^{22}=+177.0^{\circ}\left(c 1.0, \mathrm{CH}_{2} \mathrm{Cl}_{2}\right)$.

${ }^{1} \mathrm{H}$ NMR (500 MHz, $\left.\mathrm{CDCl}_{3}\right) \delta$ 7.51-7.40 (m, 3H), 7.32 (d, $\left.J=1.5 \mathrm{~Hz}, 1 \mathrm{H}\right)$, 7.29-7.27 (m, 2H), 7.26$2.24(\mathrm{~m}, 2 \mathrm{H}$, other rotamer), 7.23-2.18 (m, 1H), $7.18(\mathrm{~d}, J=1.5 \mathrm{~Hz}, 1 \mathrm{H}), 7.17-7.10(\mathrm{~m}, 2 \mathrm{H})$, 7.07$7.06(\mathrm{~m}, 1 \mathrm{H}), 7.05-7.03(\mathrm{~m}, 1 \mathrm{H}$, other rotamer $), 6.69(\mathrm{~d}, J=14.5,1 \mathrm{H}), 5.32-5.26(\mathrm{~m}, 1 \mathrm{H}), 2.31(\mathrm{~s}$, $3 \mathrm{H})$.

${ }^{13} \mathrm{C} \mathrm{NMR}\left(125 \mathrm{MHz}, \mathrm{CDCl}_{3}\right) \delta 186.2,140.9,138.6,137.7,136.6,130.5,129.2,129.10,129.05,128.7$, 127.7, 125.6, 125.2, $63.3(\mathrm{t}, J=5.9 \mathrm{~Hz}), 21.4$. (Missing one ${ }^{13} \mathrm{C}$ signal)

${ }^{19} \mathrm{~F} \mathrm{NMR}\left(282 \mathrm{MHz}, \mathrm{CDCl}_{3}\right) \delta-157.36--157.52(\mathrm{~m}, 2 \mathrm{~F}),-164.30--164.52(\mathrm{~m}, 2 \mathrm{~F}),-170.53\left(\mathrm{tt}, J_{1}\right.$ $\left.=22.00 \mathrm{~Hz}, J_{2}=5.78 \mathrm{~Hz}, 1 \mathrm{~F}\right)$.

IR (film): $v\left(\mathrm{~cm}^{-1}\right) 3371,1682,1519,1447,1395,1306,1025,980,957,915,843,768,727,697,670$, 
HRMS (ESI, $m / z$ ) calcd for $\mathrm{C}_{24} \mathrm{H}_{16} \mathrm{~F}_{5} \mathrm{~N}_{3} \mathrm{ONa}[\mathrm{M}+\mathrm{Na}]^{+}$: 480.1106 , found: 480.1106 .

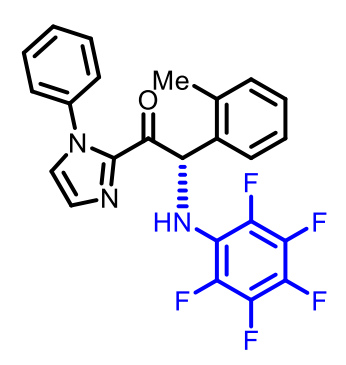

\section{(S)-2-((Perfluorophenyl)amino)-1-(1-phenyl-1H-imidazol-2-yl)-2-(o-tolyl)ethan-1-one (4ga)}

According to the general procedure, the reaction of 1-(1-phenyl-1H-imidazol-2-yl)-2-(o-tolyl) ethan1-one $1 \mathrm{~g}$ (27.6 mg, $0.10 \mathrm{mmol}), 1$-azido-2,3,4,5,6-pentafluorobenzene 2a (62.7 mg, 3.0 equiv), $\Delta$ RhS (3.5 mg, $4 \mathrm{~mol} \%),\left[\mathrm{Ru}(\mathrm{bpy})_{3}\right]\left(\mathrm{PF}_{6}\right)_{2}(2.2 \mathrm{mg}, 2.5 \mathrm{~mol} \%), \mathrm{Na}_{2} \mathrm{HPO}_{4}(2.8 \mathrm{mg}, 20 \mathrm{~mol} \%)$ and $\mathrm{H}_{2} \mathrm{O}$ (36.0 mg, 20 equiv) in acetone/DMSO $(9: 1,0.5 \mathrm{~mL}, 0.2 \mathrm{M})$ under nitrogen atmosphere with visible light for 10 hours, afforded $25.0 \mathrm{mg}(55 \%)$ of $\mathbf{4 g a}$ as a white solid. Enantiomeric excess was established by HPLC analysis using a Chiralpak AD-H column, ee = 98\% (HPLC: AD-H, 254 nm, $n$ hexane/isopropanol $=90: 10$, flow rate $1 \mathrm{~mL} / \mathrm{min}, 25^{\circ} \mathrm{C}, \mathrm{t}_{\mathrm{r}}($ major $)=6.0 \mathrm{~min}, \mathrm{t}_{\mathrm{r}}($ minor $\left.)=4.6 \mathrm{~min}\right)$. $[\alpha]_{\mathrm{D}}^{22}=+214.2^{\circ}\left(c 1.0, \mathrm{CH}_{2} \mathrm{Cl}_{2}\right)$.

${ }^{1} \mathrm{H}$ NMR (500 MHz, $\left.\mathrm{CDCl}_{3}\right) \delta$ 7.49-7.41 (m, 3H), 7.25 (d, $\left.J=0.5 \mathrm{~Hz}, 1 \mathrm{H}\right)$, 7.24-7.18 (m, 3H), 7.17$7.14(\mathrm{~m}, 2 \mathrm{H}), 7.14-7.11(\mathrm{~m}, 2 \mathrm{H}), 6.90$ (br s, 1H), 4.86 (br s, 1H), 2.72 (s, 3H).

${ }^{13} \mathrm{C}$ NMR $\left(125 \mathrm{MHz}, \mathrm{CDCl}_{3}\right) \delta 187.3,141.4,138.3,137.7,135.0,131.5,130.3,129.1,129.0,128.6$, $127.5,126.6,126.4,125.5,60.4(\mathrm{t}, J=3.6 \mathrm{~Hz}), 19.4$.

${ }^{19} \mathrm{~F}$ NMR $\left(282 \mathrm{MHz}, \mathrm{CDCl}_{3}\right) \delta-157.40--157.56(\mathrm{~m}, 2 \mathrm{~F}),-164.25--164.47(\mathrm{~m}, 2 \mathrm{~F}),-170.29\left(\mathrm{tt}, J_{1}\right.$ $\left.=22.00 \mathrm{~Hz}, J_{2}=5.64 \mathrm{~Hz}, 1 \mathrm{~F}\right)$.

IR (film): $v\left(\mathrm{~cm}^{-1}\right)$ 3366, 3128, 2923, 2855, 1692, 1597, 1514, 1464, 1402, 1351, 1307, 1247, 1211, 1152, 1120, 1086, 1011, 964, 912, 875, 844, 787, 761, 733, 691, 653, 625, 579, 561, 508.

HRMS (ESI, $m / z$ ) calcd for $\mathrm{C}_{24} \mathrm{H}_{16} \mathrm{~F}_{5} \mathrm{~N}_{3} \mathrm{ONa}[\mathrm{M}+\mathrm{Na}]^{+}: 480.1106$, found: 480.1105 . 


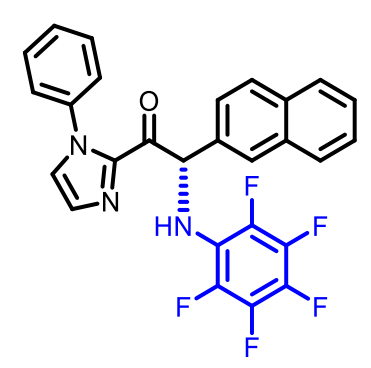

(S)-2-(Naphthalen-2-yl)-2-((perfluorophenyl)amino)-1-(1-phenyl-1H-imidazol-2-yl)ethan-1one (4ha)

According to the general procedure, the reaction of 2-(naphthalen-2-yl)-1-(1-phenyl-1H-imidazol- 2yl)ethan-1-one $\mathbf{1 h}$ (31.2 mg, $0.10 \mathrm{mmol}), 1$-azido-2,3,4,5,6-pentafluorobenzene $2 \mathbf{a}$ (62.7 $\mathrm{mg}, 3.0$ equiv), $\Delta$-RhS (3.5 mg, 4 mol\%), [Ru(bpy) 3 ] $\left(\mathrm{PF}_{6}\right)_{2}(2.2 \mathrm{mg}, 2.5 \mathrm{~mol} \%), \mathrm{Na}_{2} \mathrm{HPO}_{4}(2.8 \mathrm{mg}, 20 \mathrm{~mol} \%$ ) and $\mathrm{H}_{2} \mathrm{O}$ (36.0 mg, 20 equiv) in acetone/DMSO (9:1, $\left.0.5 \mathrm{~mL}, 0.2 \mathrm{M}\right)$ under nitrogen atmosphere with visible light for 17 hours, afforded $24.8 \mathrm{mg}(50 \%)$ of tha as a yellow oil. Enantiomeric excess was established by HPLC analysis using a Chiralpak AD-H column, ee = 95\% (HPLC: AD-H, $254 \mathrm{~nm}, n$ hexane/isopropanol $=90: 10$, flow rate $1 \mathrm{~mL} / \mathrm{min}, 25^{\circ} \mathrm{C}, \mathrm{t}_{\mathrm{r}}($ major $)=8.3 \mathrm{~min}, \mathrm{t}_{\mathrm{r}}($ minor $\left.)=6.4 \mathrm{~min}\right)$. $[\alpha]_{\mathrm{D}}^{22}=+260.2^{\circ}\left(c 1.0, \mathrm{CH}_{2} \mathrm{Cl}_{2}\right)$.

${ }^{1} \mathrm{H}$ NMR $\left(500 \mathrm{MHz}, \mathrm{CDCl}_{3}\right) \delta 7.98(\mathrm{~s}, 1 \mathrm{H}), 7.85-7.81(\mathrm{~m}, 1 \mathrm{H}), 7.80-7.76(\mathrm{~m}, 2 \mathrm{H}), 7.55\left(\mathrm{dd}, J_{1}=8.5\right.$ $\left.\mathrm{Hz}, J_{2}=1.5 \mathrm{~Hz}, 1 \mathrm{H}\right), 7.50-7.41(\mathrm{~m}, 5 \mathrm{H}), 7.33(\mathrm{~d}, J=1.0 \mathrm{~Hz}, 1 \mathrm{H}), 7.16(\mathrm{~d}, J=1.0 \mathrm{~Hz}, 1 \mathrm{H}), 7.14-7.10$ (m, 2H), 6.93-6.88 (m, 1H) 5.50-5.45 (m, 1H).

${ }^{13} \mathrm{C}$ NMR (125 MHz, $\left.\mathrm{CDCl}_{3}\right) \delta 185.8,140.8,137.6,134.2,133.3,133.1,130.6,129.1,128.8,128.21$, 128.16, 127.8, 127.6, 126.5, 126.3, 125.6, 125.0, 63.3 (t, $J=3.6 \mathrm{~Hz})$. (Missing one ${ }^{13} \mathrm{C}$ signal) ${ }^{19} \mathrm{~F}$ NMR $\left(282 \mathrm{MHz}, \mathrm{CDCl}_{3}\right) \delta-157.32--157.49(\mathrm{~m}, 2 \mathrm{~F}),-164.18--164.39(\mathrm{~m}, 2 \mathrm{~F}),-170.29\left(\mathrm{tt}, J_{l}\right.$ $\left.=22.00 \mathrm{~Hz}, J_{2}=5.92 \mathrm{~Hz}, 1 \mathrm{~F}\right)$.

IR (film): $v\left(\mathrm{~cm}^{-1}\right)$ 3367, 1687, 1597, 1517, 1448, 1398, 1305, 1266, 1180, 1154, 1123, 1099, 1022 , 971, 909, 843, 813, 734, 691, 668, 641, 610, 544, 503, 474.

HRMS (ESI, $m / z$ ) calcd for $\mathrm{C}_{27} \mathrm{H}_{16} \mathrm{~F}_{5} \mathrm{~N}_{3} \mathrm{ONa}[\mathrm{M}+\mathrm{Na}]^{+}:$516.1106, found: 516.1106.

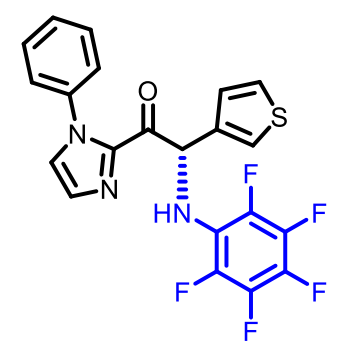




\section{(S)-2-((Perfluorophenyl)amino)-1-(1-phenyl-1H-imidazol-2-yl)-2-(thiophen-3-yl)ethan-1-one}

(4ia)

According to the general procedure, the reaction of 1-(1-phenyl-1H-imidazol-2-yl)-2-(thiophen-3yl)ethan-1-one $\mathbf{1 i}(26.8 \mathrm{mg}, 0.10 \mathrm{mmol}), 1$-azido-2,3,4,5,6-pentafluorobenzene $\mathbf{2 a}$ (62.7 mg, 3.0 equiv), $\Delta$-RhS (3.5 mg, 4 mol\%), [Ru(bpy) 3$]\left(\mathrm{PF}_{6}\right)_{2}(2.2 \mathrm{mg}, 2.5 \mathrm{~mol} \%)$, DIPEA (2.6 mg, $\left.20 \mathrm{~mol} \%\right)$ and $\mathrm{H}_{2} \mathrm{O}$ (36.0 mg, 20 equiv) in acetone/DMSO (9:1, $\left.1.0 \mathrm{~mL}, 0.1 \mathrm{M}\right)$ under nitrogen atmosphere with visible light for 40 hours, afforded $19.0 \mathrm{mg}(42 \%)$ of 4 ia as a yellow oil. Enantiomeric excess was established by HPLC analysis using a Chiralpak AD-H column, ee = 93\% (HPLC: AD-H, 254 nm, $n$ hexane/isopropanol $=90: 10$, flow rate $1 \mathrm{~mL} / \mathrm{min}, 25^{\circ} \mathrm{C}, \mathrm{t}_{\mathrm{r}}($ major $)=7.8 \mathrm{~min}, \mathrm{t}_{\mathrm{r}}($ minor $\left.)=6.2 \mathrm{~min}\right)$. $[\alpha]_{\mathrm{D}}^{22}=+123.8^{\circ}\left(c 0.4, \mathrm{CH}_{2} \mathrm{Cl}_{2}\right)$.

${ }^{1} \mathrm{H}$ NMR $\left(500 \mathrm{MHz}, \mathrm{CDCl}_{3}\right) \delta$ 7.50-7.44 (m, 3H), 7.38-7.35 (m, 1H), $7.34(\mathrm{~d}, J=1.0 \mathrm{~Hz}, 1 \mathrm{H}), 7.23$ $\left(\mathrm{dd}, J_{l}=5.0 \mathrm{~Hz}, J_{2}=3.0 \mathrm{~Hz}, 1 \mathrm{H}\right), 7.21(\mathrm{~d}, J=1.0 \mathrm{~Hz}, 1 \mathrm{H}), 7.18-7.14(\mathrm{~m}, 2 \mathrm{H}), 7.07\left(\mathrm{dd}, J_{l}=5.0 \mathrm{~Hz}\right.$, $\left.J_{2}=1.0 \mathrm{~Hz}, 1 \mathrm{H}\right), 6.83(\mathrm{~d}, J=9.5 \mathrm{~Hz}, 1 \mathrm{H}), 5.21(\mathrm{~d}, J=9.5 \mathrm{~Hz}, 1 \mathrm{H})$.

${ }^{13} \mathrm{C}$ NMR $\left(125 \mathrm{MHz}, \mathrm{CDCl}_{3}\right) \delta 185.5,140.7,137.6,137.5,130.6,129.2,129.1,127.8,126.5,126.3$, $125.6,124.6,59.3(\mathrm{t}, J=3.9 \mathrm{~Hz})$.

${ }^{19} \mathrm{~F}$ NMR $\left(282 \mathrm{MHz}, \mathrm{CDCl}_{3}\right) \delta-157.70--157.86(\mathrm{~m}, 2 \mathrm{~F}),-164.51--164.72(\mathrm{~m}, 2 \mathrm{~F}),-170.50\left(\mathrm{tt}, J_{1}\right.$ $\left.=22.14 \mathrm{~Hz}, J_{2}=5.50 \mathrm{~Hz}, 1 \mathrm{~F}\right)$.

IR (film): $v\left(\mathrm{~cm}^{-1}\right)$ 3364, 1687, 1599, 1515, 1448, 1397, 1348, 1305, 1260, 1182, 1151, 1098, 1021, 972, 912, 840, 763, 731, 693, 664, 546, 460.

HRMS (ESI, $m / z$ ) calcd for $\mathrm{C}_{21} \mathrm{H}_{12} \mathrm{~F}_{5} \mathrm{~N}_{3} \mathrm{OSNa}[\mathrm{M}+\mathrm{Na}]^{+}: 472.0513$, found: 472.0510 .

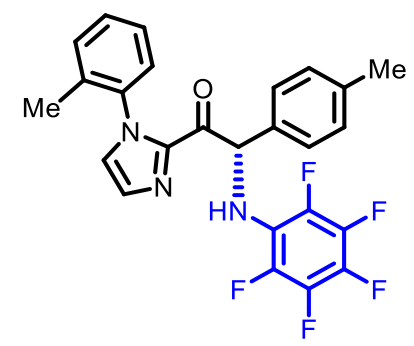

\section{(S)-2-((Perfluorophenyl)amino)-2-(p-tolyl)-1-(1-(o-tolyl)-1H-imidazol-2-yl)ethan-1-one (4ja)}

According to the general procedure, the reaction of 2-(p-tolyl)-1-(1-(o-tolyl)- $1 H$-imidazol-2yl)ethan-1-one $\mathbf{1 j}$ (29.0 mg, $0.10 \mathrm{mmol})$, 1-azido-2,3,4,5,6-pentafluorobenzene 2a (62.7 mg, 3.0 equiv), $\Delta$-RhS (3.5 mg, 4 mol\%), [Ru(bpy) 3 ](PF6) 2 (2.2 mg, $2.5 \mathrm{~mol} \%)$, DIPEA (2.6 mg, $20 \mathrm{~mol} \%$ ) 
and $\mathrm{H}_{2} \mathrm{O}$ (36.0 mg, 20 equiv) in acetone/DMSO (9:1, $\left.1.0 \mathrm{~mL}, 0.1 \mathrm{M}\right)$ under nitrogen atmosphere with visible light for 12 hours, afforded $37.5 \mathrm{mg}(80 \%)$ of $\mathbf{4 j a}$ as a yellow oil. Enantiomeric excess was established by HPLC analysis using a Chiralpak OD-H column, ee = 99.4\% (HPLC: OD-H, 254 nm, $n$-hexane/isopropanol $=90: 10$, flow rate $1 \mathrm{~mL} / \mathrm{min}, 25^{\circ} \mathrm{C}, \mathrm{t}_{\mathrm{r}}($ major $\left.)=7.1 \mathrm{~min}, \mathrm{t}_{\mathrm{r}}(\operatorname{minor})=6.0 \mathrm{~min}\right)$. $[\alpha]_{\mathrm{D}}^{22}=+241.0^{\circ}\left(c 1.0, \mathrm{CH}_{2} \mathrm{Cl}_{2}\right)$.

${ }^{1} \mathrm{H}$ NMR (500 MHz, $\left.\mathrm{CDCl}_{3}\right) \delta$ 7.42-7.27 (m, 5H), 7.26-7.19 (m, 2H), 7.10-7.03 (m, 3H), $6.80(\mathrm{~d}, J=$ $8.0 \mathrm{~Hz}, 1 \mathrm{H}$, other rotamer), 6.71-6.64 (m, 1H), 5.40-5.23 (m, 1H), 2.27 (s, 3H, other rotamer), 2.25 (s, 3H), 2.03 (s, 3H, other rotamer), $1.46(\mathrm{~s}, 3 \mathrm{H})$.

${ }^{13} \mathrm{C}$ NMR $\left(125 \mathrm{MHz}, \mathrm{CDCl}_{3}\right) \delta 186.1,186.0,141.4,141.3,138.2,138.1,137.3,137.2,134.7,134.3$, $133.7,130.83$, 130.76, 130.73, 130.68, 129.6, 129.5, 129.43, 129.37, 128.00, 127.9, 127.1, 126.7, 126.6, 126.5, 126.1, $63.0(\mathrm{t}, J=3.3 \mathrm{~Hz}), 62.8(\mathrm{t}, J=3.9 \mathrm{~Hz}), 21.11,21.08,17.2$, 16.3. (Mixture of two rotation isomers).

${ }^{19} \mathrm{~F}$ NMR $\left(282 \mathrm{MHz}, \mathrm{CDCl}_{3}\right) \delta-157.51--157.75(\mathrm{~m}, 2 \mathrm{~F}),-164.54--164.75(\mathrm{~m}, 2 \mathrm{~F}),-170.61--$ $170.96(\mathrm{~m}, 1 \mathrm{~F})$.

IR (film): $v\left(\mathrm{~cm}^{-1}\right)$ 3368, 2925, 2857, 1687, 1517, 1453, 1400, 1346, 1303, 1262, 1186, 1153, 1095, 1021, 967, 912, 851, 803, 765, 727, 653, 616, 555, 496, 455.

HRMS (ESI, $m / z$ ) calcd for $\mathrm{C}_{25} \mathrm{H}_{18} \mathrm{~F}_{5} \mathrm{~N}_{3} \mathrm{ONa}[\mathrm{M}+\mathrm{Na}]^{+}$: 494.1262, found: 494.1263.

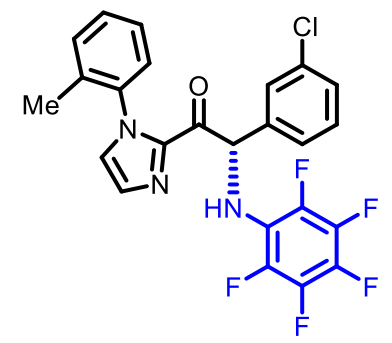

(S)-2-(3-Chlorophenyl)-2-((perfluorophenyl)amino)-1-(1-(o-tolyl)-1H-imidazol-2-yl)ethan-1one (4ka)

According to the general procedure, the reaction of 2-(3-chlorophenyl)-1-(1-(o-tolyl)-1H-imidazol2-yl)ethan-1-one 1k (31.1 mg, $0.10 \mathrm{mmol}), 1$-azido-2,3,4,5,6-pentafluorobenzene 2a (62.7 mg, 3.0 equiv), $\Delta$-RhS (3.5 mg, 4 mol\%), [Ru(bpy) 3$]\left(\mathrm{PF}_{6}\right)_{2}(2.2 \mathrm{mg}, 2.5 \mathrm{~mol} \%), \mathrm{Na}_{2} \mathrm{HPO}_{4}(2.8 \mathrm{mg}, 20 \mathrm{~mol} \%)$ and $\mathrm{H}_{2} \mathrm{O}$ (36.0 mg, 20 equiv) in acetone/DMSO (9:1, $\left.0.5 \mathrm{~mL}, 0.2 \mathrm{M}\right)$ under nitrogen atmosphere with visible light for 48 hours, afforded $23.3 \mathrm{mg}(47 \%)$ of $\mathbf{4 k a}$ as a white solid. Enantiomeric excess was 
established by HPLC analysis using a Chiralpak OD-H column, ee = 97\% (HPLC: OD-H, 254 nm, $n$-hexane/isopropanol $=95: 5$, flow rate $1 \mathrm{~mL} / \mathrm{min}, 25^{\circ} \mathrm{C}, \mathrm{t}_{\mathrm{r}}($ major $)=7.7 \mathrm{~min}, \mathrm{t}_{\mathrm{r}}($ minor $\left.)=6.9 \mathrm{~min}\right)$. $[\alpha]_{\mathrm{D}}^{22}=+162.0^{\circ}\left(c 1.0, \mathrm{CH}_{2} \mathrm{Cl}_{2}\right)$.

${ }^{1} \mathrm{H}$ NMR $\left(500 \mathrm{MHz}, \mathrm{CDCl}_{3}\right) \delta$ 7.47-7.43 (m, 1H), 7.43-7.32 (m, 4H), 7.28-7.18 (m, 4H), 7.13-7.10 $(\mathrm{m}, 1 \mathrm{H}), 6.83(\mathrm{~d}, J=8.0 \mathrm{~Hz}, 1 \mathrm{H}$, other rotamer), 6.72-6.64 $(\mathrm{m}, 1 \mathrm{H}), 5.40-5.28(\mathrm{~m}, 1 \mathrm{H}), 2.03(\mathrm{~s}, 3 \mathrm{H}$, other rotamer), $1.48(\mathrm{~s}, 3 \mathrm{H})$.

${ }^{13} \mathrm{C}$ NMR $\left(125 \mathrm{MHz}, \mathrm{CDCl}_{3}\right) \delta 185.3,185.2,141.0,140.9,139.0,138.9,137.03,136.95,134.7$, 134.64, 134.60, 134.2, 131.0, 130.94, 130.86, 130.1, 130.0, 129.6, 129.5, 128.63, 128.58, 128.2, 128.1, 127.50, 127.48, 126.8, 126.7, 126.4, 126.2, 126.0, $62.6(\mathrm{t}, J=3.8 \mathrm{~Hz}), 62.4(\mathrm{t}, J=3.5 \mathrm{~Hz})$, 17.2, 16.2. (Mixture of two rotation isomers).

${ }^{19} \mathrm{~F}$ NMR (282 MHz, $\left.\mathrm{CDCl}_{3}\right) \delta-157.89--158.16(\mathrm{~m}, 2 \mathrm{~F}),-164.29--164.51(\mathrm{~m}, 2 \mathrm{~F}),-170.25--$ $170.61(\mathrm{~m}, 1 \mathrm{~F})$.

IR (film): $v\left(\mathrm{~cm}^{-1}\right)$ 3366, 1684, 1517, 1446, 1397, 1303, 1187, 1155, 1081, 1024, 981, 956, 911, 846, $765,723,696,664,630,585,554,453$.

HRMS (ESI, $m / z$ ) calcd for $\mathrm{C}_{24} \mathrm{H}_{15} \mathrm{ClF}_{5} \mathrm{~N}_{3} \mathrm{ONa}[\mathrm{M}+\mathrm{Na}]^{+}:$514.0716, found: 514.0715.

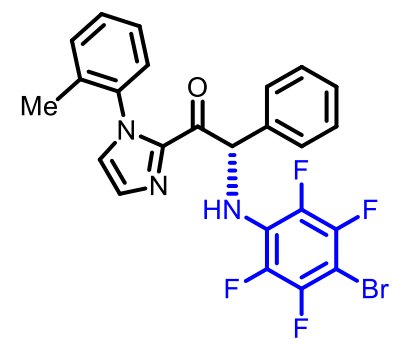

(S)-2-((4-Bromo-2,3,5,6-tetrafluorophenyl)amino)-2-phenyl-1-(1-(o-tolyl)-1H-imidazol-2yl)ethan-1-one (4bb)

According to the general procedure, the reaction of 2-phenyl-1-(1-(o-tolyl)-1H-imidazol-2- yl)ethan1-one $\mathbf{1 b}$ (27.6 mg, $0.10 \mathrm{mmol}$ ), 1-azido-4-bromo-2,3,5,6-tetrafluorobenzene $\mathbf{2 b}$ ( $80.7 \mathrm{mg}, 3.0$ equiv), $\Delta$-RhS (3.5 mg, $4 \mathrm{~mol} \%),\left[\mathrm{Ru}(\mathrm{bpy})_{3}\right]\left(\mathrm{PF}_{6}\right)_{2}(2.2 \mathrm{mg}, 2.5 \mathrm{~mol} \%), \mathrm{Na}_{2} \mathrm{HPO}_{4}(2.8 \mathrm{mg}, 20 \mathrm{~mol} \%)$ and $\mathrm{H}_{2} \mathrm{O}$ (36.0 mg, 20 equiv) in acetone/DMSO (9:1, $\left.0.5 \mathrm{~mL}, 0.2 \mathrm{M}\right)$ under nitrogen atmosphere with visible light for 5 hours, afforded $46.5 \mathrm{mg}(90 \%)$ of $\mathbf{4 b b}$ as a white solid. Enantiomeric excess was established by HPLC analysis using a Chiralpak OD-H column, ee = 99.4\% (HPLC: OD-H, $254 \mathrm{~nm}$, $n$-hexane/isopropanol $=95: 5$, flow rate $1 \mathrm{~mL} / \mathrm{min}, 25^{\circ} \mathrm{C}, \mathrm{t}_{\mathrm{r}}($ major $)=8.3 \mathrm{~min}, \mathrm{t}_{\mathrm{r}}($ minor $\left.)=7.4 \mathrm{~min}\right)$. 
$[\alpha]_{\mathrm{D}}^{22}=+150.8^{\circ}\left(c 1.0, \mathrm{CH}_{2} \mathrm{Cl}_{2}\right)$.

${ }^{1} \mathrm{H}$ NMR $\left(500 \mathrm{MHz}, \mathrm{CDCl}_{3}\right) \delta$ 7.50-7.43 (m, 2H), 7.42-7.19 (m, 8H), 7.11-7.09 (m, 1H), 6.86-6.81 (m, $1 \mathrm{H}$, other rotamer), 6.81-6.75 $(\mathrm{m}, 1 \mathrm{H}), 5.67-5.61(\mathrm{~m}, 1 \mathrm{H}), 5.60-5.56(\mathrm{~m}, 1 \mathrm{H}$, other rotamer), 2.04 (s, 3H, other rotamer), $1.41(\mathrm{~s}, 3 \mathrm{H})$.

${ }^{13} \mathrm{C}$ NMR $\left(125 \mathrm{MHz}, \mathrm{CDCl}_{3}\right) \delta$ 185.8, 185.7, 146.2-145.8 (m), 144.3-144.0 (m), 141.4-141.0 (m), 139.2-138.7 (m), 137.3-136.9 (m), 136.8, 134.7, 134.3, 130.82, 130.80, 130.77, 130.7, 129.5, 129.4, $128.84,128.76,128.38,128.35128 .1,128.0,127.21,127.18126 .7,126.6,126.4,126.0,62.5(\mathrm{t}, J=$ $3.7 \mathrm{~Hz}), 62.2(\mathrm{t}, J=3.8 \mathrm{~Hz}), 17.2,16.0$. (Mixture of two rotation isomers).

${ }^{19} \mathrm{~F}$ NMR $\left(282 \mathrm{MHz}, \mathrm{CDCl}_{3}\right) \delta-136.00--136.20(\mathrm{~m}, 2 \mathrm{~F}),-156.03--156.31(\mathrm{~m}, 2 \mathrm{~F})$.

IR (film): $v\left(\mathrm{~cm}^{-1}\right)$ 3350, 2923, 2856, 1688, 1641, 1495, 1455, 1404, 1299, 1148, 1074, 1026, 981, $948,913,858,762,736,702,675,619,579,494,454$.

HRMS (ESI, $m / z$ ) calcd for $\mathrm{C}_{24} \mathrm{H}_{16} \mathrm{BrF}_{4} \mathrm{~N}_{3} \mathrm{ONa}[\mathrm{M}+\mathrm{Na}]^{+}$: 540.0305, found: 540.0305.

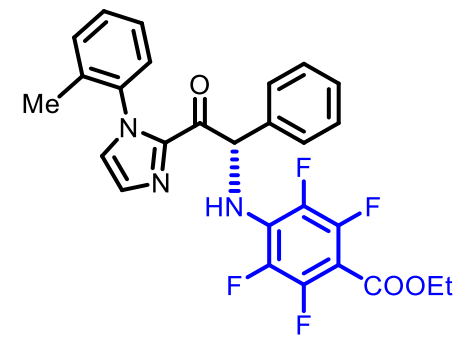

Ethyl (S)-2,3,5,6-tetrafluoro-4-((2-oxo-1-phenyl-2-(1-(o-tolyl)-1H-imidazol-2-yl)ethyl)amino) benzoate (4bc)

According to the general procedure, the reaction of 2-phenyl-1-(1-(o-tolyl)-1H-imidazol-2- yl)ethan1-one $\mathbf{1 b}$ (27.6 mg, $0.10 \mathrm{mmol}$ ), ethyl 4-azido-2,3,5,6-tetrafluorobenzoate 2c (78.9 mg, 3.0 equiv), $\Delta$-RhS (3.5 mg, $4 \mathrm{~mol} \%),\left[\mathrm{Ru}(\mathrm{bpy})_{3}\right]\left(\mathrm{PF}_{6}\right)_{2}(2.2 \mathrm{mg}, 2.5 \mathrm{~mol} \%), \mathrm{Na}_{2} \mathrm{HPO}_{4}(2.8 \mathrm{mg}, 20 \mathrm{~mol} \%)$ and $\mathrm{H}_{2} \mathrm{O}(36.0 \mathrm{mg}, 20$ equiv) in acetone/DMSO (9:1, $0.5 \mathrm{~mL}, 0.2 \mathrm{M})$ under nitrogen atmosphere with visible light for 6 hours, afforded $41.3 \mathrm{mg}(81 \%)$ of $\mathbf{4 b c}$ as a white solid. Enantiomeric excess was established by HPLC analysis using a Chiralpak AD-H column, ee = 99.6\% (HPLC: AD-H, 254 nm, $n$-hexane/isopropanol $=90: 10$, flow rate $1 \mathrm{~mL} / \mathrm{min}, 25^{\circ} \mathrm{C}, \mathrm{t}_{\mathrm{r}}($ major $\left.)=8.7 \mathrm{~min}, \mathrm{t}_{\mathrm{r}}(\operatorname{minor})=5.3 \mathrm{~min}\right)$. $[\alpha]_{\mathrm{D}}^{22}=+101.0^{\circ}\left(c 1.0, \mathrm{CH}_{2} \mathrm{Cl}_{2}\right)$.

${ }^{1} \mathrm{H}$ NMR $\left(300 \mathrm{MHz}, \mathrm{CDCl}_{3}\right) \delta$ 7.51-7.44 (m, 2H), 7.43-7.18 (m, 8H), 7.12-7.09 (m, 1H), 6.92-6.82 (m, 1H), $6.77(\mathrm{~d}, J=7.8 \mathrm{~Hz}, 1 \mathrm{H}$, other rotamer), 5.94-5.81 (m, 1H), $4.34(\mathrm{q}, J=7.1 \mathrm{~Hz}, 2 \mathrm{H}), 2.04$ (s, 
$3 \mathrm{H}$, other rotamer), $1.40(\mathrm{~s}, 3 \mathrm{H}), 1.34(\mathrm{t}, J=7.4 \mathrm{~Hz}, 3 \mathrm{H})$.

${ }^{13} \mathrm{C}$ NMR $\left(125 \mathrm{MHz}, \mathrm{CDCl}_{3}\right) \delta 185.4,185.2,160.6-160.4(\mathrm{~m}), 147.0-147.3(\mathrm{~m}), 145.3-145.0(\mathrm{~m})$, 141.1, 140.9, 138.1-137.7 (m), 137.04, 136.98, 136.7, 136.1-135.8 (m), 134.7, 134.2, 130.9, 130.81, $130.80,130.77,129.5,129.4,129.12,129.09,129.06,129.03,129.00,128.9,128.8,128.5,128.4$, 128.1, 128.0, 127.31, 127.28, 126.7, 126.6, 126.4, 126.0, $62.6(\mathrm{t}, J=3.8 \mathrm{~Hz}), 62.3(\mathrm{t}, J=3.8 \mathrm{~Hz})$, $61.6,17.2,16.0,14.1$. (Mixture of two rotation isomers).

${ }^{19} \mathrm{~F}$ NMR $\left(282 \mathrm{MHz}, \mathrm{CDCl}_{3}\right) \delta-140.71--140.86(\mathrm{~m}, 2 \mathrm{~F}),-158.78--156.97$ (m, 2F).

IR (film): $v\left(\mathrm{~cm}^{-1}\right)$ 3349, 2973, 2932, 1718, 1687, 1651, 1533, 1495, 1452, 1399, 1371, 1310, 1233, $1154,1022,986,957,913,842,789,759,699,624,561$.

HRMS (ESI, $m / z$ ) calcd for $\mathrm{C}_{27} \mathrm{H}_{21} \mathrm{~F}_{4} \mathrm{~N}_{3} \mathrm{O}_{3} \mathrm{Na}[\mathrm{M}+\mathrm{Na}]^{+}:$534.1411, found: 534.1416.

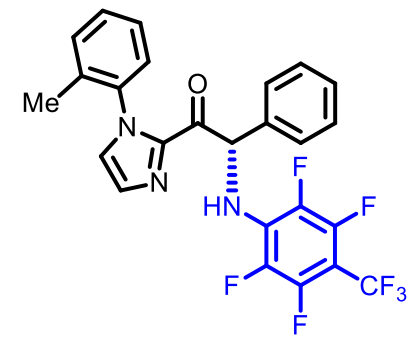

(S)-2-Phenyl-2-((2,3,5,6-tetrafluoro-4-(trifluoromethyl)phenyl)amino)-1-(1-(o-tolyl)-1H-

\section{imidazol-2-yl)ethan-1-one (4bd)}

According to the general procedure, the reaction of 2-phenyl-1-(1-(o-tolyl)-1H-imidazol-2- yl)ethan1-one $1 \mathbf{b}(27.6 \mathrm{mg}, 0.10 \mathrm{mmol})$, 1-azido-2,3,5,6-tetrafluoro-4-(trifluoromethyl)benzene $\mathbf{2 d}$ (77.7 mg, 3.0 equiv), $\Delta$-RhS (3.5 mg, $4 \mathrm{~mol} \%),\left[\mathrm{Ru}(\mathrm{bpy})_{3}\right]\left(\mathrm{PF}_{6}\right)_{2}(2.2 \mathrm{mg}, 2.5 \mathrm{~mol} \%), \mathrm{Na}_{2} \mathrm{HPO}_{4}(2.8 \mathrm{mg}, 20$ mol\%) and $\mathrm{H}_{2} \mathrm{O}$ (36.0 mg, 20 equiv) in acetone/DMSO (9:1, $\left.0.5 \mathrm{~mL}, 0.2 \mathrm{M}\right)$ under nitrogen atmosphere with visible light for 6 hours, afforded $35.6 \mathrm{mg}(70 \%)$ of $\mathbf{4 b d}$ as a yellow oil. Enantiomeric excess was established by HPLC analysis using a Chiralpak AD-H column, ee $=99 \%$ (HPLC: OD-H, $254 \mathrm{~nm}, n$-hexane/isopropanol $=90: 10$, flow rate $1 \mathrm{~mL} / \mathrm{min}, 25{ }^{\circ} \mathrm{C}, \mathrm{t}_{\mathrm{r}}$ (major) $=5.1$ $\min , \mathrm{t}_{\mathrm{r}}($ minor $\left.)=4.1 \mathrm{~min}\right) .[\alpha]_{\mathrm{D}}^{22}=+127.8^{\circ}\left(\right.$ c 1.0, $\left.\mathrm{CH}_{2} \mathrm{Cl}_{2}\right)$.

${ }^{1} \mathrm{H}$ NMR $\left(500 \mathrm{MHz}, \mathrm{CDCl}_{3}\right) \delta$ 7.51-7.44 (m, 2H), 7.43-7.19 (m, 8H), 7.12-7.09 (m, 1H), 6.91-6.87 $(\mathrm{m}, 1 \mathrm{H}), 6.87-6.82(\mathrm{~m}, 1 \mathrm{H}$, other rotamer), $6.77(\mathrm{~d}, J=8.0 \mathrm{~Hz}, 1 \mathrm{H}$, other rotamer), 5.97-5.91 (m, 1H), 5.89-5.83 (m, 1H, other rotamer), $2.04(\mathrm{~s}, 3 \mathrm{H}$, other rotamer), $1.39(\mathrm{~s}, 3 \mathrm{H})$.

${ }^{13} \mathrm{C}$ NMR $\left(125 \mathrm{MHz}, \mathrm{CDCl}_{3}\right) \delta 185.2,185.1,141.1,140.8,137.02,136.96,136.6,134.7,134.2$, 
130.93, 130.85, 130.8, 129.53, 129.47, 129.0, 128.9, 128.6, 128.5, 128.1, 128.0, 127.38, 127.36126 .8 , 126.7, 126.4, 126.0, $63.0(\mathrm{t}, J=3.6 \mathrm{~Hz}), 62.7(\mathrm{t}, J=3.9 \mathrm{~Hz}), 17.2,16.1$. (Mixture of two rotation isomers).

${ }^{19} \mathrm{~F} \mathrm{NMR}\left(282 \mathrm{MHz}, \mathrm{CDCl}_{3}\right) \delta-55.1(\mathrm{t}, J=20.87,3 \mathrm{~F}),-143.25--143.50(\mathrm{~m}, 2 \mathrm{~F}),-158.28--158.50$ $(\mathrm{m}, 2 \mathrm{~F})$.

IR (film): $v\left(\mathrm{~cm}^{-1}\right)$ 3373, 1688, 1655, 1539, 1505, 1457, 1400, 1330, 1304, 1235, 1179, 1130, 1078, 1025, 983, 957, 911, 884, 835, 764, 734, 703, 672, 626, 560, 494.

HRMS (ESI, $m / z$ ) calcd for $\mathrm{C}_{23} \mathrm{H}_{16} \mathrm{~F}_{7} \mathrm{~N}_{3} \mathrm{ONa}[\mathrm{M}+\mathrm{Na}]^{+}:$530.1074, found: 530.1075 .

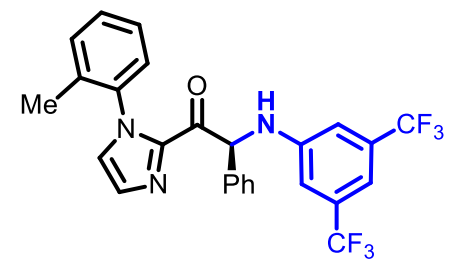

(S)-2-((3,5-Bis(trifluoromethyl)phenyl)amino)-2-phenyl-1-(1-(o-tolyl)-1H-imidazol-2-yl)ethan-

\section{1-one (4be)}

According to the general procedure, the reaction of 2-phenyl-1-(1-(o-tolyl)-1H-imidazol-2-yl) ethan1-one 1 b (27.6 mg, $0.10 \mathrm{mmol}$ ), 1-azido-3,5-bis(trifluoromethyl)benzene 2e (76.6 mg, 3.0 equiv), $\Delta$ RhS (3.5 mg, $4 \mathrm{~mol} \%$ ), [Ru(bpy) $3\left(\mathrm{PF}_{6}\right)_{2}(2.2 \mathrm{mg}, 2.5 \mathrm{~mol} \%), \mathrm{Na}_{2} \mathrm{HPO}_{4}(2.8 \mathrm{mg}, 20 \mathrm{~mol} \%)$ and $\mathrm{H}_{2} \mathrm{O}$ (36.0 mg, 20 equiv) in acetone/DMSO $(9: 1,0.5 \mathrm{~mL}, 0.2 \mathrm{M})$ under nitrogen atmosphere with visible light for 7 hours, afforded $29.8 \mathrm{mg}$ (59\%) of 4 be as a colorless oil. Enantiomeric excess was established by HPLC analysis using a Chiralpak OD-H column, ee = 98\% (HPLC: OD-H, 254 nm, $n$-hexane/isopropanol $=95: 5$, flow rate $1 \mathrm{~mL} / \mathrm{min}, 25^{\circ} \mathrm{C}, \mathrm{t}_{\mathrm{r}}($ major $\left.)=8.5 \mathrm{~min}, \mathrm{t}_{\mathrm{r}}(\operatorname{minor})=7.0 \mathrm{~min}\right)$. $[\alpha]_{\mathrm{D}}^{22}=+97.0^{\circ}\left(c 1.0, \mathrm{CH}_{2} \mathrm{Cl}_{2}\right)$.

${ }^{1} \mathrm{H}$ NMR $\left(300 \mathrm{MHz}, \mathrm{CDCl}_{3}\right) \delta$ 7.60-7.53 (m, 2H), 7.43-7.16 (m, 8H), 7.13-7.09 (m, 2H), 6.98-6.94 (m, 2H), $6.78(\mathrm{~d}, J=7.8 \mathrm{~Hz}, 1 \mathrm{H}$, other rotamer), 6.56-6.49 (m, 1H), 5.79 (d, $J=7.2 \mathrm{~Hz}, 1 \mathrm{H}), 5.73(\mathrm{~d}$, $J=7.2 \mathrm{~Hz}, 1 \mathrm{H}$, other rotamer), $2.00(\mathrm{~s}, 3 \mathrm{H}$, other rotamer), $1.40(\mathrm{~s}, 3 \mathrm{H})$.

${ }^{13} \mathrm{C}$ NMR (125 MHz, $\left.\mathrm{CDCl}_{3}\right) \delta 186.0,185.9,146.5,146.4,141.4,141.3,137.1,137.0,136.1,134.6$, 134.2, 132.3 (q, $J=97.5 \mathrm{~Hz}) 130.80,130.78,130.72,130.7,129.43,129.37,129.0,128.9,128.39$, 128.38, 128.2, 128.1, 127.4, 126.0, 123.4 (q, $J=271.1 \mathrm{~Hz}), 112.61,112.58,110.6-110.4(\mathrm{~m}), 61.9$, 61.7, 17.2, 16.0 (Mixture of two rotation isomers).

${ }^{19} \mathrm{~F} \mathrm{NMR}\left(282 \mathrm{MHz}, \mathrm{CDCl}_{3}\right) \delta-63.40(6 \mathrm{~F}),-63.40$ (other rotamer). 
IR (film): $v\left(\mathrm{~cm}^{-1}\right)$ 3381, 3066, 2927, 1674, 1624, 1501, 1450, 1394, 1274, 1172, 1126, 1028, 996, $969,927,867,762,726,690,644,606,529,405$.

HRMS (EI, $m / z$ ) calcd for $\mathrm{C}_{26} \mathrm{H}_{19} \mathrm{~F}_{6} \mathrm{~N}_{3} \mathrm{O}[\mathrm{M}]^{+}:$503.1432, found: 503.1447.

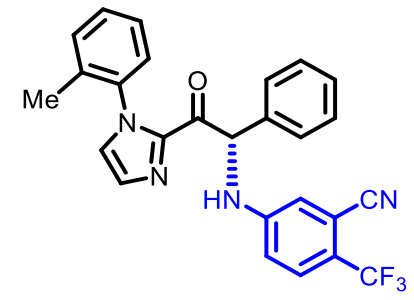

(S)-5-((2-Oxo-1-phenyl-2-(1-(o-tolyl)-1H-imidazol-2-yl)ethyl)amino)-2-

(trifluoromethyl)benzonitrile (4bf)

According to the general procedure, the reaction of 2-phenyl-1-(1-(o-tolyl)-1H-imidazol-2-yl)ethan1-one $\mathbf{1 b}$ (27.6 mg, $0.10 \mathrm{mmol}), 5$-azido-2-(trifluoromethyl)benzonitrile $\mathbf{2 f}$ (63.6 mg, 3.0 equiv), $\Delta$ RhS (3.5 mg, 4 mol\%), [Ru(bpy) $3\left(\mathrm{PF}_{6}\right)_{2}(2.2 \mathrm{mg}, 2.5 \mathrm{~mol} \%), \mathrm{Na}_{2} \mathrm{HPO}_{4}(2.8 \mathrm{mg}, 20 \mathrm{~mol} \%)$ and $\mathrm{H}_{2} \mathrm{O}$ (36.0 mg, 20 equiv) in acetone/DMSO $(9: 1,0.5 \mathrm{~mL}, 0.2 \mathrm{M})$ under nitrogen atmosphere with visible light for 6 hours, afforded $33.9 \mathrm{mg}(74 \%)$ of $\mathbf{4 b f}$ as a yellow solid. Enantiomeric excess was established by HPLC analysis using a Chiralpak AD-H column, ee = 96\% (HPLC: AD-H, 254 nm, $n$ hexane/isopropanol $=80: 20$, flow rate $1 \mathrm{~mL} / \mathrm{min}, 25^{\circ} \mathrm{C}, \mathrm{t}_{\mathrm{r}}($ major $)=8.9 \mathrm{~min}, \mathrm{t}_{\mathrm{r}}($ minor $\left.)=16.0 \mathrm{~min}\right)$. $[\alpha]_{\mathrm{D}}^{22}=+114.4^{\circ}\left(c 1.0, \mathrm{CH}_{2} \mathrm{Cl}_{2}\right)$.

${ }^{1} \mathrm{H}$ NMR $\left(500 \mathrm{MHz}, \mathrm{CDCl}_{3}\right) \delta$ 7.57-7.50 (m, 2H), 7.48-7.45 (m, 1H), 7.43-7.17 (m, 8H), 7.15-7.13 $(\mathrm{m}, 1 \mathrm{H}), 6.89\left(\mathrm{dd}, J_{1}=7.5 \mathrm{~Hz}, J_{2}=2.0 \mathrm{~Hz}, 1 \mathrm{H}\right), 6.75(\mathrm{~d}, J=8.0 \mathrm{~Hz}, 1 \mathrm{H}$, other rotamer), 6.70-6.65 (m, 1H), $6.53(\mathrm{~d}, J=6.5 \mathrm{~Hz}, 1 \mathrm{H}), 6.49(\mathrm{~d}, J=6.5 \mathrm{~Hz}, 1 \mathrm{H}$, other rotamer), 6.14-6.10 (m, 1H), 2.00 (s, $3 \mathrm{H}$, other rotamer), 1.37 (s, 3H).

${ }^{13} \mathrm{C}$ NMR (125 MHz, $\left.\mathrm{CDCl}_{3}\right) \delta 185.2,185.1,148.8,148.7,141.1,141.0,136.9,136.8,136.1,135.60$, 135.57, 134.6, 134.24, 134.15, 134.0, 130.9, 130.83, 130.79, 129.5, 129.4, 129.1, 129.0, 128.6, 128.2, 128.0, 127.6, 126.72, 126.67, 126.3, 126.0, 123.5, 121.3, 116.9, 114.6, 111.2, 96.2, 61.6, 61.3, 17.2, 16.0. (Mixture of two rotation isomers).

${ }^{19} \mathrm{~F} \mathrm{NMR}\left(282 \mathrm{MHz}, \mathrm{CDCl}_{3}\right) \delta-63.097(3 \mathrm{~F}),-63.102$ (3F, other rotamer).

IR (film): $v\left(\mathrm{~cm}^{-1}\right)$ 3336, 2922, 2223, 1697, 1608, 1522, 1497, 1447, 1402, 1352, 1274, 1172, 1131, $1025,842,768,738,701,673,557,454$.

HRMS (ESI, $m / z$ ) calcd for $\mathrm{C}_{26} \mathrm{H}_{19} \mathrm{~F}_{3} \mathrm{~N}_{4} \mathrm{ONa}[\mathrm{M}+\mathrm{Na}]^{+}$: 483.1403, found: 483.1405 . 
(N)

(S)-4-((2-Oxo-1-phenyl-2-(1-(o-tolyl)-1H-imidazol-2-yl)ethyl)amino)phthalonitrile (4bg)

According to the general procedure, the reaction of 2-phenyl-1-(1-(o-tolyl)-1H-imidazol-2- yl)ethan1-one $\mathbf{1 b}$ (27.6 mg, $0.10 \mathrm{mmol})$, 4-azidophthalonitrile $\mathbf{2 g}$ (50.7 mg, 3.0 equiv), $\Delta$-RhS (3.5 mg, 4 mol\%), $\left[\mathrm{Ru}(\mathrm{bpy})_{3}\right]\left(\mathrm{PF}_{6}\right)_{2}(2.2 \mathrm{mg}, 2.5 \mathrm{~mol} \%), \mathrm{Na}_{2} \mathrm{HPO}_{4}(2.8 \mathrm{mg}, 20 \mathrm{~mol} \%)$ and $\mathrm{H}_{2} \mathrm{O}(36.0 \mathrm{mg}, 20$ equiv) in acetone/DMSO $(9: 1,0.5 \mathrm{~mL}, 0.2 \mathrm{M})$ under nitrogen atmosphere with visible light for 6 hours, afforded $37.0 \mathrm{mg}(89 \%)$ of $\mathbf{4 b g}$ as a yellow solid. Enantiomeric excess was established by HPLC analysis using a Chiralpak AD-H column, ee $=99 \%$ (HPLC: AD-H, 254 nm, $n$ hexane/isopropanol $=60: 40$, flow rate $1 \mathrm{~mL} / \mathrm{min}, 25^{\circ} \mathrm{C}, \mathrm{t}_{\mathrm{r}}($ major $)=8.2 \mathrm{~min}, \mathrm{t}_{\mathrm{r}}($ minor $\left.)=17.3 \mathrm{~min}\right)$. $[\alpha]_{\mathrm{D}}^{22}=+111.4^{\circ}\left(c 1.0, \mathrm{CH}_{2} \mathrm{Cl}_{2}\right)$.

${ }^{1} \mathrm{H}$ NMR (500 MHz, $\left.\mathrm{CDCl}_{3}\right) \delta$ 7.56-7.50 (m, 2H), 7.44-7.37 (m, 2H), 7.37-7.23 (m, 5H), 7.22-7.16 (m, 2H), 7.15-7.13 (m, 1H), 6.86-6.83 (m, 1H), 6.78-6.72 (m, 1H), 6.78-6.72 (m, 1H, other rotamer), $6.50(\mathrm{~d}, J=6.5 \mathrm{~Hz}, 1 \mathrm{H}), 6.46(\mathrm{~d}, J=6.5 \mathrm{~Hz}, 1 \mathrm{H}$, other rotamer $), 6.21-6.15(\mathrm{~m}, 1 \mathrm{H}), 2.01(\mathrm{~s}, 3 \mathrm{H}$, other rotamer), $1.36(\mathrm{~s}, 3 \mathrm{H})$.

${ }^{13} \mathrm{C}$ NMR $\left(125 \mathrm{MHz}, \mathrm{CDCl}_{3}\right) \delta 184.9,184.8,148.9,148.8,141.0,140.9,136.9,136.8,135.3,135.2$, 134.6, 134.5, 134.1, 130.94, 130.86, 130.82, 130.79, 129.5, 129.4, 129.14, 129.10, 128.7, 128.1, 128.0, 127.7, 126.70, 126.66, 126.3, 126.0, 117.0, 116.6, 116.4, 115.8, 102.2, 61.5, 61.3, 17.2, 15.9. (Mixture of two rotation isomers)

IR (film): $v\left(\mathrm{~cm}^{-1}\right)$ 3366, 3063, 2923, 2220, 1685, 1596, 1514, 1454, 1398, 1346, 1304, 1257, 1023, $968,910,832,764,731,703,673,521,492,453$,

HRMS (ESI, $m / z$ ) calcd for $\mathrm{C}_{26} \mathrm{H}_{19} \mathrm{~N}_{5} \mathrm{ONa}[\mathrm{M}+\mathrm{Na}]^{+}$: 440.1482, found: 440.1482 . 
<smiles>Cc1ccccc1-n1ccnc1C(=O)C(Nc1c(F)c(F)c(C(F)(F)F)c(F)c1F)c1ccccc1</smiles>

(S)-2-((4-(Azidodifluoromethyl)-2,3,5,6-tetrafluorophenyl)amino)-2-phenyl-1-(1-(o-tolyl)-1Himidazol-2-yl)ethan-1-one (4bh)

According to the general procedure, the reaction of 2-phenyl-1-(1-(o-tolyl)-1H-imidazol-2-yl) ethan1-one $\mathbf{1 b}$ (27.6 mg, $0.10 \mathrm{mmol})$, 1-azido-4-(azidodifluoromethyl)-2,3,5,6-tetrafluorobenzene $\mathbf{2 h}$ (84.6 mg, 3.0 equiv), $\Delta-\mathbf{R h S}(3.5 \mathrm{mg}, 4 \mathrm{~mol} \%),\left[\mathrm{Ru}(\mathrm{bpy})_{3}\right]\left(\mathrm{PF}_{6}\right)_{2}(2.2 \mathrm{mg}, 2.5 \mathrm{~mol} \%), \mathrm{Na}_{2} \mathrm{HPO}_{4}(2.8 \mathrm{mg}$, $20 \mathrm{~mol} \%$ ) and $\mathrm{H}_{2} \mathrm{O}$ (36.0 mg, 20 equiv) in acetone/DMSO (9:1, $\left.0.5 \mathrm{~mL}, 0.2 \mathrm{M}\right)$ under nitrogen atmosphere with visible light for 11 hours, afforded $25.5 \mathrm{mg}(48 \%)$ of $\mathbf{4 b h}$ as a colorless oil. Enantiomeric excess was established by HPLC analysis using a Chiralpak OD-H column, ee $=98 \%$ (HPLC: OD-H, $254 \mathrm{~nm}, n$-hexane/isopropanol $=95: 5$, flow rate $1 \mathrm{~mL} / \mathrm{min}, 25^{\circ} \mathrm{C}, \mathrm{t}_{\mathrm{r}}$ (major) $=8.8$ $\min , \mathrm{t}_{\mathrm{r}}($ minor $\left.)=7.6 \mathrm{~min}\right) .[\alpha]_{\mathrm{D}}^{22}=+128.2^{\circ}\left(\right.$ c 1.0, $\left.\mathrm{CH}_{2} \mathrm{Cl}_{2}\right)$.

${ }^{1} \mathrm{H}$ NMR $\left(500 \mathrm{MHz}, \mathrm{CDCl}_{3}\right) \delta$ 7.50-7.43 (m, 2H), 7.42-7.18 (m, 8H), 7.11-7.09 (m, 1H), 6.90-6.85 (m, 1H), 6.85-6.81 (m, 1H, other rotamer), $6.76(\mathrm{~d}, J=8.0 \mathrm{~Hz}, 1 \mathrm{H}$, other rotamer), 5.90-5.85 (m, 1H), 5.83-5.78 (m, $1 \mathrm{H}$, other rotamer), $2.03(\mathrm{~s}, 3 \mathrm{H}$, other rotamer), $1.39(\mathrm{~s}, 3 \mathrm{H})$.

${ }^{13} \mathrm{C} \mathrm{NMR}\left(125 \mathrm{MHz}, \mathrm{CDCl}_{3}\right) \delta 185.3,185.2,141.1,140.9,137.05,136.98,136.7,134.7,134.2,130.9$, $130.84,130.81,129.52,129.46,128.94,128.85,128.1,128.0,127.4,127.3,126.75,126.65,126.4$, 126.0, $62.6(\mathrm{t}, J=3.4 \mathrm{~Hz}), 62.2(\mathrm{t}, J=3.7 \mathrm{~Hz}), 17.2,16.0$ (Mixture of two rotation isomers).

${ }^{19} \mathrm{~F}$ NMR $\left(282 \mathrm{MHz}, \mathrm{CDCl}_{3}\right) \delta-62.93(\mathrm{t}, J=24.82,2 \mathrm{~F}),-142.24--142.54(\mathrm{~m}, 2 \mathrm{~F}),-157.67--$ $157.90(\mathrm{~m}, 2 \mathrm{~F})$.

IR (film): $v\left(\mathrm{~cm}^{-1}\right)$ 3373, 2962, 2927, 2145, 1689, 1655, 1536, 1500, 1456, 1428, 1400, 1320, 1275 , 1229, 1148, 1028, 979, 953, 912, 865, 786, 763, 730, 697, 670, 626, 562, 488, 454, 393.

HRMS (ESI, $m / z$ ) calcd for $\mathrm{C}_{25} \mathrm{H}_{16} \mathrm{~F}_{6} \mathrm{~N}_{6} \mathrm{ONa}[\mathrm{M}+\mathrm{Na}]^{+}: 553.1182$, found: 553.1182 .

The structure of $\mathbf{4 b h}$ was further confirmed by ${ }^{1} \mathrm{H}-\left\{{ }^{19} \mathrm{~F}\right\}$ NMR shown below: 


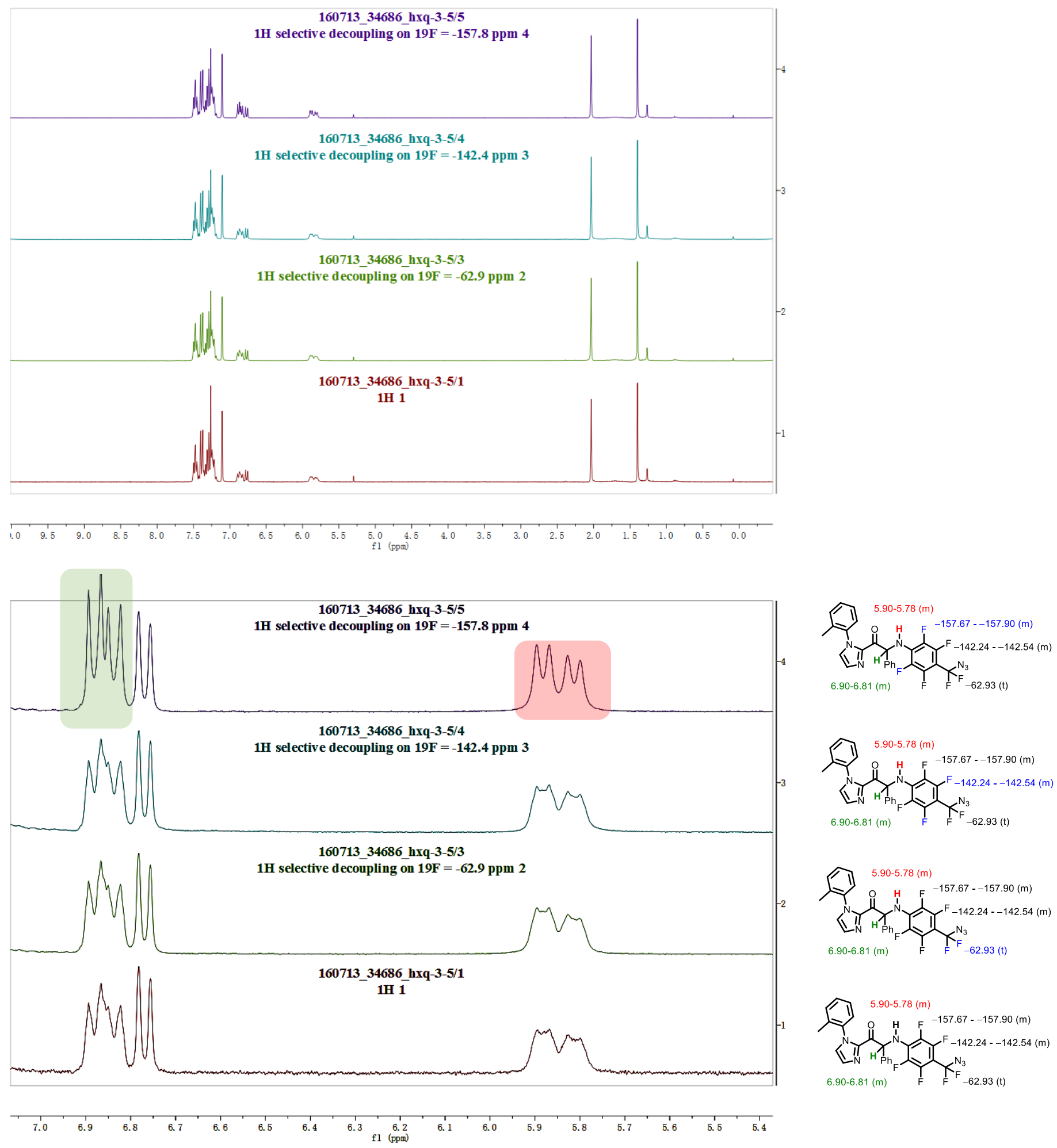




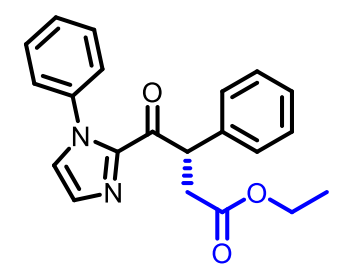

Ethyl (S)-4-oxo-3-phenyl-4-(1-phenyl-1H-imidazol-2-yl)butanoate (5aa)

According to the general procedure, the reaction of 2-phenyl-1-(1-phenyl-1H-imidazol-2-yl) ethan1-one 1a (26.2 mg, $0.10 \mathrm{mmol}$ ), ethyl 2-diazoacetate 3a (34.2 mg, 3.0 equiv), $\Delta$-RhS (3.5 mg, 4 mol\%), $\left[\mathrm{Ru}(\mathrm{bpy})_{3}\right]\left(\mathrm{PF}_{6}\right)_{2}(1.3 \mathrm{mg}, 1.5 \mathrm{~mol} \%), \mathrm{Na}_{2} \mathrm{HPO}_{4}(2.8 \mathrm{mg}, 20 \mathrm{~mol} \%)$ and $\mathrm{H}_{2} \mathrm{O}(36.0 \mathrm{mg}, 20$ equiv) in acetone/DMSO (9:1, 0.5 mL, 0.2 M) under nitrogen atmosphere with visible light for 15 hours, afforded $32.7 \mathrm{mg}$ (94\%) of 5aa as a yellow solid. Enantiomeric excess was established by HPLC analysis using a Chiralpak OD-H column, ee = 92\% (HPLC: OD-H, 254 nm, $n$ hexane/isopropanol $=90: 10$, flow rate $1 \mathrm{~mL} / \mathrm{min}, 25^{\circ} \mathrm{C}, \mathrm{t}_{\mathrm{r}}($ major $)=15.0 \mathrm{~min}, \mathrm{t}_{\mathrm{r}}($ minor $\left.)=11.1 \mathrm{~min}\right)$. $[\alpha]_{\mathrm{D}}^{22}=+213.6^{\circ}\left(c 1.0, \mathrm{CH}_{2} \mathrm{Cl}_{2}\right)$.

${ }^{1} \mathrm{H}$ NMR (500 MHz, $\left.\mathrm{CDCl}_{3}\right) \delta$ 7.44-7.39 (m, 5H), 7.32-7.27 (m, 2H), 7.27 (d, J=1.5 Hz, 1H), 7.25$7.21(\mathrm{~m}, 1 \mathrm{H}), 7.19-7.15(\mathrm{~m}, 2 \mathrm{H}), 7.11(\mathrm{~d}, J=1.0 \mathrm{~Hz}, 1 \mathrm{H}), 5.60\left(\mathrm{dd}, J_{l}=10.5 \mathrm{~Hz}, J_{2}=5.0 \mathrm{~Hz}, 1 \mathrm{H}\right)$, $4.07\left(\mathrm{qd}, J_{1}=7.0 \mathrm{~Hz}, J_{2}=1.0 \mathrm{~Hz}, 2 \mathrm{H}\right), 3.30\left(\mathrm{dd}, J_{l}=17.0 \mathrm{~Hz}, J_{2}=10.5 \mathrm{~Hz}, 1 \mathrm{H}\right), 2.70\left(\mathrm{dd}, J_{l}=17.0\right.$ $\left.\mathrm{Hz}, J_{2}=5.0 \mathrm{~Hz}, 1 \mathrm{H}\right), 1.16(\mathrm{t}, J=7.3 \mathrm{~Hz}, 3 \mathrm{H})$.

${ }^{13} \mathrm{C}$ NMR $\left(125 \mathrm{MHz}, \mathrm{CDCl}_{3}\right) \delta 189.4,171.8,142.4,138.3,137.6,129.9,128.9,128.7,128.6,127.3$, 127.0, 125.6, 60.6, 48.8, 37.4, 14.1. (Missing one ${ }^{13} \mathrm{C}$ signal)

IR (film): $v\left(\mathrm{~cm}^{-1}\right)$ 2980, 2930, 1722, 1678, 1495, 1449, 1401, 1373, 1329, 1302, 1245, 1187, 1152, $1097,1029,939,906,756,694,585,530$.

HRMS (ESI, $m / z$ ) calcd for $\mathrm{C}_{21} \mathrm{H}_{21} \mathrm{~N}_{2} \mathrm{O}_{3}[\mathrm{M}+\mathrm{H}]^{+}: 349.1547$, found: 349.1548 .

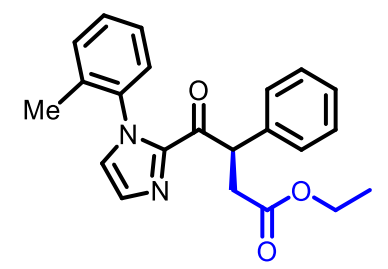

\section{Ethyl $(R)-4-0 x o-3-p h e n y l-4-(1-(o-t o l y l)-1 H$-imidazol-2-yl)butanoate (5ba)}

According to the general procedure, the reaction of 2-phenyl-1-(1-(o-tolyl)-1H-imidazol-2-yl) ethan1-one $\mathbf{1 b}$ (27.6 mg, $0.10 \mathrm{mmol})$, ethyl 2-diazoacetate 3a (34.2 mg, 3.0 equiv), $\Lambda$-RhS (3.5 mg, 4 mol\%), [Ru(bpy) $3\left(\mathrm{PF}_{6}\right)_{2}(1.3 \mathrm{mg}, 1.5 \mathrm{~mol} \%), \mathrm{Na}_{2} \mathrm{HPO}_{4}(2.8 \mathrm{mg}, 20 \mathrm{~mol} \%)$ and $\mathrm{H}_{2} \mathrm{O}(36.0 \mathrm{mg}, 20$ 
equiv) in acetone/DMSO (9:1, $0.5 \mathrm{~mL}, 0.2 \mathrm{M})$ under nitrogen atmosphere with visible light for 15 hours, afforded $35.9 \mathrm{mg}$ (99\%) of $\mathbf{5 b a}$ as a yellow oil. Enantiomeric excess was established by HPLC analysis using a Chiralpak OD-H column, ee = 97\% (HPLC: OD-H, 254 nm, $n$-hexane/isopropanol $=90: 10$, flow rate $1 \mathrm{~mL} / \mathrm{min}, 25^{\circ} \mathrm{C}, \mathrm{t}_{\mathrm{r}}($ major $)=9.8 \mathrm{~min}, \mathrm{t}_{\mathrm{r}}($ minor $\left.)=11.7 \mathrm{~min}\right) .[\alpha]_{\mathrm{D}}{ }^{22}=-238.4^{\circ}(c$ 1.0, $\mathrm{CH}_{2} \mathrm{Cl}_{2}$ ).

${ }^{1} \mathrm{H}$ NMR $\left(500 \mathrm{MHz}, \mathrm{CDCl}_{3}\right) \delta 7.43-7.18(\mathrm{~m}, 10 \mathrm{H}), 7.03(\mathrm{~d}, J=1.0 \mathrm{~Hz}, 1 \mathrm{H}), 6.90(\mathrm{~d}, J=8.0 \mathrm{~Hz}, 1 \mathrm{H}$, other rotamer), 5.65-5.58 (m, 1H), 4.10-4.03 (m, 2H), 3.34-3.25 (m, 1H), 2.74-2.64 (m, 1H), $2.04(\mathrm{~s}$, $3 \mathrm{H}), 1.55$ ( $\mathrm{s}, 3 \mathrm{H}$, other rotamer), 1.20-1.14 (m, 3H).

${ }^{13} \mathrm{C}$ NMR $\left(125 \mathrm{MHz}, \mathrm{CDCl}_{3}\right) \delta 189.35,189.25,171.6,142.9,142.8,137.84,137.80,137.7,137.6$, $134.9,134.3,130.6,130.5,130.2,130.1,129.0,128.9$, 128.7 , 128.61, 128.56, 128.5, 127.23, 127.19, $126.5,126.41,126.38,126.1,60.5,48.61,48.58,37.4,37.0,17.1,16.4,14.05,14.02$. (Mixture of two rotation isomers)

IR (film): $v\left(\mathrm{~cm}^{-1}\right)$ 3111, 3061, 2981, 2931, 1729, 1682, 1594, 1495, 1453, 1403, 1375, 1305, 1243, $1178,1093,1026,941,909,848,762,699,670,587,529,455$.

HRMS (ESI, $m / z$ ) calcd for $\mathrm{C}_{22} \mathrm{H}_{22} \mathrm{~N}_{2} \mathrm{O}_{3} \mathrm{Na}[\mathrm{M}+\mathrm{Na}]^{+}: 385.1523$, found: 385.1525

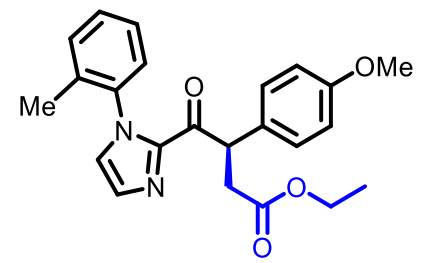

Ethyl $(R)-3-(4-m e t h o x y p h e n y l)-4-0 x o-4-(1-(o-t o l y l)-1 H$-imidazol-2-yl)butanoate (5la)

According to the general procedure, the reaction of 2-(4-methoxyphenyl)-1-(1-(o-tolyl)-1Himidazol-2-yl)ethan-1-one 11 (30.6 mg, $0.10 \mathrm{mmol}$ ), ethyl 2-diazoacetate 3a (34.2 mg, 3.0 equiv), $\Lambda$ RhS (3.5 mg, 4 mol\%), [Ru(bpy) 3 ( $\left.\mathrm{PF}_{6}\right)_{2}(1.3 \mathrm{mg}, 1.5 \mathrm{~mol} \%), \mathrm{Na}_{2} \mathrm{HPO}_{4}(2.8 \mathrm{mg}, 20 \mathrm{~mol} \%)$ and $\mathrm{H}_{2} \mathrm{O}$ (36.0 mg, 20 equiv) in acetone/DMSO (9:1, $0.5 \mathrm{~mL}, 0.2 \mathrm{M})$ under nitrogen atmosphere with visible light for 15 hours, afforded $38.1 \mathrm{mg}(97 \%)$ of $\mathbf{5 l a}$ as a yellow solid. Enantiomeric excess was established by HPLC analysis using a Chiralpak IC column, ee = 95\% (HPLC: IC, 254 nm, $n$ hexane/isopropanol = 80:20, flow rate $1 \mathrm{~mL} / \mathrm{min}, 25^{\circ} \mathrm{C}, \mathrm{t}_{\mathrm{r}}($ major $\left.)=13.1 \mathrm{~min}, \mathrm{t}_{\mathrm{r}}(\operatorname{minor})=14.8 \mathrm{~min}\right)$. $[\alpha]_{\mathrm{D}}^{22}=-239.4^{\circ}\left(c 1.0, \mathrm{CH}_{2} \mathrm{Cl}_{2}\right)$.

${ }^{1} \mathrm{H}$ NMR $\left(500 \mathrm{MHz}, \mathrm{CDCl}_{3}\right) \delta$ 7.38-7.26 (m, 5H), 7.25-7.18 (m, 2H), 7.03-7.01 (m, 1H), $6.89(\mathrm{~d}, J=$ $8.0 \mathrm{~Hz}, 1 \mathrm{H}$, other rotamer), 6.84-6.78 (m, 2H), 5.58-5.51 (m, 1H), 4.09-4.02 (m, 2H), $3.76(\mathrm{~s}, 3 \mathrm{H})$, 
3.29-3.20 (m, 1H), 2.71-2.61 (m, 1H), $2.03(\mathrm{~s}, 3 \mathrm{H}), 1.57$ (s, 3H, other rotamer), 1.20-1.13 (m, 3H). ${ }^{13} \mathrm{C} \mathrm{NMR}\left(125 \mathrm{MHz}, \mathrm{CDCl}_{3}\right) \delta 189.5,189.4,171.7,158.8,143.0,142.9,137.9,134.9,134.3,130.6$, 130.08, 130.06, 129.7, 129.6, 129.0, 128.9, 126.5, 126.44, 126.39, 126.33, 126.31, 126.1, 114.1, 114.0, $60.5,55.2,47.83,47.76,37.4,37.1,17.2,16.5,14.1,14.0$. (Mixture of two rotation isomers) IR (film): $v\left(\mathrm{~cm}^{-1}\right)$ 2971, 2933, 1728, 1674, 1606, 1507, 1452, 1340, 1302, 1243, 1178, 1106, 1091, 1024, 940, 906, 848, 766, 719, 536.

HRMS (ESI, $m / z$ ) calcd for $\mathrm{C}_{23} \mathrm{H}_{24} \mathrm{~N}_{2} \mathrm{O}_{4} \mathrm{Na}[\mathrm{M}+\mathrm{Na}]^{+}$: 415.1628, found: 415.1629 .

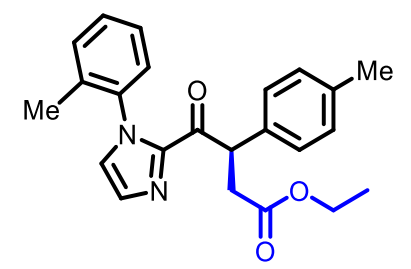

\section{Ethyl (R)-4-oxo-3-(p-tolyl)-4-(1-(o-tolyl)-1H-imidazol-2-yl)butanoate (5ja)}

According to the general procedure, the reaction of 2-(p-tolyl)-1-(1-(o-tolyl)-1H-imidazol- 2yl)ethan-1-one $\mathbf{1 j}$ (29.0 mg, $0.10 \mathrm{mmol})$, ethyl 2-diazoacetate $\mathbf{3 a}(34.2 \mathrm{mg}, 3.0$ equiv), $\Lambda$ - $\mathbf{R h S}$ (3.5 $\mathrm{mg}, 4 \mathrm{~mol} \%),\left[\mathrm{Ru}(\mathrm{bpy})_{3}\right]\left(\mathrm{PF}_{6}\right)_{2}(2.2 \mathrm{mg}, 2.5 \mathrm{~mol} \%), \mathrm{Na}_{2} \mathrm{HPO}_{4}(2.8 \mathrm{mg}, 20 \mathrm{~mol} \%)$ and $\mathrm{H}_{2} \mathrm{O}(36.0 \mathrm{mg}$, 20 equiv) in acetone/DMSO (9:1, $2.0 \mathrm{~mL}, 0.05 \mathrm{M})$ under nitrogen atmosphere with visible light for 24 hours, afforded $35.5 \mathrm{mg}$ (94\%) of $\mathbf{5 j a}$ as a yellow solid. Enantiomeric excess was established by HPLC analysis using a Chiralpak OD-H column, ee $=96 \%$ (HPLC: OD-H, 254 nm, $n$ hexane/isopropanol = 95:5, flow rate $1 \mathrm{~mL} / \mathrm{min}, 25^{\circ} \mathrm{C}, \mathrm{t}_{\mathrm{r}}($ major $)=10.5 \mathrm{~min}, \mathrm{t}_{\mathrm{r}}($ minor $\left.)=13.2 \mathrm{~min}\right)$. $[\alpha]_{\mathrm{D}}^{22}=-272.8^{\circ}\left(c 1.0, \mathrm{CH}_{2} \mathrm{Cl}_{2}\right)$.

${ }^{1} \mathrm{H}$ NMR $\left(500 \mathrm{MHz}, \mathrm{CDCl}_{3}\right) \delta 7.39-7.21(\mathrm{~m}, 7 \mathrm{H}), 7.12-7.06(\mathrm{~m}, 2 \mathrm{H}), 7.03-7.01(\mathrm{~m}, 1 \mathrm{H}), 6.91(\mathrm{~d}, J=$ 8.0 Hz, 1H, other rotamer), 5.60-5.53 (m, 1H), 4.10-4.02 (m, 2H), 3.31-3.24 (m, 1H), 2.71-2.62 (m, 1H), $2.30(\mathrm{~s}, 3 \mathrm{H}), 2.29(\mathrm{~s}, 3 \mathrm{H}$, other rotamer), $2.04(\mathrm{~s}, 3 \mathrm{H}), 1.59(\mathrm{~s}, 3 \mathrm{H}$, other rotamer), 1.21-1.14 (m, $3 \mathrm{H})$.

${ }^{13} \mathrm{C}$ NMR $\left(125 \mathrm{MHz}, \mathrm{CDCl}_{3}\right) \delta 189.5,189.4,171.7,142.3,142.8,137.9,137.8,136.8,134.9,134.6$, $134.5,134.3,130.6,130.5,130.11,130.09,129.4,129.3,128.95,128.89,128.4,126.5,126.41,126.35$, 126.30, 126.29, 126.1, 60.4, 48.21, 48.20, 37.4, 37.1, 21.0, 17.2, 16.6, 14.05, 14.01. (Mixture of two rotation isomers)

IR (film): $v\left(\mathrm{~cm}^{-1}\right)$ 3172, 2981, 2924, 1731, 1680, 1500, 1445, 1405, 1373, 1321, 1179, 1032, 942, $907,767,717,534$. 
HRMS (ESI, $m / z$ ) calcd for $\mathrm{C}_{23} \mathrm{H}_{24} \mathrm{~N}_{2} \mathrm{O}_{3} \mathrm{Na}[\mathrm{M}+\mathrm{Na}]^{+}: 399.1679$, found: 399.1677 .

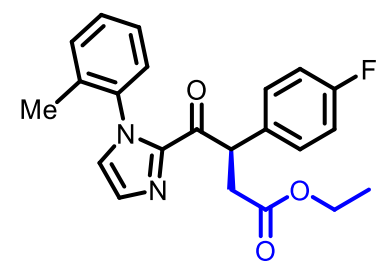

Ethyl (R)-3-(4-fluorophenyl)-4-oxo-4-(1-(o-tolyl)-1H-imidazol-2-yl)butanoate (5ma)

According to the general procedure, the reaction of 2-(4-fluorophenyl)-1-(1-(o-tolyl)- $1 H$-imidazol2-yl)ethan-1-one $\mathbf{1 m}$ (29.4 mg, $0.10 \mathrm{mmol}$ ), ethyl 2-diazoacetate $\mathbf{3 a}$ (34.2 mg, 3.0 equiv), $\Lambda$-RhS (3.5 $\mathrm{mg}, 4 \mathrm{~mol} \%)$, [Ru(bpy) $\left.)_{3}\right]\left(\mathrm{PF}_{6}\right)_{2}(1.3 \mathrm{mg}, 1.5 \mathrm{~mol} \%), \mathrm{Na}_{2} \mathrm{HPO}_{4}(2.8 \mathrm{mg}, 20 \mathrm{~mol} \%)$ and $\mathrm{H}_{2} \mathrm{O}(36.0 \mathrm{mg}$, 20 equiv) in acetone/DMSO (9:1, $1.0 \mathrm{~mL}, 0.1 \mathrm{M})$ under nitrogen atmosphere with visible light for 16 hours, afforded $36.5 \mathrm{mg}(96 \%)$ of $\mathbf{5 m a}$ as a yellow solid. Enantiomeric excess was established by HPLC analysis using a Chiralpak OD-H column, ee =98\% (HPLC: OD-H, 254 nm, $n$ hexane/isopropanol $=90: 10$, flow rate $1 \mathrm{~mL} / \mathrm{min}, 25^{\circ} \mathrm{C}, \mathrm{t}_{\mathrm{r}}($ major $)=7.6 \mathrm{~min}, \mathrm{t}_{\mathrm{r}}($ minor $\left.)=8.7 \mathrm{~min}\right)$. $[\alpha]_{\mathrm{D}}^{22}=-205.8^{\circ}\left(c 1.0, \mathrm{CH}_{2} \mathrm{Cl}_{2}\right)$.

${ }^{1} \mathrm{H}$ NMR $\left(500 \mathrm{MHz}, \mathrm{CDCl}_{3}\right) \delta$ 7.40-7.26 (m, 5H), 7.25-7.17 (m, 2H), 7.04-7.02 (m, 1H), 6.99-6.92 $(\mathrm{m}, 2 \mathrm{H}), 6.90\left(\mathrm{dd}, J_{l}=8.0 \mathrm{~Hz}, J_{2}=1.0 \mathrm{~Hz}, 1 \mathrm{H}\right.$, other rotamer $), 5.62-5.54(\mathrm{~m}, 1 \mathrm{H}), 4.08-4.01(\mathrm{~m}, 2 \mathrm{H})$, 3.28-3.20 (m, 1H), 2.71-2.60 (m, 1H), $2.02(\mathrm{~s}, 3 \mathrm{H}), 1.56(\mathrm{~s}, 3 \mathrm{H}$, other rotamer), 1.19-1.12 (m, 3H). ${ }^{13} \mathrm{C}$ NMR (125 MHz, $\left.\mathrm{CDCl}_{3}\right) \delta 189.2,189.1,171.51,171.49,163.0,161.0,142.7,142.6,137.79$, $137.75,134.9,134.2,133.41,133.39,133.35,133.32,130.7,130.6,130.23,130.21,130.16,130.1$, 129.1, 129.0, 126.6, 126.5, 126.4, 126.0, 115.64, 115.59, 115.5, 115.4, 60.6, 47.8, 47.7, 37.4, 37.0, 17.1, 16.5, 14.1, 14.0. (Mixture of two rotation isomers)

${ }^{19} \mathrm{~F} \mathrm{NMR}\left(282 \mathrm{MHz}, \mathrm{CDCl}_{3}\right) \delta-115.32(1 \mathrm{~F}),-115.39$ (1F, other rotamer).

IR (film): $v\left(\mathrm{~cm}^{-1}\right)$ 3119, 2985, 2926, 1723, 1683, 1502, 1454, 1403, 1376, 1313, 1225, 1185, 1159, 1096, 1022, 941, 908, 842, 803, 766, 716, 544, 492, 454.

HRMS (ESI, $m / z$ ) calcd for $\mathrm{C}_{22} \mathrm{H}_{21} \mathrm{FN}_{2} \mathrm{O}_{3} \mathrm{Na}[\mathrm{M}+\mathrm{Na}]^{+}$: 403.1428 , found: 403.1425 .

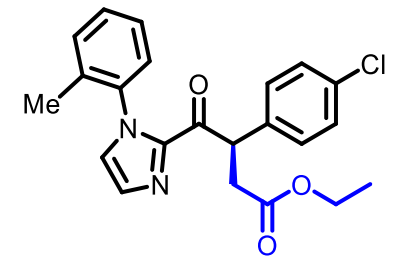




\section{Ethyl $(R)-3-(4-c h l o r o p h e n y l)-4-0 x o-4-(1-(o-t o l y l)-1 H$-imidazol-2-yl)butanoate (5na)}

According to the general procedure, the reaction of 2-(4-chlorophenyl)-1-(1-(o-tolyl)-1H-imidazol2-yl)ethan-1-one $\mathbf{1 n}(31.1 \mathrm{mg}, 0.10 \mathrm{mmol})$, ethyl 2-diazoacetate 3a (34.2 mg, 3.0 equiv), $\Lambda$-RhS (3.5 $\mathrm{mg}, 4 \mathrm{~mol} \%)$, [Ru(bpy) 3 ]( $\left.\mathrm{PF}_{6}\right)_{2}(2.2 \mathrm{mg}, 2.5 \mathrm{~mol} \%), \mathrm{Na}_{2} \mathrm{HPO}_{4}(2.8 \mathrm{mg}, 20 \mathrm{~mol} \%)$ and $\mathrm{H}_{2} \mathrm{O}(36.0 \mathrm{mg}$, 20 equiv) in acetone/DMSO (9:1, $2.0 \mathrm{~mL}, 0.05 \mathrm{M})$ under nitrogen atmosphere with visible light for 22 hours, afforded $39.0 \mathrm{mg}(98 \%)$ of $\mathbf{5 n a}$ as a white solid. Enantiomeric excess was established by HPLC analysis using a Chiralpak IC column, ee = 95\% (HPLC: IC, $254 \mathrm{~nm}, n$-hexane/isopropanol =

$85: 15$, flow rate $1 \mathrm{~mL} / \mathrm{min}, 25^{\circ} \mathrm{C}, \mathrm{t}_{\mathrm{r}}($ major $)=7.3 \mathrm{~min}, \mathrm{t}_{\mathrm{r}}($ minor $\left.)=8.7 \mathrm{~min}\right) .[\alpha]_{\mathrm{D}}^{22}=-208.4^{\circ}(c 1.0$, $\mathrm{CH}_{2} \mathrm{Cl}_{2}$ ).

${ }^{1} \mathrm{H}$ NMR (500 MHz, $\left.\mathrm{CDCl}_{3}\right) \delta$ 7.39-7.26 (m, 5H), 7.26-7.17 (m, 4H), 7.04-7.03 (m, 1H), $6.91\left(\mathrm{dd}, J_{1}\right.$ $=8.0 \mathrm{~Hz}, J_{2}=1.0 \mathrm{~Hz}, 1 \mathrm{H}$, other rotamer $), 5.61-5.54(\mathrm{~m}, 1 \mathrm{H}), 4.08-4.01(\mathrm{~m}, 2 \mathrm{H}), 3.16-3.06(\mathrm{~m}, 1 \mathrm{H})$, 2.72-2.62 (m, 1H), $2.04(\mathrm{~s}, 3 \mathrm{H}), 1.81$ (s, 3H, other rotamer), 1.20-1.14 (m, 3H).

${ }^{13} \mathrm{C}$ NMR $\left(125 \mathrm{MHz}, \mathrm{CDCl}_{3}\right) \delta 189.0,188.9,171.3,171.2,142.80,142.77,137.83,137.81,136.1$, 135.9, 135.0, 134.2, 134.1, 134.0, 130.7, 130.6, 130.45, 130.41, 130.2, 129.1, 129.0, 128.6, 128.5, $128.43,128.40,126.9,126.8,126.7,126.55,126.50,126.43,126.37,126.1,60.6,45.7,45.6,36.7$, 36.5, 17.2, 16.9, 14.03, 14.00. (Mixture of two rotation isomers)

IR (film): $v\left(\mathrm{~cm}^{-1}\right)$ 2974, 2925, 1731, 1682, 1493, 1458, 1401, 1374, 1254, 1185, 1148, 1087, 1035 , $943,906,769,717,531$.

HRMS (ESI, $m / z$ ) calcd for $\mathrm{C}_{22} \mathrm{H}_{21} \mathrm{ClN}_{2} \mathrm{O}_{3} \mathrm{Na}[\mathrm{M}+\mathrm{Na}]^{+}: 419.1133$, found: 419.1131 .

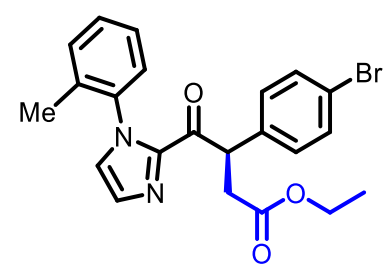

Ethyl (R)-3-(4-bromophenyl)-4-oxo-4-(1-(o-tolyl)-1H-imidazol-2-yl)butanoate (5oa)

According to the general procedure, the reaction of 2-(4-bromophenyl)-1-(1-(o-tolyl)-1H-imidazol2-yl)ethan-1-one 10 (35.5 mg, $0.10 \mathrm{mmol}$ ), ethyl 2-diazoacetate $\mathbf{3 a}(34.2 \mathrm{mg}, 3.0$ equiv), $\Lambda$ - $\mathbf{R h S}$ (3.5 mg, $4 \mathrm{~mol} \%$ ), [Ru(bpy) 3 ]( $\left.\mathrm{PF}_{6}\right)_{2}(2.2 \mathrm{mg}, 2.5 \mathrm{~mol} \%), \mathrm{Na}_{2} \mathrm{HPO}_{4}(2.8 \mathrm{mg}, 20 \mathrm{~mol} \%)$ and $\mathrm{H}_{2} \mathrm{O}(36.0 \mathrm{mg}$, 20 equiv) in acetone/DMSO (9:1, $2.0 \mathrm{~mL}, 0.05 \mathrm{M})$ under nitrogen atmosphere with visible light for 38 hours, afforded $41.0 \mathrm{mg}(93 \%)$ of $\mathbf{5 0 a}$ as a white solid. Enantiomeric excess was established by HPLC analysis using a Chiralpak IC column, ee = 95\% (HPLC: IC, $254 \mathrm{~nm}, n$-hexane/isopropanol = 
$85: 15$, flow rate $1 \mathrm{~mL} / \mathrm{min}, 25^{\circ} \mathrm{C}, \mathrm{t}_{\mathrm{r}}$ (major $)=7.8 \mathrm{~min}, \mathrm{t}_{\mathrm{r}}($ minor $\left.)=9.1 \mathrm{~min}\right) .[\alpha]_{\mathrm{D}}{ }^{22}=-183.2^{\circ}(c 1.0$, $\mathrm{CH}_{2} \mathrm{Cl}_{2}$ ).

${ }^{1} \mathrm{H}$ NMR $\left(500 \mathrm{MHz}, \mathrm{CDCl}_{3}\right) \delta$ 7.42-7.17 (m, 9H), 7.05-7.02 (m, 1H), $6.91(\mathrm{~d}, J=8.0 \mathrm{~Hz}, 1 \mathrm{H}$, other rotamer), 5.60-5.53 (m, 1H), 4.08-4.02 (m, 2H), 3.27-3.19 (m, 1H), 2.70-2.61 (m, 1H), $2.01(\mathrm{~s}, 3 \mathrm{H})$, 1.59 (s, 3H, other rotamer), 1.19-1.12 (m, 3H).

${ }^{13} \mathrm{C} \mathrm{NMR}\left(125 \mathrm{MHz}, \mathrm{CDCl}_{3}\right) \delta 188.9,188.8,171.43,171.41,142.6,142.5,137.74,137.71,136.20$, $136.17,134.9$, 134.2, 133.20, 133.19, 130.7, 130.6, 130.24, 130.21, 130.0, 129.12, 129.06, 128.9, $128.8,126.7,126.63,126.58,126.5,126.4,126.0,60.6,47.97,47.95,37.2$, 36.9, 17.1, 16.6, 14.1, 14.0. (Mixture of two rotation isomers)

IR (film): $v\left(\mathrm{~cm}^{-1}\right)$ 2980, 2929, 1730, 1683, 1491, 1454, 1403, 1374, 1309, 1240, 1178, 1014, 941, 909, 766, 722, 532.

HRMS (ESI, $m / z$ ) calcd for $\mathrm{C}_{22} \mathrm{H}_{21} \mathrm{BrN}_{2} \mathrm{O}_{3} \mathrm{Na}[\mathrm{M}+\mathrm{Na}]^{+}$: 463.0628 , found: 463.0624 .

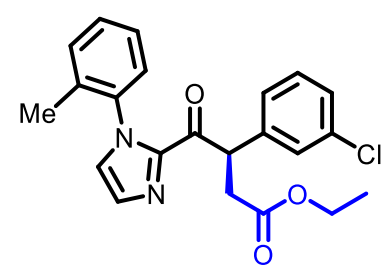

Ethyl (R)-3-(3-chlorophenyl)-4-oxo-4-(1-(o-tolyl)-1H-imidazol-2-yl)butanoate (5ka)

According to the general procedure, the reaction of 2-(3-chlorophenyl)-1-(1-(o-tolyl)-1H-imidazol2-yl)ethan-1-one $\mathbf{1 k}(31.1 \mathrm{mg}, 0.10 \mathrm{mmol})$, ethyl 2-diazoacetate $\mathbf{3 a}(34.2 \mathrm{mg}, 3.0$ equiv), $\Lambda$ - $\mathbf{R h S}$ (3.5 mg, $4 \mathrm{~mol} \%$ ), [Ru(bpy) 3 ] $\left(\mathrm{PF}_{6}\right)_{2}(1.3 \mathrm{mg}, 1.5 \mathrm{~mol} \%), \mathrm{Na}_{2} \mathrm{HPO}_{4}(2.8 \mathrm{mg}, 20 \mathrm{~mol} \%)$ and $\mathrm{H}_{2} \mathrm{O}(36.0 \mathrm{mg}$, 20 equiv) in acetone/DMSO $(9: 1,0.5 \mathrm{~mL}, 0.2 \mathrm{M})$ under nitrogen atmosphere with visible light for 26 hours, afforded $36.5 \mathrm{mg}$ (92\%) of $\mathbf{5 k a}$ as a yellow oil. Enantiomeric excess was established by HPLC analysis using a Chiralpak IC column, ee = 95\% (HPLC: IC, $254 \mathrm{~nm}, n$-hexane/isopropanol = 80:20, flow rate $1 \mathrm{~mL} / \mathrm{min}, 25^{\circ} \mathrm{C}, \mathrm{t}_{\mathrm{r}}$ (major) $=7.1 \mathrm{~min}, \mathrm{t}_{\mathrm{r}}($ minor $\left.)=12.2 \mathrm{~min}\right)$. $[\alpha]_{\mathrm{D}}{ }^{22}=-223.0^{\circ}(c 1.0$, $\mathrm{CH}_{2} \mathrm{Cl}_{2}$ ).

${ }^{1} \mathrm{H}$ NMR $\left(500 \mathrm{MHz}, \mathrm{CDCl}_{3}\right) \delta$ 7.40-7.18 $(\mathrm{m}, 9 \mathrm{H}), 7.06-7.04(\mathrm{~m}, 1 \mathrm{H}), 6.31\left(\mathrm{dd}, J_{1}=8.0 \mathrm{~Hz}, J_{2}=1.0\right.$ $\mathrm{Hz}, 1 \mathrm{H}$, other rotamer), 5.61-5.55 (m, 1H), 4.09-4.02 (m, 2H), 3.28-3.21 (m, 1H), 2.71-2.62 (m, 1H), $2.02(\mathrm{~s}, 3 \mathrm{H}), 1.62$ (s, 3H, other rotamer), 1.20-1.13 (m, 3H).

${ }^{13} \mathrm{C} \mathrm{NMR}\left(125 \mathrm{MHz}, \mathrm{CDCl}_{3}\right) \delta 188.70,188.65,171.4,171.3,142.7,142.6,139.72,139.69,137.73$, $137.69,134.7,134.40,134.38,134.2$, 130.7 130.34, 130.31, 129.91, 129.88, 129.12, 129.06, 128.64, 
$128.59,127.5,126.88,126.85,126.69,126.67,126.6,126.5,126.4,126.1,60.6,48.22,48.20,37.3$, $36.9,17.1,16.5,14.1,14.0$. (Mixture of two rotation isomers)

IR (film): $v\left(\mathrm{~cm}^{-1}\right)$ 2982, 2930, 1730, 1683, 1454, 1402, 1376, 1304, 1241, 1180, 1088, 1025, 942, 908, 766, 724, 690, 454.

HRMS (ESI, $m / z$ ) calcd for $\mathrm{C}_{22} \mathrm{H}_{21} \mathrm{ClN}_{2} \mathrm{O}_{3} \mathrm{Na}[\mathrm{M}+\mathrm{Na}]^{+}: 419.1133$, found: 419.1131 .

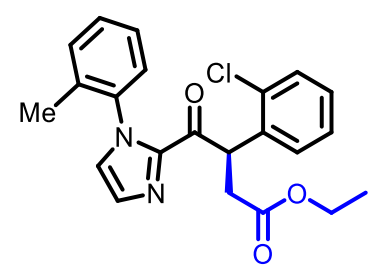

\section{Ethyl (R)-3-(2-chlorophenyl)-4-oxo-4-(1-(o-tolyl)-1H-imidazol-2-yl)butanoate (5pa)}

According to the general procedure, the reaction of 2-(2-chlorophenyl)-1-(1-(o-tolyl)-1H-imidazol2-yl)ethan-1-one $\mathbf{1 p}$ (31.1 mg, $0.10 \mathrm{mmol}$ ), ethyl 2-diazoacetate $\mathbf{3 a}(34.2 \mathrm{mg}, 3.0$ equiv), $\Lambda$ - $\mathbf{R h S}$ (3.5 $\mathrm{mg}, 4 \mathrm{~mol} \%)$, [Ru(bpy) $3\left(\mathrm{PF}_{6}\right)_{2}(1.3 \mathrm{mg}, 1.5 \mathrm{~mol} \%), \mathrm{Na}_{2} \mathrm{HPO}_{4}(2.8 \mathrm{mg}, 20 \mathrm{~mol} \%)$ and $\mathrm{H}_{2} \mathrm{O}(36.0 \mathrm{mg}$, 20 equiv) in acetone/DMSO $(9: 1,0.5 \mathrm{~mL}, 0.2 \mathrm{M})$ under nitrogen atmosphere with visible light for 15 hours, afforded $32.2 \mathrm{mg}(81 \%)$ of $\mathbf{5 p a}$ as a yellow oil. Enantiomeric excess was established by HPLC analysis using a Chiralpak IC column, ee = 97\% (HPLC: IC, $254 \mathrm{~nm}, n$-hexane/isopropanol = 85:15, flow rate $1 \mathrm{~mL} / \mathrm{min}, 25^{\circ} \mathrm{C}, \mathrm{t}_{\mathrm{r}}($ major $)=12.9 \mathrm{~min}, \mathrm{t}_{\mathrm{r}}($ minor $\left.)=15.6 \mathrm{~min}\right) .[\alpha]_{\mathrm{D}}{ }^{22}=-309.8^{\circ}(c 1.0$, $\mathrm{CH}_{2} \mathrm{Cl}_{2}$ ).

${ }^{1} \mathrm{H}$ NMR $\left(500 \mathrm{MHz}, \mathrm{CDCl}_{3}\right) \delta$ 7.43-7.21 (m, 6H), 7.20-7.08 (m, 3H), 7.04-7.02 (m, 1H), 7.01 (d, $J=$ $8.0 \mathrm{~Hz}, 1 \mathrm{H}$, other rotamer), 6.03-5.95 (m, 1H), 4.50-4.03 (m, 2H), 3.16-3.06 (m, 1H), 2.72-2.62 (m, 1H), 2.04 (s, 3H), 1.81 (s, 3H, other rotamer), 1.20-1.14 (m, 3H).

${ }^{13} \mathrm{C}$ NMR $\left(125 \mathrm{MHz}, \mathrm{CDCl}_{3}\right) \delta 189.0,188.9,171.3,171.2,142.80,142.77,137.83,137.81,136.1$, 135.9, 135.0, 134.2, 134.1, 134.0, 130.7, 130.6, 130.45, 130.41, 130.2, 129.1, 129.0, 128.6, 128.5, $128.43,128.40,126.9,126.8,126.7,126.55,126.50,126.43,126.37,126.1,60.6,45.7,45.6,36.7$, $36.5,17.2,16.9,14.03,14.00$. (Mixture of two rotation isomers)

IR (film): $v\left(\mathrm{~cm}^{-1}\right)$ 2982, 2929, 1731, 1683, 1451, 1403, 1375, 1299, 1242, 1180, 1033, 940, 909, 759, $727,458$.

HRMS (ESI, $m / z$ ) calcd for $\mathrm{C}_{22} \mathrm{H}_{22} \mathrm{ClN}_{2} \mathrm{O}_{3}[\mathrm{M}+\mathrm{H}]^{+}:$397.1313, found: 397.1315 . 
(1)

Ethyl (R)-3-(naphthalen-2-yl)-4-oxo-4-(1-(o-tolyl)-1H-imidazol-2-yl)butanoate (5qa)

According to the general procedure, the reaction of 2-(naphthalen-2-yl)-1-(1-(o-tolyl)-1H-imidazol2-yl)ethan-1-one 1q (32.6 mg, $0.10 \mathrm{mmol}$ ), ethyl 2-diazoacetate 3a (34.2 mg, 3.0 equiv), $\Lambda$-RhS (3.5 mg, $4 \mathrm{~mol} \%$ ), [Ru(bpy) $)_{3}\left(\mathrm{PF}_{6}\right)_{2}(1.3 \mathrm{mg}, 1.5 \mathrm{~mol} \%), \mathrm{Na}_{2} \mathrm{HPO}_{4}(2.8 \mathrm{mg}, 20 \mathrm{~mol} \%)$ and $\mathrm{H}_{2} \mathrm{O}(36.0 \mathrm{mg}$, 20 equiv) in acetone/DMSO $(9: 1,0.5 \mathrm{~mL}, 0.2 \mathrm{M})$ under nitrogen atmosphere with visible light for 15 hours, afforded $35.4 \mathrm{mg}(86 \%)$ of $\mathbf{5 q a}$ as a colorless oil. Enantiomeric excess was established by HPLC analysis using a Chiralpak IC column, ee = 95\% (HPLC: IC, $254 \mathrm{~nm}, n$-hexane/isopropanol = $85: 15$, flow rate $1 \mathrm{~mL} / \mathrm{min}, 25^{\circ} \mathrm{C}, \mathrm{t}_{\mathrm{r}}($ major $)=15.3 \mathrm{~min}, \mathrm{t}_{\mathrm{r}}($ minor $\left.)=17.7 \mathrm{~min}\right) .[\alpha]_{\mathrm{D}}{ }^{22}=-297.6^{\circ}(c$ $1.0, \mathrm{CH}_{2} \mathrm{Cl}_{2}$ ).

${ }^{1} \mathrm{H}$ NMR $\left(500 \mathrm{MHz}, \mathrm{CDCl}_{3}\right) \delta 7.83(\mathrm{~d}, J=12.5 \mathrm{~Hz}, 1 \mathrm{H}), 7.79-7.74(\mathrm{~m}, 3 \mathrm{H}), 7.56-7.50(\mathrm{~m}, 1 \mathrm{H}), 7.46-$ $7.40(\mathrm{~m}, 2 \mathrm{H}), 7.38-7.27(\mathrm{~m}, 3 \mathrm{H}), 7.24-7.17(\mathrm{~m}, 2 \mathrm{H}), 7.01-6.98(\mathrm{~m}, 1 \mathrm{H}), 6.85(\mathrm{~d}, J=8.0 \mathrm{~Hz}, 1 \mathrm{H}$, other rotamer), 5.80-5.73 (m, 1H), 4.10-4.03 (m, 2H), 3.41-3.32 (m, 1H), 2.81-2.72 (m, 1H), $2.05(\mathrm{~s}, 3 \mathrm{H})$, 1.52 (s, 3H, other rotamer), 1.20-1.13 (m, 3H).

${ }^{13} \mathrm{C}$ NMR $\left(125 \mathrm{MHz}, \mathrm{CDCl}_{3}\right) \delta 189.2$ 189.1, 171.7, 171.6, 142.9, 142.8, 137.8, 135.2, 134.9, 134.3, $133.4,132.5,130.61,130.58,130.22,130.16,129.1,129.0,128.40,128.35,127.9,127.8,127.6$, $127.5,126.6,126.52,126.46,126.4,126.1,126.0,125.87,125.85,60.6,48.80,48.77,37.5,37.2,17.2$, 16.6, 14.09, 14.05. (Mixture of two rotation isomers)

IR (film): $v\left(\mathrm{~cm}^{-1}\right)$ 3056, 2981, 2929, 1729, 1682, 1498, 1453, 1403, 1376, 1262, 1241, 1178, 1154, 1024, 941, 908, 816, 763, 728, 479.

HRMS (ESI, $m / z$ ) calcd for $\mathrm{C}_{26} \mathrm{H}_{24} \mathrm{~N}_{2} \mathrm{O}_{3} \mathrm{Na}[\mathrm{M}+\mathrm{Na}]^{+}$: 435.1679, found: 435.1678 .

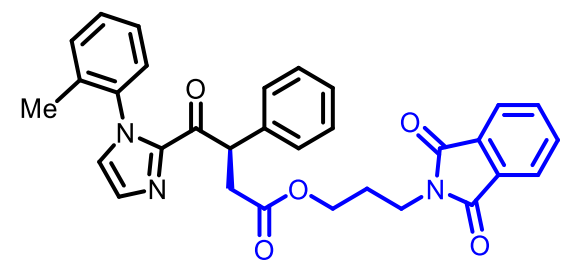

3-(1,3-Dioxoisoindolin-2-yl)propyl $(R)-4-0 x 0-3-p h e n y l-4-(1-(o-t o l y l)-1 H$-imidazol-2-yl)butanoate $(5 b b)$ 
According to the general procedure, the reaction of 2-phenyl-1-(1-(o-tolyl)-1H-imidazol-2-yl) ethan1-one $\mathbf{1 b}$ (27.6 mg, $0.10 \mathrm{mmol}$ ), 3-(1,3-dioxoisoindolin-2-yl)propyl 2-diazoacetate $3 \mathbf{b}$ (82.0 mg, 3.0 equiv), $\Lambda$-RhS (3.5 mg, 4 mol\%), [Ru(bpy) $)_{3}\left(\mathrm{PF}_{6}\right)_{2}(1.3 \mathrm{mg}, 1.5 \mathrm{~mol} \%), \mathrm{Na}_{2} \mathrm{HPO}_{4}(2.8 \mathrm{mg}, 20 \mathrm{~mol} \%)$ and $\mathrm{H}_{2} \mathrm{O}$ (36.0 mg, 20 equiv) in acetone/DMSO (9:1, $\left.0.5 \mathrm{~mL}, 0.2 \mathrm{M}\right)$ under nitrogen atmosphere with visible light for 16 hours, afforded $51.3 \mathrm{mg}(98 \%)$ of $\mathbf{5 b b}$ as a white solid. Enantiomeric excess was established by HPLC analysis using a Chiralpak IC column, ee $=96 \%$ (HPLC: IC, 254 nm, $n$ hexane/isopropanol $=50: 50$, flow rate $0.5 \mathrm{~mL} / \mathrm{min}, 25^{\circ} \mathrm{C}, \mathrm{t}_{\mathrm{r}}$ (major) $=58.0 \mathrm{~min}, \mathrm{t}_{\mathrm{r}}($ minor $)=50.5$ $\min ) .[\alpha]_{\mathrm{D}}^{22}=-106.8^{\circ}\left(c 1.0, \mathrm{CH}_{2} \mathrm{Cl}_{2}\right)$.

${ }^{1} \mathrm{H}$ NMR $\left(500 \mathrm{MHz}, \mathrm{CDCl}_{3}\right) \delta$ 7.87-7.80 (m, 2H), 7.75-7.66 (m, 2H), 7.38-7.23 (m, 7H), 7.23-7.17 (m, 3H), 7.01-7.00 (m, 1H), $6.87(\mathrm{~d}, J=7.5 \mathrm{~Hz}, 1 \mathrm{H}$, other rotamer), 5.61-5.54 (m, 1H), 4.09-3.98 (m, 2H), 3.76-3.67 (m, 2H), 3.29-3.19 (m, 1H), 2.68-2.59 (m, 1H), 2.01 (s, 3H), 1.98-1.90 (m, 2H), 1.52 (s, $3 \mathrm{H}$, other rotamer).

${ }^{13} \mathrm{C}$ NMR $\left(125 \mathrm{MHz}, \mathrm{CDCl}_{3}\right) \delta 189.2,189.1,171.6,1$ 171.60, 168.2, 142.9, 142.8, 137.83, 137.80, 137.6, 137.5, 134.9, 134.3, 134.1, 133.9, 132.0, 130.65, 130.56, 130.1, 129.02, 128.96, 128.70, 128.66, 128.6, 127.3, 127.2, 126.54, 126.50, 126.44, 126.39, 126.1, 123.3, 62.0, 48.59, 48.55, 37.2, 36.7, 35.0, 34.9, 27.5, 17.2, 16.5. (Mixture of two rotation isomers)

IR (film): $v\left(\mathrm{~cm}^{-1}\right)$ 2953, 2928, 1771, 1708, 1683, 1496, 1445, 1398, 1243, 1172, 1086, 1046, 941, $907,765,717,524,466$.

HRMS (ESI, $m / z$ ) calcd for $\mathrm{C}_{31} \mathrm{H}_{27} \mathrm{~N}_{3} \mathrm{O}_{5} \mathrm{Na}[\mathrm{M}+\mathrm{Na}]^{+}$: 544.1843 , found: 544.1848 .

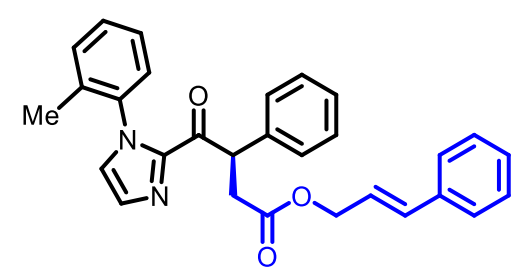

\section{Cinnamyl $(R)-4-o x o-3-p h e n y l-4-(1-(o-t o l y l)-1 H$-imidazol-2-yl)butanoate (5bc)}

According to the general procedure, the reaction of 2-phenyl-1-(1-(o-tolyl)-1H-imidazol-2-yl) ethan1-one $\mathbf{1 b}$ (27.6 mg, $0.10 \mathrm{mmol})$, cinnamyl 2-diazoacetate $3 \mathbf{c}(60.7 \mathrm{mg}, 3.0$ equiv), $\Lambda$-RhS (3.5 mg, 4 mol\%), $\left[\mathrm{Ru}(\mathrm{bpy})_{3}\right]\left(\mathrm{PF}_{6}\right)_{2}(1.3 \mathrm{mg}, 1.5 \mathrm{~mol} \%), \mathrm{Na}_{2} \mathrm{HPO}_{4}(2.8 \mathrm{mg}, 20 \mathrm{~mol} \%)$ and $\mathrm{H}_{2} \mathrm{O}(36.0 \mathrm{mg}, 20$ equiv) in acetone/DMSO (9:1, 0.5 mL, 0.2 M) under nitrogen atmosphere with visible light for 10 hours, afforded $26.3 \mathrm{mg}$ (58\%) of $\mathbf{5 b c}$ as a yellow oil. Enantiomeric excess was established by HPLC analysis using a Chiralpak IC column, ee $=98 \%$ (HPLC: IC, $254 \mathrm{~nm}, n$-hexane/isopropanol = 85:15, 
flow rate $1 \mathrm{~mL} / \mathrm{min}, 25^{\circ} \mathrm{C}, \mathrm{t}_{\mathrm{r}}($ major $)=14.9 \mathrm{~min}, \mathrm{t}_{\mathrm{r}}($ minor $\left.)=17.8 \mathrm{~min}\right) .[\alpha]_{\mathrm{D}}{ }^{22}=-168.6^{\circ}(c 1.0$, $\mathrm{CH}_{2} \mathrm{Cl}_{2}$ ).

${ }^{1} \mathrm{H}$ NMR $\left(500 \mathrm{MHz}, \mathrm{CDCl}_{3}\right) \delta$ 7.42-7.36 (m, 2H), 7.36-7.29 (m, 6H), 7.29-7.24 (m, 4H), 7.24-7.17 $(\mathrm{m}, 3 \mathrm{H}), 7.17-7.14\left(\mathrm{~m}, 1 \mathrm{H}\right.$, other rotamer), $7.00\left(\mathrm{dd}, J_{l}=3.5 \mathrm{~Hz}, J_{2}=1.0 \mathrm{~Hz}, 1 \mathrm{H}\right), 6.89\left(\mathrm{dd}, J_{1}=8.0\right.$ $\mathrm{Hz}, J_{2}=1.0 \mathrm{~Hz}, 1 \mathrm{H}$, other rotamer), $6.57(\mathrm{~d}, J=15.5 \mathrm{~Hz}, 1 \mathrm{H}), 6.56(\mathrm{~d}, J=15.5 \mathrm{~Hz}, 1 \mathrm{H}$, other rotamer), 6.21-6.12 (m, 1H), 5.67-5.60 (m, 1H), 4.72-4.61 (m, 2H), 3.39-3.30 (m, 1H), 2.79-2.70 (m, 1H), 1.98 $(\mathrm{s}, 3 \mathrm{H}), 1.54(\mathrm{~s}, 3 \mathrm{H}$, other rotamer).

${ }^{13} \mathrm{C}$ NMR $\left(125 \mathrm{MHz}, \mathrm{CDCl}_{3}\right) \delta 189.27,189.18,171.5,171.4,142.9,142.8,137.84,137.79,137.6$, $137.5,136.2$, 134.9, 134.3, 133.93, 133.85, 130.61, 130.55, 130.21, 130.20, 129.0, 128.9, 128.73, $128.68,128.59,128.57,128.51,128.48,128.0,127.30,127.27,126.6,126.52$, 126.46, 126.44, 126.42, 126.39, 126.0, 123.1, 123.0, 65.13, 65.12, 48.7, 48.6, 37.41, 37.0, 17.1, 16.5. (Mixture of two rotation isomers)

IR (film): $v\left(\mathrm{~cm}^{-1}\right)$ 3059, 3030, 2927, 1732, 1682, 1495, 1451, 1404, 1308, 1242, 1161, 1091, 965, 939, 907, 759, 732, 695, 546, 454.

HRMS (ESI, $m / z$ ) calcd for $\mathrm{C}_{29} \mathrm{H}_{26} \mathrm{~N}_{2} \mathrm{O}_{3} \mathrm{Na}[\mathrm{M}+\mathrm{Na}]^{+}:$473.1836, found: 473.1837 .

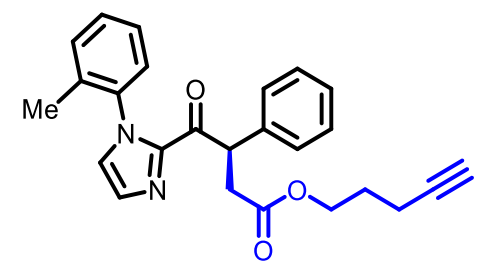

\section{Pent-4-yn-1-yl (R)-4-oxo-3-phenyl-4-(1-(o-tolyl)-1H-imidazol-2-yl)butanoate (5bd)}

According to the general procedure, the reaction of 2-phenyl-1-(1-(o-tolyl)-1H-imidazol-2-yl) ethan1-one $\mathbf{1 b}$ (27.6 mg, $0.10 \mathrm{mmol}$ ), pent-4-yn-1-yl 2-diazoacetate $\mathbf{3 d}$ (45.7 mg, 3.0 equiv), $\Lambda$ - RhS (3.5 mg, $4 \mathrm{~mol} \%$ ), [Ru(bpy) 3 ](PF 6$)_{2}(1.3 \mathrm{mg}, 1.5 \mathrm{~mol} \%), \mathrm{Na}_{2} \mathrm{HPO}_{4}(2.8 \mathrm{mg}, 20 \mathrm{~mol} \%)$ and $\mathrm{H}_{2} \mathrm{O}(36.0 \mathrm{mg}$, 20 equiv) in acetone/DMSO $(9: 1,0.5 \mathrm{~mL}, 0.2 \mathrm{M})$ under nitrogen atmosphere with visible light for 18 hours, afforded $36.0 \mathrm{mg}$ (90\%) of $\mathbf{5 b d}$ as a yellow oil. Enantiomeric excess was established by HPLC analysis using a Chiralpak OD-H column, ee = 96\% (HPLC: OD-H, $254 \mathrm{~nm}, n$-hexane/isopropanol $=90: 10$, flow rate $1 \mathrm{~mL} / \mathrm{min}, 25^{\circ} \mathrm{C}, \mathrm{t}_{\mathrm{r}}($ major $)=12.2 \mathrm{~min}, \mathrm{t}_{\mathrm{r}}($ minor $\left.)=14.6 \mathrm{~min}\right) .[\alpha]_{\mathrm{D}}{ }^{22}=-201.4^{\circ}$ (c $1.0, \mathrm{CH}_{2} \mathrm{Cl}_{2}$ ).

${ }^{1} \mathrm{H}$ NMR $\left(500 \mathrm{MHz}, \mathrm{CDCl}_{3}\right) \delta$ 7.42-7.26 (m, 7H), 7.25-7.19 (m, 3H), 7.04-7.03 (m, 1H), $6.89(\mathrm{~d}, J=$ $7.5 \mathrm{~Hz}, 1 \mathrm{H}$, other rotamer), 5.65-5.57 (m, 1H), 4.17-4.07 (m, 2H), 3.36-3.29 (m, 1H), 2.75-2.66 (m, 
$1 \mathrm{H}), 2.22-2.13(\mathrm{~m}, 2 \mathrm{H}), 2.04(\mathrm{~s}, 3 \mathrm{H}), 1.97-1.93(\mathrm{~m}, 1 \mathrm{H}), 1.80-1.73(\mathrm{~m}, 2 \mathrm{H}), 1.55(\mathrm{~s}, 3 \mathrm{H}$, other rotamer).

${ }^{13} \mathrm{C}$ NMR (125 MHz, $\left.\mathrm{CDCl}_{3}\right) \delta 189.2,189.1$ 171.6, 171.5, 142.8, 142.7, 137.78, 137.76, 137.6, 137.4, 134.8, 134.3, 130.6, 130.5, 130.2 130.1, 129.0, 128.9, 128.70, 128.66, 128.5, 127.29, 127.25, 126.51, $126.48,126.43,126.40,126.39,126.0,83.0,68.9,63.1,63.0,48.64,48.56,37.3,36.9,27.42,27.38$, 17.2, 16.4, 15.05, 15.00. (Mixture of two rotation isomers)

IR (film): $v\left(\mathrm{~cm}^{-1}\right)$ 3289, 2959, 2927, 1731, 1682, 1495, 1453, 1403, 1362, 1306, 1243, 1170, 1089, 1026, 941, 908, 763, 700, 639, 525.

HRMS (ESI, $m / z$ ) calcd for $\mathrm{C}_{25} \mathrm{H}_{24} \mathrm{~N}_{2} \mathrm{O}_{3} \mathrm{Na}[\mathrm{M}+\mathrm{Na}]^{+}$: 423.1679, found: 423.1679 .

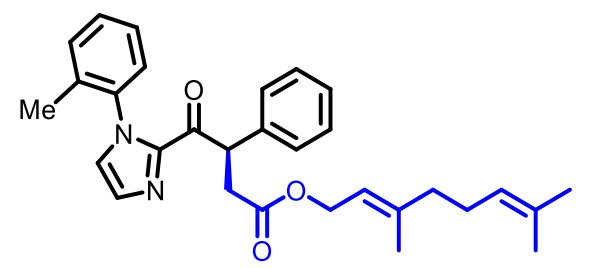

(E)-3,7-Dimethylocta-2,6-dien-1-yl (R)-4-oxo-3-phenyl-4-(1-(o-tolyl)-1H-imidazol-2-yl)butanoate (5be)

According to the general procedure, the reaction of 2-phenyl-1-(1-(o-tolyl)-1H-imidazol-2-yl) ethan1-one $\mathbf{1 b}$ (27.6 mg, $0.10 \mathrm{mmol}$ ), (E)-3,7-dimethylocta-2,6-dien-1-yl 2-diazoacetate 3e (66.7 mg, 3.0 equiv), $\Lambda$-RhS (3.5 mg, 4 mol\%), [Ru(bpy) 3 ] $\left(\mathrm{PF}_{6}\right)_{2}(1.3 \mathrm{mg}, 1.5 \mathrm{~mol} \%), \mathrm{Na}_{2} \mathrm{HPO}_{4}(2.8 \mathrm{mg}, 20 \mathrm{~mol} \%)$ and $\mathrm{H}_{2} \mathrm{O}$ (36.0 mg, 20 equiv) in acetone/DMSO (9:1, $\left.0.5 \mathrm{~mL}, 0.2 \mathrm{M}\right)$ under nitrogen atmosphere with visible light for 24 hours, afforded $38.6 \mathrm{mg}$ (82\%) of 5be as a yellow oil. Enantiomeric excess was established by HPLC analysis using a Chiralpak OD-H column, ee = 96\% (HPLC: OD-H, 254 nm, $n$-hexane/isopropanol $=90: 10$, flow rate $1 \mathrm{~mL} / \mathrm{min}, 25^{\circ} \mathrm{C}, \mathrm{t}_{\mathrm{r}}($ major $\left.)=7.7 \mathrm{~min}, \mathrm{t}_{\mathrm{r}}(\operatorname{minor})=9.1 \mathrm{~min}\right)$. $[\alpha]_{\mathrm{D}}^{22}=-170.2^{\circ}\left(c 0.4, \mathrm{CH}_{2} \mathrm{Cl}_{2}\right)$.

${ }^{1} \mathrm{H}$ NMR $\left(500 \mathrm{MHz}, \mathrm{CDCl}_{3}\right) \delta$ 7.42-7.24 (m, 7H), 7.24-7.17 (m, 3H), 7.03-7.01 (m, 1H), $6.89(\mathrm{~d}, J=$ $7.5 \mathrm{~Hz}, 1 \mathrm{H}$, other rotamer), 5.64-5.57 (m, 1H), 5.27-5.22 (m, 1H), 5.10-5.05 (m, 1H), 4.55-4.49 (m, 2H), 3.35-3.26 (m, 1H), 2.75-2.65 (m, 1H), 2.11-1.97 (m, 4H), $2.04(\mathrm{~s}, 3 \mathrm{H}), 1.69$ (s, 3H), $1.65(\mathrm{~s}, 3 \mathrm{H})$, $1.64(\mathrm{~s}, 3 \mathrm{H}$, other rotamer), $1.61(\mathrm{~s}, 3 \mathrm{H}), 1.55$ (s, 3H, other rotamer).

${ }^{13} \mathrm{C}$ NMR $\left(125 \mathrm{MHz}, \mathrm{CDCl}_{3}\right) \delta 189.4,189.3,171.7,143.0,142.8,142.1,142.0,137.9,137.8,137.7$, 137.6, 134.9, 134.3, 131.8, 130.60, 130.56, 130.2, 130.1, 129.0, 128.9, 128.7, 128.63, 128.59, 128.58, 127.23, 127.20, 126.5, 126.45, 126.39, 126.37, 126.1, 123.7, 118.2, 118.1, 61.50, 48.65, 48.62, 39.5, 
37.4, 37.0, 26.2, 25.7, 17.7, 17.2, 16.5, 16.4. (Mixture of two rotation isomers)

IR (film): $v\left(\mathrm{~cm}^{-1}\right)$ 2968, 2922, 2858, 1731, 1685, 1496, 1451, 1405, 1310, 1240, 1167, 940, 908, 763, $738,700,547$.

HRMS (ESI, $m / z$ ) calcd for $\mathrm{C}_{30} \mathrm{H}_{34} \mathrm{~N}_{2} \mathrm{O}_{3} \mathrm{Na}[\mathrm{M}+\mathrm{Na}]^{+}$: 493.2462, found: 493.2462 .

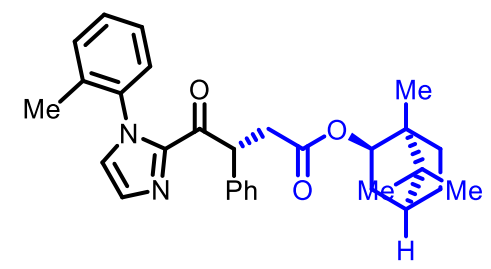

$(1 S, 2 R, 4 S)-1,7,7-T r i m e t h y l b i c y c l o[2.2 .1]$ heptan-2-yl $(R)-4-o x o-3-p h e n y l-4-(1-(o-t o l y l)-1 H$ imidazol-2-yl)butanoate (5bf)

According to the general procedure, the reaction of 2-phenyl-1-(1-(o-tolyl)-1H-imidazol-2-yl) ethan1-one $\mathbf{1 b}(27.6 \mathrm{mg}, 0.10 \mathrm{mmol}),(1 S, 2 R, 4 S)$-1,7,7-trimethylbicyclo[2.2.1]heptan-2-yl 2-diazoacetate 3f (66.7 mg, 3.0 equiv), $\Lambda$-RhS (3.5 mg, 4 mol\%), [Ru(bpy) $)_{3}\left(\mathrm{PF}_{6}\right)_{2}(1.3 \mathrm{mg}, 1.5 \mathrm{~mol} \%), \mathrm{Na}_{2} \mathrm{HPO}_{4}$ (2.8 mg, $20 \mathrm{~mol} \%$ ) and $\mathrm{H}_{2} \mathrm{O}(36.0 \mathrm{mg}, 20$ equiv) in acetone/DMSO (9:1, $0.5 \mathrm{~mL}, 0.2 \mathrm{M})$ under nitrogen atmosphere with visible light for 24 hours, afforded $43.4 \mathrm{mg}$ (92\%) of $\mathbf{5 b f}$ as a yellow solid. Diasteromer ratio was established by ${ }^{1} \mathrm{H}$ NMR and HPLC analysis using a Chiralpak OD-H column, d.r. > 98:2 (HPLC: OD-H, $254 \mathrm{~nm}, n$-hexane/isopropanol = 99:1, flow rate $1 \mathrm{~mL} / \mathrm{min}, 25^{\circ} \mathrm{C}, \mathrm{t}_{\mathrm{r}}$ (major) $=17.8 \min , \mathrm{t}_{\mathrm{r}}($ minor $\left.)=25.5 \mathrm{~min}\right) .[\alpha]_{\mathrm{D}}^{22}=-180.0^{\circ}\left(c 1.0, \mathrm{CH}_{2} \mathrm{Cl}_{2}\right)$.

${ }^{1} \mathrm{H}$ NMR $\left(500 \mathrm{MHz}, \mathrm{CDCl}_{3}\right) \delta$ 7.44-7.17 (m, 10H), 7.04-7.01 (m, 1H), $6.86(\mathrm{~d}, J=8.0 \mathrm{~Hz}, 1 \mathrm{H}$, other rotamer), 5.67-5.50 (m, 1H), 4.87-4.76 (m, 1H), 3.39-3.25 (m, 1H), 2.79-2.70 (m, 1H), 2.32-2.21 (m, $1 \mathrm{H}), 2.05(\mathrm{~s}, 3 \mathrm{H}), 1.89-1.60(\mathrm{~m}, 3 \mathrm{H}), 1.52(\mathrm{~s}, 3 \mathrm{H}$, other rotamer), 1.30-1.06 (m, 2H), $1.51(\mathrm{~s}, 3 \mathrm{H}$, other rotamer), 0.95-0.83 (m, 7H), $0.72(\mathrm{~s}, 3 \mathrm{H}), 0.70$ (s, 3H, other rotamer).

${ }^{13} \mathrm{C}$ NMR $\left(125 \mathrm{MHz}, \mathrm{CDCl}_{3}\right) \delta 189.3,189.2,171.9,171.8,142.89,142.87,137.81,137.79,137.6$, $137.5,134.8,134.3,130.6,130.5,130.13,130.09,129.0,128.9,128.8,128.7,128.62$, 128.60, 128.58, 127.22, 127.19, 126.5, 126.42, 126.35, 126.1, 80.2, 48.8, 48.61, 48.59, 48.5, 47.70, 47.66, 44.74, $44.73,37.5,37.1,36.45,36.35,27.82$, 27.77, 27.0, 26.9, 19.6, 18.7, 17.2, 16.4, 13.3, 13.28. (Mixture of two rotation isomers)

IR (film): $v\left(\mathrm{~cm}^{-1}\right)$ 2953, 2878, 1728, 1684, 1495, 1453, 1404, 1306, 1253, 1181, 1155, 1022, 942 , $910,763,731,703,549$.

HRMS (ESI, $m / z$ ) calcd for $\mathrm{C}_{30} \mathrm{H}_{34} \mathrm{~N}_{2} \mathrm{O}_{3} \mathrm{Na}[\mathrm{M}+\mathrm{Na}]^{+}$: 493.2462, found: 493.2462 . 


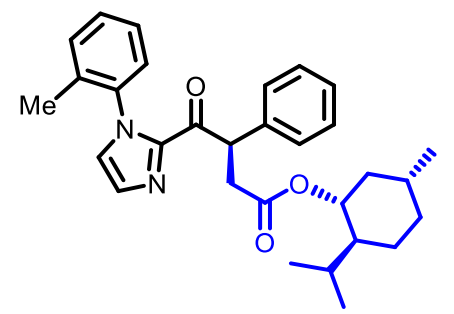

$(1 R, 2 S, 5 R)-2-I s o p r o p y l-5-m e t h y l c y c l o h e x y l ~(R)-4-0 x 0-3-p h e n y l-4-(1-(o-t o l y l)-1 H$-imidazol-2-yl)

\section{butanoate (5bg)}

According to the general procedure, the reaction of 2-phenyl-1-(1-(o-tolyl)-1H-imidazol-2-yl) ethan1-one $\mathbf{1 b}$ (27.6 mg, $0.10 \mathrm{mmol}),(1 R, 2 S, 5 R)$-2-isopropyl-5-methylcyclohexyl 2-diazoacetate $\mathbf{3 g}(67.2$ mg, 3.0 equiv), $\Lambda$-RhS (3.5 mg, 4 mol\%), [Ru(bpy) $\left.)_{3}\right]\left(\mathrm{PF}_{6}\right)_{2}(1.3 \mathrm{mg}, 1.5 \mathrm{~mol} \%), \mathrm{Na}_{2} \mathrm{HPO}_{4}(2.8 \mathrm{mg}$, $20 \mathrm{~mol} \%$ ) and $\mathrm{H}_{2} \mathrm{O}$ (36.0 mg, 20 equiv) in acetone/DMSO (9:1, $\left.0.5 \mathrm{~mL}, 0.2 \mathrm{M}\right)$ under nitrogen atmosphere with visible light for 13 hours, afforded $44.2 \mathrm{mg}$ (93\%) of $\mathbf{5 b g}$ as a colorless oil. Diasteromer ratio was established by ${ }^{1} \mathrm{H}$ NMR and HPLC analysis using a Chiralpak IC column, d.r. > 99:1 (HPLC: IC, $254 \mathrm{~nm}, n$-hexane/isopropanol = 98:2, flow rate $1 \mathrm{~mL} / \mathrm{min}, 25^{\circ} \mathrm{C}$, $\mathrm{t}_{\mathrm{r}}$ (major) $=30.4$ $\min , \mathrm{t}_{\mathrm{r}}($ minor $\left.)=22.0 \mathrm{~min}\right) .[\alpha]_{\mathrm{D}}^{22}=-220.6^{\circ}\left(c 0.4, \mathrm{CH}_{2} \mathrm{Cl}_{2}\right)$.

${ }^{1} \mathrm{H}$ NMR (500 MHz, $\left.\mathrm{CDCl}_{3}\right) \delta$ 7.42-7.16 (m, 10H), 7.03-7.00 (m, 1H), $6.84(\mathrm{~d}, J=7.5 \mathrm{~Hz}, 1 \mathrm{H}$, other rotamer), 5.62-5.54 (m, 1H), 4.62-4.53 $(\mathrm{m}, 1 \mathrm{H}), 3.30-3.17(\mathrm{~m}, 1 \mathrm{H}), 2.74-2.65(\mathrm{~m}, 1 \mathrm{H}), 2.03(\mathrm{~s}, 3 \mathrm{H})$, 1.88-1.83 (m, 1H), 1.66-1.60 (m, 2H), 1.51 (s, 3H, other rotamer), 1.47-1.23 (m, 3H), 1.02-0.75 (m, 9H), 0.66-0.60 (m, 3H).

${ }^{13} \mathrm{C}$ NMR $\left(125 \mathrm{MHz}, \mathrm{CDCl}_{3}\right) \delta 189.3,171.3,171.3,143.0,142.9,137.8,137.6,137.5,134.9,134.3$, 130.6, 130.14, 130.10, 129.03, 128.96, 128.68, 128.66, 128.6, 127.3, 127.2, 126.47, 126.45, 126.40, $126.37,126.1,74.49,74.47,49.0,48.8,46.8,46.7,40.7,37.7,37.3,34.2,31.3,26.03,25.97,23.33$, 23.26, 21.99, 21.95, 20.73, 20.68, 17.3, 16.4, 16.21, 16.15. (Mixture of two rotation isomers) IR (film): $v\left(\mathrm{~cm}^{-1}\right)$ 2954, 2926, 2867, 1726, 1684, 1496, 1454, 1405, 1373, 1306, 1246, 1178, 1153, 1091, 980, 941, 909, 763, 730, 702, 549.

HRMS (ESI, $m / z$ ) calcd for $\mathrm{C}_{30} \mathrm{H}_{36} \mathrm{~N}_{2} \mathrm{O}_{3} \mathrm{Na}[\mathrm{M}+\mathrm{Na}]^{+}$: 495.2618, found: 495.2619 .

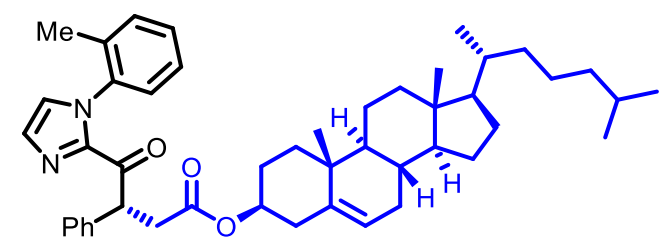


2,3,4,7,8,9,10,11,12,13,14,15,16,17-tetradecahydro-1H-cyclopenta[a]phenanthren-3-yl $(R)-4$ oxo-3-phenyl-4-(1-(o- tolyl)-1H-imidazol-2-yl)butanoate (5bh)

According to the general procedure, the reaction of 2-phenyl-1-(1-(o-tolyl)-1H-imidazol-2-yl) ethan1-one 1b (41.4 mg, $0.15 \mathrm{mmol})$, cholesteryl 2-diazoacetate $3 \mathbf{h}$ (45.4 mg, $0.10 \mathrm{mmol}), \Lambda$-RhS (3.5 mg, $4 \mathrm{~mol} \%),\left[\mathrm{Ru}(\mathrm{bpy})_{3}\right]\left(\mathrm{PF}_{6}\right)_{2}(2.2 \mathrm{mg}, 2.5 \mathrm{~mol} \%), \mathrm{Na}_{2} \mathrm{HPO}_{4}(2.8 \mathrm{mg}, 20 \mathrm{~mol} \%)$ and $\mathrm{H}_{2} \mathrm{O}(36.0 \mathrm{mg}, 20$ equiv) in acetone/DMSO (9:1, $2.0 \mathrm{~mL}, 0.05 \mathrm{M})$ under nitrogen atmosphere with visible light for 60 hours, afforded $58.1 \mathrm{mg}(83 \%)$ of $\mathbf{5 b h}$ as a white solid. Diasteromer ratio was established by ${ }^{1} \mathrm{H}$ NMR and HPLC analysis using a Chiralpak IC column, d.r. > 97:3 (HPLC: IC, 254 nm, nhexane/isopropanol $=85: 15$, flow rate $1 \mathrm{~mL} / \mathrm{min}, 25^{\circ} \mathrm{C}, \mathrm{t}_{\mathrm{r}}($ major $)=15.5 \mathrm{~min}, \mathrm{t}_{\mathrm{r}}($ minor $\left.)=8.9 \mathrm{~min}\right)$. $[\alpha]_{\mathrm{D}}^{22}=-116.6^{\circ}\left(c 0.4, \mathrm{CH}_{2} \mathrm{Cl}_{2}\right)$.

${ }^{1} \mathrm{H}$ NMR $\left(500 \mathrm{MHz}, \mathrm{CDCl}_{3}\right) \delta$ 7.42-7.17 (m, 10H), 7.03-7.01 (m, 1H), $6.88(\mathrm{~d}, J=7.5 \mathrm{~Hz}, 1 \mathrm{H}$, other rotamer), 5.62-5.56 (m, 1H), 5.35-5.31 (m, 1H), 4.56-4.57 (m, 1H), 3.30-3.22 (m, 1H), 2.73-2.62 (m, 1H), 2.25-2.17 (m, 2H), 2.04 (s, 3H), 2.03-1.90 (m, 2H), 1.88-1.70 (m, 3H), 1.60-0.98 (m, 21H), 1.54 (s, 3H, other rotamer), $0.98(\mathrm{~s}, 3 \mathrm{H}), 0.97(\mathrm{~s}, 3 \mathrm{H}$, other rotamer $), 0.91(\mathrm{~d}, J=6.5 \mathrm{~Hz}, 3 \mathrm{H}), 0.87(\mathrm{~d}, J=$ $6.5 \mathrm{~Hz}, 3 \mathrm{H}), 0.86(\mathrm{~d}, J=7.0 \mathrm{~Hz}, 3 \mathrm{H}), 0.67$ (s, 3H).

${ }^{13} \mathrm{C}$ NMR (125 MHz, $\left.\mathrm{CDCl}_{3}\right) \delta 189.4,189.3,171.1,171.0,142.9,142.8,139.7,139.6,137.85,137.83$, $137.7,137.6$, 134.9, 134.3, 130.61, 130.59, 130.10, 130.07, 129.02, 128.96, 128.7, 128.6, 127.23, 127.20, 126.5, 126.4, 126.1, 122.50, 122.46, 74.25, 74.23, 56.6, 56.1, 49.93, 49.92, 48.7, 48.6, 42.3, 39.7, 39.5, 37.92, 37.87, 37.8, 37.4, 36.9, 36.52, 36.50, 36.1, 35.8, 31.9, 31.84, 31.79, 28.20, 28.0, 27.6, 27.5, 24.2, 23.8, 22.8, 22.5, 21.0, 19.3, 18.7, 17.3, 16.5, 11.8. (Mixture of two rotation isomers) IR (film): $v\left(\mathrm{~cm}^{-1}\right)$ 2939, 2864, 1730, 1685, 1496, 1458, 1406, 1373, 1245, 1176, 1007, 940, 909, 763, $730,703,547$.

HRMS (ESI, $m / z$ ) calcd for $\mathrm{C}_{47} \mathrm{H}_{62} \mathrm{~N}_{2} \mathrm{O}_{3} \mathrm{Na}[\mathrm{M}+\mathrm{Na}]^{+}:$725.4653, found: 725.4666 . 


\section{Removal of Directing Group}
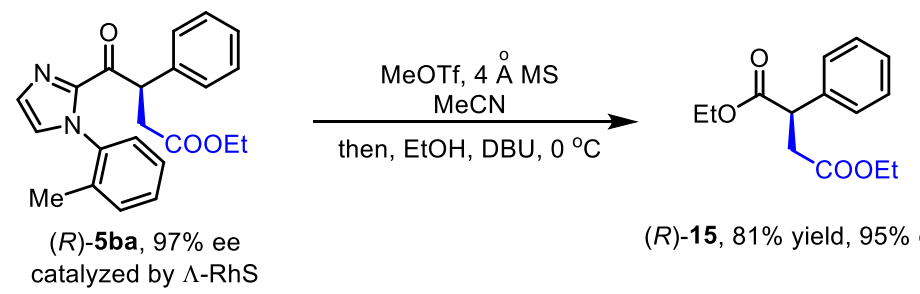

$(\mathrm{S} 14)$

$(R)-15,81 \%$ yield, $95 \%$ ee

The directing imidazole moiety was cleaved according to our previous report with slight modification. ${ }^{19} 4$ A $\mathrm{MS}(169 \mathrm{mg}, 100 \mathrm{mg} / 0.1 \mathrm{mmol}$ of $\mathbf{5 b a})$ was added to a solution of $(R)-5 \mathbf{b a}(61$ $\mathrm{mg}, 0.169 \mathrm{mmol})$ in $\mathrm{CH}_{3} \mathrm{CN}(0.1 \mathrm{M})$ under nitrogen atmosphere. The suspension was stirred vigorously under a positive pressure of nitrogen for $3 \mathrm{~h}$ at $0{ }^{\circ} \mathrm{C}$. Then methyl trifluoromethansulfonate (30.5 mg, $0.186 \mathrm{mmol}, 1.1$ equiv) was added dropwise at $0{ }^{\circ} \mathrm{C}$. After being stirred at $0{ }^{\circ} \mathrm{C}$ for $6 \mathrm{~h}$, EtOH (1.0 mL) and DBU (28.3 mg, $0.186 \mathrm{mmol}, 1.1$ equiv) were subsequently added to the reaction mixture at $0{ }^{\circ} \mathrm{C}$. After being stirred at $0{ }^{\circ} \mathrm{C}$ for $60 \mathrm{~min}, 10 \mathrm{~mL}$ of saturated $\mathrm{NaHCO}_{3}$ aqueous solution was added. And the mixture was extracted with DCM, washed with $\mathrm{NaHCO}_{3}$ aqueous solution, water. The organic layer was dried and the solvent was evaporated and the residue was purified by flash chromatography on silica gel $($ EtOAc/ $n$-hexane $=1: 50)$ to give $34.1 \mathrm{mg}(R)-\mathbf{1 5}(81 \%)$ as a colorless oil.

Enantiomeric excess of $(R)$-15 was established by HPLC analysis using a Chiralpak AD-H column, 95\% ee (HPLC: AD-H, $n$-hexane/isopropanol = 95:5, flow rate $1 \mathrm{~mL} / \mathrm{min}, 25^{\circ} \mathrm{C}, \mathrm{t}_{\mathrm{r}}$ (major) $=7.0 \mathrm{~min}$, $\left.\mathrm{t}_{\mathrm{r}}(\operatorname{minor})=8.0 \mathrm{~min}\right) .[\alpha]_{\mathrm{D}}^{22}=-97.6^{\circ}\left(c 1.0, \mathrm{CH}_{2} \mathrm{Cl}_{2}\right)$.

Literature report $^{20}$ for $(S)$-15: 77\% ee (HPLC: AD-H, $n$-hexane/isopropanol = 95:5, flow rate 1 $\mathrm{mL} / \mathrm{min}$, Retention times: $6.69 \mathrm{~min}[(R)$-enantiomer $], 7.68 \mathrm{~min}[(S)$-enantiomer $] .[\alpha]_{\mathrm{D}}{ }^{29}=+51.25(\mathrm{c}$ $1.19, \mathrm{CHCl}_{3}$ ). All other spectroscopic data of $\mathbf{1 5}$ are in agreement with literature report. ${ }^{20}$ 


\section{Enantioselectivities as Determined by Chiral HPLC}

Enantiomeric purities of the reaction products were determined with a Daicel Chiralpak AD-H, ODH, IC $(250 \times 4.6 \mathrm{~mm})$ HPLC column on an Agilent 1200 or 1260 Series or Shimadzu Lc-2030c HPLC System using $n$-hexane/isopropanol as a mobile phase. The column temperature was $25^{\circ} \mathrm{C}$ and UVabsorption was measured at $254 \mathrm{~nm}$.

$\mathrm{mV}$

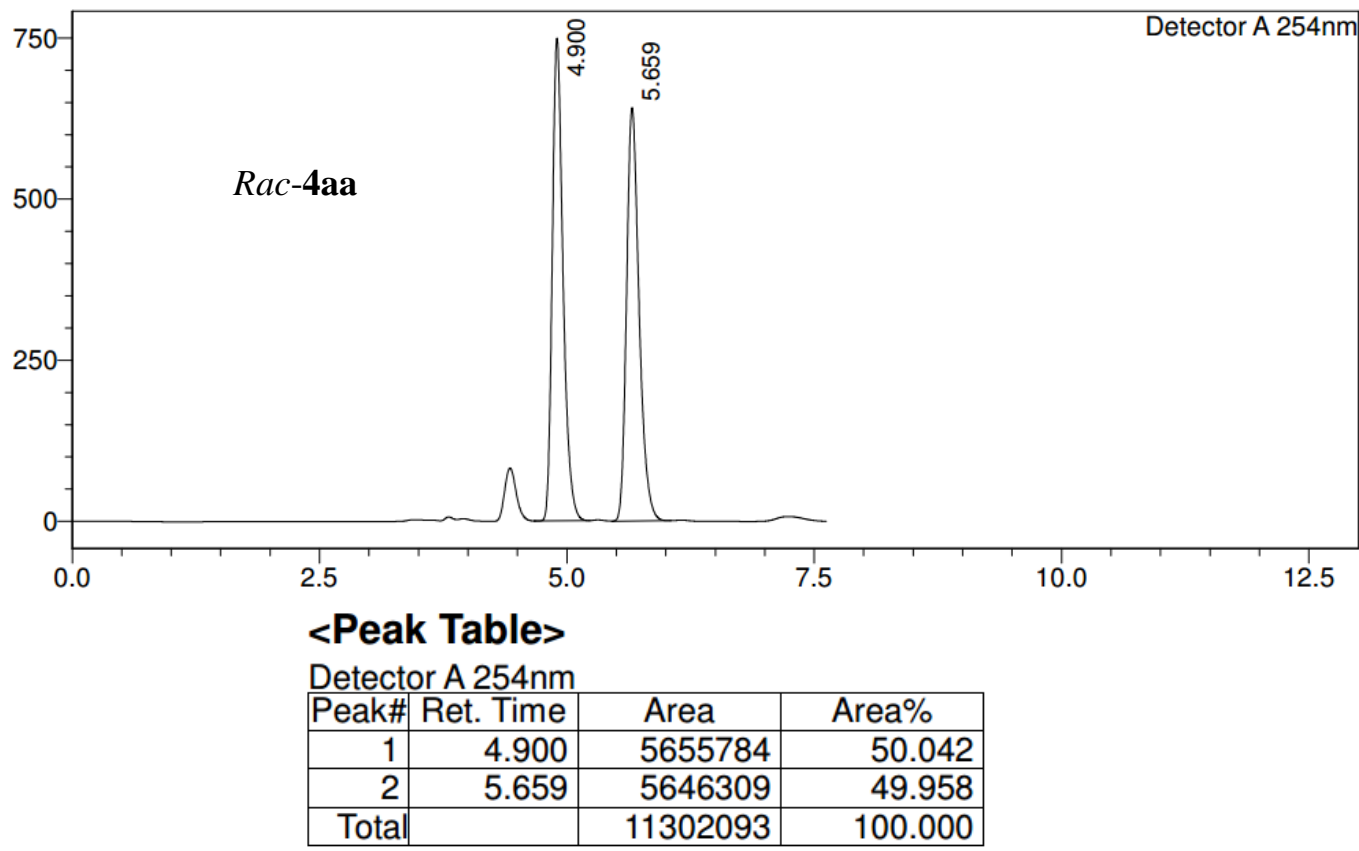

$\mathrm{mV}$

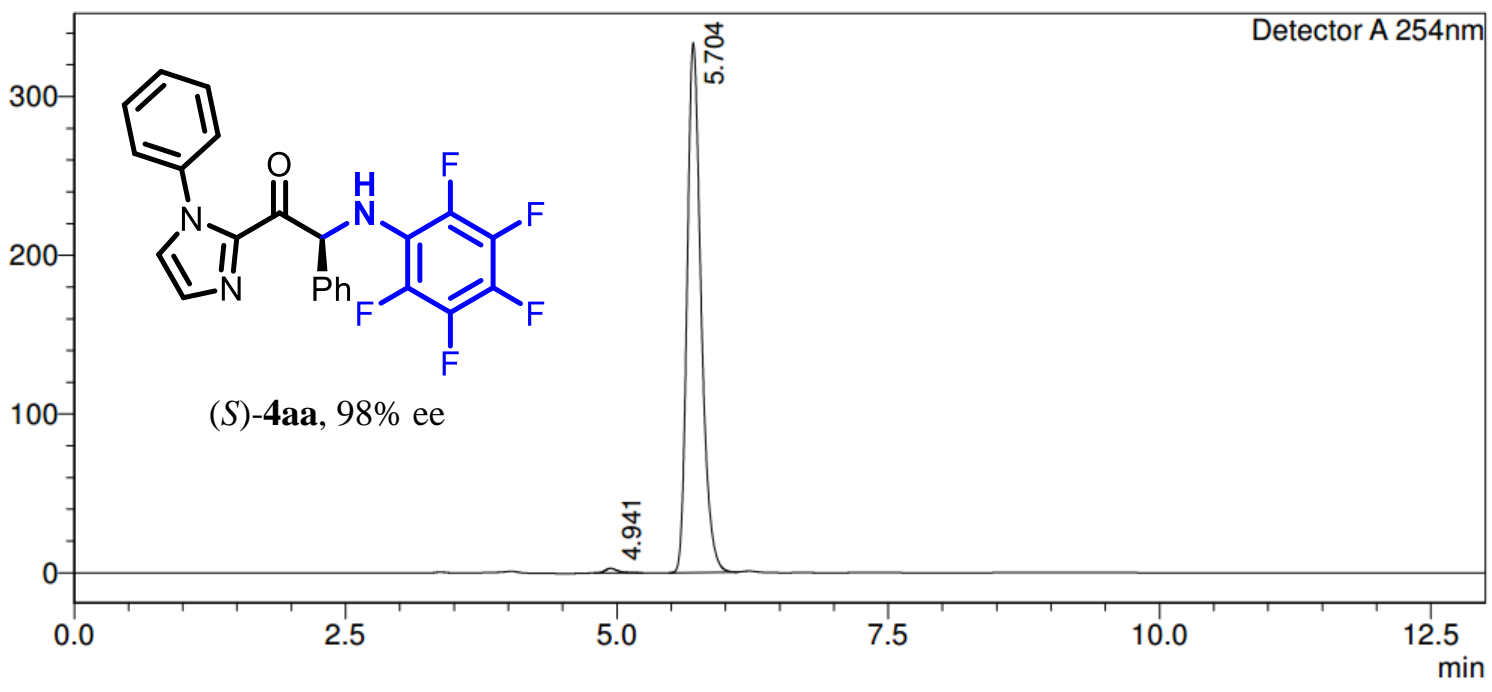

\begin{tabular}{|c|c|c|c|}
\hline \multicolumn{4}{|c|}{$54 \mathrm{~nm}$} \\
\hline Peak\# & Ret. Time & Area & Area $\%$ \\
\hline 1 & 4.941 & 23510 & 0.780 \\
\hline 2 & 5.704 & 2991241 & 99.220 \\
\hline Total & & 3014751 & 100.000 \\
\hline
\end{tabular}

Figure S6. HPLC traces of rac-4aa (reference) and (S)-4aa. 


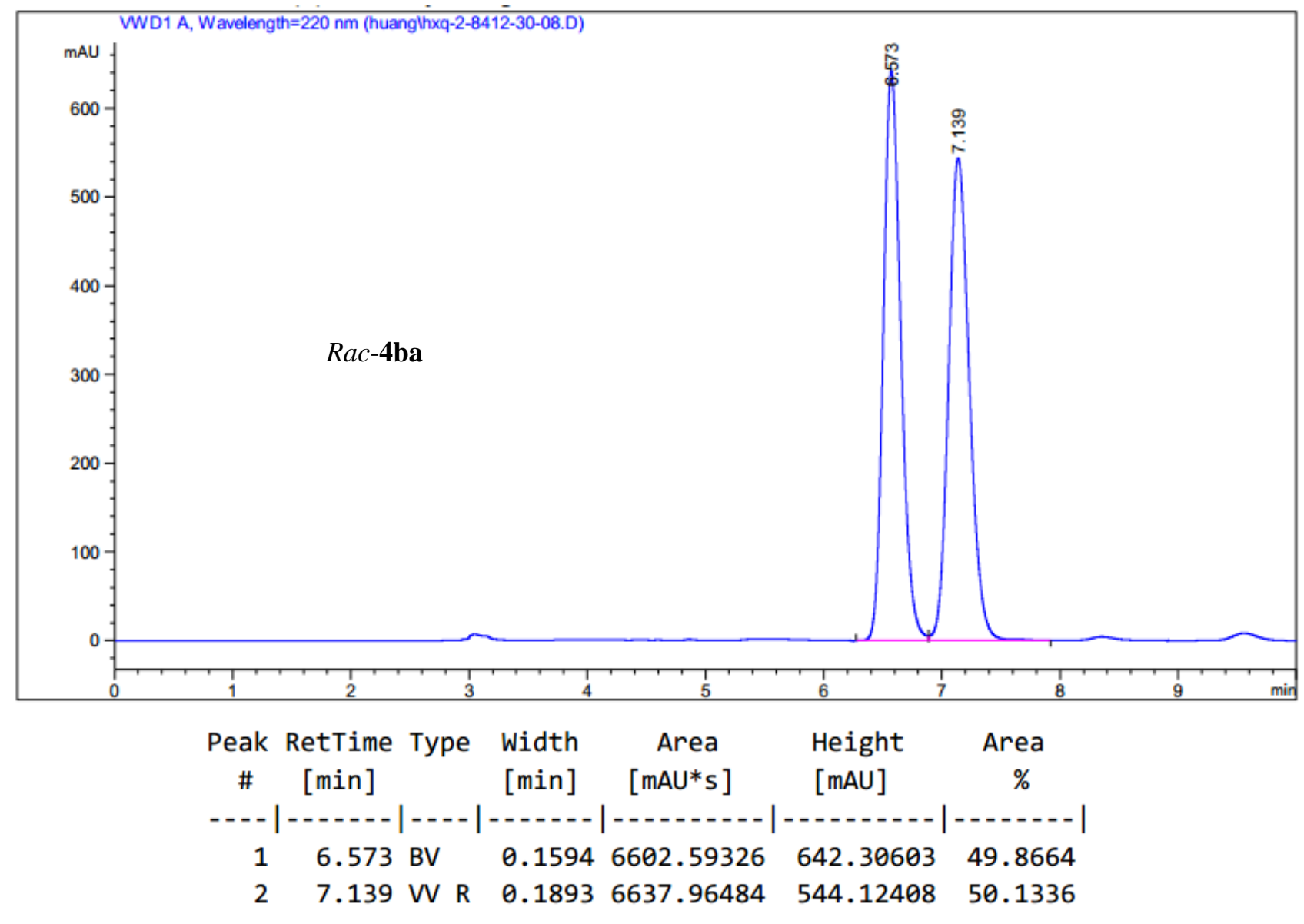

$\mathrm{mV}$

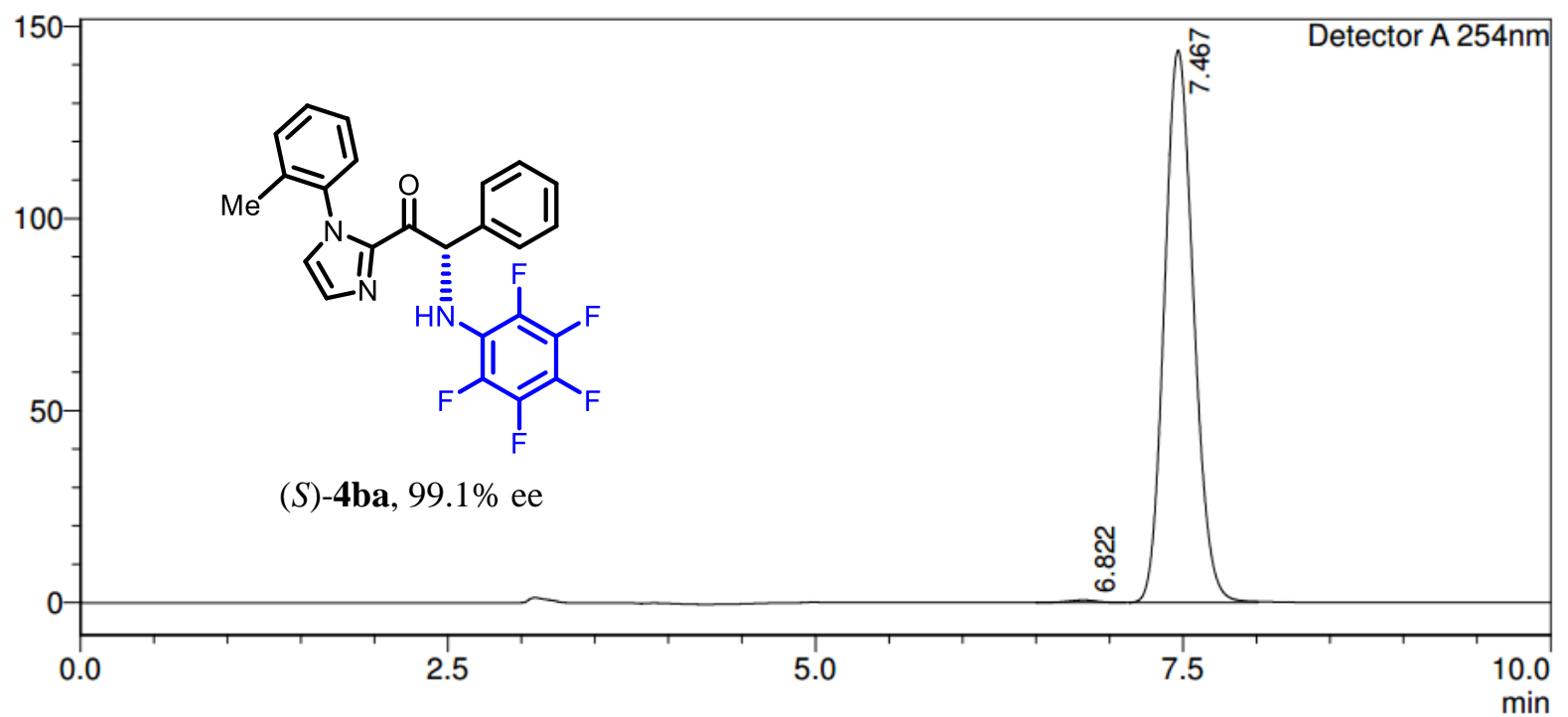

$<$ Peak Table>

\begin{tabular}{|c|c|c|c|}
\hline \multicolumn{4}{|c|}{ Detector A 254nm } \\
\hline 1 & 6.822 & 8758 & 0.439 \\
\hline 2 & 7.467 & 1986670 & 99.561 \\
\hline Total & & 1995428 & 100.000 \\
\hline
\end{tabular}

Figure S7. HPLC traces of $r a c-4 b a$ (reference) and (S)-4ba. 
$\mathrm{mV}$

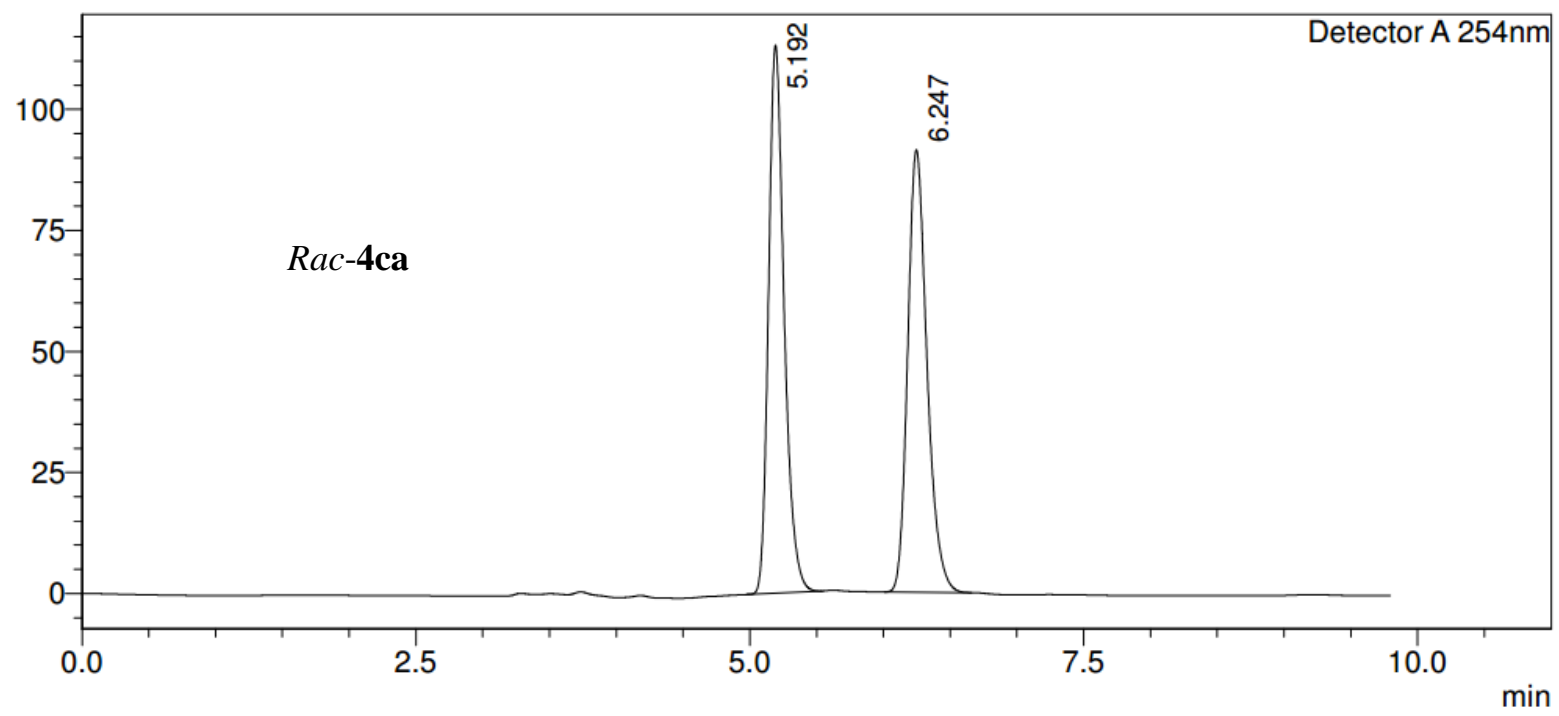

$<$ Peak Table>

Detector A 254nm

\begin{tabular}{|r|r|r|r|}
\hline Peak\# & Ret. Time & \multicolumn{1}{c|}{ Area } & \multicolumn{1}{c|}{ Area\% } \\
\hline 1 & 5.192 & 923178 & 50.150 \\
\hline 2 & 6.247 & 917651 & 49.850 \\
\hline Total & & 1840829 & 100.000 \\
\hline
\end{tabular}

$\mathrm{mV}$

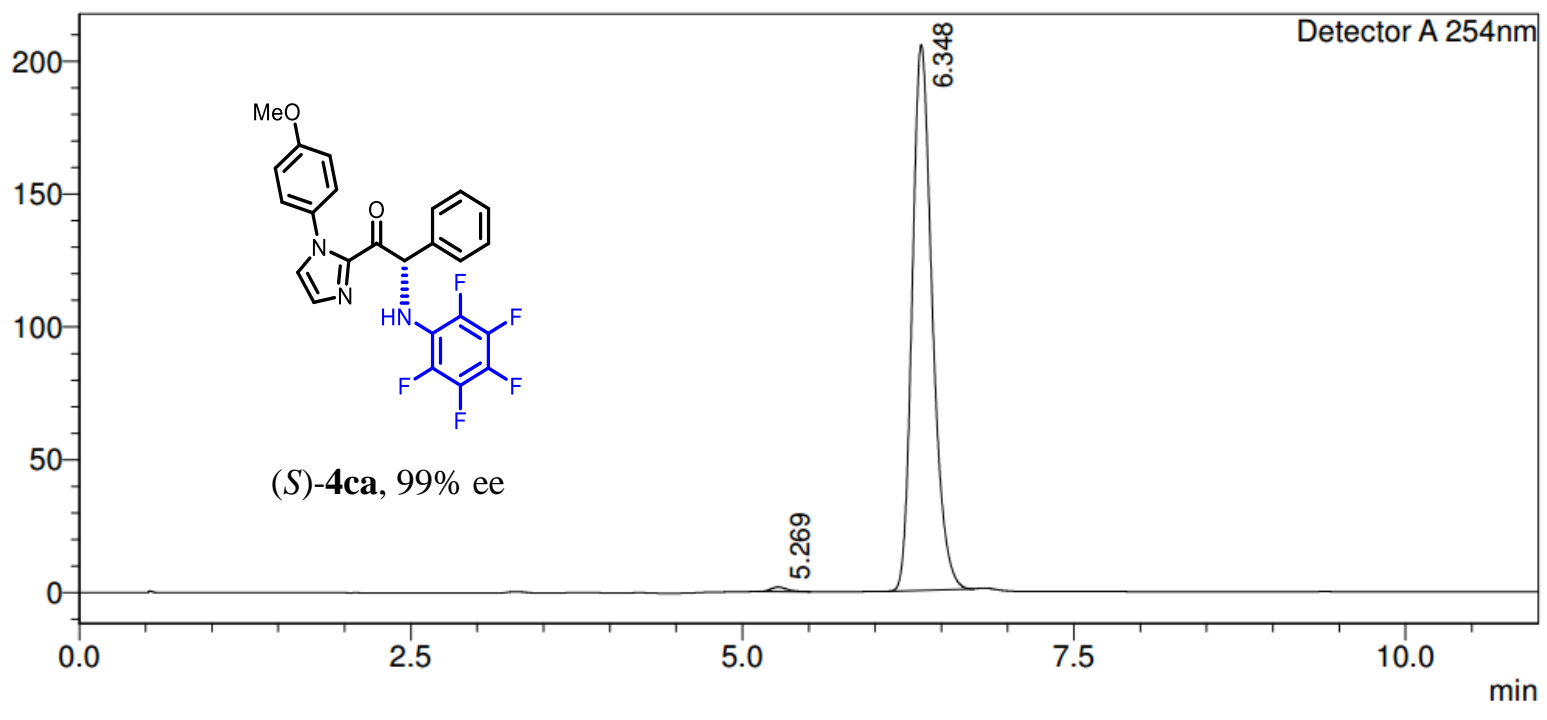

$<$ Peak Table>

Detector A 254nm

\begin{tabular}{|r|r|r|r|}
\hline Peak\# & Ret. Time & \multicolumn{1}{c|}{ Area } & \multicolumn{1}{c|}{ Area\% } \\
\hline 1 & 5.269 & 14044 & 0.646 \\
\hline 2 & 6.348 & 2161068 & 99.354 \\
\hline Total & & 2175112 & 100.000 \\
\hline
\end{tabular}

Figure S8. HPLC traces of rac-4ca (reference) and (S)-4ca. 
$\mathrm{mV}$

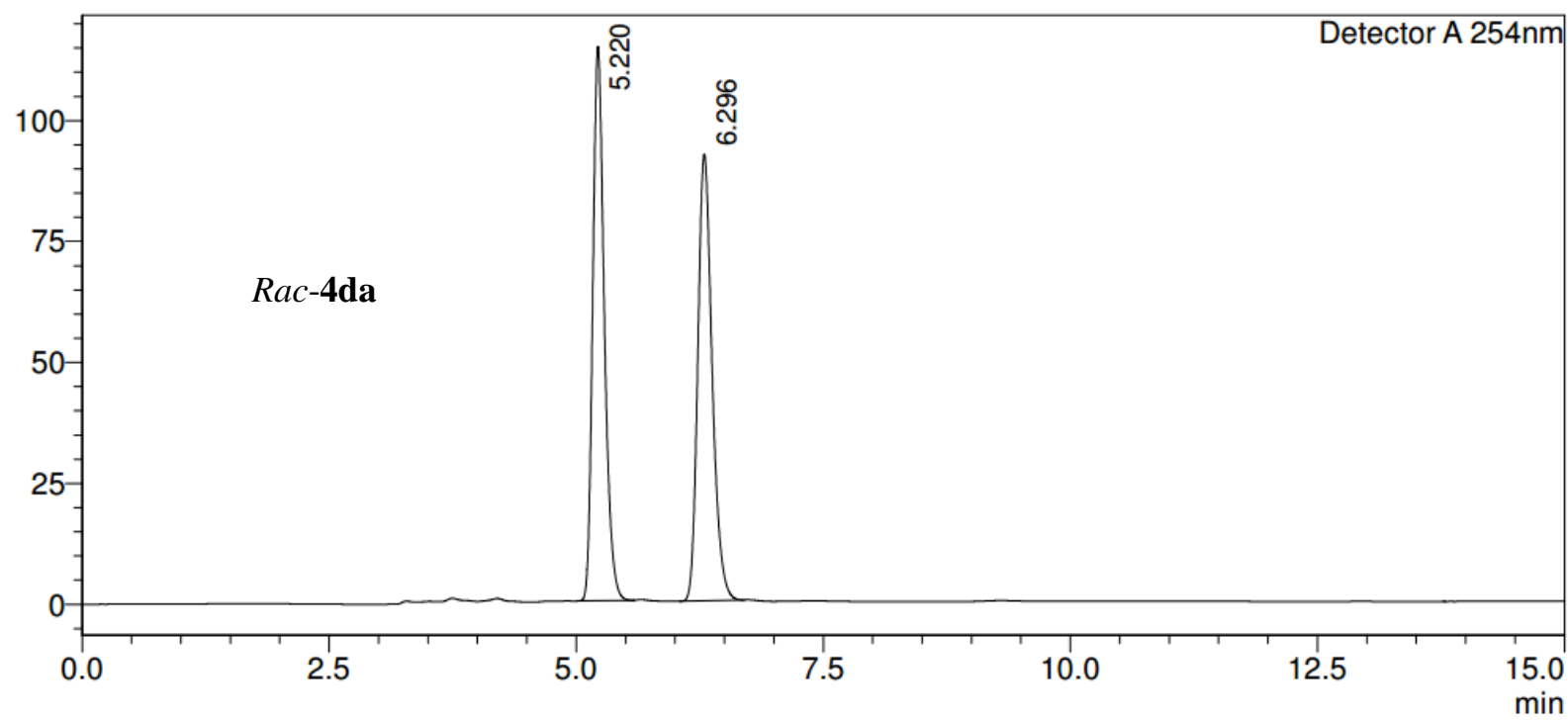

<Peak Table>

Detector A 254nm

\begin{tabular}{|r|r|r|r|}
\hline Peak\# & Ret. Time & \multicolumn{1}{c|}{ Area } & \multicolumn{1}{c|}{ Area\% } \\
\hline 1 & 5.220 & 932942 & 50.121 \\
\hline 2 & 6.296 & 928434 & 49.879 \\
\hline Total & & 1861376 & 100.000 \\
\hline
\end{tabular}

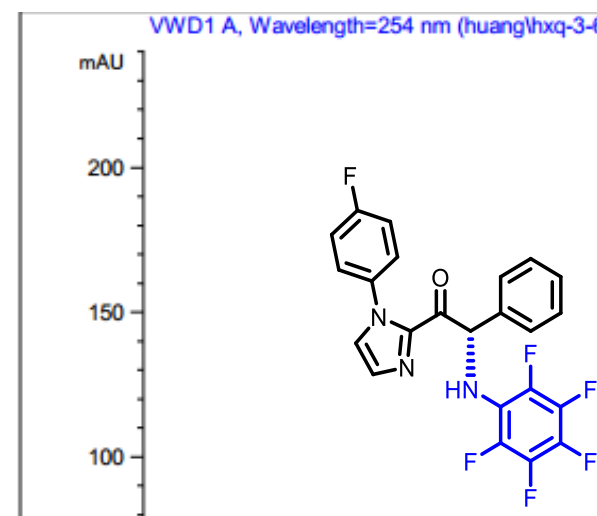

(S)-4da, $98 \%$ ee
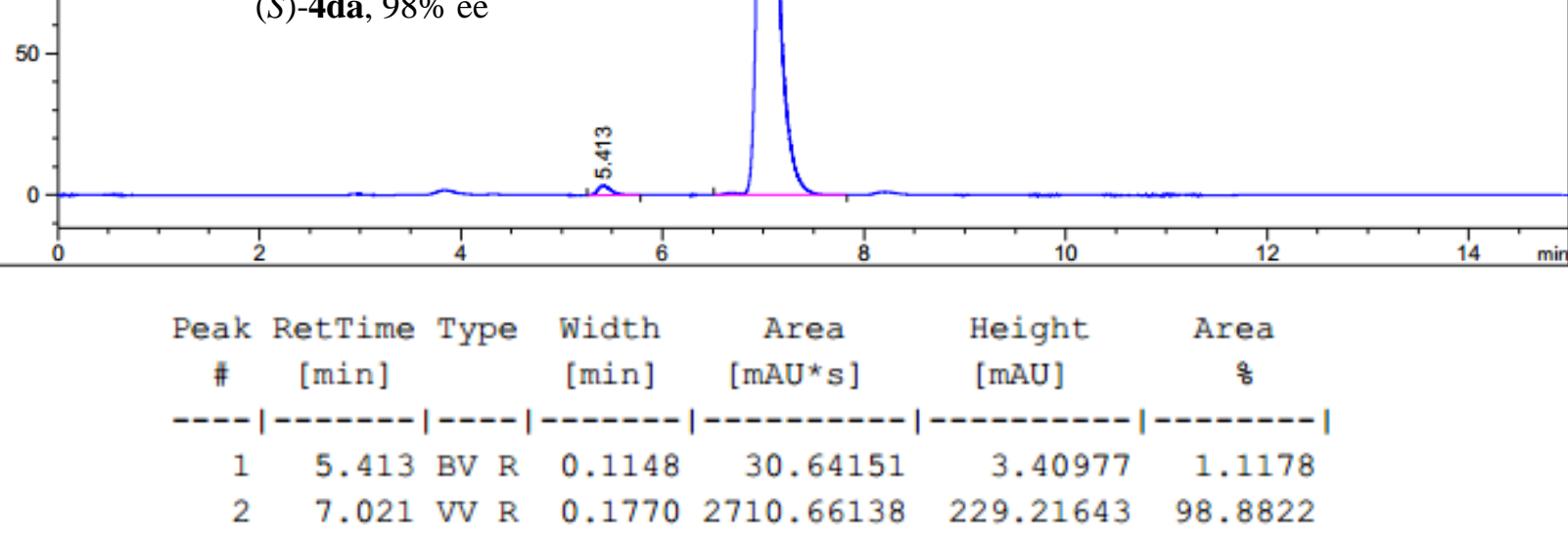

Totals : $\quad 2741.30289 \quad 232.62620$

Figure S9. HPLC traces of rac-4da (reference) and (S)-4da. 
$\mathrm{mV}$

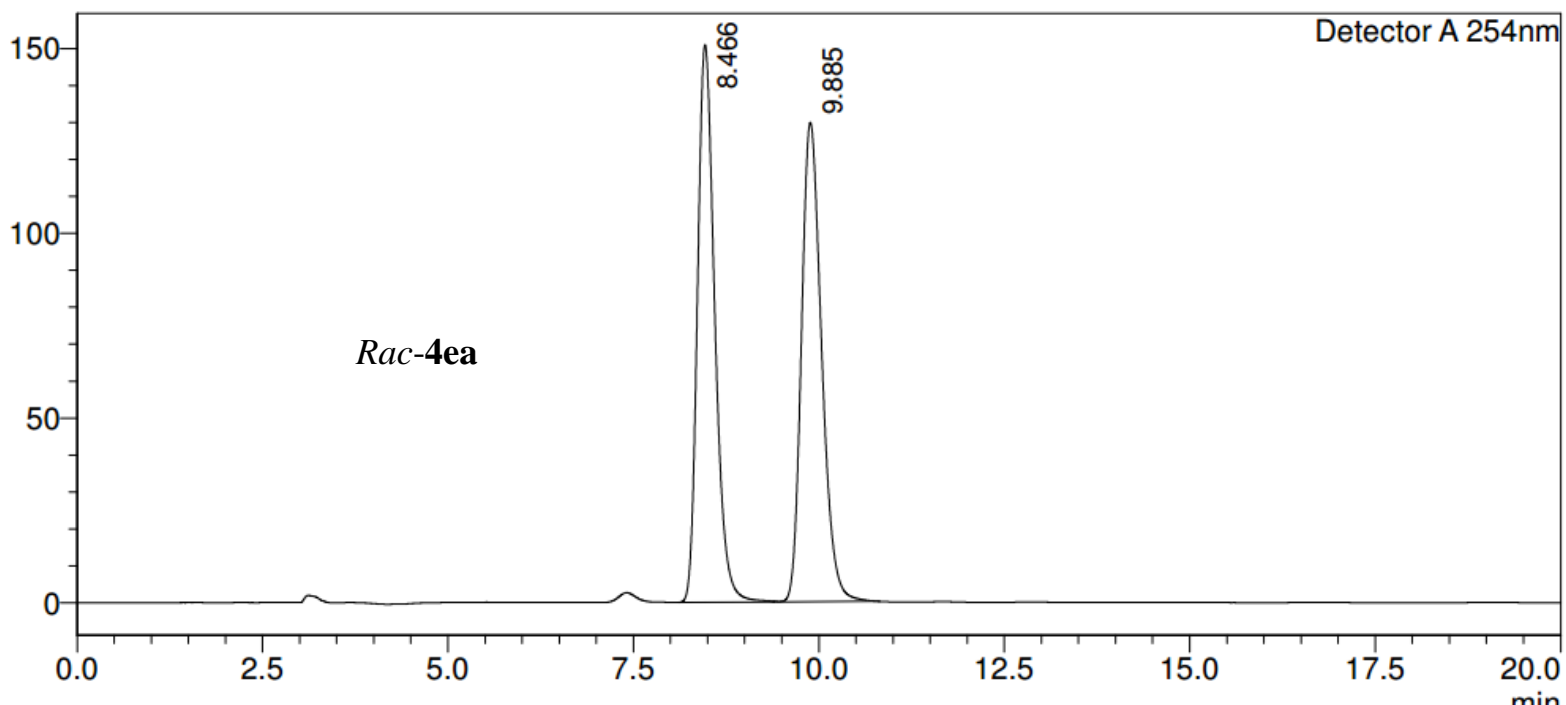

$<$ Peak Table>

Detector A 254nm

\begin{tabular}{|c|c|c|c|}
\hline Peak\# & Ret. Time & Area & Area\% \\
\hline 1 & 8.466 & 2447131 & 49.962 \\
\hline 2 & 9.885 & 2450820 & 50.038 \\
\hline Total & & 4897951 & 100.000 \\
\hline
\end{tabular}

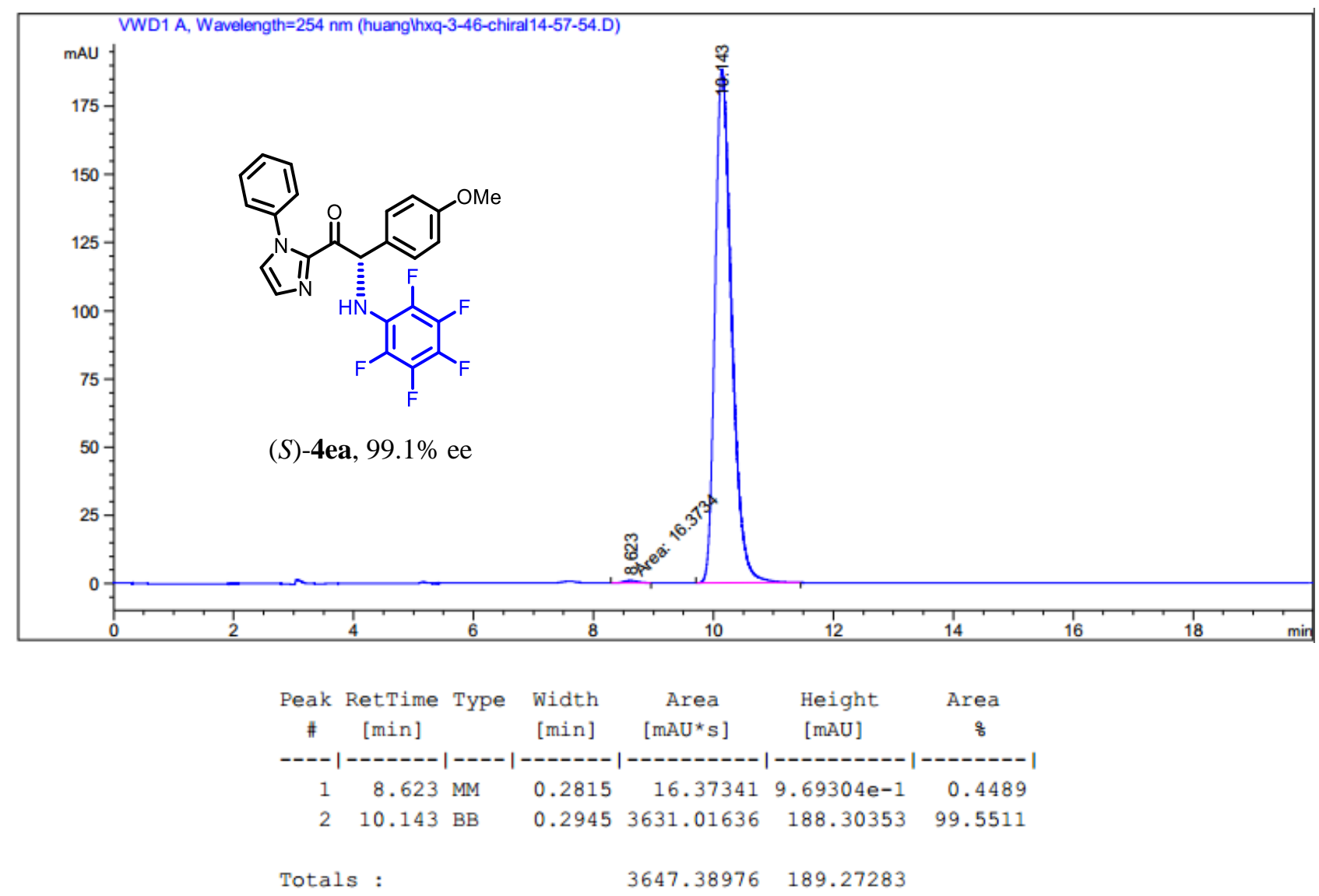

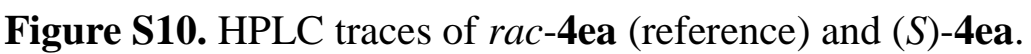



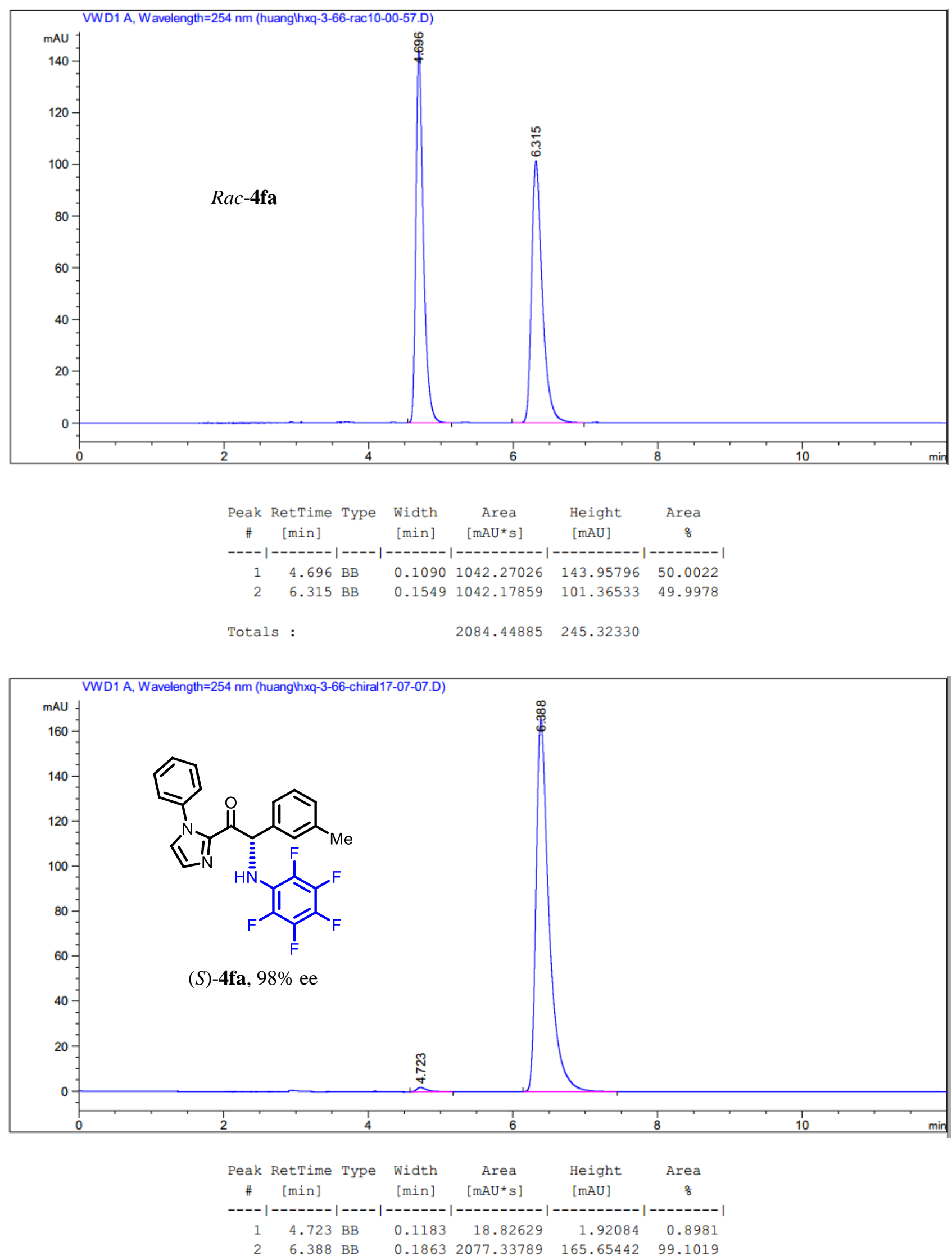

Figure S11. HPLC traces of rac-4fa (reference) and $(S)-\mathbf{4 f a}$. 

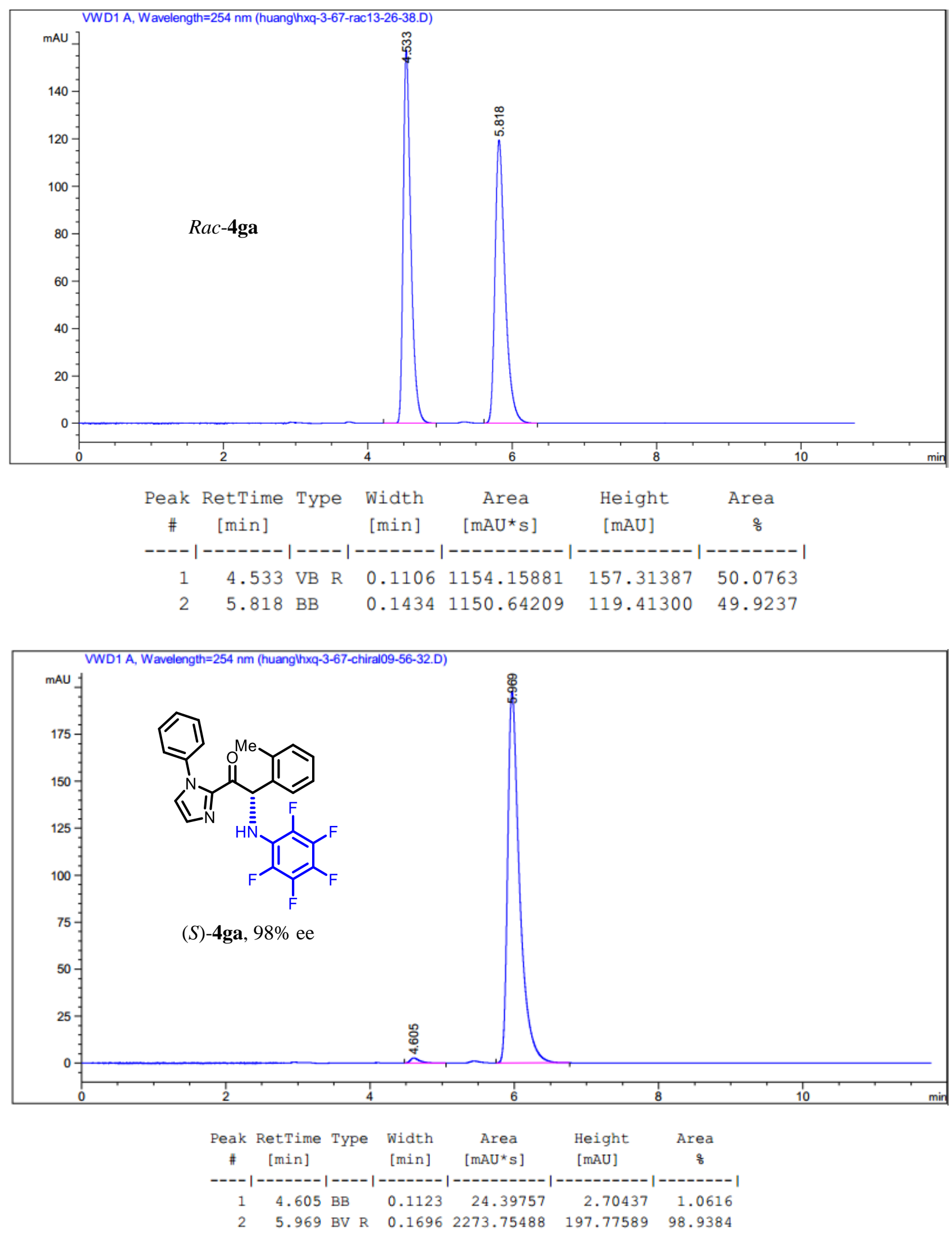

Figure S12. HPLC traces of $r a c-4 g a(r e f e r e n c e)$ and $(S)-4 g a$. 


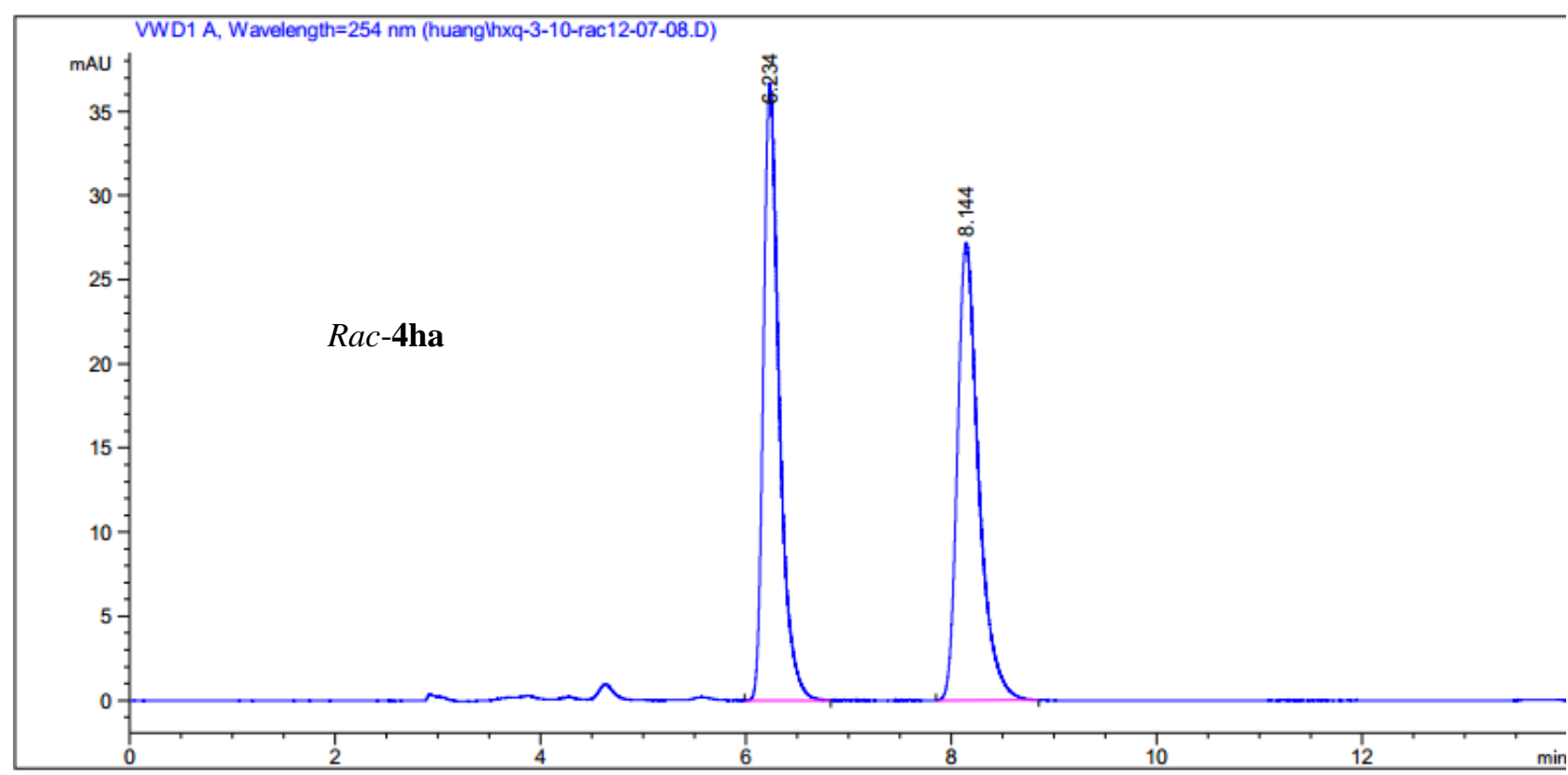

\begin{tabular}{|c|c|c|c|c|c|c|}
\hline $\begin{array}{c}\text { Peak } \\
\#\end{array}$ & $\begin{array}{c}\text { RetTime } \\
\text { [min] }\end{array}$ & Type & $\begin{array}{l}\text { Width } \\
\text { [min] }\end{array}$ & $\begin{array}{c}\text { Area } \\
{\left[\mathrm{mAU}{ }^{*} \mathrm{~s}\right]}\end{array}$ & $\begin{array}{l}\text { Height } \\
\text { [mAU] }\end{array}$ & $\begin{array}{c}\text { Area } \\
\frac{8}{6}\end{array}$ \\
\hline-7 & --- & & - & --ー-ー-ー-ー- & --- & --- \\
\hline 1 & 6.234 & $\mathrm{BB}$ & 0.1570 & 391.23090 & 36.67200 & 50.1275 \\
\hline 2 & 8.144 & BB & 0.2051 & 389.24060 & 27.17608 & 49.8725 \\
\hline
\end{tabular}

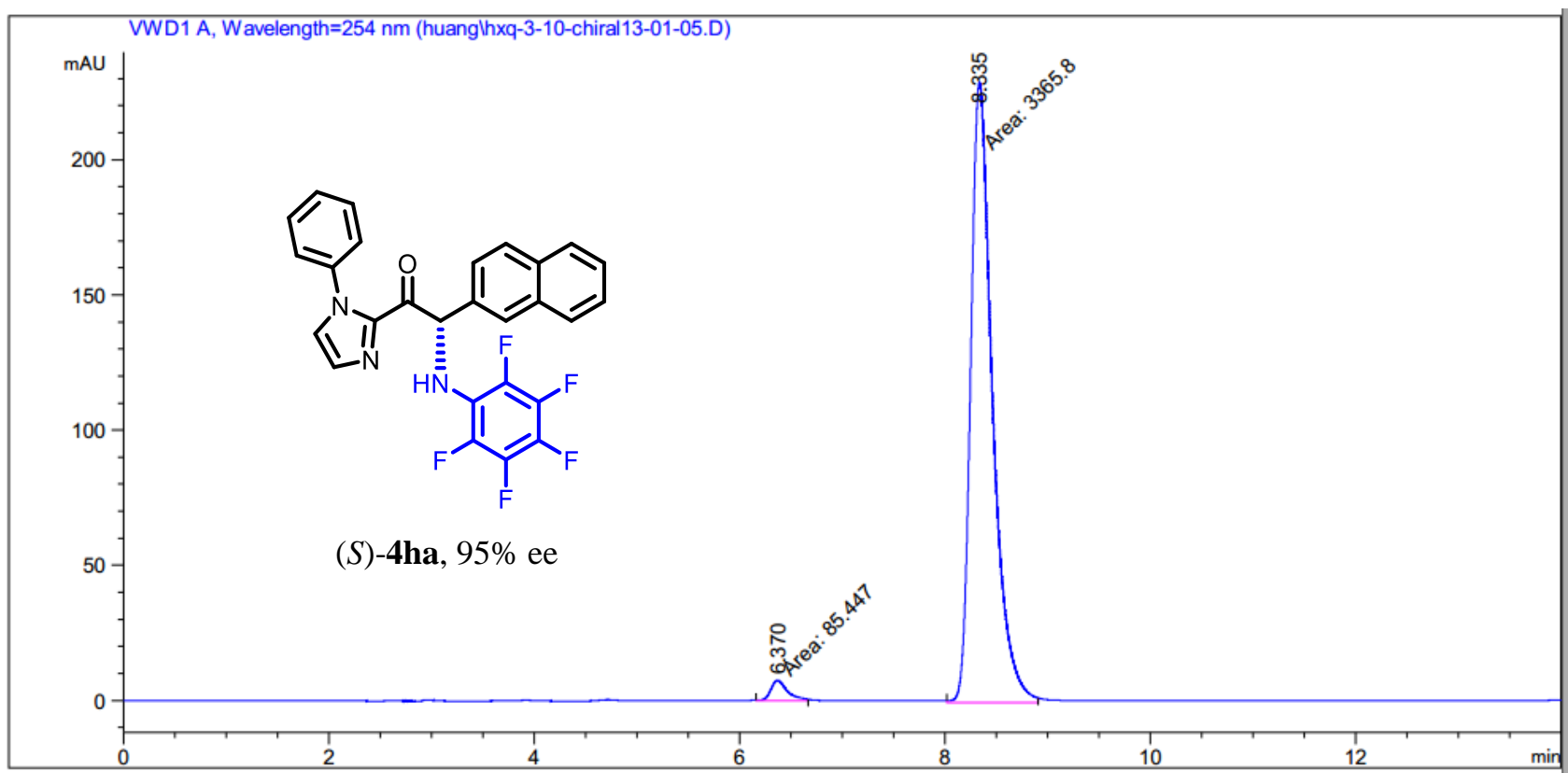

\begin{tabular}{|c|c|c|c|c|c|c|}
\hline $\begin{array}{c}\text { Peak } \\
\#\end{array}$ & $\begin{array}{c}\text { RetTime } \\
\text { [min] }\end{array}$ & Type & $\begin{array}{l}\text { Width } \\
\text { [min] }\end{array}$ & $\begin{array}{c}\text { Area } \\
{\left[\mathrm{mAU}{ }^{\star} \mathrm{s}\right]}\end{array}$ & $\begin{array}{l}\text { Height } \\
\text { [mAU] }\end{array}$ & $\begin{array}{c}\text { Area } \\
\frac{8}{8}\end{array}$ \\
\hline & & & & ---------- & $---------\mid$ & $--------\mid$ \\
\hline 1 & & $\mathbb{M}$ & 0.1895 & 85.44697 & 7.51552 & 758 \\
\hline 2 & 8.335 & MM & 0.2446 & 3365.80078 & 229.34956 & 97.5242 \\
\hline
\end{tabular}

Figure S13. HPLC traces of $\mathrm{rac}-\mathbf{4 h a}$ (reference) and (S)-5ha. 

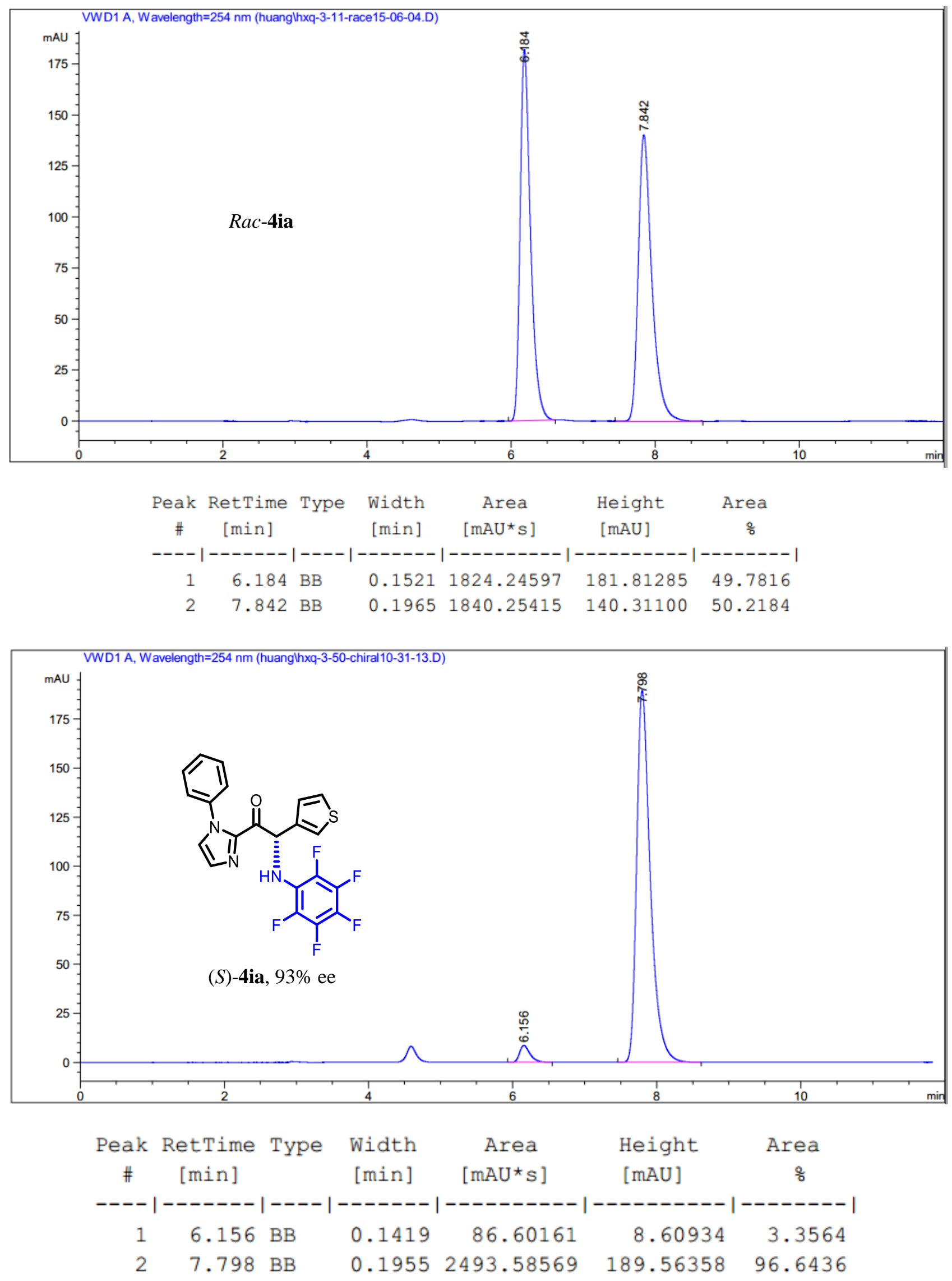

Figure S14. HPLC traces of rac-4ia (reference) and (S)-4ia. 

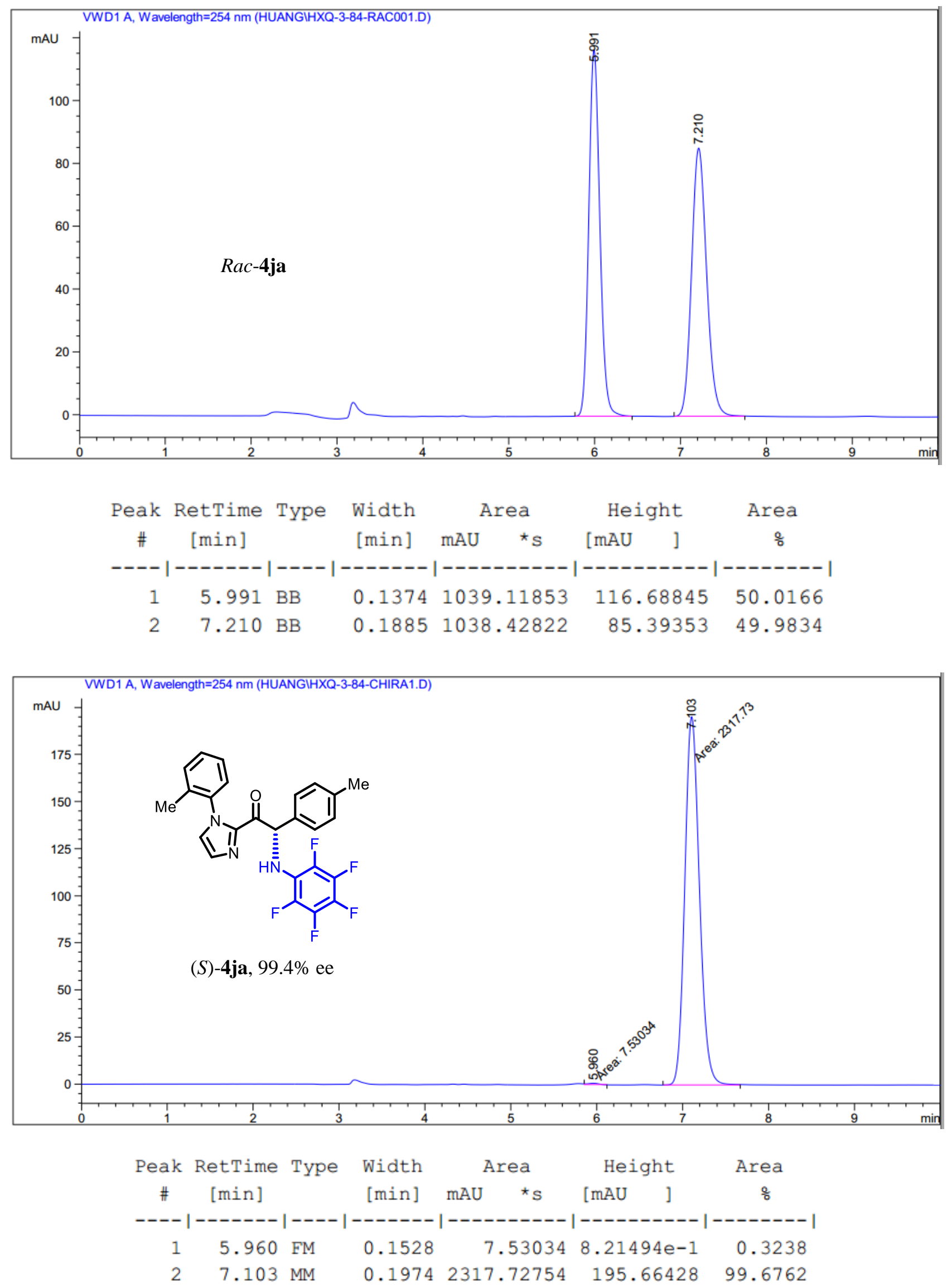

Figure S15. HPLC traces of rac-4ja (reference) and (S)-4ja. 

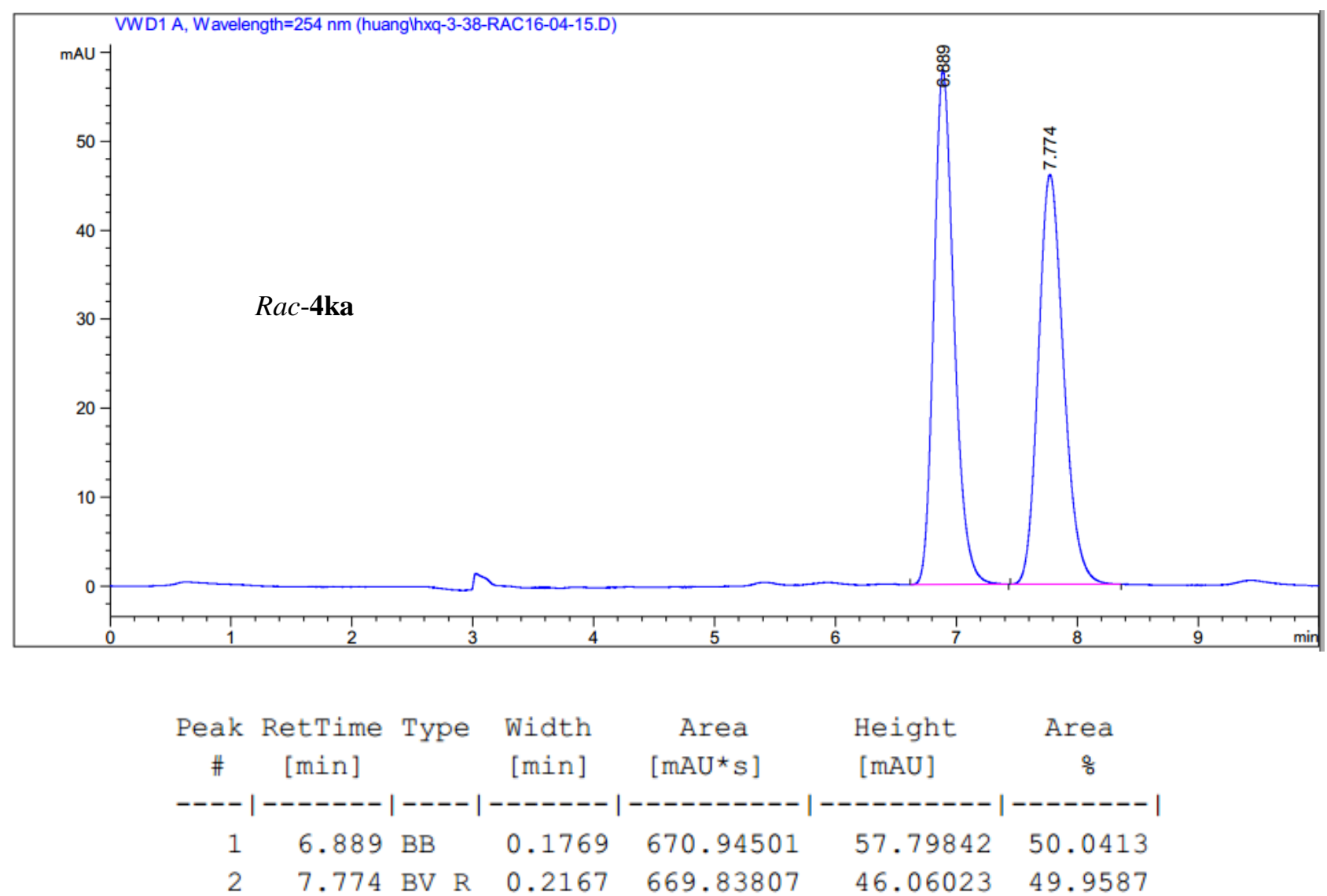

$\mathrm{mV}$

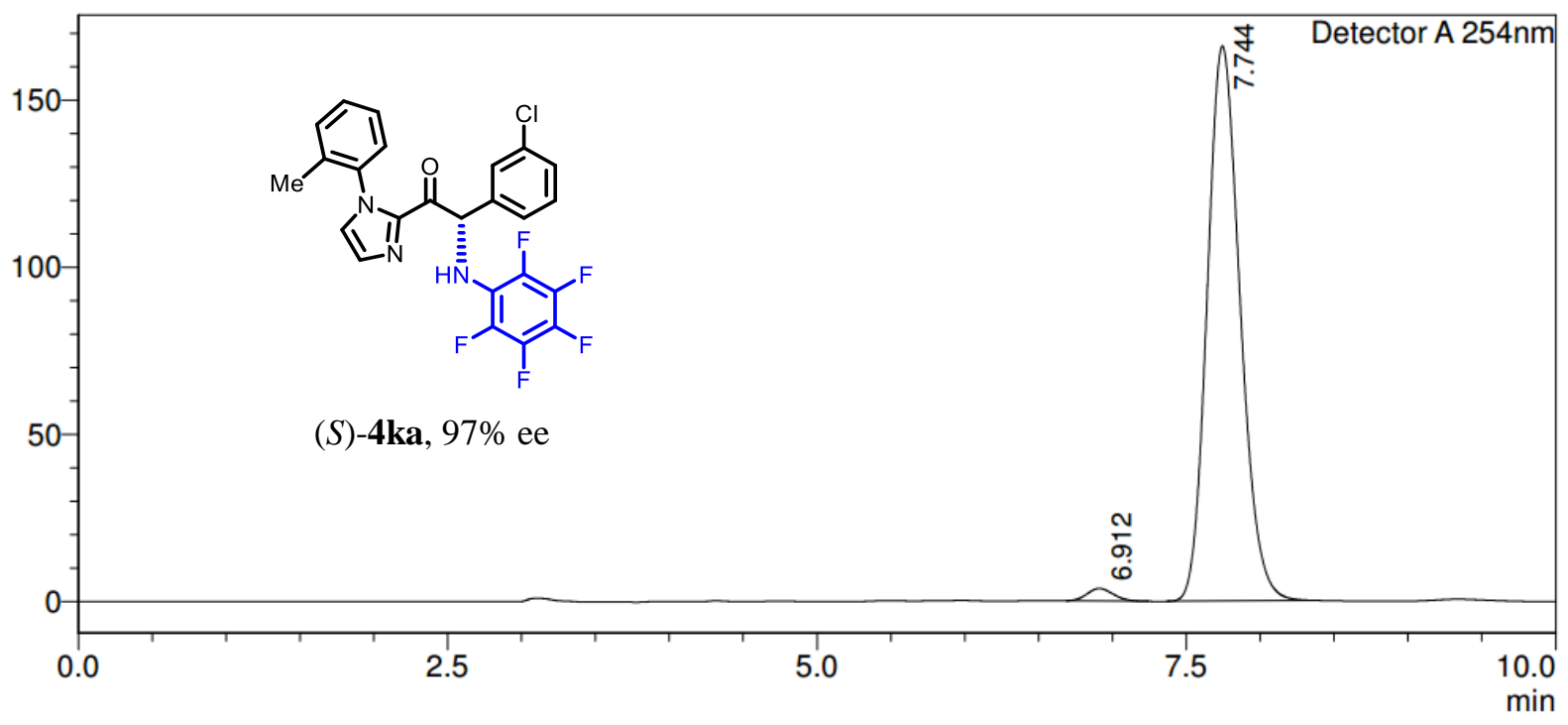

$<$ Peak Table>

Detector A 254nm

\begin{tabular}{|r|r|r|r|}
\hline Peak\# & Ret. Time & \multicolumn{1}{|c|}{ Area } & \multicolumn{1}{c|}{ Area\% } \\
\hline 1 & 6.912 & 45760 & 1.745 \\
\hline 2 & 7.744 & 2576344 & 98.255 \\
\hline Total & & 2622103 & 100.000 \\
\hline
\end{tabular}

Figure S16. HPLC traces of rac-4ka (reference) and (S)-4ka. 

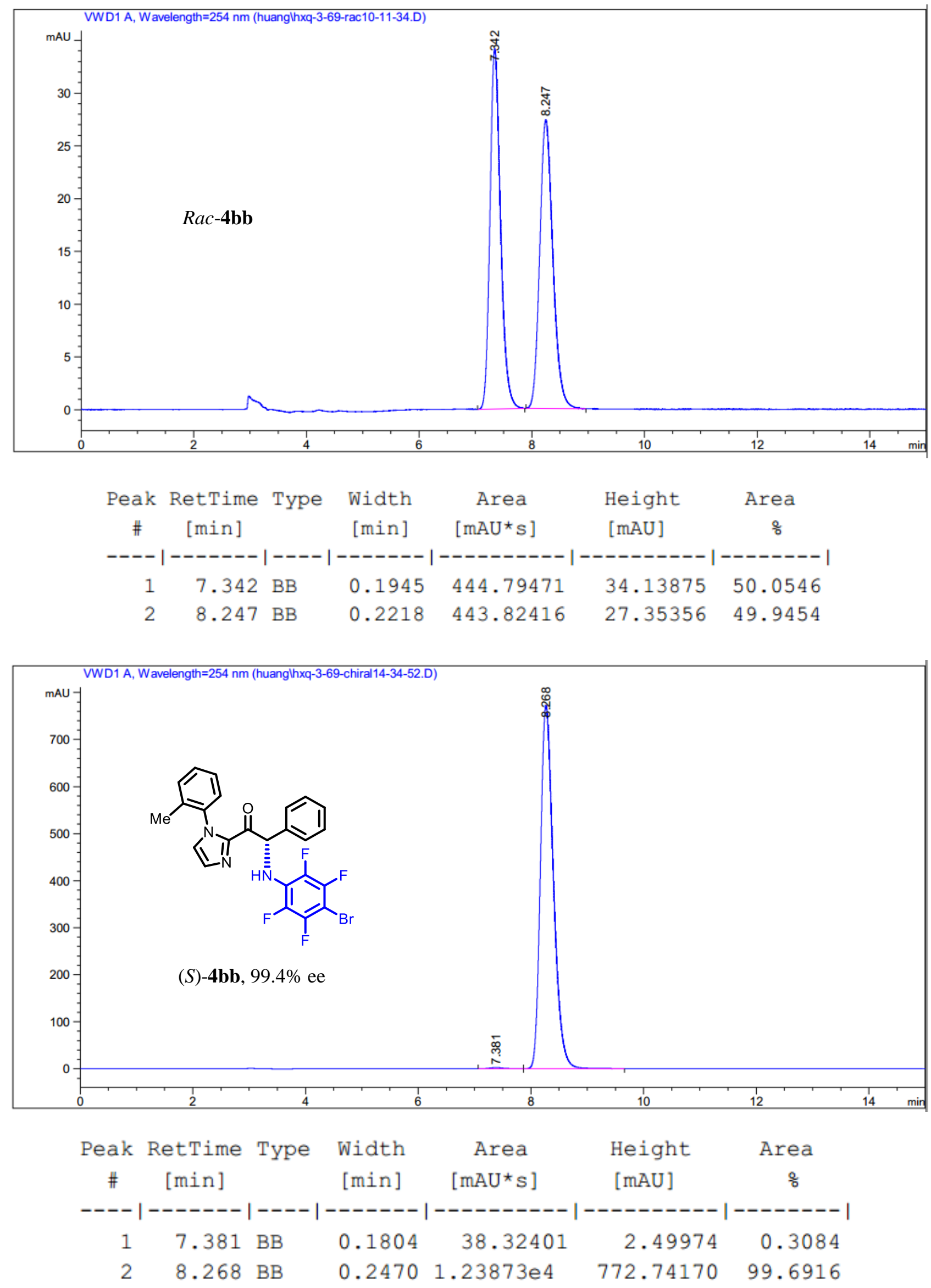

Figure S17. HPLC traces of $r a c-4 \mathbf{b b}$ (reference) and (S)-4bb. 

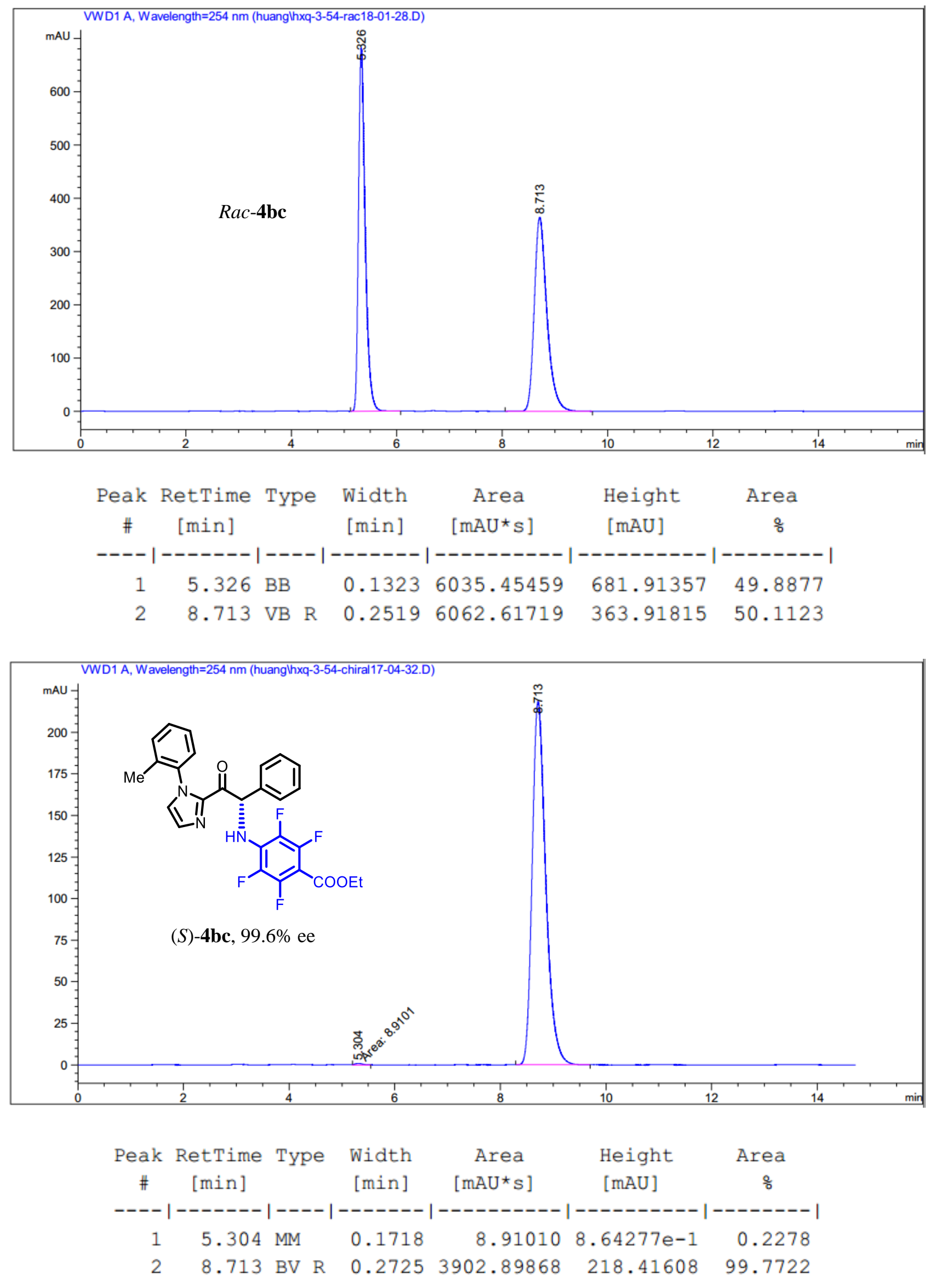

Figure S18. HPLC traces of $r a c-4 b c$ (reference) and (S)-4bc. 

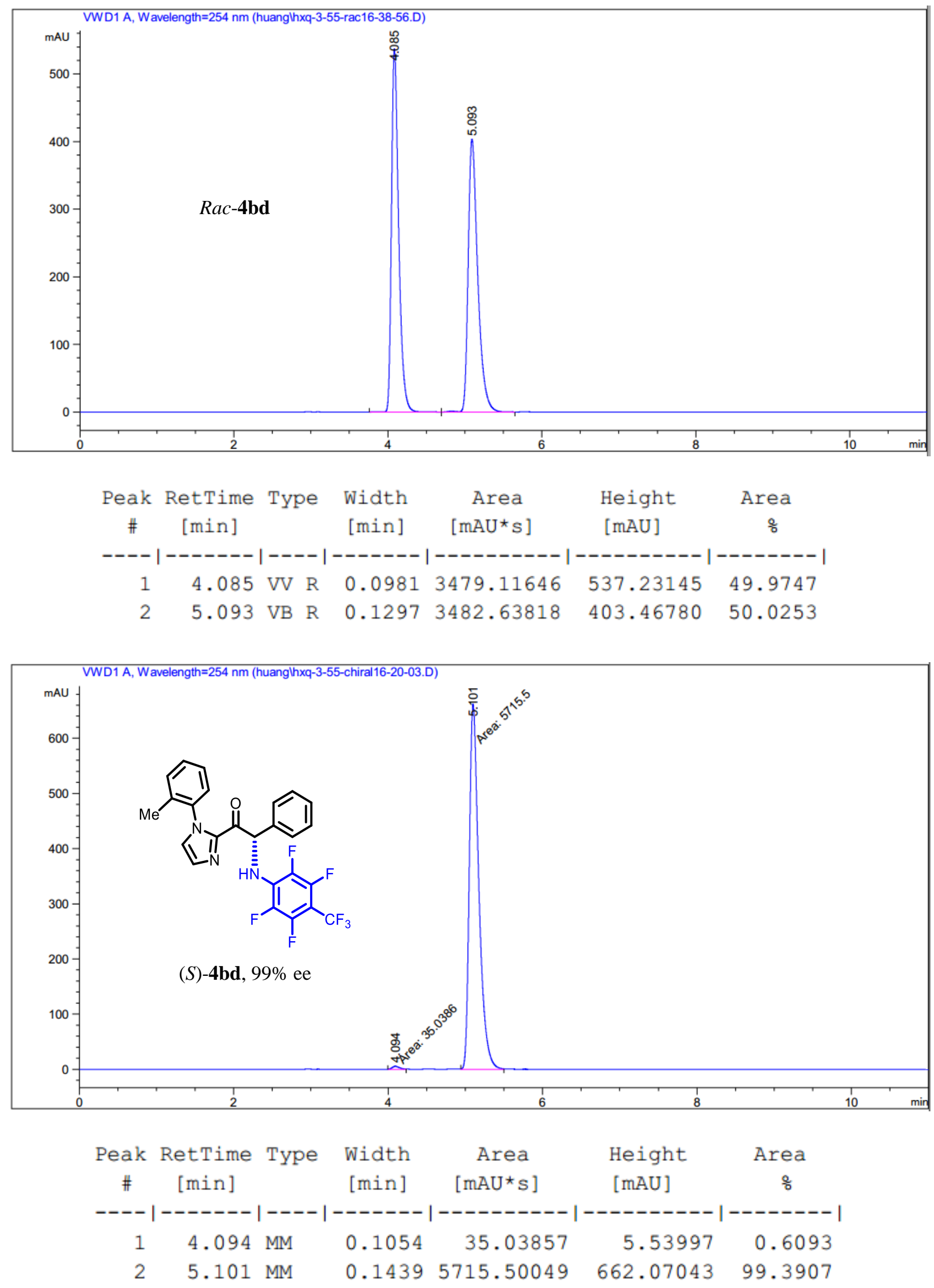

Figure S19. HPLC traces of $r a c-4 b d$ (reference) and (S)-4bd. 
$\mathrm{mV}$

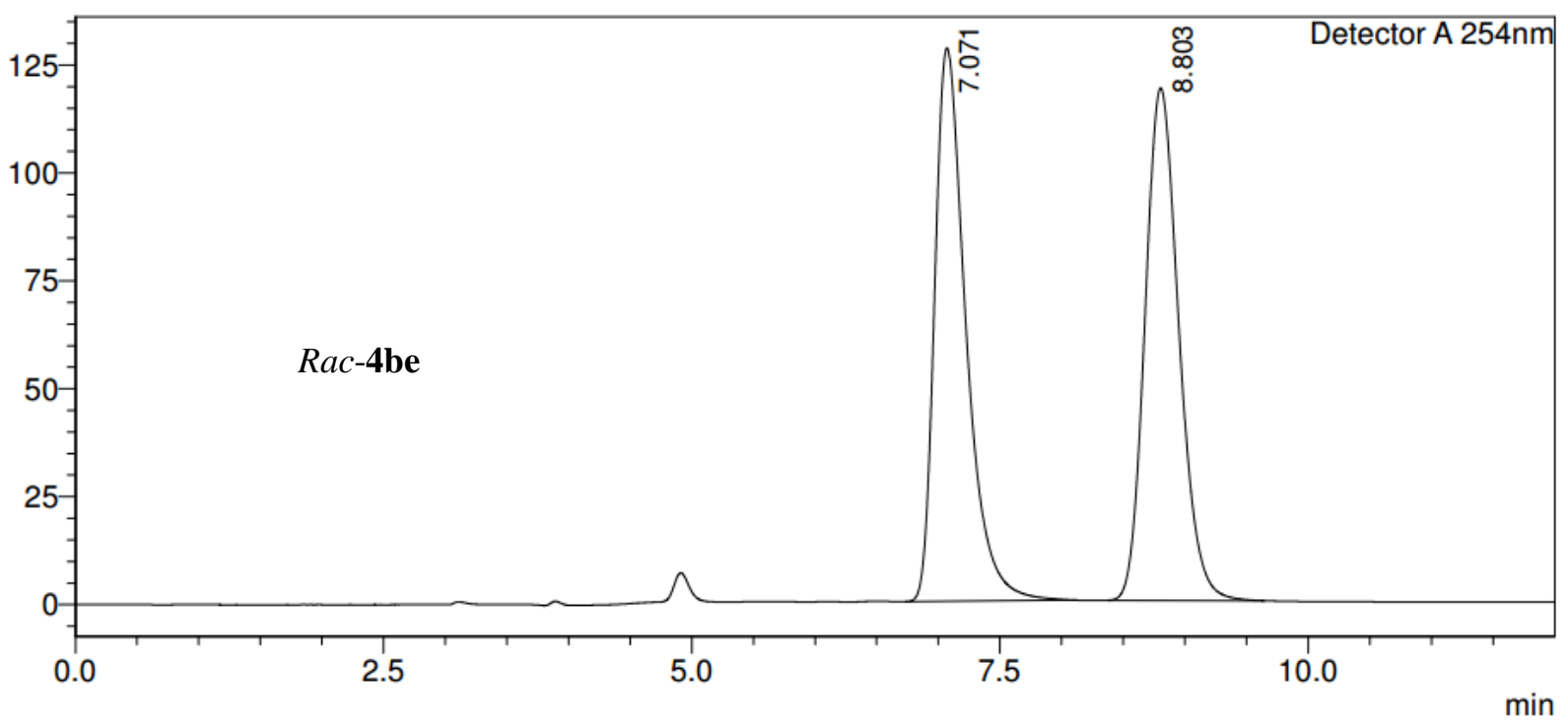

$<$ Peak Table>

Detector A 254nm

\begin{tabular}{|r|r|r|r|}
\hline Peak\# & Ret. Time & Area & \multicolumn{1}{c|}{ Area\% } \\
\hline 1 & 7.071 & 2257837 & 49.931 \\
\hline 2 & 8.803 & 2264107 & 50.069 \\
\hline Total & & 4521944 & 100.000 \\
\hline
\end{tabular}

$\mathrm{mV}$

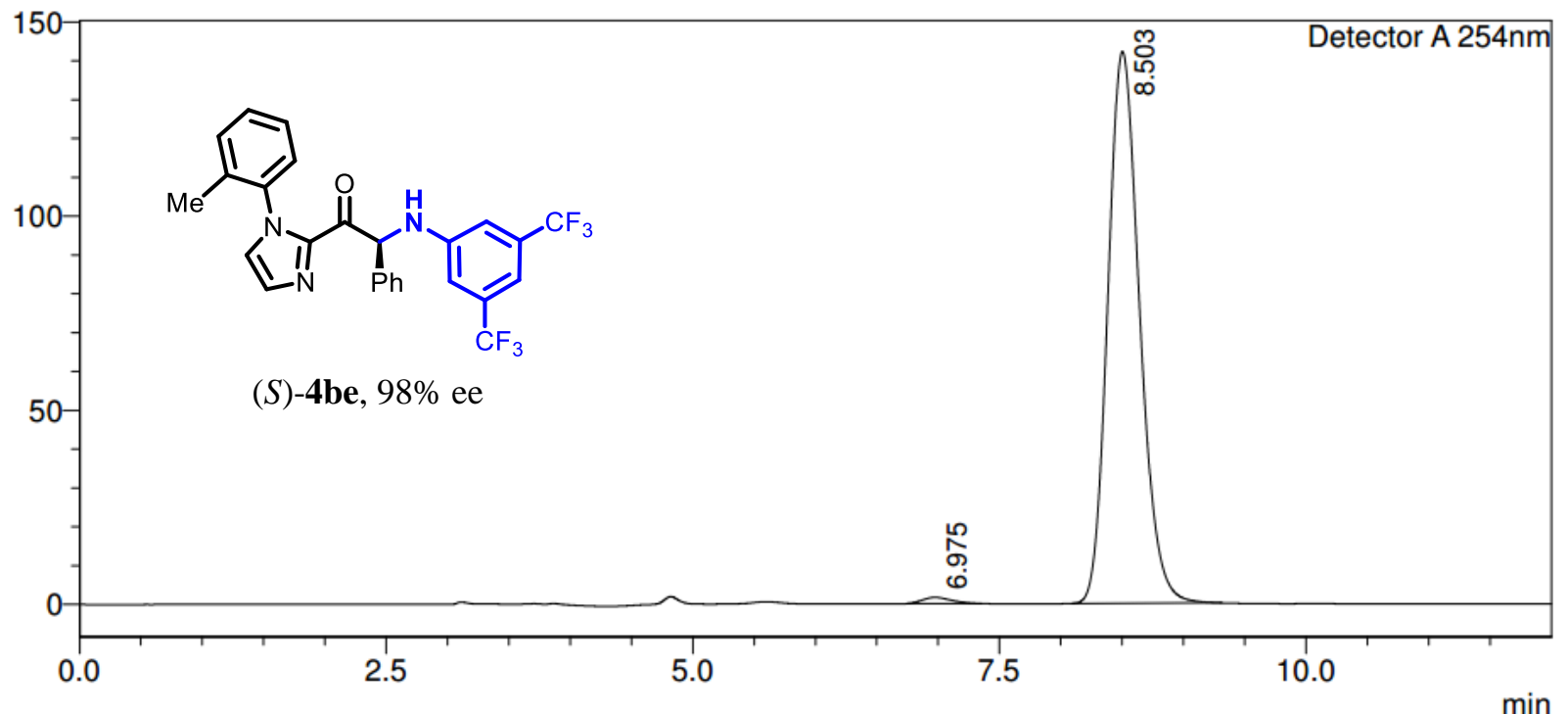

$<$ Peak Table>

Detector A 254nm

\begin{tabular}{|r|r|r|r|}
\hline Peak\# & Ret. Time & \multicolumn{1}{|c|}{ Area } & \multicolumn{1}{c|}{ Area\% } \\
\hline 1 & 6.975 & 25835 & 1.000 \\
\hline 2 & 8.503 & 2558683 & 99.000 \\
\hline Total & & 2584518 & 100.000 \\
\hline
\end{tabular}

Figure S20. HPLC traces of rac-4be (reference) and $(S)$-4be. 

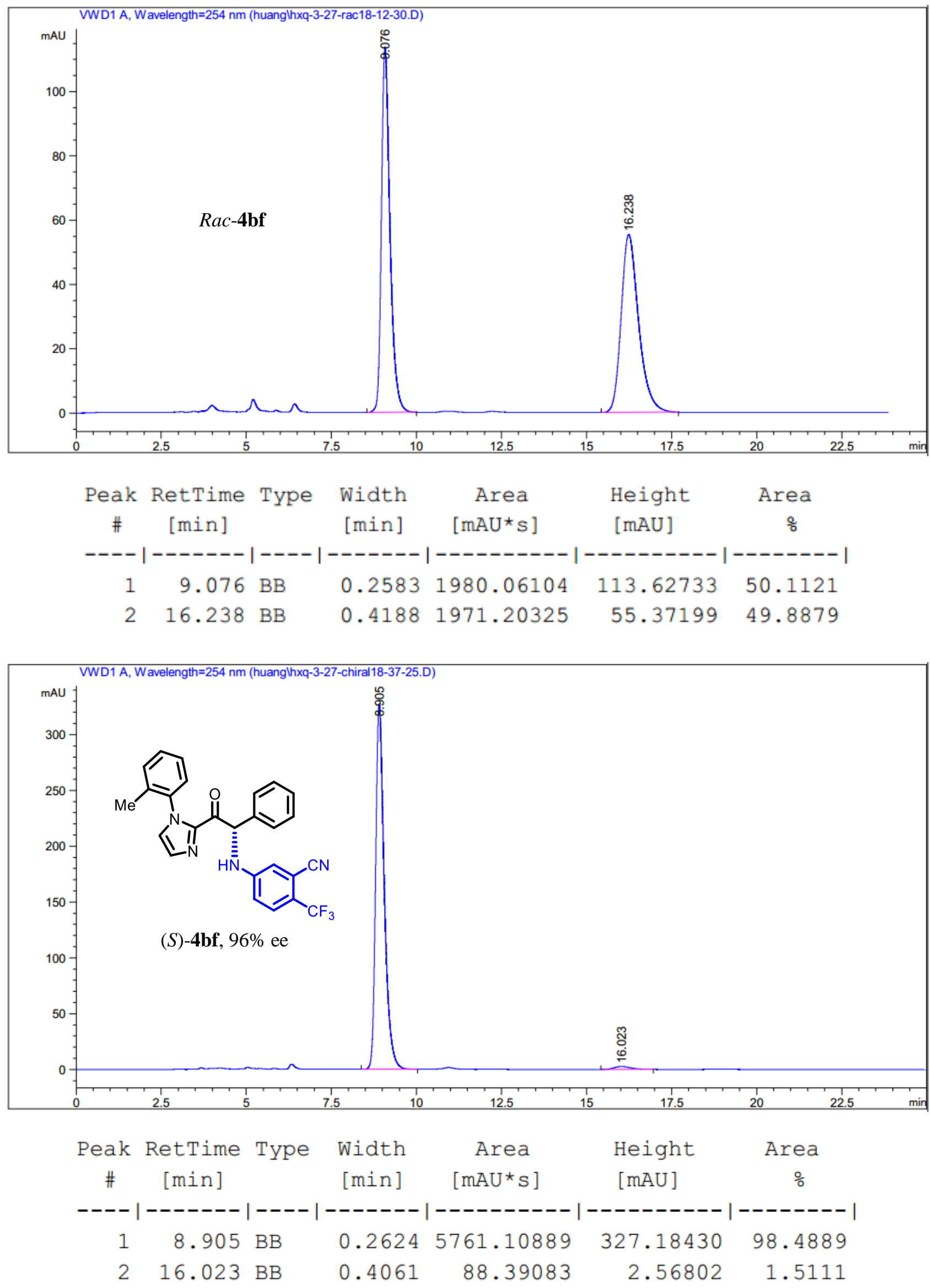

Figure S21. HPLC traces of $r a c-\mathbf{4 b f}$ (reference) and (S)-4bf. 


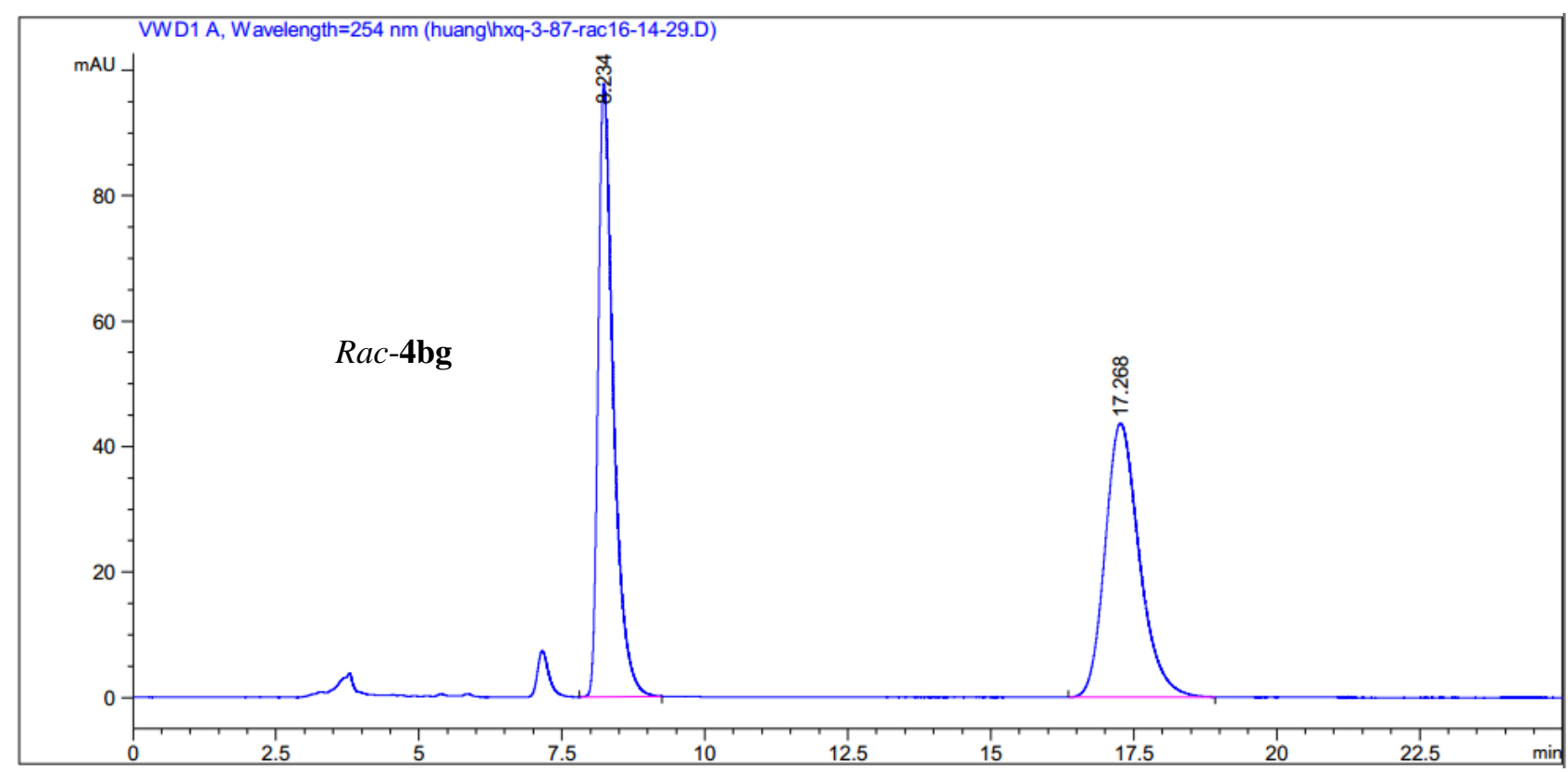

\begin{tabular}{|c|c|c|c|c|c|c|}
\hline \# & $\begin{array}{c}\text { RetTime } \\
\text { [min] }\end{array}$ & ype & $\begin{array}{l}\text { Width } \\
\text { [min] }\end{array}$ & $\begin{array}{c}\text { Area } \\
{\left[\mathrm{mAU}{ }^{\star} \mathrm{S}\right]}\end{array}$ & $\begin{array}{l}\text { Height } \\
\text { [mAU] }\end{array}$ & $\begin{array}{c}\text { Area } \\
\frac{\%}{6}\end{array}$ \\
\hline & & & & ------- & ---- & -- \\
\hline 1 & & & & 1811 & & 49 \\
\hline 2 & 68 & & 1 & 1814.40271 & 43.67960 & 0351 \\
\hline
\end{tabular}
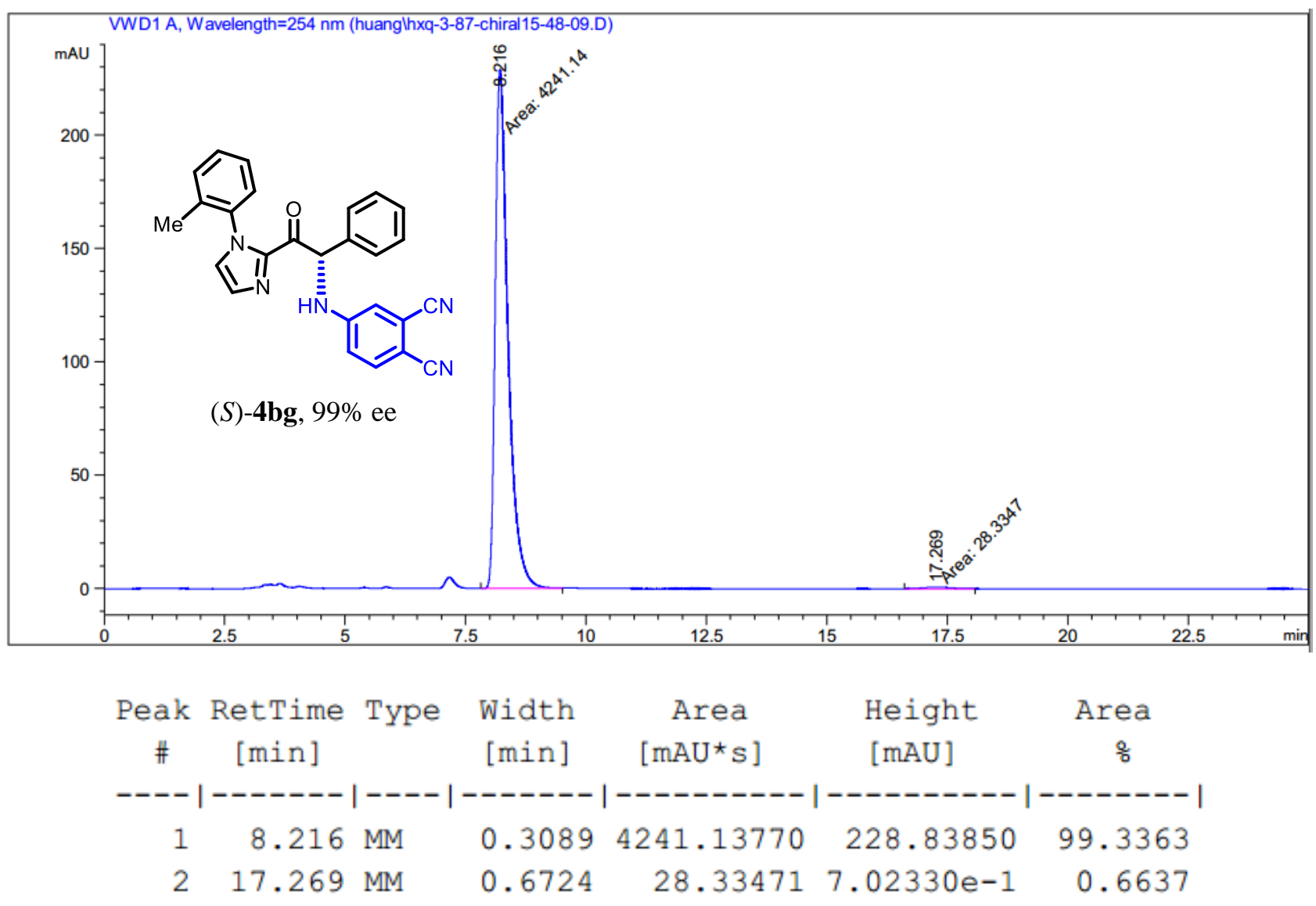

Figure S22. HPLC traces of $r a c-4 b g$ (reference) and (S)-4bg. 
$\mathrm{mV}$

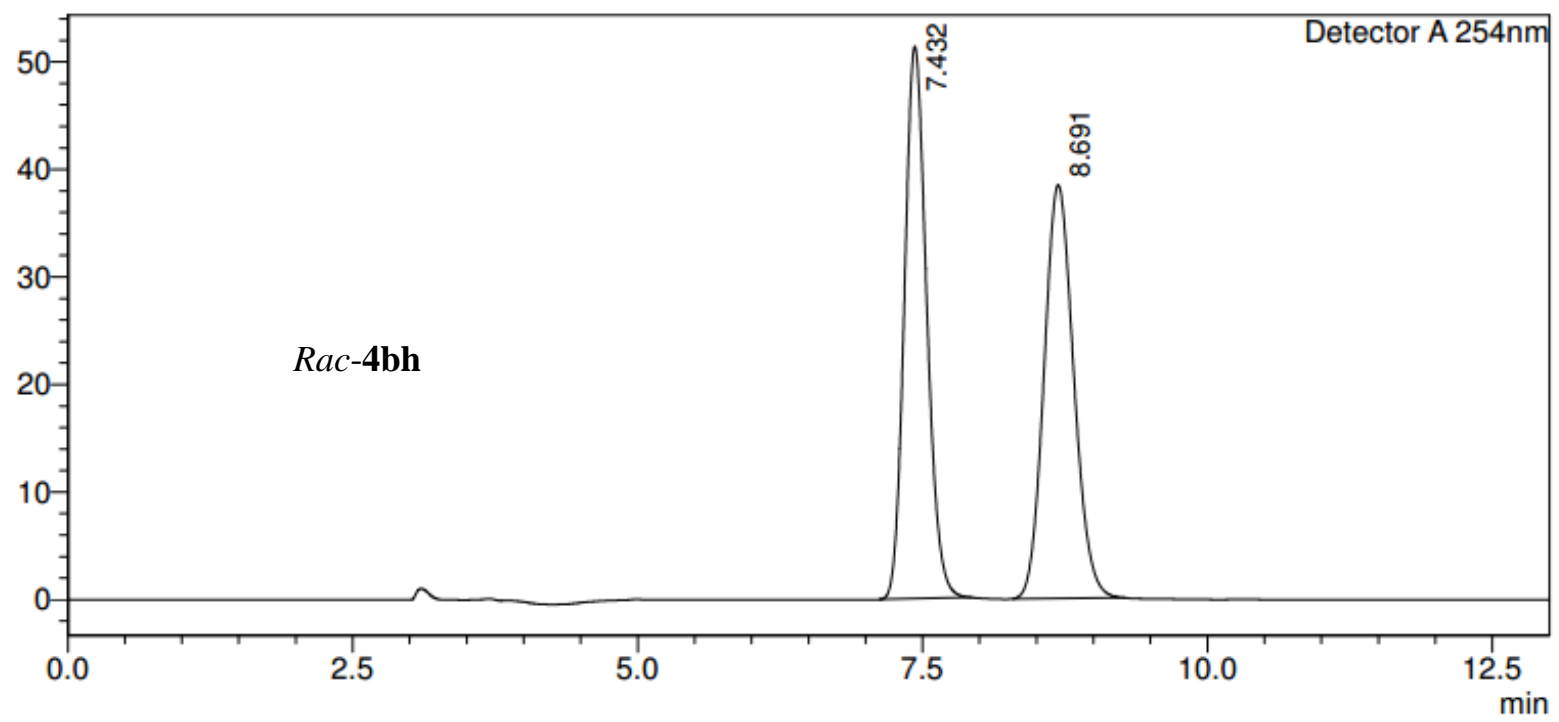

<Peak Table>

Detector A 254nm

\begin{tabular}{|c|c|c|}
\hline Peak\# Ret. Time & Area & Area\% \\
\hline
\end{tabular}

\begin{tabular}{|c|c|c|c|}
\hline & ...... & & 年 \\
\hline 1 & 7.432 & 696315 & 50.044 \\
\hline 2 & 8.6 & 695077 & \\
\hline th & & 1391392 & 10 \\
\hline
\end{tabular}

$\mathrm{mV}$

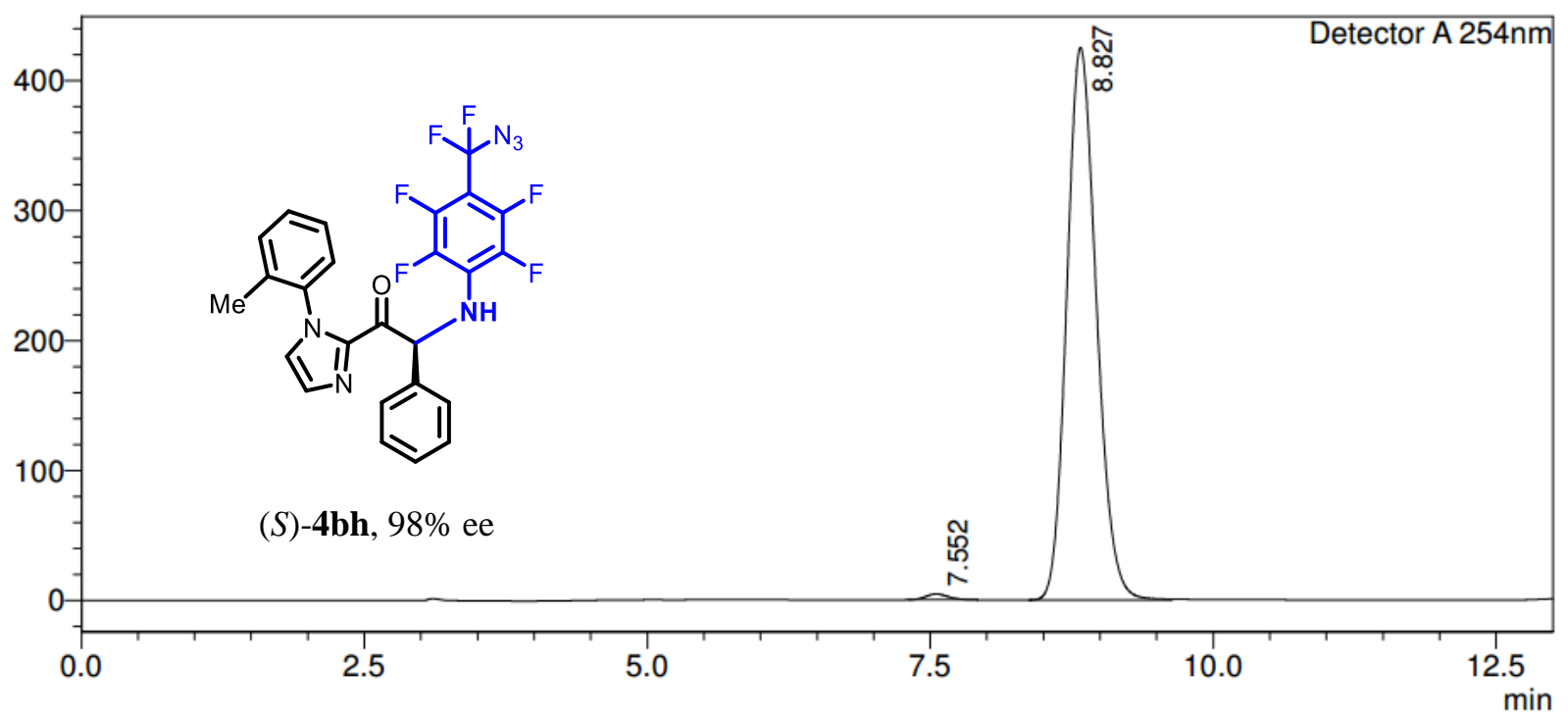

$<$ Peak Table>

Detector A 254nm

\begin{tabular}{|c|c|c|c|}
\hline Peak\# & Ret. Time & Area & Area\% \\
\hline 1 & 7.552 & 62340 & 0.797 \\
\hline 2 & 8.827 & 7762066 & 99.203 \\
\hline Tota & & 7824406 & 100.000 \\
\hline
\end{tabular}

Figure S23. HPLC traces of $r a c-4 b h$ (reference) and (S)-4bh. 

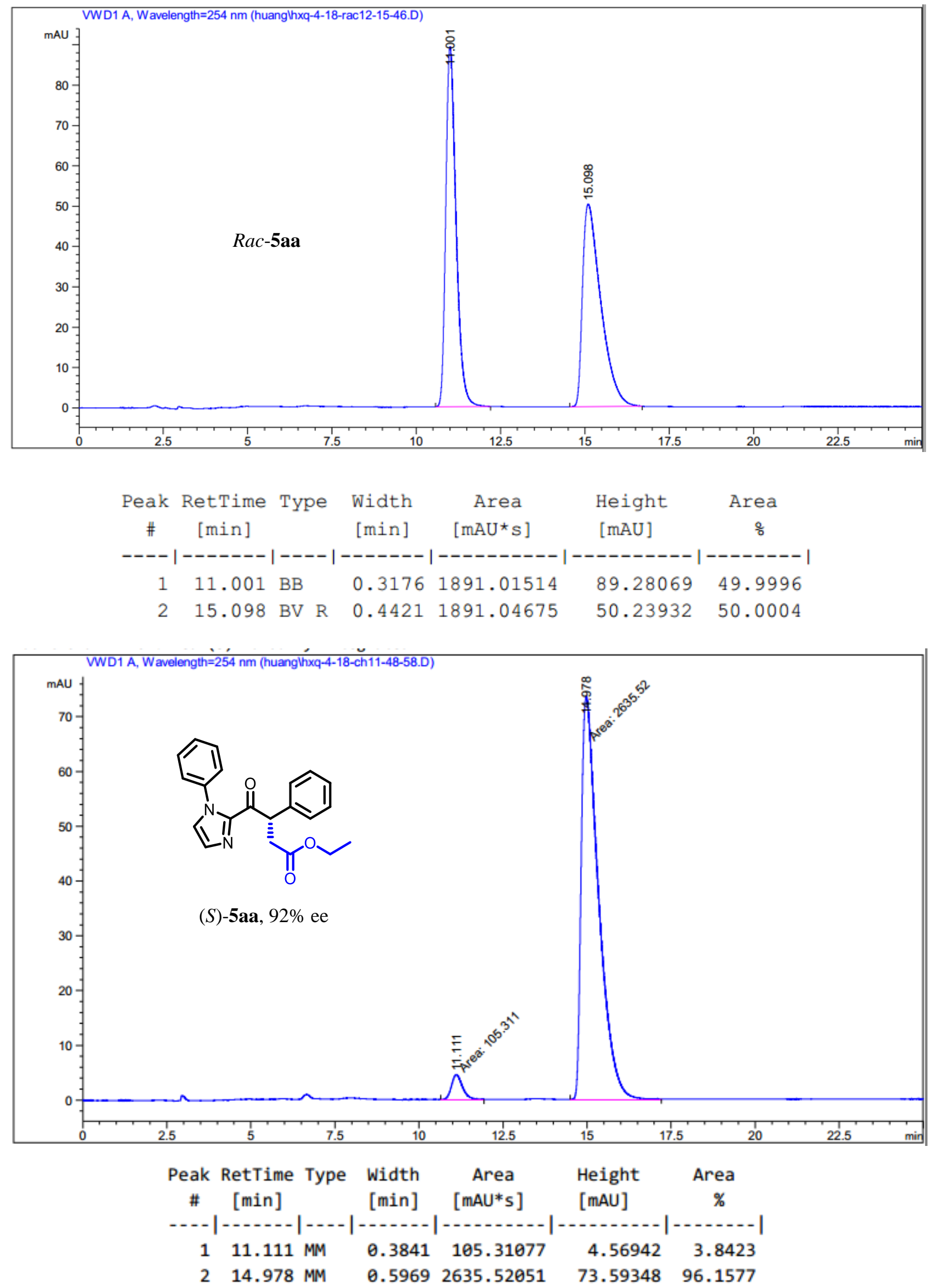

Figure S24. HPLC traces of rac-5aa (reference) and $(S)$-5aa. 

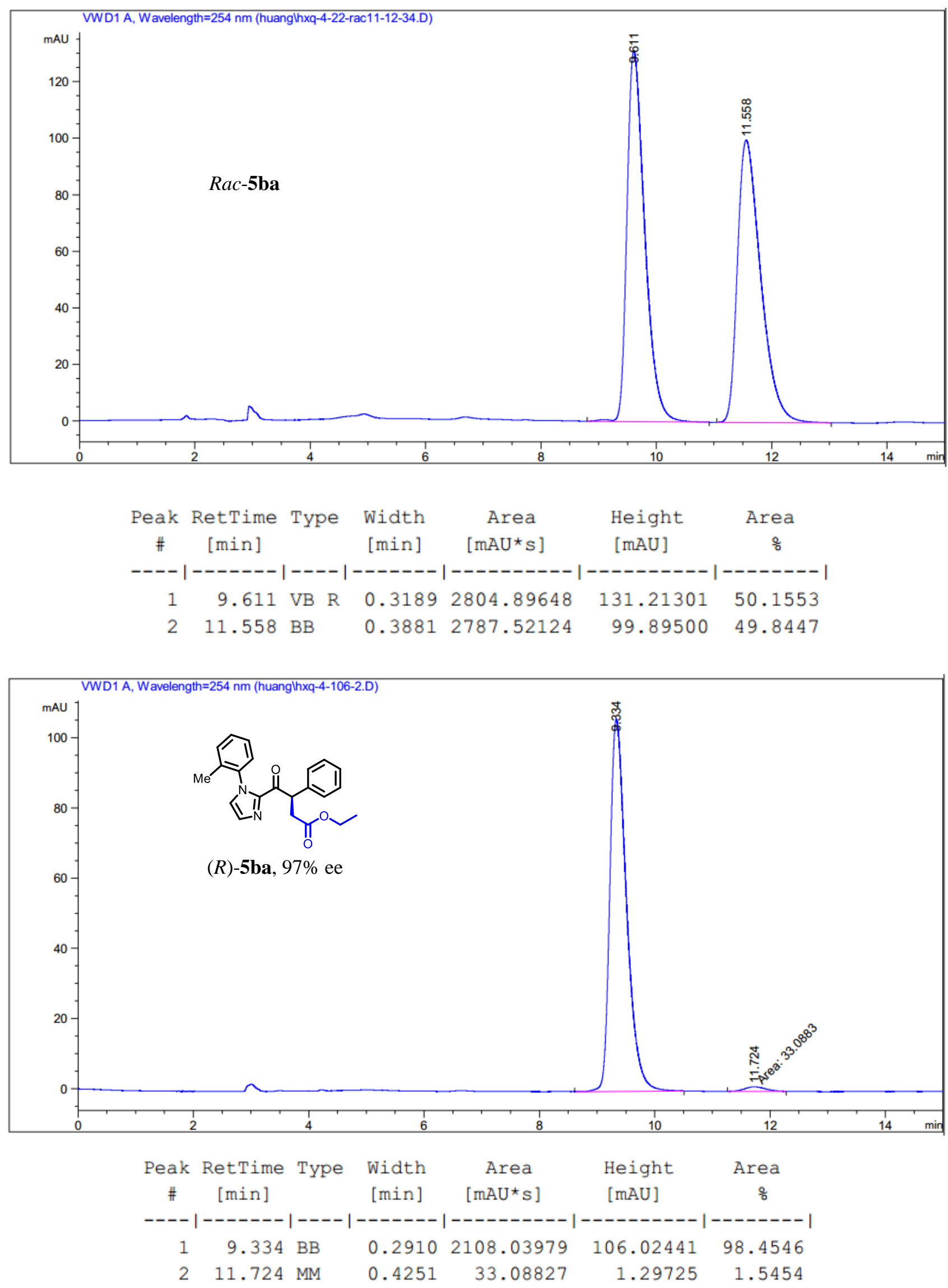

Figure S25. HPLC traces of rac-5ba (reference) and (R)-5ba. 


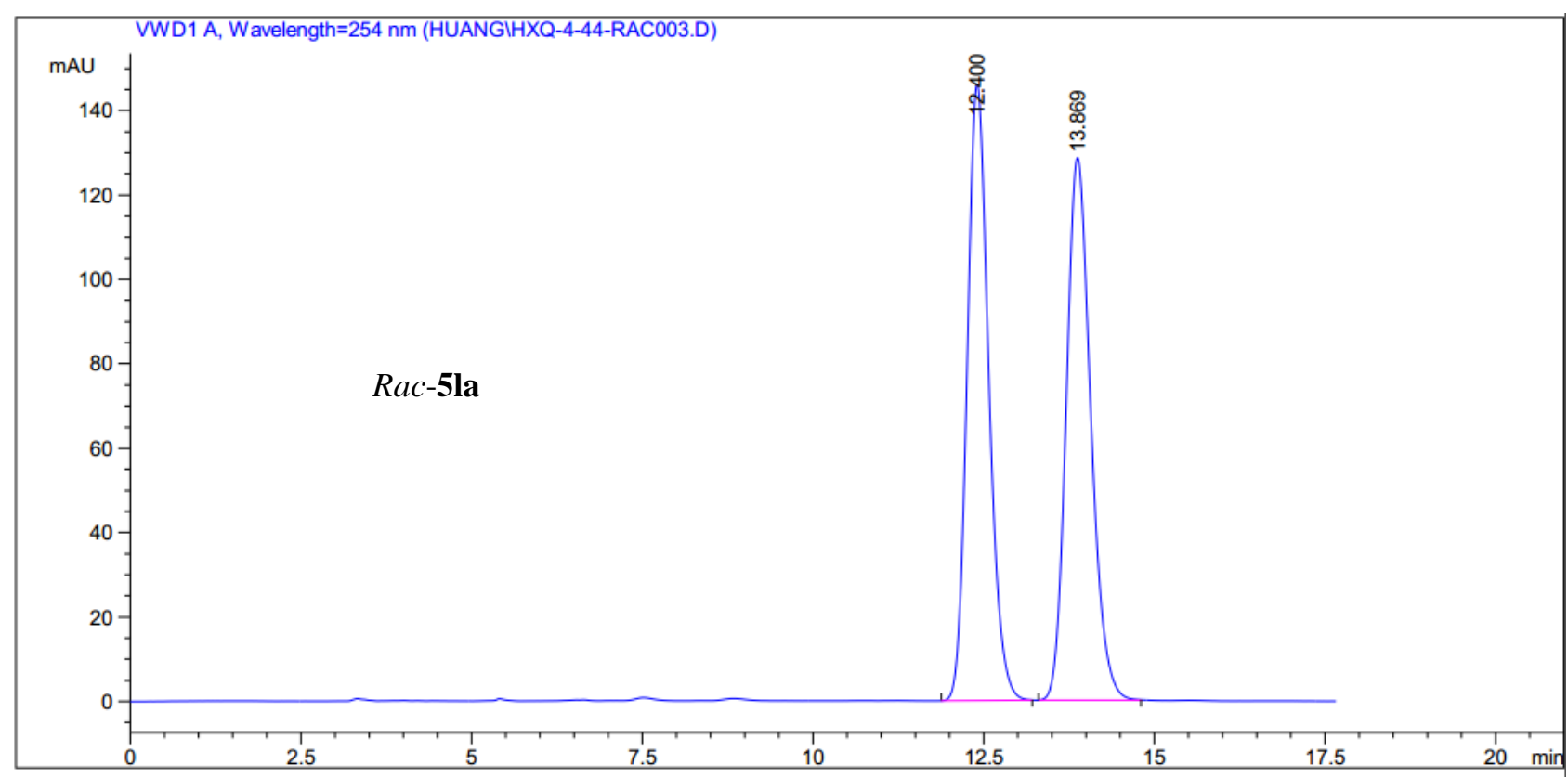

\begin{tabular}{|c|c|c|c|c|c|c|c|}
\hline Peak & RetTime & Type & Width & & & Height & Area \\
\hline \# & [min] & & [min] & $\mathrm{mAU}$ & ${ }^{*} \mathrm{~S}$ & {$[\mathrm{mAU}$} & $\frac{2}{0}$ \\
\hline- & & & & & 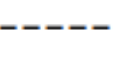 & --- & $=-$ \\
\hline 1 & 12 & B & 6 & 320 & 1748 & 146.11 & 114 \\
\hline 2 & 13.869 & $\mathrm{BB}$ & 40 & 320 & 95483 & 128.59326 & 9886 \\
\hline
\end{tabular}

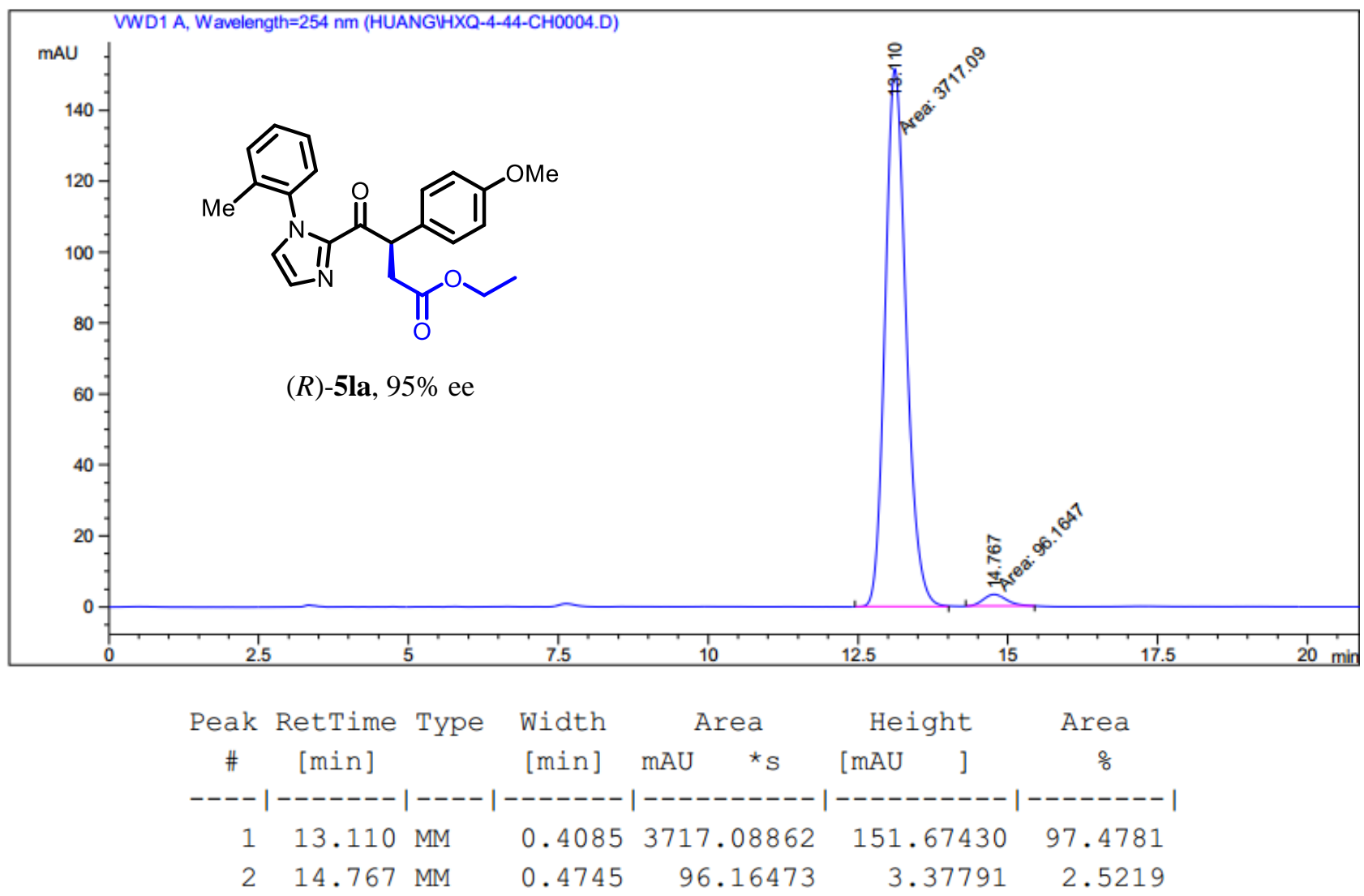

Figure S26. HPLC traces of rac-5la (reference) and (R)-5la. 

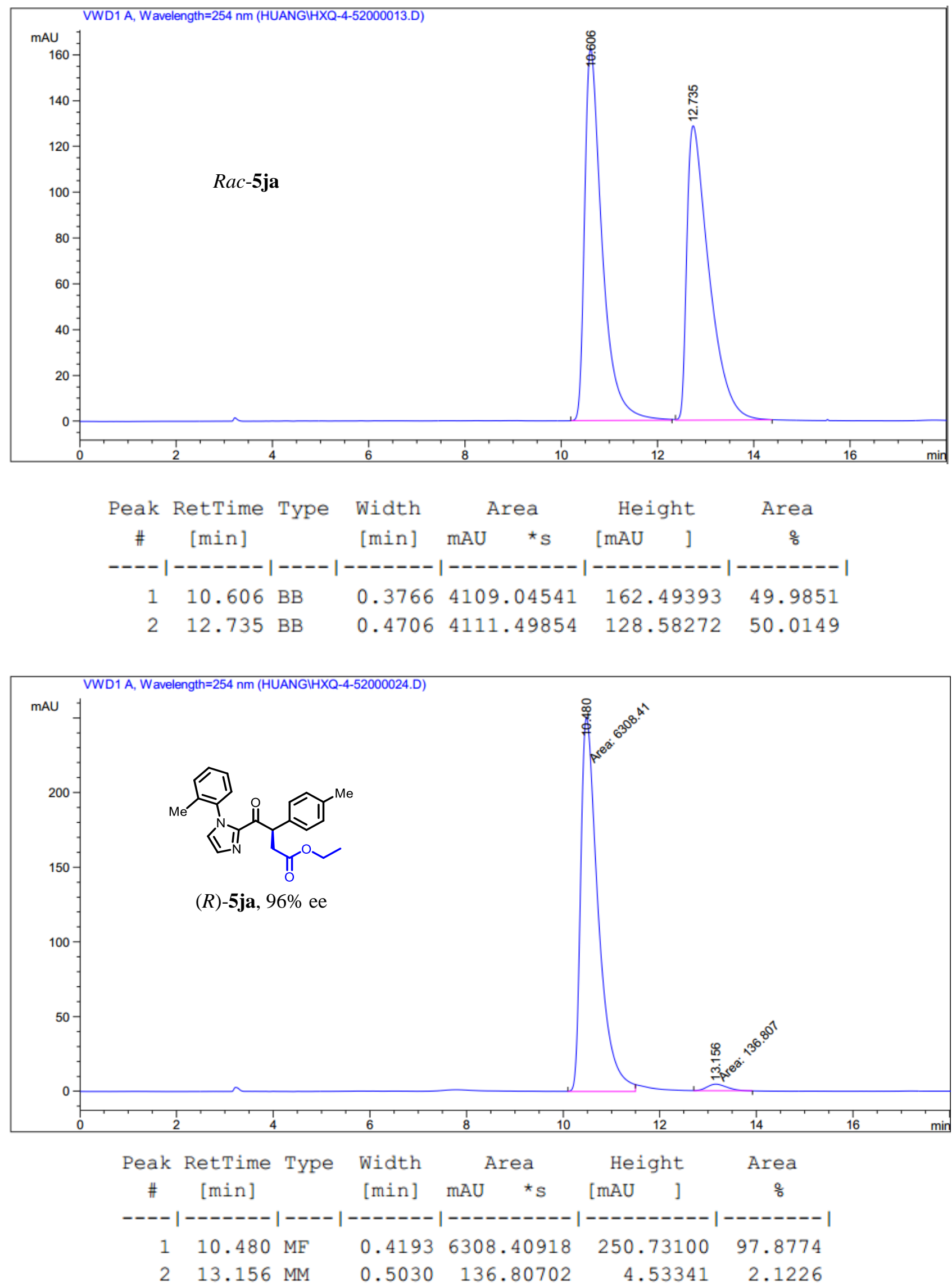

Figure S27. HPLC traces of rac-5ja (reference) and (R)-5ja. 

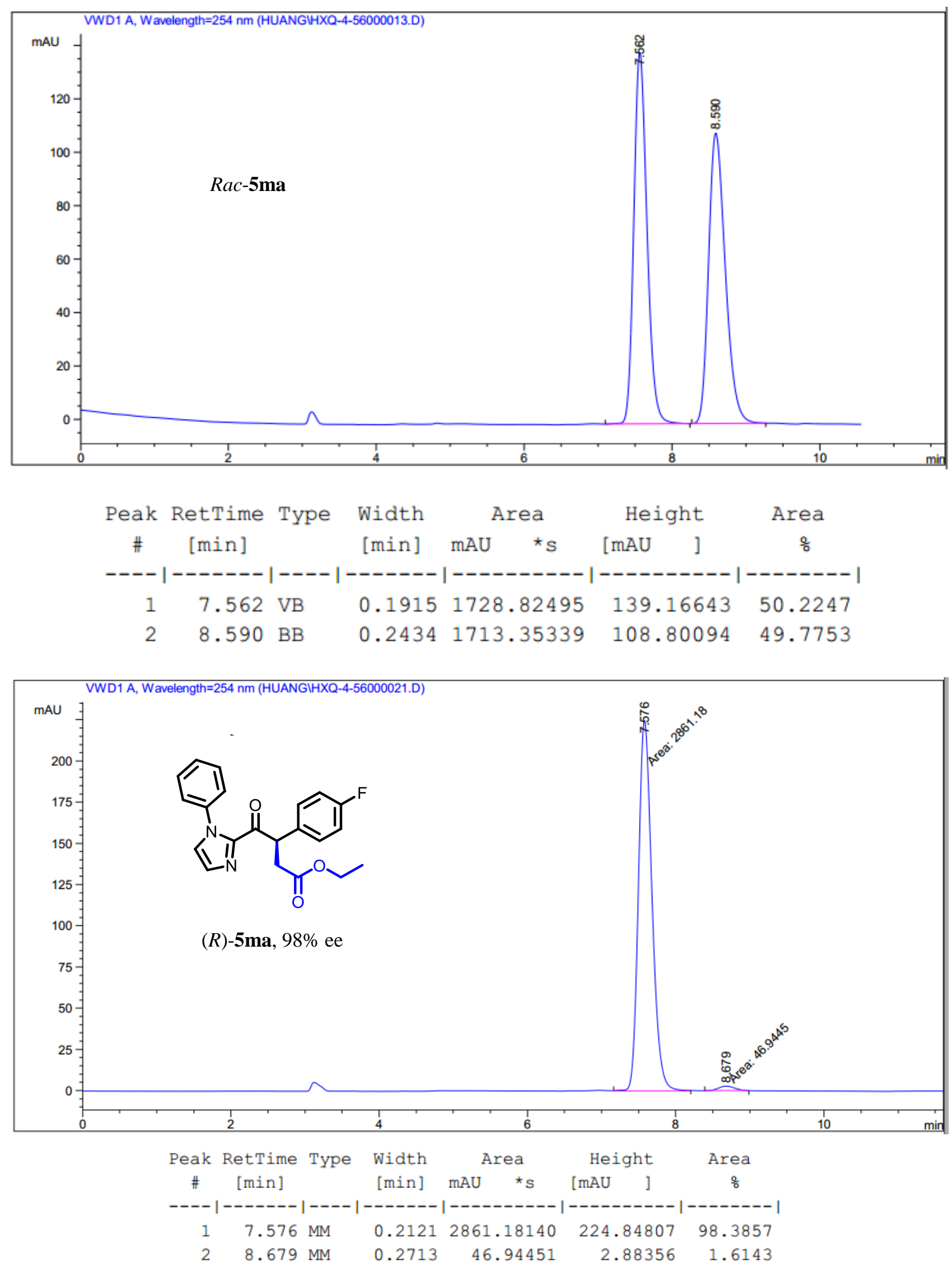

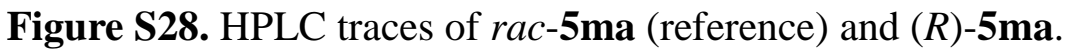



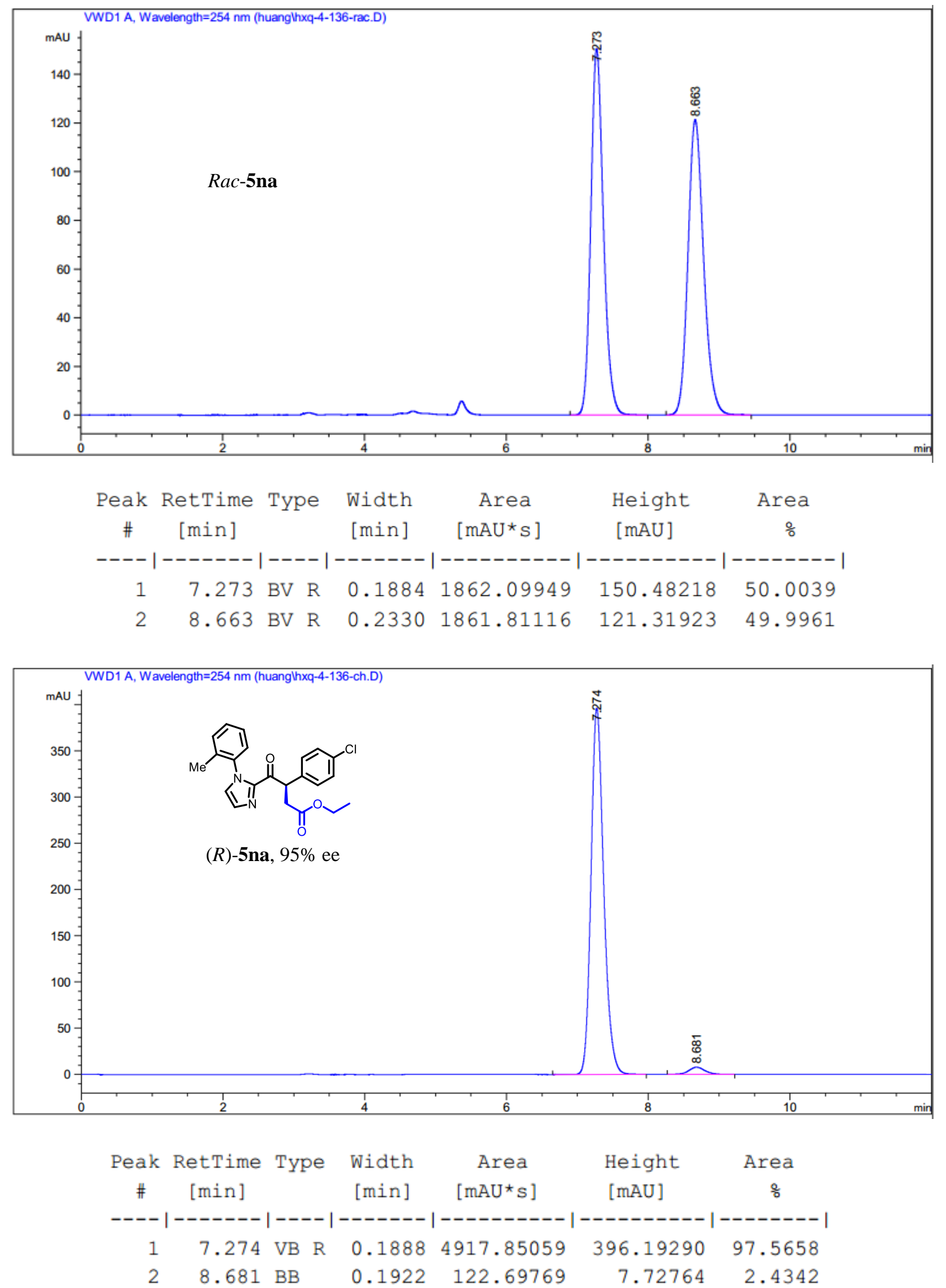

Figure S29. HPLC traces of $r a c-5 n a(r e f e r e n c e)$ and $(R)-5$ na. 

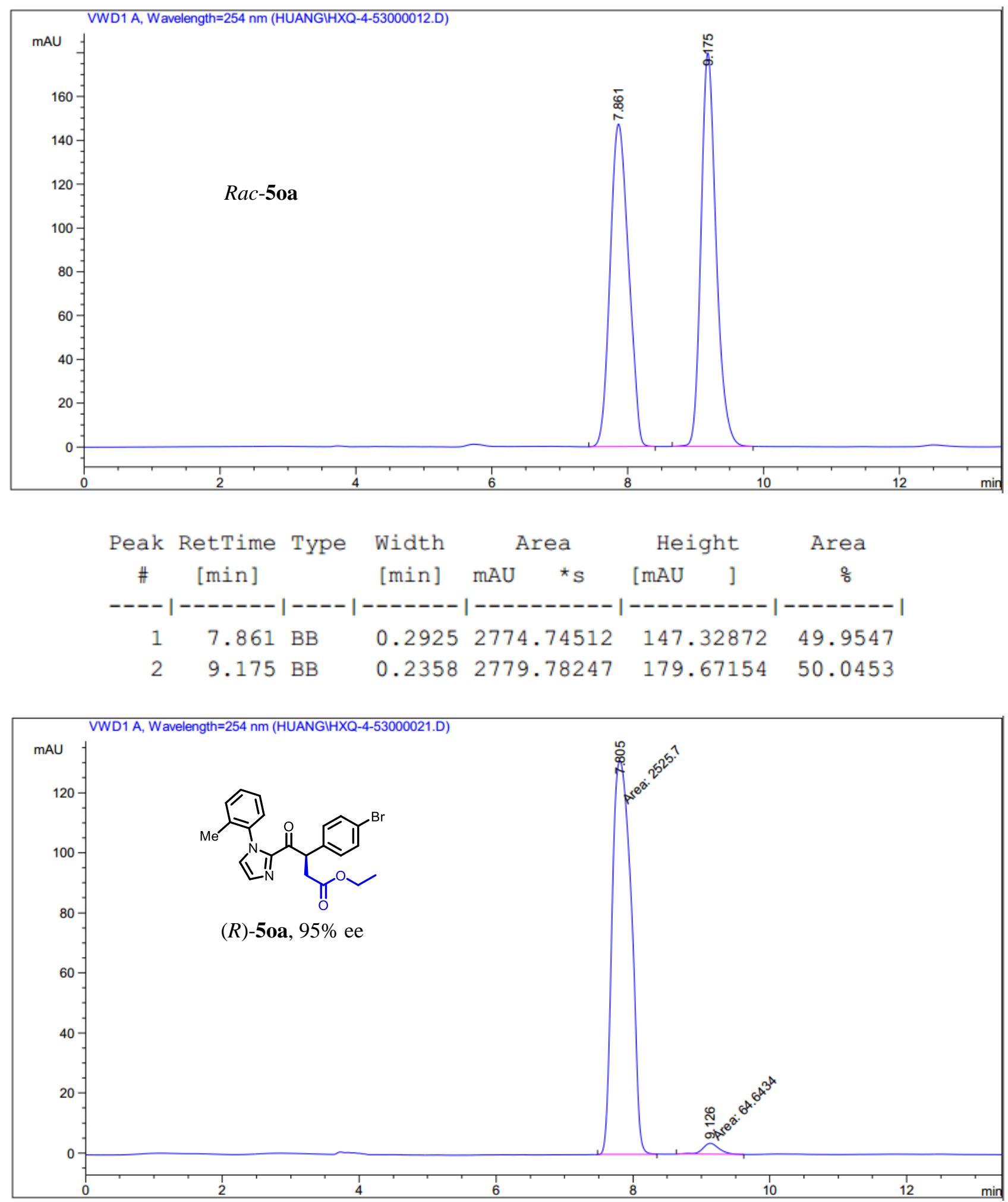

$\begin{array}{cccccc}\begin{array}{c}\text { Peak RetTime Type } \\ \text { Width }\end{array} & \begin{array}{c}\text { Area } \\ \text { [min] }\end{array} & \begin{array}{c}\text { Height } \\ \text { [min] }\end{array} & \text { Area } \\ - \text { mAU }_{\mathrm{S}} & {[\mathrm{mAU}]} & \% \\ 1 & 7.805 \mathrm{MM} & 0.3211 & 2525.69629 & 131.09987 & 97.5044 \\ 2 & 9.126 \mathrm{MM} & 0.2925 & 64.64340 & 3.68310 & 2.4956\end{array}$

Figure S30. HPLC traces of rac-50a (reference) and (R)-5oa. 

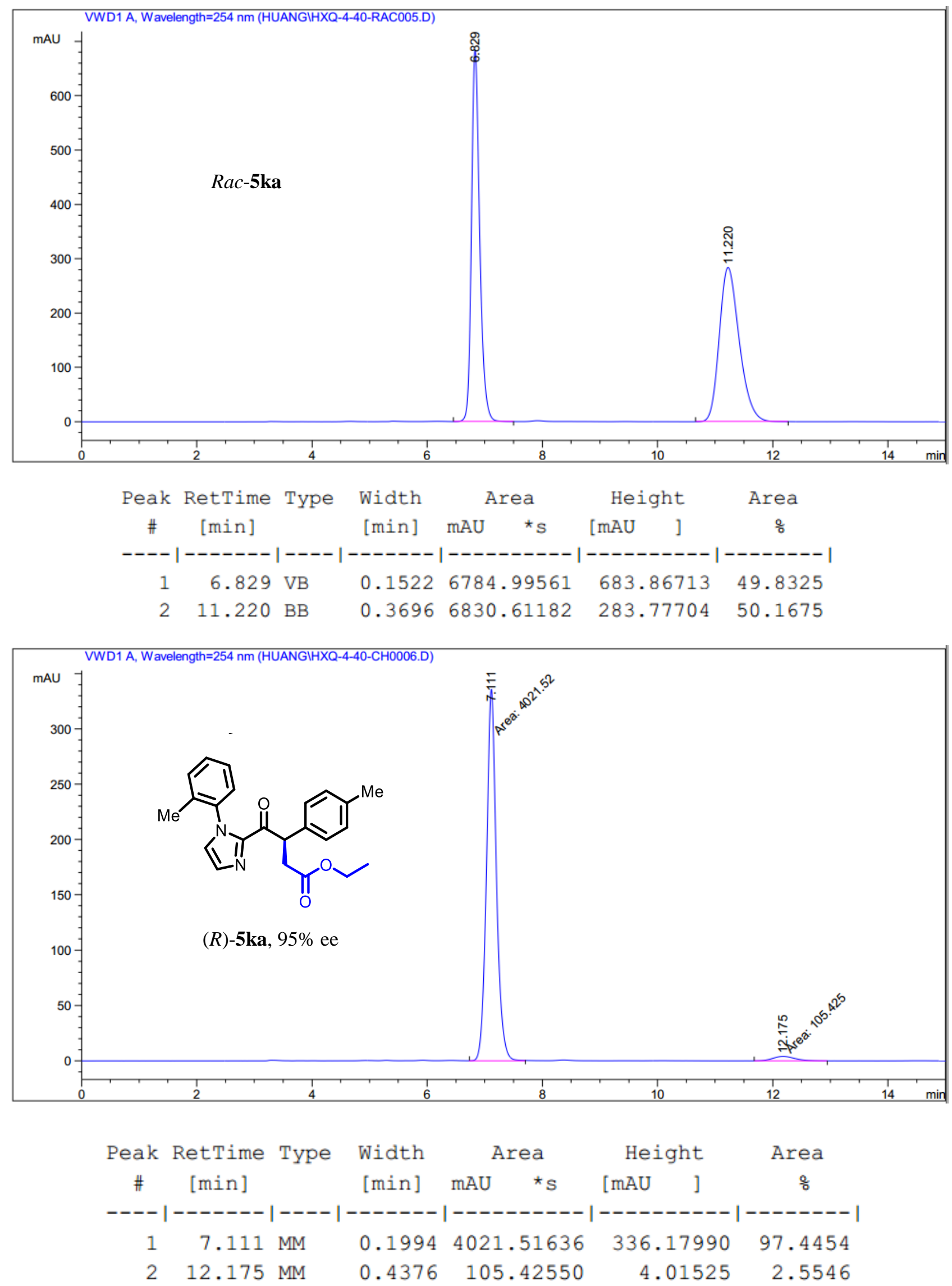

Figure S31. HPLC traces of $r a c-5 \mathbf{k a}$ (reference) and (R)-5ka. 

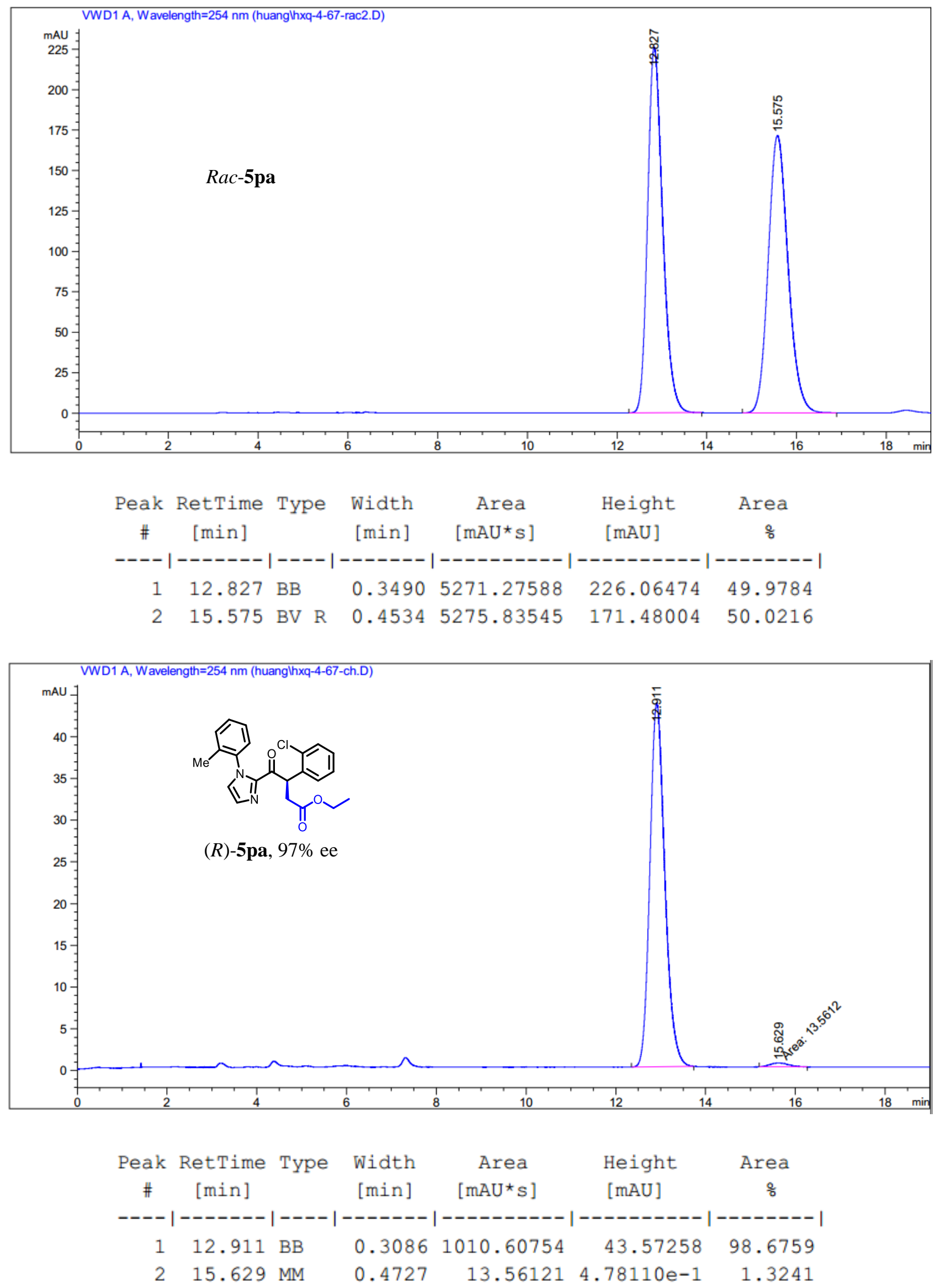

Figure S32. HPLC traces of $r a c-5 p a(r e f e r e n c e)$ and $(R)-5 p a$. 

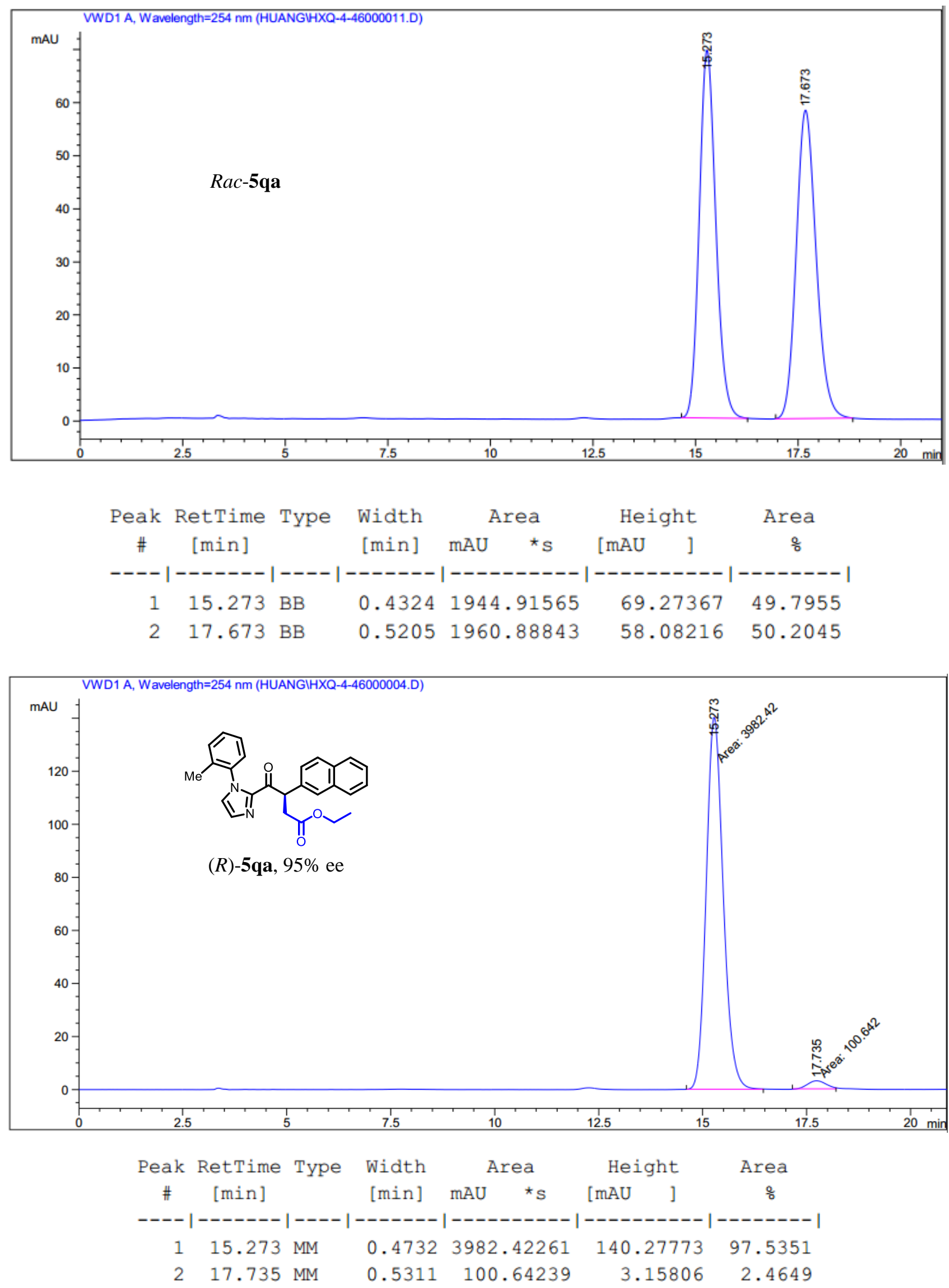

Figure S33. HPLC traces of $r a c-5 q \mathbf{a}$ (reference) and (R)-5qa. 

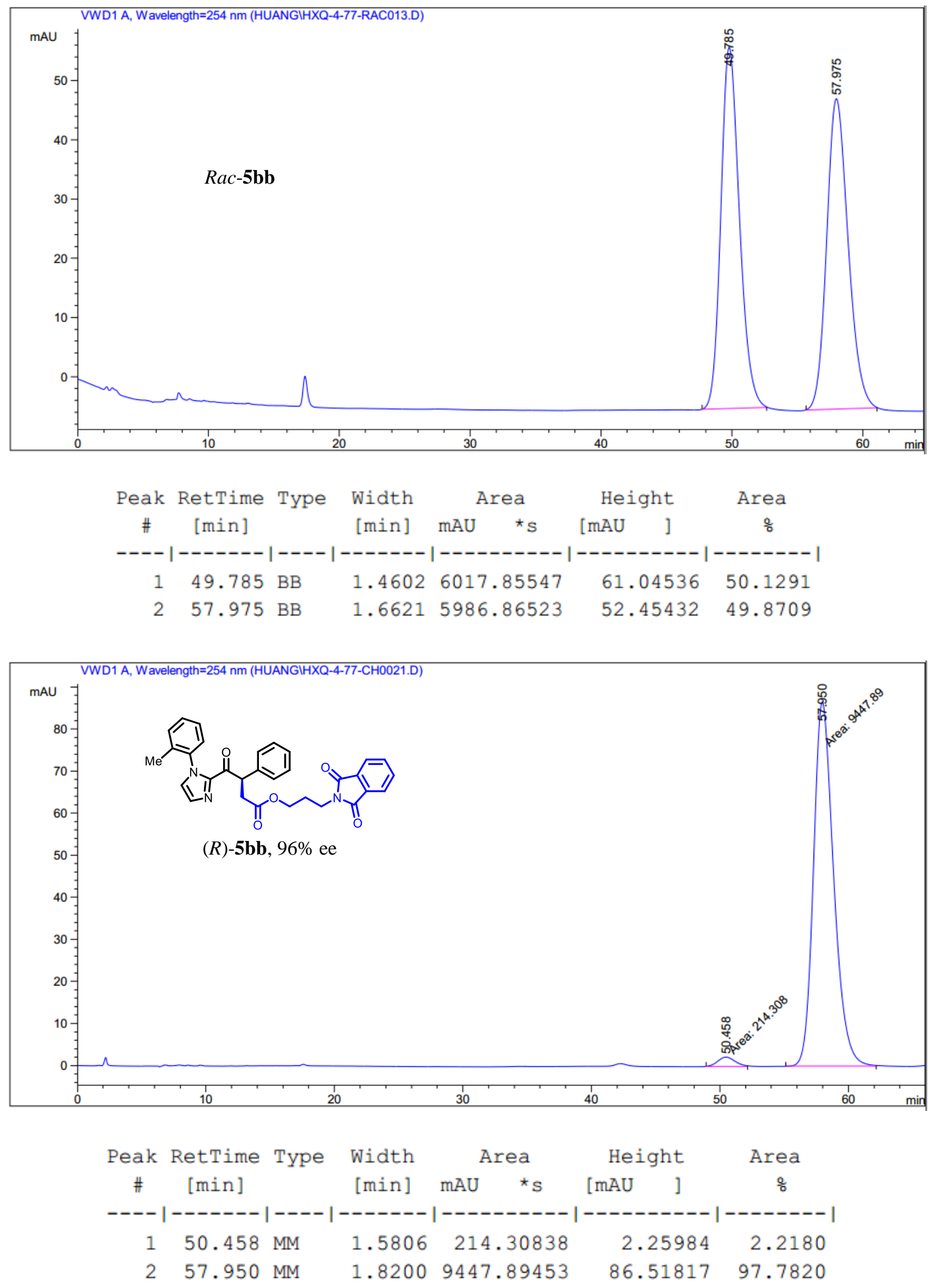

Figure S34. HPLC traces of $r a c-5 b b$ (reference) and ( $R$ )-5bb. 

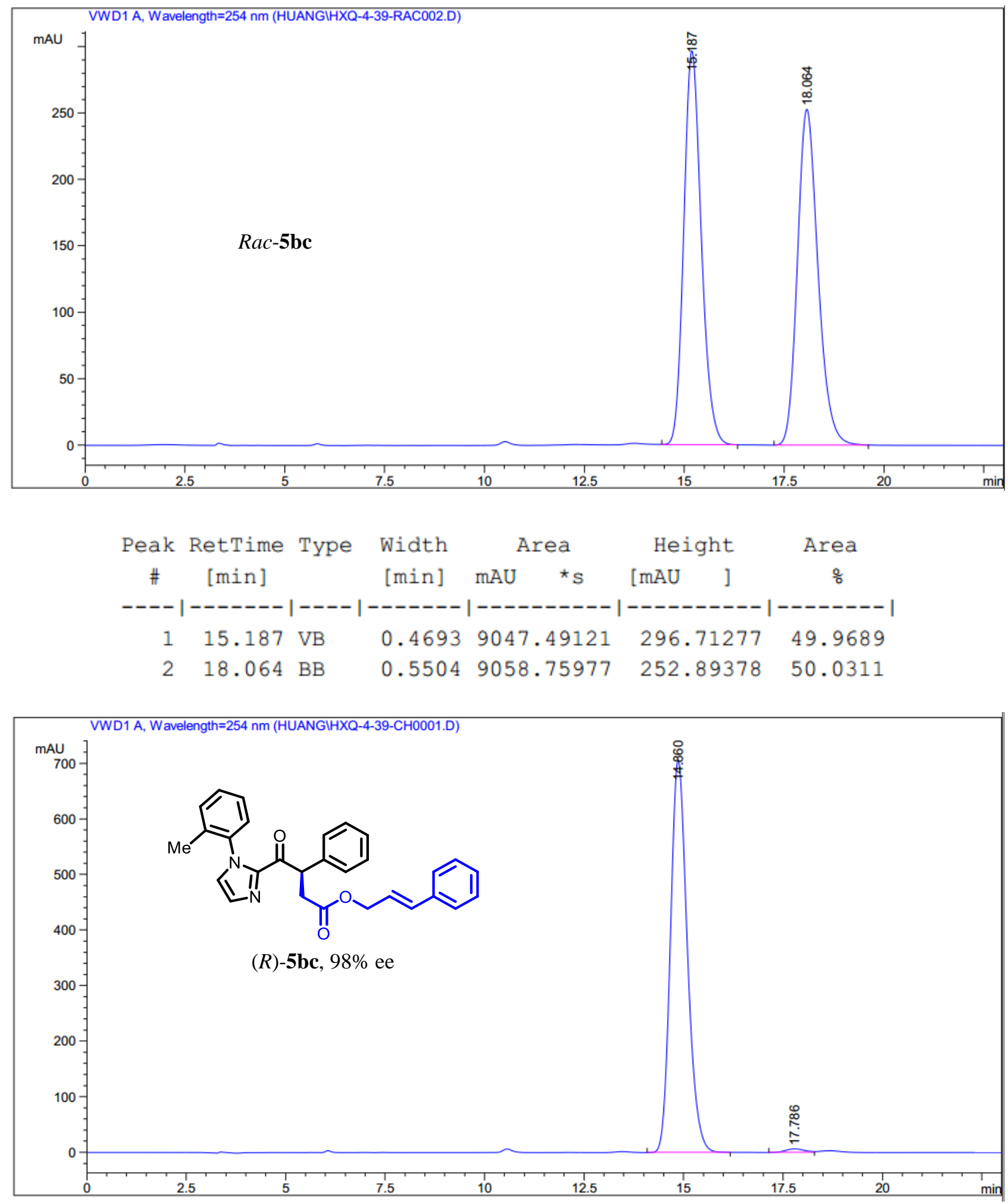

\begin{tabular}{|c|c|c|c|c|c|c|}
\hline Peak & RetTime & Type & Width & Area & Height & Area \\
\hline \# & [min] & & {$[\mathrm{min}]$} & $\mathrm{mAU}$ & {$[\mathrm{mAU}$} & 8 \\
\hline & -- & & ----- & |--------- & --------- & ---- \\
\hline 1 & 14.860 & VB & 0.4525 & $2.07813 \mathrm{e} 4$ & 706.21075 & 98.9366 \\
\hline 2 & 786 & $\mathrm{~V}$ & 0.5198 & 223.35866 & 6.55629 & .0634 \\
\hline
\end{tabular}

Figure S35. HPLC traces of $r a c-5 b c$ (reference) and (R)-5bc. 

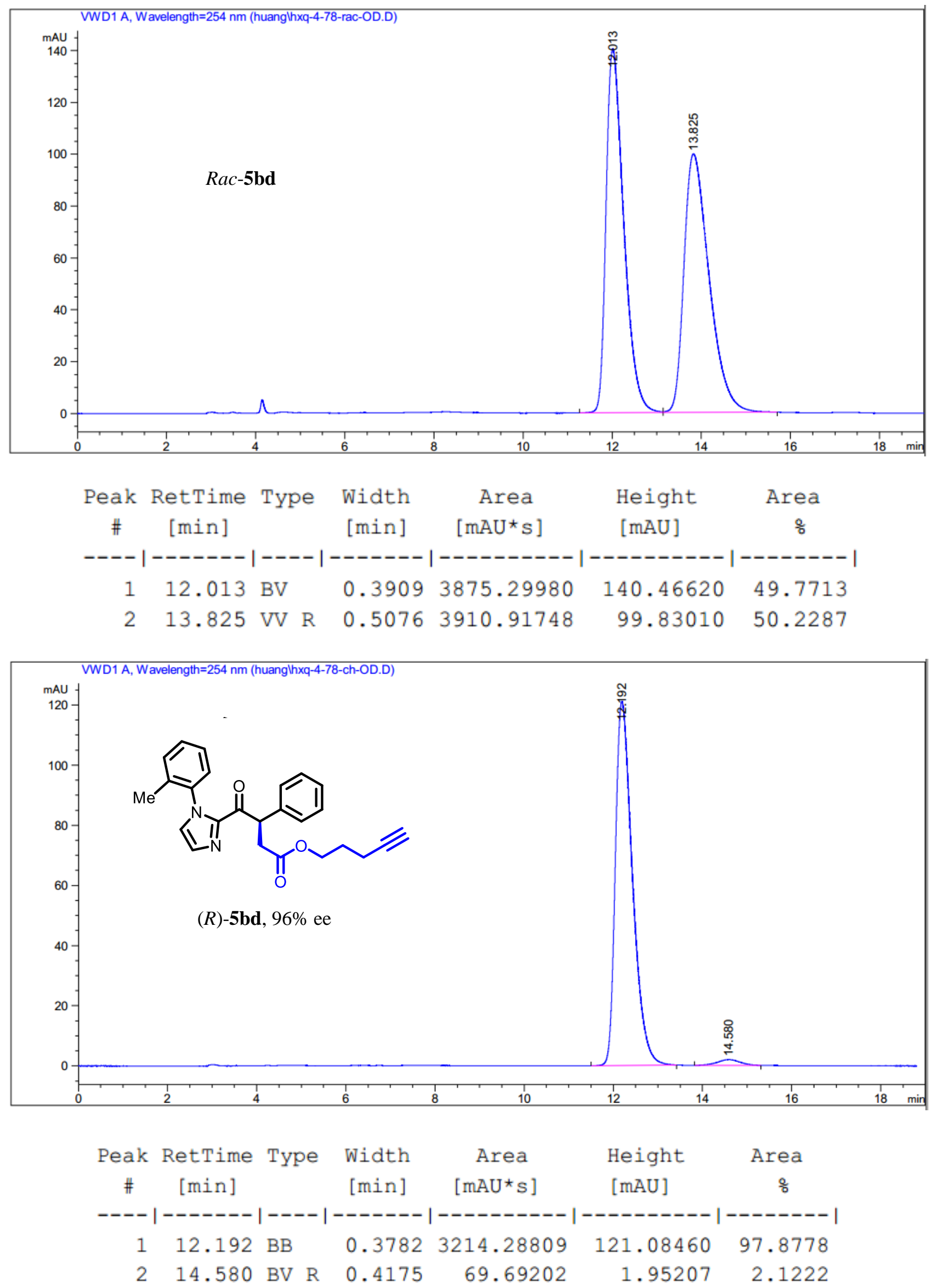

Figure S36. HPLC traces of $r a c-5 b d$ (reference) and (R)-5bd. 

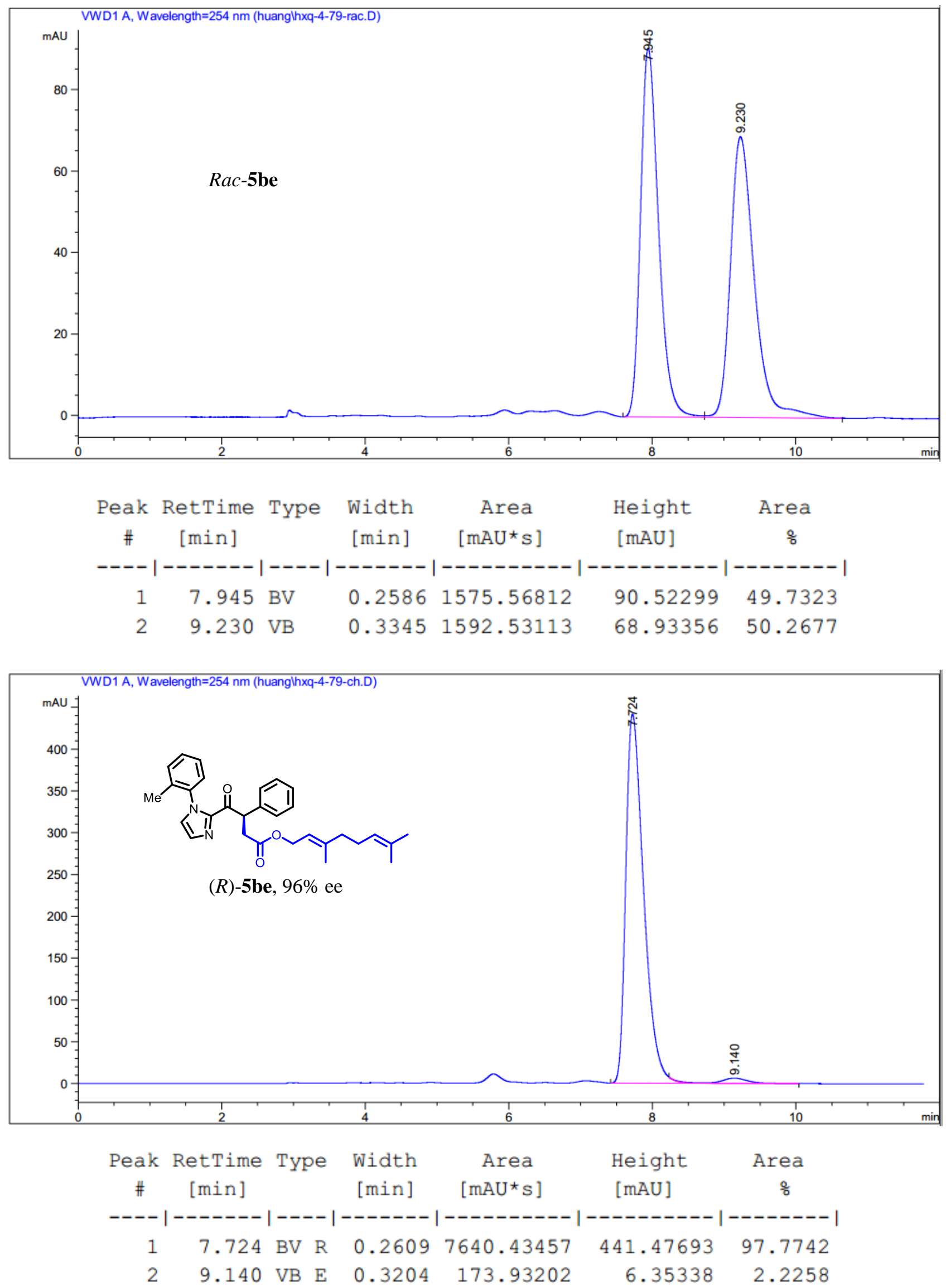

Figure S37. HPLC traces of $\mathrm{rac}$-5be (reference) and ( $R$ )-5be. 
$\mathrm{mV}$

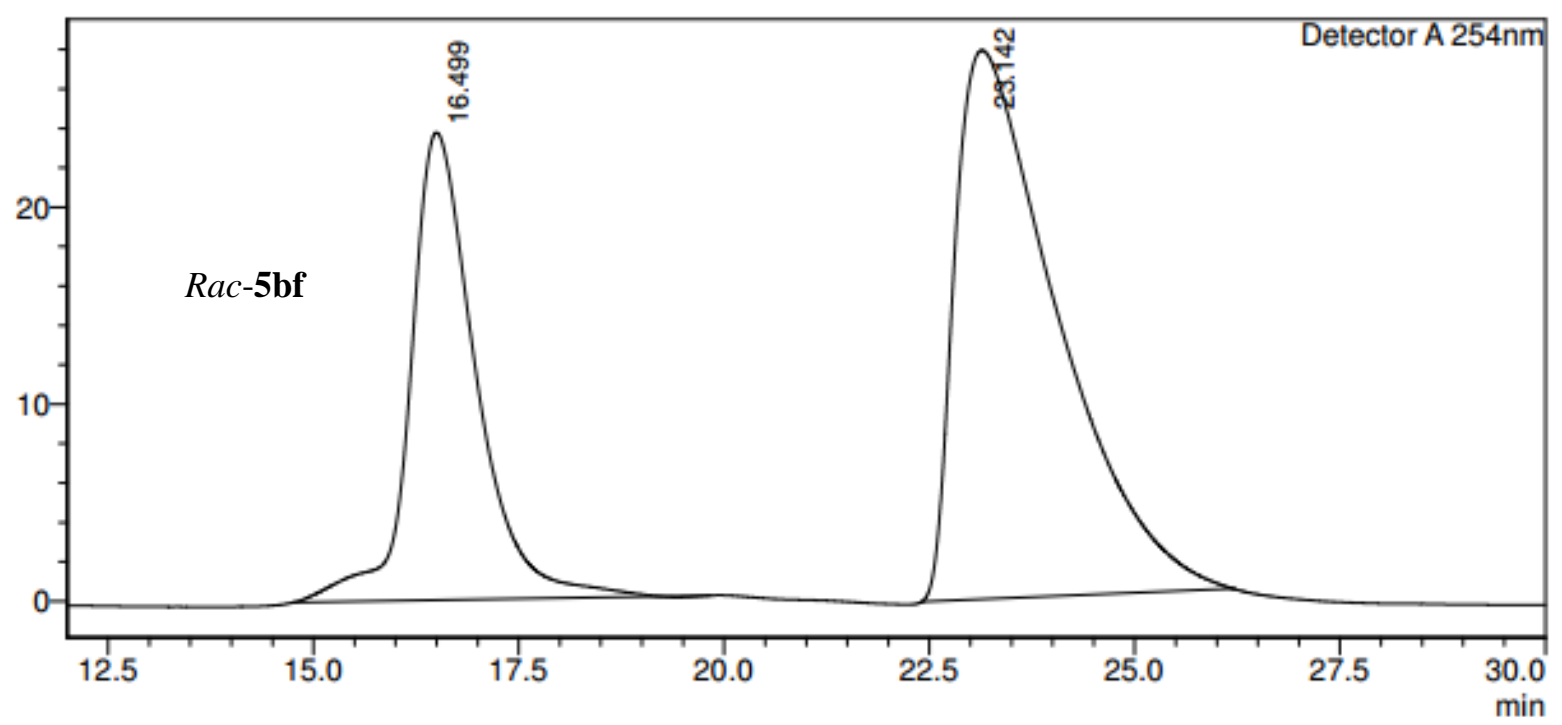

$<$ Peak Table>

Detector A 254nm

\begin{tabular}{|c|c|c|c|}
\hline Peak\# & Ret. Time & Area & Area\% \\
\hline 1 & 16.499 & 1326110 & 35.416 \\
\hline 2 & 23.142 & 2418227 & 64.584 \\
\hline Tota & & 3744337 & 100.000 \\
\hline
\end{tabular}

$\mathrm{mV}$

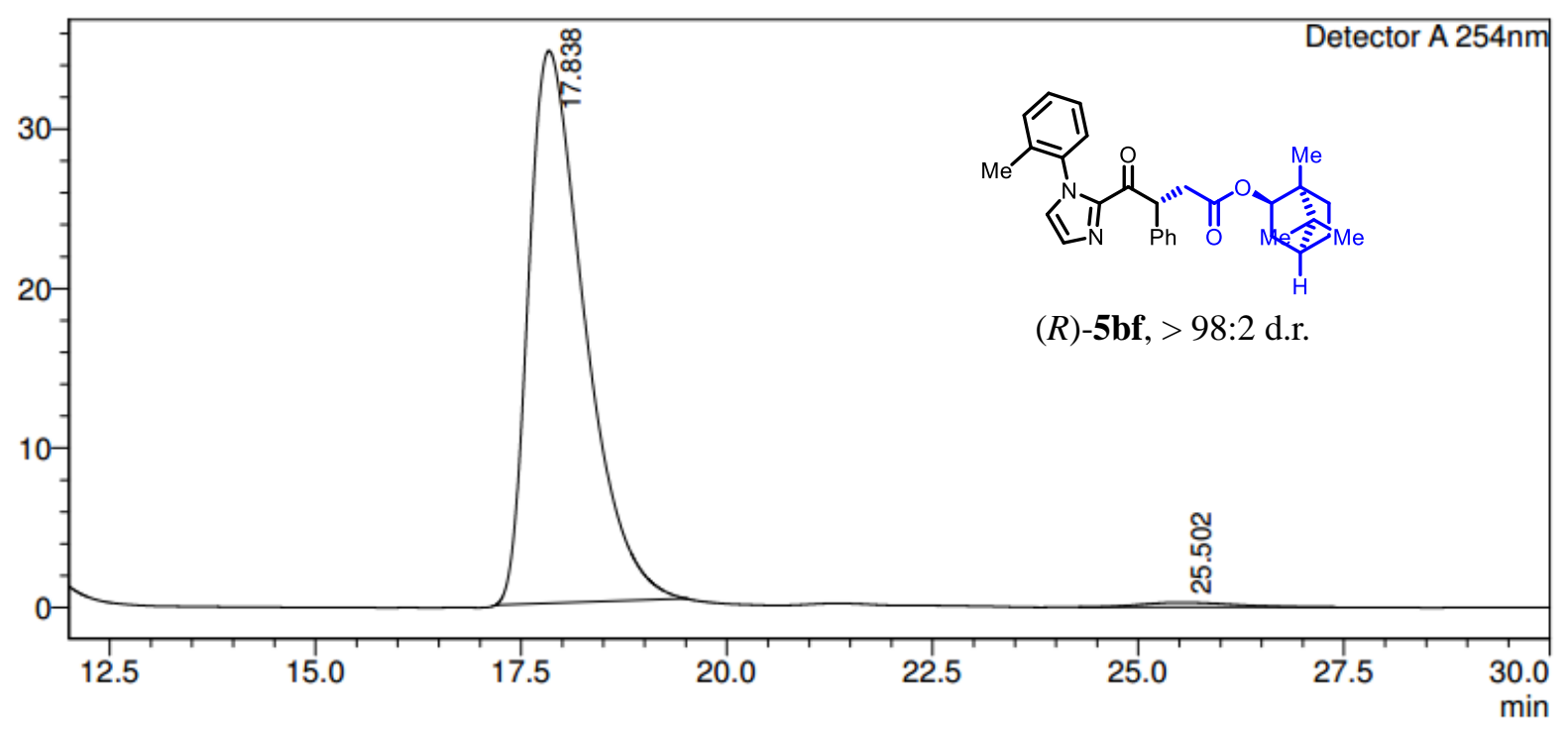

<Peak Table>

\begin{tabular}{|c|c|c|c|}
\hline \multirow{2}{*}{\multicolumn{4}{|c|}{$\begin{array}{l}\text { Detector A 254nm } \\
\text { Peak\#|Ret. Time }\end{array}$}} \\
\hline & Ret. Time & Area & Area\% \\
\hline 1 & 17.8 & 1636703 & 98.593 \\
\hline 2 & 25.502 & 23359 & 1.407 \\
\hline
\end{tabular}

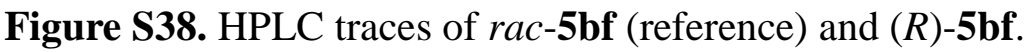



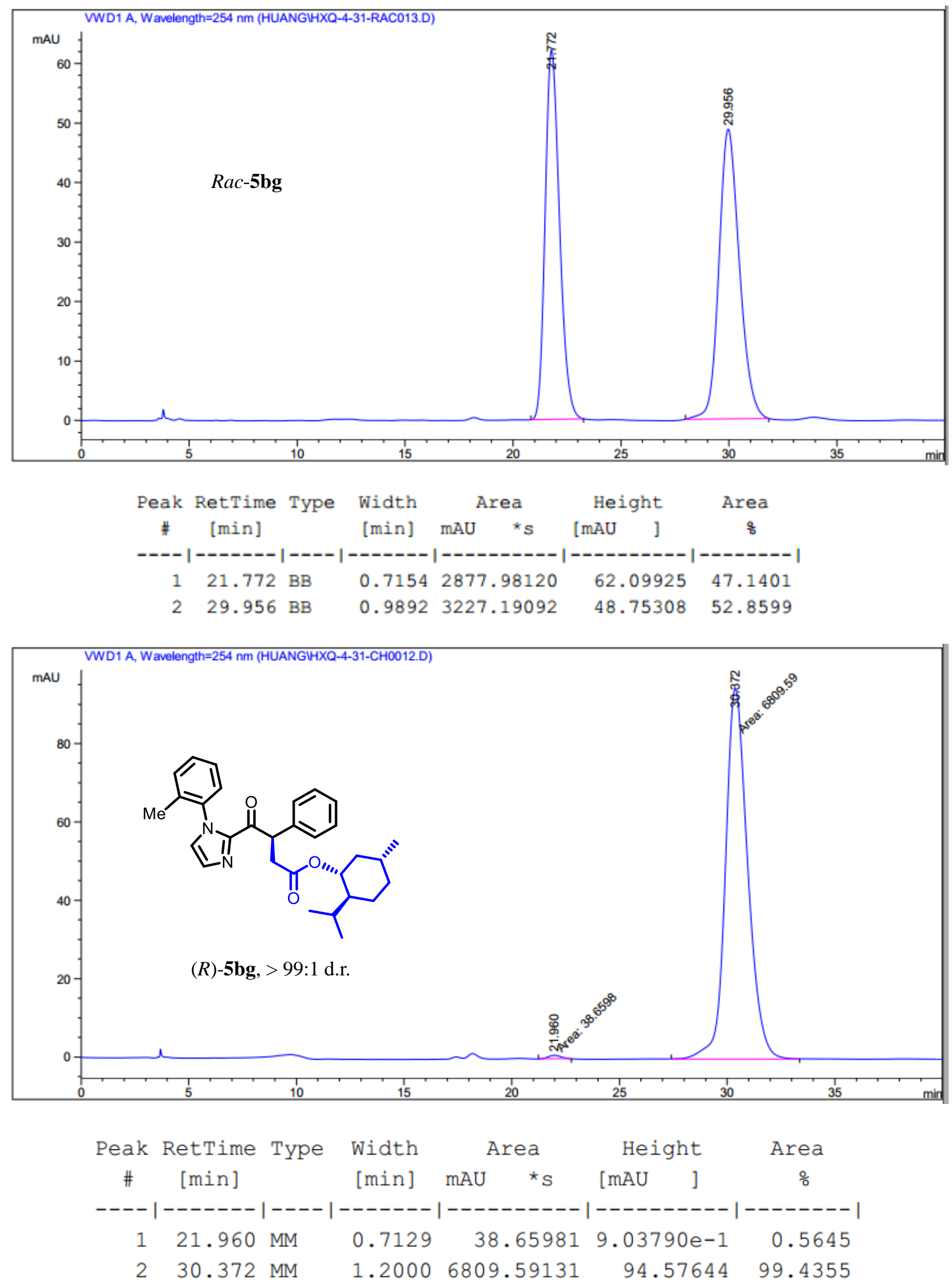

Figure S39. HPLC traces of $r a c-5 b g$ (reference) and (R)-5bg. 

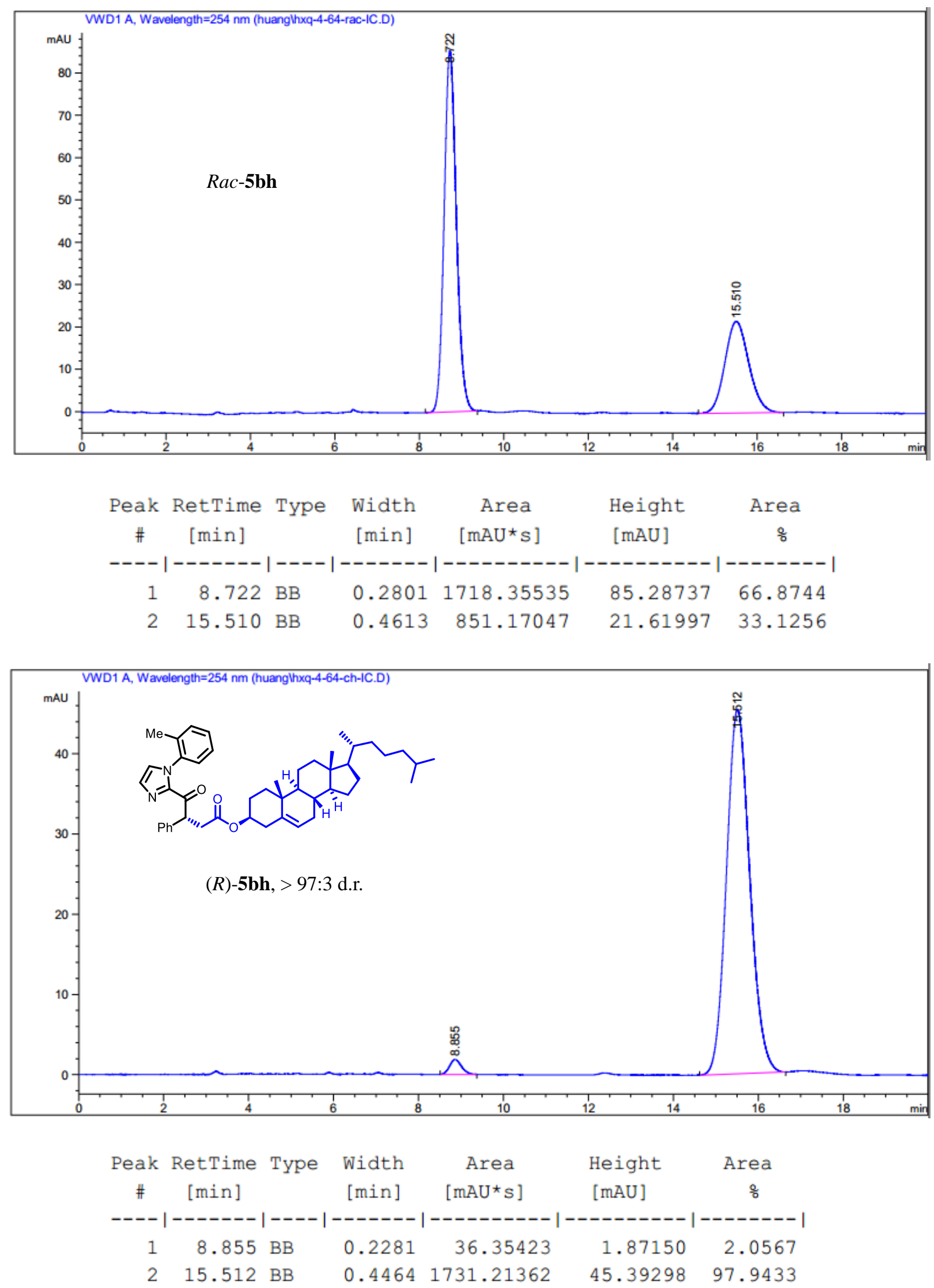

Figure S40. HPLC traces of $r a c-5 b h$ (reference) and (R)-5bh. 

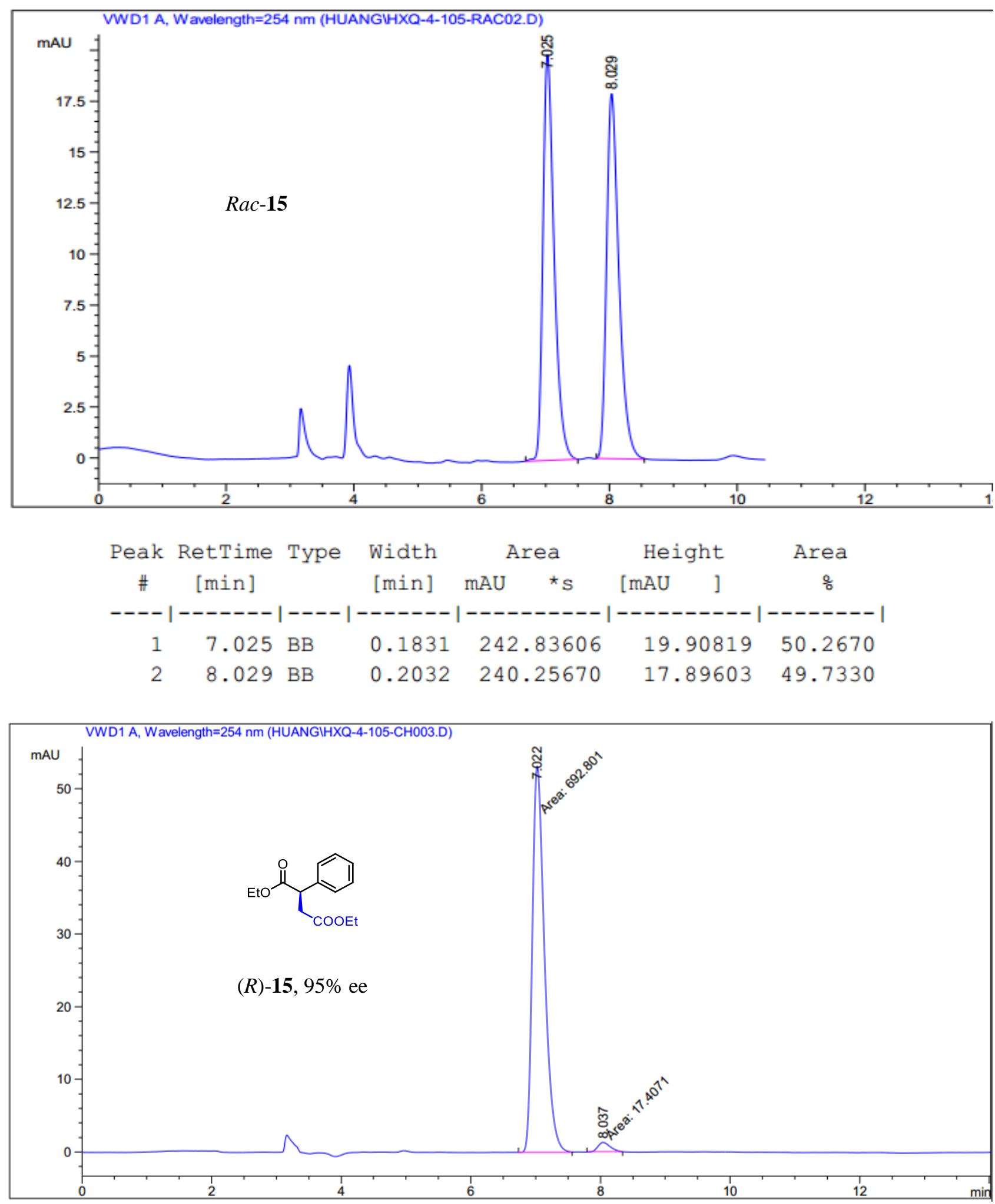

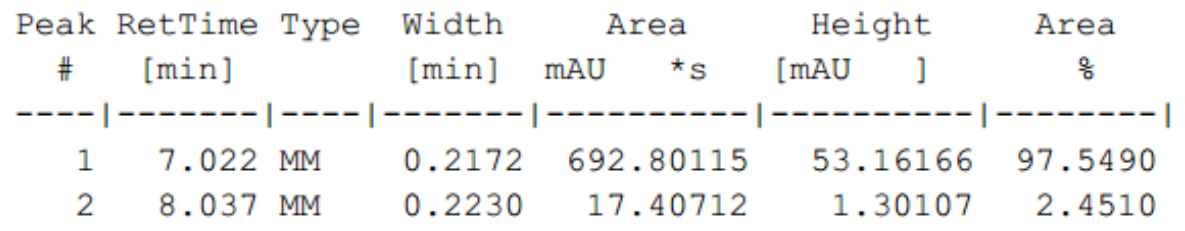

Figure S41. HPLC traces of rac-15 (reference) and (R)-15. 


\section{Single-Crystal X-Ray Diffraction Studies}

Single crystals of $\mathbf{R h}$-enolate suitable for X-ray diffraction were obtained by slow diffusion from a solution of Rh-enolate $(20 \mathrm{mg})$ in DCM $(1.0 \mathrm{~mL})$ layered with $n$-hexane $(0.5 \mathrm{~mL})$ at room temperature for several days in a NMR tube.

Single crystals of 5oa suitable for X-ray diffraction were obtained by slow diffusion from a solution of $50 a(30 \mathrm{mg})$ in DCM $(0.5 \mathrm{~mL})$ layered with $n$-hexane $(0.5 \mathrm{~mL})$ at $-20{ }^{\circ} \mathrm{C}$ for several days in a NMR tube.

X-ray data were collected with a Bruker 3 circuit D8 Quest diffractometer with MoK $\alpha$ radiation (microfocus tube with multilayer optics) and Photon 100 CMOS detector at $100 \mathrm{~K}$. Scaling and

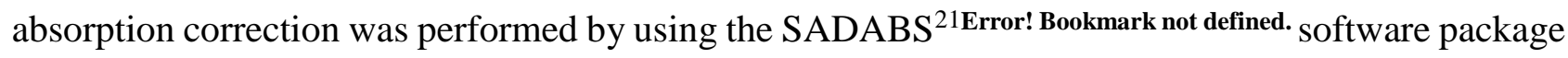
of Bruker. Structures were solved using direct methods in SHELXT ${ }^{22}$ and refined using the full matrix least squares procedure in SHELXL-2014 ${ }^{23}$. The hydrogen atoms were placed in calculated positions and refined as riding on their respective $\mathrm{C}$ atom, and $\operatorname{Uiso}(\mathrm{H})$ was set at $1.2 \mathrm{Ueq}\left(\mathrm{Csp}^{2}\right)$ and 1.5 $\mathrm{Ueq}\left(\mathrm{Csp}^{3}\right)$. Disorder was refined using restraints for both the geometry and the anisotropic displacement factors. The absolute configuration of $\mathbf{5 0 a}$ has been determined. ${ }^{24}$

Crystal structure, data and details of the structure determination for $\mathbf{R h}$-enolate are presented in the Figure S42 and Table S9.

Crystal structure, data and details of the structure determination for $\mathbf{5 0 a}$ are presented in the Figure S43 and Table S10. 


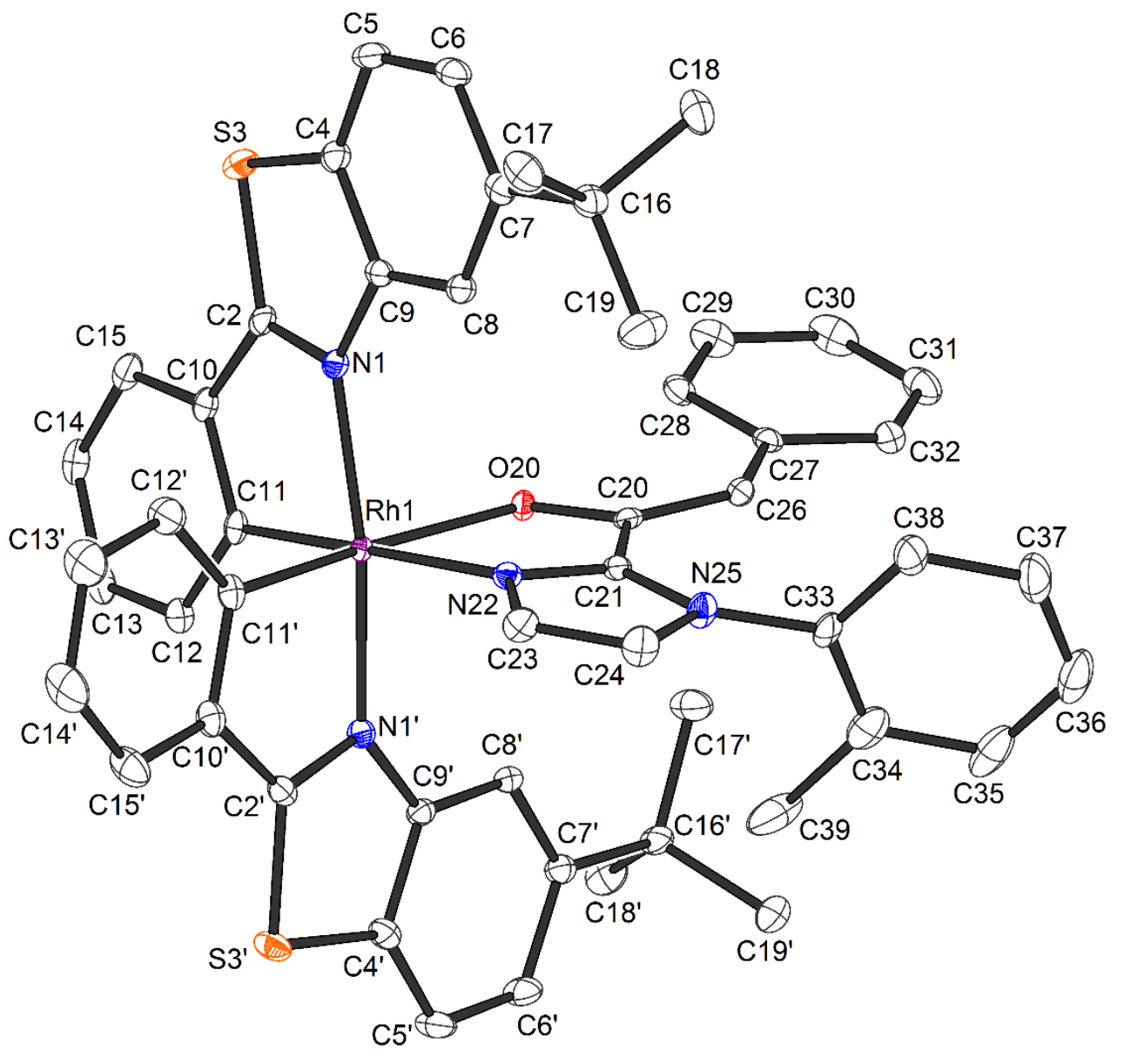

Figure S42. Crystal structure of Rh-enolate. ORTEP drawing with $30 \%$ probability thermal ellipsoids. 
Table S9. Crystal Data and Structure Refinement for Rh-enolate

Crystal data

Identification code

Habitus, colour

Crystal size

Crystal system

Space group

Unit cell dimensions

Volume

Cell determination

Empirical formula

Moiety formula

Formula weight

Density (calculated)

Absorption coefficient

$\mathrm{F}(000)$

Data collection:

Diffractometer type

Wavelength

Temperature

Theta range for data collection

Index ranges

Data collection software

Cell refinement software

Data reduction software

Solution and refinement:

Reflections collected

Independent reflections

Completeness to theta $=25.242^{\circ}$

Observed reflections

Reflections used for refinement

Absorption correction

Max. and min. transmission

Largest diff. peak and hole

Solution

Refinement

Treatment of hydrogen atoms

Programs used

Data / restraints / parameters

Goodness-of-fit on $\mathrm{F}^{2}$

$\mathrm{R}$ index (all data)

$\mathrm{R}$ index conventional [I $>2 \operatorname{sigma}(\mathrm{I})]$
hxqC31_0m

nugget, light green

$0.23 \times 0.13 \times 0.10 \mathrm{~mm}^{3}$

Monoclinic

$\mathrm{P} 21 / \mathrm{n} \quad \mathrm{Z}=4$

$\mathrm{a}=11.6759(5) \AA \quad \alpha=90^{\circ}$.

$\mathrm{b}=23.0296(9) \AA \quad \beta=92.6600(10)^{\circ}$.

$\mathrm{c}=16.4573(7) \AA \quad \gamma=90^{\circ}$.

4420.5(3) $\AA^{3}$

9634 peaks with Theta 2.4 to $27.5^{\circ}$.

$\mathrm{C}_{52} \mathrm{H}_{47} \mathrm{~N}_{4} \mathrm{O}$ Rh $\mathrm{S}_{2}$

$\mathrm{C}_{52} \mathrm{H}_{47} \mathrm{~N}_{4} \mathrm{O}$ Rh $\mathrm{S}_{2}$

910.96

$1.369 \mathrm{Mg} / \mathrm{m}^{3}$

$0.524 \mathrm{~mm}^{-1}$

1888

Bruker D8 QUEST area detector

$0.71073 \AA$

100(2) K

2.159 to $27.558^{\circ}$.

$-15<=\mathrm{h}<=15,-29<=\mathrm{k}<=29,-21<=1<=21$

APEX3 (Bruker AXS Inc., 2015) 25

SAINT V8.35A (Bruker AXS Inc., 2015)26

SAINT V8.35A (Bruker AXS Inc., 2015)

155552

$10199[\mathrm{R}(\mathrm{int})=0.0382]$

$99.9 \%$

9143[I > 2(I)]

10199

Semi-empirical from equivalents

0.95 and 0.90

0.413 and -0.701 e. $\AA^{-3}$

Direct methods

Full-matrix least-squares on $\mathrm{F}^{2}$

Calculated positions, constr. ref.

XT V2014/1 (Bruker AXS Inc., 2014) ${ }^{27}$

SHELXL-2014/7 (Sheldrick, 2014) ${ }^{28}$

DIAMOND (Crystal Impact) ${ }^{29}$

ShelXle (Hübschle, Sheldrick, Dittrich, 2011) ${ }^{30}$

10199 / 303 / 583

1.052

$\mathrm{wR} 2=0.0556$

$\mathrm{R} 1=0.0227$ 


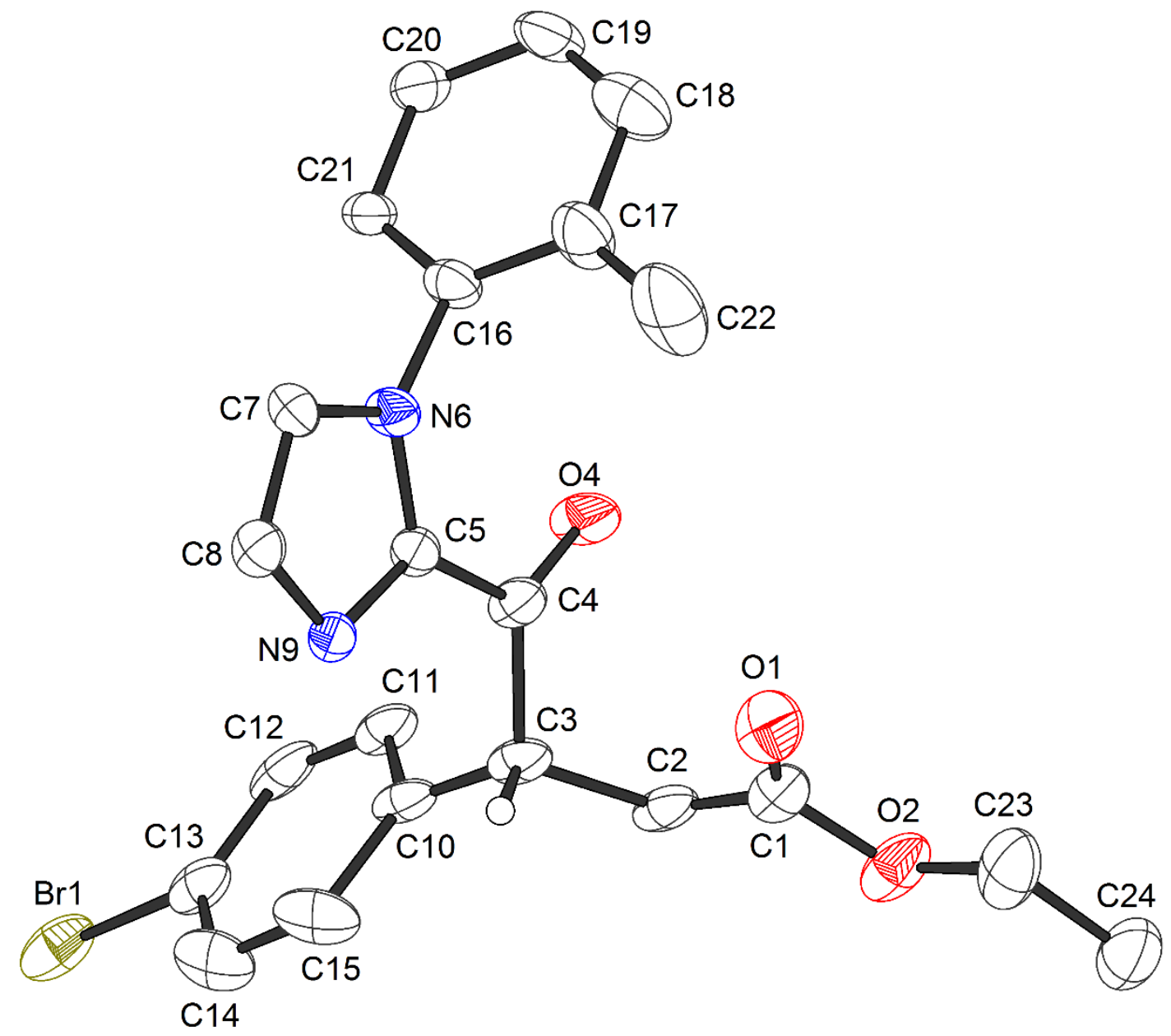

Figure S43. Crystal structure of 5oa. ORTEP drawing with 30\% probability thermal ellipsoids. 
Table S10. Crystal Data and Structure Refinement for 5oa

Crystal data

Identification code

Habitus, colour

Crystal size

Crystal system

Space group

Unit cell dimensions

Volume

Cell determination

Empirical formula

Moiety formula

Formula weight

Density (calculated)

Absorption coefficient

$\mathrm{F}(000)$

Data collection:

Diffractometer type

Wavelength

Temperature

Theta range for data collection

Index ranges

Data collection software

Cell refinement software

Data reduction software

Solution and refinement:

Reflections collected

Independent reflections

Completeness to theta $=25.242^{\circ}$

Observed reflections

Reflections used for refinement

Extinction coefficient

Absorption correction

Max. and min. transmission

Flack parameter (absolute struct.)

Largest diff. peak and hole

Solution

Refinement

Treatment of hydrogen atoms

Programs used

Data / restraints / parameters

Goodness-of-fit on $\mathrm{F}^{2}$

$\mathrm{R}$ index (all data)

$\mathrm{R}$ index conventional [I $>2 \operatorname{sigma}(\mathrm{I})]$
hxqD53_0m

nugget, colourless

$0.27 \times 0.22 \times 0.15 \mathrm{~mm}^{3}$

Orthorhombic

$\mathrm{P} 22_{1} 2_{1}$

$\mathrm{a}=9.8543(4) \AA$

$\mathrm{Z}=4$

$\mathrm{b}=13.1873(6) \AA$

$\mathrm{c}=15.4684(7) \AA$

2010.14(15) $\AA^{3}$

$\alpha=90^{\circ}$.

$\beta=90^{\circ}$.

$\gamma=90^{\circ}$.

9961 peaks with Theta 2.5 to $25.3^{\circ}$.

$\mathrm{C}_{22} \mathrm{H}_{21} \mathrm{Br} \mathrm{N}_{2} \mathrm{O}_{3}$

$\mathrm{C}_{22} \mathrm{H}_{21} \mathrm{Br} \mathrm{N}_{2} \mathrm{O}_{3}$

441.32

$1.458 \mathrm{Mg} / \mathrm{m}^{3}$

$2.070 \mathrm{~mm}^{-1}$

904

Bruker D8 QUEST area detector

$0.71073 \AA$

100(2) K

2.451 to $25.288^{\circ}$.

$-11<=\mathrm{h}<=11,-13<=\mathrm{k}<=15,-18<=1<=18$

APEX3 (Bruker AXS Inc., 2015) ${ }^{25}$

SAINT V8.35A (Bruker AXS Inc., 2015) ${ }^{26}$

SAINT V8.35A (Bruker AXS Inc., 2015)

29129

$3633[\mathrm{R}(\mathrm{int})=0.0358]$

$100.0 \%$

$3507[\mathrm{I}>2(\mathrm{I})]$

3633

$\mathrm{X}=0.0018(4)$

Semi-empirical from equivalents

0.75 and 0.65

$0.005(4)^{31}$

0.720 and -0.920 e. $\AA^{-3}$

Direct methods

Full-matrix least-squares on $\mathrm{F}^{2}$

Calculated positions, constr. ref.

XT V2014/1 (Bruker AXS Inc., 2014)27

SHELXL-2014/7 (Sheldrick, 2014) ${ }^{28}$

DIAMOND (Crystal Impact) ${ }^{29}$

ShelXle (Hübschle, Sheldrick, Dittrich, 2011) 30 $^{30}$

3633 / 756 / 366

1.152

$\mathrm{wR} 2=0.0787$

$\mathrm{R} 1=0.0376$ 
10. NMR Spectra of New Compounds
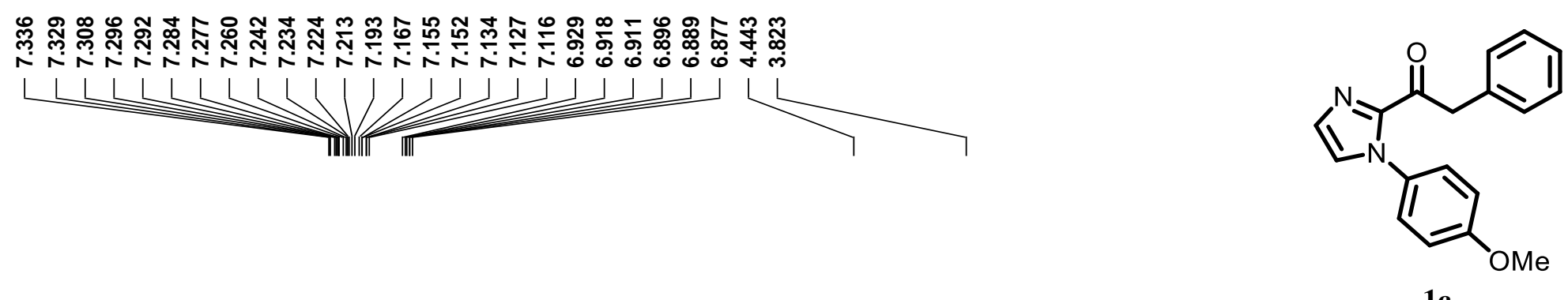

${ }^{1} \mathrm{H}$ NMR $\left(300 \mathrm{MHz}, \mathrm{CDCl}_{3}\right.$ )

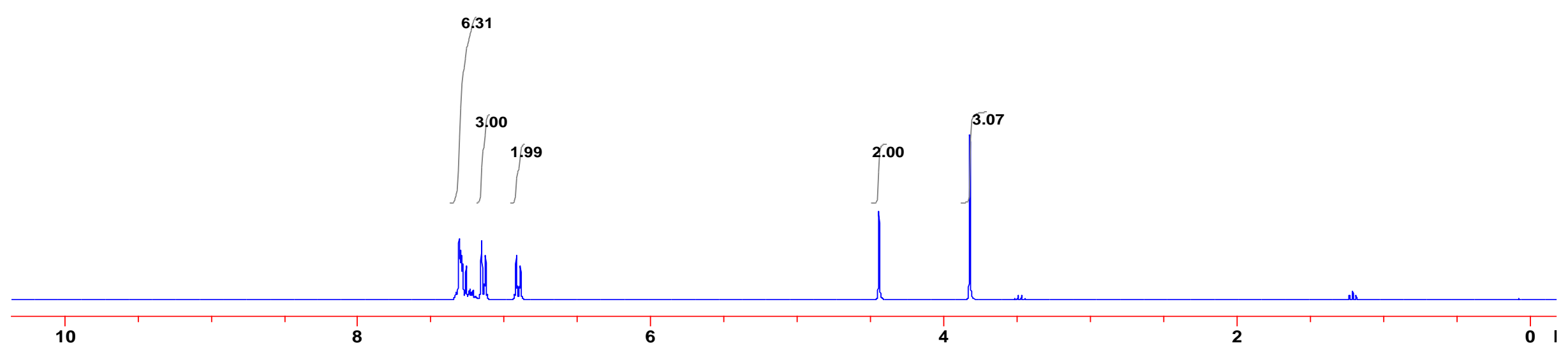




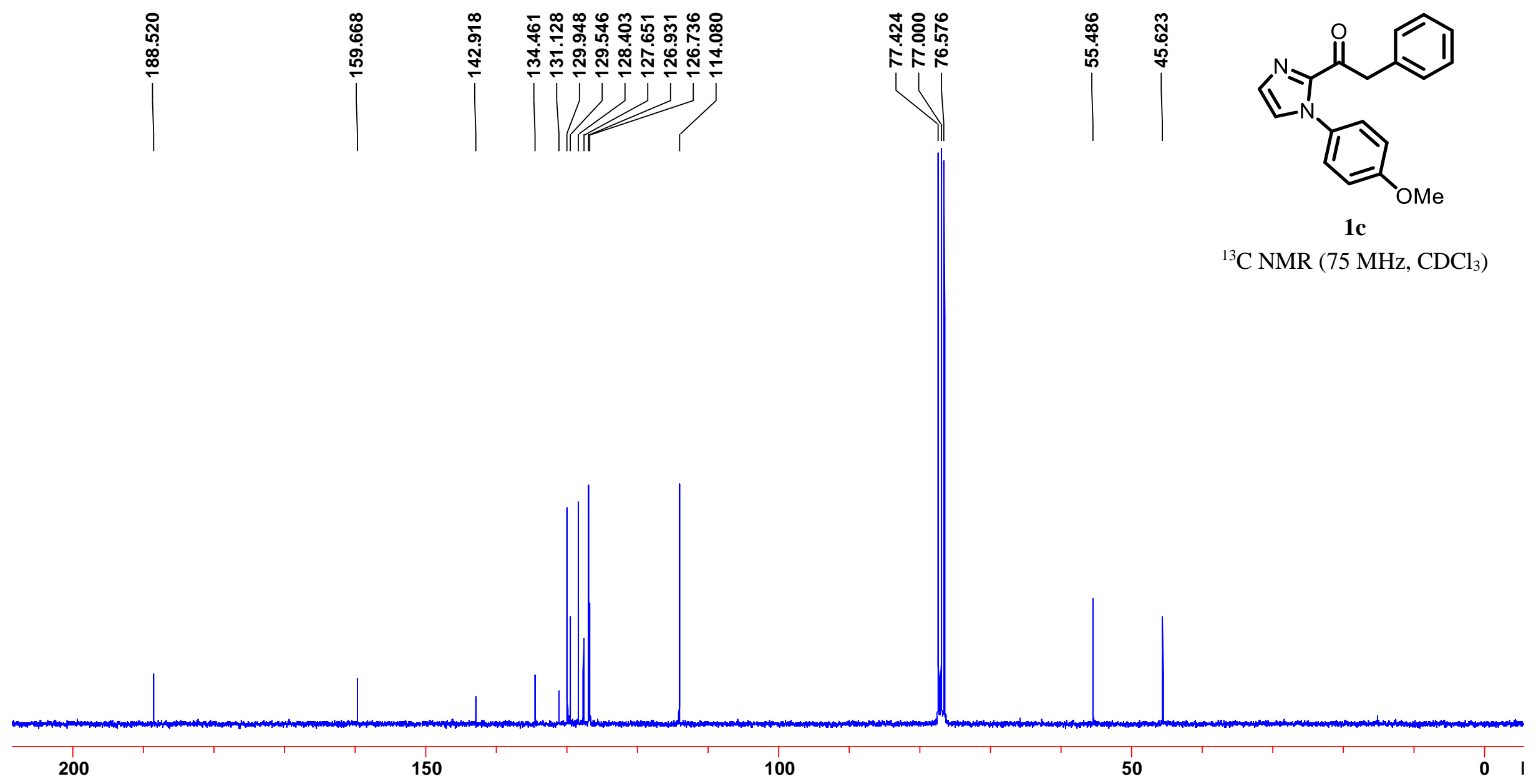




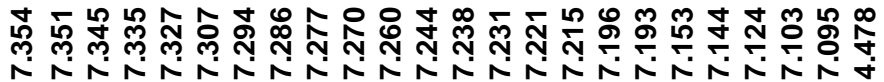

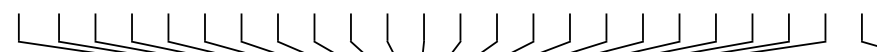

11lाI

9.01

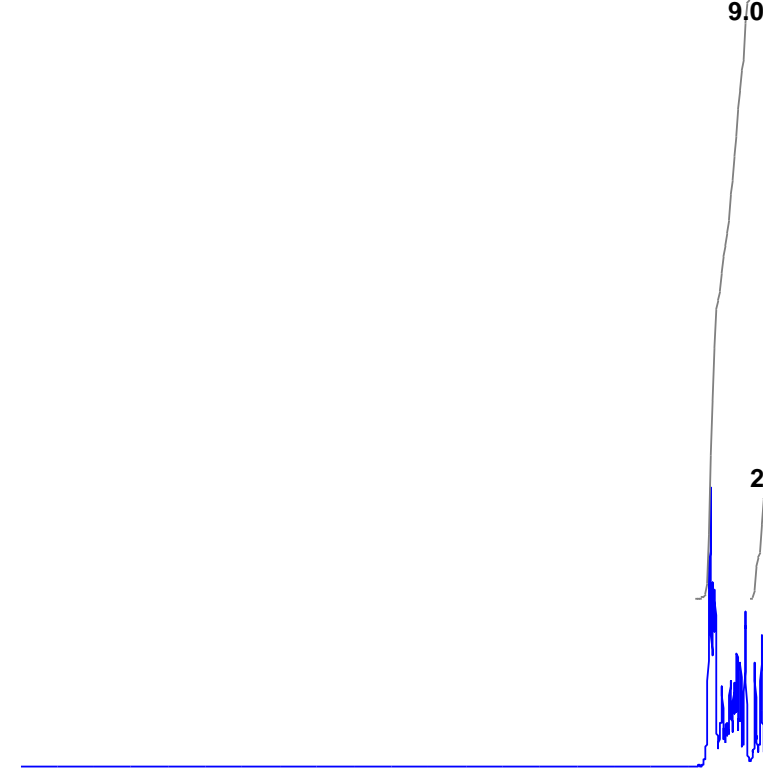

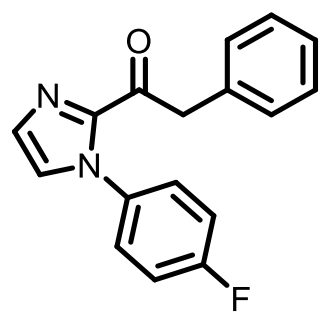

1d

${ }^{1} \mathrm{H}$ NMR $\left(300 \mathrm{MHz}, \mathrm{CDCl}_{3}\right)$ 


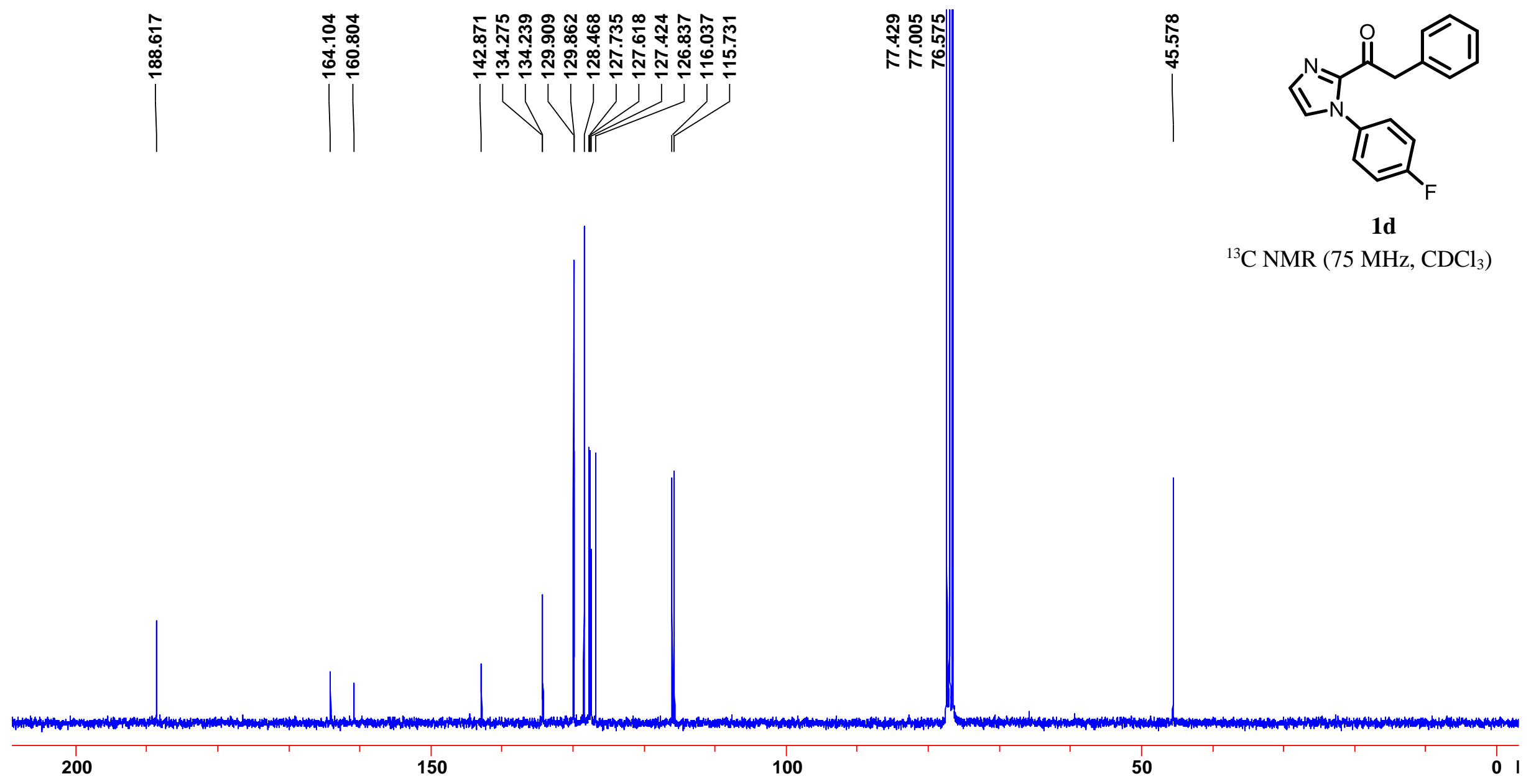




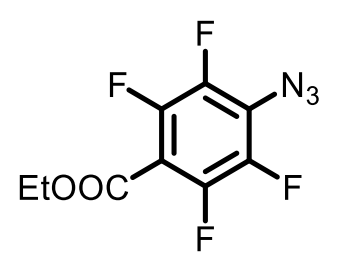

2c

${ }^{1} \mathrm{H}$ NMR $\left(500 \mathrm{MHz}, \mathrm{CDCl}_{3}\right)$

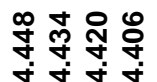

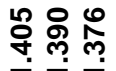

(1)

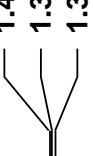

V

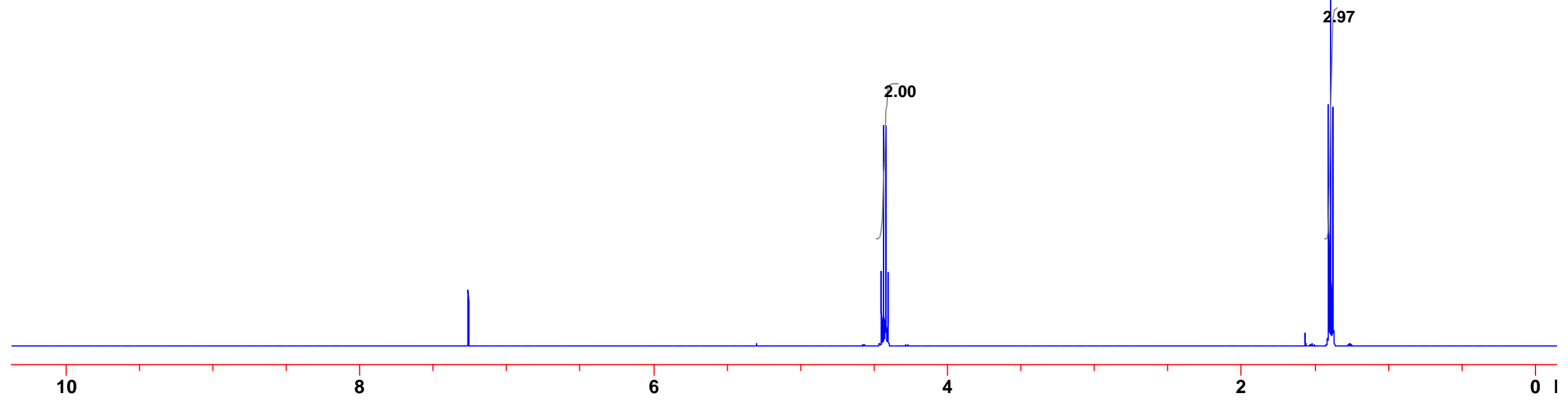



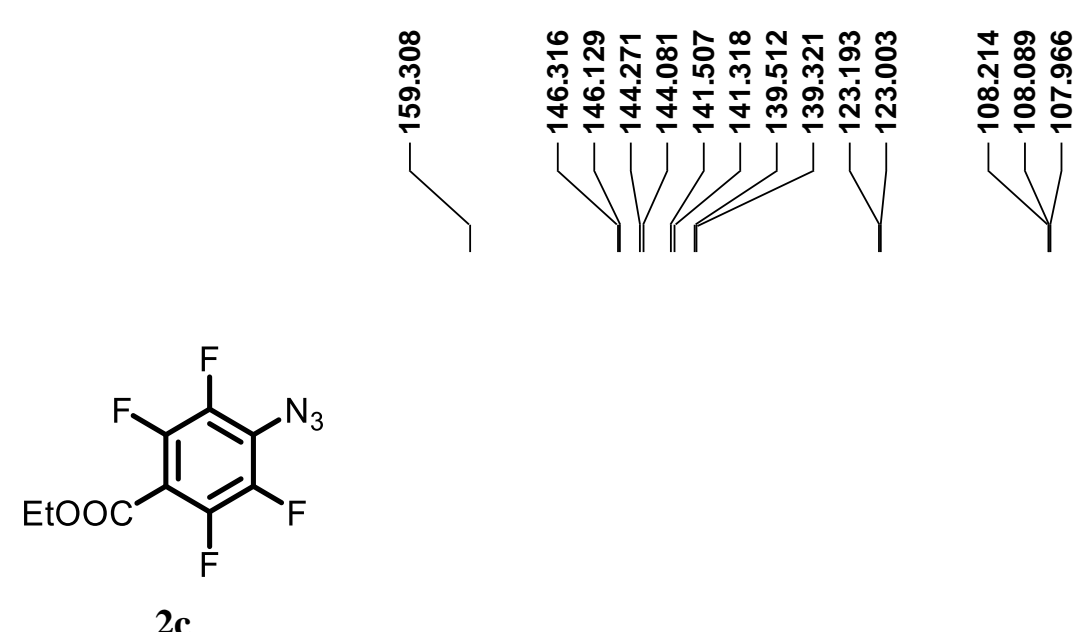

${ }^{13} \mathrm{C} \mathrm{NMR}\left(125 \mathrm{MHz}, \mathrm{CDCl}_{3}\right)$

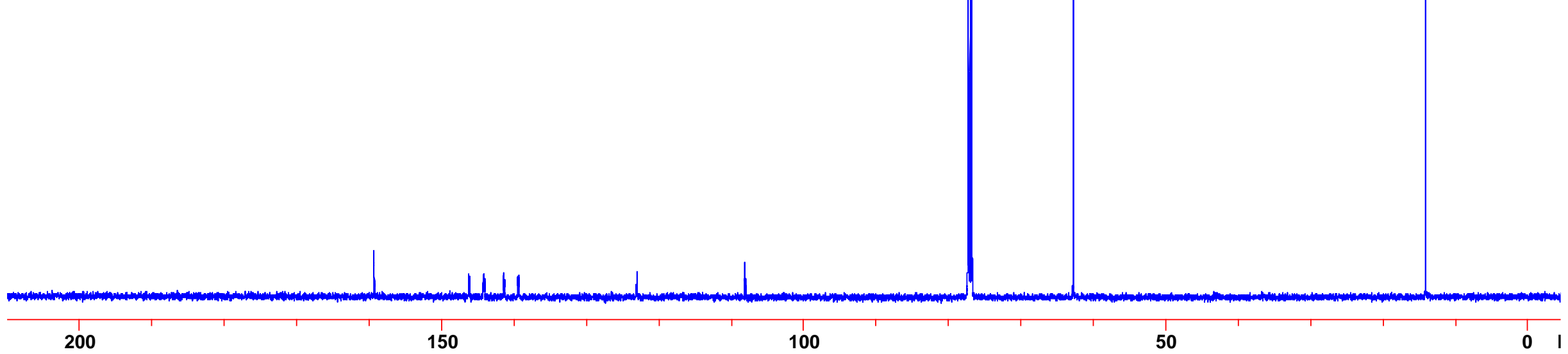



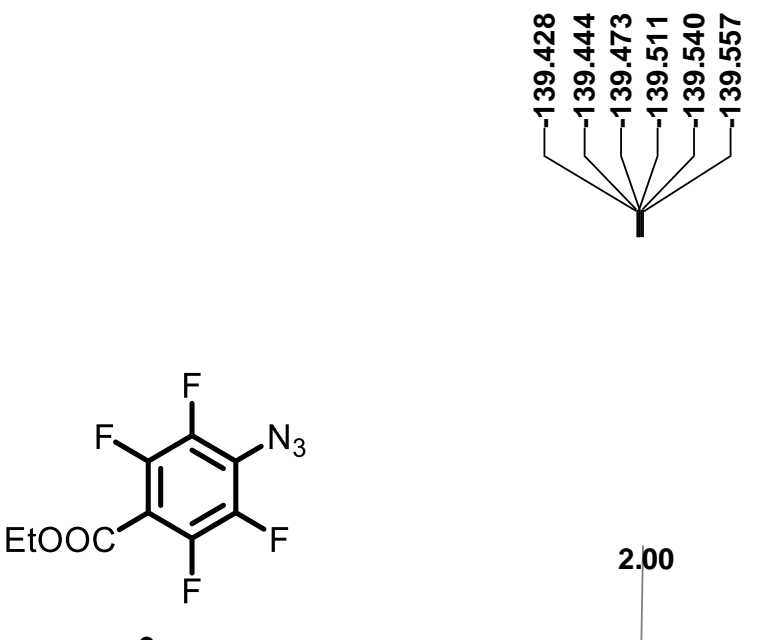

$2 c$

${ }^{19} \mathrm{~F}$ NMR $\left(282 \mathrm{MHz}, \mathrm{CDCl}_{3}\right)$
2.00

1.96

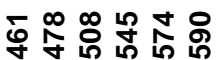

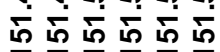

(T)

$\psi$

60 


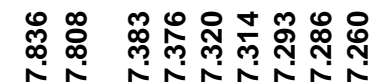

(r)

,

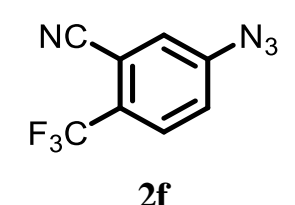

${ }^{1} \mathrm{H}$ NMR (300 MHz, $\mathrm{CDCl}_{3}$ )

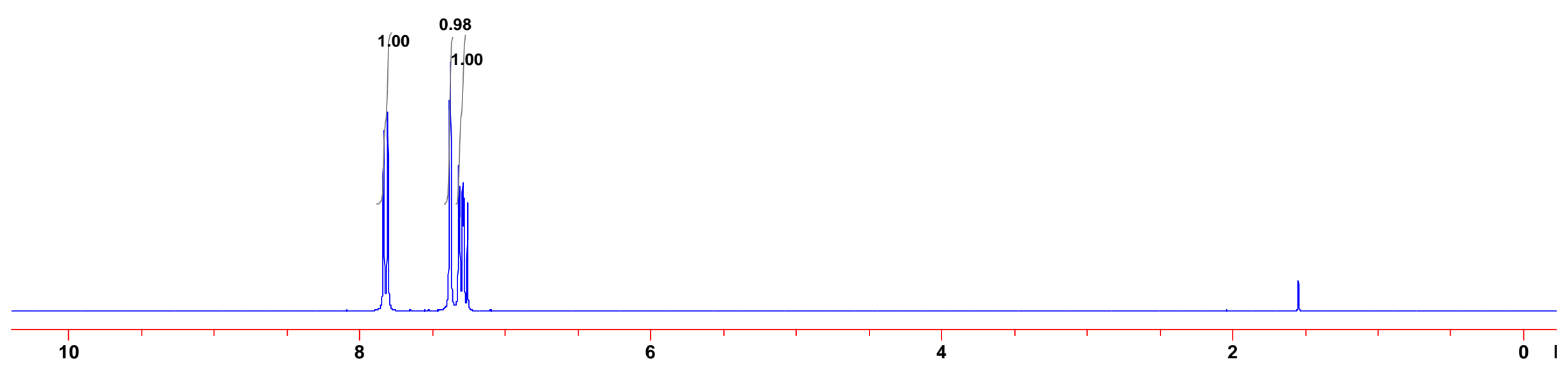



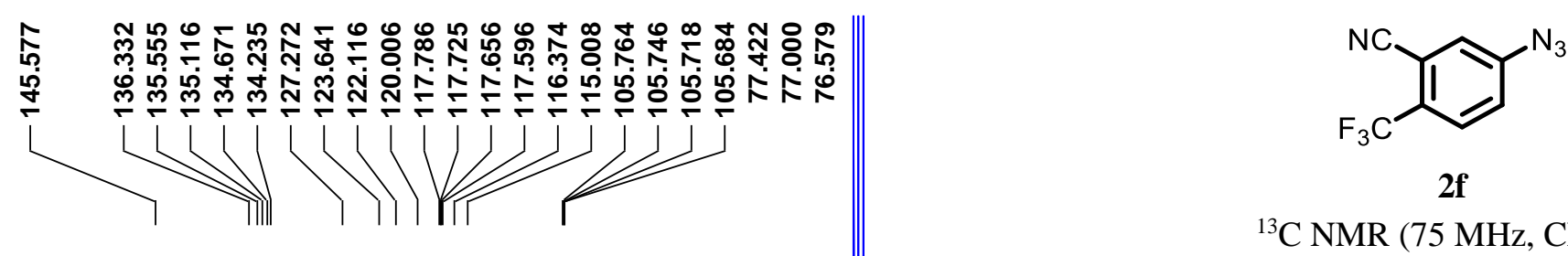

${ }^{13} \mathrm{C}$ NMR $\left(75 \mathrm{MHz}, \mathrm{CDCl}_{3}\right.$ )

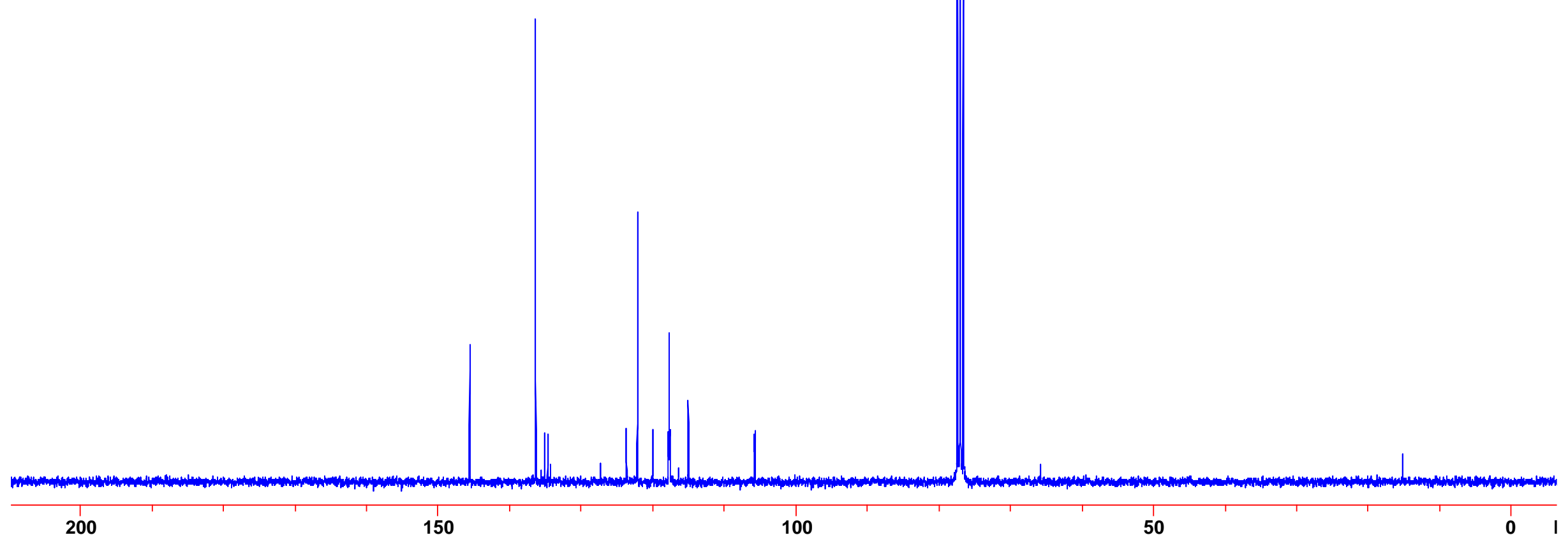




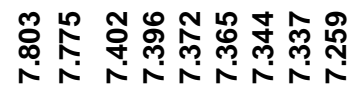

L)

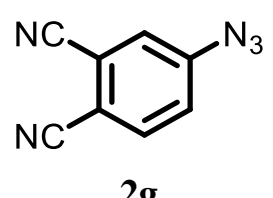

${ }^{1} \mathrm{H}$ NMR $\left(300 \mathrm{MHz}, \mathrm{CDCl}_{3}\right)$

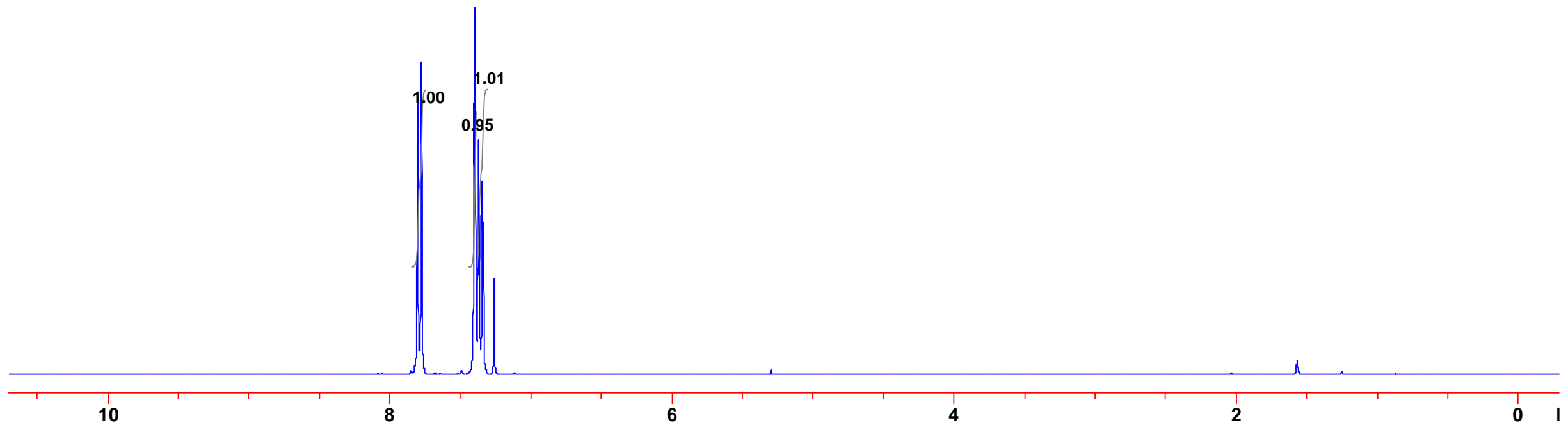



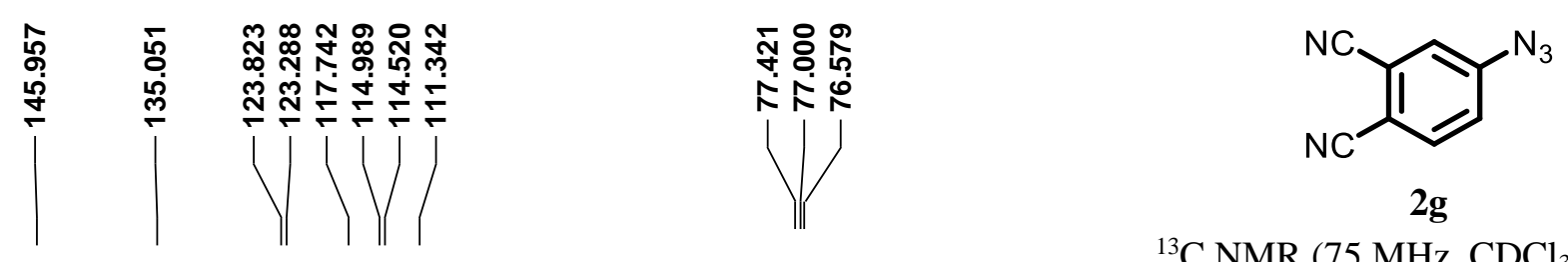

${ }^{13} \mathrm{C}$ NMR $\left(75 \mathrm{MHz}, \mathrm{CDCl}_{3}\right.$ )

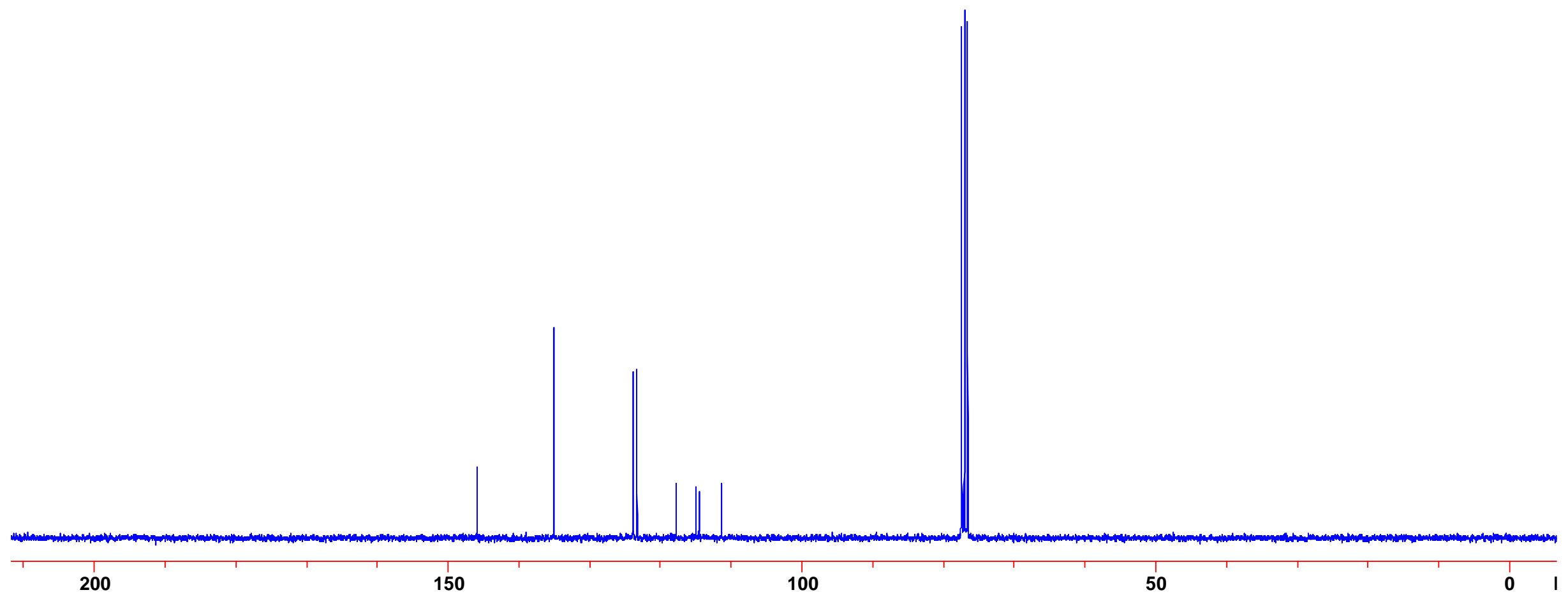




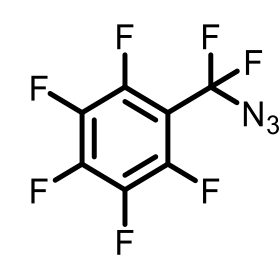

2h

${ }^{19} \mathrm{~F}$ NMR $\left(282 \mathrm{MHz}, \mathrm{CDCl}_{3}\right)$

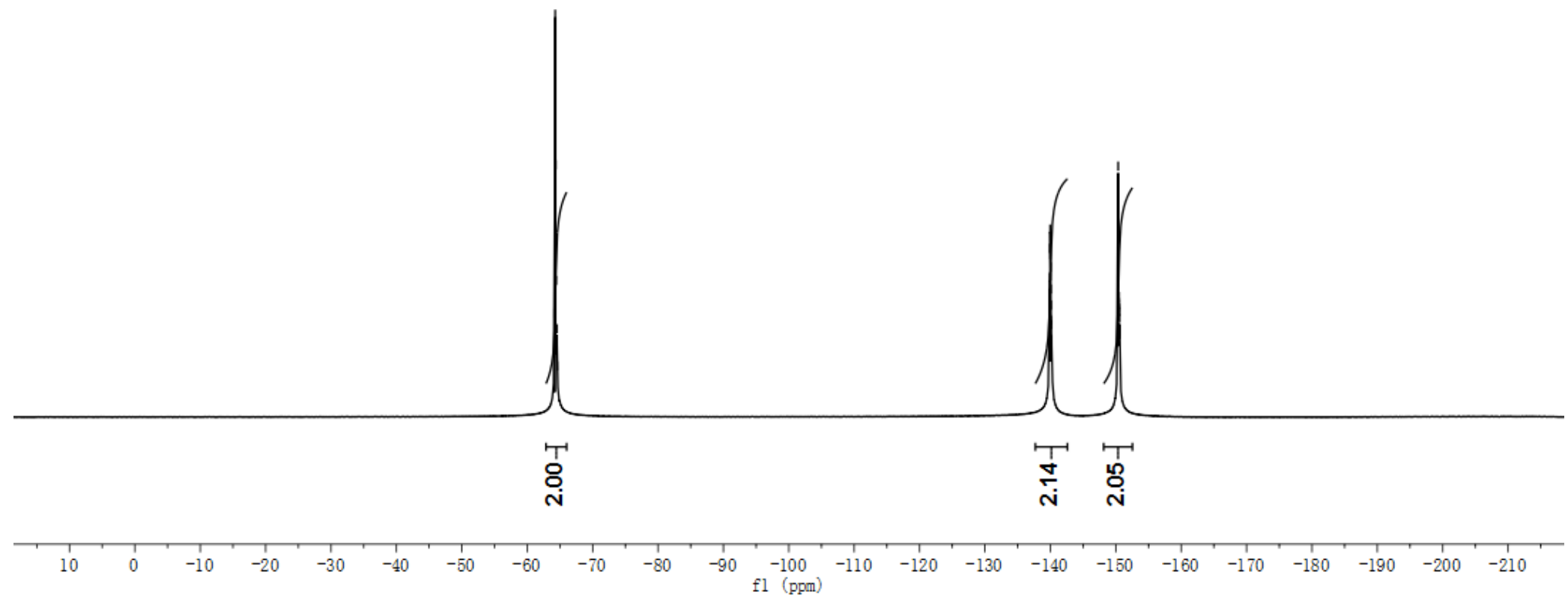

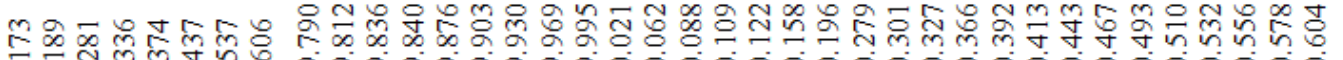

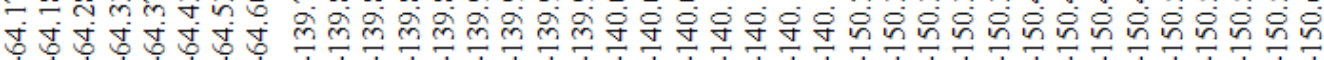



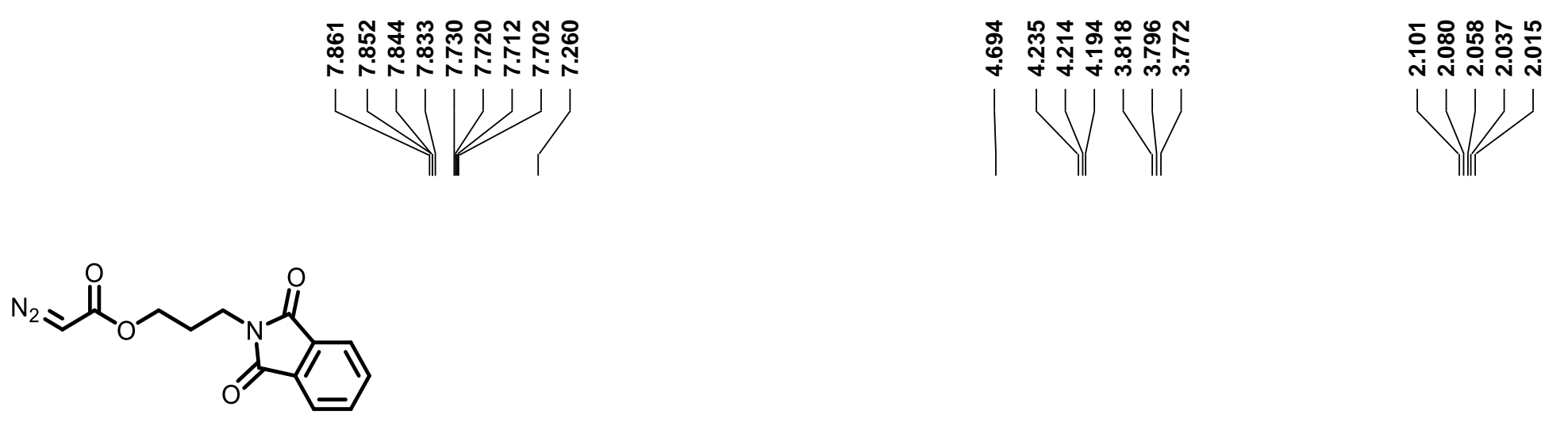

3b

${ }^{1} \mathrm{H}$ NMR $\left(300 \mathrm{MHz}, \mathrm{CDCl}_{3}\right)$

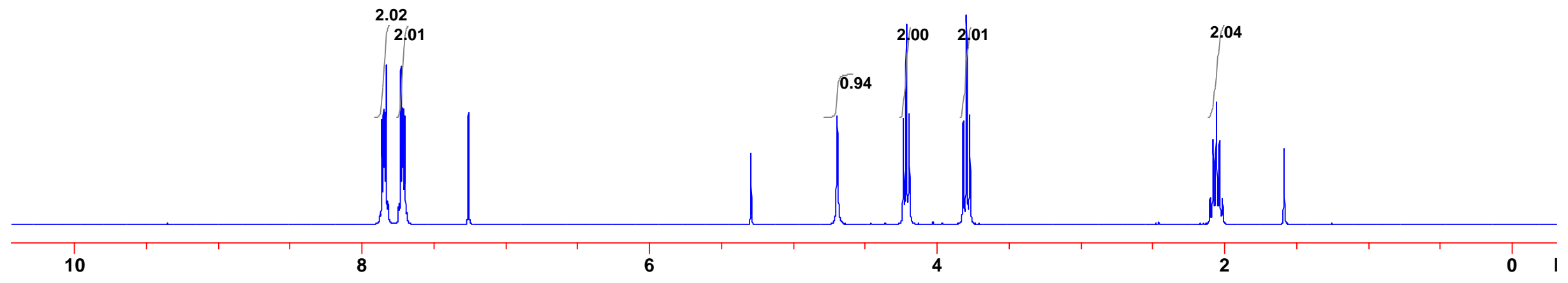



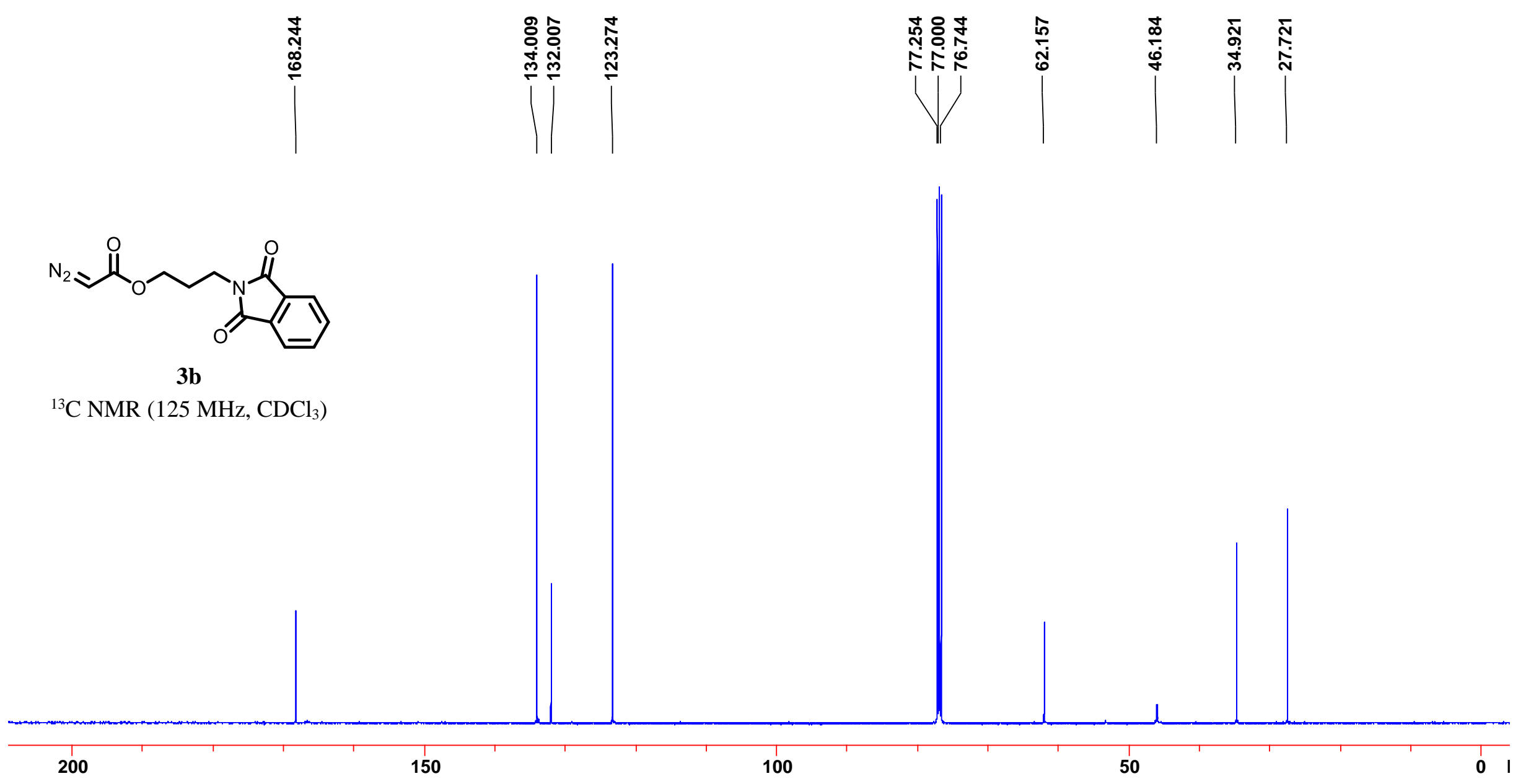


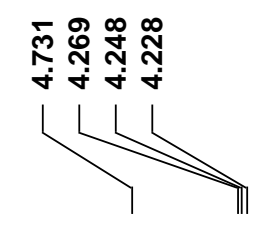

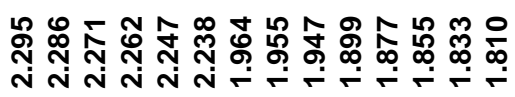
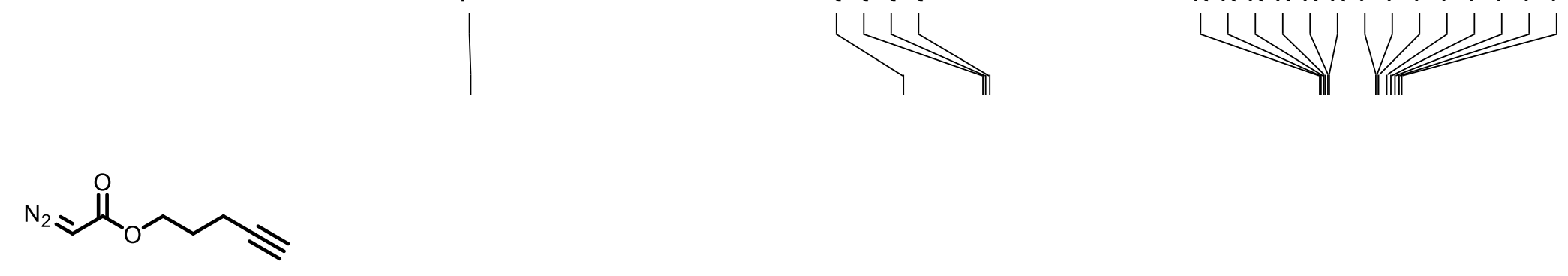

3d

${ }^{1} \mathrm{H}$ NMR (300 MHz, $\mathrm{CDCl}_{3}$ )

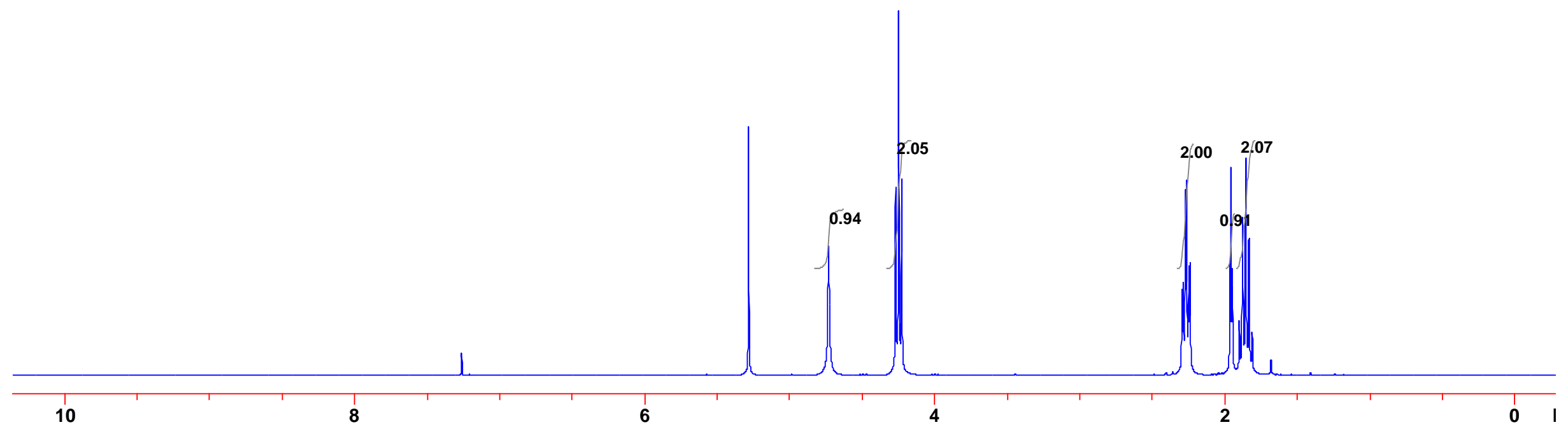




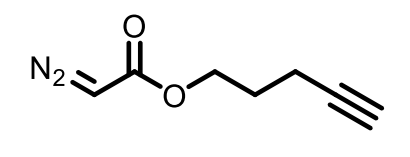

3d

${ }^{13} \mathrm{C}$ NMR (75 MHz, $\mathrm{CDCl}_{3}$ )

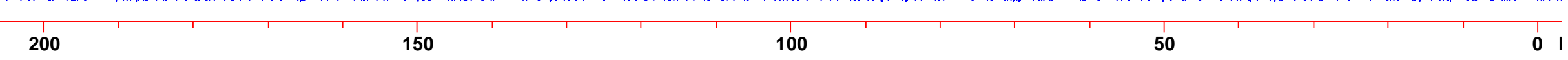




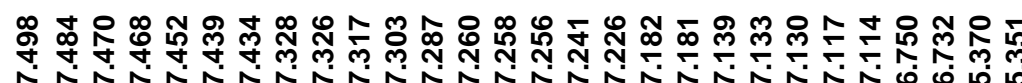

LLLLLLLU

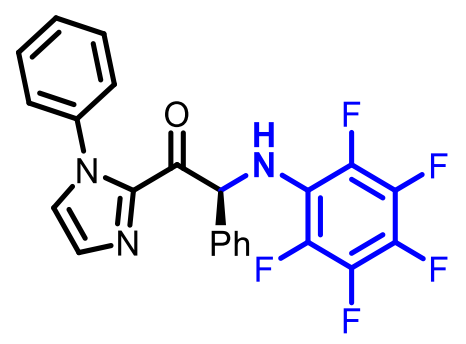

$4 a a$

${ }^{1} \mathrm{H}$ NMR (500 MHz, $\mathrm{CDCl}_{3}$ )

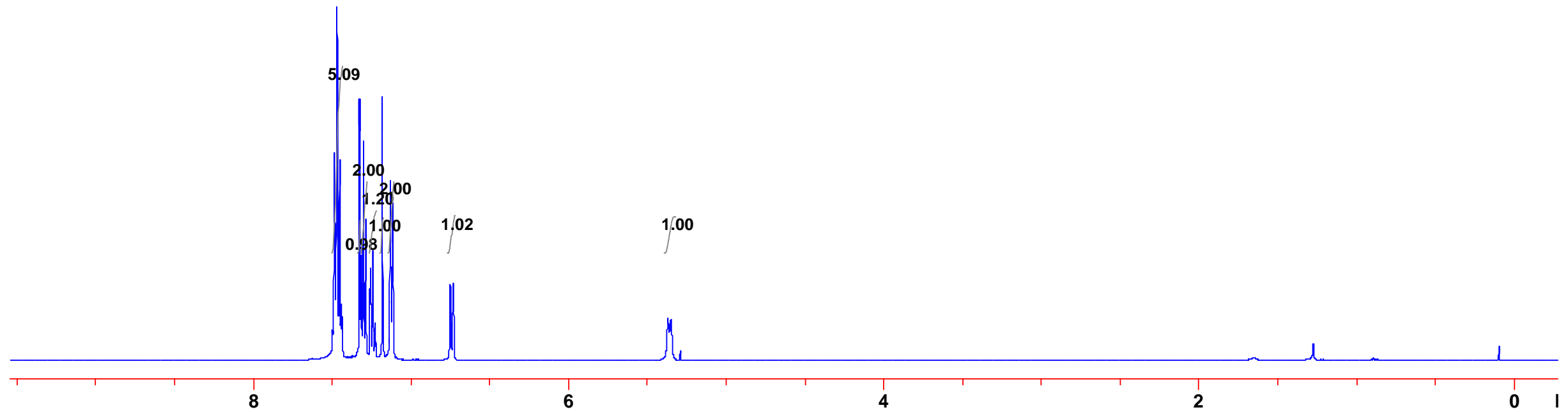




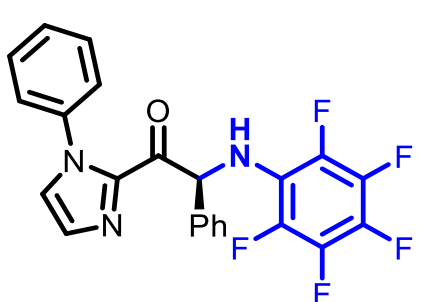

$4 a a$

${ }^{13} \mathrm{C}$ NMR (125 MHz, $\mathrm{CDCl}_{3}$ )

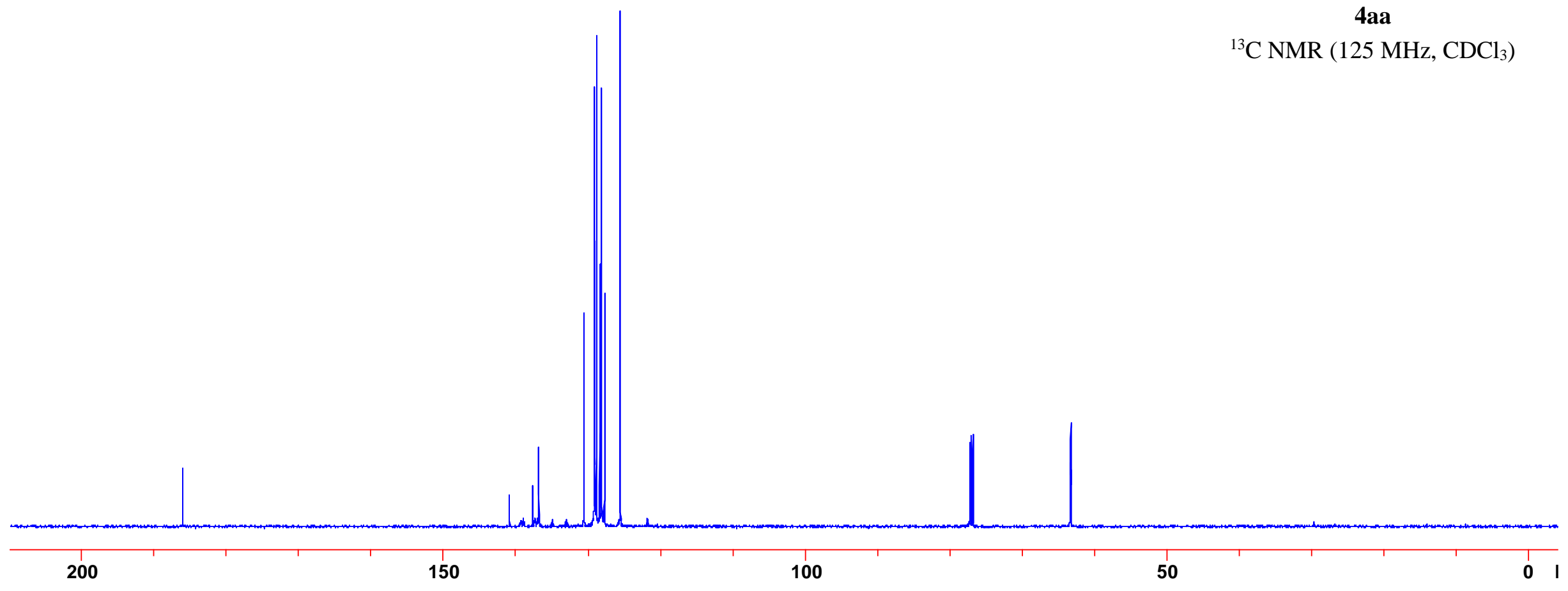


צr กิ

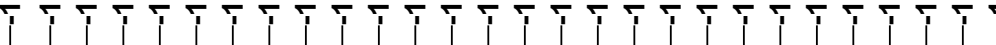

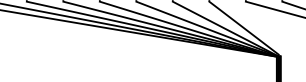

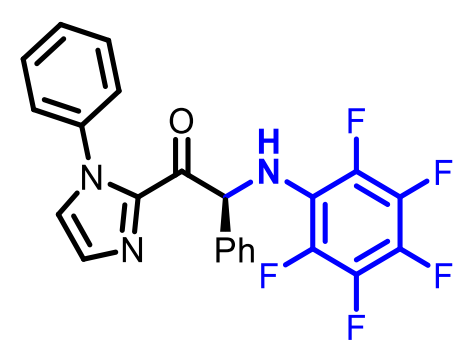

$4 a a$

${ }^{19} \mathrm{~F}$ NMR $\left(282 \mathrm{MHz}, \mathrm{CDCl}_{3}\right)$

2.18

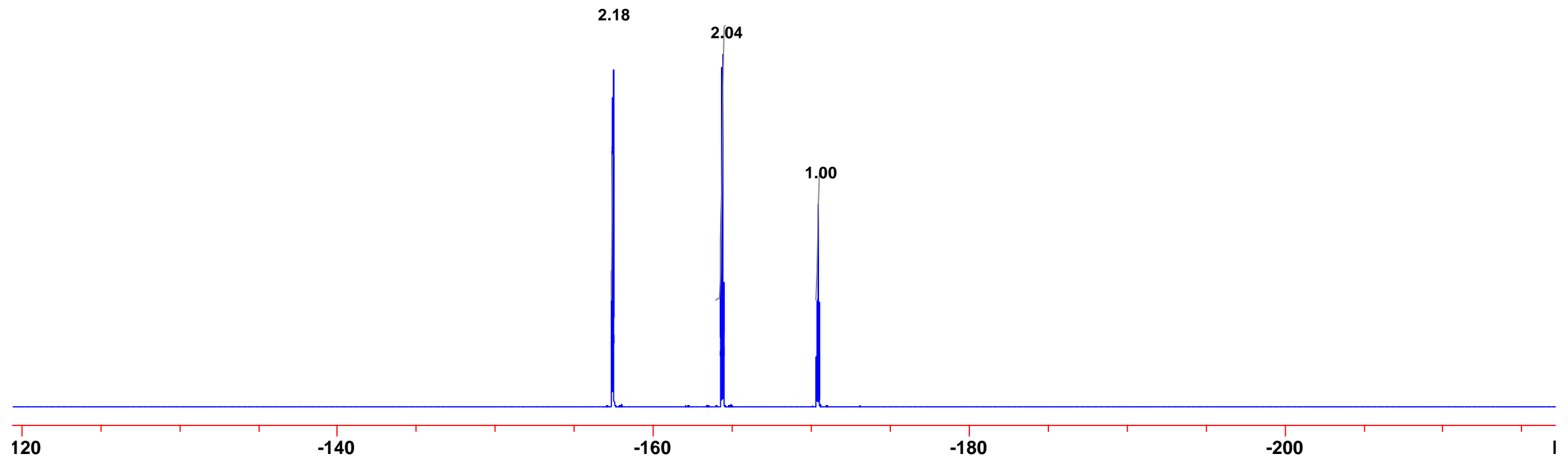




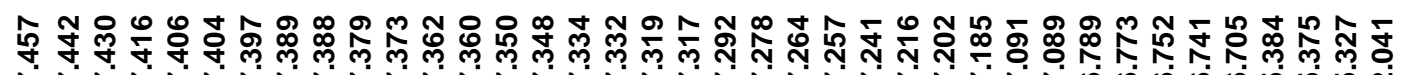

(1)

LLLLLLLLLLLULJJ」」」」」」」」」」」」」」」」」

$\pi$

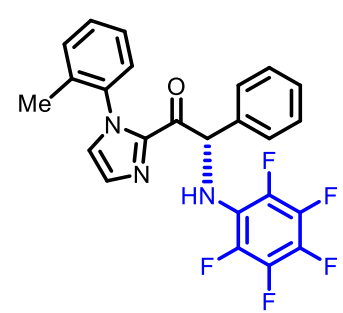

$4 \mathrm{ba}$

${ }^{1} \mathrm{H}$ NMR $\left(500 \mathrm{MHz}, \mathrm{CDCl}_{3}\right.$ )
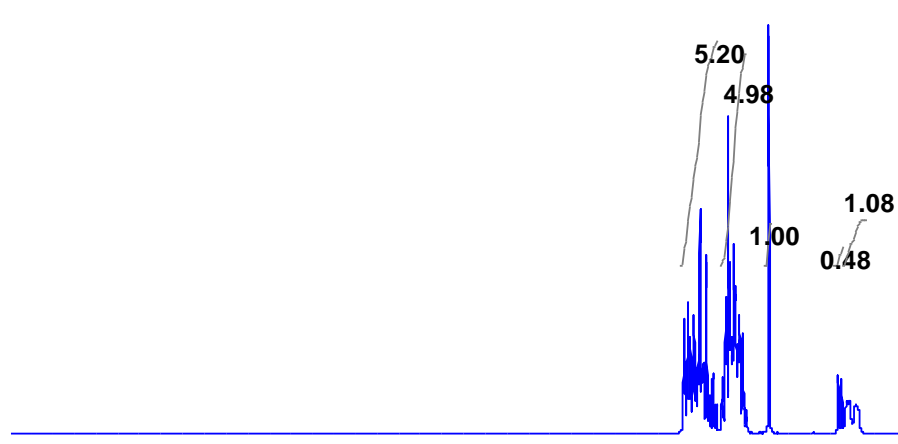

0.99 

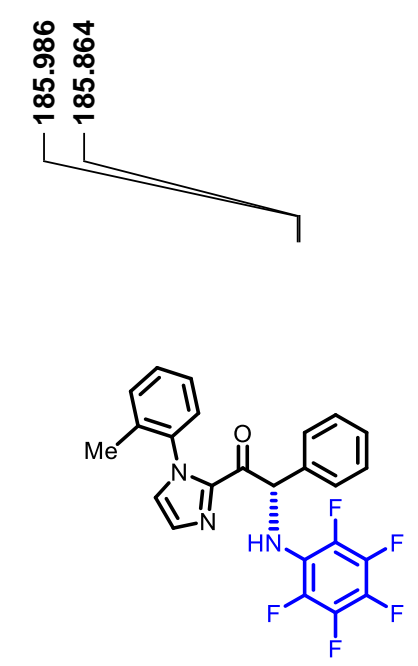

4 ba

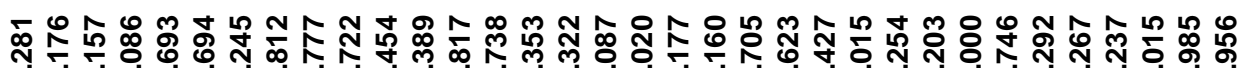

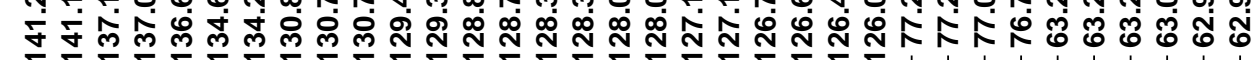

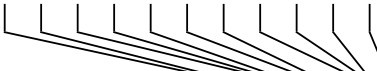

(ㄱ

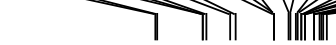

${ }^{13} \mathrm{C}$ NMR (125 MHz, $\left.\mathrm{CDCl}_{3}\right)$

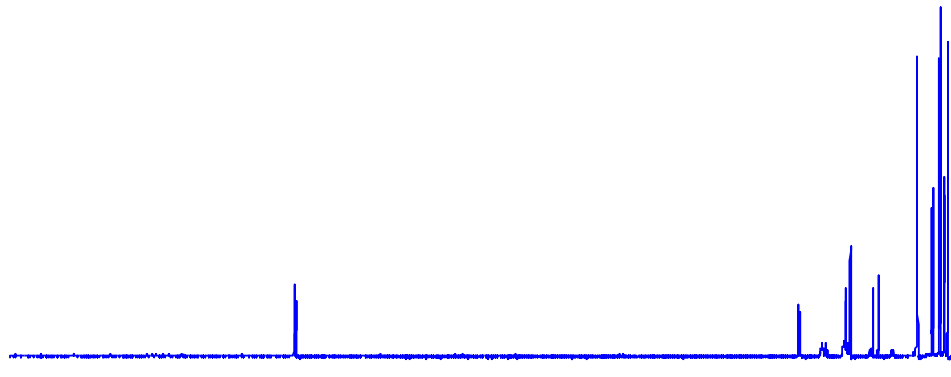

200

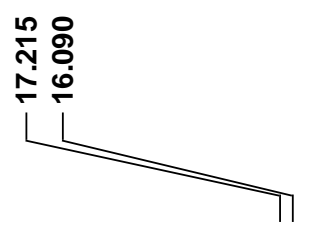



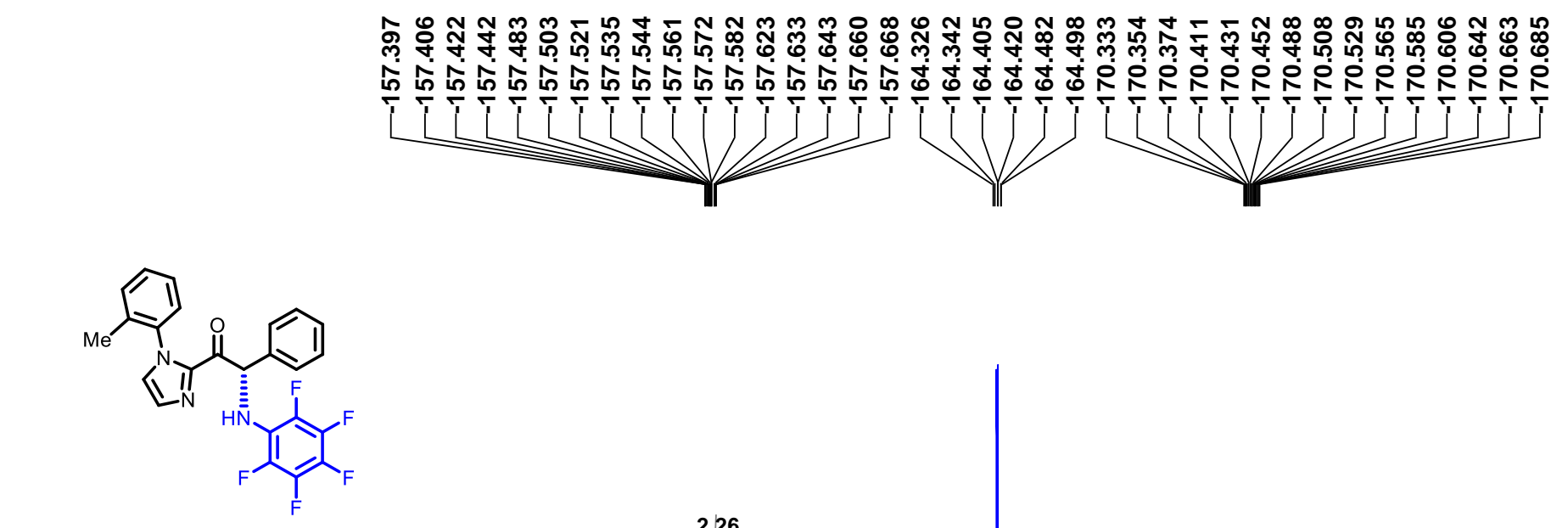

$4 \mathrm{ba}$

${ }^{19} \mathrm{~F}$ NMR (282 MHz, $\left.\mathrm{CDCl}_{3}\right)$

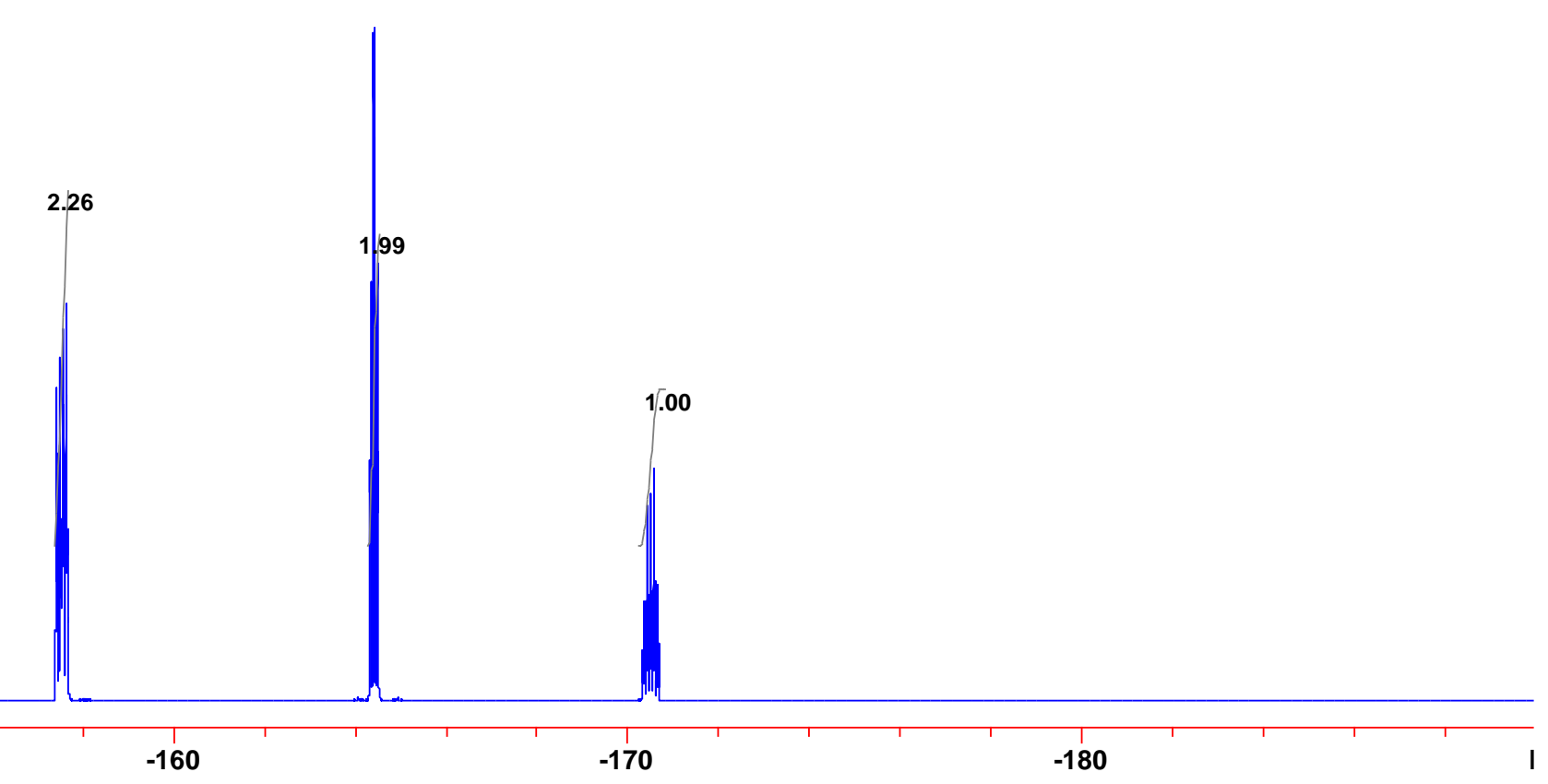




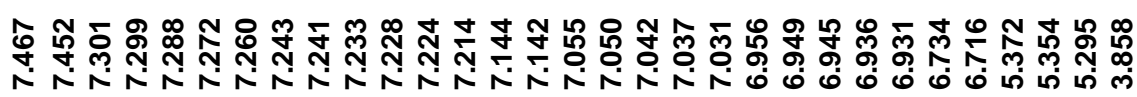

(IIII)
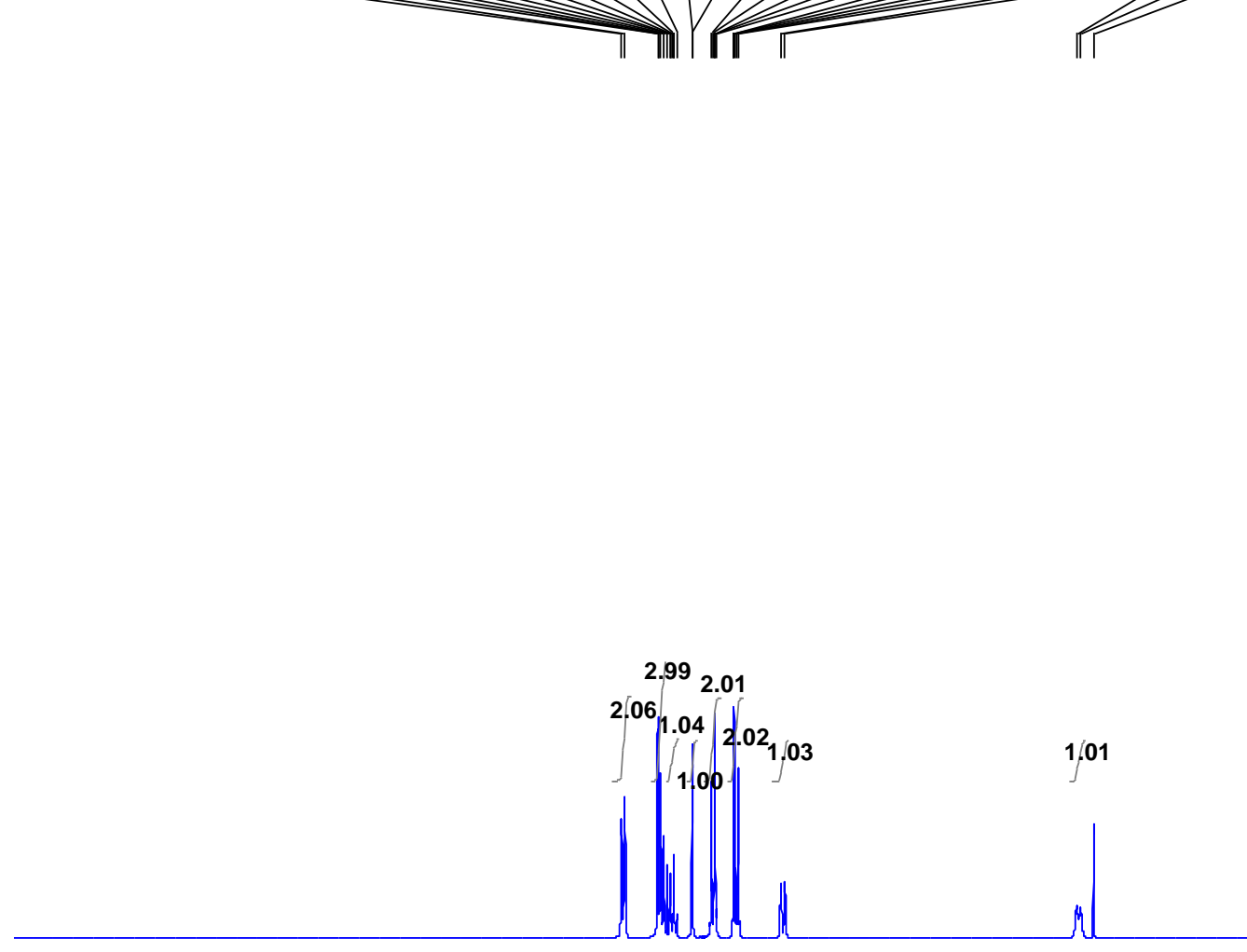

2.992 .01

2.06 1.04 2. 2.02 1.03

1.01

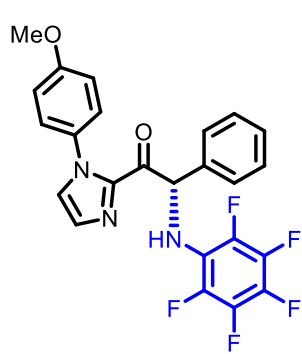

4ca

${ }^{1} \mathrm{H} \mathrm{NMR}\left(500 \mathrm{MHz}, \mathrm{CDCl}_{3}\right)$ 


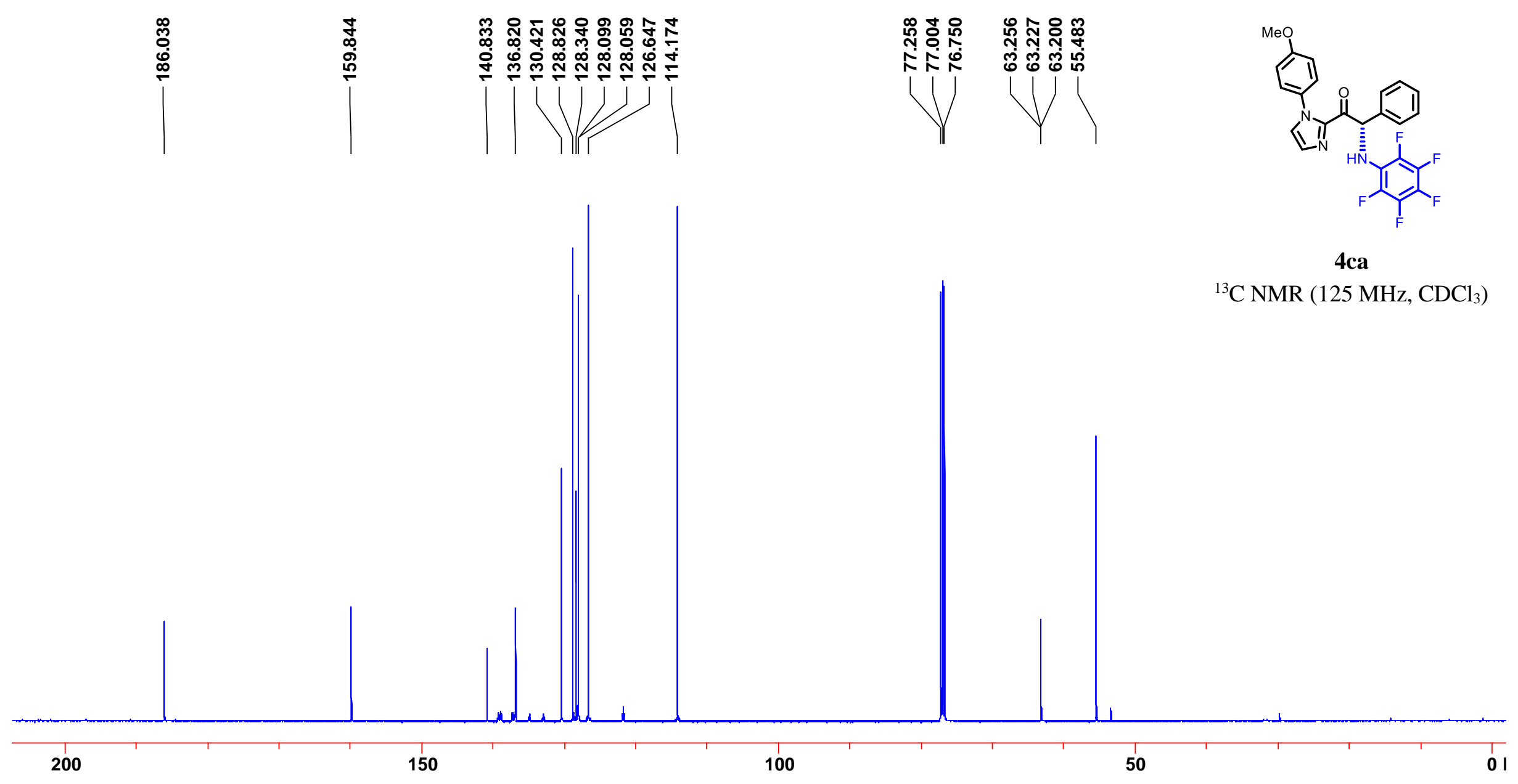




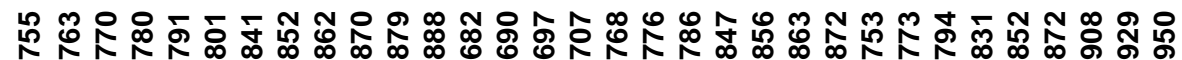

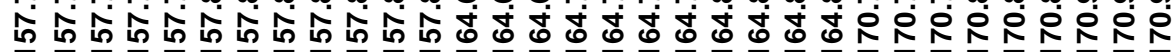

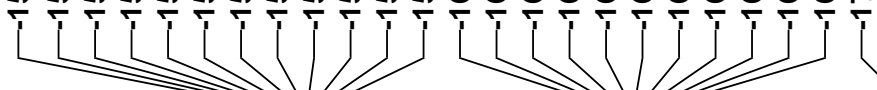
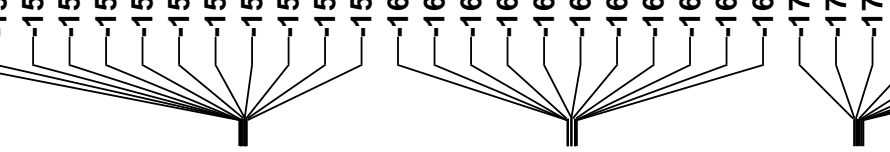

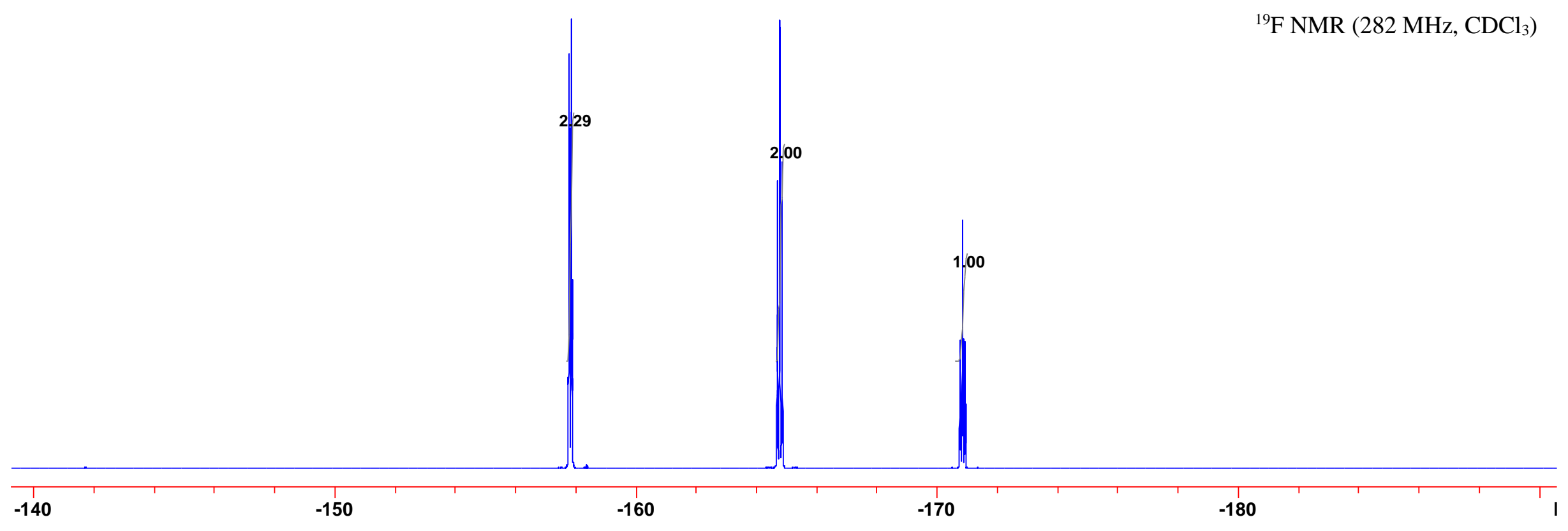

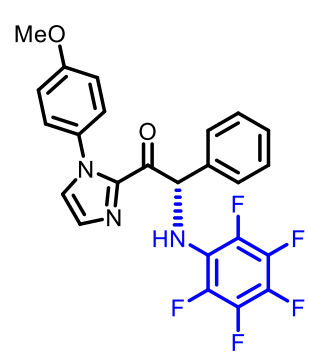

$4 \mathrm{ca}$

${ }^{19} \mathrm{~F}$ NMR $\left(282 \mathrm{MHz}, \mathrm{CDCl}_{3}\right)$ 

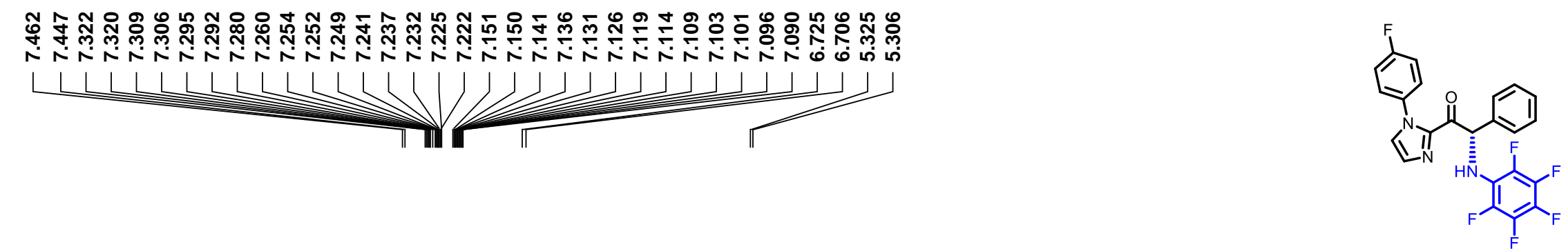

4da

${ }^{1} \mathrm{H}$ NMR (500 MHz, $\mathrm{CDCl}_{3}$ )

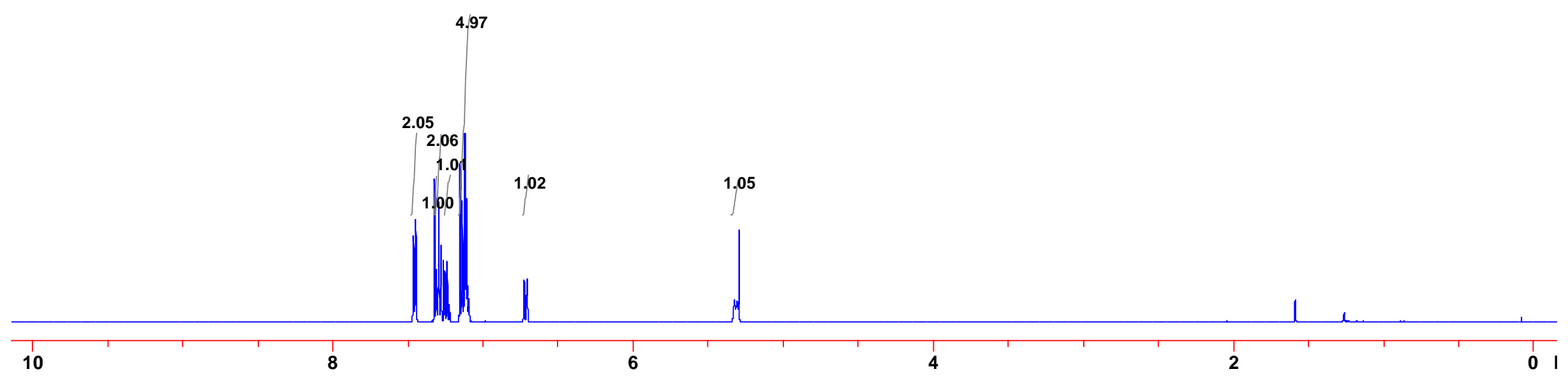




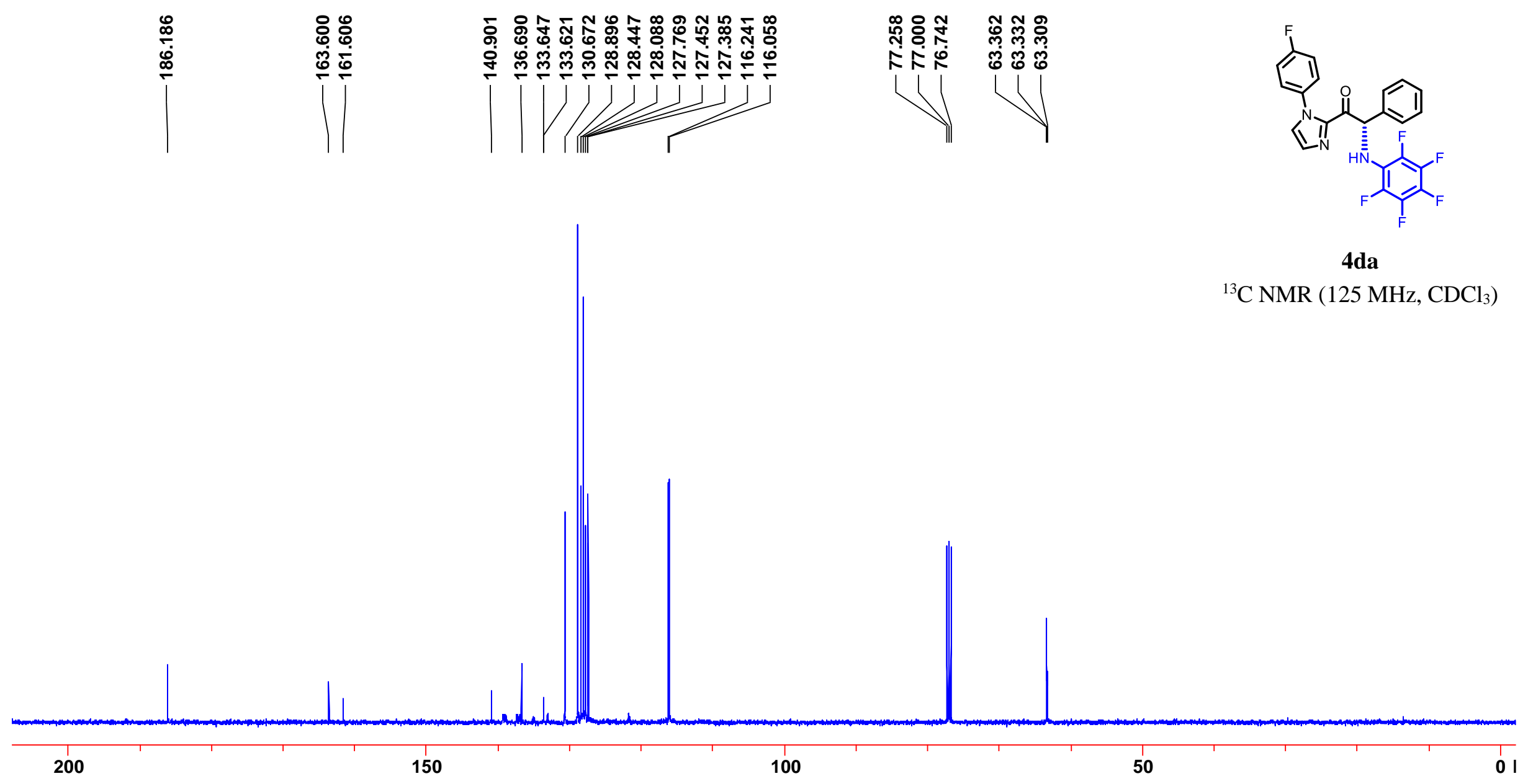




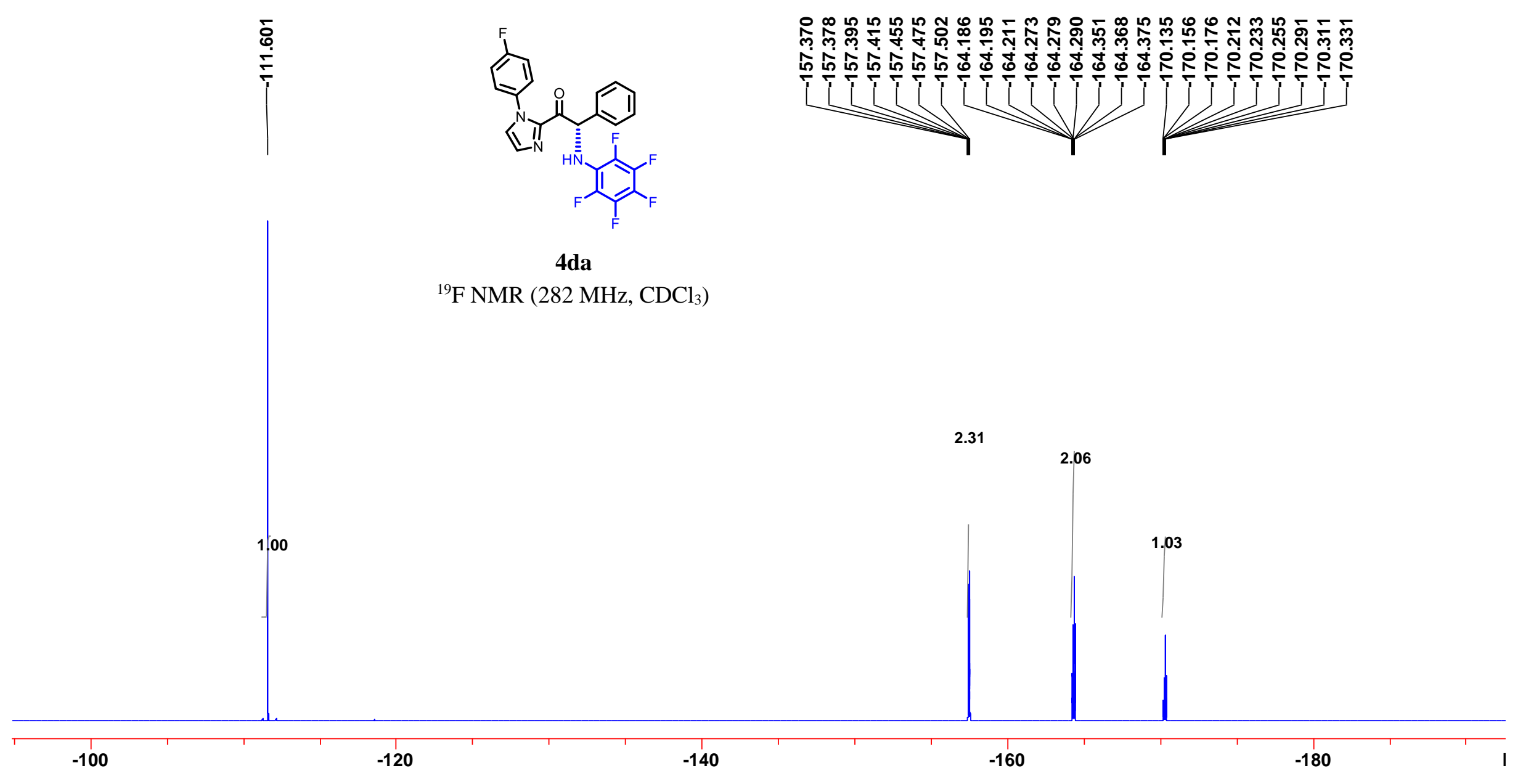




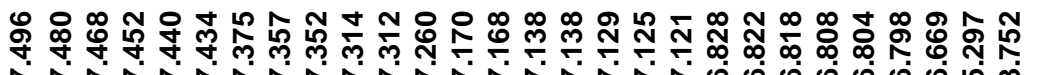

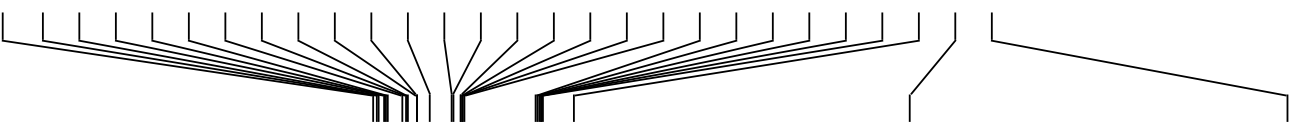

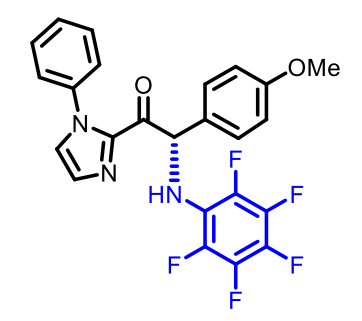

4 ea

${ }^{1} \mathrm{H}$ NMR $\left(500 \mathrm{MHz}, \mathrm{CDCl}_{3}\right)$

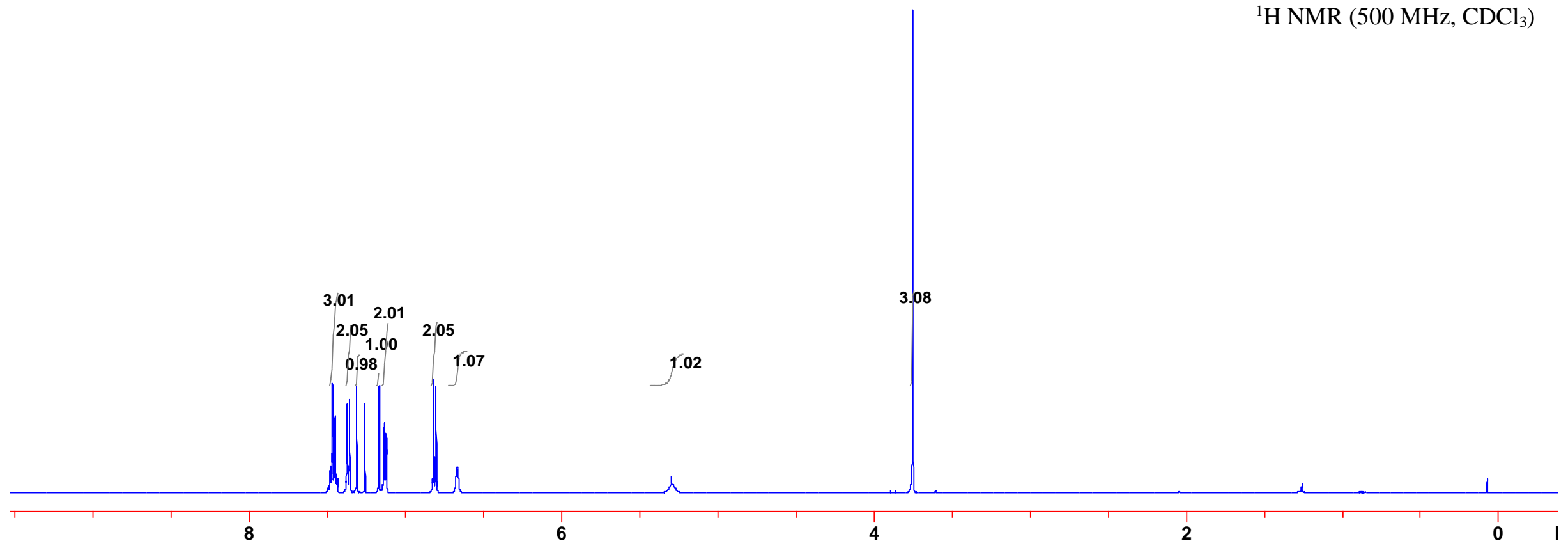



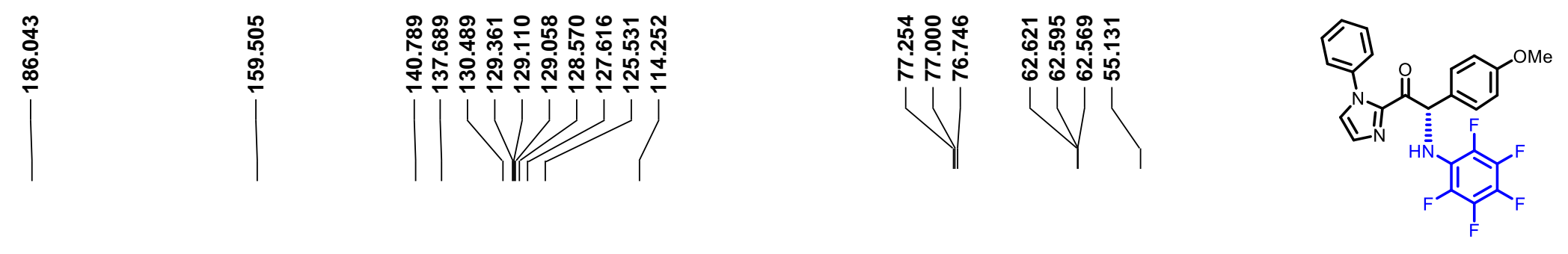

4ea

${ }^{13} \mathrm{C}$ NMR $\left(125 \mathrm{MHz}, \mathrm{CDCl}_{3}\right)$

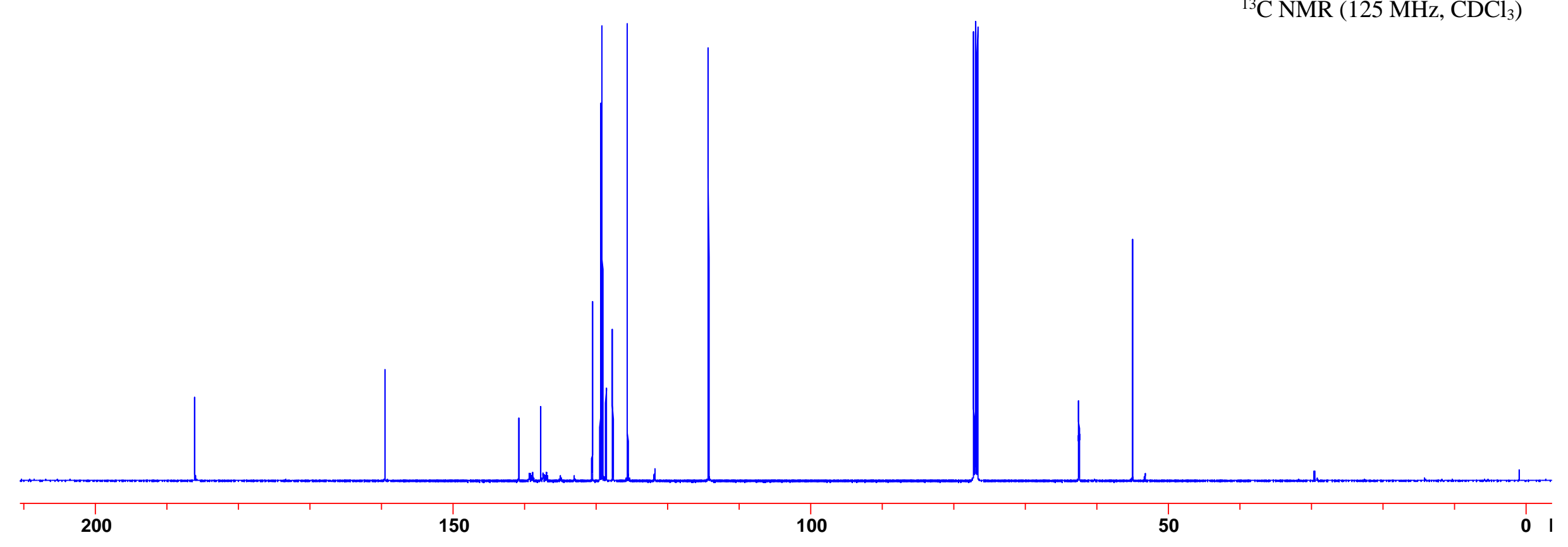



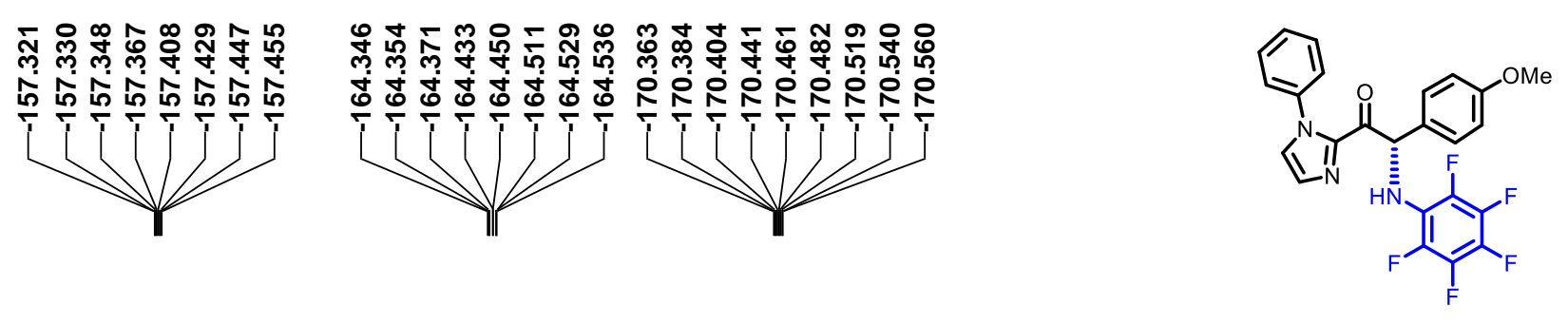

$4 \mathrm{ea}$

${ }^{19} \mathrm{~F}$ NMR (282 MHz, $\left.\mathrm{CDCl}_{3}\right)$

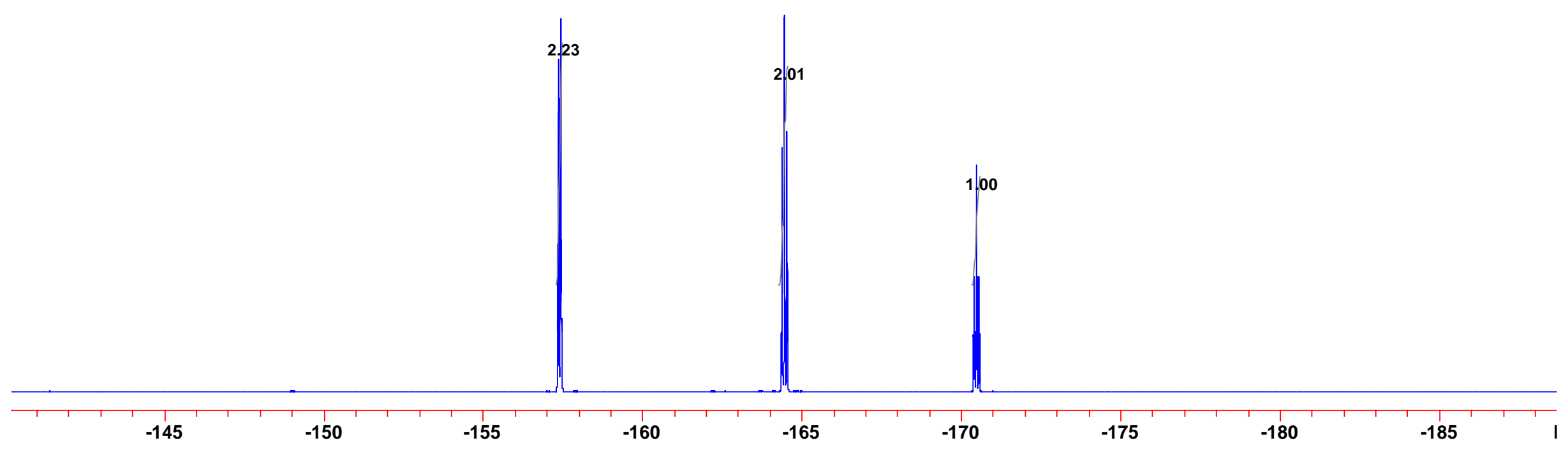




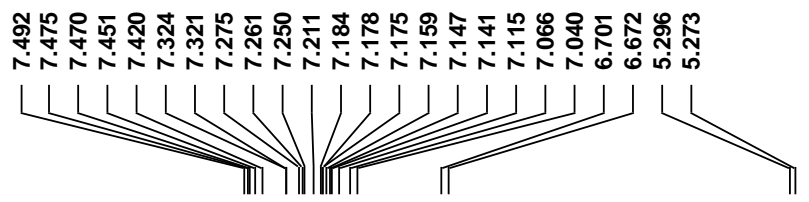

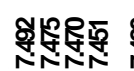

盤

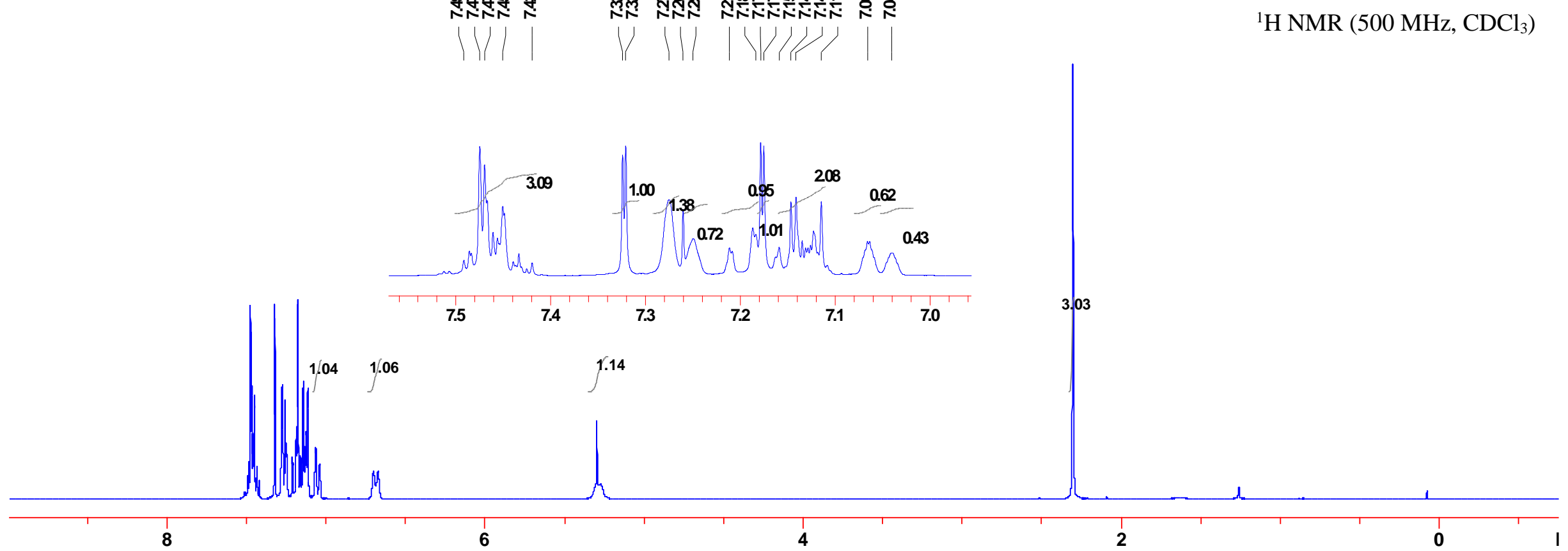

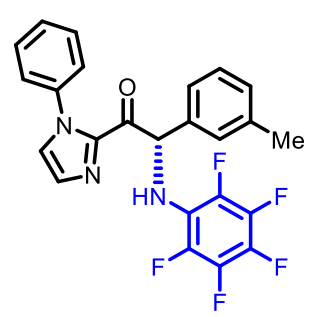

$4 f a$

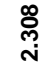

1 

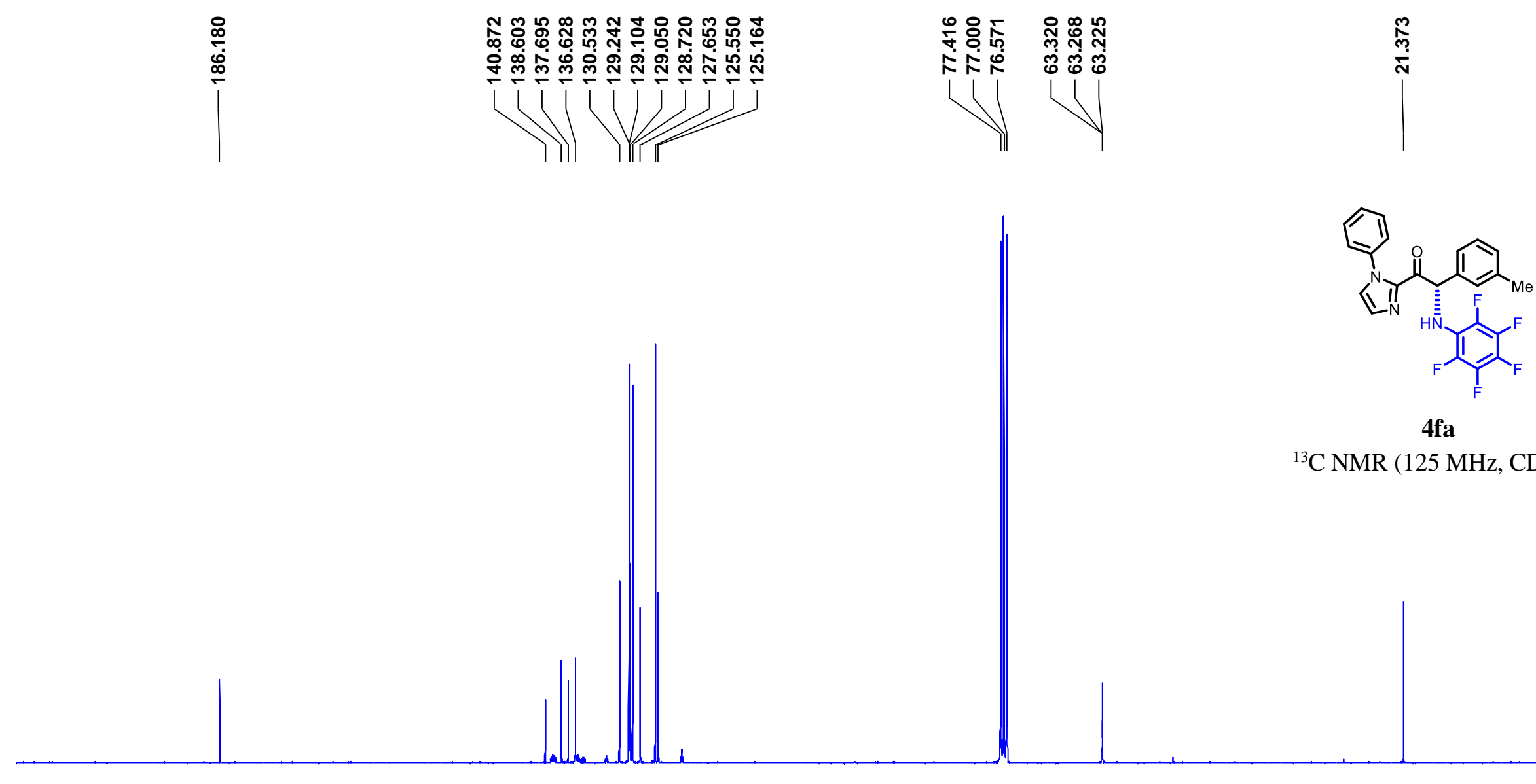

4 a

${ }^{13} \mathrm{C}$ NMR (125 MHz, $\left.\mathrm{CDCl}_{3}\right)$ 

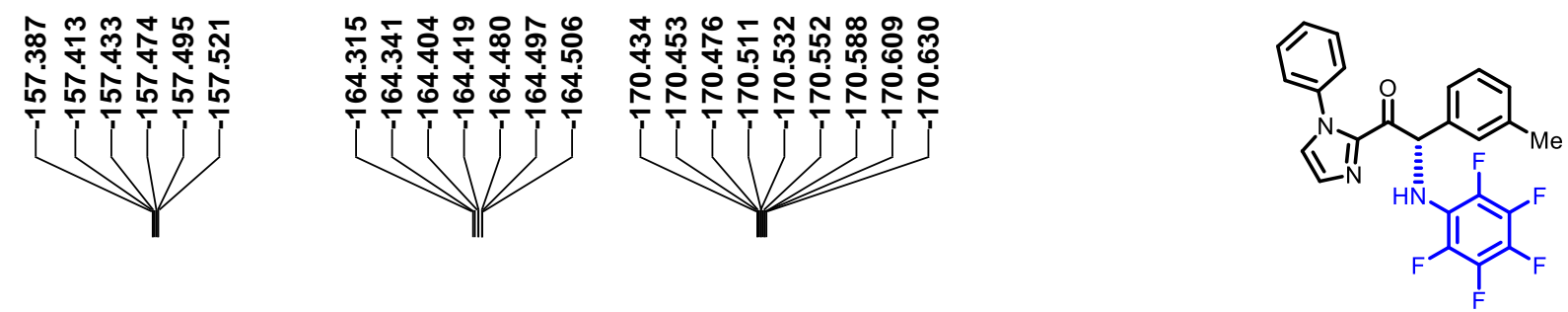

$4 \mathrm{fa}$

${ }^{19} \mathrm{~F}$ NMR (282 MHz, $\mathrm{CDCl}_{3}$ )

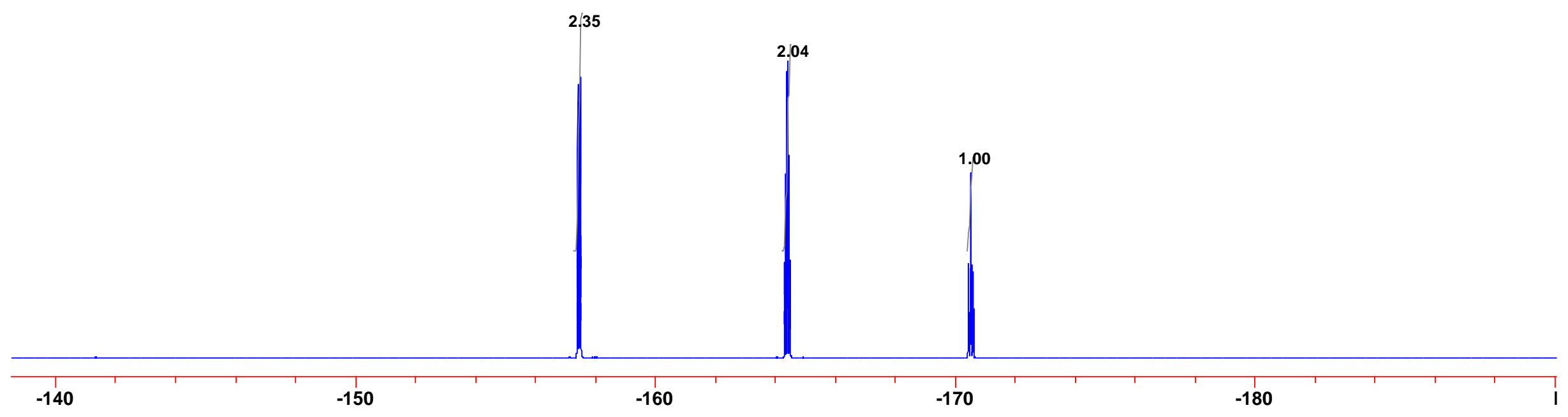



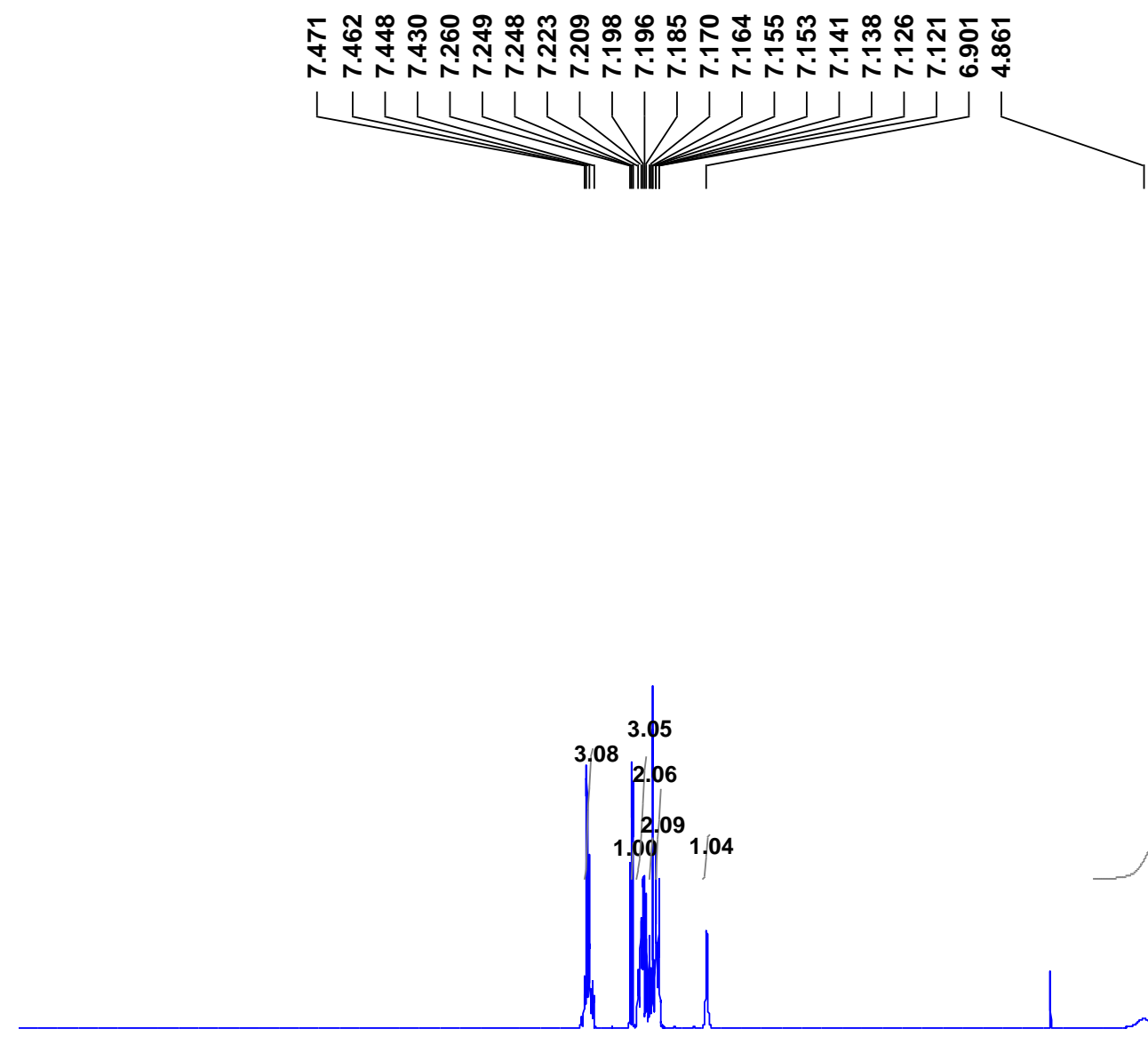

10

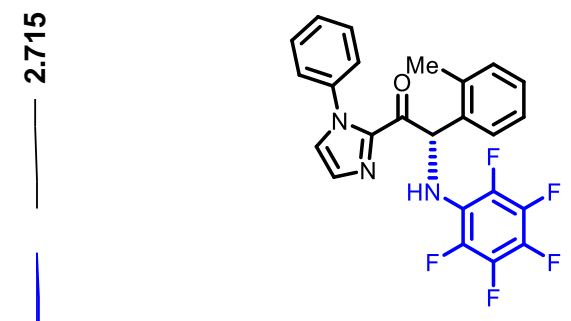

4 ga

${ }^{1} \mathrm{H}$ NMR (500 MHz, $\mathrm{CDCl}_{3}$ ) 


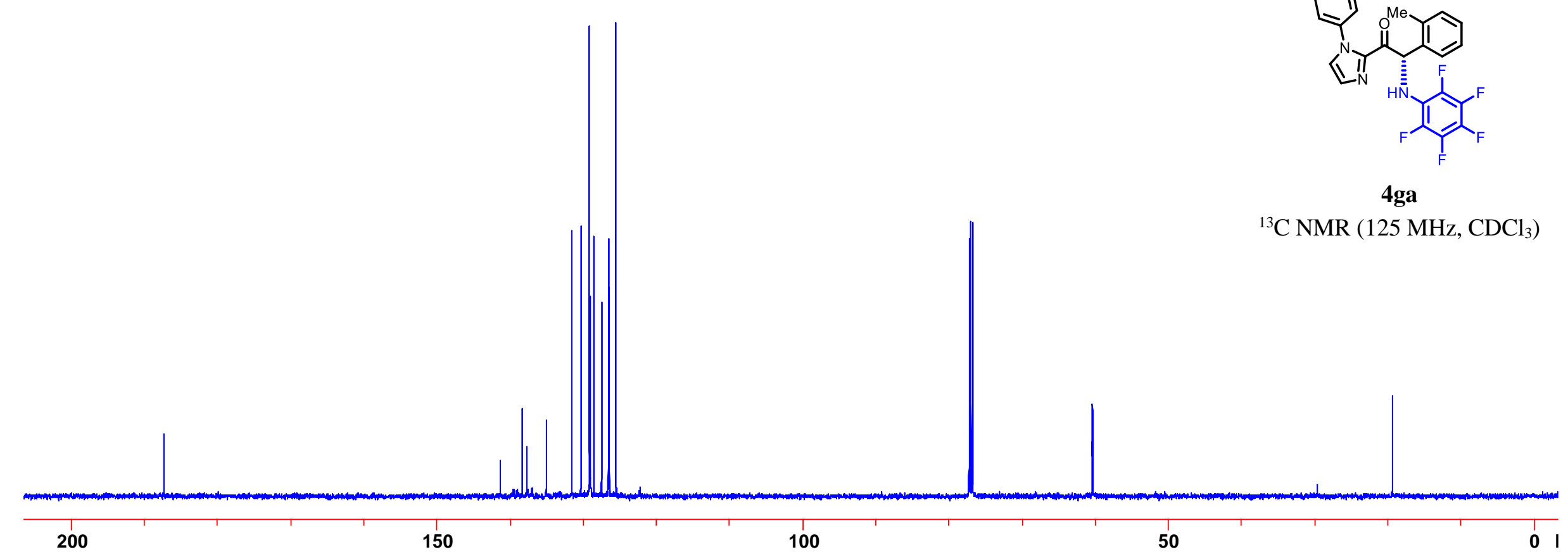



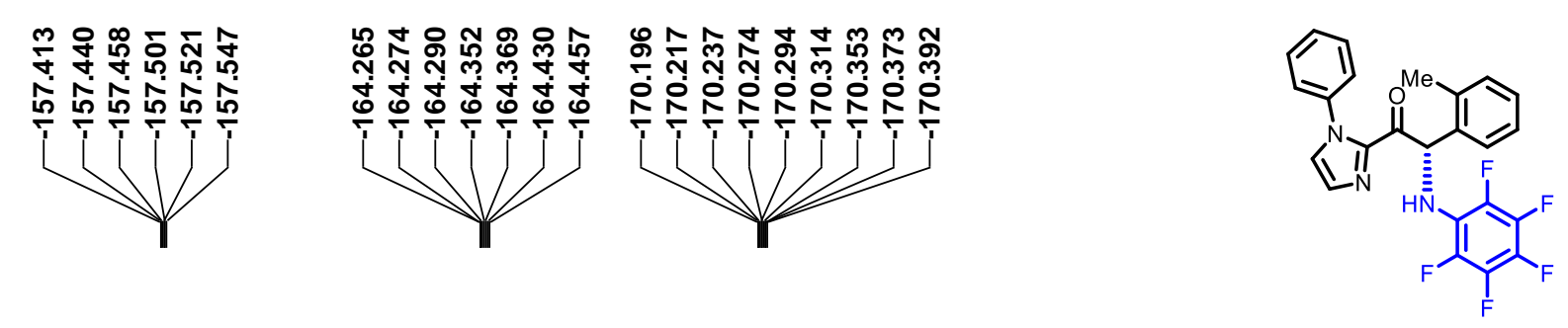

4ga

${ }^{19} \mathrm{~F}$ NMR (282 MHz, $\mathrm{CDCl}_{3}$ )

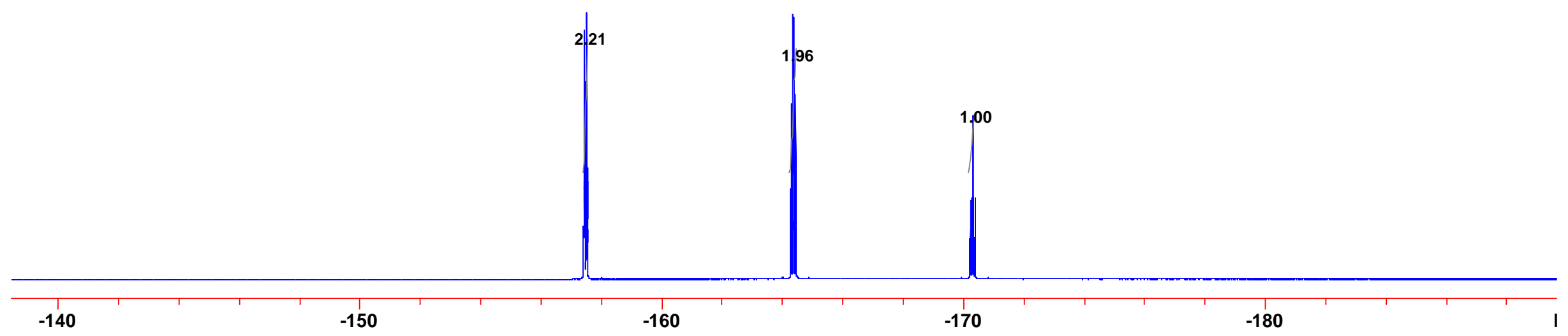




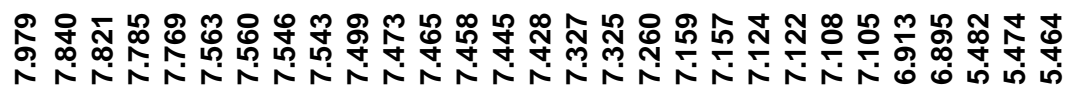

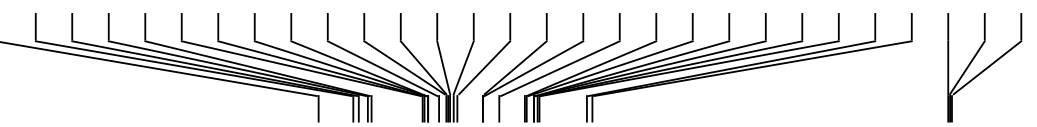

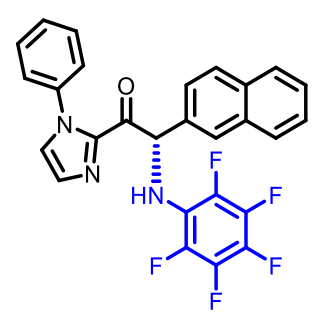

4ha

${ }^{1} \mathrm{H}$ NMR $\left(500 \mathrm{MHz}, \mathrm{CDCl}_{3}\right)$

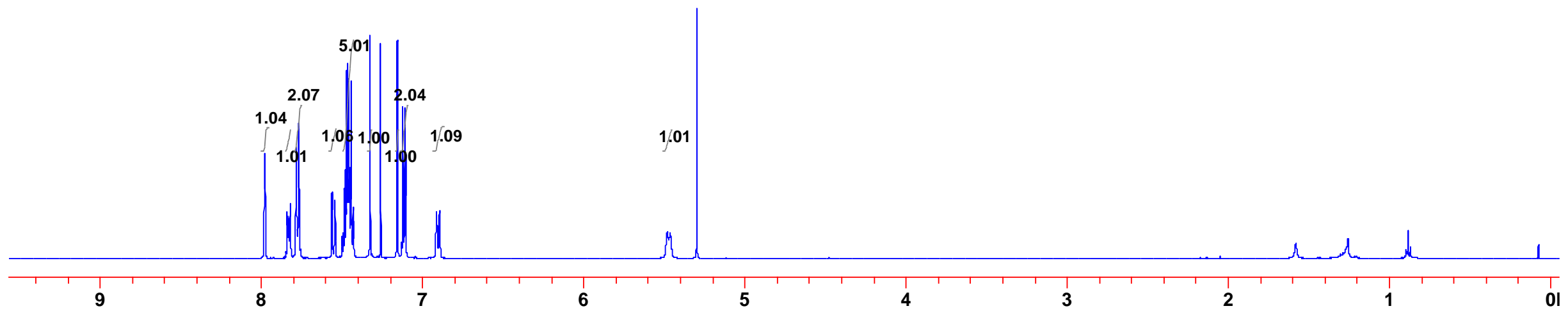




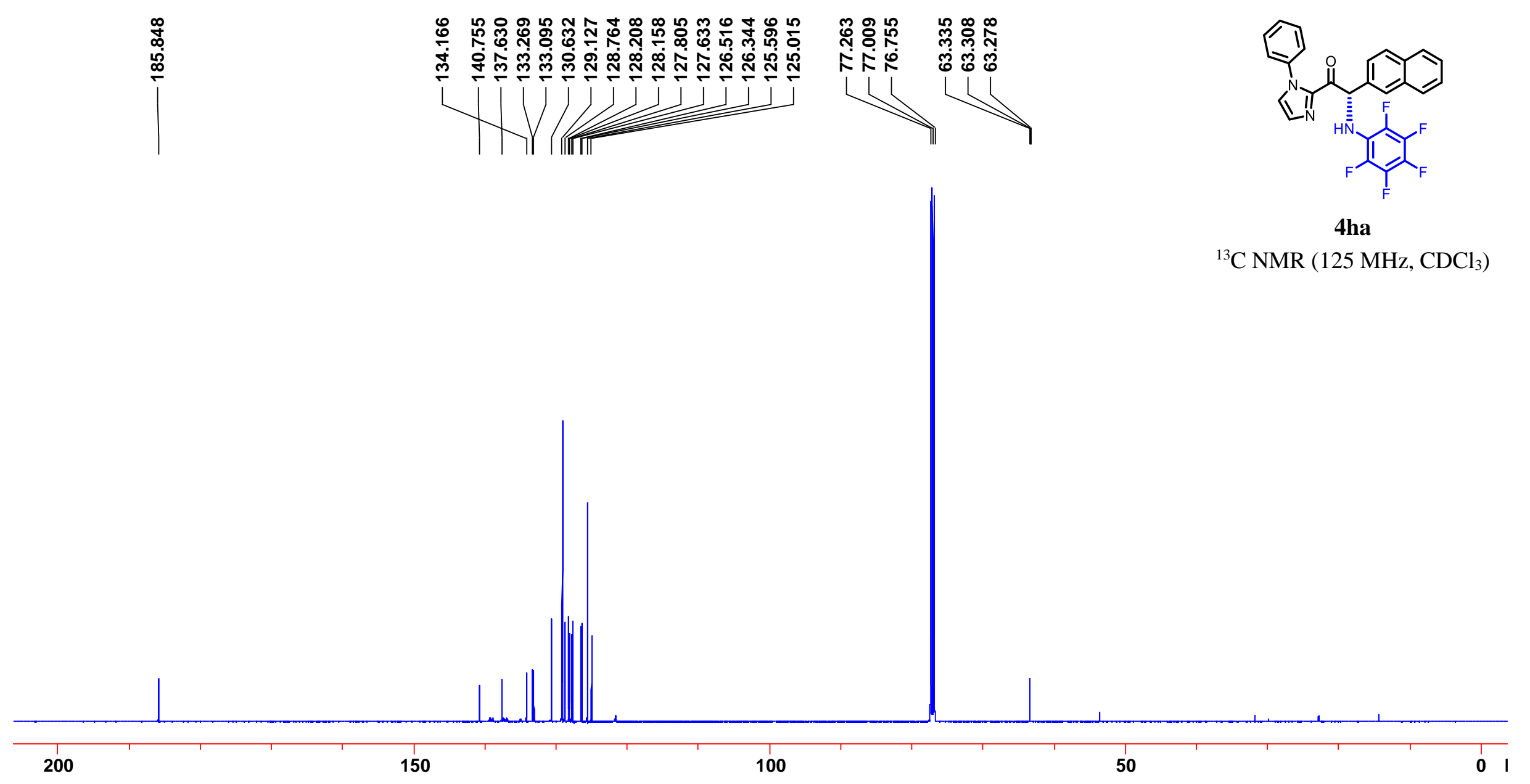



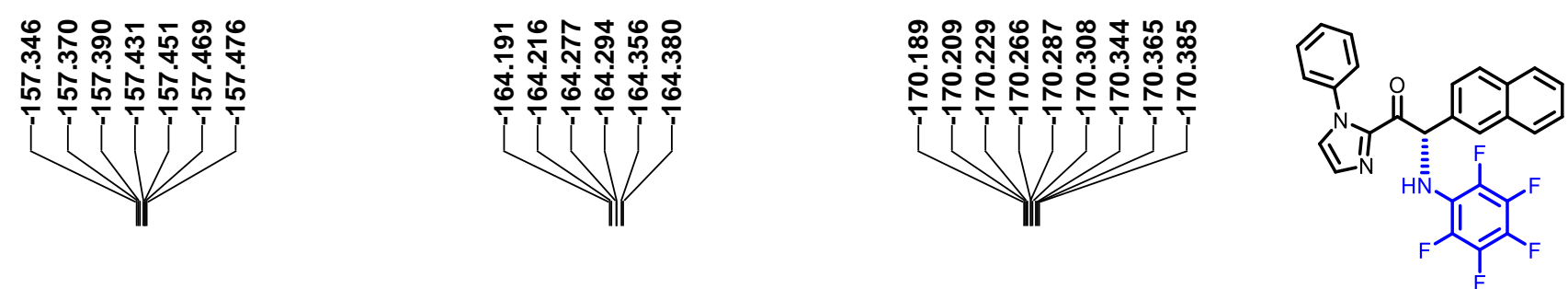

4ha

${ }^{19} \mathrm{~F}$ NMR (282 MHz, $\mathrm{CDCl}_{3}$ )

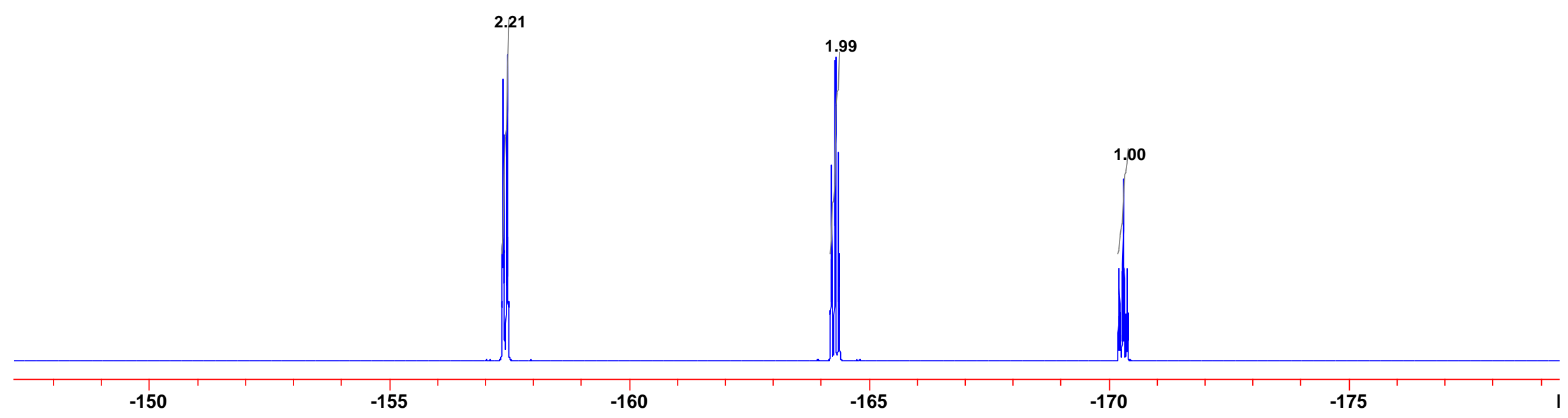




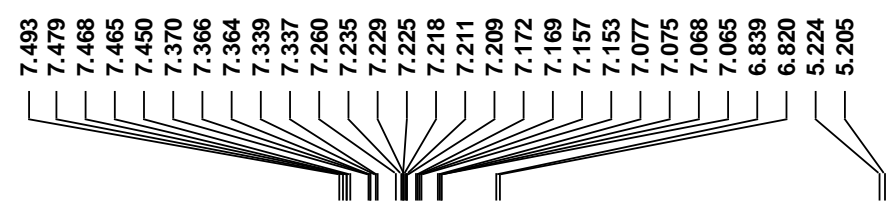

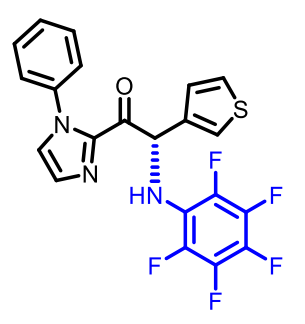

4ia

${ }^{1} \mathrm{H}$ NMR $\left(500 \mathrm{MHz}, \mathrm{CDCl}_{3}\right.$ )

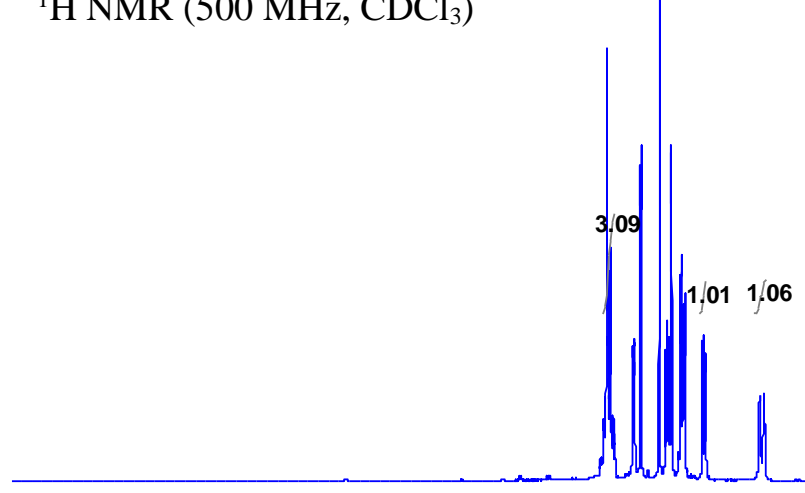

8

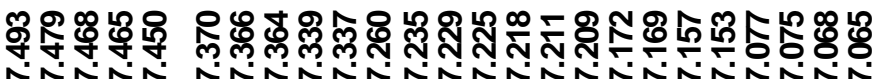

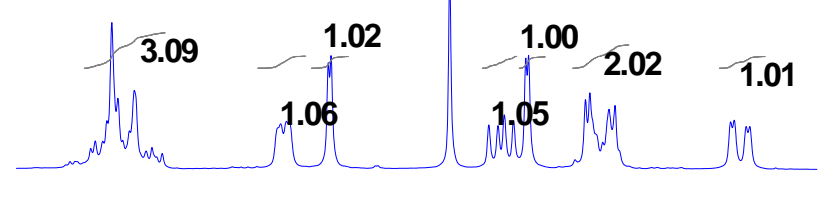

1.03

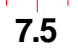

7.4

7.3

7.2

7.1

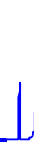




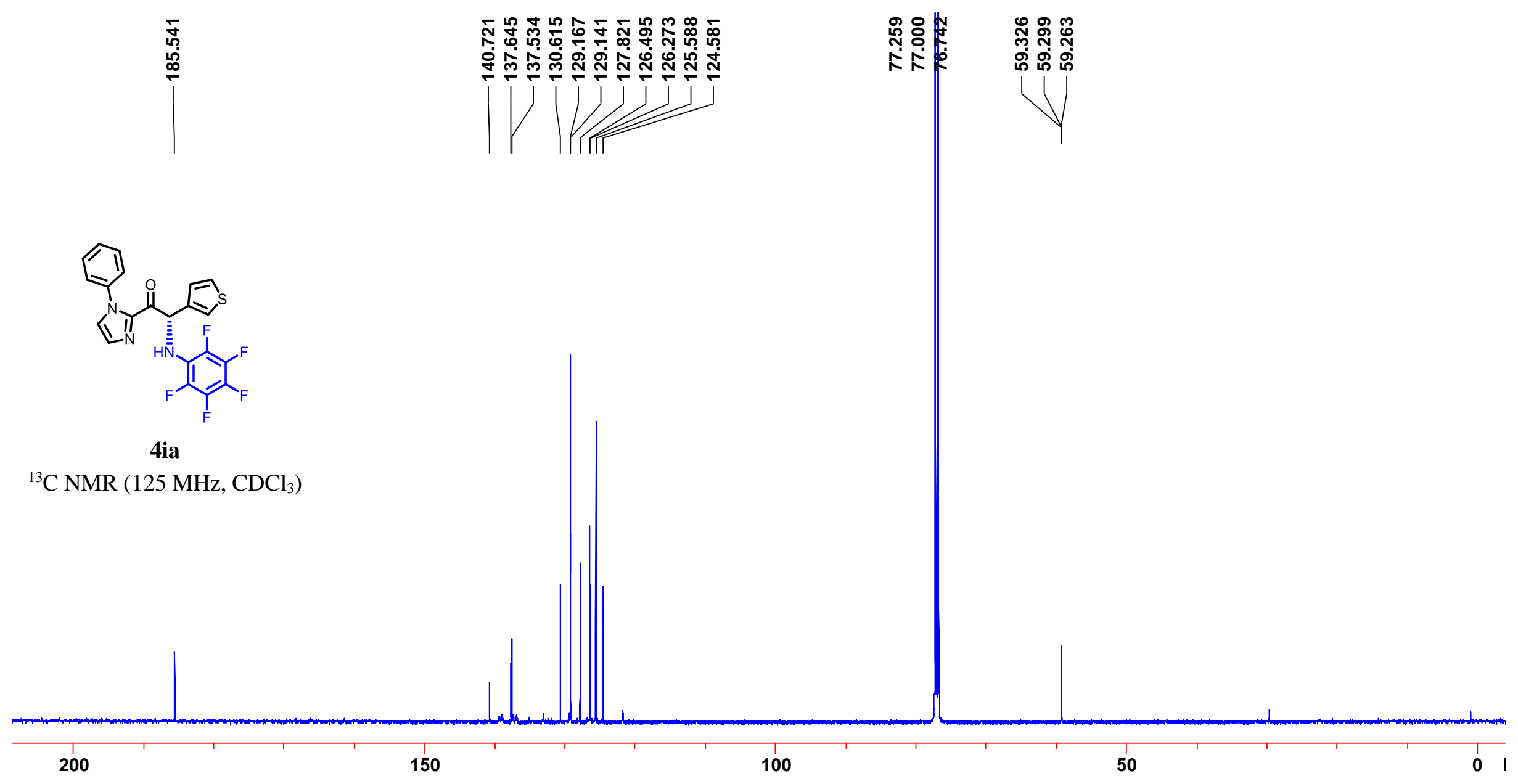



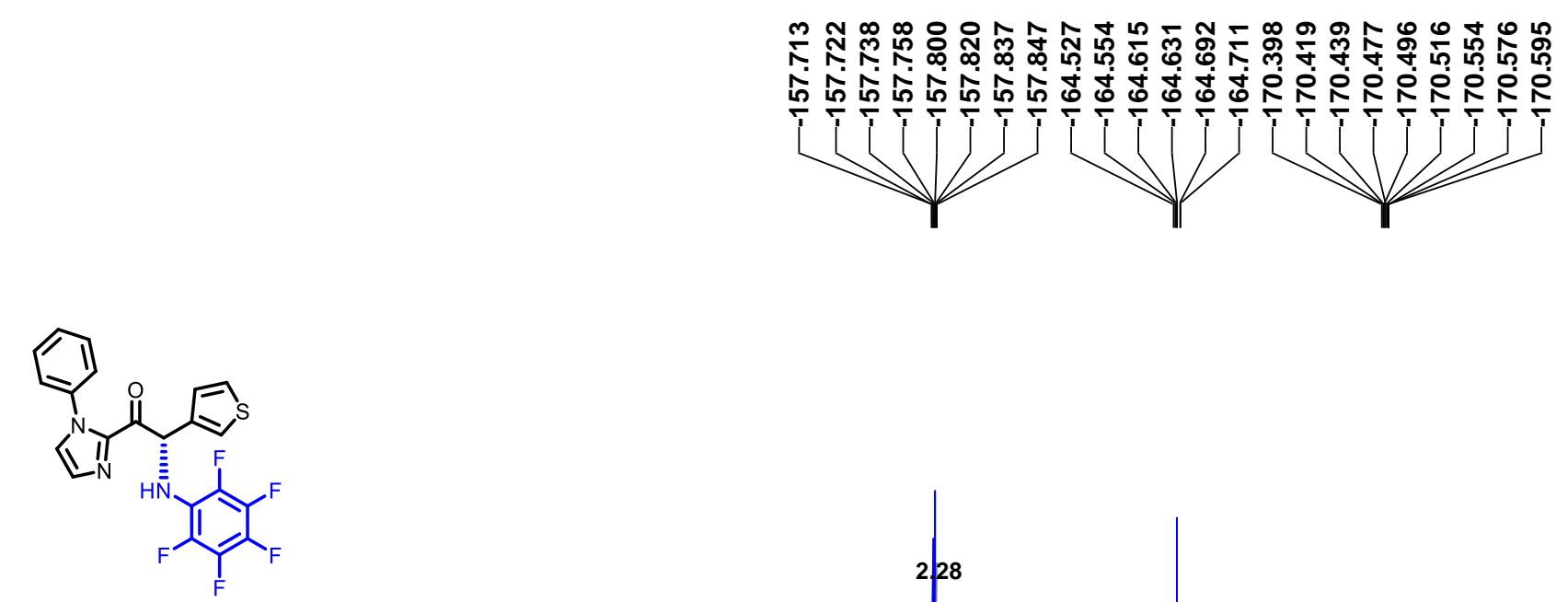

4ia

${ }^{19} \mathrm{~F}$ NMR (282 MHz, $\mathrm{CDCl}_{3}$ )

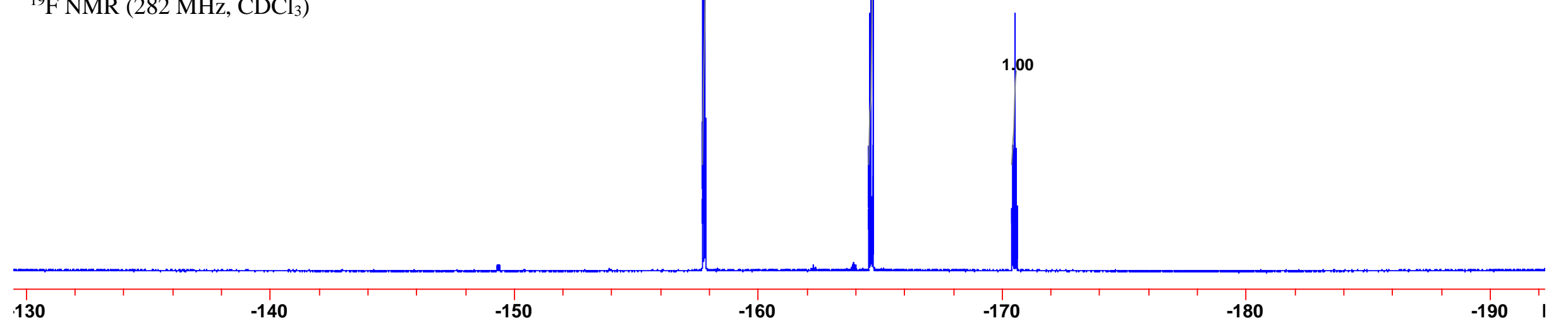




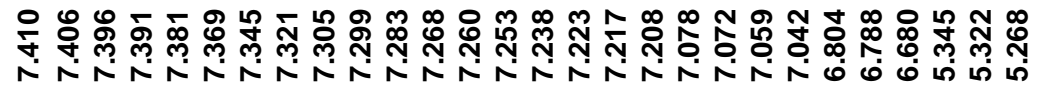

$\ldots$
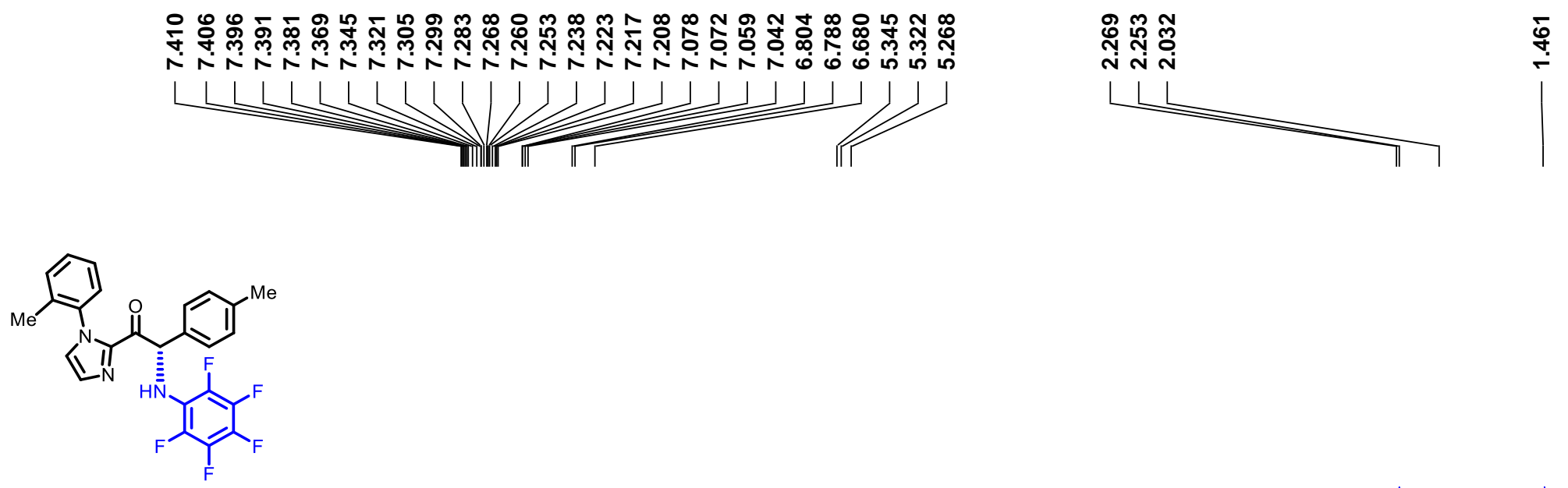

$4 \mathbf{j a}$

${ }^{1} \mathrm{H} \mathrm{NMR}\left(500 \mathrm{MHz}, \mathrm{CDCl}_{3}\right)$

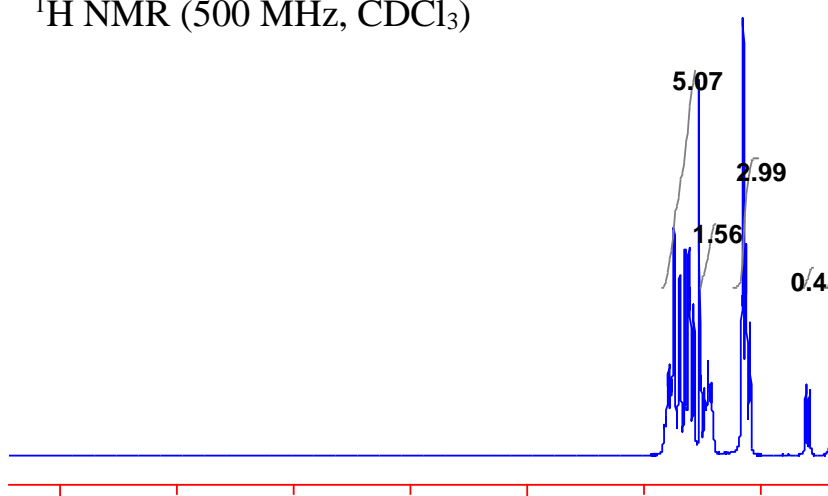

$0.48^{1.02}$

1.06

10

6

4

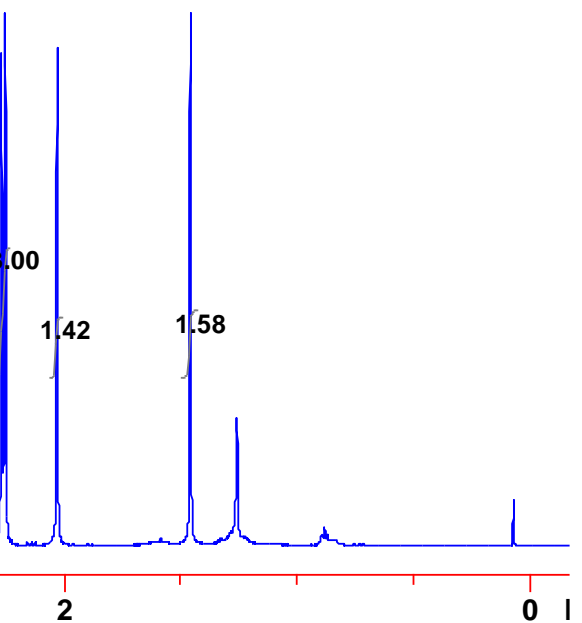




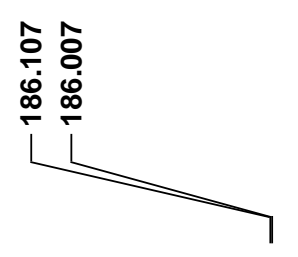

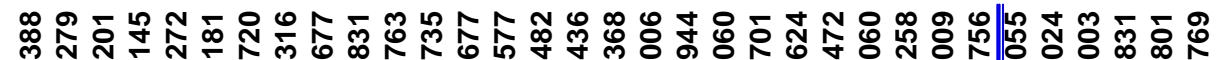

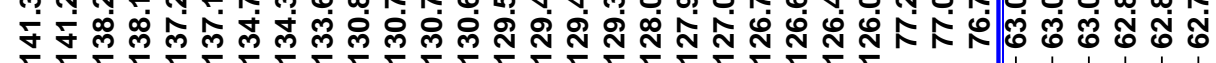

ㅇํㅇ 옹 ్ㅠ융

( 711 ,

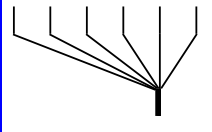

ลัก ㄷํㄴ
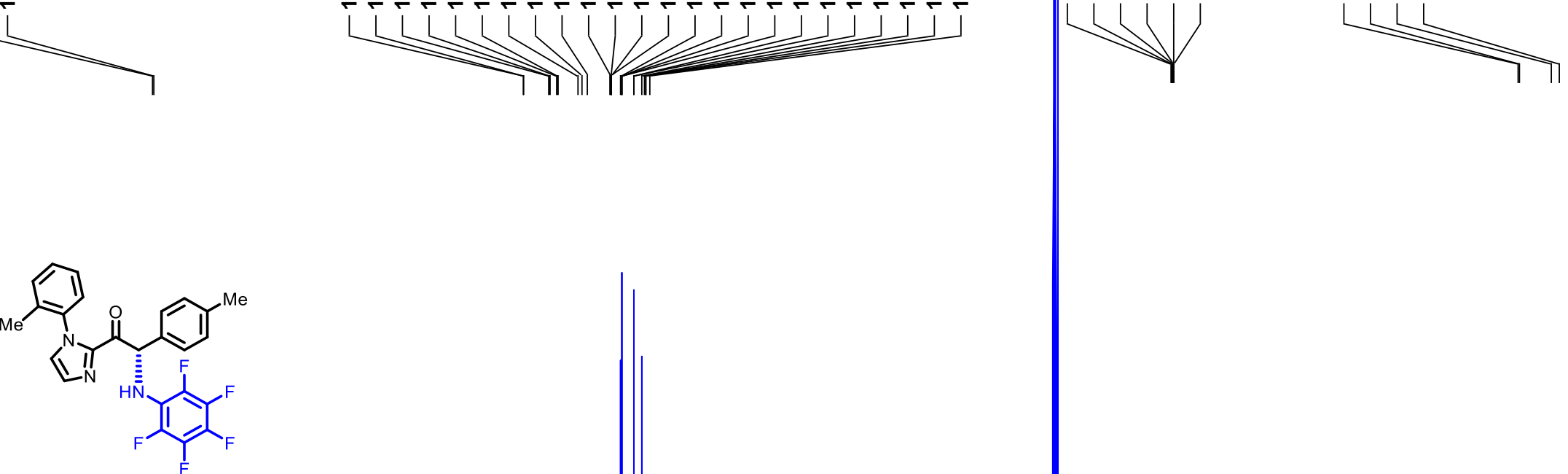

4 ja

${ }^{13} \mathrm{C}$ NMR (125 MHz, $\left.\mathrm{CDCl}_{3}\right)$
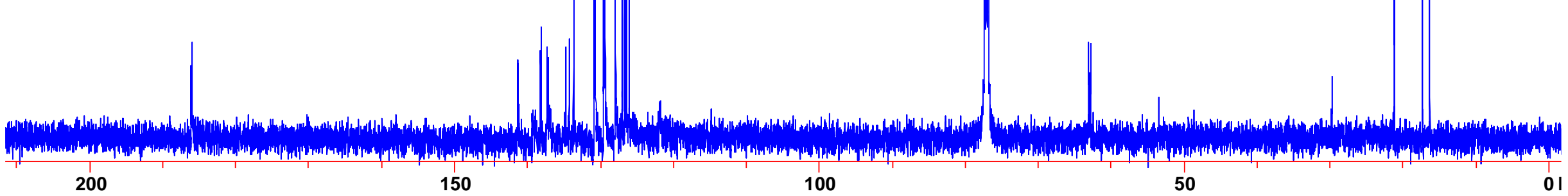


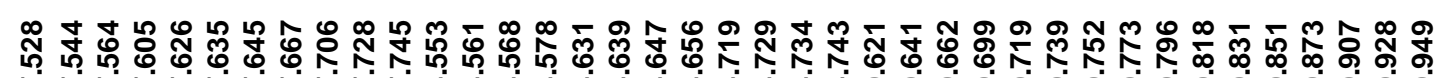

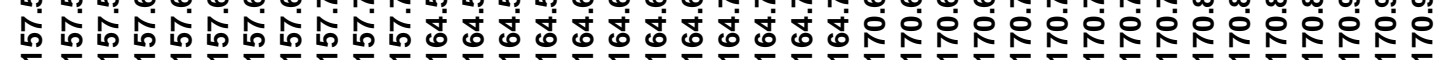

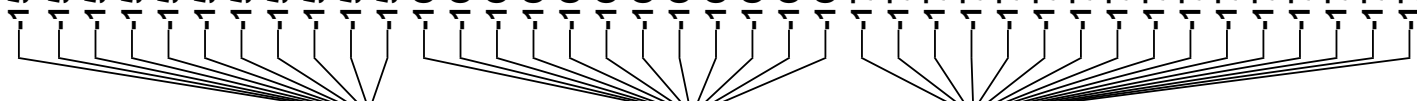

$\ldots$

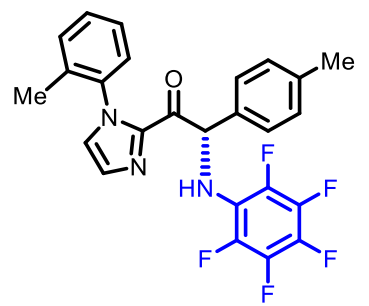

4 ja

${ }^{19} \mathrm{~F}$ NMR $\left(282 \mathrm{MHz}, \mathrm{CDCl}_{3}\right)$

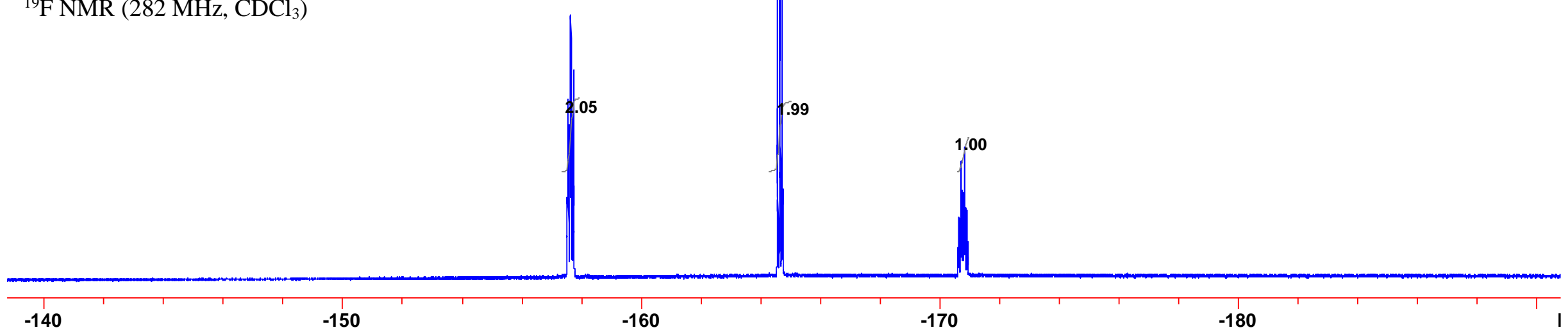




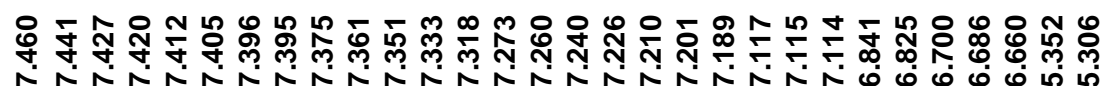

(L)

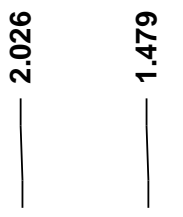

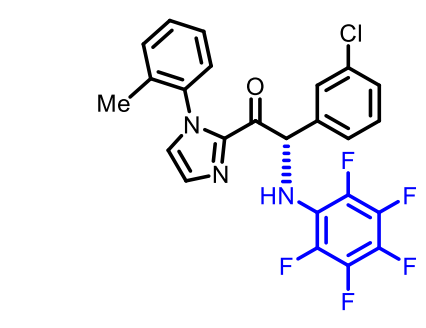

$4 \mathbf{k a}$

${ }^{1} \mathrm{H}$ NMR (500 MHz, $\mathrm{CDCl}_{3}$ )
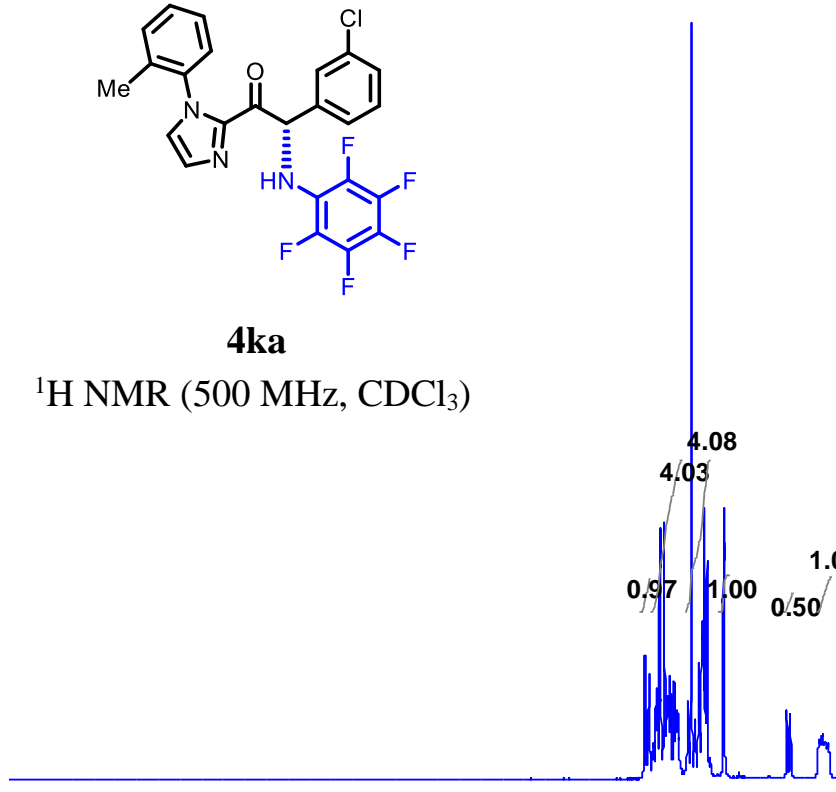

03

10

8

4

2

$0 \quad 1$ 


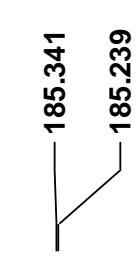

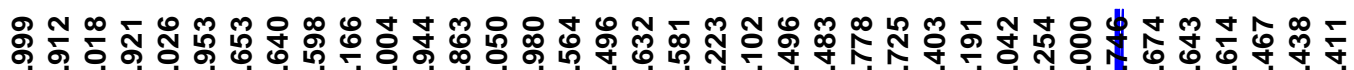

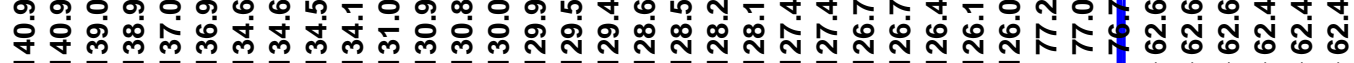
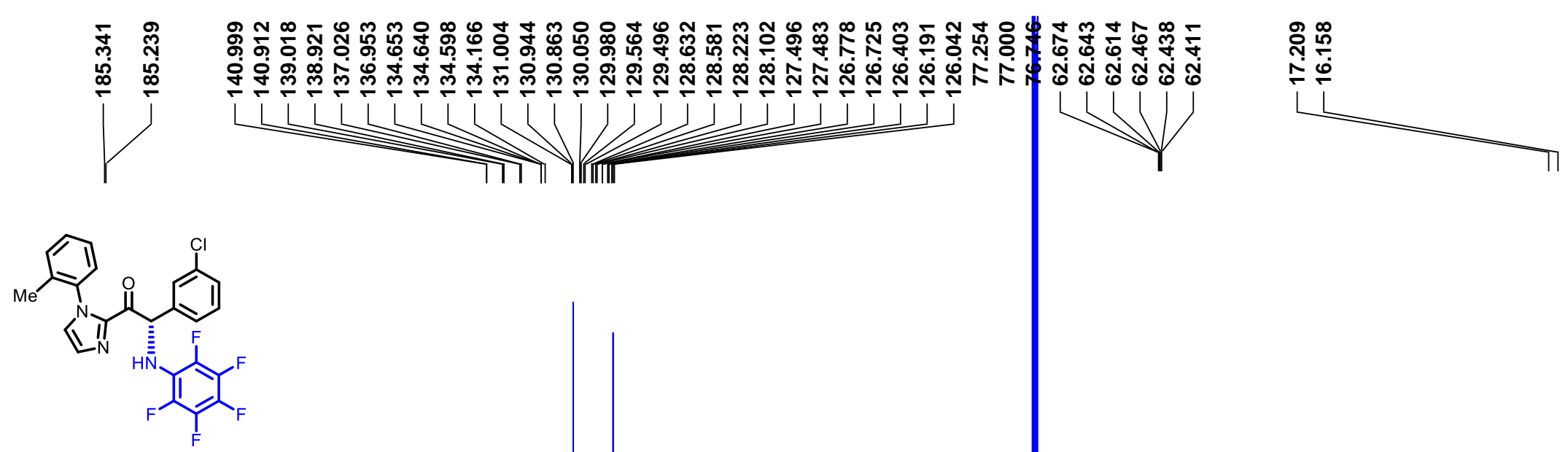

$4 \mathrm{ka}$

${ }^{13} \mathrm{C}$ NMR (125 MHz, $\left.\mathrm{CDCl}_{3}\right)$

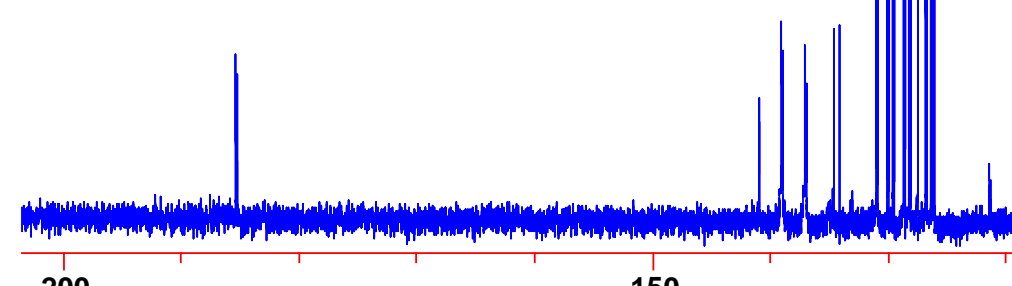




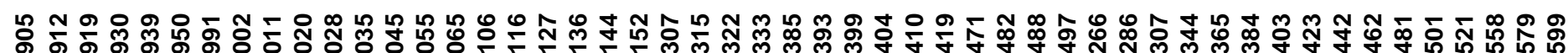

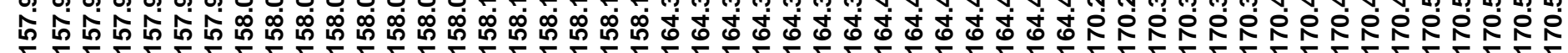

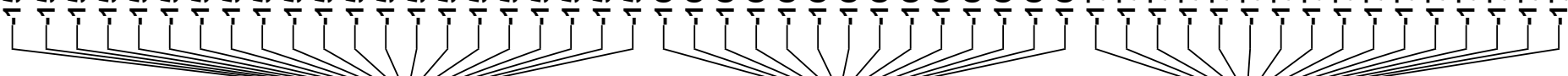

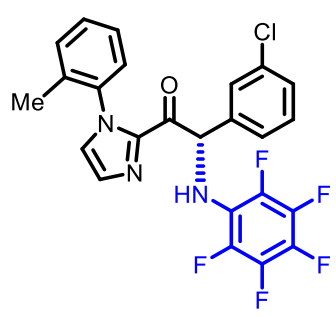

$4 \mathbf{k a}$

${ }^{19} \mathrm{~F}$ NMR $\left(282 \mathrm{MHz}, \mathrm{CDCl}_{3}\right)$
2.22
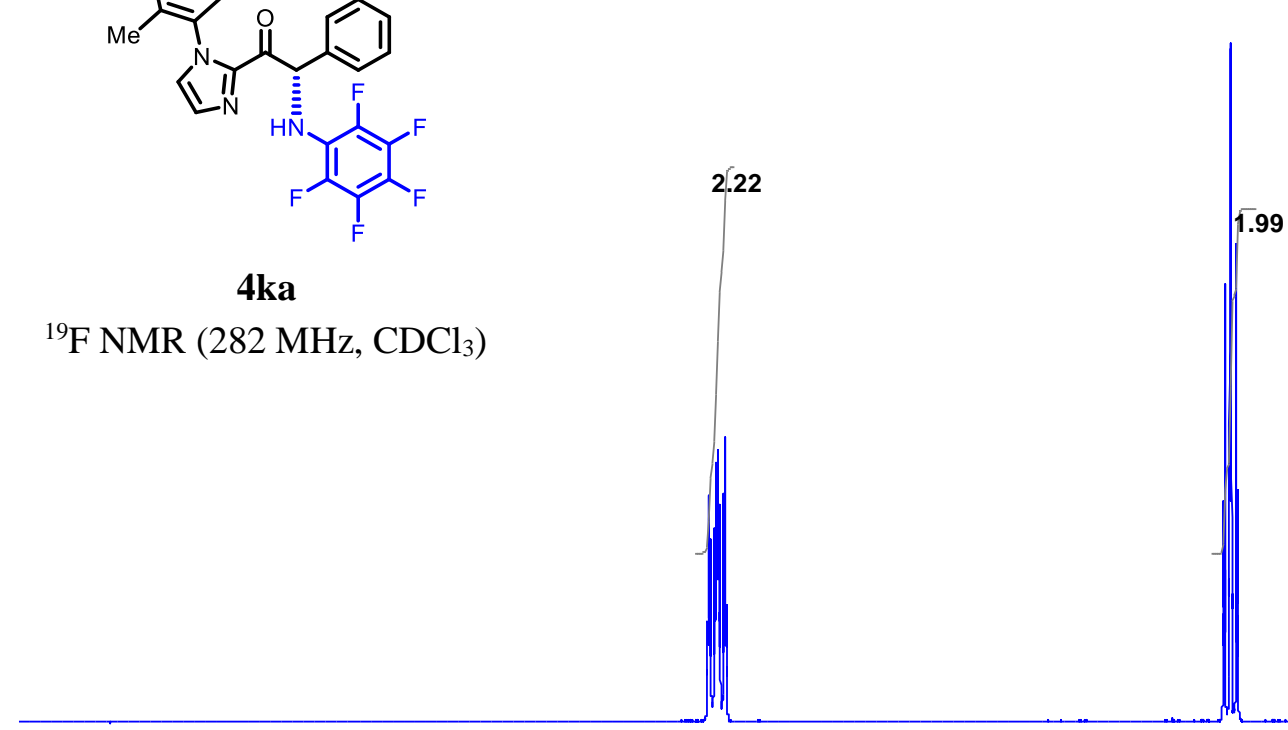

1.00

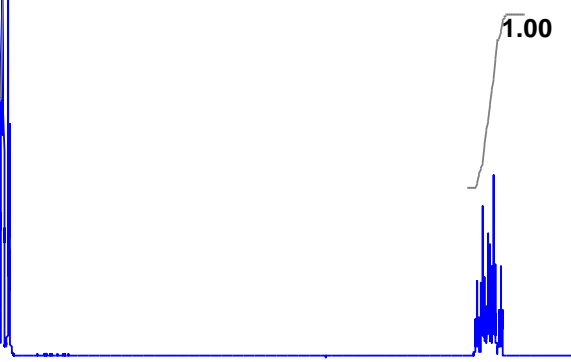

$-160$

$-165$

$-170$

$-175$ 


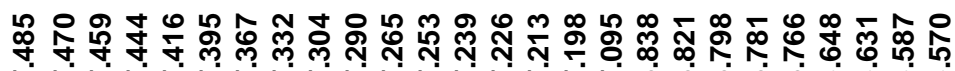

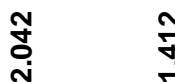
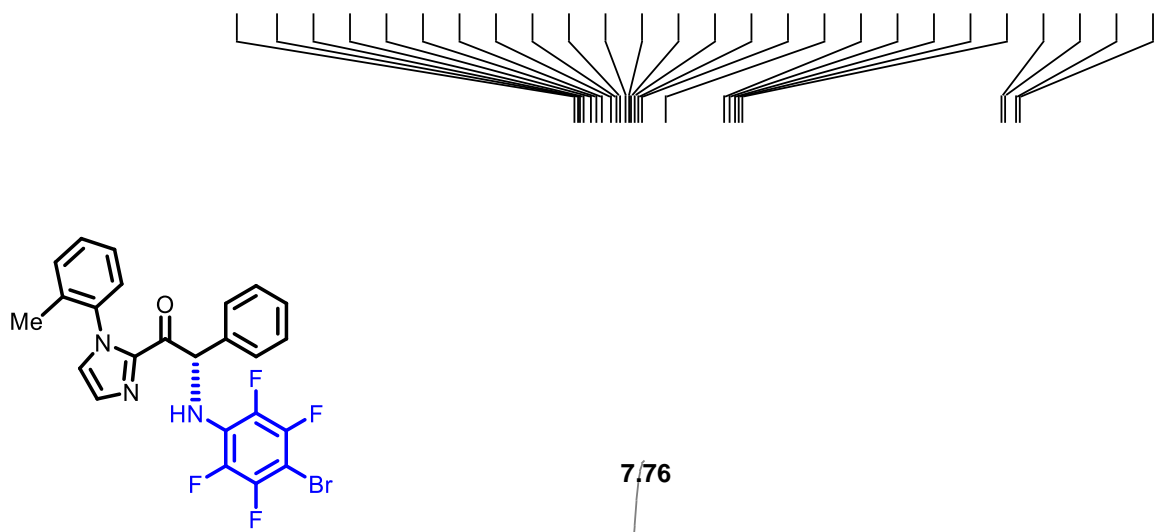

$4 \mathrm{bb}$

${ }^{1} \mathrm{H}$ NMR (500 MHz, $\mathrm{CDCl}_{3}$ )

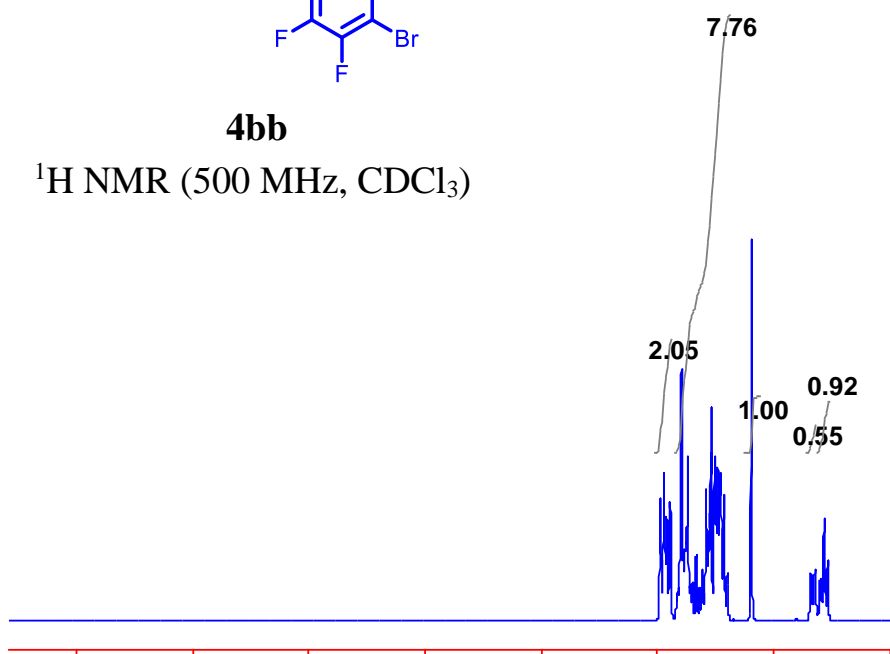

0.55

10

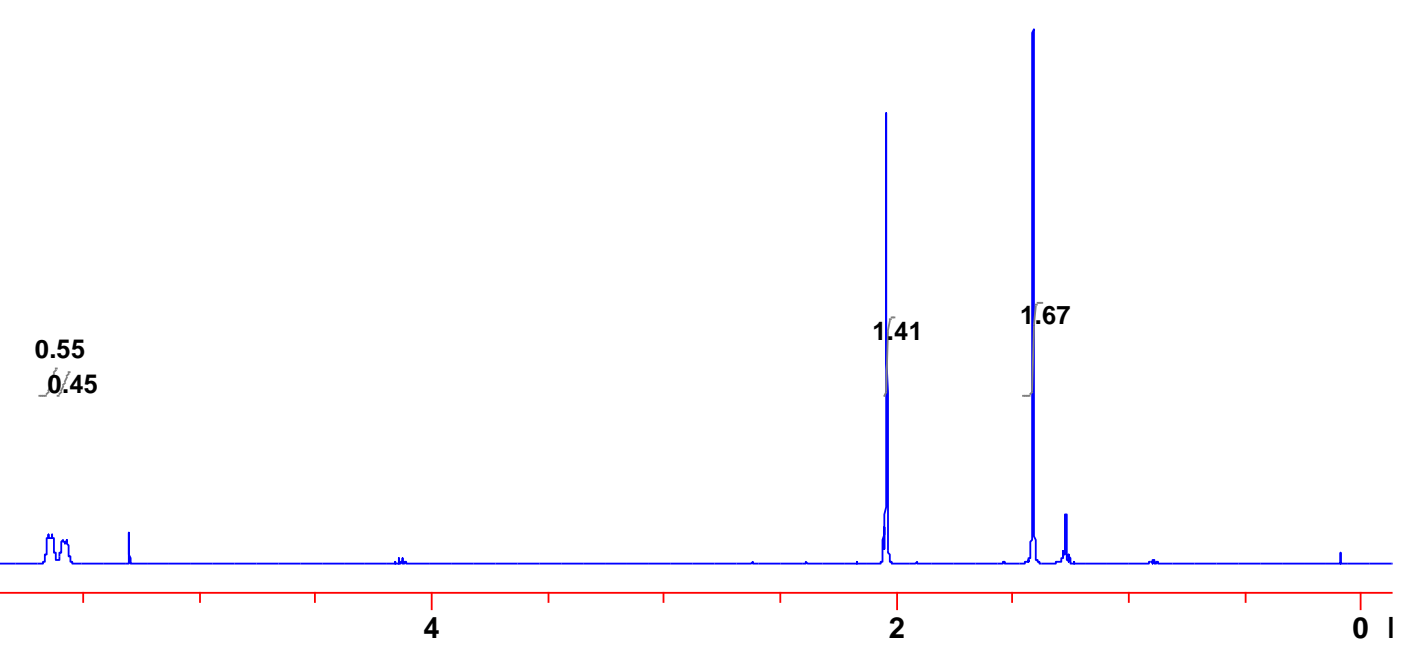



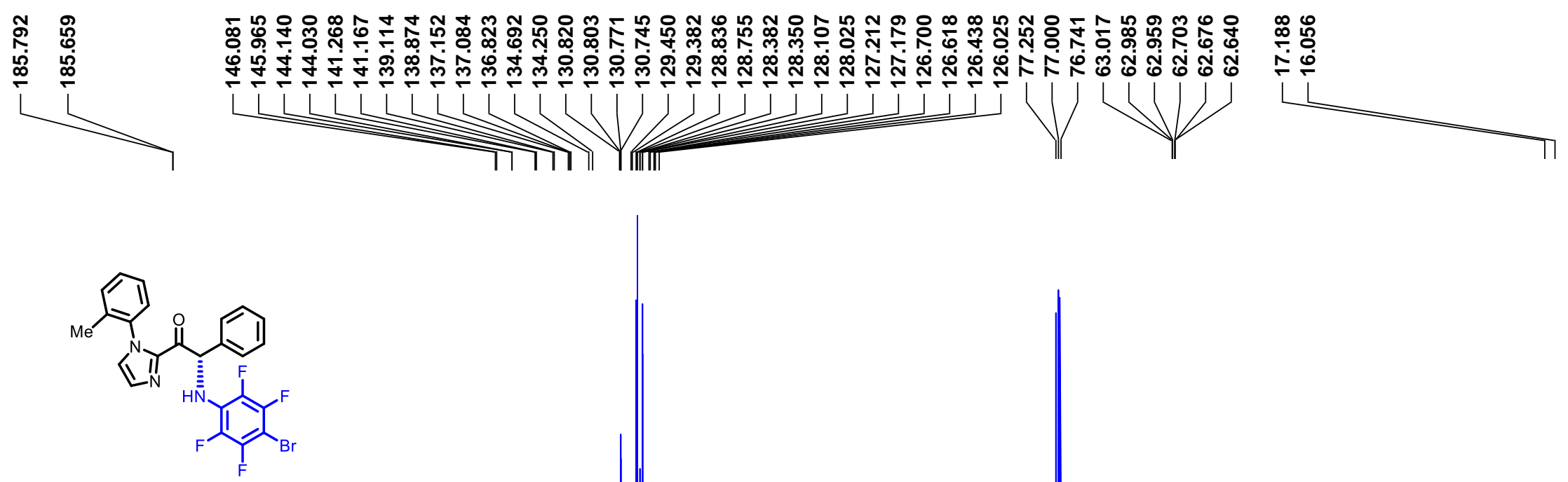

$4 \mathrm{bb}$

${ }^{13} \mathrm{C}$ NMR $\left(282 \mathrm{MHz}, \mathrm{CDCl}_{3}\right)$

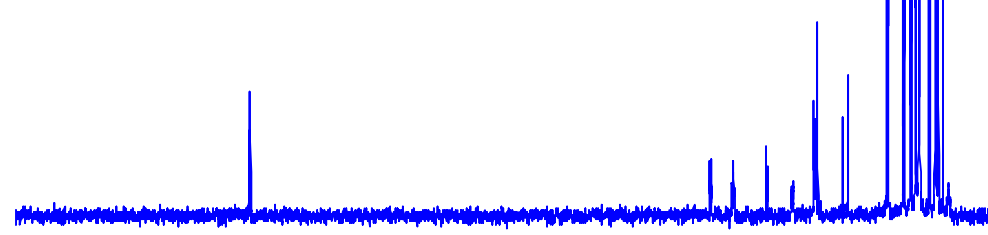

200 

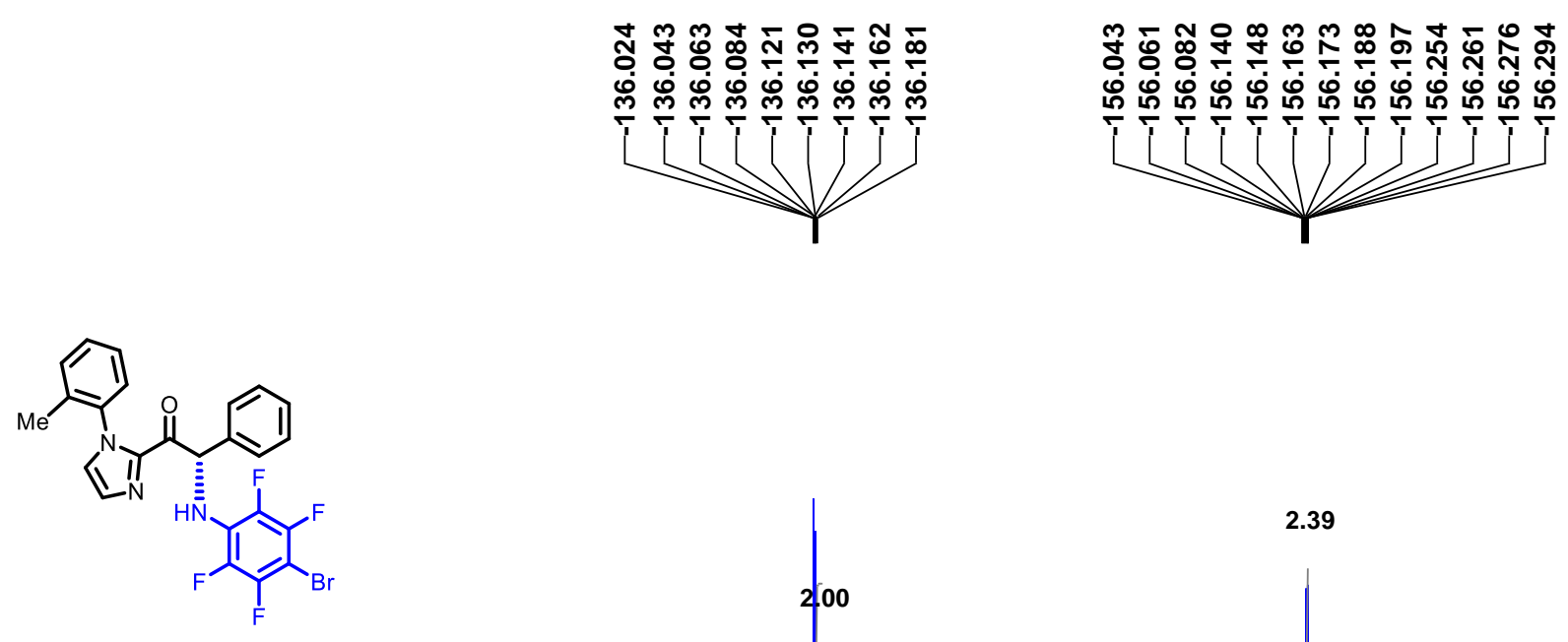

$4 \mathrm{bb}$

${ }^{19} \mathrm{~F}$ NMR (282 MHz, $\left.\mathrm{CDCl}_{3}\right)$

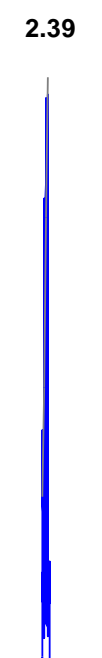

00

$-120$

$-140$

$-160$

$-180$ 


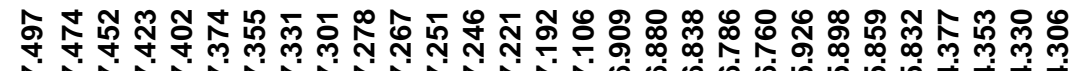

(1)

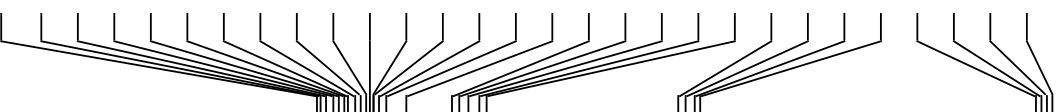

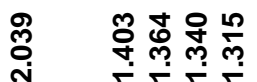

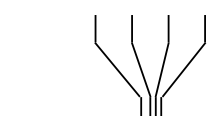

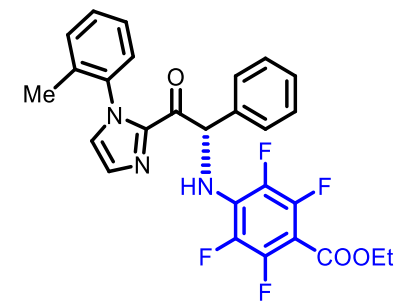

4 bc

${ }^{1} \mathrm{H} \mathrm{NMR}\left(300 \mathrm{MHz}, \mathrm{CDCl}_{3}\right.$ )

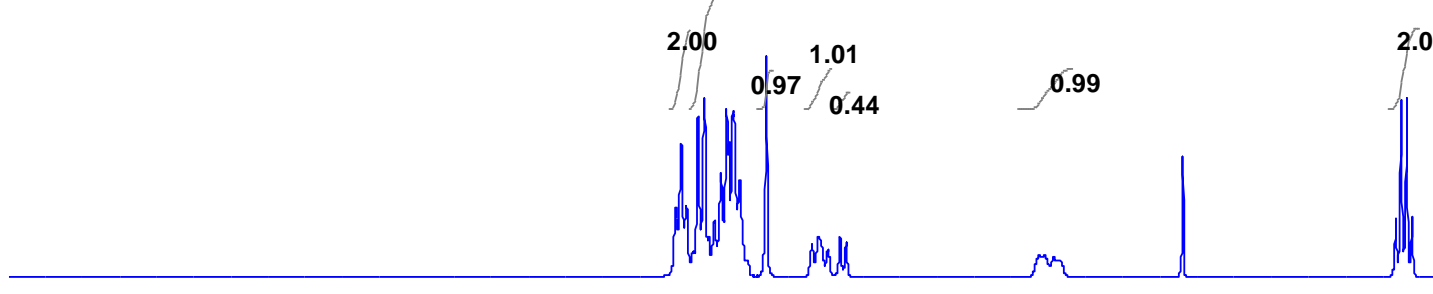

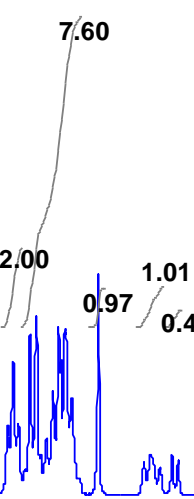

6
10

8

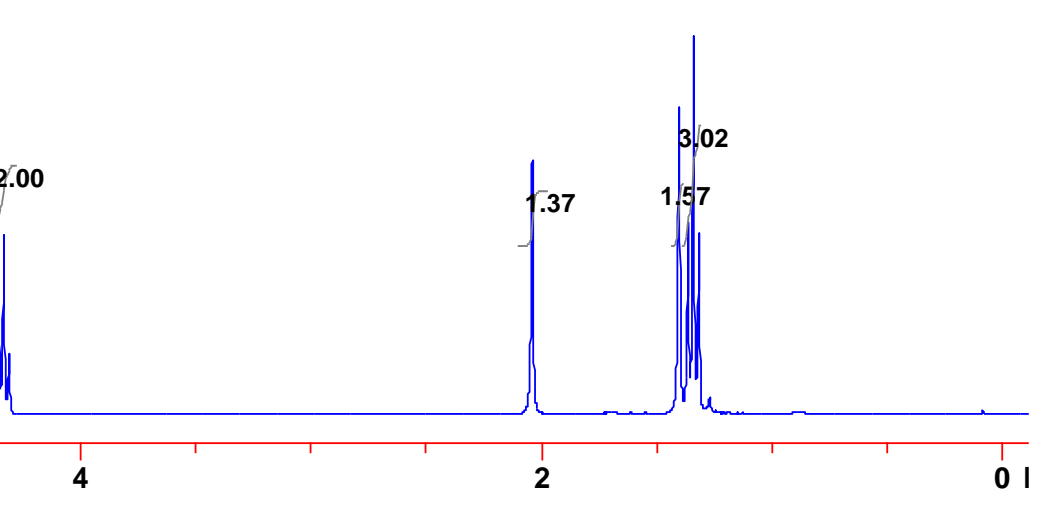



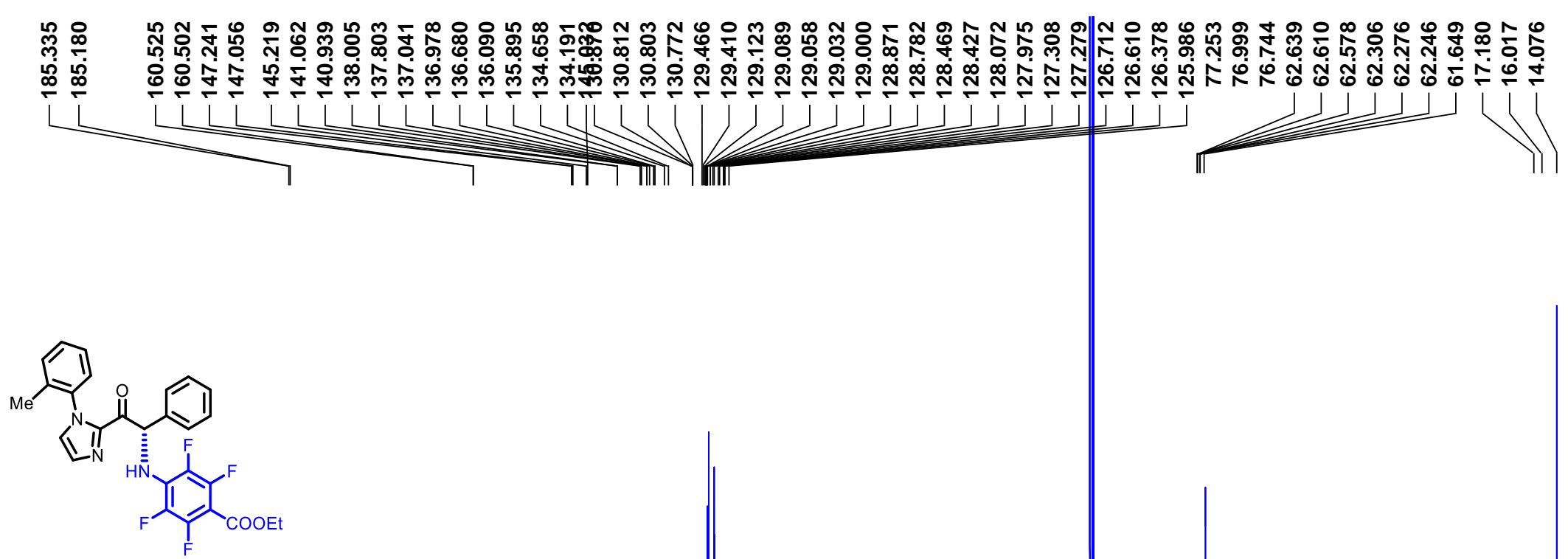

4 bc

${ }^{13} \mathrm{C}$ NMR $\left(125 \mathrm{MHz}, \mathrm{CDCl}_{3}\right)$

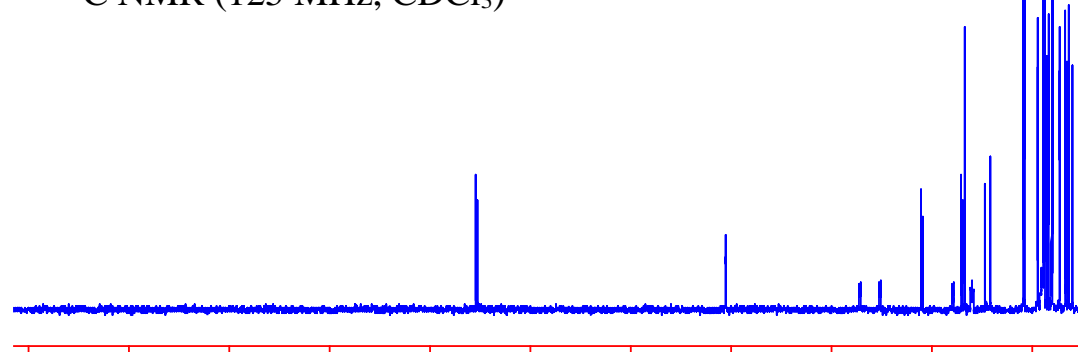

200

150

100

50 

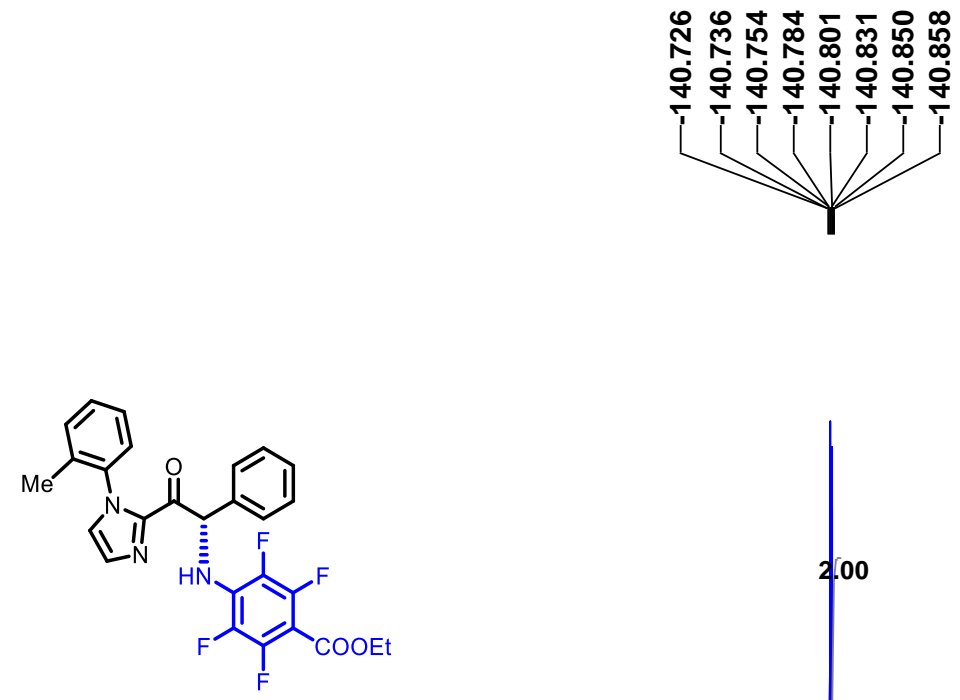

$4 b c$

${ }^{19} \mathrm{~F}$ NMR $\left(282 \mathrm{MHz}, \mathrm{CDCl}_{3}\right)$

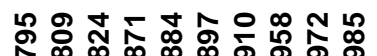

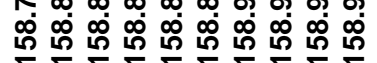

lijij

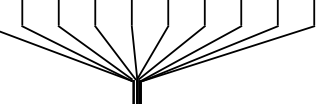

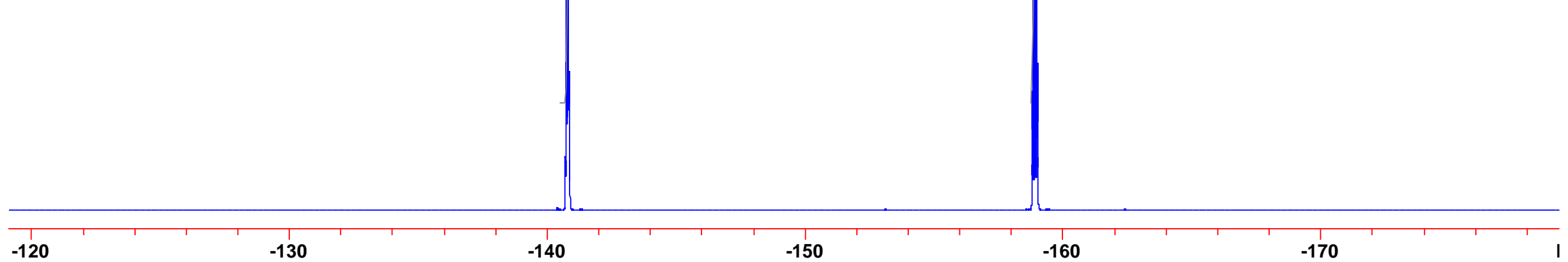




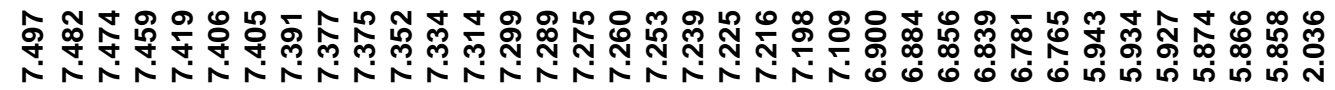

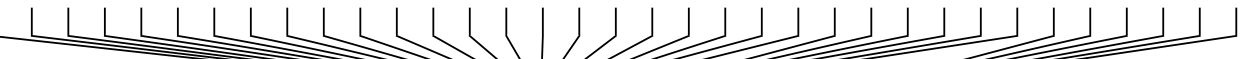

11111\|ा\|ा $\pi$

$\pi$

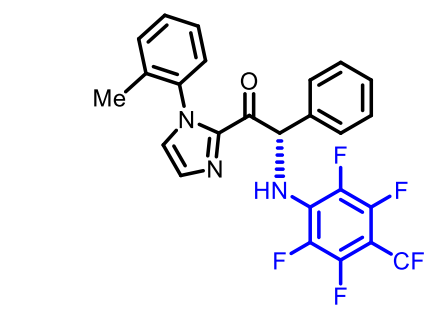

4 bd

${ }^{1} \mathrm{H}$ NMR $\left(500 \mathrm{MHz}, \mathrm{CDCl}_{3}\right.$ )

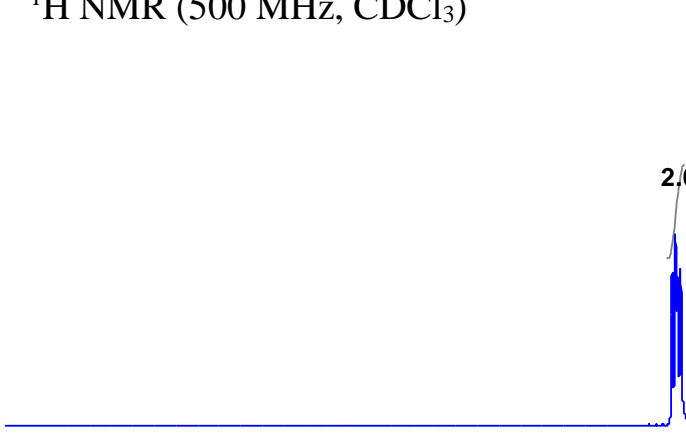

10

7.72

0.54

000.49

W

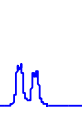

6

4 


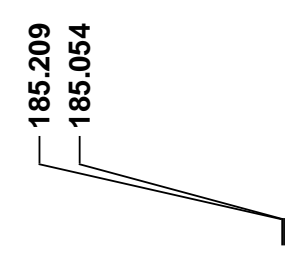

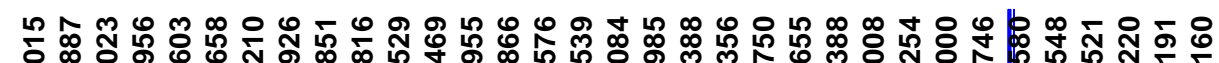

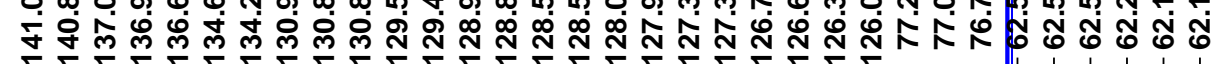

พ1
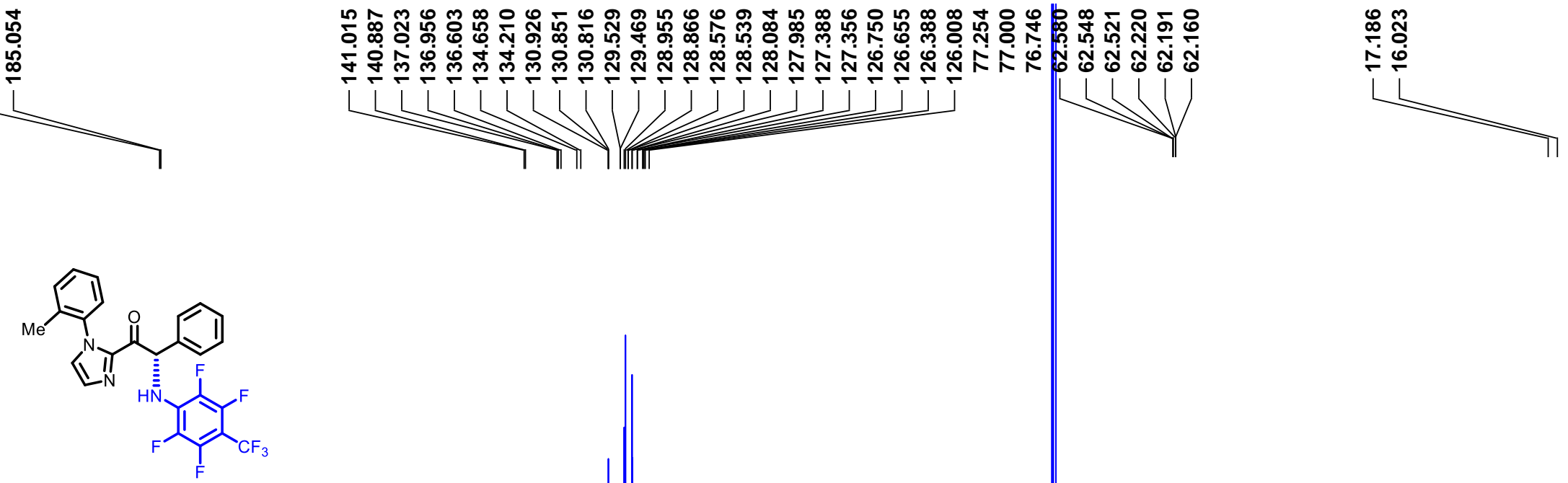

4 bd

${ }^{13} \mathrm{C}$ NMR (125 MHz, $\left.\mathrm{CDCl}_{3}\right)$

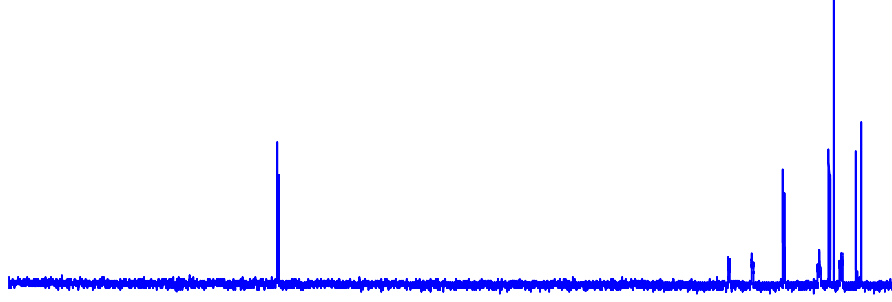

200 


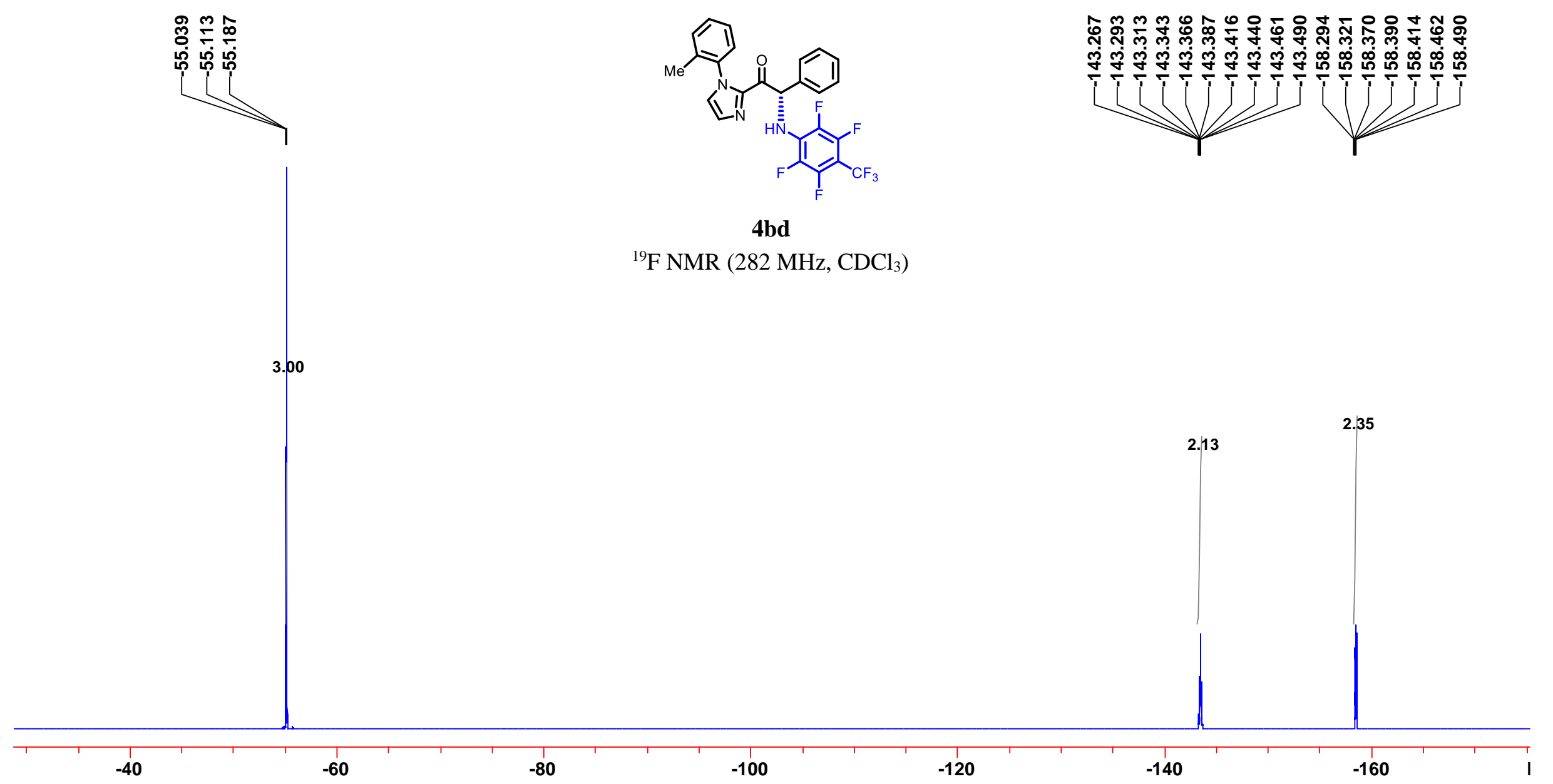



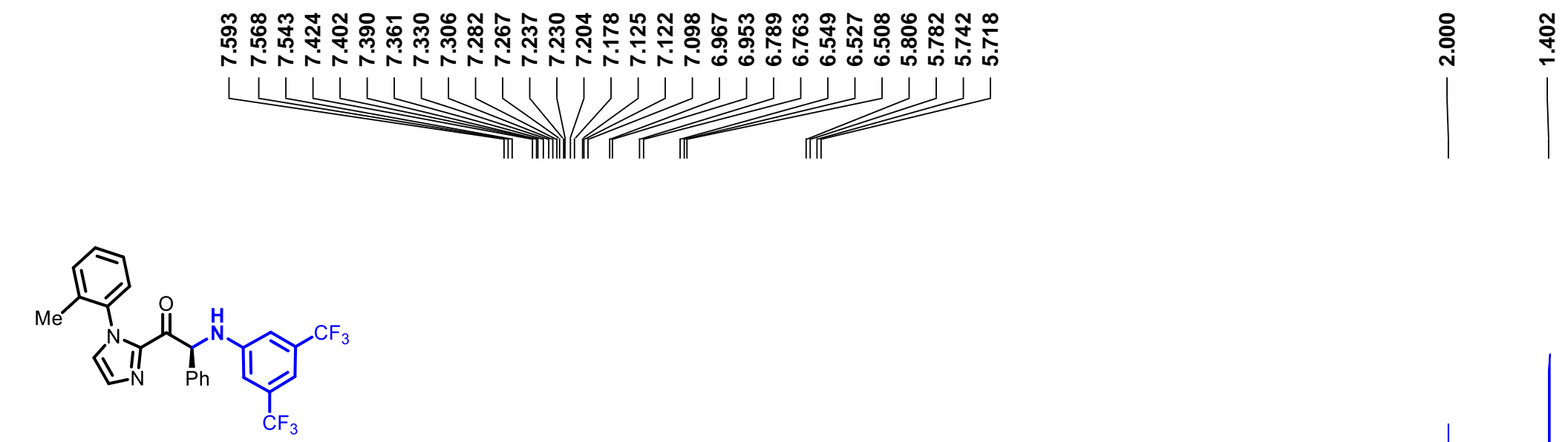

4 be

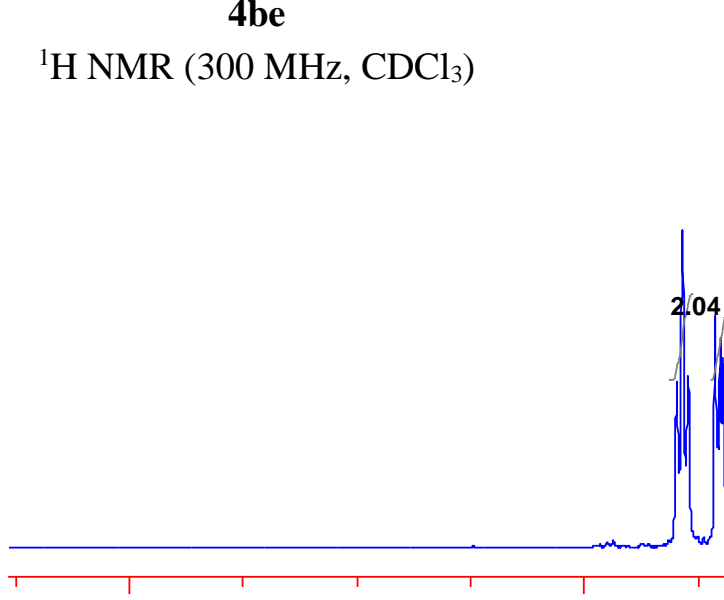

${ }^{1} \mathrm{H}$ NMR (300 MHz, $\mathrm{CDCl}_{3}$ )

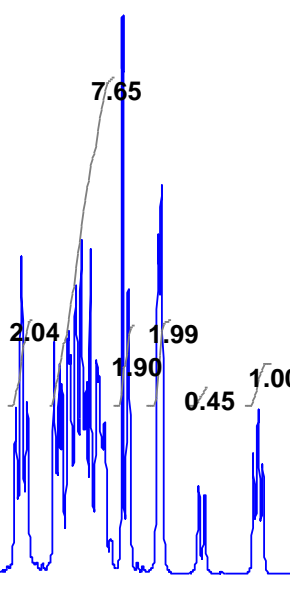

10 8

0.45

ty 


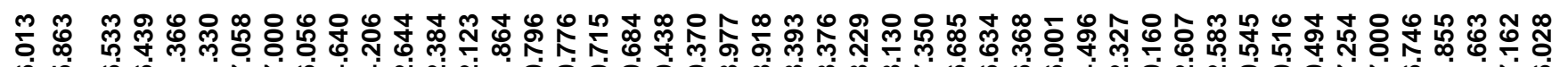

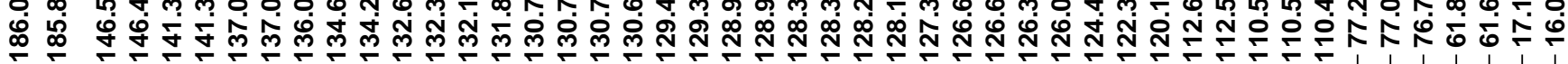

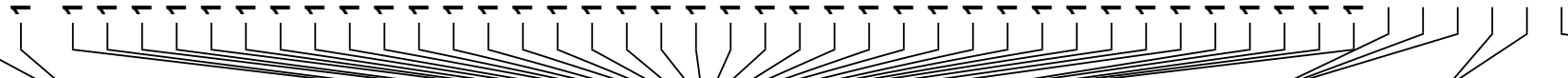

4

1711 II 1 ता

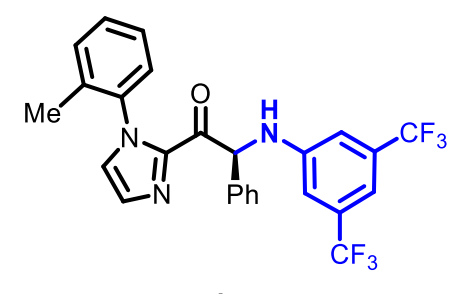

4be

${ }^{13} \mathrm{C} \mathrm{NMR}\left(125 \mathrm{MHz}, \mathrm{CDCl}_{3}\right)$

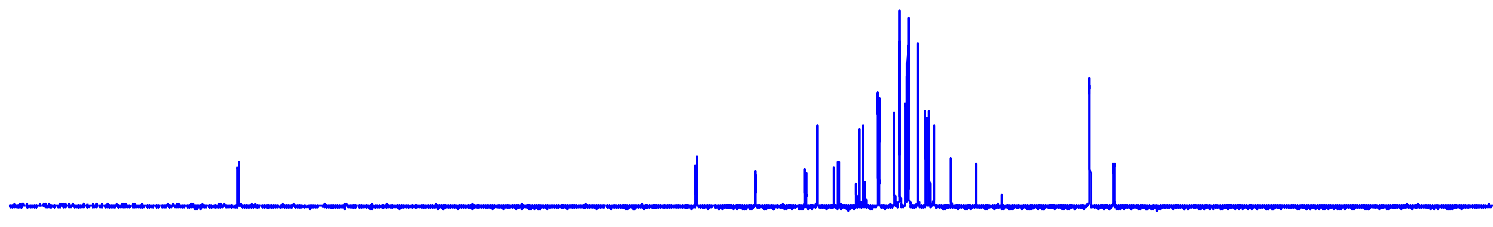

200

150

100

50

01 


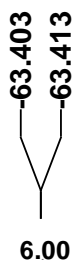

(1)

4be

${ }^{19} \mathrm{~F}$ NMR $\left(282 \mathrm{MHz}, \mathrm{CDCl}_{3}\right)$

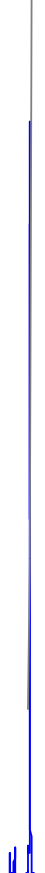

$-45$

$-50$

$-55$

$-60$

$-65$

$-70$

$-75$

$-80$

$-85$ 


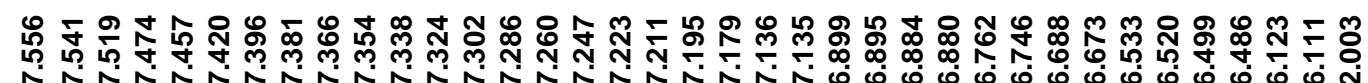

LLLLLLLLLLULUL

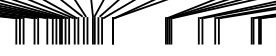

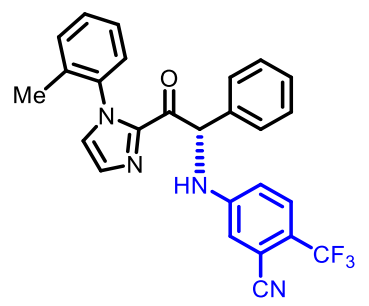

$4 \mathrm{bf}$

${ }^{1} \mathrm{H} \mathrm{NMR}\left(500 \mathrm{MHz}, \mathrm{CDCl}_{3}\right)$

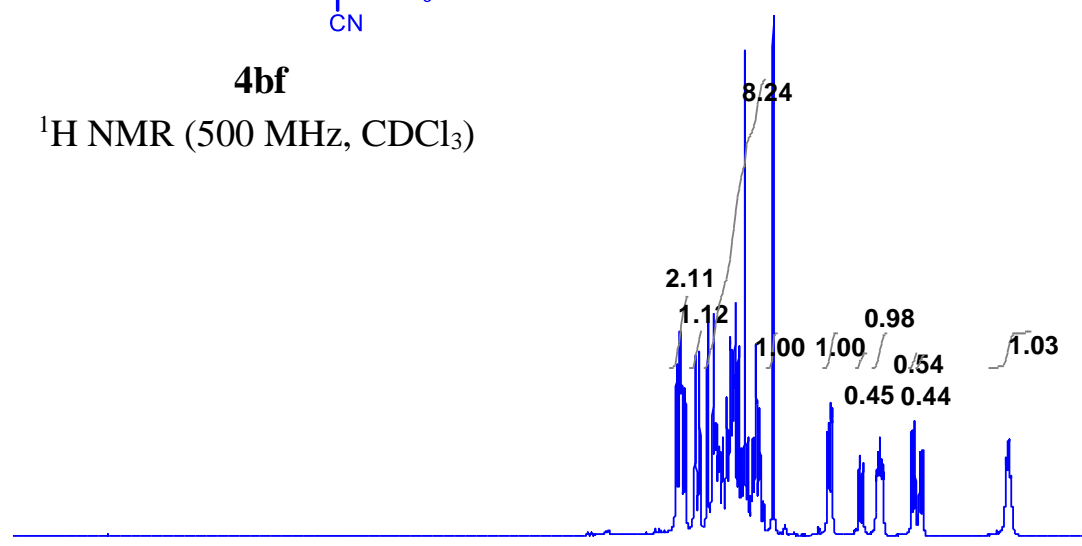

10

4

2 


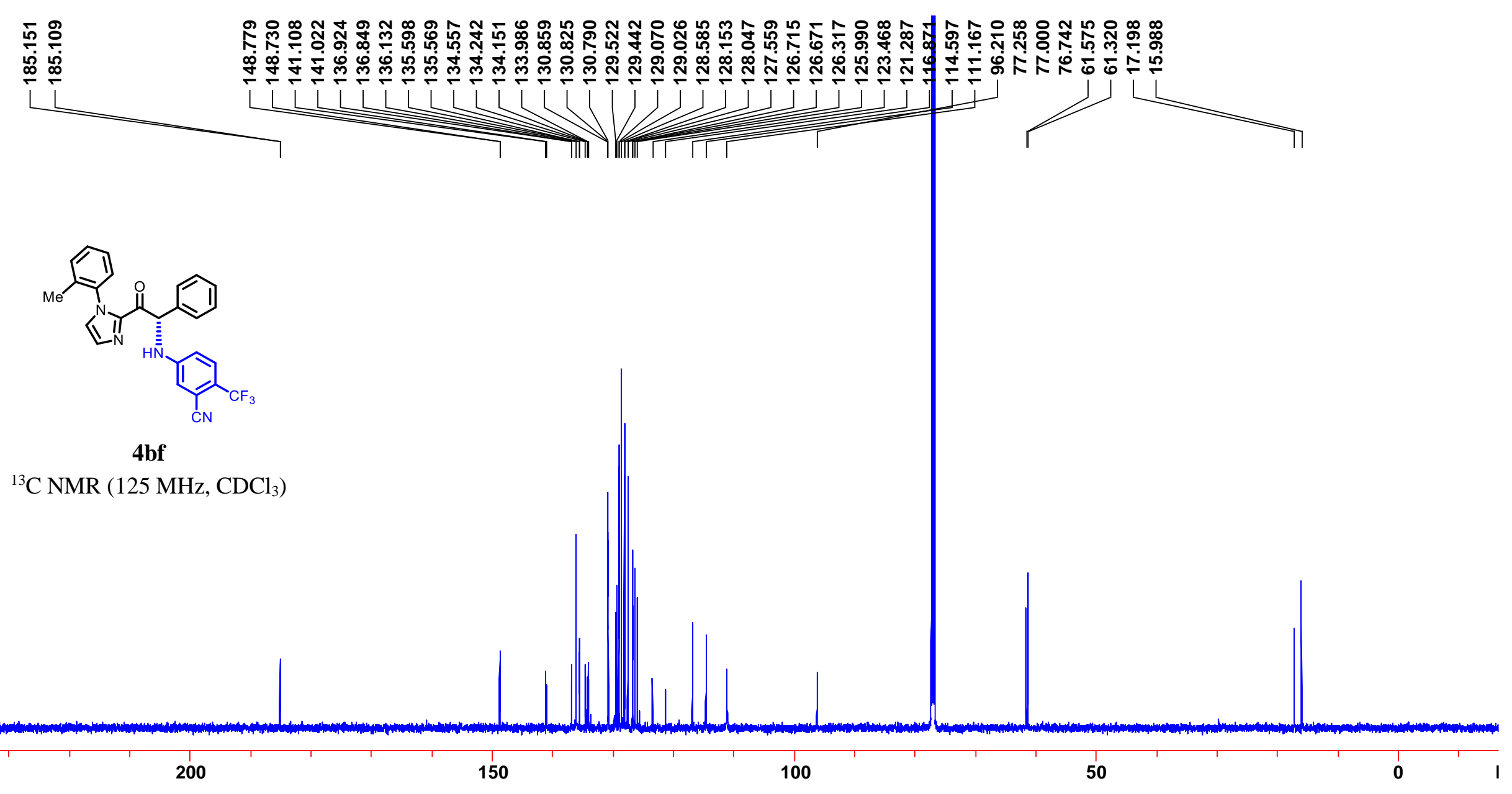



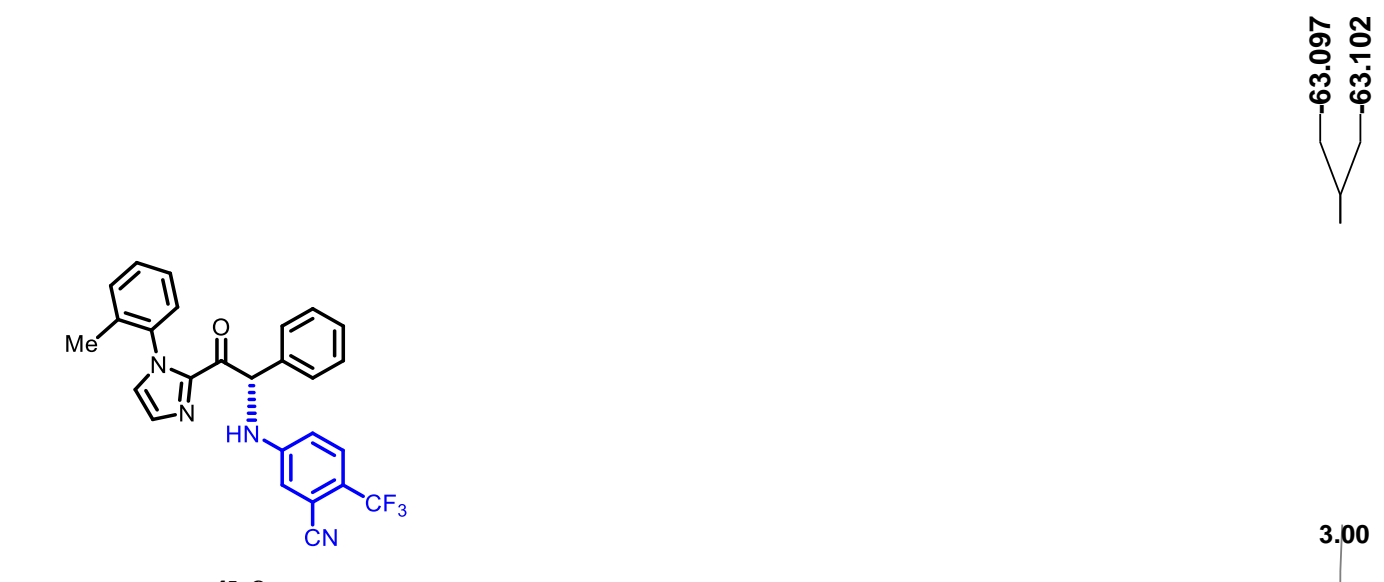

3.00

${ }^{19} \mathrm{~F} \mathrm{NMR}\left(282 \mathrm{MHz}, \mathrm{CDCl}_{3}\right)$

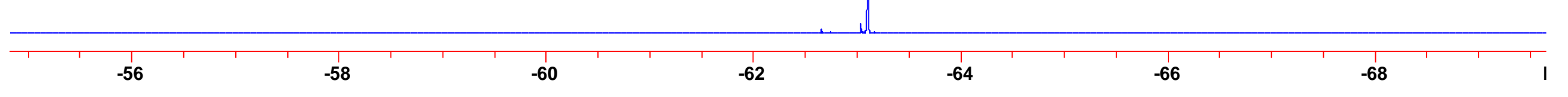




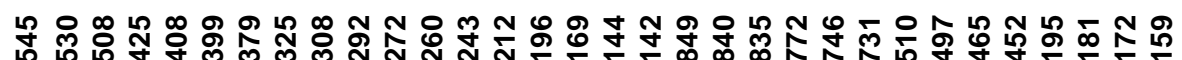

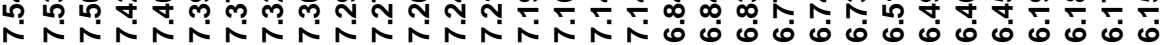

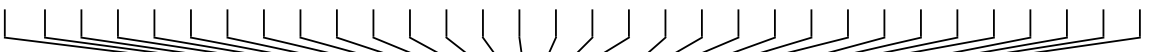

IIIIIII

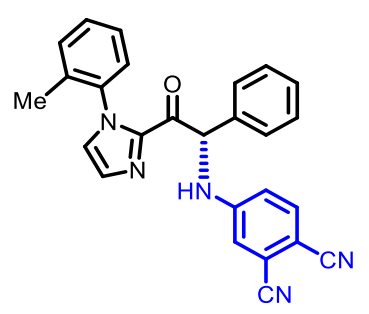

4 bg

${ }^{1} \mathrm{H} \mathrm{NMR}\left(500 \mathrm{MHz}, \mathrm{CDCl}_{3}\right)$
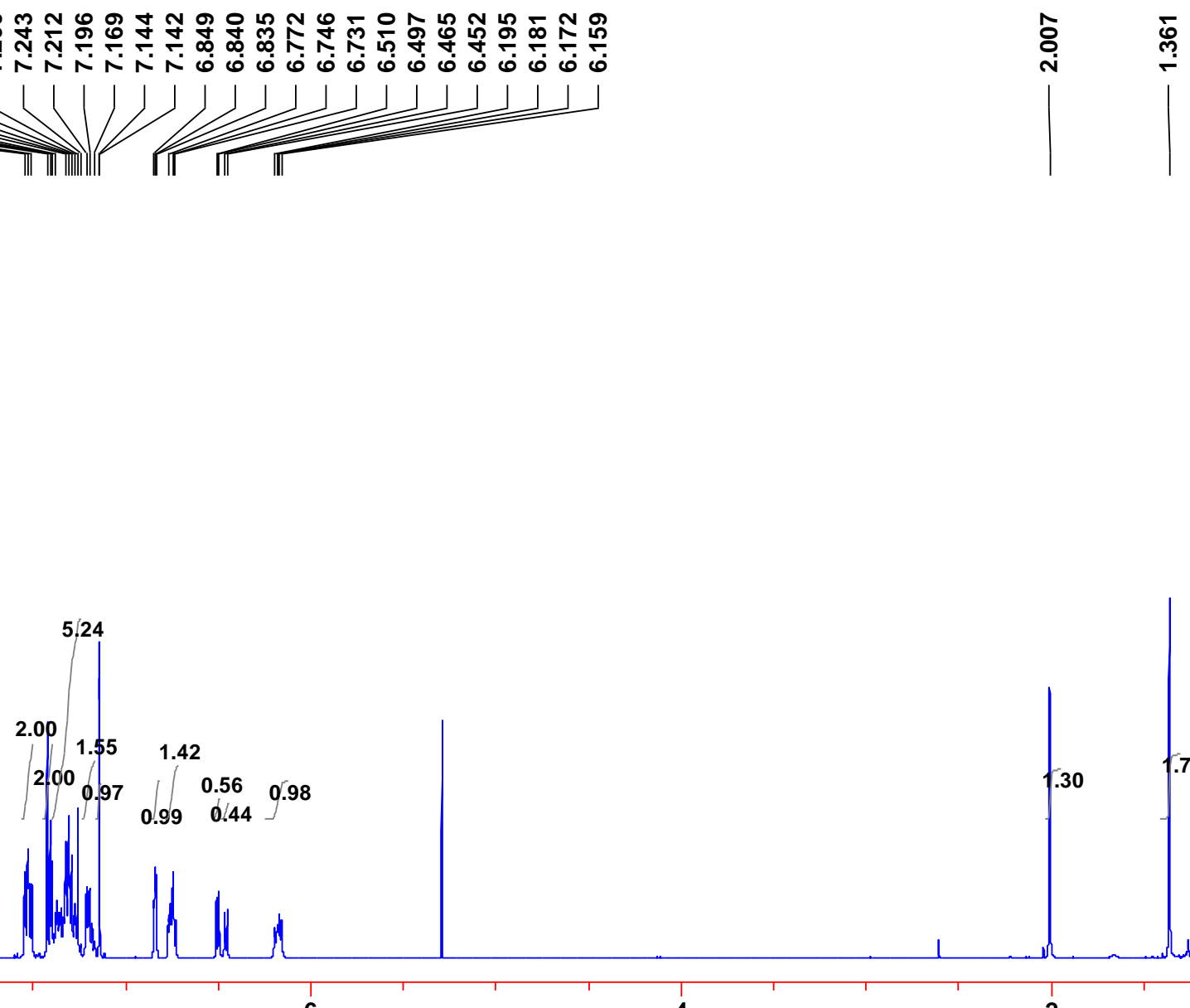

8

4

01 


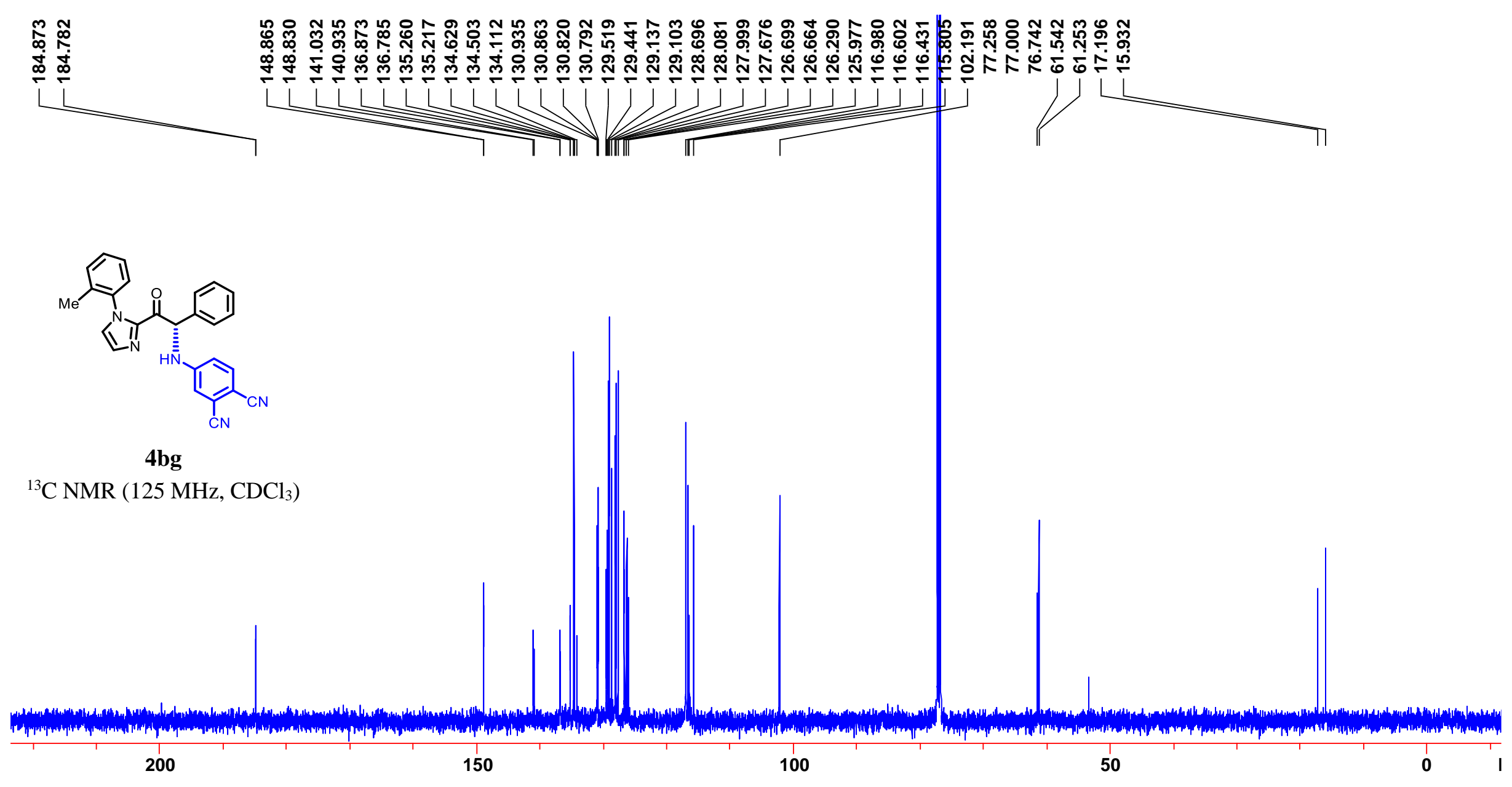




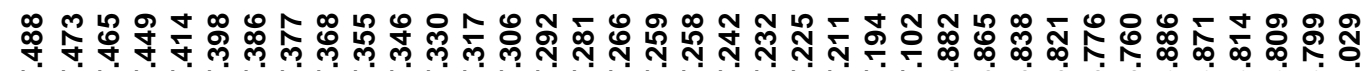

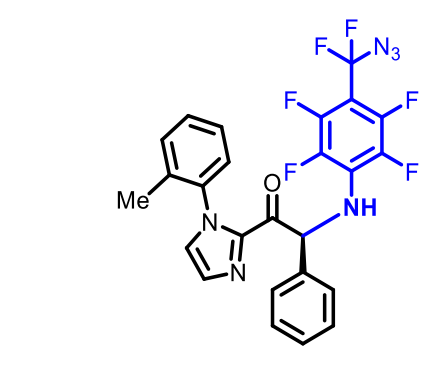

$4 \mathrm{bh}$

${ }^{1} \mathrm{H}$ NMR (500 MHz, $\mathrm{CDCl}_{3}$ )

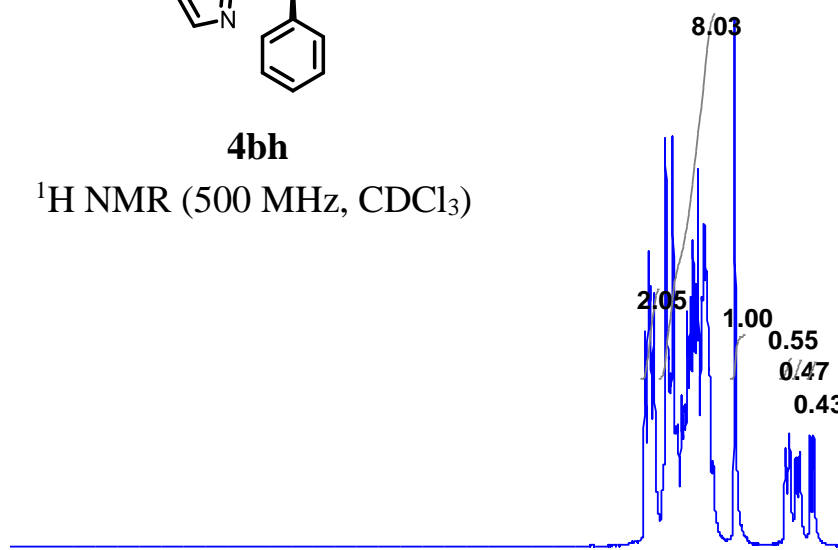

10

8

0.57

0.47

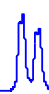

6

4

2

0 I 

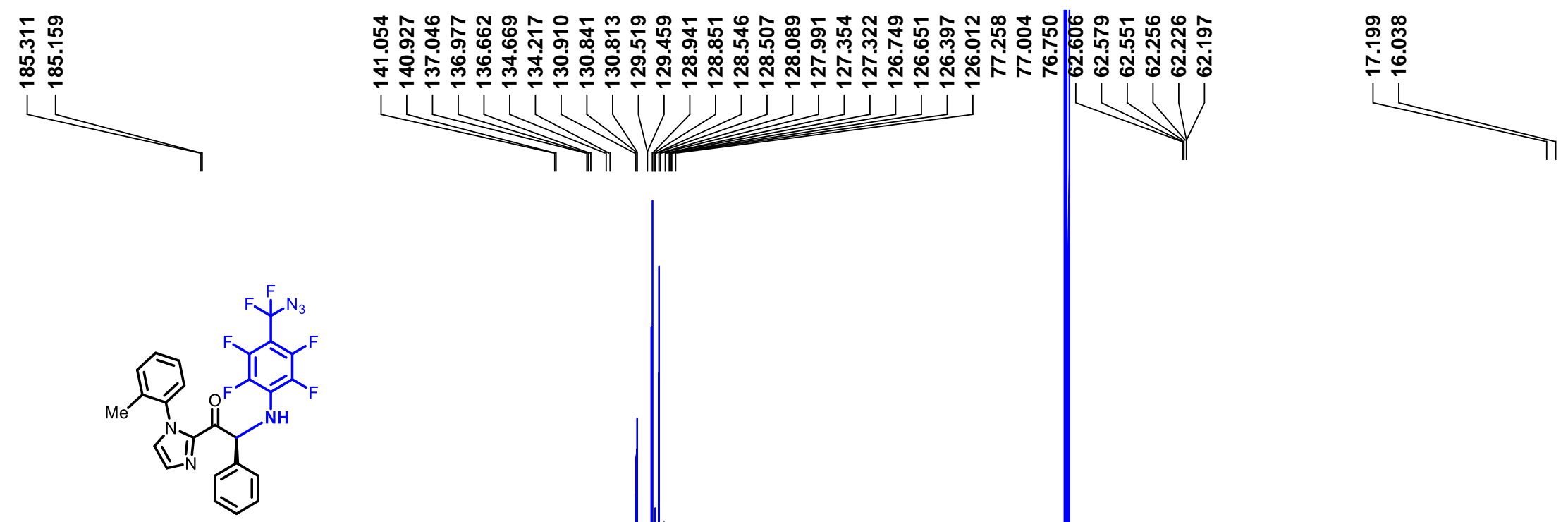

4bh

${ }^{13} \mathrm{C}$ NMR (125 MHz, $\left.\mathrm{CDCl}_{3}\right)$

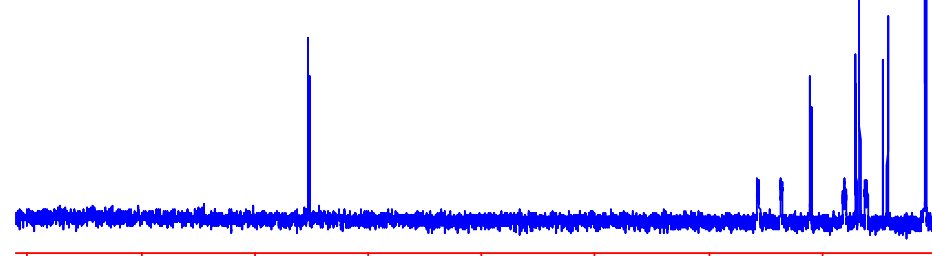




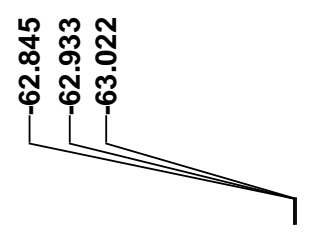

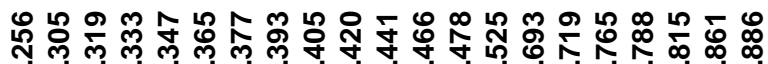

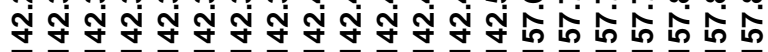
更
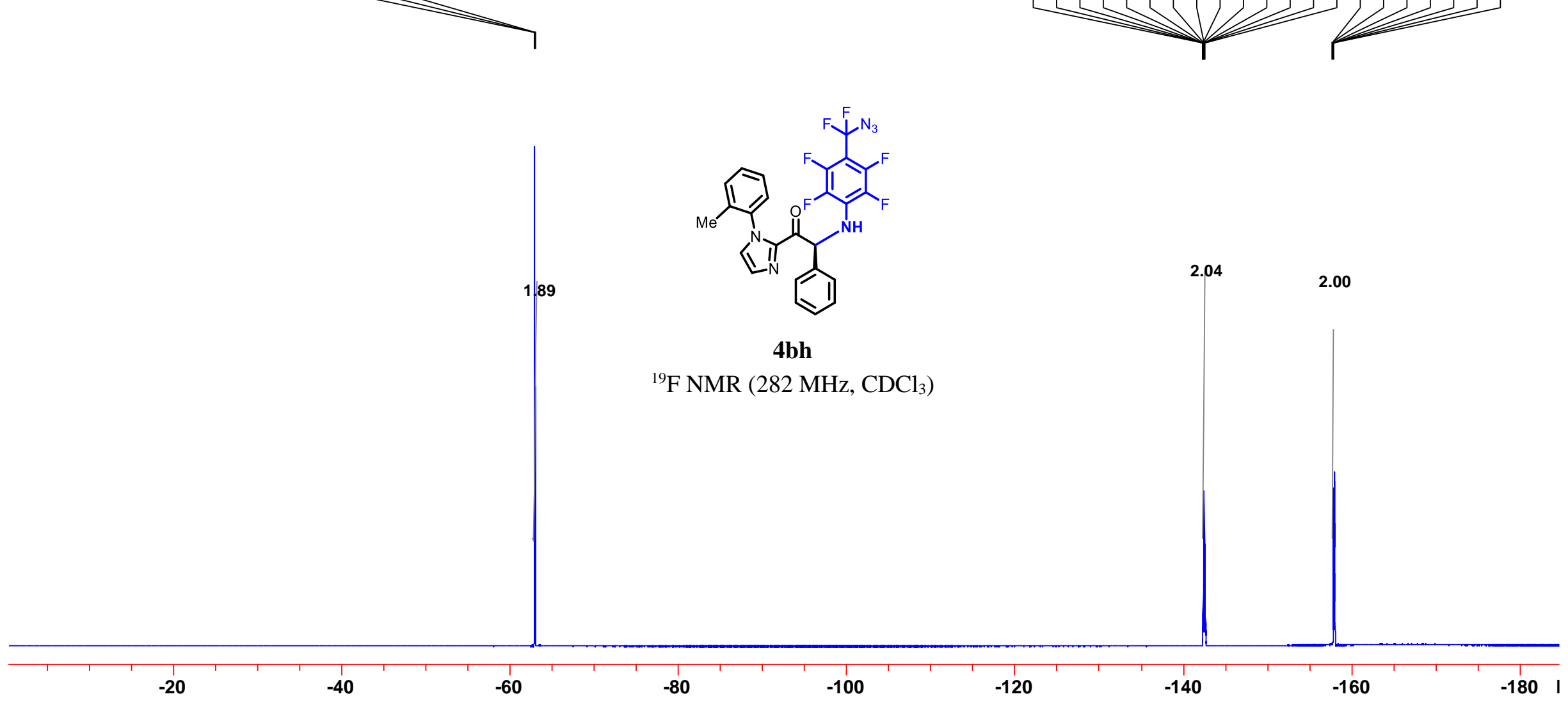


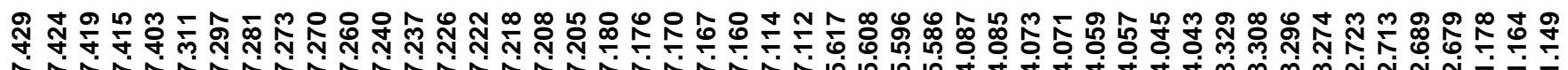
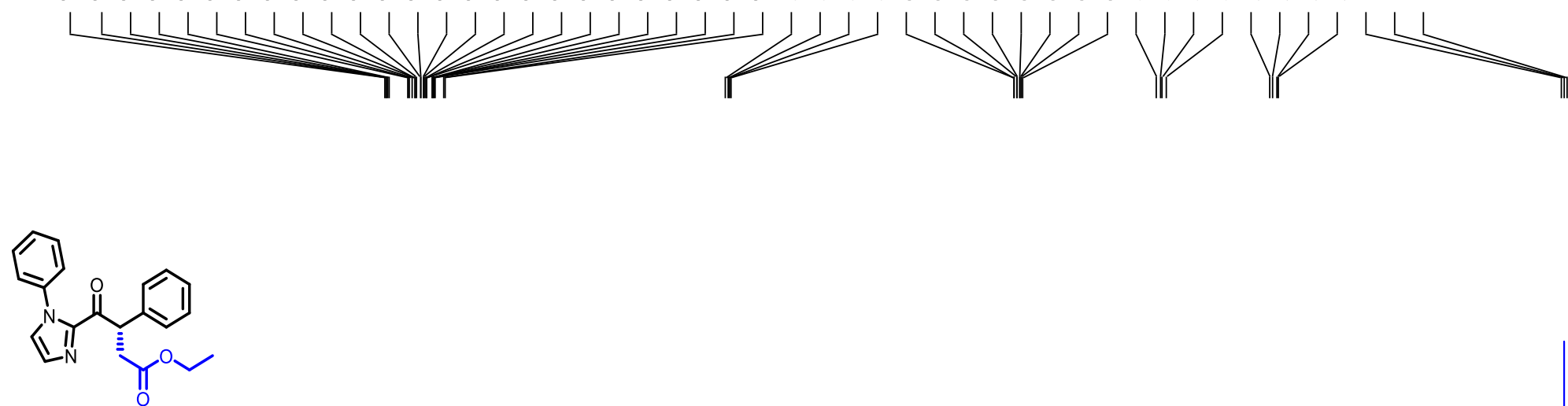

5aa

${ }^{1} \mathrm{H}$ NMR $\left(500 \mathrm{MHz}, \mathrm{CDCl}_{3}\right)$

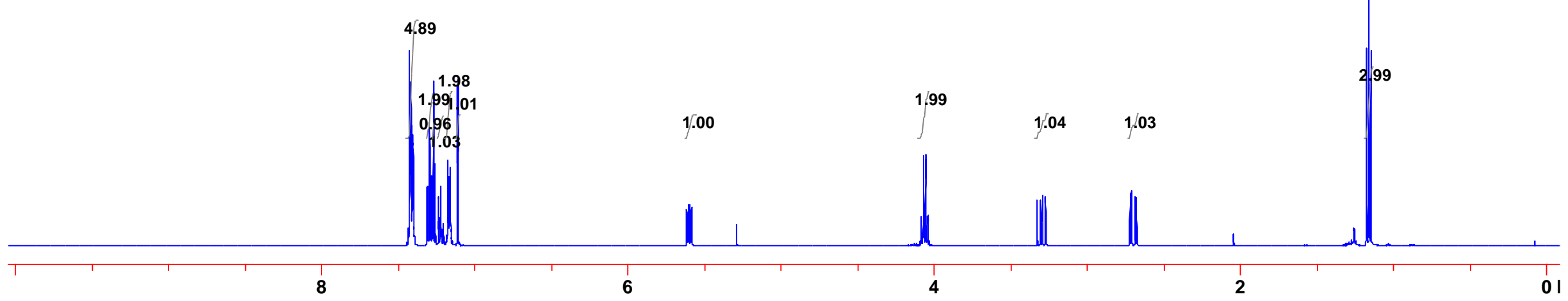




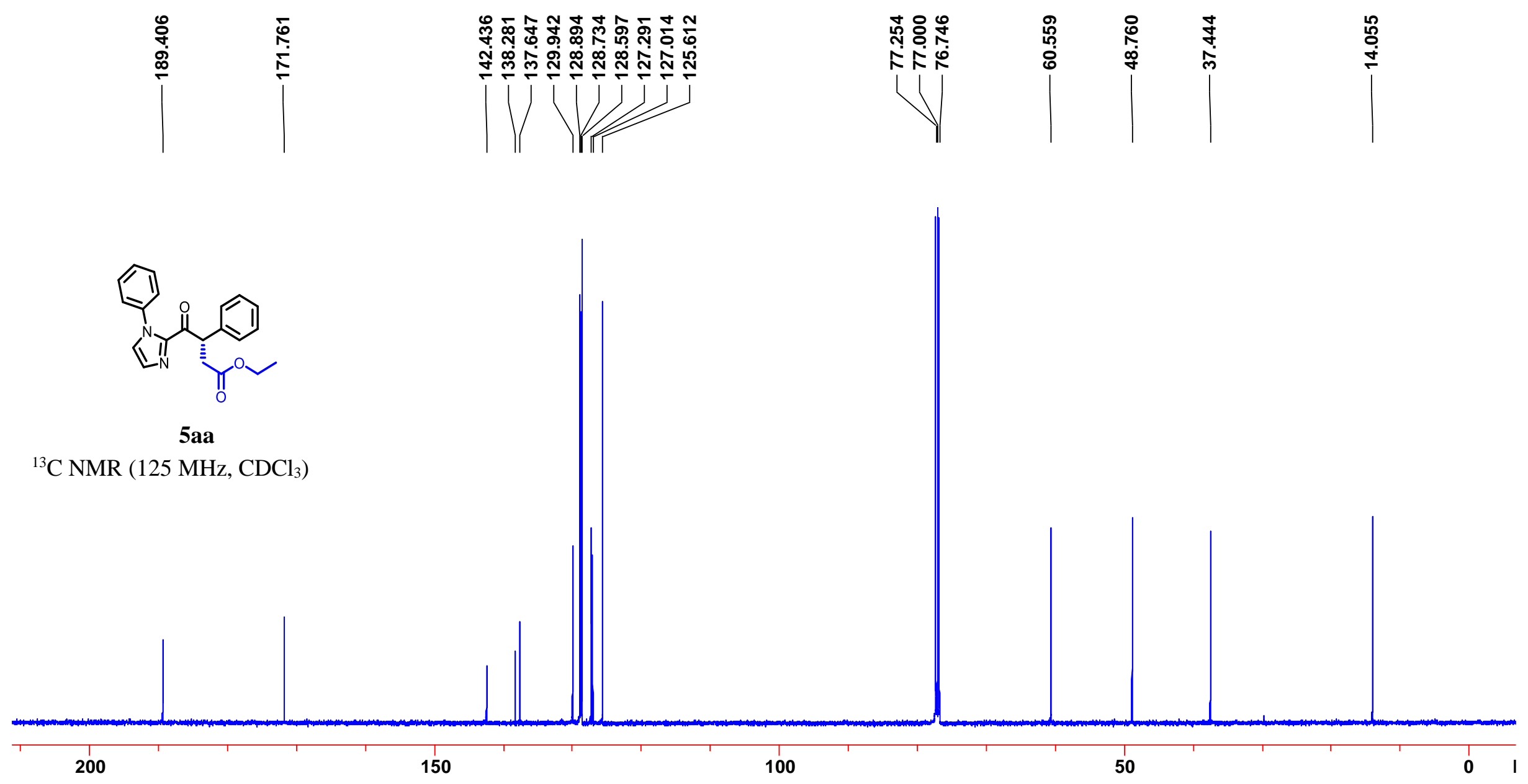



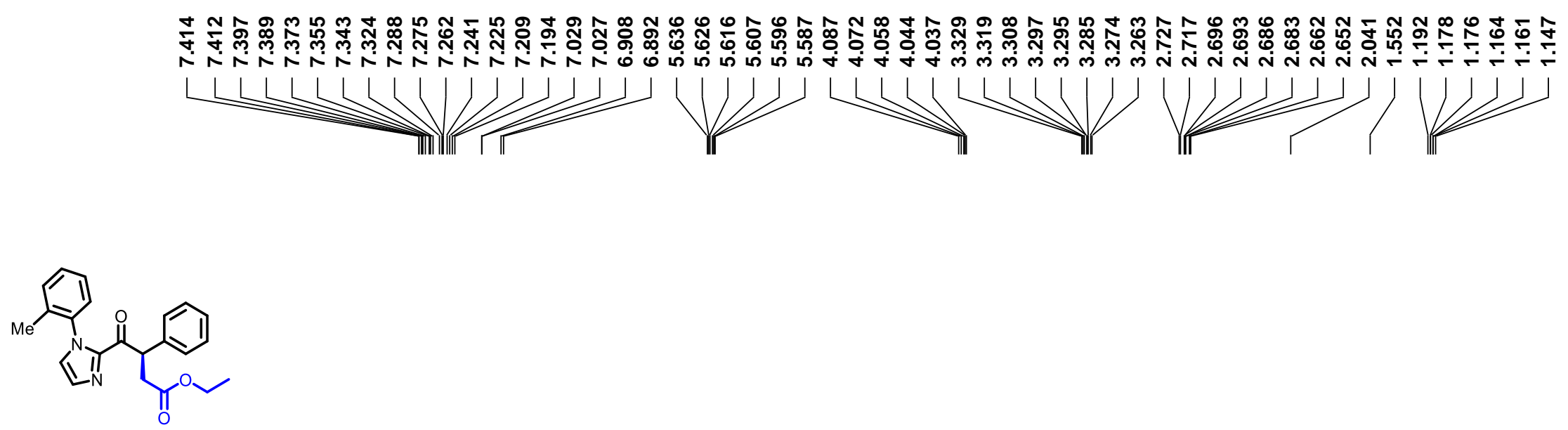

5 ba

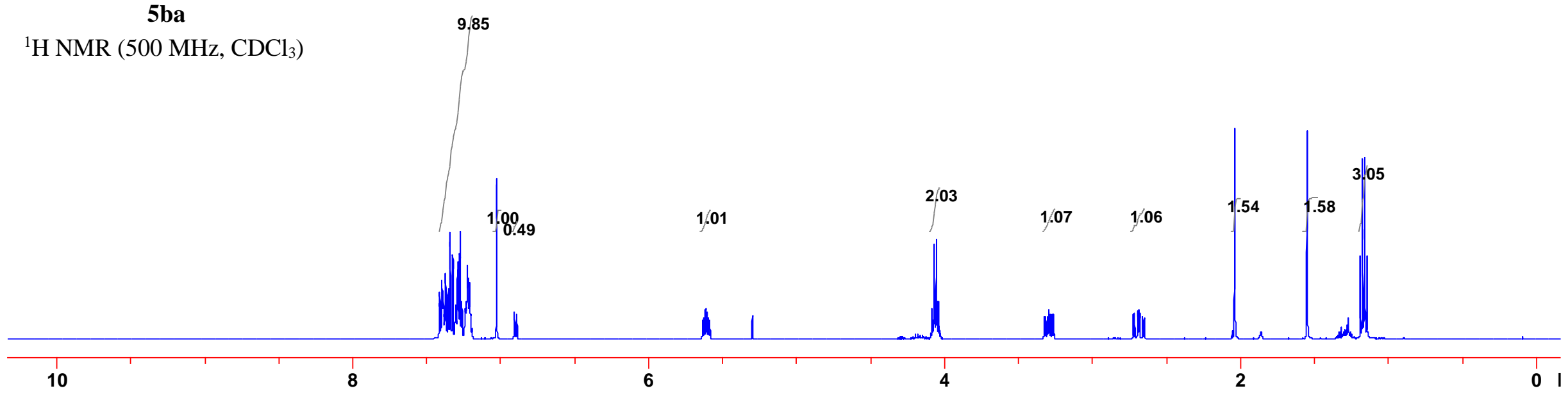




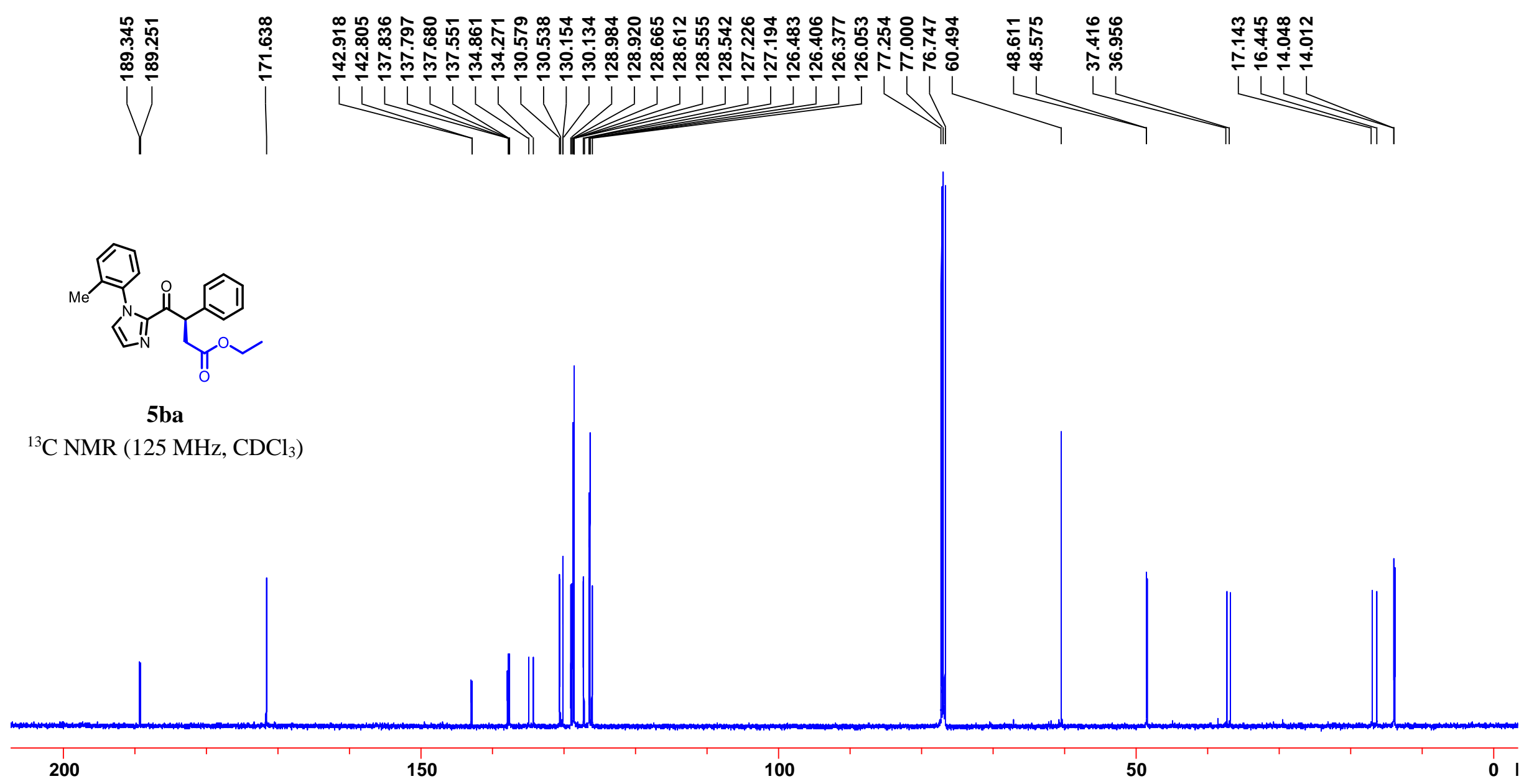




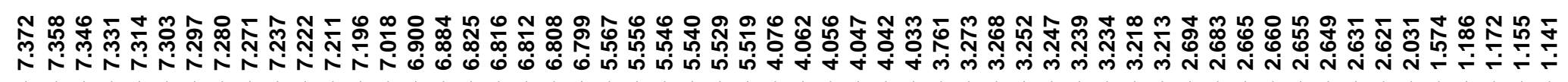

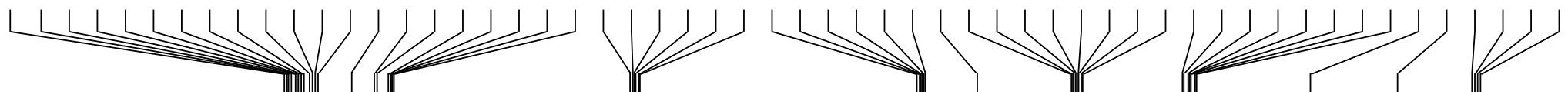

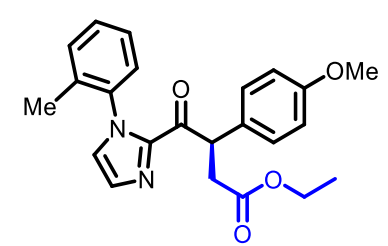

5la

${ }^{1} \mathrm{H} \mathrm{NMR}\left(500 \mathrm{MHz}, \mathrm{CDCl}_{3}\right)$

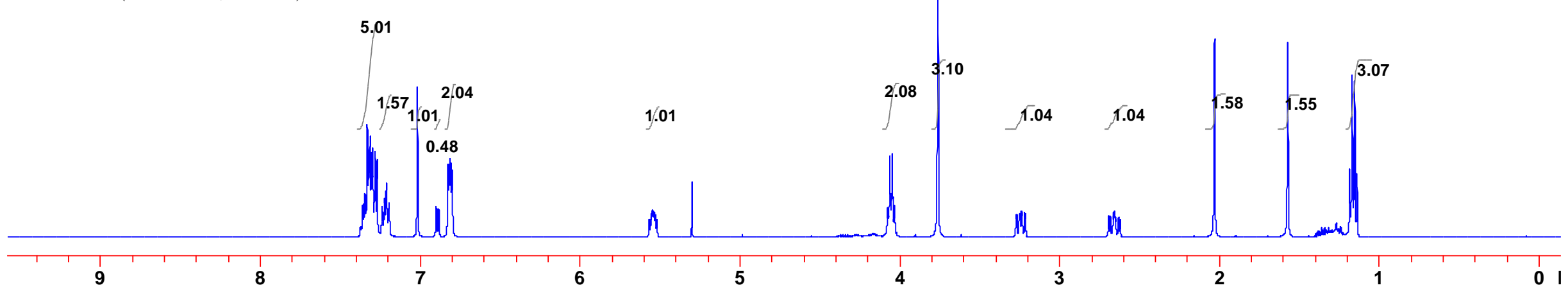



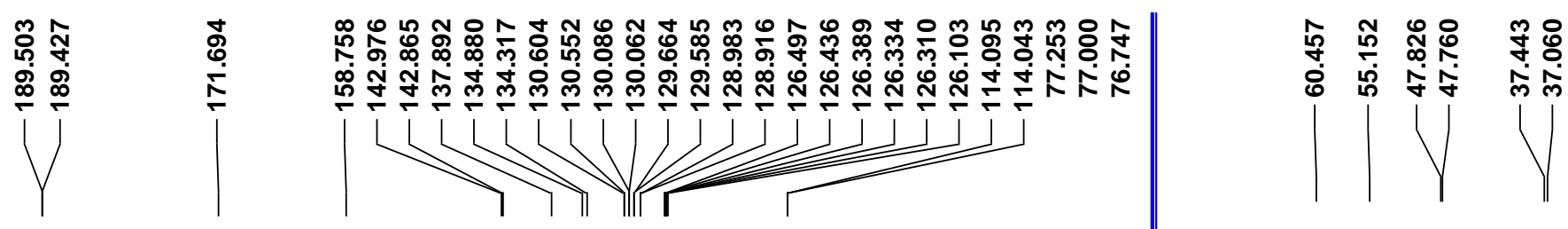

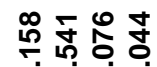

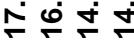

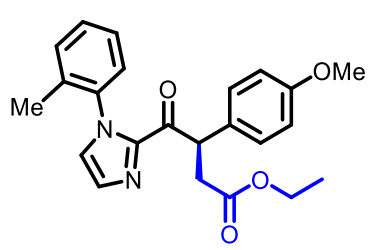

5la

${ }^{13} \mathrm{C}$ NMR (125 MHz, $\left.\mathrm{CDCl}_{3}\right)$ 


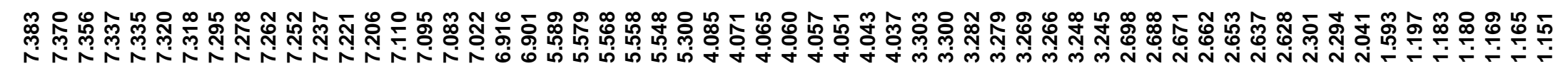

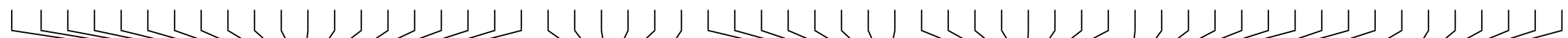

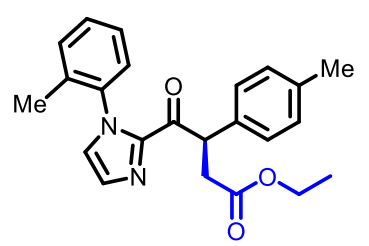

5 ja

${ }^{1} \mathrm{H}$ NMR $\left(500 \mathrm{MHz}, \mathrm{CDCl}_{3}\right)$

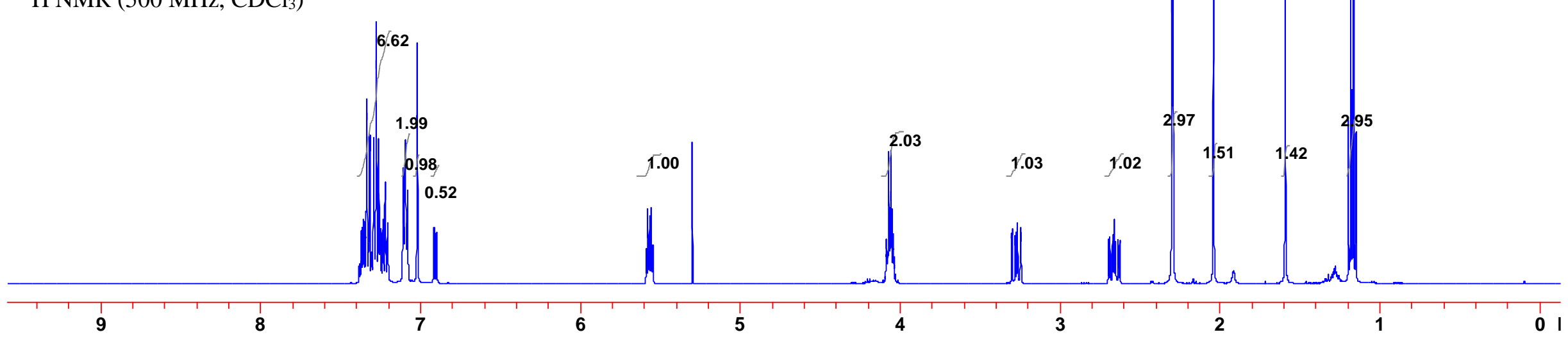


|⿱宀十九⿺

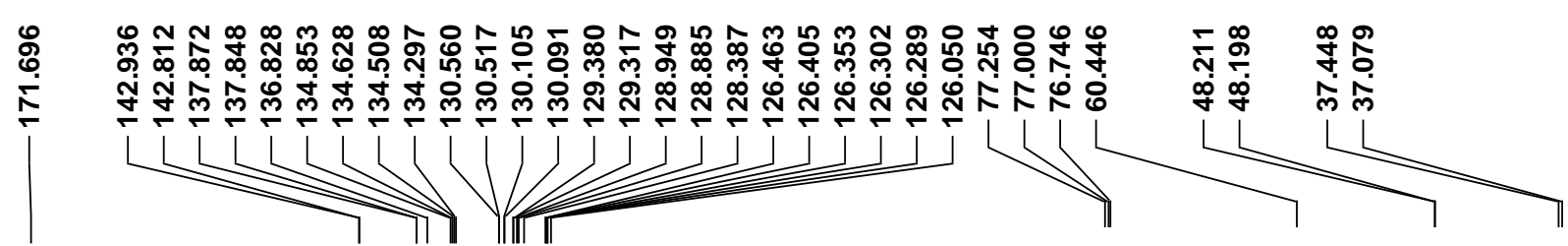

종을

舟덩

)

V

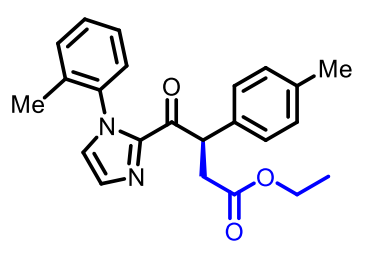

5ja

${ }^{13} \mathrm{C}$ NMR (125 MHz, $\mathrm{CDCl}_{3}$ )

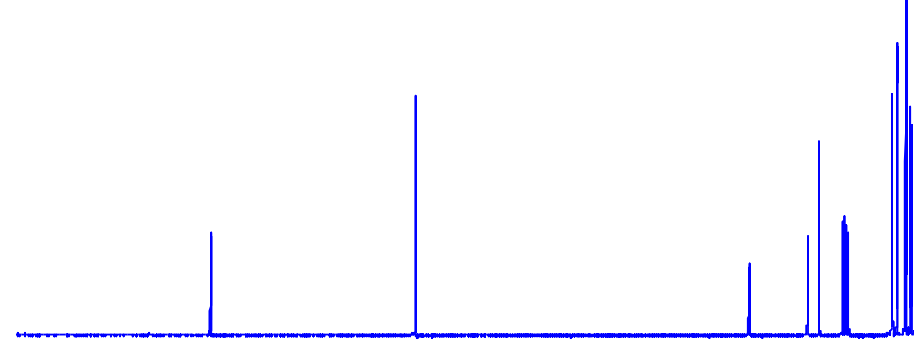

200

150

100

50

01 


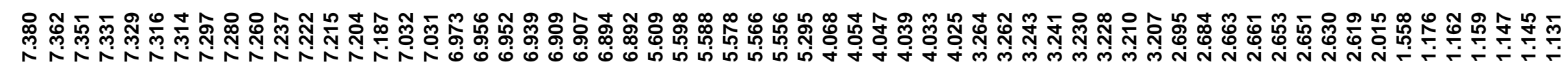

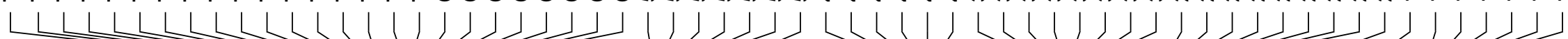

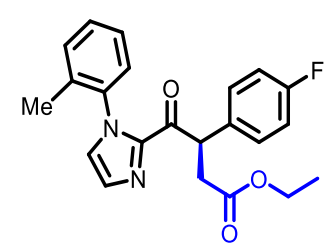

$5 \mathrm{ma}$

${ }^{1} \mathrm{H} \mathrm{NMR}\left(500 \mathrm{MHz}, \mathrm{CDCl}_{3}\right)$

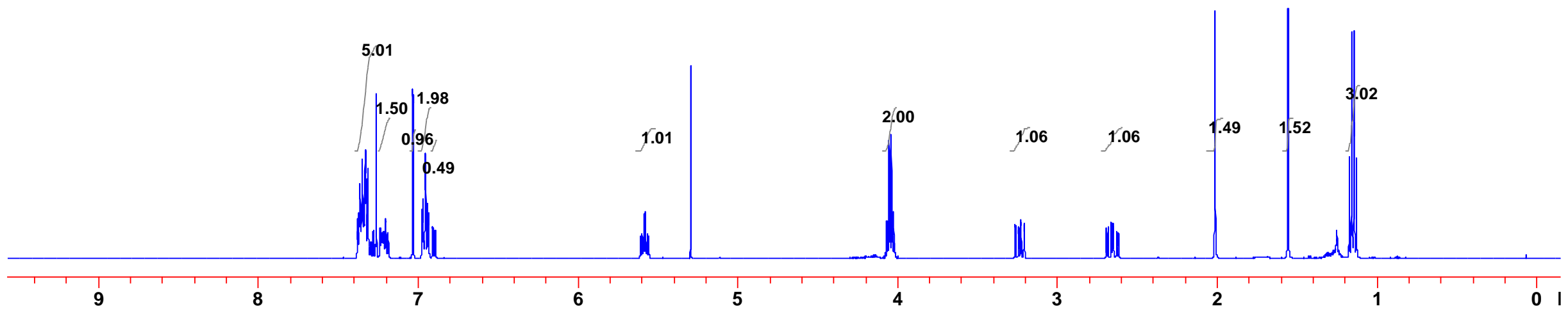




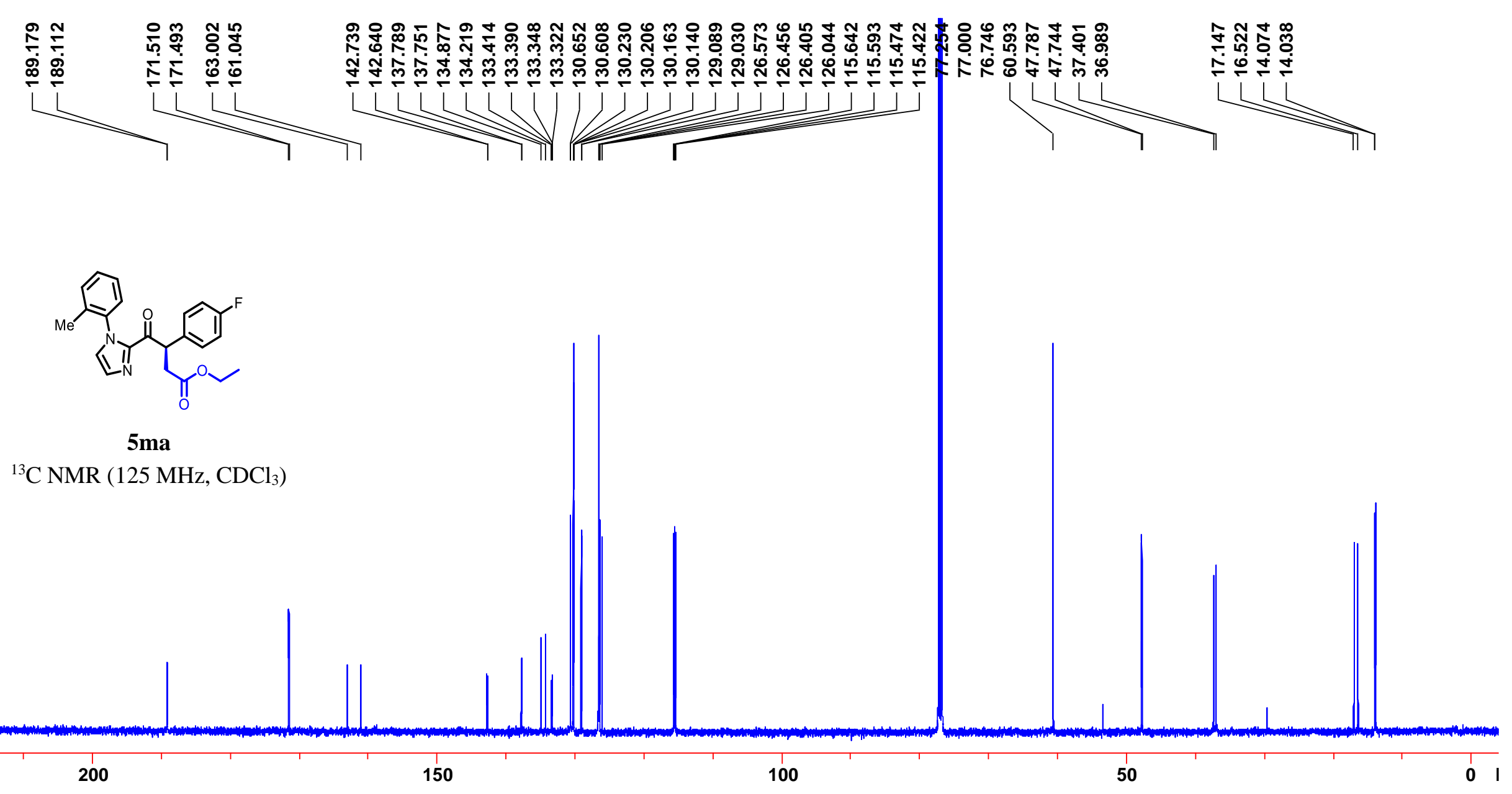


议

西

j

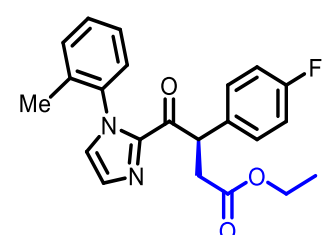

$5 \mathrm{ma}$

${ }^{19} \mathrm{~F} \mathrm{NMR} \mathrm{(282} \mathrm{MHz,} \mathrm{CDCl}_{3}$ )

1.00

$-120$

$-125$ 


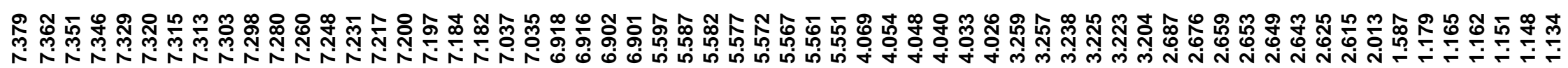

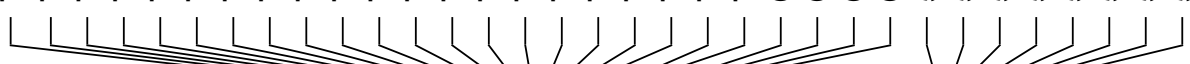
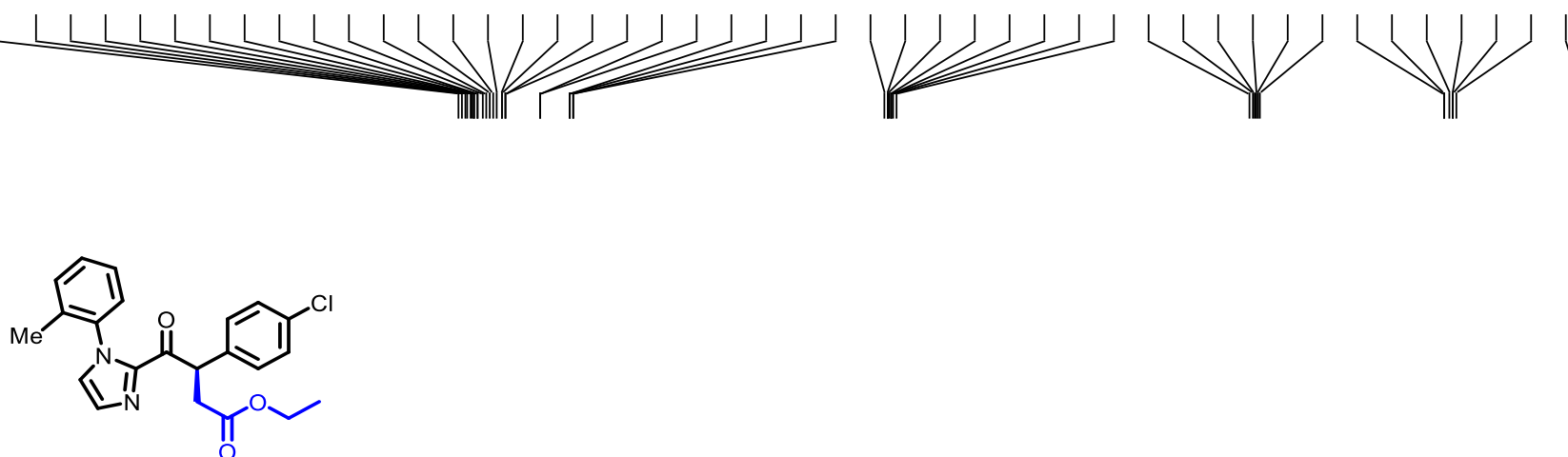

5na

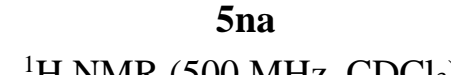

${ }^{1} \mathrm{H} \mathrm{NMR}\left(500 \mathrm{MHz}, \mathrm{CDCl}_{3}\right)$

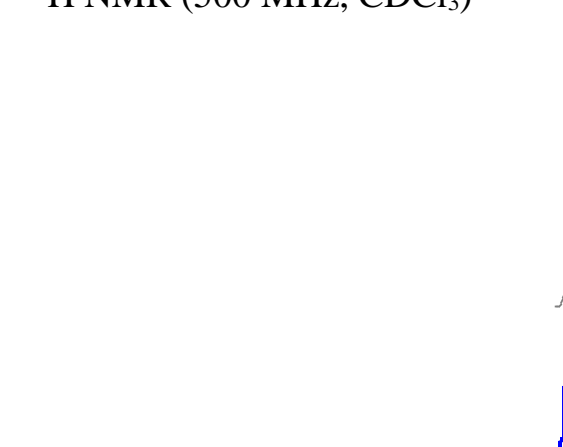

8
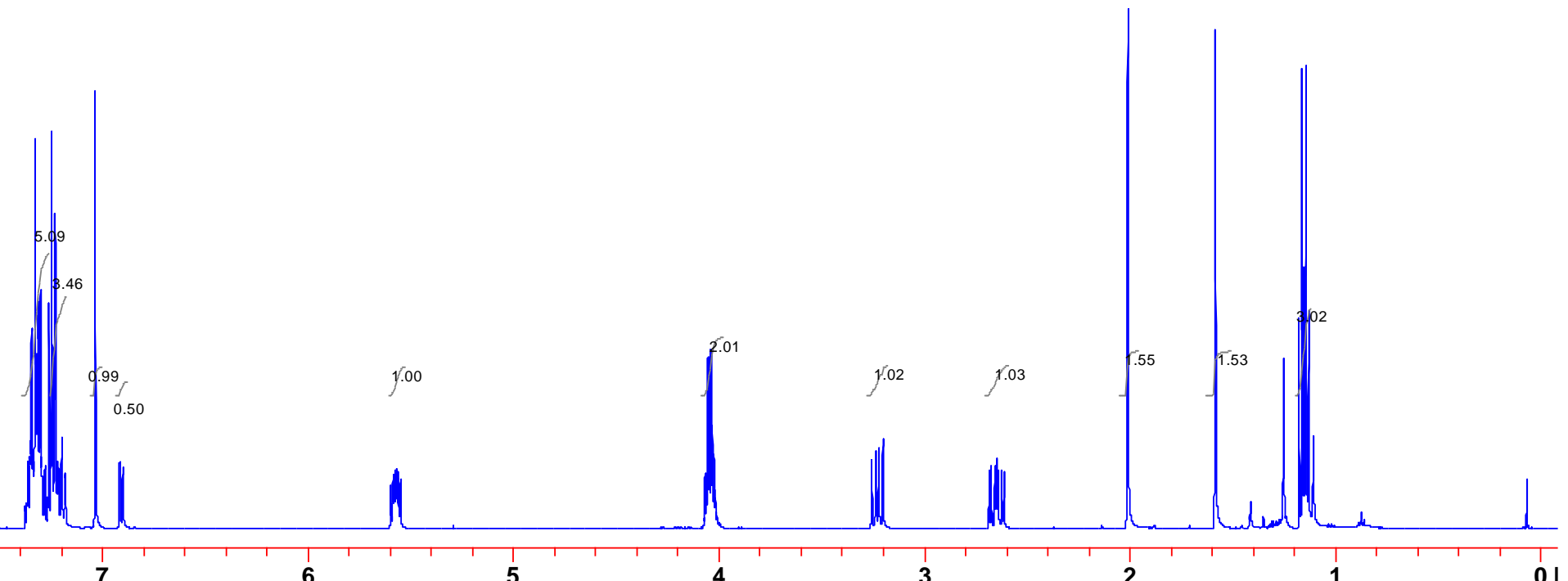

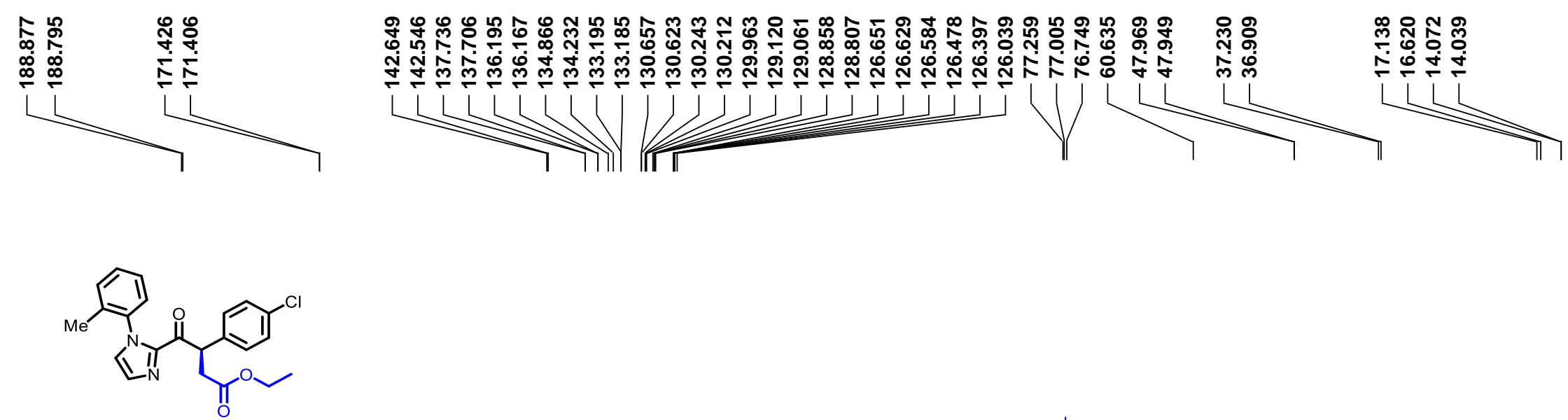

5na

${ }^{13} \mathrm{C}$ NMR (125 MHz, $\mathrm{CDCl}_{3}$ )

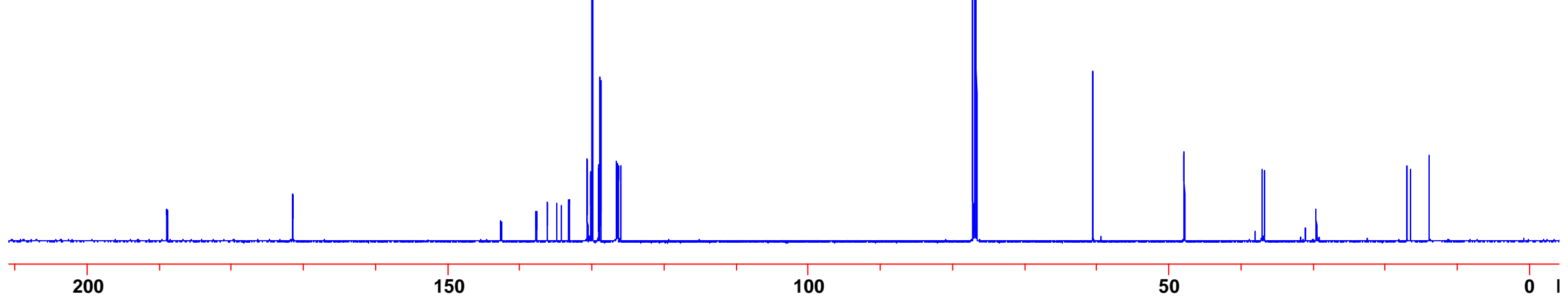




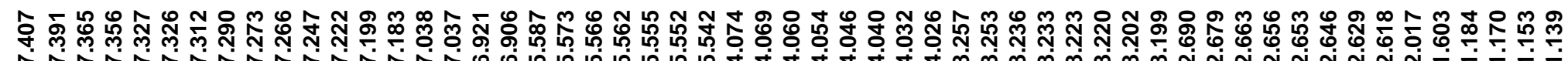

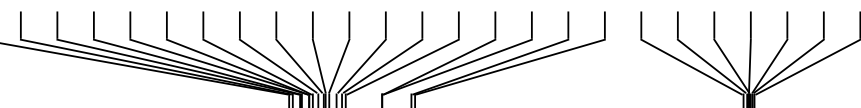
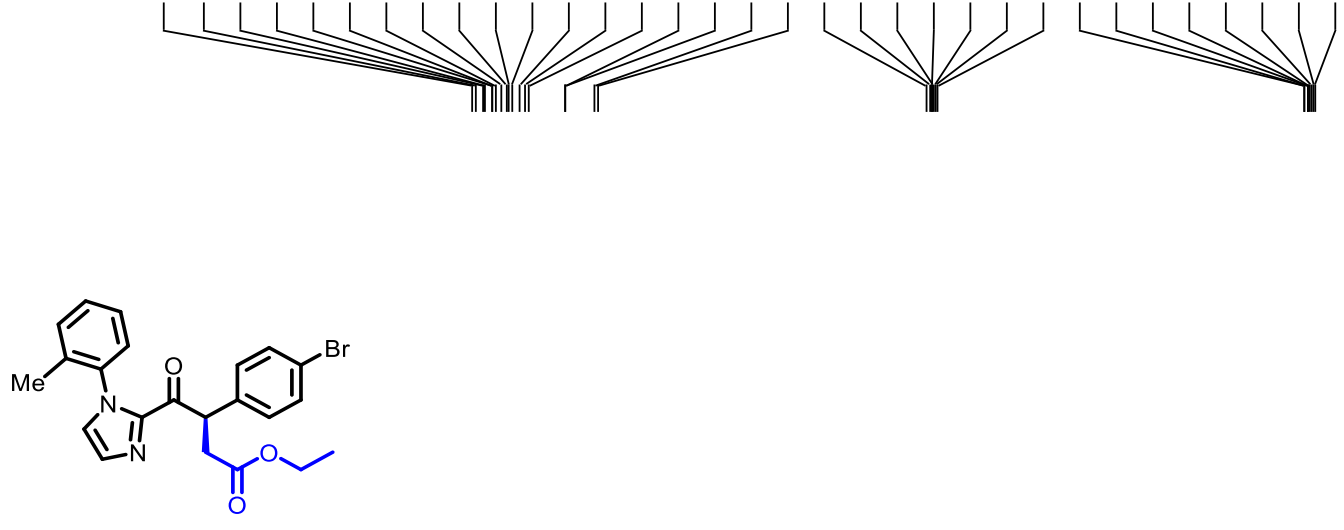

$50 a$

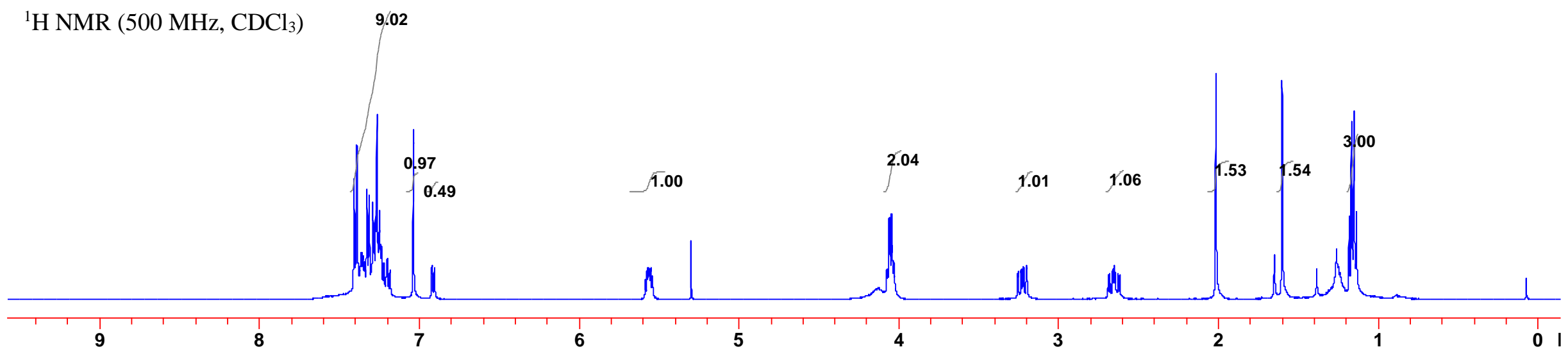




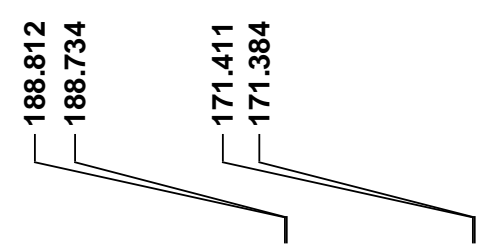

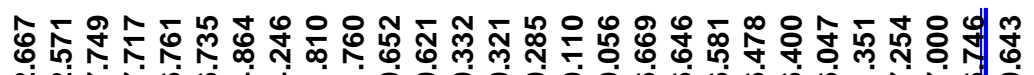

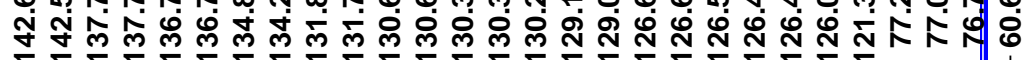
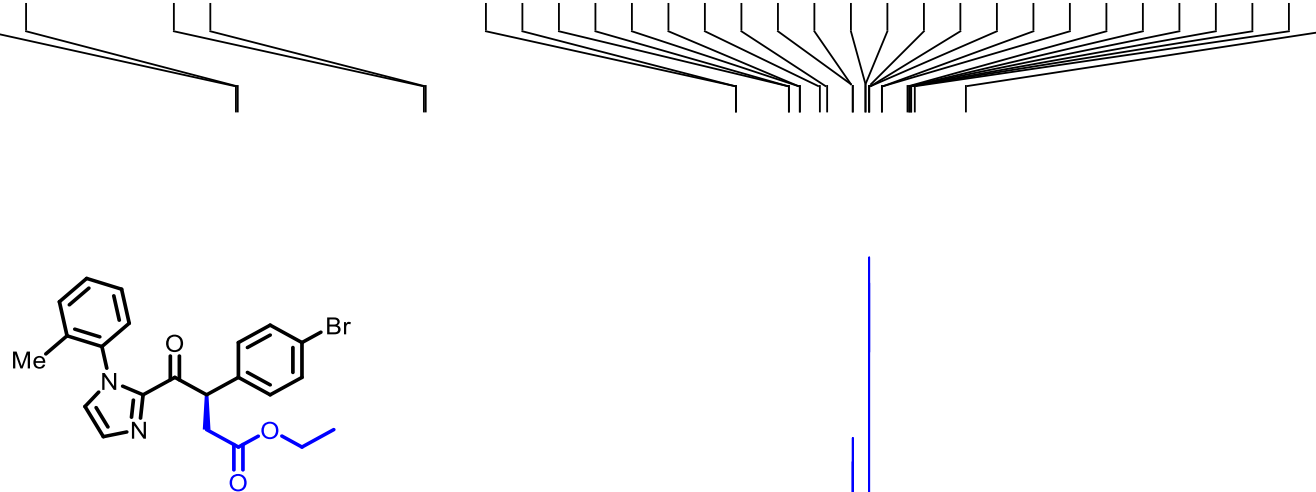

$50 a$

${ }^{13} \mathrm{C}$ NMR (125 MHz, $\left.\mathrm{CDCl}_{3}\right)$

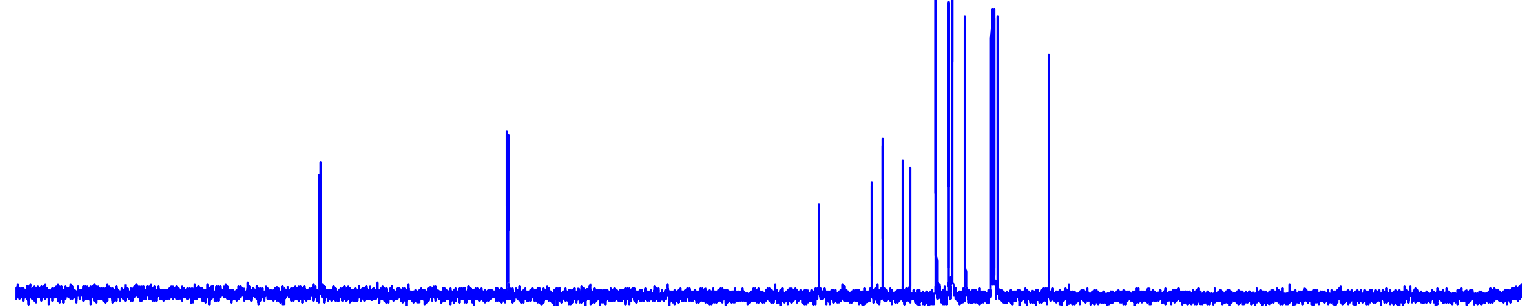



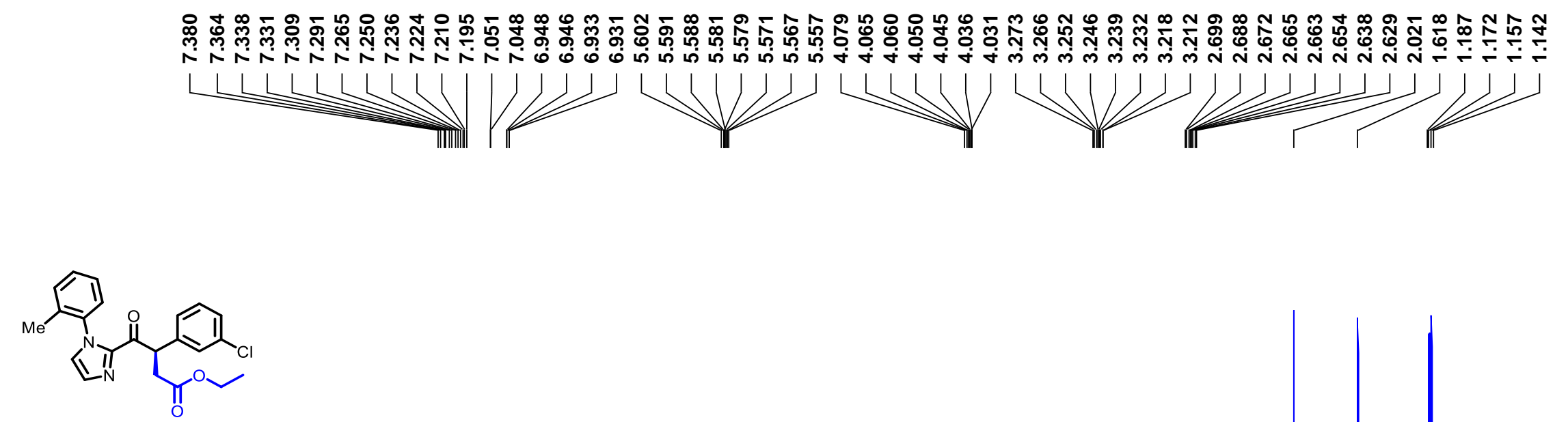

5 ka

${ }^{1} \mathrm{H}$ NMR $\left(500 \mathrm{MHz}, \mathrm{CDCl}_{3}\right)$
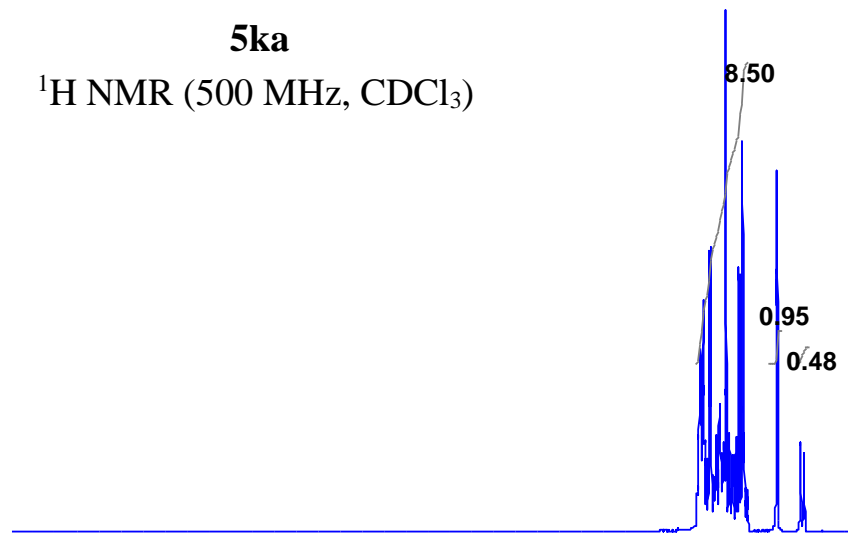

8

6

4

2 


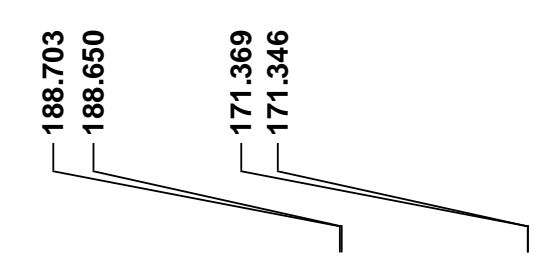

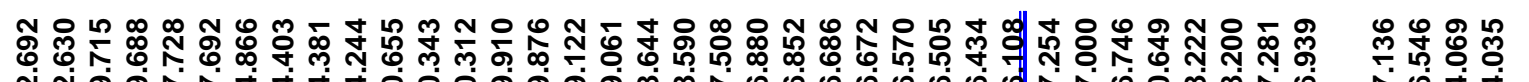

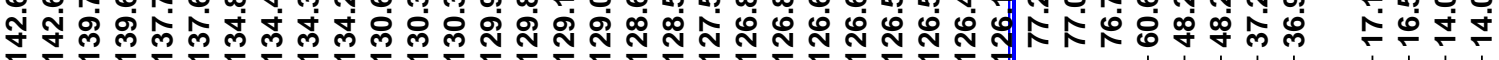
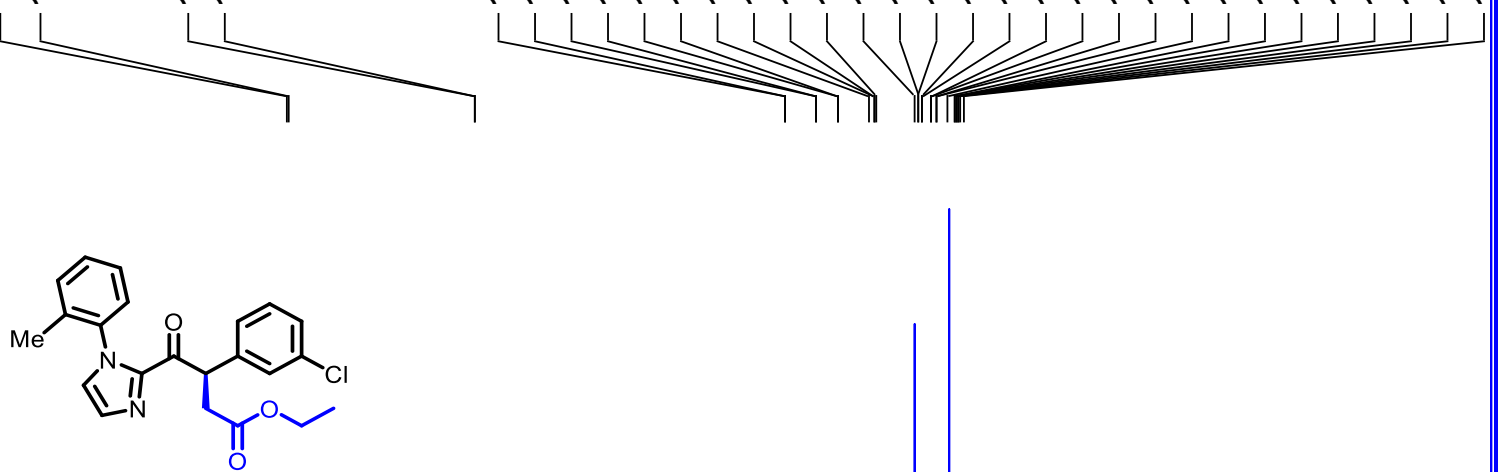

5ka

${ }^{13} \mathrm{C}$ NMR $\left(125 \mathrm{MHz}, \mathrm{CDCl}_{3}\right)$

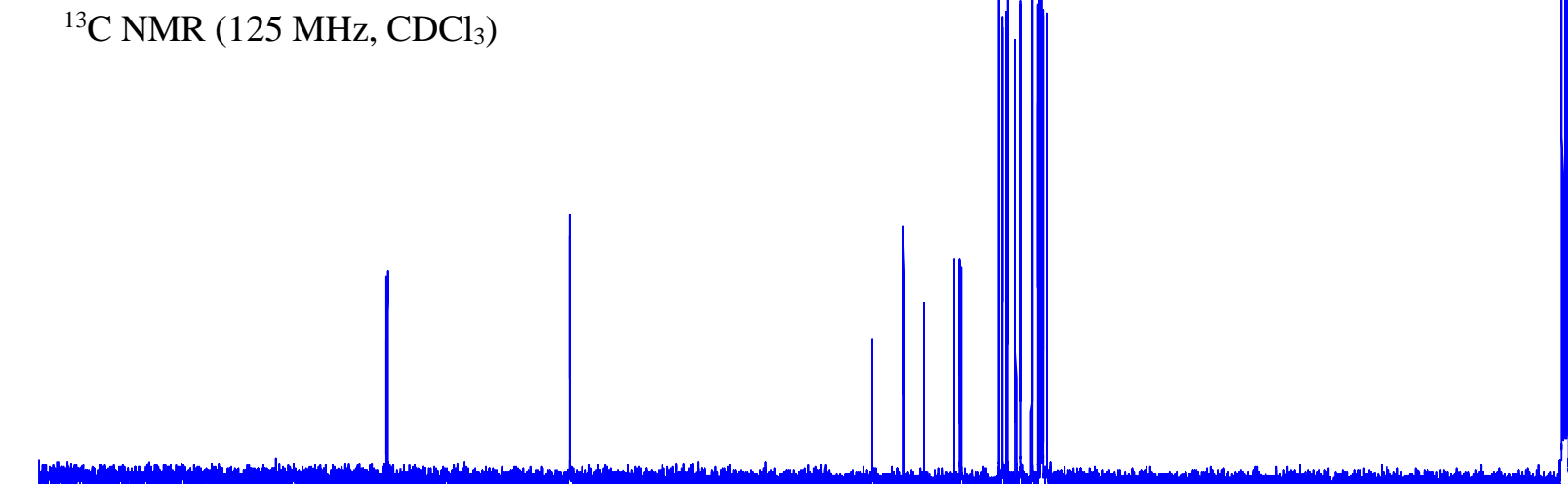




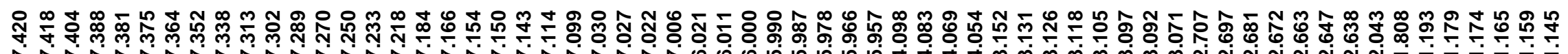
1IIIIIII

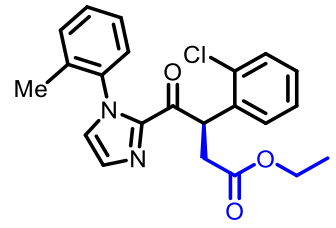

5 pa

${ }^{1} \mathrm{H}$ NMR (500 MHz, $\mathrm{CDCl}_{3}$ )

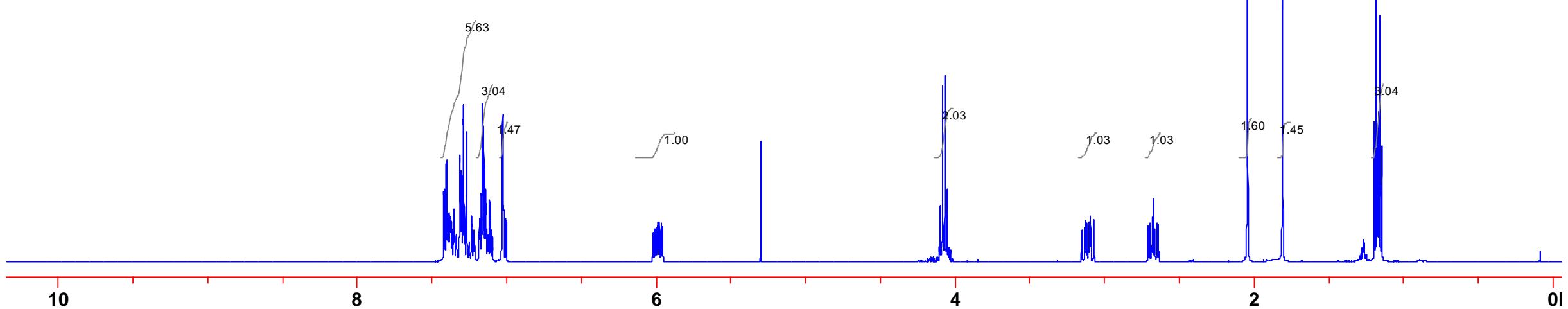




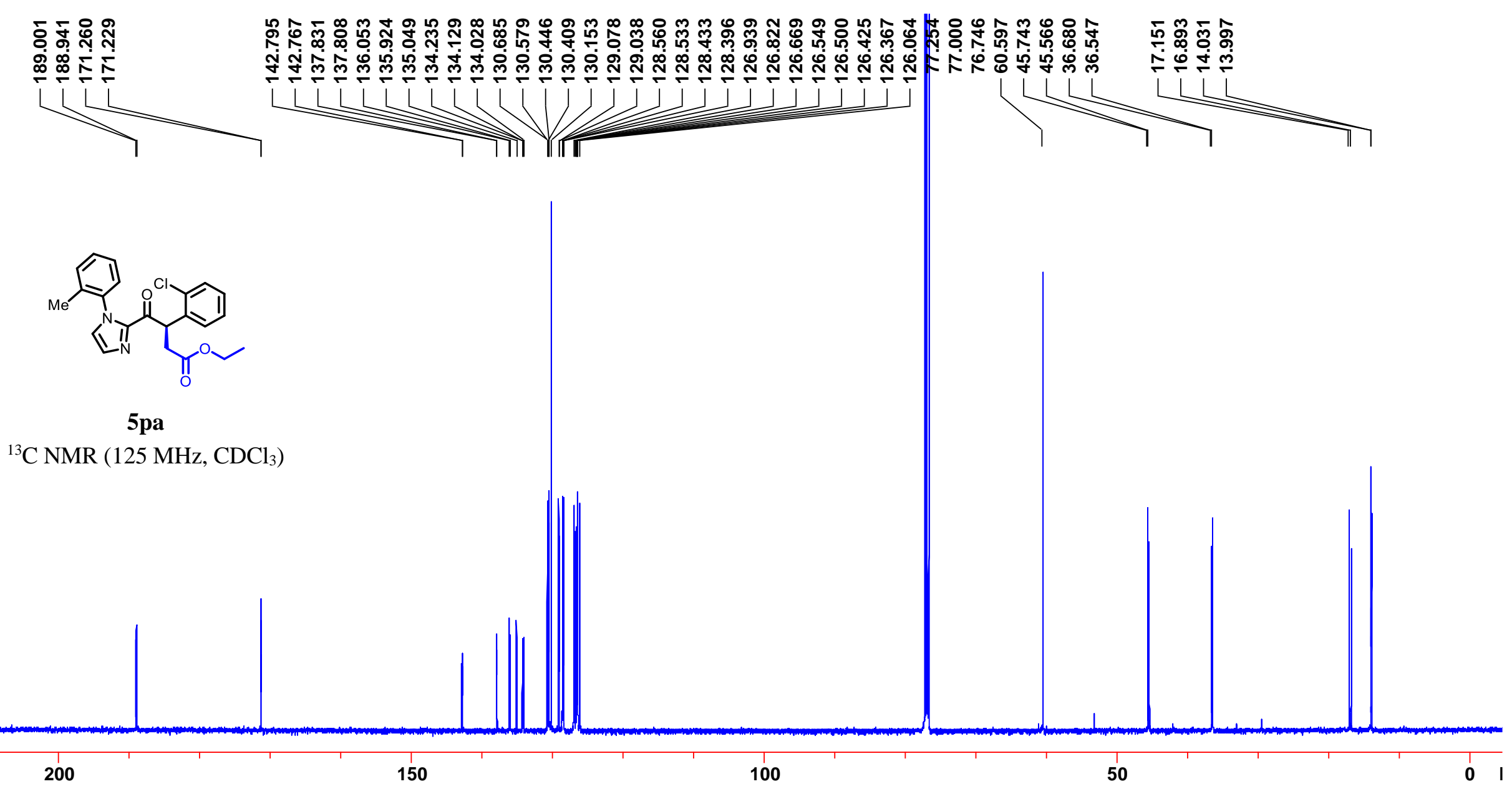




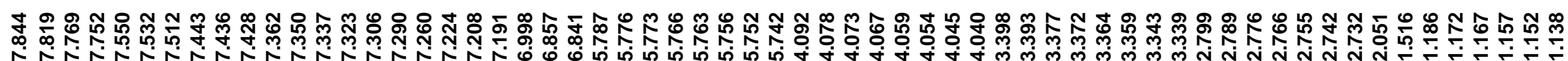
(n)

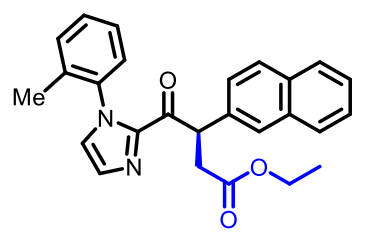

5qa

${ }^{1} \mathrm{H} \mathrm{NMR}\left(500 \mathrm{MHz}, \mathrm{CDCl}_{3}\right)$

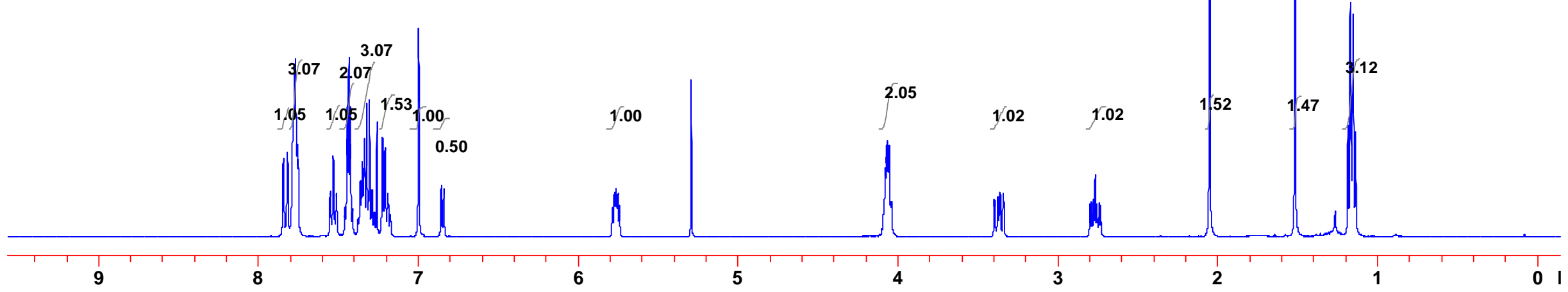



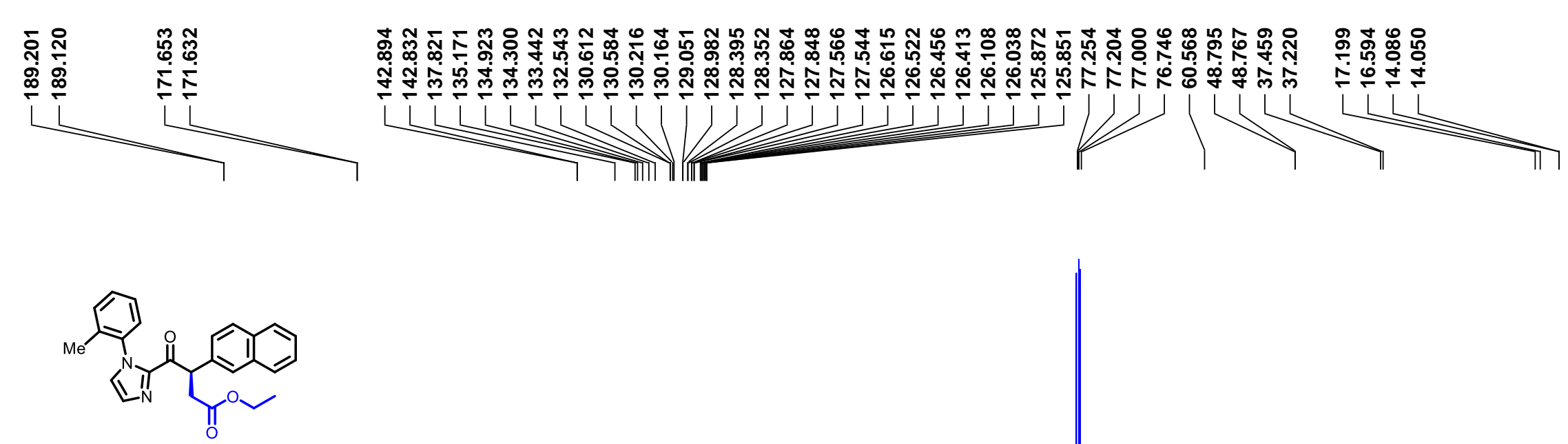

$5 q \mathbf{a}$

${ }^{13} \mathrm{C} \mathrm{NMR}\left(125 \mathrm{MHz}, \mathrm{CDCl}_{3}\right)$

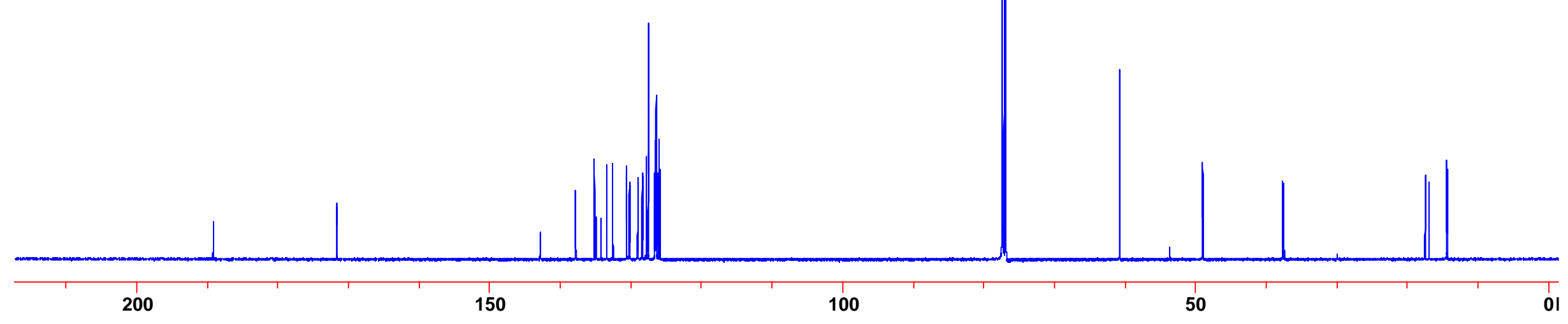




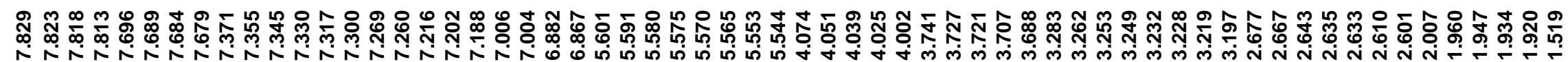

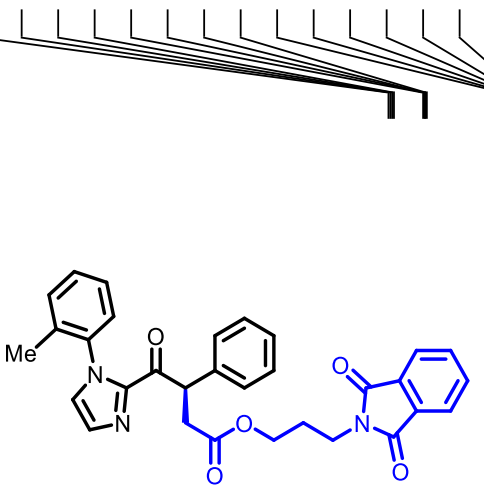

5bb

${ }^{1} \mathrm{H}$ NMR (500 MHz, $\mathrm{CDCl}_{3}$ )

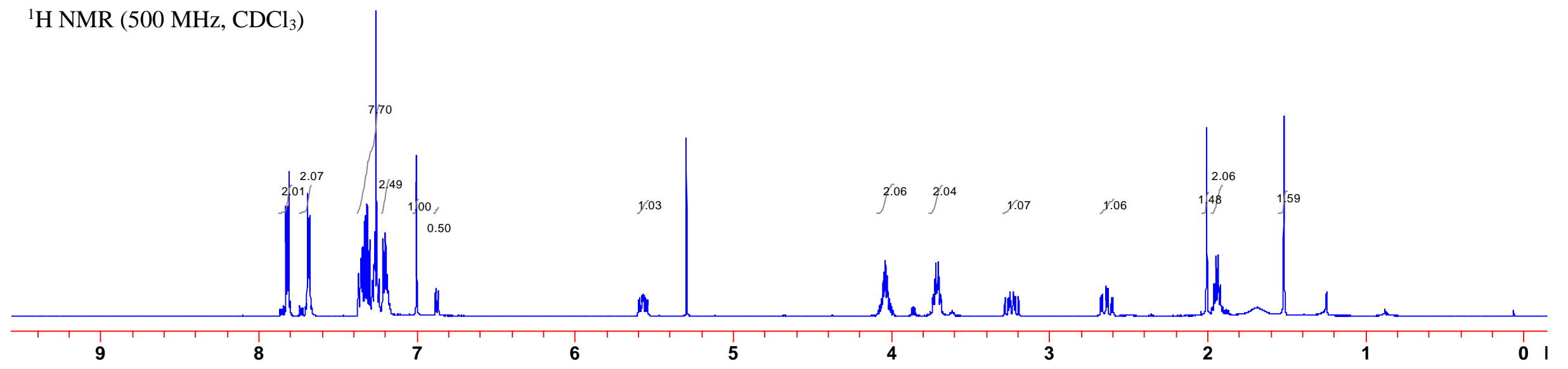



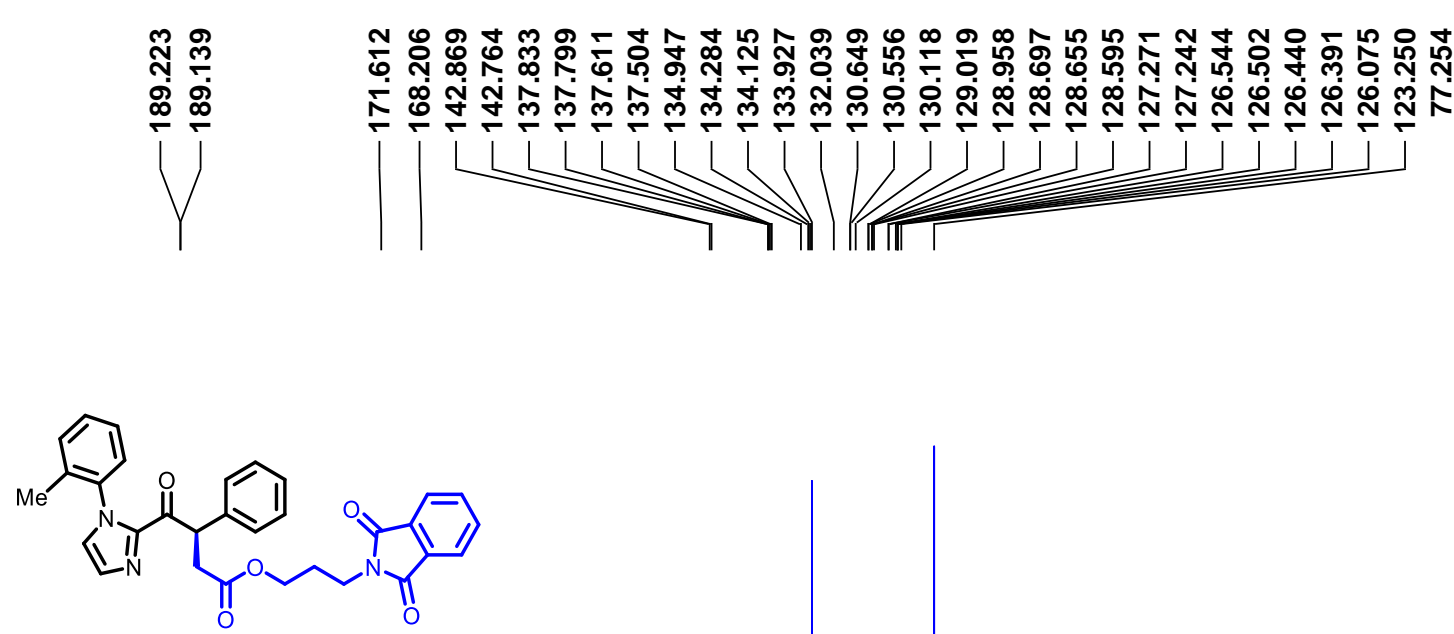

5 bb

${ }^{13} \mathrm{C}$ NMR (125 MHz, $\mathrm{CDCl}_{3}$ ) 


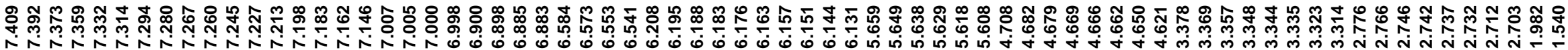

LLLL L LLLLLLLLLLLLLLLLLLLLLLLL」L L L L |ाIIIIIII |
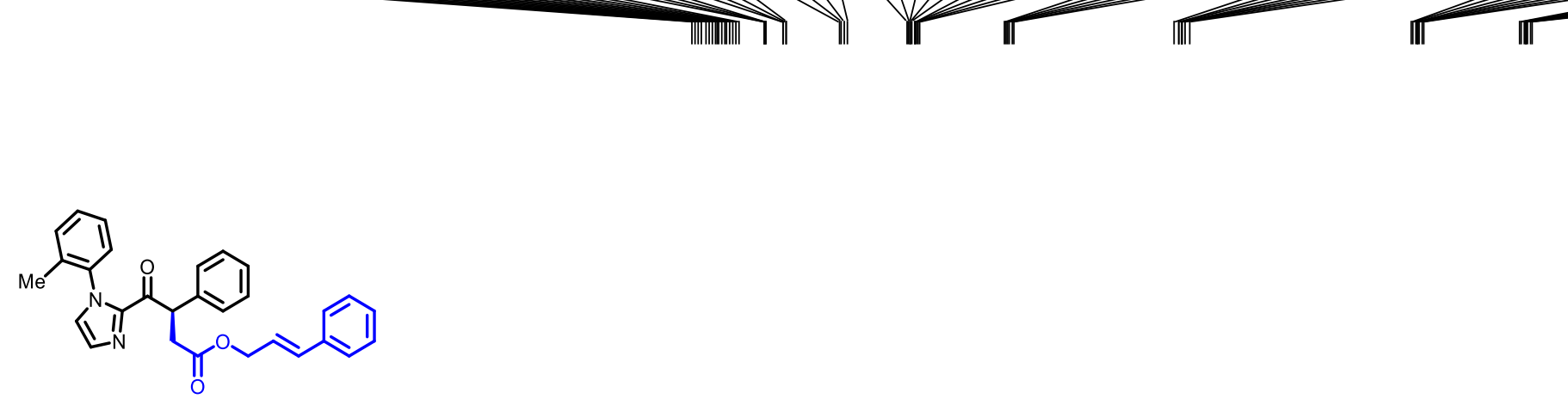

$5 \mathbf{b c}$

${ }^{1} \mathrm{H}$ NMR $\left(500 \mathrm{MHz}, \mathrm{CDCl}_{3}\right)$

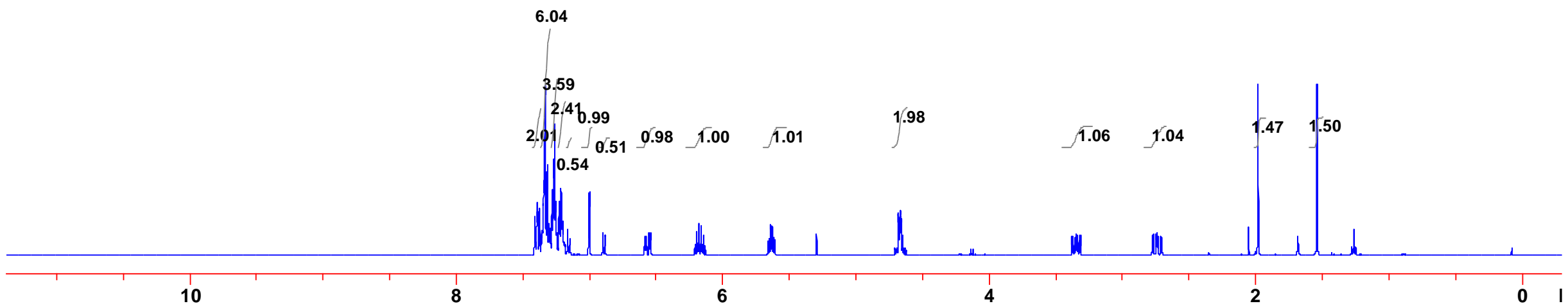




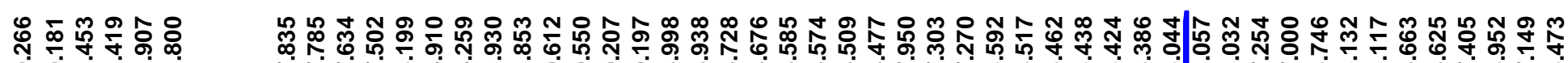

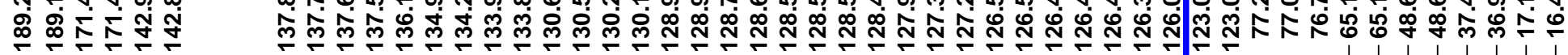

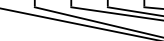
7
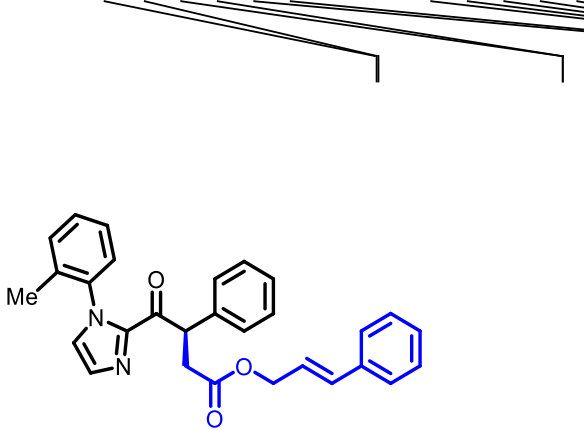

$5 \mathbf{b c}$

${ }^{13} \mathrm{C}$ NMR (125 MHz, $\left.\mathrm{CDCl}_{3}\right)$

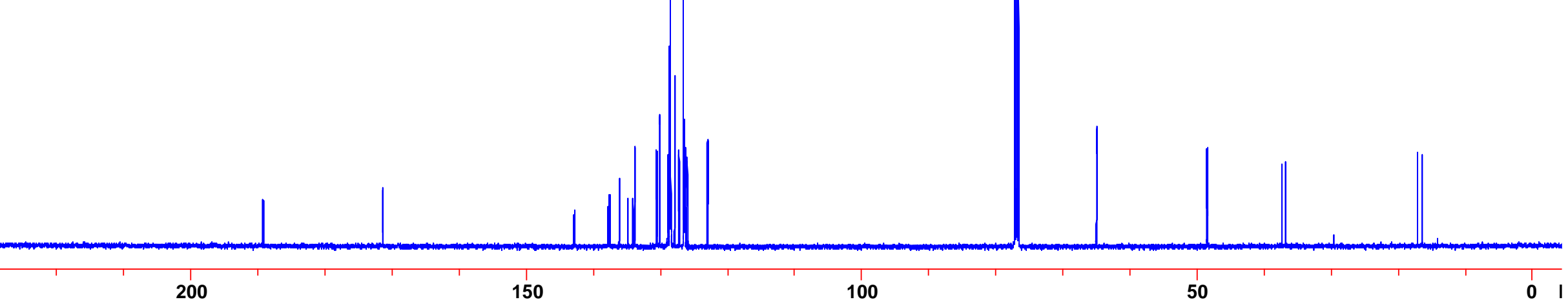




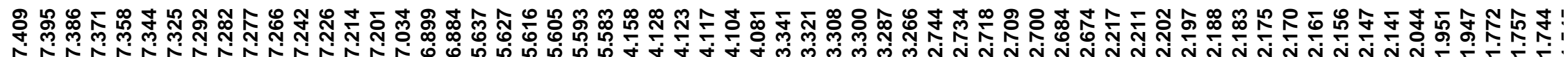

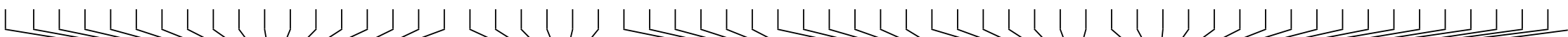
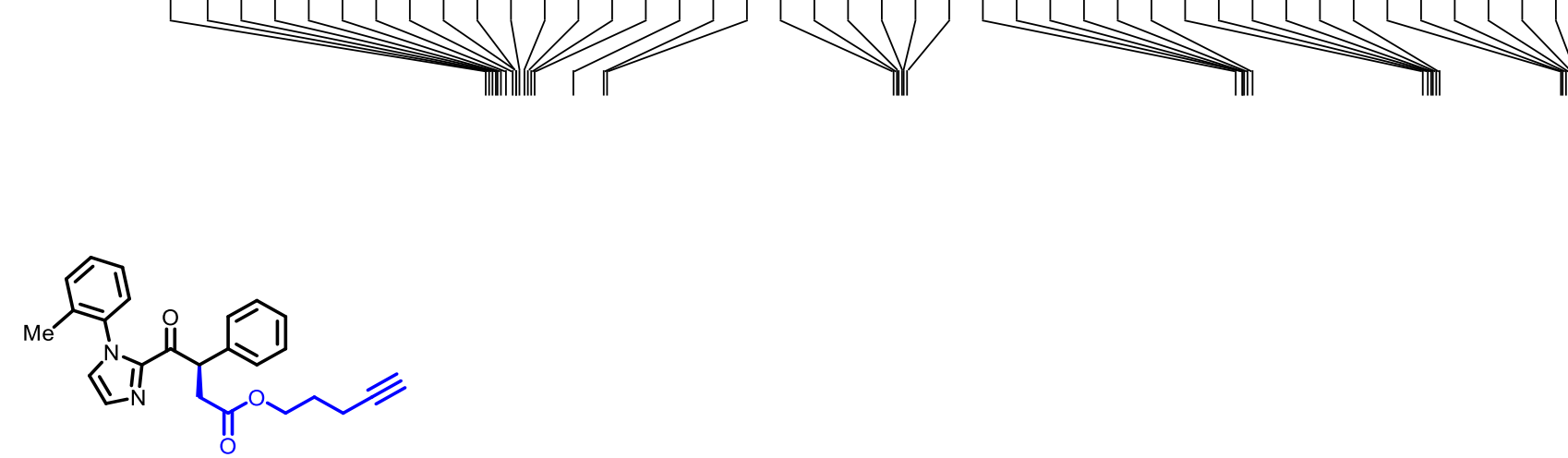

5bd

${ }^{1} \mathrm{H}$ NMR $\left(500 \mathrm{MHz}, \mathrm{CDCl}_{3}\right)$

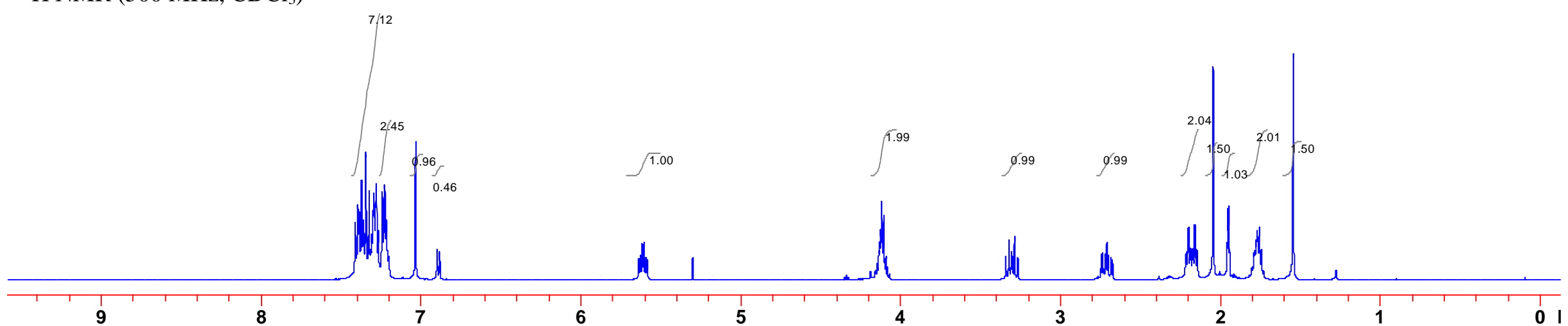




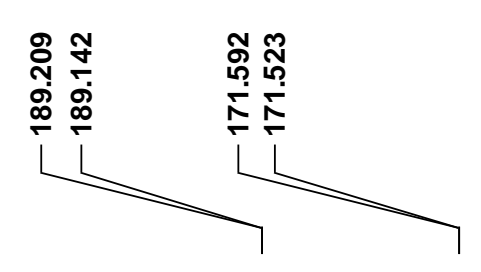

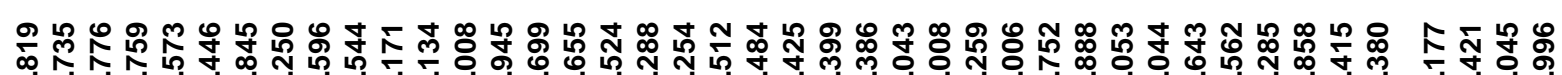

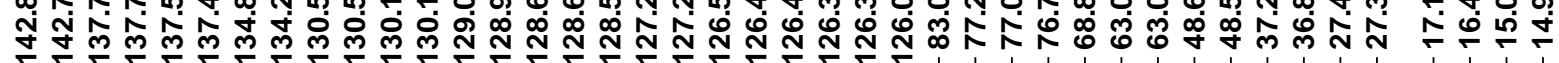

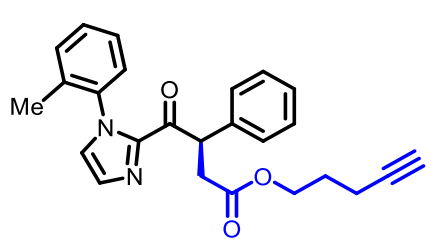

5bd

${ }^{13} \mathrm{C}$ NMR (125 MHz, $\mathrm{CDCl}_{3}$ )

150

100

50 


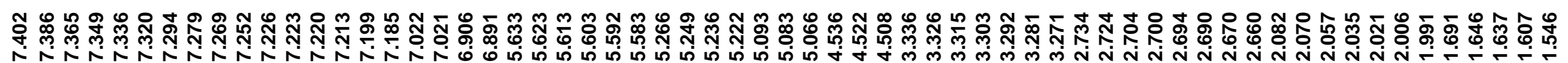
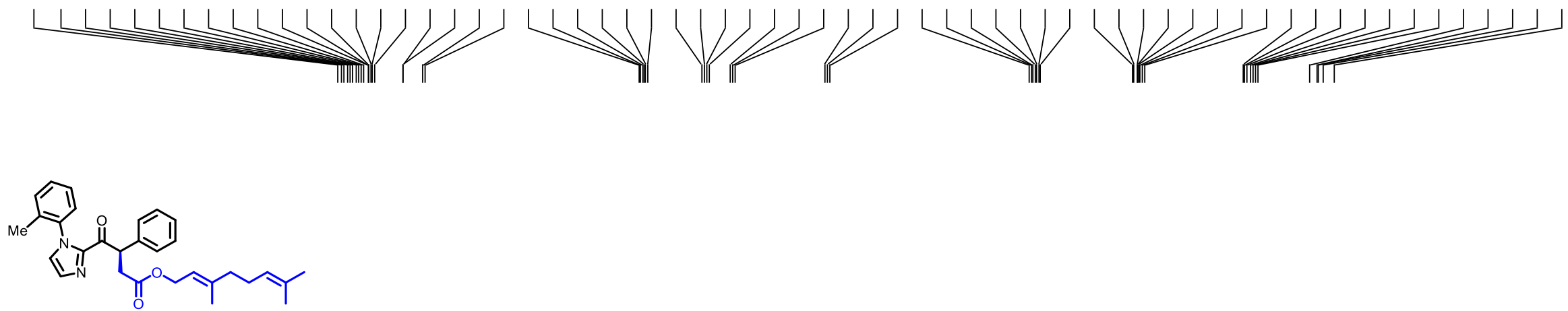

5be

${ }^{1} \mathrm{H} \mathrm{NMR}\left(500 \mathrm{MHz}, \mathrm{CDCl}_{3}\right.$ )

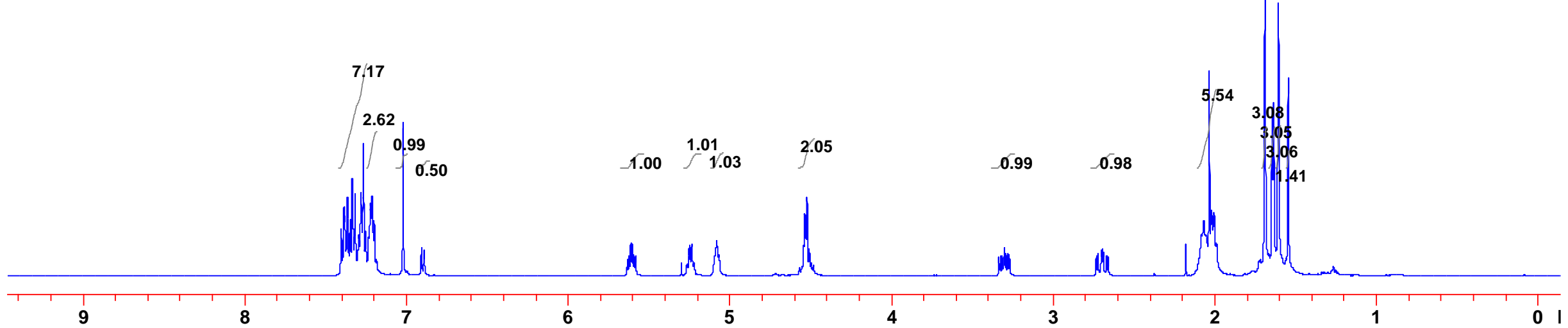




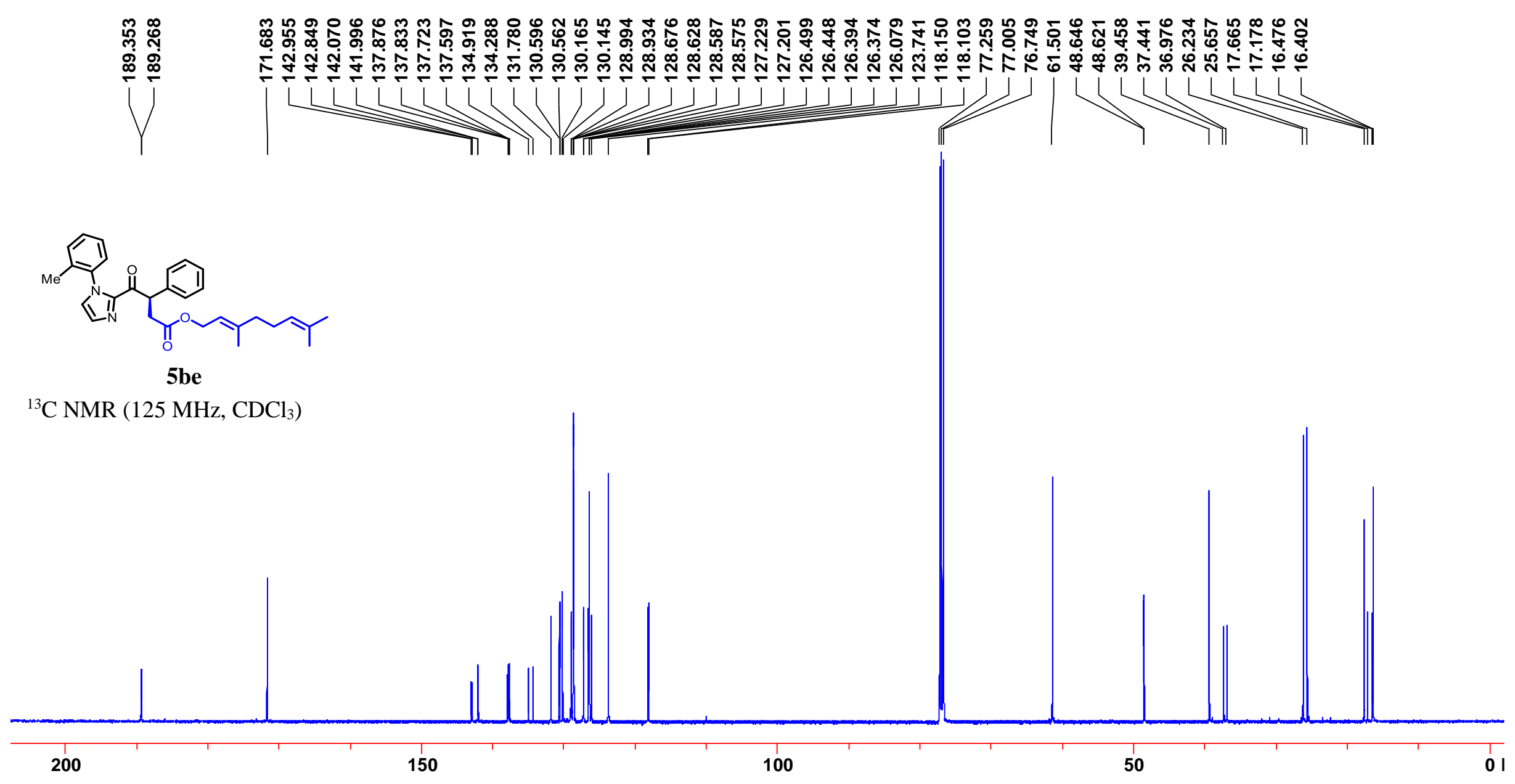




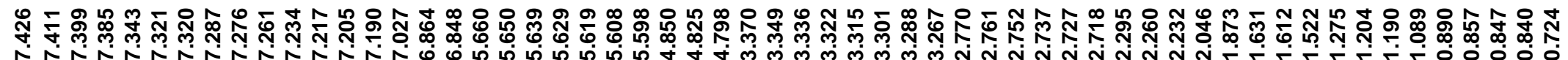

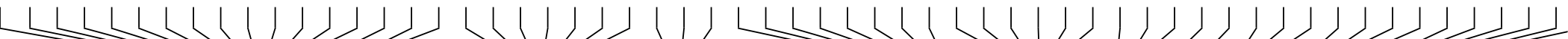

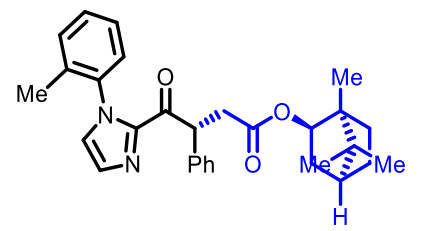

$5 b f$

${ }^{1} \mathrm{H} \mathrm{NMR}\left(500 \mathrm{MHz}, \mathrm{CDCl}_{3}\right)$

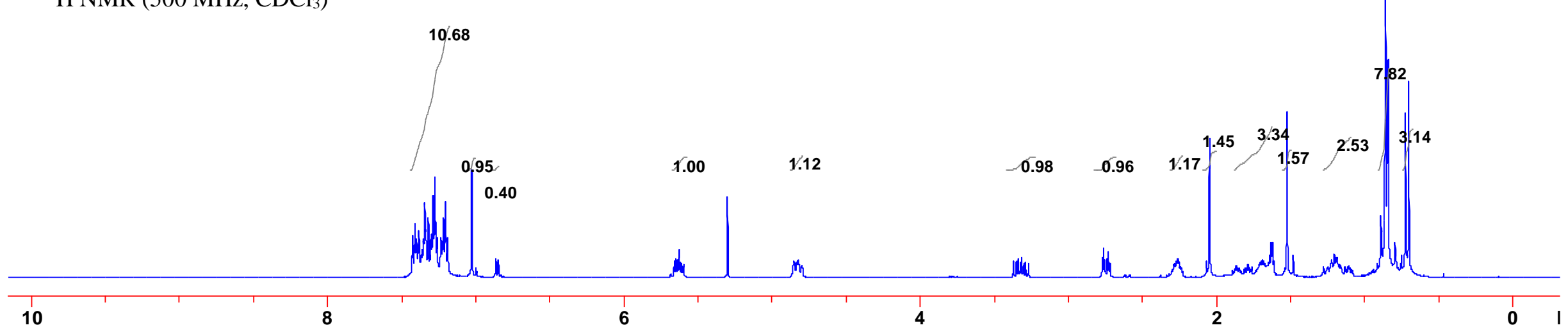



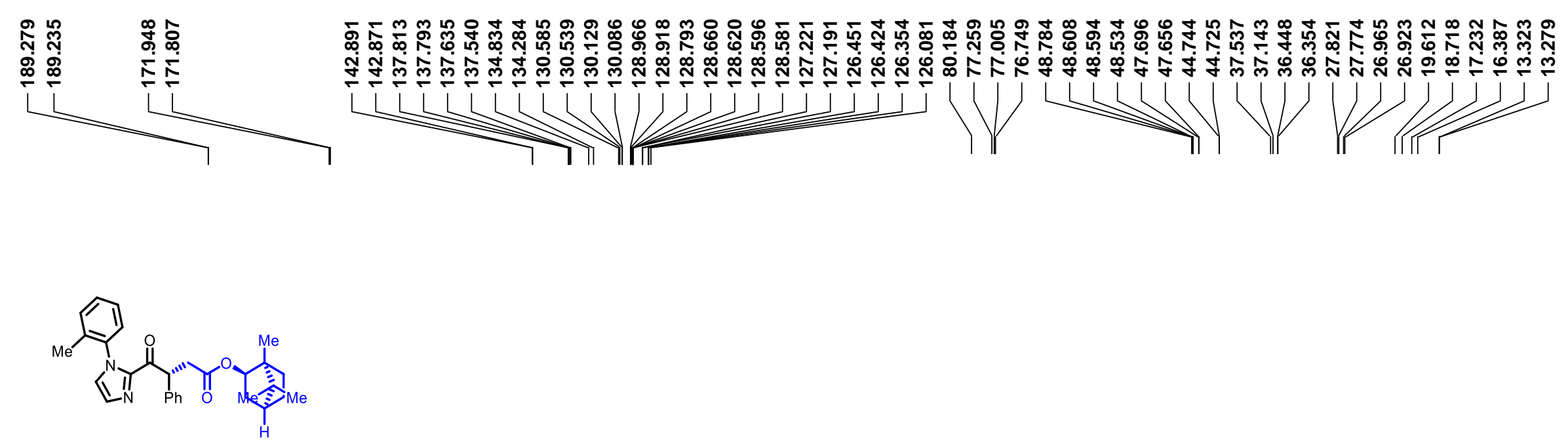

5 bf

${ }^{13} \mathrm{C}$ NMR (125 MHz, $\left.\mathrm{CDCl}_{3}\right)$

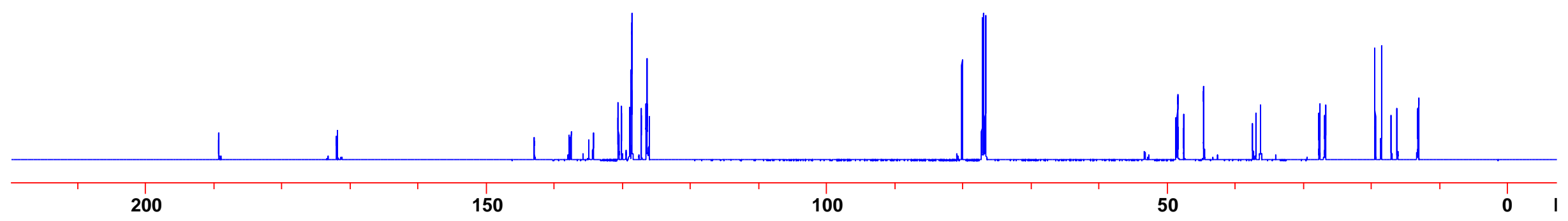




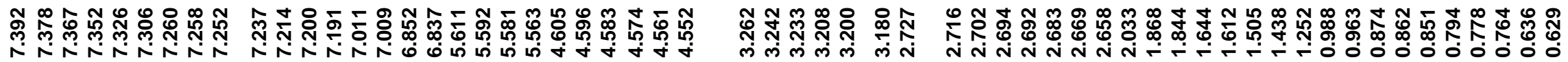

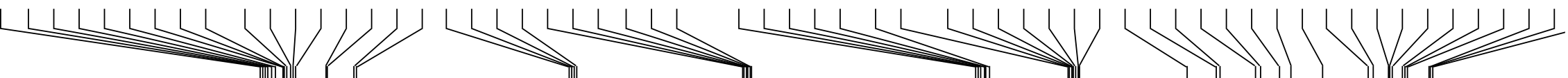

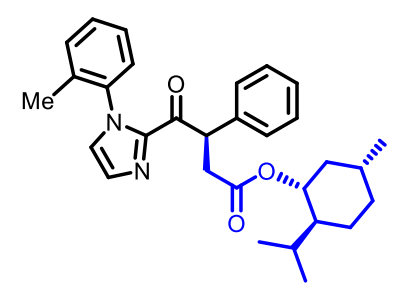

5 bg

${ }^{1} \mathrm{H} \mathrm{NMR}\left(500 \mathrm{MHz}, \mathrm{CDCl}_{3}\right)$

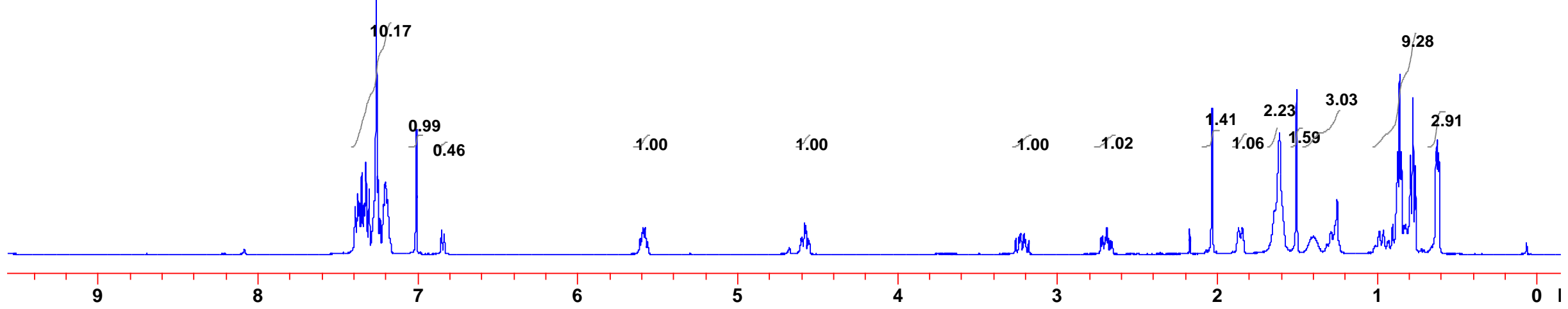




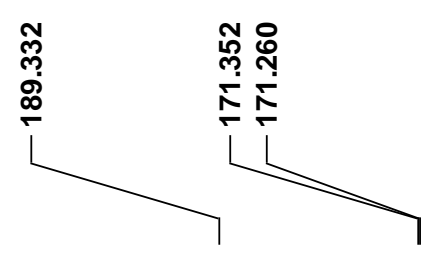

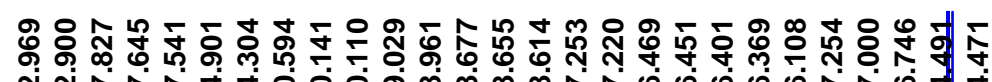

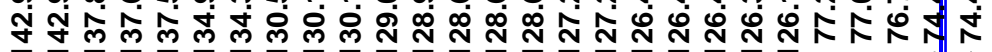

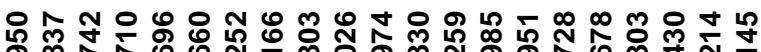

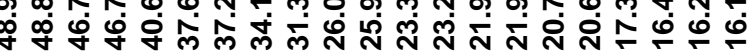

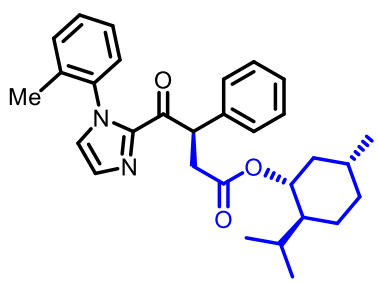

5 bg

${ }^{13} \mathrm{C}$ NMR $\left(125 \mathrm{MHz}, \mathrm{CDCl}_{3}\right)$

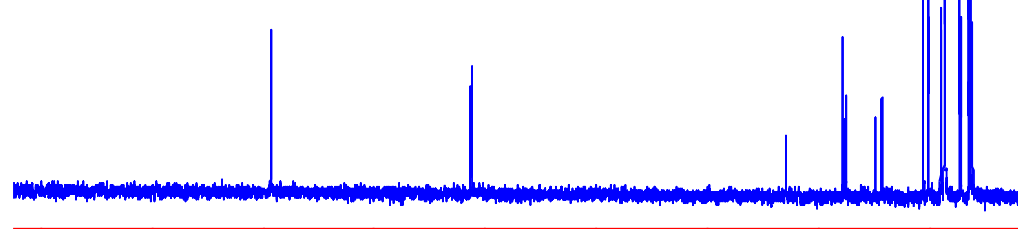




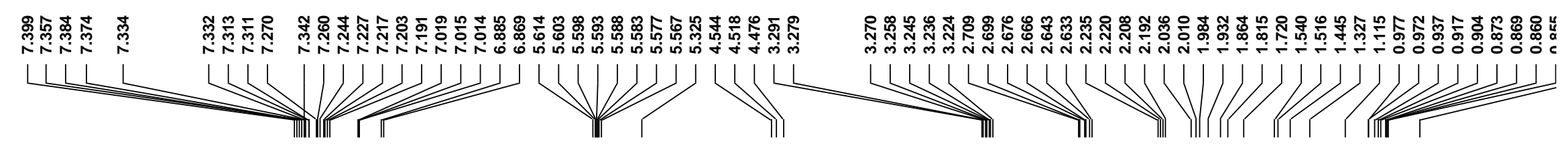

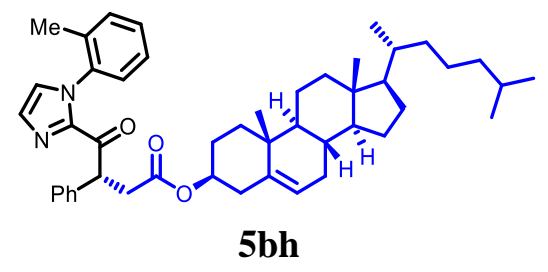

${ }^{1} \mathrm{H}$ NMR (500 MHz, $\mathrm{CDCl}_{3}$ )

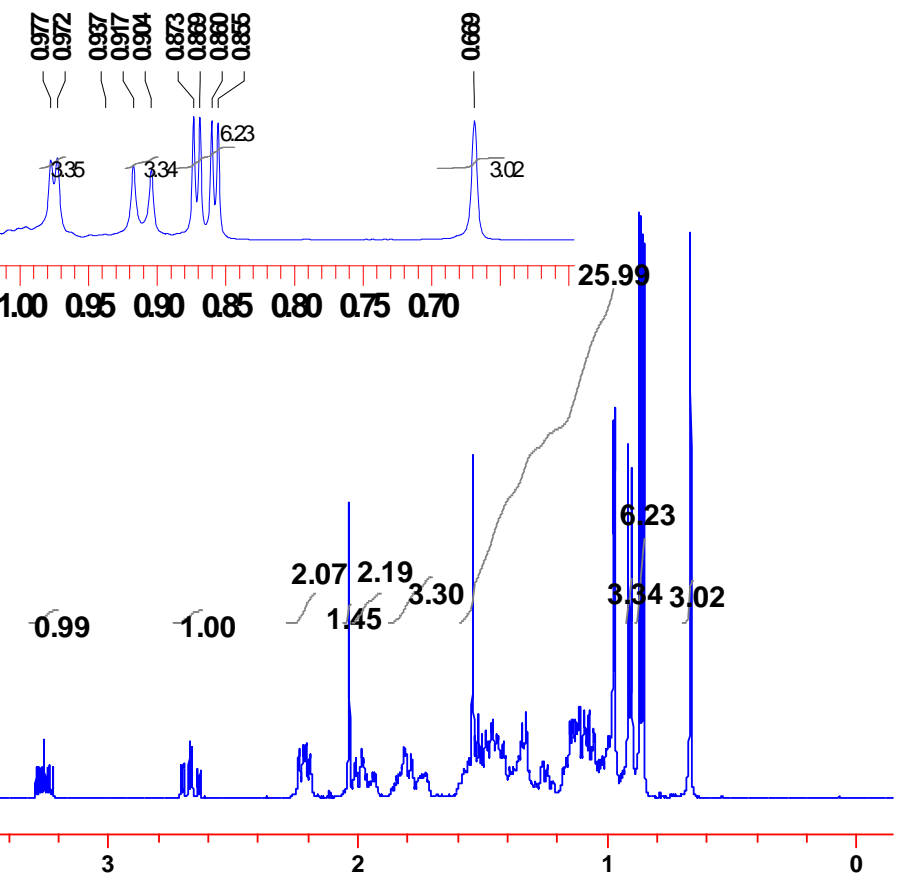




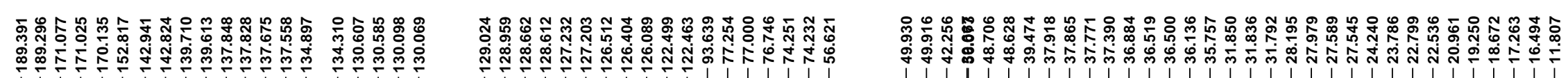
LLLLLLLLLLUL LUJJJJJJJ
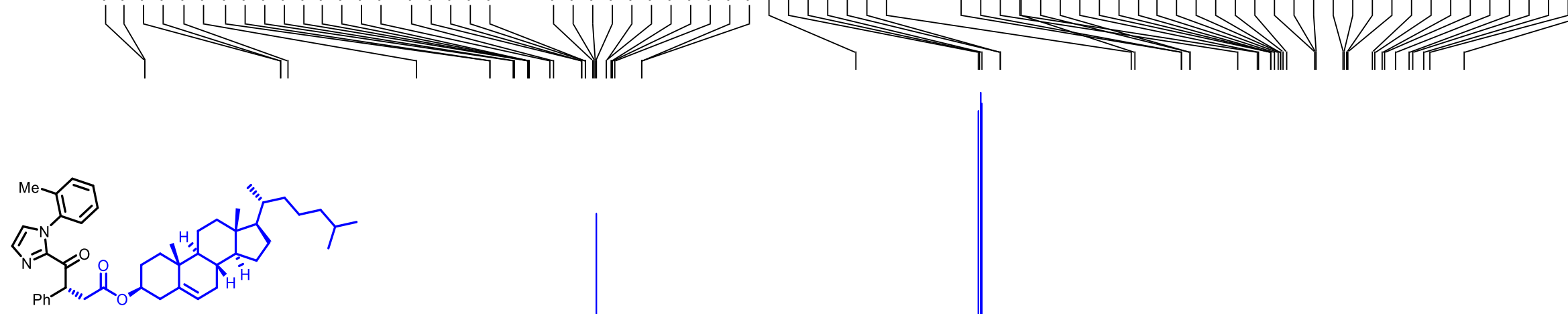

5bh

${ }^{13} \mathrm{C} \mathrm{NMR}\left(125 \mathrm{MHz}, \mathrm{CDCl}_{3}\right)$

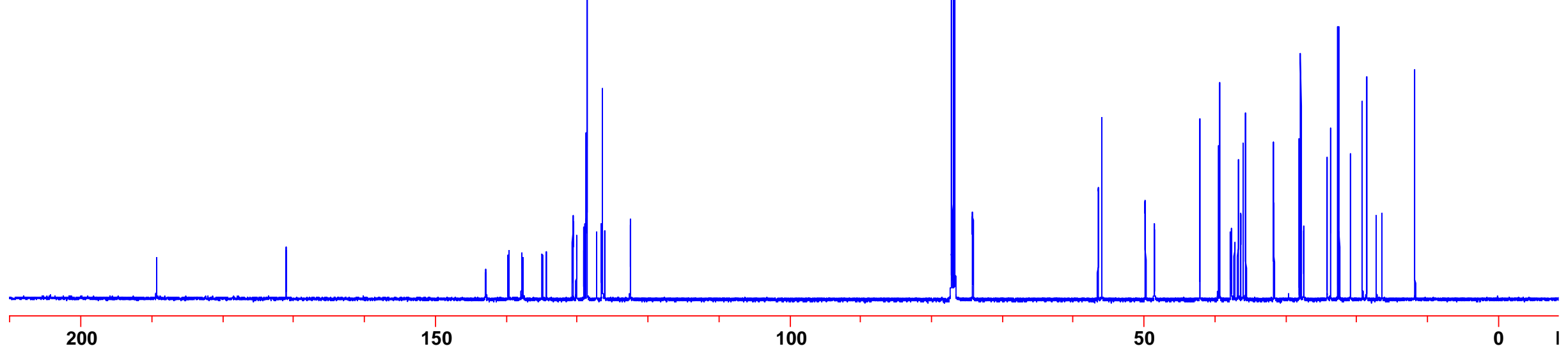




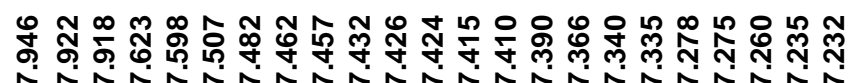

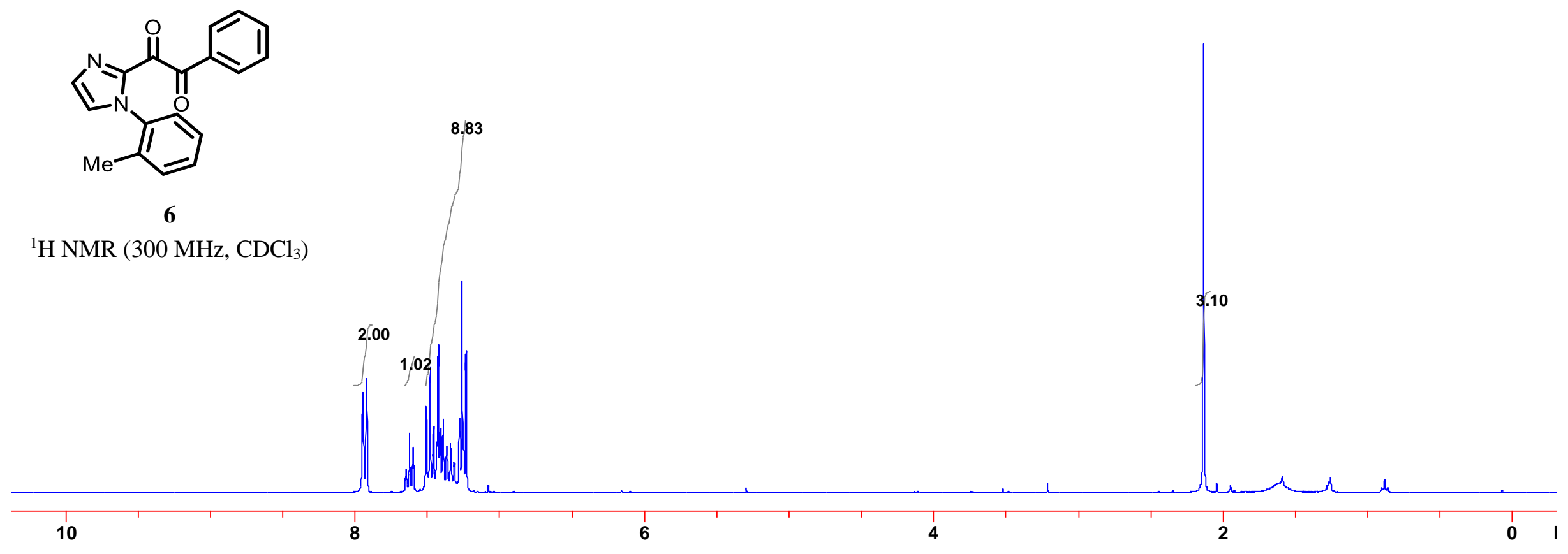




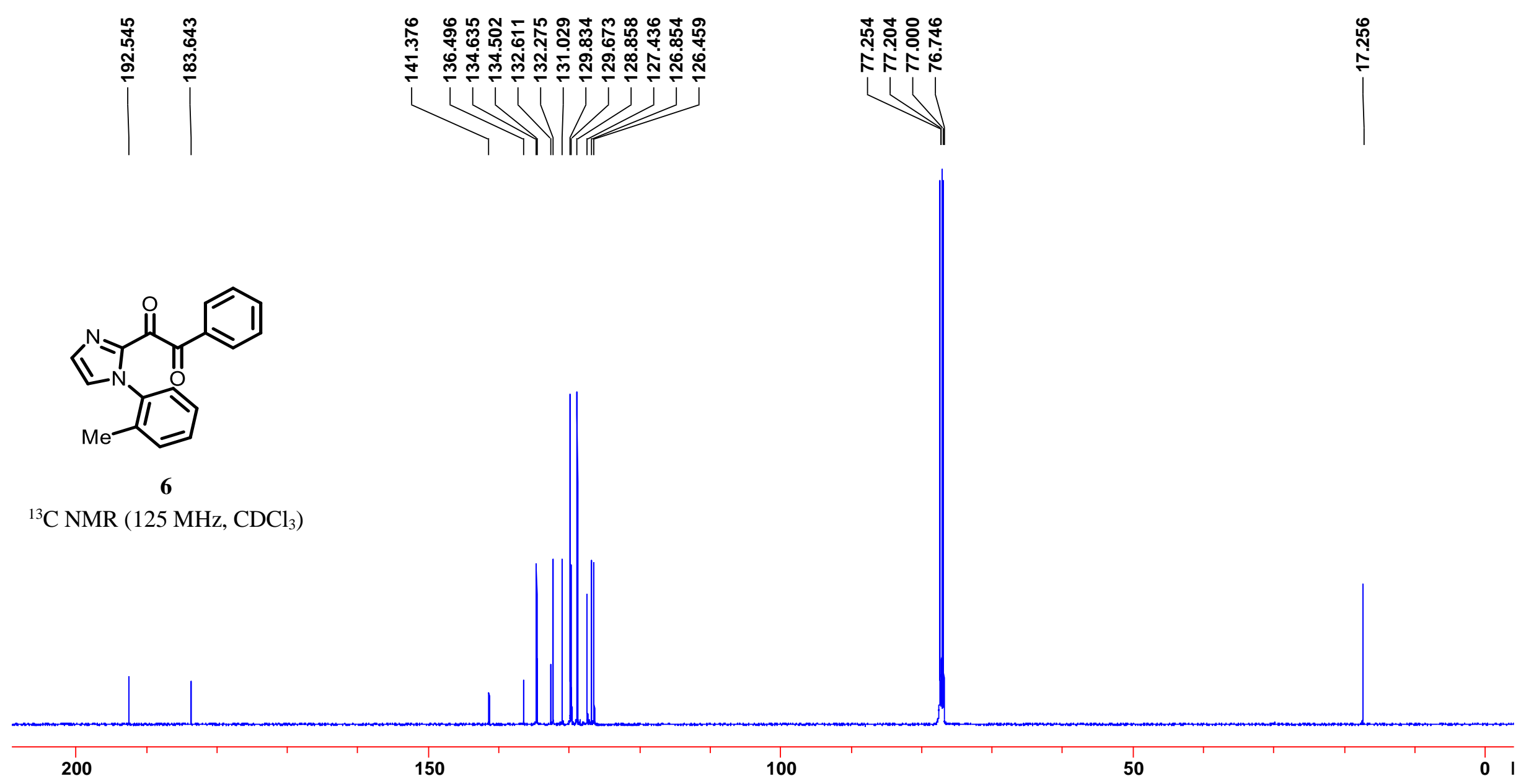




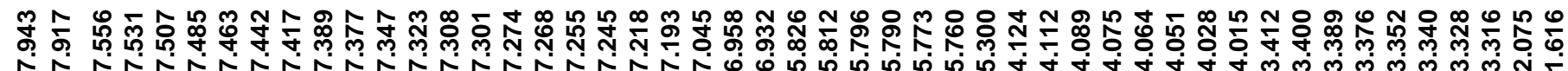

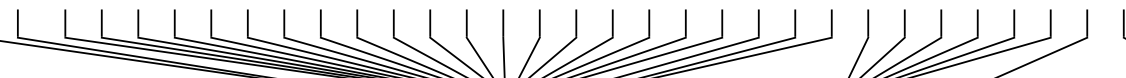
(n)

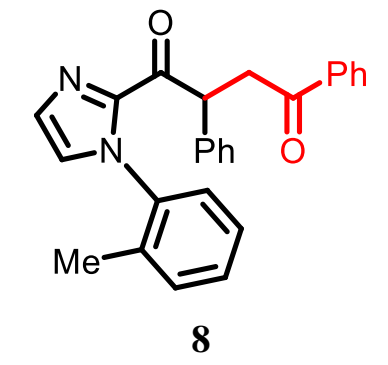

${ }^{1} \mathrm{H}$ NMR $\left(300 \mathrm{MHz}, \mathrm{CDCl}_{3}\right)$

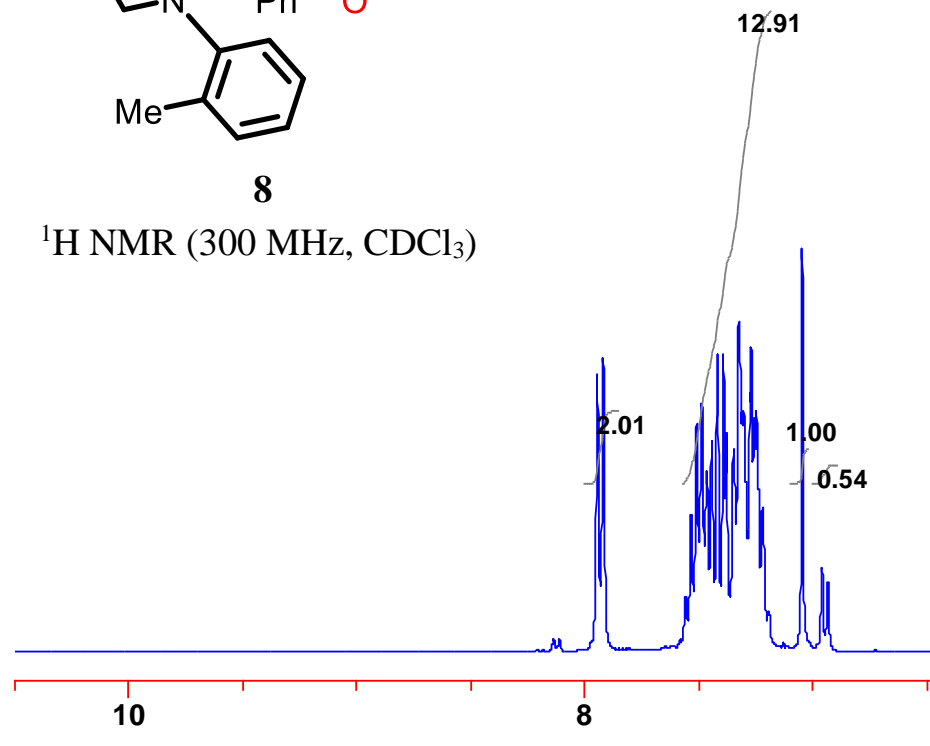

1291

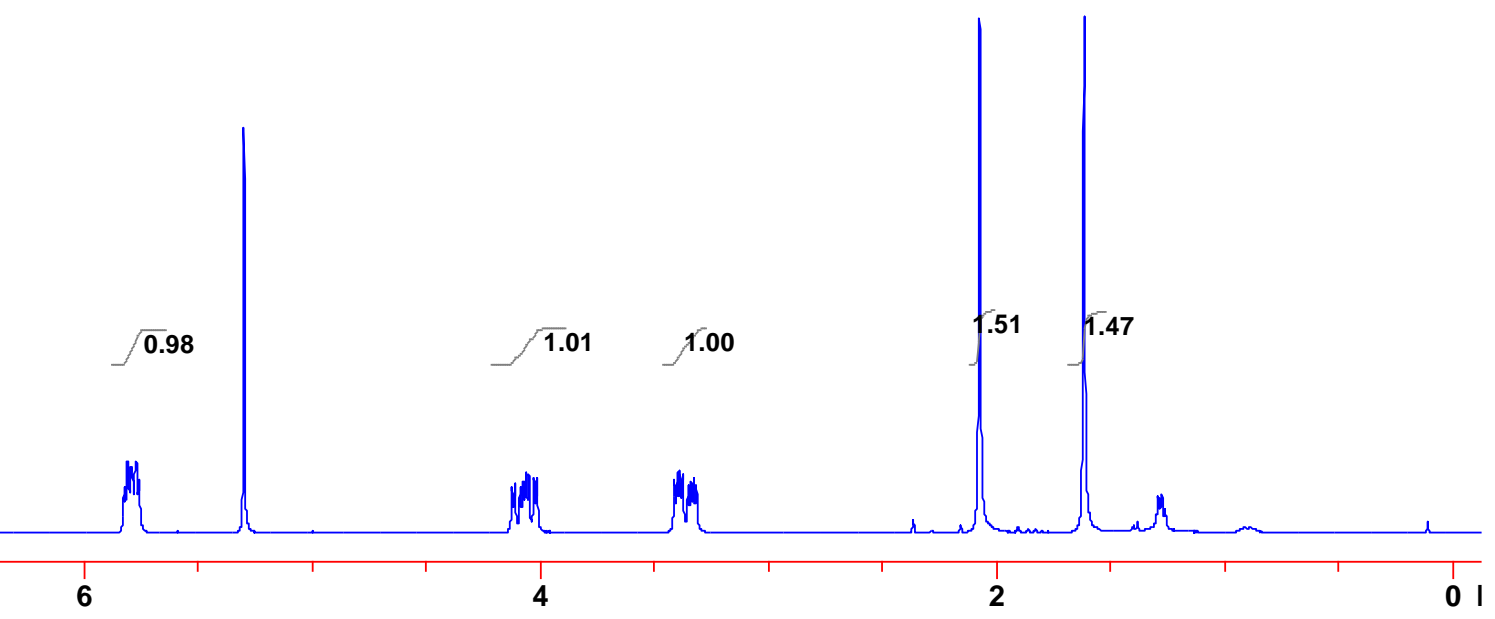



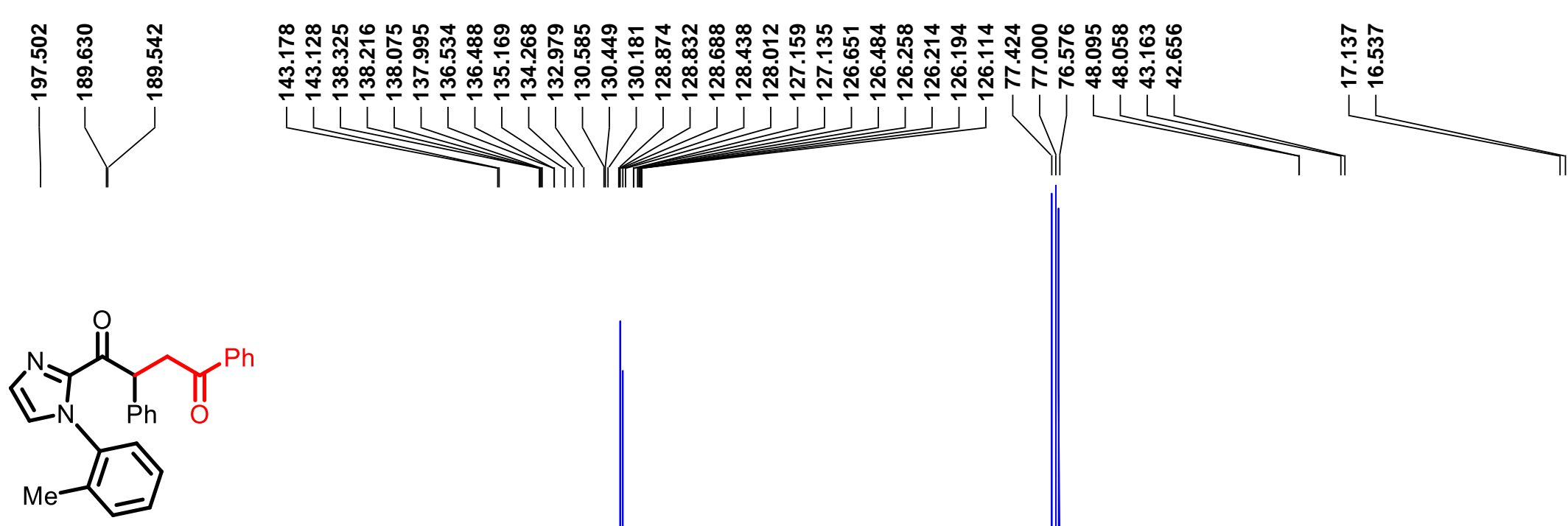

8

${ }^{13} \mathrm{C}$ NMR $\left(75 \mathrm{MHz}, \mathrm{CDCl}_{3}\right.$ )

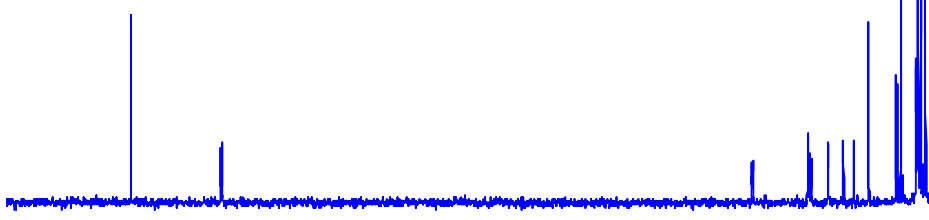

200 


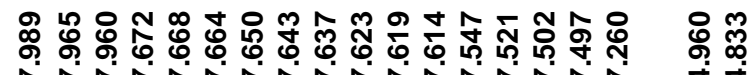

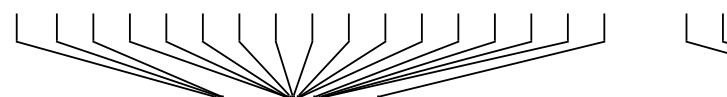

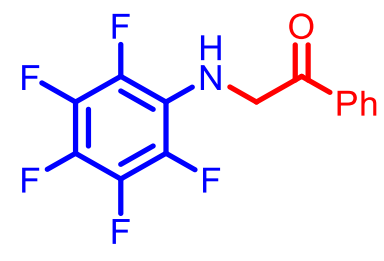

9

${ }^{1} \mathrm{H}$ NMR $\left(300 \mathrm{MHz}, \mathrm{CDCl}_{3}\right)$

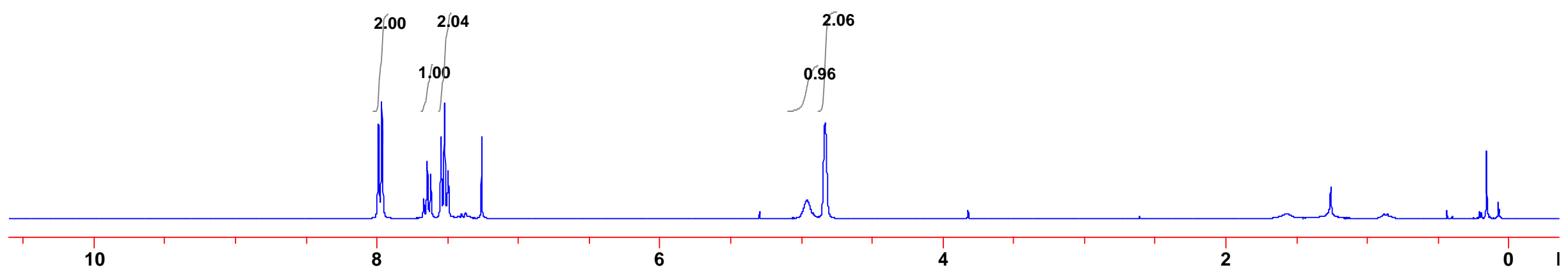



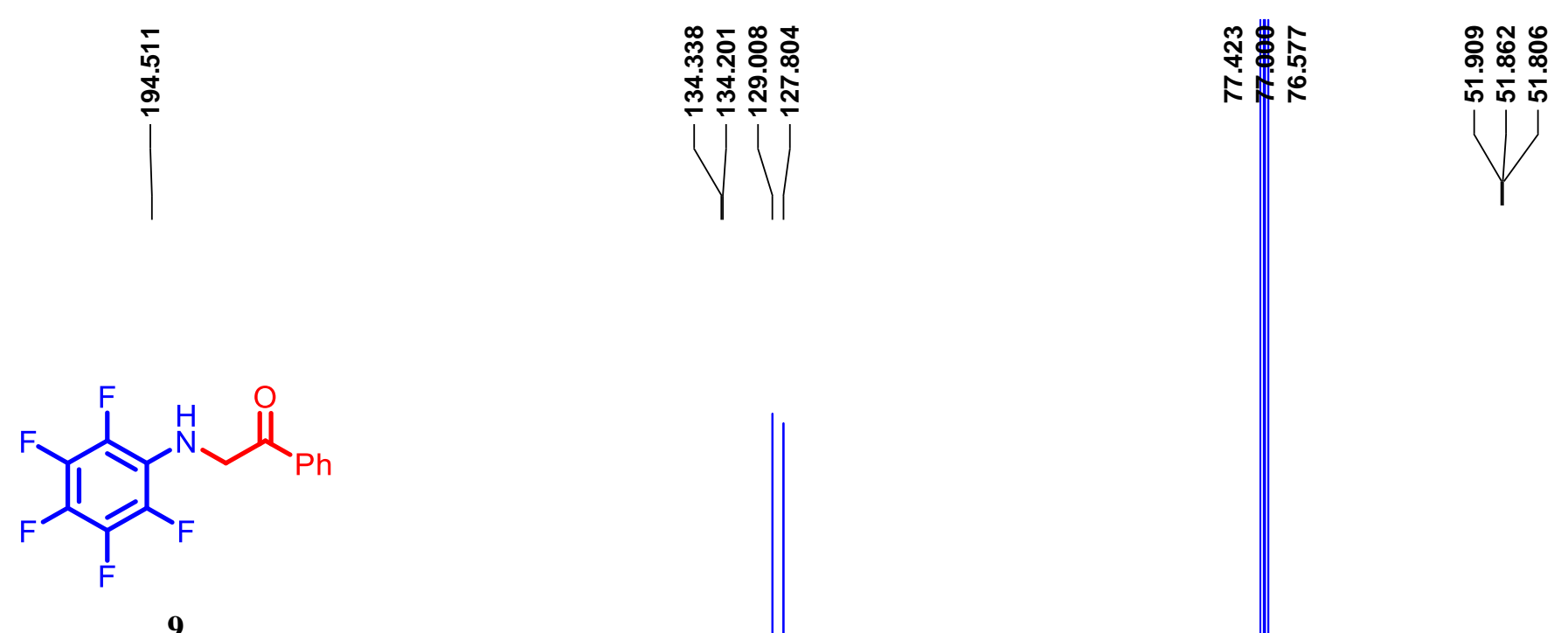

${ }^{13} \mathrm{C} \mathrm{NMR}\left(75 \mathrm{MHz}, \mathrm{CDCl}_{3}\right)$

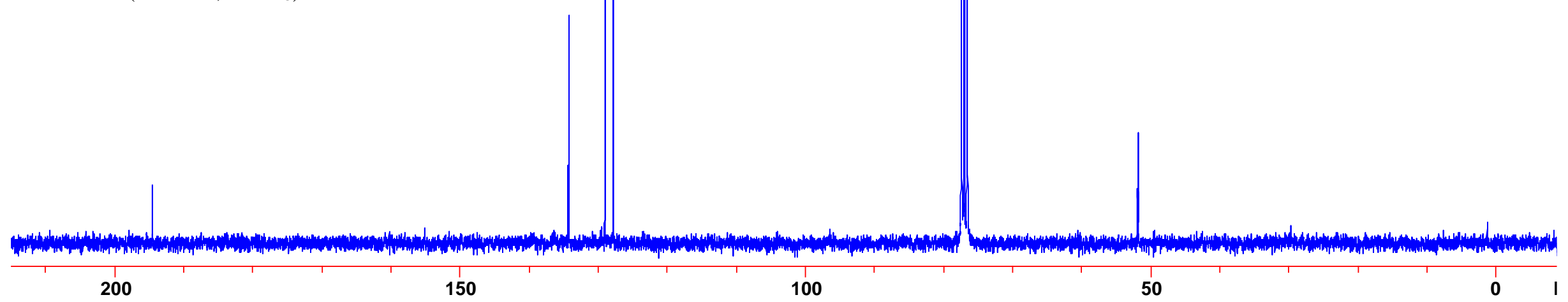



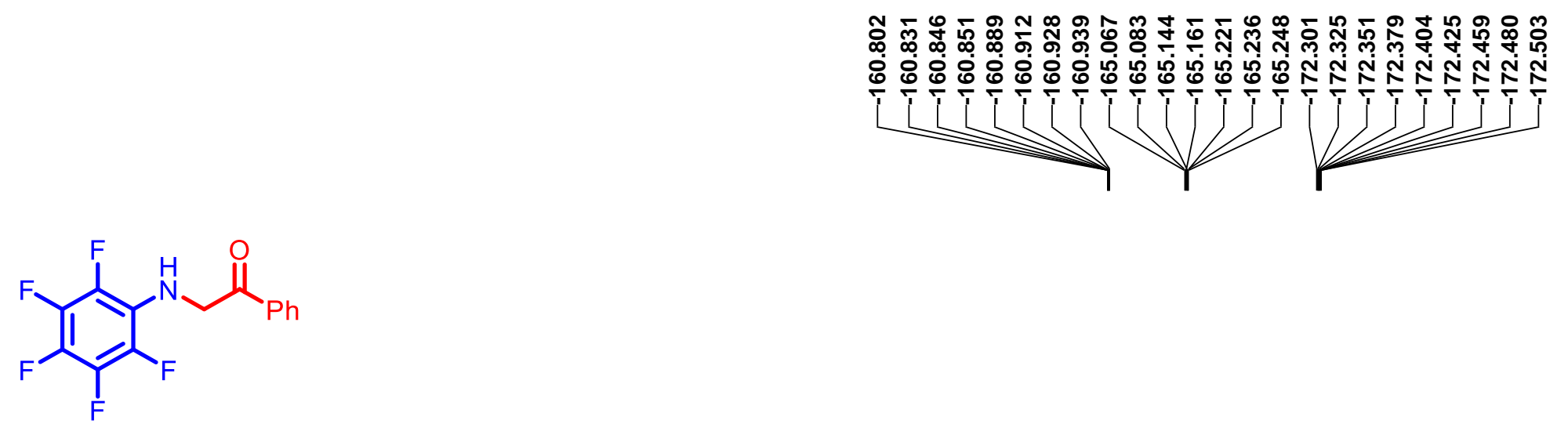

9

${ }^{19} \mathrm{~F}$ NMR $\left(282 \mathrm{MHz}, \mathrm{CDCl}_{3}\right)$

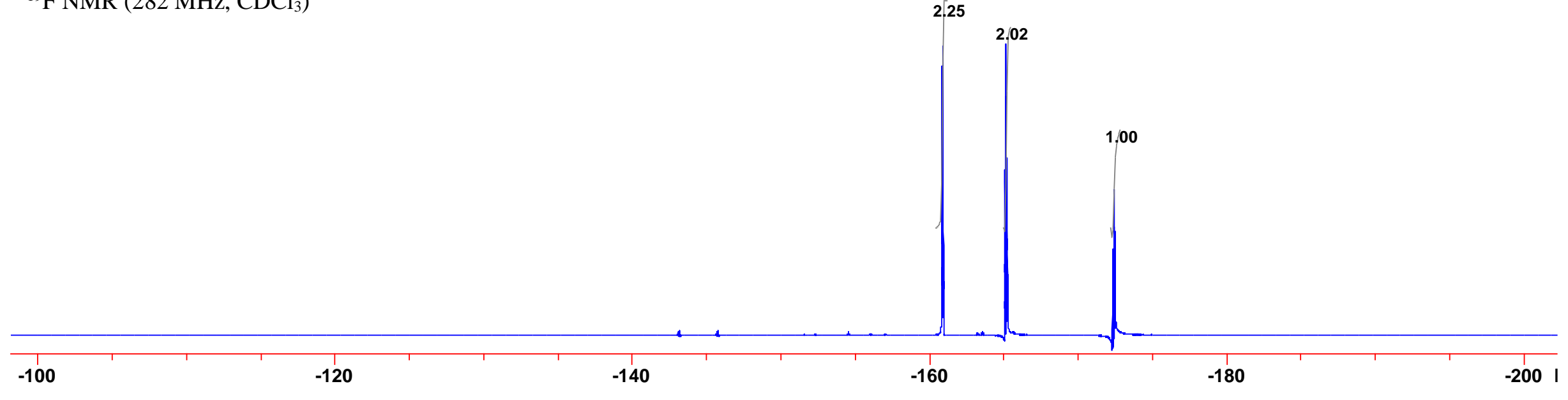




\section{References}

(1) Huo, H.; Shen, X.; Wang, C.; Zhang, L.; Rose, P.; Chen, L.-A.; Harms, K.; Marsch, M.; Hilt, G.; Meggers, E. Nature 2014, 515, 100.

(2) Wang, C.; Chen, L.-A.; Huo, H.; Shen, X.; Harms, K.; Gong, L.; Meggers, E. Chem. Sci. 2015, $6,1094$.

(3) Ma, J.; Shen, X.; Harms, K.; Meggers, E. Dalton Trans. 2016, 45, 8320.

(4) (a) Wang, C.; Zheng, Y.; Huo, H.; Röse, P.; Zhang, L.; Harms, K.; Hilt, G.; Meggers, E. Chem. Eur. J. 2015, 21, 7355. (b) Huo, H.; Wang, C.; Harms, K.; Meggers, E. J. Am. Chem. Soc. 2015, 137, 9551. (c) Shen, X.; Harms, K.; Marsch, M.; Meggers, E. Chem. Eur. J. 2016, 22, 9102.

(5) Jin, L.-M.; Xu, X.; Lu, H.; Cui, X.; Wojtas, L.; Zhang, X. P. Angew. Chem., Int. Ed. 2013, 52, 5309.

(6) Ryu, J.; Shin, K.; Park, S. H.; Kim, J. Y.; Chang, S. Angew. Chem., Int. Ed. 2012, 51, 9904.

(7) Chapyshev, S. V. Chem. Heterocycl. Compd. 2001, 37, 968.

(8) Zhao, W.; Li, H.; Zhang, J.; Cao, S. Chin. J. Chem. 2011, 29, 2763.

(9) (a) Toma, T.; Shimokawa, J.; Fukuyama, T. Org. Lett. 2007, 9, 3195-3197. (b) Kaupang, Å.; Bonge-Hansen, T. Beilstein J. Org. Chem. 2013, 9, 1407.

(10) Berkessel, A.; Kaiser, P.; Lex, J. Chem. Eur. J. 2003, 9, 4746.

(11) Bai, X.-F.; Xu, L.-W.; Zheng, L.-S.; Jiang, J.-X.; Lai, G.-Q.; Shang, J.-Y. Chem. Eur. J. 2012, 18,8174 .

(12) Sumino, S.; Fusano, A.; Ryu, I. Org. Lett. 2013, 15, 2826.

(13) Scholz, S. O.; Farney, E. P.; Kim, S.; Bates, D. M; Yoon, T. P. Angew. Chem., Int. Ed. 2016, 55, 2239.

(14) Liu, Q.; Yi, H.; Liu, J.; Yang, Y.; Zhang, X.; Zeng, Z.; Lei, A. Chem. Eur. J. 2013, 19, 5120.

(15) (a) Fan, J.-H.; Wei, W.-T.; Zhou, M.-B.; Song, R.-J.; Li, J.-H. Angew. Chem., Int. Ed. 2014, 53, 6650. (b) Wang, S.; Huang, X.; Li, B.; Ge, Z.; Wang, X.; Li, R. Tetrahedron 2015, 71, 1869.

(16) Julià, L.; Bosch, M. P.; Rodriguez, S.; Guerrero, A. J. Org. Chem. 2000, 65, 5098. 
(17) (a) Prier, C. K.; Rankic, D. A.; MacMillan, D. W. C. Chem. Rev. 2013, 113, 5322-5363. (b) Bonn,

A. G.; Yushchenko, O.; Vauthey, E.; Wenger, O. S. Inorg. Chem. 2016, 55, 2894.

(18) Cismesia, M. A.; Yoon, T. P. Chem. Sci. 2015, 6, 5426.

(19) Huo, H.; Harms, K.; Meggers, E. J. Am. Chem. Soc. 2016, 138, 6936.

(20) Chung, Y.-C.; Janmanchi, D.; Wu, H.-L. Org. Lett. 2012, 14, 2766.

(21) SADABS. Bruker AXS area detector scaling and absorption correction, Bruker AXS Inc., Madison, Wisconsin, USA, 2014.

(22) Sheldrick, G. M. Acta Cryst. A 2015, 71, 3.

(23) Sheldrick, G. M. Acta Cryst. C 2015, 71, 3.

(24) Parsons, S.; Flack, H.; Wagner, T. Acta Cryst. B 2013, 69, 249.

(25) APEX3, Bruker AXS Inc., Madison, Wisconsin, USA, 2015.

(26) SAINT, Bruker AXS Inc., Madison, Wisconsin, USA, 2015.

(27) Sheldrick, G. M. Acta Cryst. A 2015, 71, 3.

(28) Sheldrick, G. M. Acta Cryst. C 2015, 71, 3.

(29) Brandenburg, K. Diamond - Crystal and Molecular Structure Visualization, Crystal Impact Dr. Putz, H. \& Dr. Brandenburg K. GbR, Bonn, Germany, 2014.

(30) Hübschle, C. B.; Sheldrick, G. M.; Dittrich, B. J. Appl. Crystallog. 2011, 44, 1281.

(31) Parsons, S.; Flack, H. D.; Wagner, T. Acta Cryst. B 2013, 69, 249. 